JOINT TRANSPORTATION RESEARCH PROGRAM

FHWA/IN/JTRP-2010/10

Final Report

DEVELOPMENT OF INTERNALLY CURED

CONCRETE FOR INCREASED SERVICE LIFE

\author{
J. Schlitter \\ R. Henkensiefken \\ J. Castro \\ K. Raoufi \\ J. Weiss
}

October 2010 


\section{TECHNICAL Summary}

INDOT Research

Technology Transfer and Project Implementation Information

TRB Subject Code:

Publication No.: FHWA/IN/JTRP-2010/10, SPR-3211

October 2010

Final Report

\section{Development of Internally Cured Concrete for Increased Service Life}

\section{Introduction}

The main objective of this study was to develop internally cured concrete for use in the state of Indiana.

The scope of the research included characterization of locally available lightweight aggregate for use in internal curing. Experimental measurements were performed on these mixtures to assess autogenous shrinkage, drying shrinkage, plastic shrinkage cracking, drying shrinkage cracking, autogenous shrinkage cracking, water absorption, compressive strength, elastic modulus, tensile strength, thermal cracking and freeze-thaw resistance.

Highlights of the testing program included the use of new methods to measure autogenous shrinkage from the time of set (This has since become known as ASTM C-1698). In addition a new testing device was developed that allowed a mixtures susceptibility to be tested.

\section{Findings}

The following conclusions can be drawn based on the results from this study:

- It is important the lightweight aggregate (LWA): 1) be able to provide a sufficient volume of water (e.g., $24 \mathrm{hr}$ absorption), has a structure that allows the water to be released to the paste as needed (i.e., enables a large volume $90 \%+$ to be desorbed by $92 \%$ $\mathrm{RH})$, and is small enough so that they can be appropriately spaced in the matrix (i.e., fine aggregate is preferred).

- A reduction or elimination of autogenous shrinkage is observed for internally cured mixtures. This reduces or eliminates the potential for early age cracking.

\section{Implementation}

The results of this investigation indicate that internally cured concrete has great potential for use in transportation structures. This occurs specifically due to the reduced potential for shrinkage and thermal cracking, the reduced fluid transport, and the increased densification of the matrix.
- Plastic shrinkage cracking is reduced in internally cured concrete.

- Internally cured concrete has a higher degree of hydration resulting in reduced water absorption.

- Internally cured concrete mixtures are less susceptible to early age thermal cracking.

- Large scale testing showed a reduction in the cracking potential when internal curing was used. 
these materials to be used and specified more widely in Indiana. It is recommended that a training video be developed that highlights the benefits of this material, describes its use, and

\section{Contact}

For more information:

Prof. Jason Weiss

Principal Investigator

School of Civil Engineering

Purdue University

West Lafayette, IN 47907

Phone: (765) 494-2215

wjweiss@purdue.edu discusses important features associated with placement and testing

Indiana Department of Transportation

Division of Research

1205 Montgomery Street

P.O. Box 2279

West Lafayette, IN 47906

Phone: (765) 463-1521

Fax: (765) 497-1665

\section{Purdue University}

Joint Transportation Research Program

School of Civil Engineering

West Lafayette, IN 47907-1284

Phone: (765) 494-9310

Fax: (765) 496-1105 
Final Report

JTRP SPR-3211

\title{
Development of Internally Cured Concrete for Increased Service Life
}

\author{
John Schlitter, P.E., S.E. \\ Graduate Research Assistant \\ Ryan Henkensiefken \\ Graduate Research Assistant \\ Javier Castro \\ Graduate Research Assistant \\ Kambiz Raoufi \\ Graduate Research Assistant \\ Dr. Jason Weiss \\ Professor of Civil Engineering \\ School of Civil Engineering \\ Purdue University \\ West Lafayette, IN 47907
}

And

Tommy Nantung, Ph.D., P.E.

Section Manager

Indiana Department of Transportation

Joint Transportation Research Program

Project No. C-36-37PP

File No. $05-08-42$

SPR-3211

The contents of this paper reflect the views of the authors, who are responsible for the facts and the accuracy of the data presented herin, and do not necessarily reflect the official views or policies of the Indiana Department of Transportation, nor do the contents constitute a standard, specification, or regulation.

October 2010 


\begin{tabular}{|c|c|c|}
\hline $\begin{array}{l}\text { 1. Report No. } \\
\text { FHWA/IN/JTRP-2010/10 }\end{array}$ & 2. Government Accession No. & 3. Recipient's Catalog No. \\
\hline \multirow{2}{*}{\multicolumn{2}{|c|}{$\begin{array}{l}\text { 4. Title and Subtitle } \\
\text { Development of Internally Cured Concrete for Increased Service Life }\end{array}$}} & $\begin{array}{l}\text { 5. Report Date } \\
\text { October } 2010\end{array}$ \\
\hline & & 6. Performing Organization Code \\
\hline \multicolumn{2}{|c|}{$\begin{array}{l}\text { 7. Author(s) } \\
\text { Schlitter, J., Henkensiefken, R., Castro, J., Raoufi, K., and Weiss, J }\end{array}$} & $\begin{array}{l}\text { 8. Performing Organization Report No. } \\
\text { FHWA/IN/JTRP-2010/10 }\end{array}$ \\
\hline \multirow{2}{*}{\multicolumn{2}{|c|}{$\begin{array}{l}\text { 9. Performing Organization Name and Address } \\
\text { Joint Transportation Research Program } \\
\text { Purdue University } \\
550 \text { Stadium Mall Drive } \\
\text { West Lafayette, IN 47907-2051 }\end{array}$}} & 10. Work Unit No. \\
\hline & & $\begin{array}{l}\text { 11. Contract or Grant No. } \\
\text { SPR-3211 }\end{array}$ \\
\hline \multirow{2}{*}{\multicolumn{2}{|c|}{$\begin{array}{l}\text { 12. Sponsoring Agency Name and Address } \\
\text { Indiana Department of Transportation } \\
\text { State Office Building } \\
100 \text { North Senate Avenue } \\
\text { Indianapolis, IN } 46204\end{array}$}} & $\begin{array}{l}\text { 13. Type of Report and Period Covered } \\
\text { Final Report }\end{array}$ \\
\hline & & 14. Sponsoring Agency Code \\
\hline
\end{tabular}

\section{Abstract}

Higher strength, lower water to cement ratio (w/c) concrete has been advocated over the last two decades due to its increased strength and reduced permeability. The lower w/c of these concretes makes them susceptible to autogenous shrinkage. This autogenous shrinkage can be significant and can be a contributing factor to early age cracking. Internal curing was investigated as a potential method to improve the durability of concrete pavements and bridge decks. Prewetted lightweight aggregate was used to supply water to the hydrating cement paste. This additional water can counteract the hindered strength development, suspended hydration, autogenous shrinkage, and early age cracking.

An overview of the concepts behind internal curing was presented. It is important the internal curing agent (lightweight aggregate (LWA) in this case): be able to provide a sufficient volume of water, has a structure that allows the water to be released to the paste as needed, and is small enough so that they can be appropriately spaced in the matrix. Local materials were used. Before concrete could be prepared the locally produced LWA was characterized to determine absorption and desorption properties. Concrete mixtures were prepared for concrete with and without internal curing. A constant aggregate volume was maintained. Tests performed on these mixtures were designed to measure: autogenous shrinkage, drying shrinkage, plastic shrinkage cracking, drying shrinkage cracking, autogenous shrinkage cracking, water absorption, compressive strength, elastic modulus, tensile strength, thermal cracking and freeze-thaw resistance. Internally cured mixtures showed less autogenous shrinkage. In addition they were less likely to crack due to plastic, autogenous, and drying effects. Internal curing reduced the water absorption and potential for freeze-thaw damage. Further, internal curing allowed a greater temperature swing in the concrete before cracking would occur.

Internally cured concrete mixtures could enable INDOT to produce more durable concrete pavements and structures that are less susceptible to cracking and have improved transport properties thereby providing great potential for more sustainable, cost-effective construction.

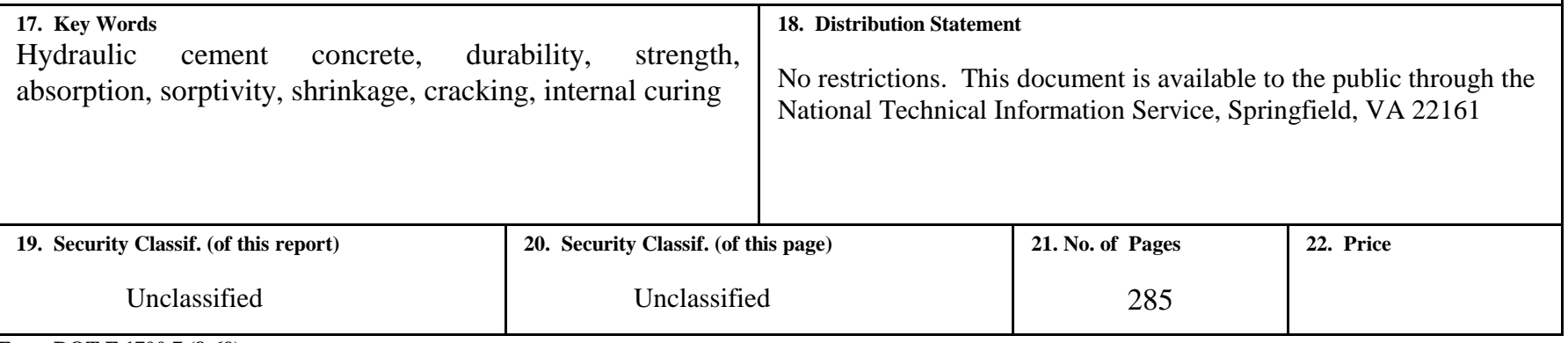




\section{ACKNOWLEDGEMENTS}

The authors gratefully acknowledge the guidance provided by Dr. Dale P. Bentz from the Building and Fire Research Laboratory, National Institute of Standards and Technology.

This work was conducted in the Charles Pankow Concrete Materials Laboratory at Purdue University. As such, the authors gratefully acknowledge the support which has made this laboratory and its operation possible. 
TABLE OF CONTENTS

Page

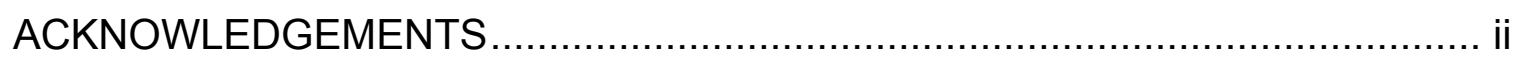

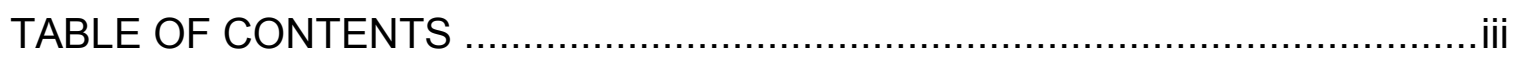

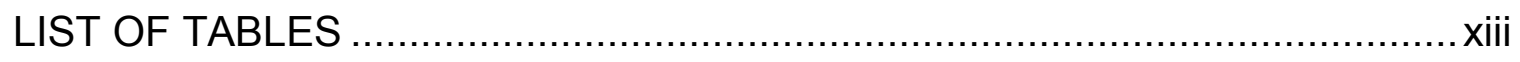

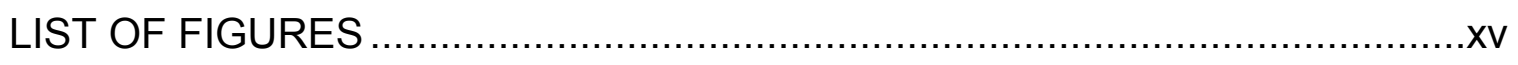

LIST OF ABBREVIATIONS AND VARIABLES ............................................xxiv

CHAPTER 1. INTRODUCTION, OBJECTIVES, AND APPROACH ................... 1

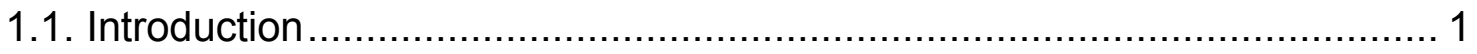

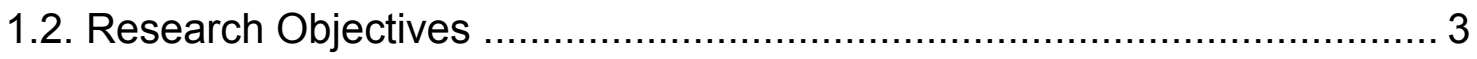

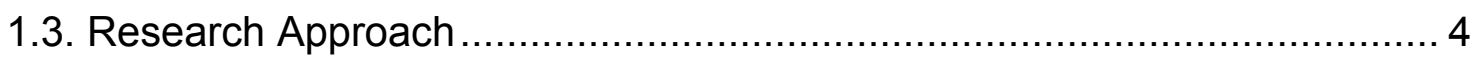

1.3.1. Task 1: Literature Review ........................................................... 4

1.3.2. Task 2: Characterization of Local Materials ...................................... 5

1.3.3. Task 3: Evaluating Self-Curing Concrete Made with Local Materials .. 5

1.3.4. Tasks 4 and 5: Full Scale Trial Batch and Testing............................. 8

CHAPTER 2. CHARACTERIZATION OF LOCAL MATERIALS ......................... 9

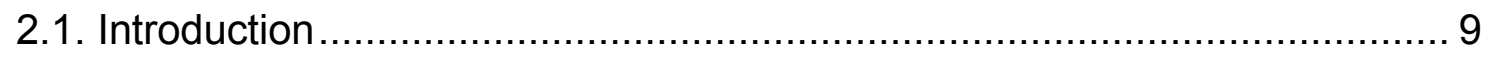

2.2. Techniques for Determining the Absorption of LWA ............................... 10

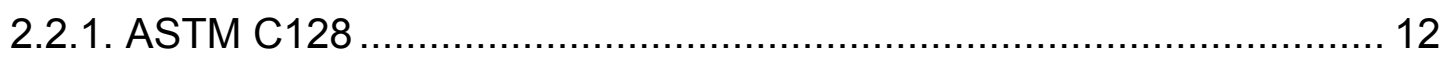

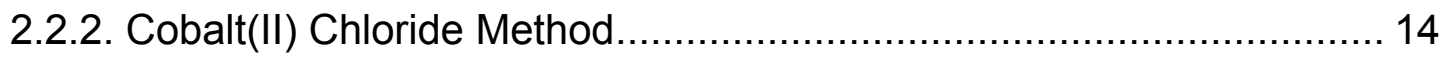

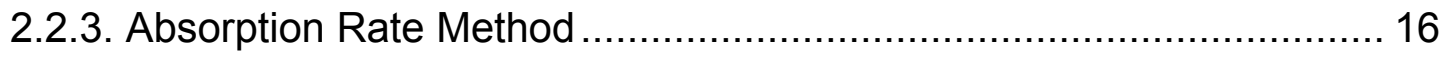

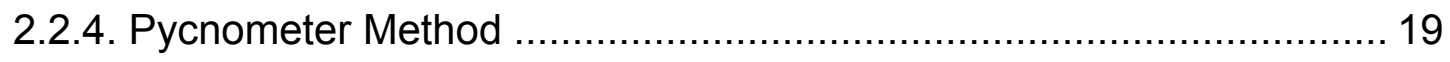


2.2.5. Comments and Recommendations ........................................... 20

2.3. Techniques for Determining the Pore Size Distribution ......................... 20

2.3.1. Mercury Intrusion Porosimetry ................................................. 21

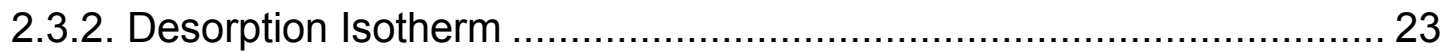

2.4. Implications of These Properties on Shrinkage .................................... 24

2.4.1. Materials and Testing Procedures ............................................... 24

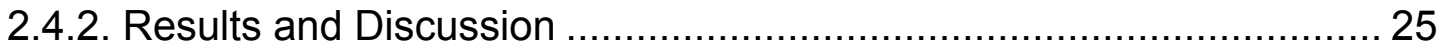

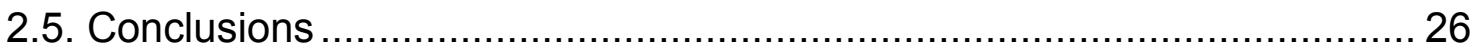

CHAPTER 3. MECHANISMS OF INTERNAL CURING …............................... 28

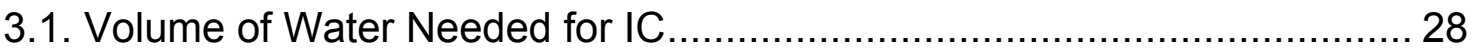

3.2. Ability of Water to Leave the LWA …….............................................. 31

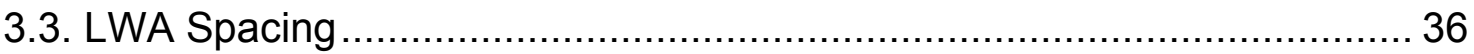

3.4. The Drying Mechanisms in Sealed and Unsealed Systems ................... 39

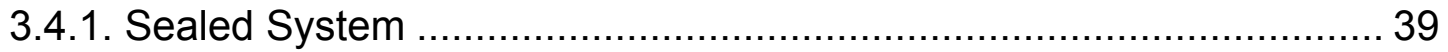

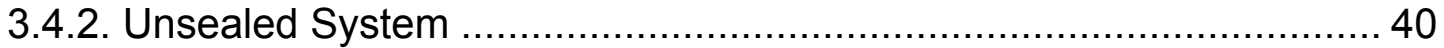

CHAPTER 4. MONITORING WATER MOVEMENT FROM PREWETTED LIGHTWEIGHT AGGREGATE USING X-RAY ABSORPTION ....................... 42

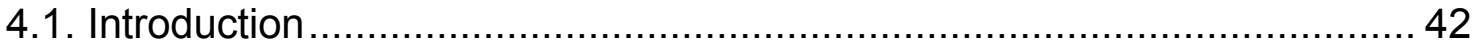

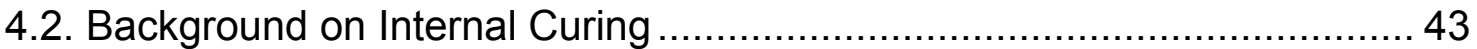

4.3. Background on the Detection of Water Movement Using X-ray Absorption45

4.4. Previous Studies Using X-ray Absorption .......................................... 46

4.5. Improvement to Measuring Techniques ……..................................... 47

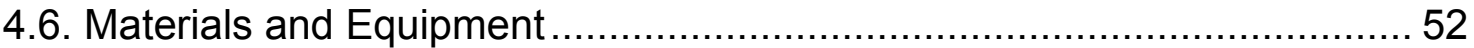

4.6.1. Materials and Mixture Proportions ................................................ 52 
4.6.2. Description of the X-ray Absorption Equipment …........................... 52

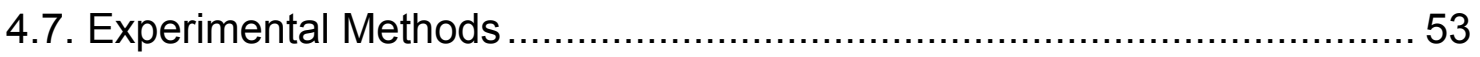

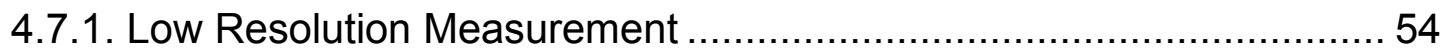

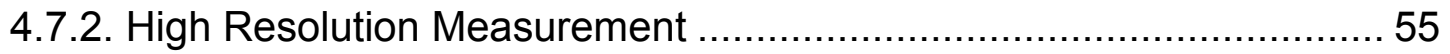

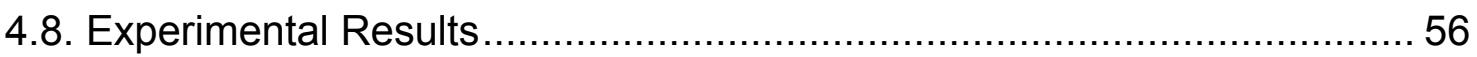

4.8.1. Low Resolution Measurement ……………….............................. 56

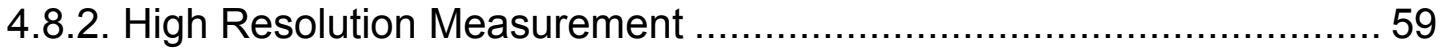

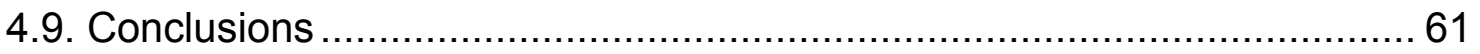

CHAPTER 5. DISCUSSION ON THE SHRINKAGE OF THE PASTE IN MORTAR CONTAINING PREWETTED LIGHTWEIGHT AGGREGATE............63

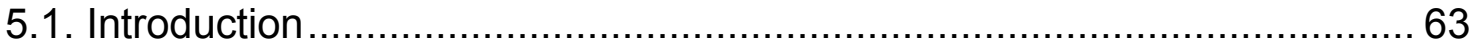

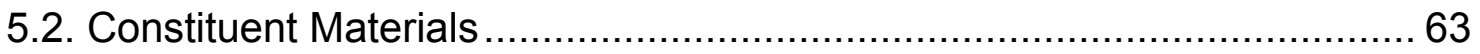

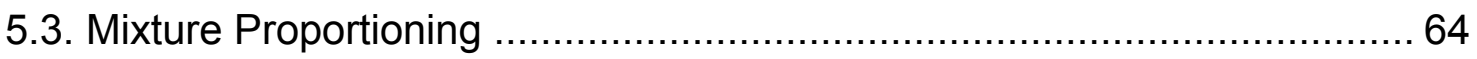

5.4. Mixing Procedure

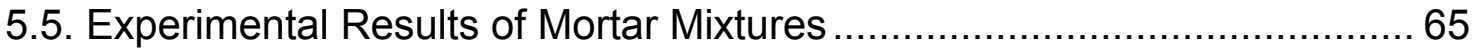

5.6. Modeling Concrete Shrinkage from Pickett's Equation .......................... 67

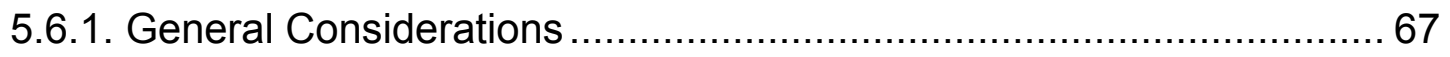

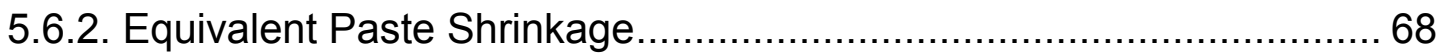

5.7. Describing Shrinkage Using Modified Mackenzie's Equation .................. 69

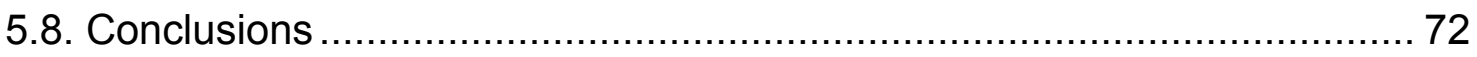

CHAPTER 6. MEASURING VOLUME CHANGE AND CRACKING UNDER SEALED AND DRYING CONDITIONS ……….................................. 74

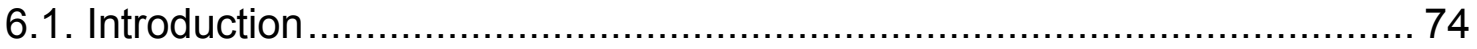

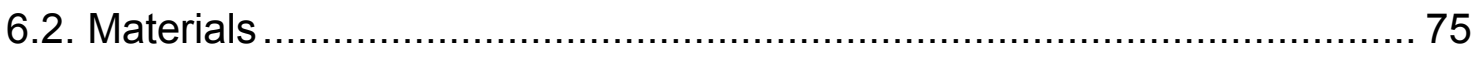

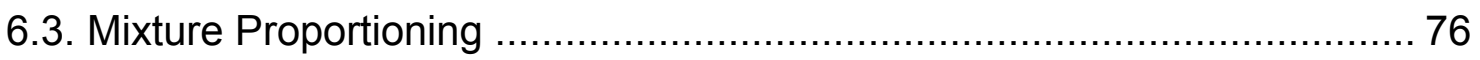




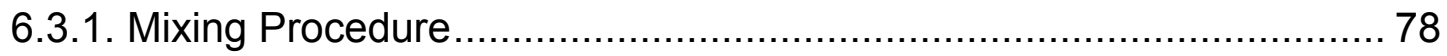

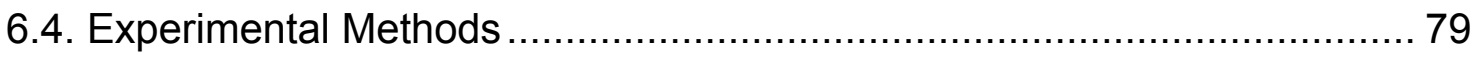

6.4.1. Unrestrained Volumetric Change during the First $24 \mathrm{~h} . . \ldots \ldots \ldots \ldots \ldots \ldots . . . . . . . . . .79$

6.4.2. Unrestrained Volumetric Change \& Mass Loss after $24 \mathrm{~h}$.................. 79

6.4.3. Measurements of Restrained Shrinkage ........................................ 80

6.4.4. Internal Relative Humidity of Sealed Specimens ............................... 80

6.5. Experimental Results and Discussion of Internal Curing Mixtures............ 81

6.5.1. Different LWA Volume Replacements on Free Shrinkage .................. 81

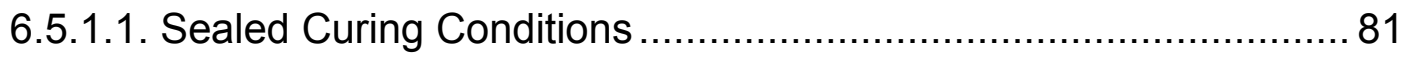

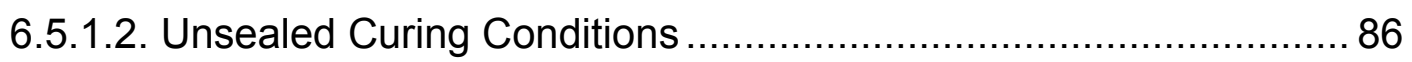

6.5.2. Different LWA Volume Replacements on Restrained Shrinkage ........ 89

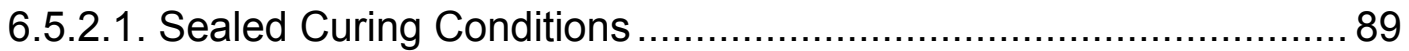

6.5.2.2. Unsealed Curing Conditions .................................................... 91

6.5.3. Different Volume Replacements on Internal Relative Humidity .......... 93

6.5.4. Effect of Particle Spacing on Internal Curing Effectiveness ............... 95

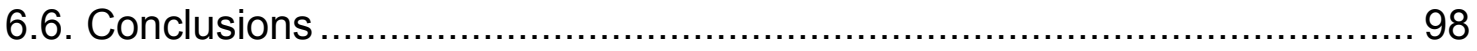

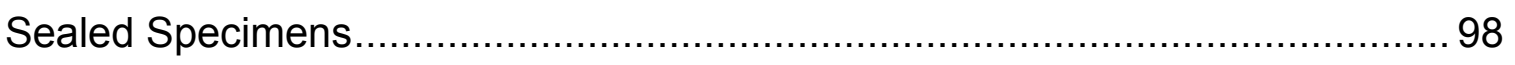

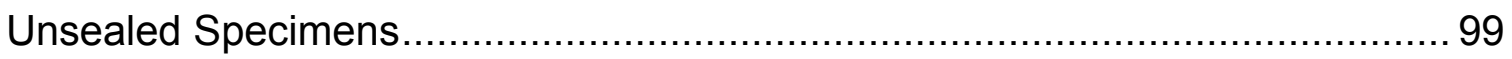

CHAPTER 7. PLASTIC SHRINKAGE CRACKING IN MIXTURES MADE WITH PREWETTED LIGHTWEIGHT AGGREGATE.......................................... 100

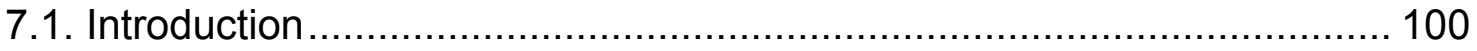

7.2. Mechanisms of Plastic Shrinkage ................................................... 100

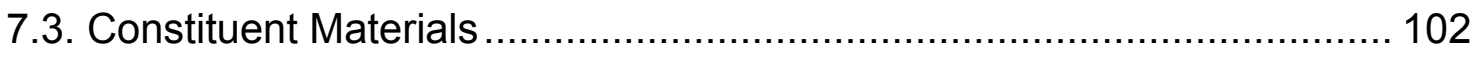

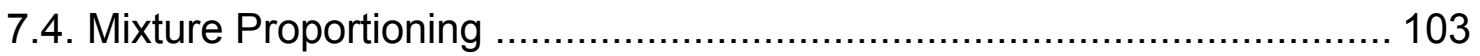

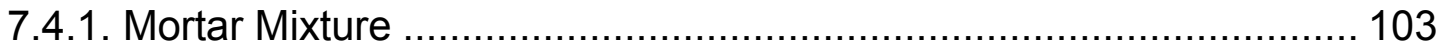




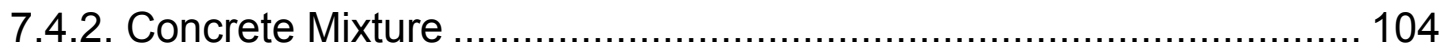

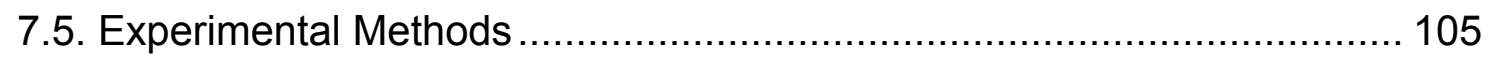

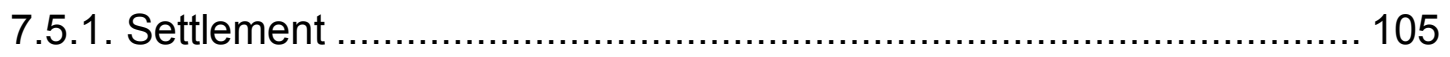

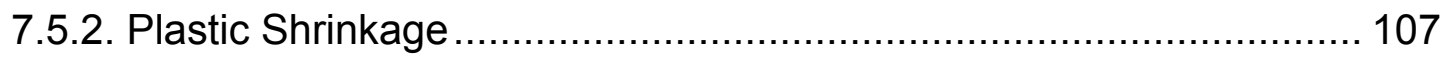

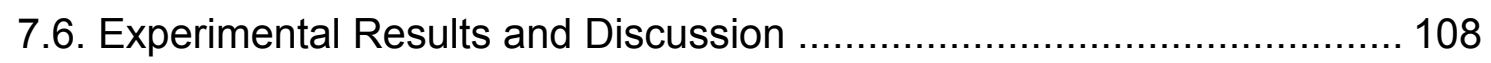

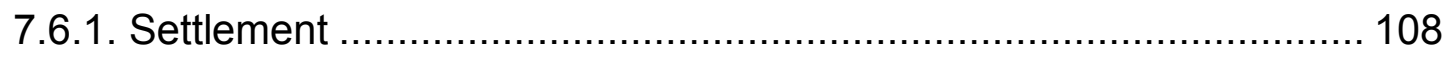

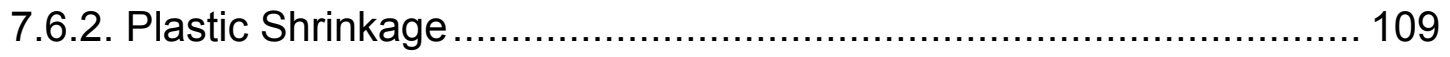

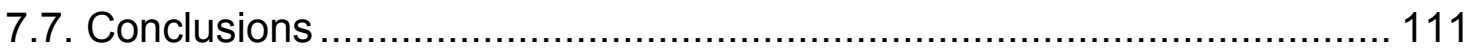

CHAPTER 8. MONITORING WATER ABSORPTION IN CEMENTITIOUS SYSTEMS MADE WITH PREWETTED LIGHTWEIGHT AGGREGATE ..........113

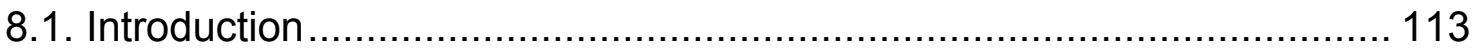

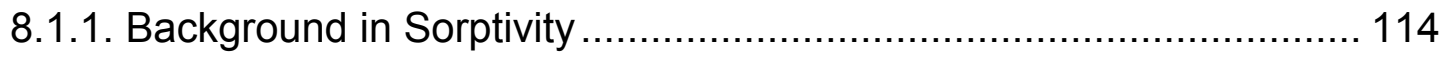

8.1.2. Background on the Effects of Internal Curing on Sorptivity.............. 115

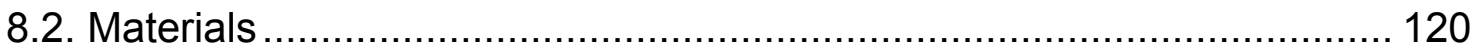

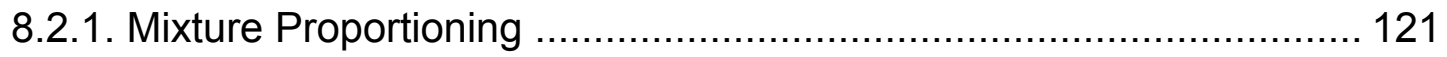

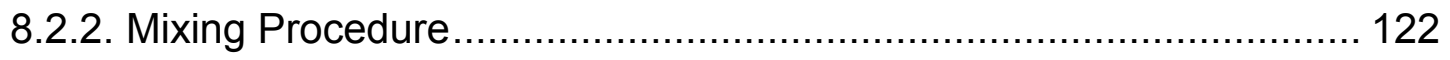

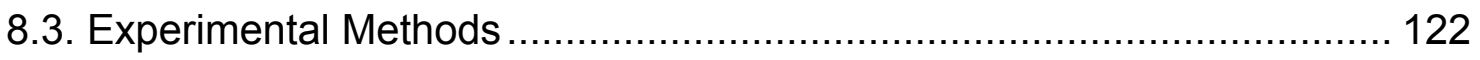

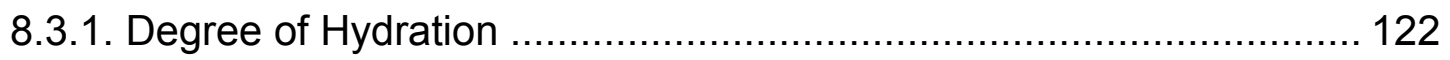

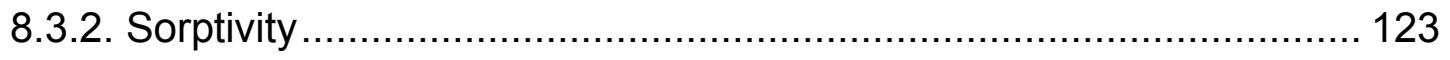

8.3.3. Electrical Impedance Spectroscopy............................................. 123

8.4. Experimental Results and Discussion ............................................ 124

8.4.1. Effects of Internal Curing on the Degree of Hydration ..................... 124

8.4.2. Effects of Internal Curing on Initial and Secondary Sorptivity .......... 125 
8.4.3. Effects of Internal Curing on the Amount of Absorbed Water During the

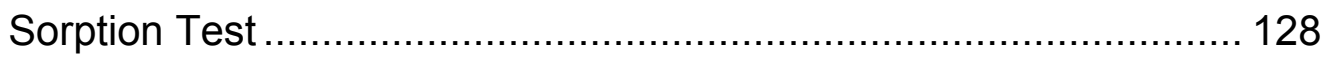

8.4.4. Effects of Available Space on Both Sorptivity and Total Amount of

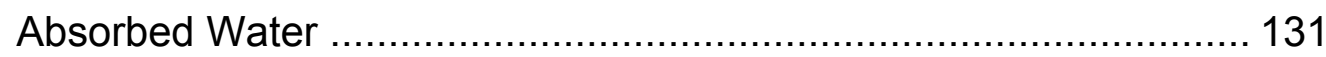

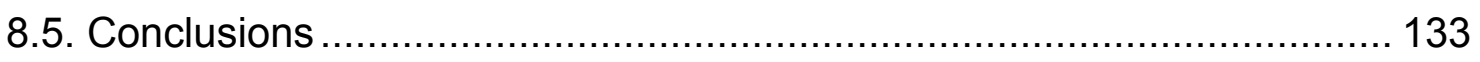

CHAPTER 9. Mechanical Properties of IC Systems Made with Prewetted

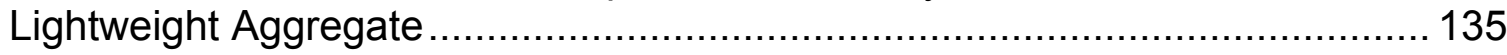

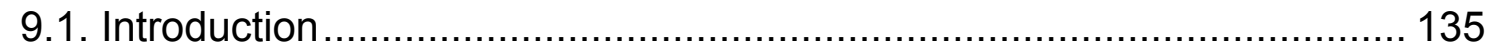

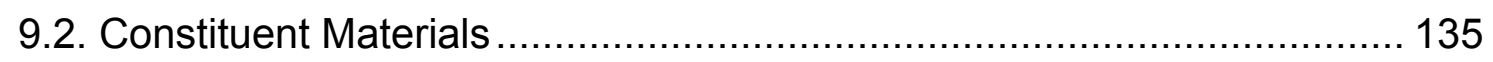

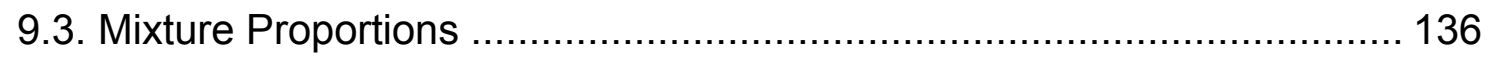

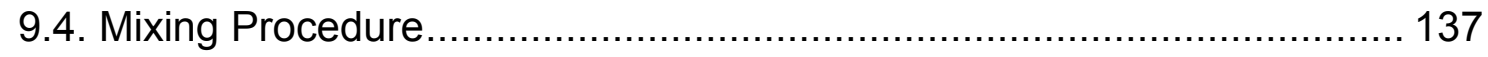

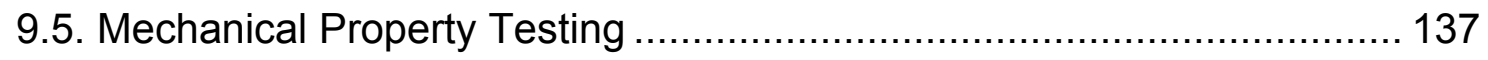

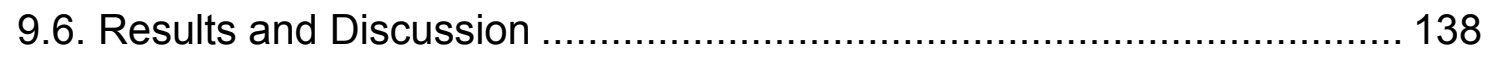

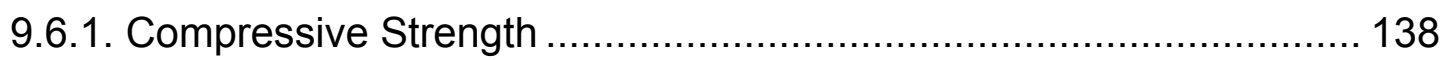

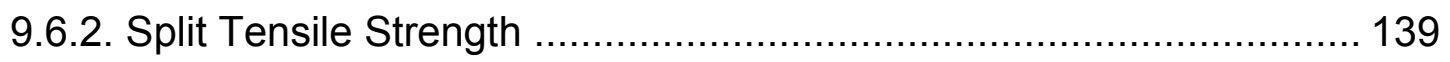

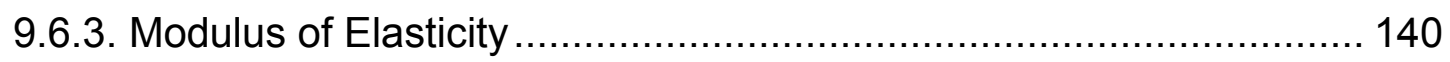

9.7. Mechanical Properties Conclusions ..................................................... 141

CHAPTER 10. The Effect of Temperature Change on IC Systems Made with

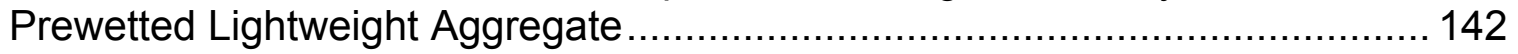

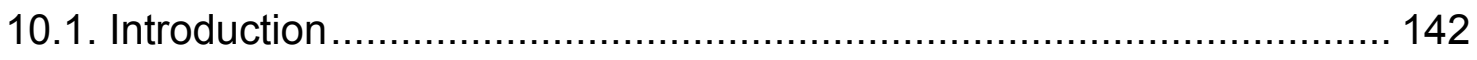

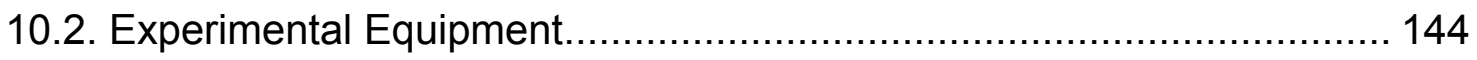

10.2.1. Dual Restraining Rings Geometry and Construction ..................... 144

10.2.2. Dual Ring Instrumentation and Data Acquisition ........................... 147

10.2.3. Strain Gauge Temperature Calibration ....................................... 148

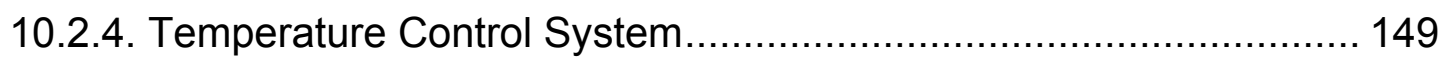




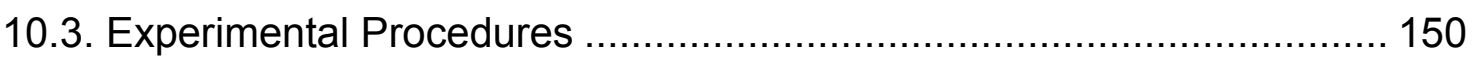

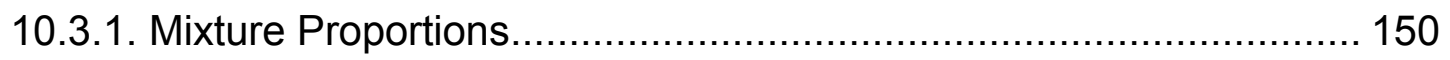

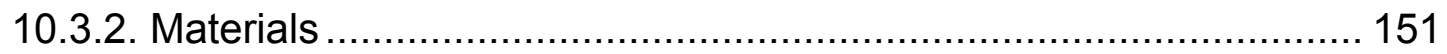

10.3.3. Dual Ring Specimen Mixing, Casting, and Testing Procedure ....... 152

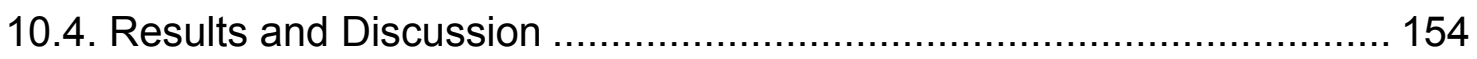

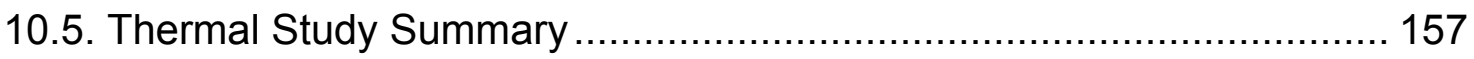

CHAPTER 11. Evaluating internally cured mixtures with a temperature drop

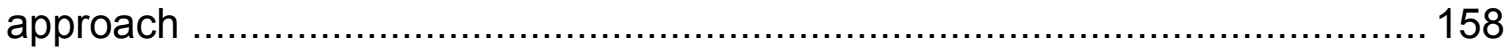

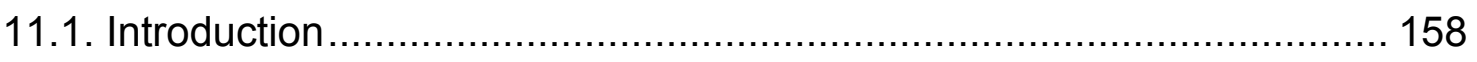

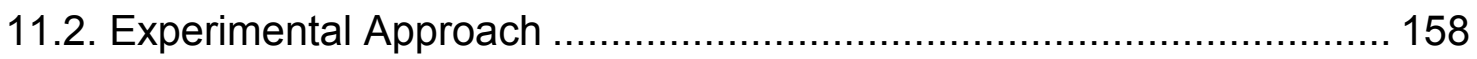

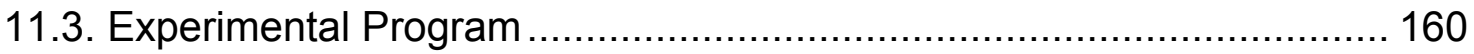

11.3.1. Mixture Proportions................................................................. 160

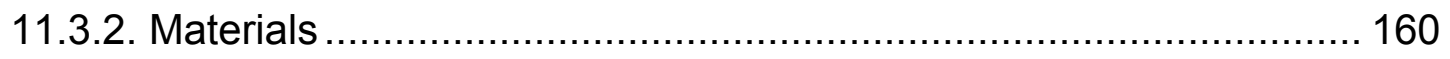

11.3.3. Dual Ring Testing Protocol ....................................................... 161

11.3.4. Split Tensile, Compressive Strength, and Elastic Modulus Tests ... 162

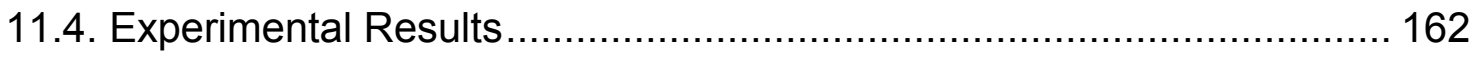

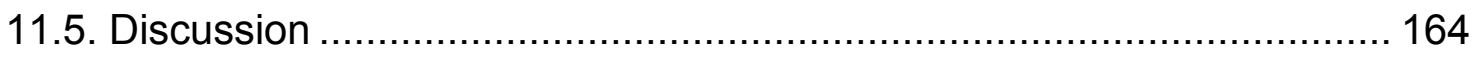

11.6. Summary of Temperature Drop Approach ........................................ 168

CHAPTER 12. Freeze thaw resistance of mixtures containing prewetted

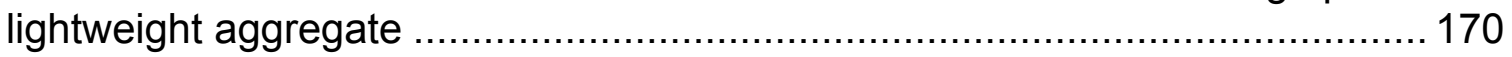

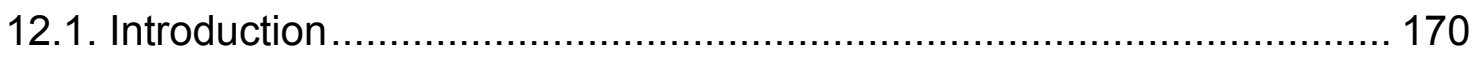

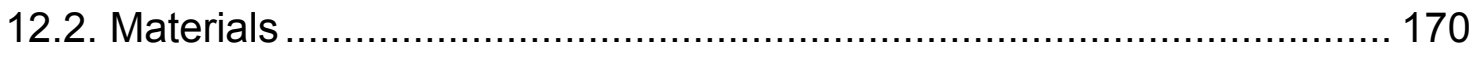

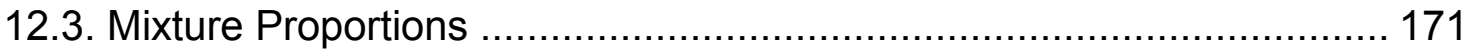

12.4. Mixing and Freeze Thaw Testing Procedure ................................... 172

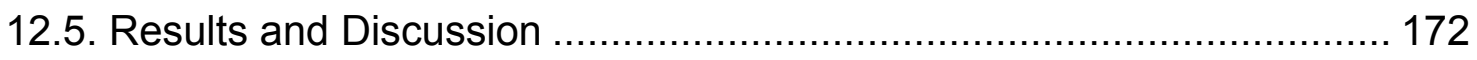


12.6. Conclusions

CHAPTER 13. Full scale batching and testing of internally cured mixtures using prewetted lightweight aggregate. 175

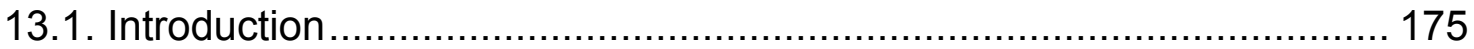

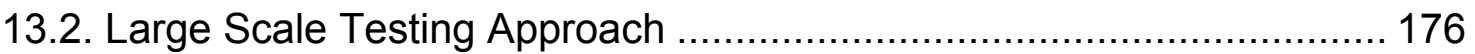

13.3. Design of the Large Scale Restrained Slab Test ............................... 177

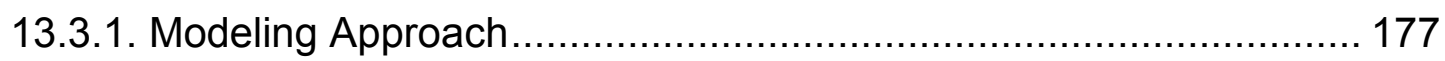

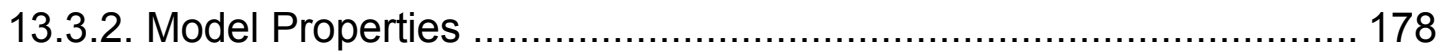

13.3.3. Modeled Concrete Properties Used to Design Large Scale Test

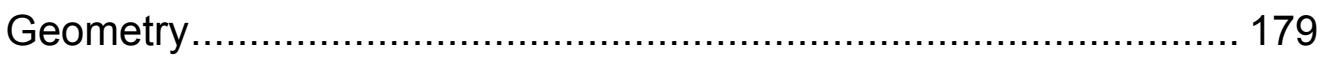

13.3.4. Results of Modeling and Selected Geometry.............................. 181

13.3.4.1. Influence of Restraining Slab Base Thickness......................... 182

13.3.4.2. Influence of Test Slab Length .............................................. 185

13.3.4.3. Influence of Restraining Base Elastic Modulus ........................ 186

13.3.5. Summary of Large Scale Test Design ....................................... 188

13.4. Large Scale Testing of Internally Cured Proportions .......................... 188

13.4.1. Restraining Base Slab Construction ........................................... 188

13.4.2. Mixture Proportioning for Test Slabs .......................................... 189

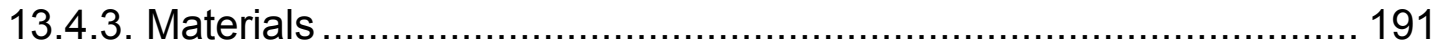

13.4.4. Mixing Procedure................................................................ 192

13.4.5. Large Scale Casting Procedure .................................................. 192

13.4.6. Experimental Results and Discussion ......................................... 194

13.5. Large Scale Testing Conclusions ..................................................... 197

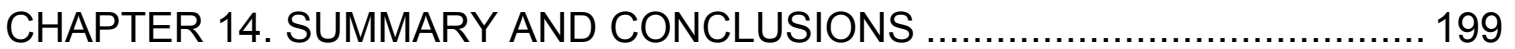

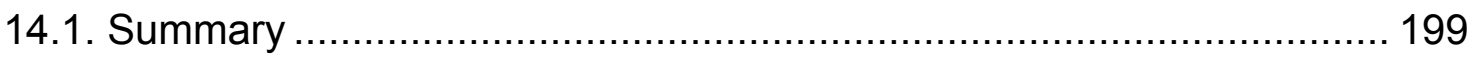




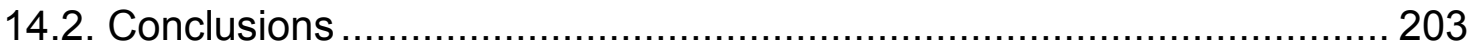

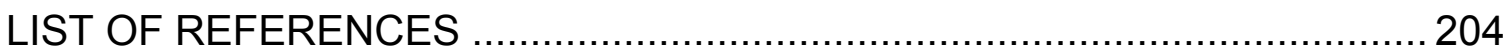

Appendix A. Calculation of LWA needed from Equation 2-1 ...................... 218

Appendix B. Unrestrained Shrinkage Measurements ................................ 220

Figure B-1 - All unrestrained, sealed shrinkage measurements .................... 221

Figure B-2 - Unrestrained, sealed shrinkage for $0.0 \%$ mixture ..................... 221

Figure B-3 - Unrestrained, sealed shrinkage for 7.3\%k mixture..................... 222

Figure B-4 - Unrestrained, sealed shrinkage for 11.0\%k mixture.................... 222

Figure B-5 - Unrestrained, sealed shrinkage 14.3\%k mixture........................ 223

Figure B-6 - Unrestrained, sealed shrinkage for 18.3\%k mixture.................... 223

Figure B-7 - Unrestrained, sealed shrinkage 25.3\%k mixture ........................ 224

Figure B-8 - Unrestrained, sealed shrinkage for 29.3\%k mixture.................... 224

Figure B-9 - Unrestrained, sealed shrinkage for 33.0\%k mixture................... 225

Figure B-10 - All unrestrained, unsealed shrinkage measurements ............... 227

Figure B-11 - Unrestrained, unsealed shrinkage for 0.0\% mixture ................ 227

Figure B-12 - Unrestrained, unsealed shrinkage for 7.3\%k mixture ................. 228

Figure B-13 - Unrestrained, unsealed shrinkage for $11.0 \%$ k mixture ............... 228

Figure B-14 - Unrestrained, unsealed shrinkage for 14.3\%k mixture............... 229

Figure B-15 - Unrestrained, unsealed shrinkage for 18.3\%k mixture............... 229

Figure B-16 - Unrestrained, unsealed shrinkage for 25.3\%k mixture.............. 230

Figure B-17 - Unrestrained, unsealed shrinkage for 29.3\%k mixture ............... 230

Figure B-18 - Unrestrained, unsealed shrinkage for 33.0\%k mixture............... 231

Appendix C. Restrained Shrinkage Measurements ..................................... 232

Figure C-1 - All restrained, sealed shrinkage measurements ....................... 238

Figure C-2 - Restrained, sealed shrinkage for $0.0 \%$ mixture ....................... 238

Figure C-3 - Restrained, sealed shrinkage for 3.8\%k mixture ...................... 239

Figure C-4 - Restrained, sealed shrinkage for 7.3\%k mixture ....................... 239

Figure C-5 - Restrained, sealed shrinkage for $11.0 \%$ k mixture ...................... 240

Figure C-6 - Restrained, sealed shrinkage for 14.3\%k mixture...................... 240

Figure C-7 - Restrained, sealed shrinkage for 25.3\%k mixture...................... 241 
Figure C-8 - All restrained, unsealed shrinkage measurements 247

Figure C-9 - Restrained, unsealed shrinkage for $0.0 \%$ mixture 247

Figure C-10 - Restrained, unsealed shrinkage for $7.3 \% \mathrm{k}$ mixture 248

Figure C-11 - Restrained, unsealed shrinkage for $11.0 \% \mathrm{k}$ mixture. 248

Figure C-12 - Restrained, unsealed shrinkage for $14.3 \% \mathrm{k}$ mixture. 249

Figure C-13 - Restrained, unsealed shrinkage for $25.3 \% \mathrm{k}$ mixture 249

Figure C-14 - Restrained, unsealed shrinkage for 29.3\%k mixture................. 250

Figure C-15 - Restrained, unsealed shrinkage for 33.0\%k mixture................. 250

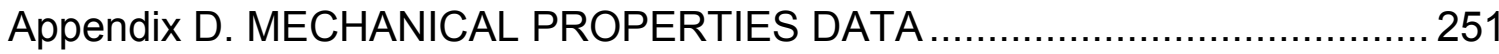

Table D-1 - Mixture M-0 Mechanical Properties........................................ 251

Table D-2 - Mixture M-11 Mechanical Properties.......................................... 251

Table D-3 - Mixture M-24 Mechanical Properties........................................ 251

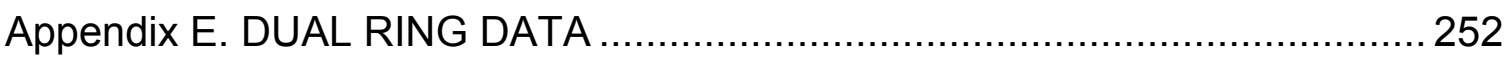

Table E-1 - Mixture M-0 7-Day Mechanical Properties ................................... 252

Table E-2 - Mixture M-11 7-Day Mechanical Properties ................................ 252

Table E-3 - Mixture M-24 7-Day Mechanical Properties …............................. 252

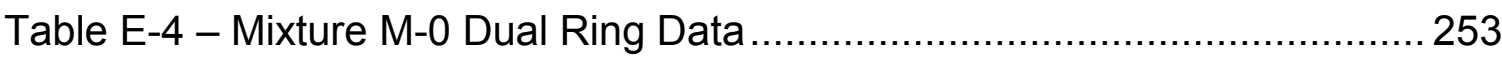

Table E-5 - Mixture M-11 Dual Ring Data .................................................... 253

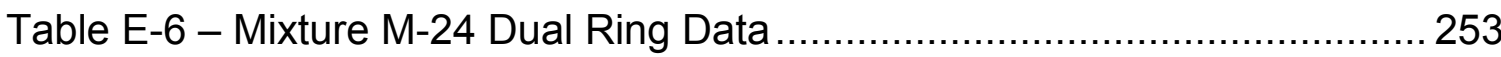

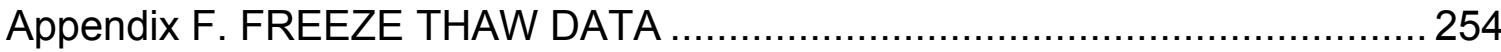

Table F-1 - Mixture M-0 Freeze Thaw Data ............................................... 254

Table F-2 - Mixture M-11 Sealed Cure Freeze Thaw Data ............................ 255

Table F-3 - Mixture M-24 Sealed Cure Freeze Thaw Data ............................ 256

Appendix G. LARGE SCALE UNRESTRAINED SHRINKAGE MEASUREMENTS

Table G-1 - Large Scale Restraining Slab 1 Unrestrained Shrinkage ….......... 257

Table G-2 - Large Scale Restraining Slab 4 Unrestrained Shrinkage .............. 257

Table G-3 - Large Scale Mixture C-0 Unrestrained Shrinkage ....................... 257

Table G-4 - Large Scale Mixture C-21 Unrestrained Shrinkage...................... 258 


\section{LIST OF TABLES}

Table

Page

Table 2-1 - Absorptions of different LWA using different methods................... 14

Table 2-2 - Mixture proportions for LWA variability study …........................... 25

Table 5-1 - Mixture proportions for modeling study..................................... 64

Table 5-2 - Kelvin Radius from internal RH and pore size distribution...............72

Table 6-1 - Mixture proportions for volume change and cracking study............ 77

Table 7-1 - Mixture proportions of mortar for settlement study ...................... 103

Table 7-2 - Mixture proportions of concrete for plastic shrinkage study.......... 104

Table 7-3 - Crack reducing ratios for each LWA replacement ....................... 111

Table 8-1 - Mixture proportions for water absorption study .......................... 118

Table 9-1 - Mixture proportions for mechanical properties study ................... 136

Table 10-1 - Mixture Proportions for Temperature Study............................... 151

Table 11-1 -Mixture Proportions for Temperature Drop Approach................... 160

Table 12-1 - Mixture Proportions for Freeze Thaw Study ............................. 171

Table 13-1 - Mixture Proportions for Restraining Base Slab .......................... 189

Table 13-2 -Mixture Proportions for Large Scale Testing ............................. 191

Table 13-3 - Coarse Aggregate Gradation for Large Scale Testing (Indiana \#9)

Appendix B Tables 
Table B-1 - All unrestrained shrinkage measurements in sealed conditions.... 220

Table B-2 - All unrestrained shrinkage measurements in unsealed conditions 226 Appendix C Tables

Table C-1 - Restrained, sealed measurements for $0.0 \%$ mixture .................. 232

Table C-2 - Restrained, sealed measurements for 3.8\%k mixture ................. 233

Table C-3 - Restrained, sealed measurements for 7.3\%k mixture ................. 234

Table C-4 - Restrained, sealed measurements for $11.0 \%$ k mixture................. 235

Table C-5 - Restrained, sealed measurements for $14.3 \%$ k mixture ................ 236

Table C-6 - Restrained, sealed measurements for 25.3\%k mixture ............... 237

Table C-7 - Restrained, unsealed measurements for $0.0 \%$ mixture ................ 242

Table C-8 - Restrained, unsealed measurements for 7.3\%k mixture .............. 242

Table C-9 - Restrained, unsealed measurements for $11.0 \% \mathrm{k}$ mixture ............ 243

Table C-10 - Restrained, unsealed measurements for $14.3 \% \mathrm{k}$ mixture............ 243

Table C-11 - Restrained, unsealed measurements for $25.3 \% \mathrm{k}$ mixture........... 244

Table C-12 - Restrained, unsealed measurements for $29.3 \% \mathrm{k}$ mixture........... 245

Table C-13 - Restrained, unsealed measurements for 33.0\%k mixture........... 246 


\section{LIST OF FIGURES}

Figure

Page

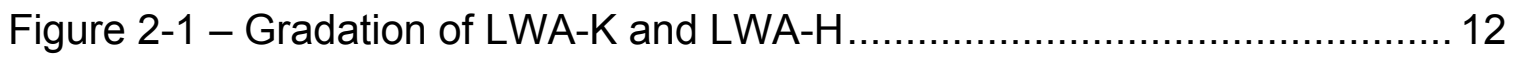

Figure 2-2 - Slight slumping of LWA using the standard cone test in ASTM C128. The slight slumping indicates no surface moisture on the LWA

Figure 2-3 - Average hue of blue at different moisture contents for LWA-K using

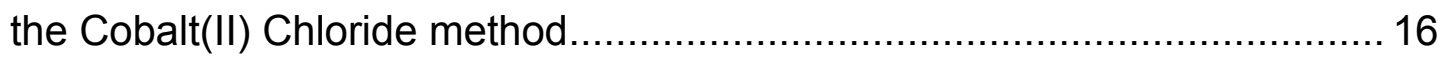

Figure 2-4 - Experimental Setup for the absorption rate method ................... 17

Figure 2-5 - (a) the absorption of different grain sizes of LWA-K along with a graded sample, (b) the absorption of the graded sample along with a graded sample reconstructed for the individual grain size absorption, (c) absorption divided by the particle diameter as a function of time (d) the absorption of LWA-K as a function of the square root of time 18

Figure 2-6 - Pycnometer used to determine absorption of LWA ..................... 20

Figure 2-7 - Pore size distribution from mercury intrusion porosimetry for (a) LWA-K, LWA-H, and a $24 \mathrm{~h}$ old cement paste with a w/c of 0.30 and (b) two different LWA-K samples showing the variation in pores of LWA particles... 22

Figure 2-8 - (a) desorption isotherm of different aggregate, and (b) the pore size distribution of the LWA determined from the desorption isotherm ............... 24

Figure 2-9 - Unrestrained shrinkage measurements for mortar made with different LWA during the first $96 \mathrm{~h}$ 26 
Figure 3-1 - Chemical shrinkage and autogenous shrinkage volumes during hydration of a paste with a $w / c$ of 0.30 (Henkensiefken et al. 2008f). 29

Figure 3-2 - Pore size distribution for cement paste with a w/c of 0.30 at three different ages and for two different LWA measured using mercury intrusion porosimetry. 33

Figure 3-3 - Desorption isotherm of two different LWA 33

Figure 3-4 - Conceptual illustration of the menisci formation in sealed and unsealed specimens made of plain and SLWA concrete (Henkensiefken et al. 2008e) 36

Figure 3-5 - Volume fraction of paste within a specified distance of a SLWA. This plot does not show whether water can reach the paste. Volume and travel distance of water need to be considered to determine this 38

Figure 3-6 - Illustrations showing the protected paste volume concept of two mixtures with similar LWA replacements volumes, but one replacement is of (a) coarse aggregate, and the other of (b) fine aggregate (Henkensiefken 2008a) 39

Figure 3-7 - Illustration of drying mechanisms in sealed and unsealed systems: (a) Sealed - only internal drying, (b) Only external drying, (c) Unsealed internal plus external drying (Radlinska et al. 2008b) 41

Figure 4-1 - Chemical shrinkage and autogenous shrinkage volumes during hydration of a paste with a $w / c$ of 0.30 44

Figure 4-2 - Samples (used for determining travel distance of water) mounted on rotating table inside $x$-ray chamber 47

Figure 4-3 - Schematic (used for detecting travel distance of water) of (a) misaligned sample and (b) a properly oriented sample 48 
Figure 4-4 - X-ray image (used for detecting travel distance of water) of samples rotated to orientations of (a) -10.0 degrees, (b) -5.0 degrees, (c) -2.5 degrees and (d) 0.0 degrees 49

Figure 4-5 - Thickness of the LWA/paste interface as sample is rotated 50

Figure 4-6 - Thickness of LWA/paste interface for different angles of orientation 50

Figure 4-7 - (a) A schematic illustration of the geometry of the experimental setup (not to scale) and (b) influence of the angle of orientation on the fraction of the $x$-ray beam that contains LWA 51

Figure 4-8 - (a) A schematic illustration of the geometry of the experimental setup (not to scale) and (b) schematic of sample holder with precut LWA and paste (used to detect water movement near the time of set) 55

Figure 4-9 - (a) X-ray measurements showing water movement from the LWA during the first day, and (b) X-ray measurements during first $6.5 \mathrm{~h}$ for determining when water leaves the LWA 58

Figure 4-10 - Difference in counts normalized to initial counts averaged from 2.0 $\mathrm{mm}$ to $2.4 \mathrm{~mm}$ in the LWA as a function of age 58

Figure 4-11 - Moisture movement from LWA into the cement paste................. 60

Figure 4-12 - Protected paste volume at different particle replacement sizes ... 61

Figure 5-1 - Autogenous shrinkage measurements for mortar specimens ........ 65

Figure 5-2 - Chemical shrinkage and autogenous shrinkage measurements showing when water depletes from LWA ............................................... 66

Figure 5-3 - Plain paste measurement and modeling results of plain and LWA

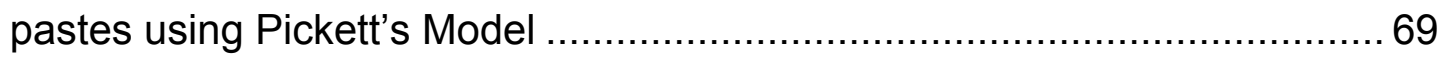

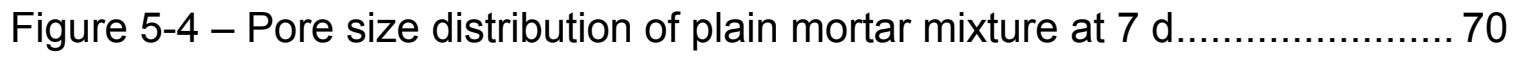

Figure 5-5 - Affected pore size distribution of plain mortar mixture ................... 71 
Figure 6-1 - Mixture proportions on a volumetric basis . 78

Figure 6-2 - Free shrinkage results of plain and LWA-K mortar mixtures in sealed curing conditions during the first $7 \mathrm{~d}$

Figure 6-3 - Free shrinkage results of plain and LWA-K mortar mixtures in sealed curing conditions. 82

Figure 6-4 - Void creation in cement paste and the time when water depletes from the LWA-K as volume replacements increase. The $18.3 \% \mathrm{k}, 25.3 \% \mathrm{k}$, $29.3 \% \mathrm{k}$ and $33.0 \% \mathrm{k}$ mixtures don't deplete of water before $7 \mathrm{~d}$ 84

Figure 6-5 - Time of water depletion from the SLWA versus the time when the mixtures begin to shrink as measured by the corrugated tubes and sealed prisms. 85

Figure 6-6 - $7 \mathrm{~d}$ free shrinkage measurements plotted as a function of the replacement volume of LWA-K ( 3 samples are shown for each mixture) ..... 86

Figure 6-7 - Free shrinkage results of plain and LWA-K mortar mixtures in unsealed curing conditions...... 87

Figure 6-8 - Mass loss of LWA-K free shrinkage samples in unsealed curing conditions..... 88

Figure 6-9 - 28 d mass loss measurements for unsealed free shrinkage measurements plotted as a function of the replacement volume of LWA-K . 89

Figure 6-10 - Restrained shrinkage results of plain and LWA-K mortar mixtures in sealed curing conditions (In each case, a typical response is shown from the three samples tested). Sharp vertical rise in strain curve indicates occurrence of cracking in the specimen 90

Figure 6-11 - Time of cracking in LWA-K mixtures plotted as a function of replacement volume ( 3 samples are shown for each mixture). 91

Figure 6-12 - Restrained shrinkage results of plain and LWA-K mortar mixtures in unsealed curing conditions (In each case, a typical response is shown from 
the three samples tested). Sharp vertical rise in strain curve indicates

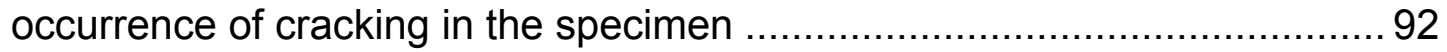

Figure 6-13 - Strain at cracking in LWA-K mixtures plotted as a function of replacement volume ( 3 samples are shown for each mixture).................... 93

Figure 6-14 - Internal relative humidity measurements for the sealed LWA-K mixtures

Figure 6-15 - Kelvin Radius and capillary stress as determined from $\mathrm{RH}$ measurements as a function of replacement volume of LWA-K at $7 \mathrm{~d}$......... 95

Figure 6-16 - Free shrinkage results of a comparison between LWA-K and LWA$\mathrm{H}$ LWA in sealed curing conditions 96

Figure 6-17 - Restrained shrinkage results of a comparison between LWA-K and LWA-H LWA in unsealed curing conditions 97

Figure 6-18 - Internal relative humidity results of a comparison between LWA-K

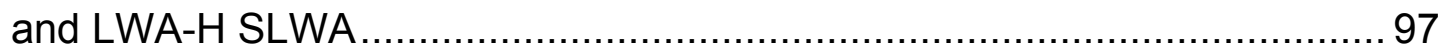

Figure 7-1 - Laser setup for settlement test (Qi et al. 2005) ......................... 106

Figure 7-2 - Geometry of the specimens used for plastic shrinkage investigation

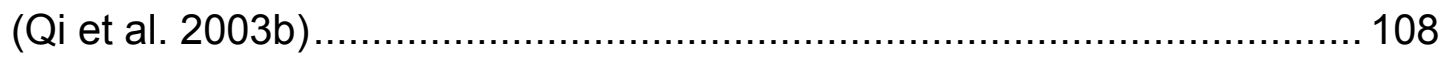

Figure 7-3 - Settlement results for mortar samples..................................... 109

Figure 7-4 - Cumulative distribution of crack width occurrences in concrete with different replacement volumes of LWA ................................................. 110

Figure 7-5 - Probability of cracks widths being smaller than $0.0 \mathrm{~mm}$ and $0.2 \mathrm{~mm}$ at different LWA replacement volumes ................................................. 111

Figure 8-1 - Powers model showing the phase distribution for (a) 55/0.30, (b) $11.0 \% \mathrm{k}$ and (c) $25.3 \% \mathrm{k}$ mixtures, and (d) the maximum theoretical degree of hydration as a function of LWA volume 119

Figure 8-2 - ITZ percolation as a function of replacement volume of LWA ...... 120 
Figure 8-3 - Degree of hydration 125

Figure 8-4 - Sorption test on samples cured for (a) 1d, (b) $7 \mathrm{~d}$, (c) $28 \mathrm{~d}$, and (d) $90 \mathrm{~d}$ 127

Figure 8-5 - (a) Initial rate of absorption, and (b) secondary rate of absorption for samples cured for different periods 128

Figure 8-6 - Cumulated absorbed water for samples at $8 \mathrm{~d}$ 129

Figure 8-7 - The equivalent $w / c$ determined from (a) cumulated absorbed water at $8 \mathrm{~d}$ on samples cured for $28 \mathrm{~d}$ and (b) conductivity. Porosity as a function of the (c) cumulated absorbed water at $8 \mathrm{~d}$ on samples cured for $28 \mathrm{~d}$ and (d) conductivity

Figure 8-8 - Relationship between open porosity and (a) initial sorptivity and (b) total absorbed water after 8 days of sorption test.

Figure 8-9 - (a) Long term sorption of samples cured for $28 \mathrm{~d}$ and (b) the total absorption for the samples after vacuum saturation 133

Figure 9-1 - Compressive Strength ....................................................... 139

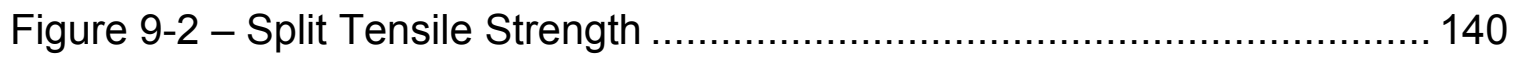

Figure 9-3 - Modulus Of Elasticity ........................................................... 141

Figure 10-1 - Geometry of the Dual Ring Test .......................................... 145

Figure 10-2 - Ring in Insulation Chamber with Top Insulation Removed .......... 145

Figure 10-3 - Effect of Invar Ring Thickness on DOR.................................. 147

Figure 10-4 - Inner Ring Thermal Correction ............................................. 149

Figure 10-5 - Outer ring Thermal Correction ............................................. 149

Figure 10-6 - Applied Temperature in Dual Ring Testing.............................. 153

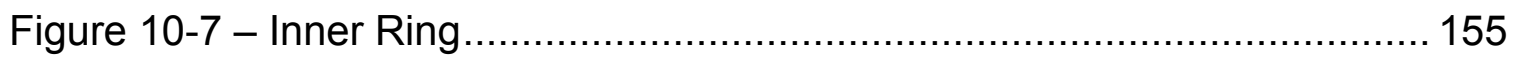

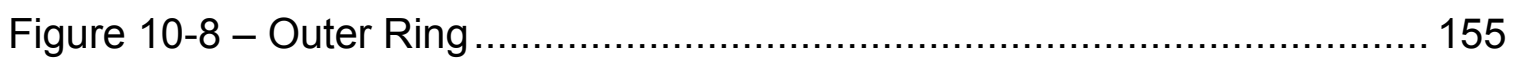




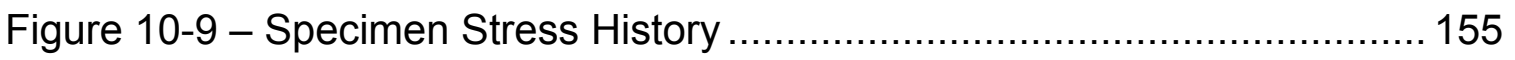

Figure 11-1 - Example Temperature Reduction ......................................... 159

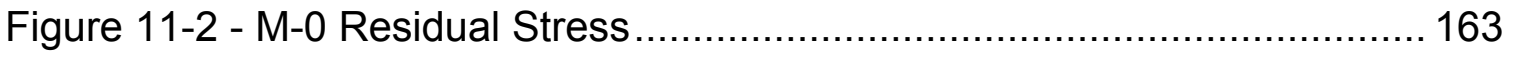

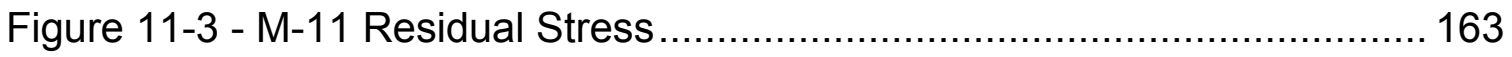

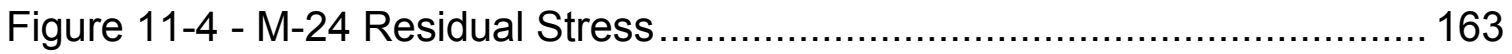

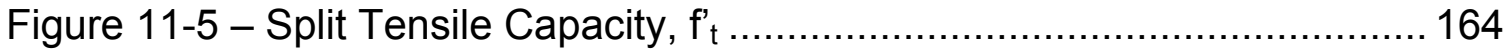

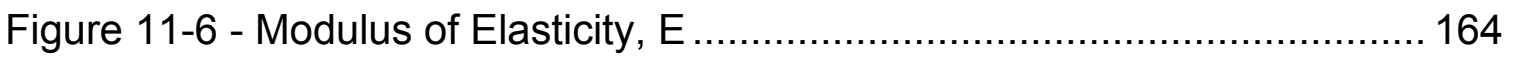

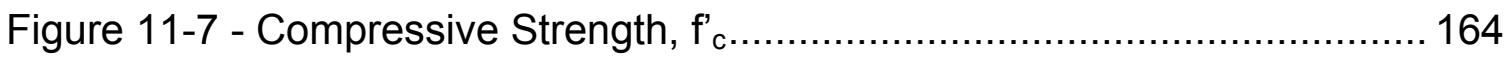

Figure 11-8 - Required Temperature Change to Induce Cracking ….............. 166

Figure 11-9 - Cracking Stress History .......................................................... 167

Figure 11-10 - Reserve Stress Capacity ................................................. 167

Figure 11-11 - Factor of Safety To Cracking ……..................................... 168

Figure 12-1 - Relative Dynamic Modulus .................................................. 173

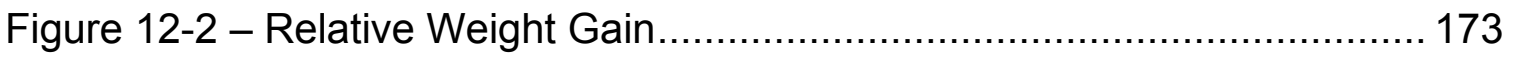

Figure 13-1 - Basic Test Geometry (a) Longitudinal Cross Section View ......... 179

Figure 13-2 - Elastic Modulus and Tensile Strength Used in Model ............... 180

Figure 13-3 -Autogenous Shrinkage Used in Model ..................................... 180

Figure 13-4 - Longitudinal Section at End of Slabs Depicting Shrinkage and

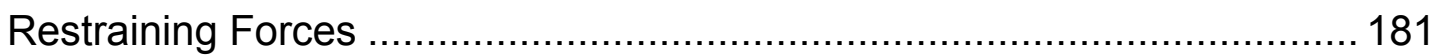

Figure 13-5 - Exaggerated Longitudinal Section View of Slab Curvature During

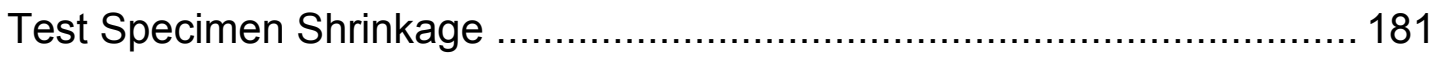

Figure 13-6 - Restraining Base Thickness Influence on (a) Deflection (b)

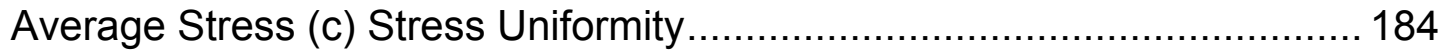

Figure 13-7 - Effect of Restraining Base Thickness on Degree of Restraint..... 185 
Figure 13-8 - Test Slab Length influence on (a) Vertical Deflection (b) Average Stress (c) Stress Uniformity 187

Figure 13-9 - Effect of Restraining Slab Modulus on Vertical Deflection at End of Slab 188

Figure 13-10 - Photo of Formwork Prepared for Large Scale Test Slab..... 193

Figure 13-11 - Photo of Finished Test Slab 193

Figure 13-12 - Mixture C-0 Test Slab Crack at 12 Day ................................. 196

Figure 13-13 - Mixture C-21 Test Slab Crack at 42 day................................ 197

Figure 13-14 - C-0 Crack Width History .................................................... 197

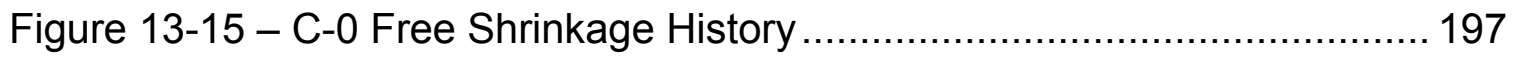

Figure 13-16 - C-21 Free Shrinkage History ……...................................... 197

Appendix B Figures

Figure B-1 - All unrestrained, sealed shrinkage measurements .................... 221

Figure B-2 - Unrestrained, sealed shrinkage for 0.0\% mixture ..................... 221

Figure B-3 - Unrestrained, sealed shrinkage for 7.3\%k mixture..................... 222

Figure B-4 - Unrestrained, sealed shrinkage for $11.0 \%$ k mixture................... 222

Figure B-5 - Unrestrained, sealed shrinkage 14.3\%k mixture ......................... 223

Figure B-6 - Unrestrained, sealed shrinkage for 18.3\%k mixture.................... 223

Figure B-7 - Unrestrained, sealed shrinkage 25.3\%k mixture....................... 224

Figure B-8 - Unrestrained, sealed shrinkage for 29.3\%k mixture................... 224

Figure B-9 - Unrestrained, sealed shrinkage for 33.0\%k mixture.................... 225

Figure B-10 - All unrestrained, unsealed shrinkage measurements ............... 227

Figure B-11 - Unrestrained, unsealed shrinkage for 0.0\% mixture ................ 227

Figure B-12 - Unrestrained, unsealed shrinkage for 7.3\%k mixture ................ 228 
Figure B-13 - Unrestrained, unsealed shrinkage for $11.0 \%$ k mixture .............. 228

Figure B-14 - Unrestrained, unsealed shrinkage for 14.3\%k mixture.............. 229

Figure B-15 - Unrestrained, unsealed shrinkage for $18.3 \%$ k mixture............... 229

Figure B-16 - Unrestrained, unsealed shrinkage for 25.3\%k mixture .............. 230

Figure B-17 - Unrestrained, unsealed shrinkage for 29.3\%k mixture .............. 230

Figure B-18 - Unrestrained, unsealed shrinkage for 33.0\%k mixture.............. 231

Appendix C Figures

Figure C-1 - All restrained, sealed shrinkage measurements ........................ 238

Figure C-2 - Restrained, sealed shrinkage for $0.0 \%$ mixture ........................ 238

Figure C-3 - Restrained, sealed shrinkage for 3.8\%k mixture ….................... 239

Figure C-4 - Restrained, sealed shrinkage for 7.3\%k mixture ...................... 239

Figure C-5 - Restrained, sealed shrinkage for $11.0 \% \mathrm{k}$ mixture...................... 240

Figure C-6 - Restrained, sealed shrinkage for $14.3 \% \mathrm{k}$ mixture ....................... 240

Figure C-7 - Restrained, sealed shrinkage for 25.3\%k mixture...................... 241

Figure C-8 - All restrained, unsealed shrinkage measurements .................... 247

Figure C-9 - Restrained, unsealed shrinkage for $0.0 \%$ mixture .................... 247

Figure C-10 - Restrained, unsealed shrinkage for 7.3\%k mixture................... 248

Figure C-11 - Restrained, unsealed shrinkage for $11.0 \% \mathrm{k}$ mixture ................. 248

Figure C-12 - Restrained, unsealed shrinkage for 14.3\%k mixture................ 249

Figure C-13 - Restrained, unsealed shrinkage for 25.3\%k mixture................ 249

Figure C-14 - Restrained, unsealed shrinkage for $29.3 \% k$ mixture.................. 250

Figure C-15 - Restrained, unsealed shrinkage for 33.0\%k mixture.................. 250 


\section{LIST OF ABBREVIATIONS AND VARIABLES}

\begin{tabular}{|c|c|c|}
\hline $\mathrm{Al}_{2} \mathrm{O}_{3}$ & Aluminum oxide & (chemical) \\
\hline$\alpha_{\max }$ & Expected maximum degree of hydration & (ratio) \\
\hline ASTM & American society for testing and materials & (Organization) \\
\hline $\mathrm{C}_{2} \mathrm{~S}$ & Dicalcium silicate & (chemical) \\
\hline $\mathrm{C}_{3} \mathrm{~A}$ & Tricalcium aluminate & (chemical) \\
\hline $\mathrm{C}_{3} \mathrm{~S}$ & Tricalcium silicate & (chemical) \\
\hline $\mathrm{C}_{4} \mathrm{AF}$ & Tetracalcium Aluminoferrite & (chemical) \\
\hline $\mathrm{CaO}$ & Calcium oxide & (chemical) \\
\hline CCD & Charged coupled device & (equipment) \\
\hline CCR & Crack reducing ratio & (ratio) \\
\hline$C_{f}$ & Cement content & $\left(\mathrm{kg} / \mathrm{m}^{3}\right)$ \\
\hline $\mathrm{CoCL}_{2}$ & Cobalt(II) chloride & (chemical) \\
\hline CRCA & Crushed returned concrete aggregate & (material) \\
\hline CS & Chemical shrinkage & $\left(\mathrm{g} / \mathrm{g}_{\text {cement }}\right)$ \\
\hline$d$ & Pore diameter & $(\mathrm{m})$ \\
\hline$\varepsilon_{\mathrm{p}}$ & Shrinkage strain of concrete & $(\mu \mathrm{m} / \mathrm{m})$ \\
\hline$\varepsilon_{\mathrm{p}}$ & Shrinkage strain of cement paste & $(\mu \mathrm{m} / \mathrm{m})$ \\
\hline$E_{a}$ & Elastic modulus of the aggregate & $(\mathrm{MPa})$ \\
\hline$E_{c}$ & Elastic modulus of the concrete & $(\mathrm{MPa})$ \\
\hline
\end{tabular}


FDD Focus to detector distance

$\mathrm{Fe}_{2} \mathrm{O}_{3} \quad$ Iron oxide

(chemical)

$\gamma \quad$ surface tension

$(\mathrm{N} / \mathrm{m})$

HDPE High density polyethylene

(material)

HPC high performance concrete

(material)

HRWRA High range water reducing admixture

(material)

IC

Internal curing

(method)

Imeasured Transmitted x-ray intensity

(counts/second)

Io Incident $x$-ray intensity

(counts/second)

$\mathrm{K} \quad$ Paste of mortar bulk modulus

$\mathrm{keV} \quad$ kiloelectron volt $(\mathrm{keV})$

$\mathrm{K}_{\mathrm{s}} \quad$ Solid skeleton bulk modulus

LVDT Linear variable differential transformer

(equipment)

LWA Lightweight aggregate

(material)

LWA-H Haydite lightweight aggregate

(material)

LWA-K Kenlite lightweight aggregate

(material)

$\mathrm{MgO} \quad$ Magnesium oxide

(chemical)

MIP

Mercury intrusion porosimetry

(equipment)

MLWA Mass of dry lightweight aggregate

$\left(\mathrm{kg} / \mathrm{m}^{3}\right)$

n

Aggregate stiffness term

(unitless)

$\mathrm{n}_{\mathrm{FA}} \quad$ Aggregate stiffness term for fine aggregate

(unitless)

$\mathrm{n}_{\mathrm{FA}}$

Aggregate stiffness term for fine lightweight aggregate

(unitless)

$\mathrm{Na}_{2} \mathrm{O} \quad$ Sodium oxide

(chemical) 


\begin{tabular}{|c|c|c|}
\hline NIST & National institute of standards and technology & (Organization) \\
\hline ODD & Object to detector distance & $(\mathrm{mm})$ \\
\hline OPC & Ordinary portland cement & (material) \\
\hline$P$ & Pressure & $(\mathrm{Pa})$ \\
\hline$\phi_{\text {LWA }}$ & Absorption capacity of lightweight aggregate & $(\%)$ \\
\hline$\mu \mathrm{A}$ & Microampere & $(\mu \mathrm{A})$ \\
\hline$\mu_{\text {LWA }}$ & Linear attenuation coefficient of lightweight aggregate & $\left(\mathrm{cm}^{-1}\right)$ \\
\hline$\mu \mathrm{m}$ & Micrometer & $(\mu \mathrm{m})$ \\
\hline$\mu_{\text {Paste }}$ & Linear attenuation coefficient of cement paste & $\left(\mathrm{cm}^{-1}\right)$ \\
\hline$\mu_{\mathrm{v}}$ & Linear attenuation coefficient of vapor voids & $\left(\mathrm{cm}^{-1}\right)$ \\
\hline$\mu_{\mathrm{w}}$ & Linear attenuation coefficient of water & $\left(\mathrm{cm}^{-1}\right)$ \\
\hline r & radius of curvature of the menisci & (m) \\
\hline $\mathrm{R}$ & Universal gas constant & $(\mathrm{J} / \mathrm{mol} \cdot \mathrm{K})$ \\
\hline $\mathrm{RH}$ & Relative humidity & $(\%)$ \\
\hline S & Degree of saturation & (unitless) \\
\hline$\sigma_{\text {cap }}$ & Capillary pressure & $(\mathrm{Pa})$ \\
\hline $\mathrm{SiO}_{2}$ & Silicon dioxide & (chemical) \\
\hline SLWA & Prewetted lightweight aggregate & (material) \\
\hline $\mathrm{SO}_{3}$ & Sulfur trioxide & (chemical) \\
\hline$t$ & thickness of the sample & $(\mathrm{cm})$ \\
\hline $\mathrm{T}$ & Thermodynamic temperature & $(\mathrm{K})$ \\
\hline$\Theta$ & Liquid-solid contact angle & (radians) \\
\hline $\mathrm{v}_{\mathrm{c}}$ & Poisson's ratio of concrete & (unitless) \\
\hline
\end{tabular}




$\begin{array}{ll}V_{a} & \text { Poisson's ratio of aggregate } \\ V_{F A} & \text { Volume fraction of fine aggregate } \\ V_{L W A} & \text { volume proportions of lightweight aggregate } \\ V_{m} & \text { Molar volume of the pore solution } \\ V_{\text {Paste }} & \text { volume proportions of cement paste } \\ V_{V} & \text { volume proportions of vapor voids } \\ V_{W} & \text { volume proportions of water } \\ W / c & \text { Water-to-cement ratio }\end{array}$

(unitless)

$\left(\mathrm{m}^{3} / \mathrm{mol}\right)$

(mass fraction) 


\section{CHAPTER 1. INTRODUCTION, OBJECTIVES, AND APPROACH}

\subsection{Introduction}

Water is a necessary component of the cement hydration reaction. The hydration reaction is responsible for the conversion of the gray cement powder into the binding cement paste which gives concrete its strength. It is well known in concrete construction that a 'proper curing period' is essential at early ages to enable the concrete to gain strength, to reduce shrinkage, and to develop a structure that will make the concrete sufficiently durable.

While water curing supplies additional water that the concrete can absorb at early ages when it undergoes the largest part of the hydration reaction, practical considerations frequently do not allow structures to be ponded with water for the first two weeks or month after construction. As a compromise many pavements and bridge components are coated with a 'curing compound' after casting which slows or eliminates the loss of water from the concrete, however it does not provide any additional water. The application of this curing compound can be problematic for two reasons. First, it is difficult to determine whether it has been applied correctly or with sufficient attention until it is too late. Second, and more importantly in higher performance concretes (HPC), the lack of water addition leads to concretes that are 'water-starved' causing them to undergo selfdesiccation (RILEM Report 41 2007). 
This self-desiccation results in concrete that 'dries out internally' resulting in hindered strength development, reduced durability, and the potential for autogenous shrinkage and cracking. Self-desiccation occurs even in concretes where the curing compound has been applied correctly. The concept of selfdesiccation has been a topic of recent interest in the concrete community and has even led to a series of workshops specifically devoted to this topic (1997, 1999, 2002, 2005).

Weber and Reinhardt (Weber et al. 1995) showed that additives could be used to store internal water in concrete which can be used to 'self-cure' the concrete. Since that time it has been shown that certain cellulose fibers (Mohr et al. June 2005), lightweight aggregates (Zhutovsky 2002; Lura 2003; Holm et al. August 2000), and absorbent polymers (Bentz et al. 2004) can be used as these additives. While these technologies are rapidly being proposed in literature, they are not rapidly added to concrete specifications. In addition, these materials are not always designed from a scientific perspective; consequently these materials are not always designed in the most efficient fashion.

Lightweight aggregate has been used in concrete for over a century as a method to reduce the self-weight (ESCSI 1971), however its use as an internal curing (IC) agent is a more recent development (Philleo 1991; Jensen et al. 2001b; Bentz et al. 2008b). Research for the last decade has focused on the laboratory measurements of autogenous deformation of these mixtures (Jensen 2005). State Departments of Transportations (DOTs) are beginning to consider the beneficial effects of using internal curing (Delatte et al. 2007; Weiss et al. 2007). However more needs to be understood about these materials to take them from laboratory concepts to field realities. 
In 2007 RILEM released a comprehensive State-of-the-Art Report (STAR) on internal curing which primarily focused on unrestrained autogenous deformation (RILEM 2007). The STAR report is one of the first documents to compile the major research effort that has taken place in this area. Although this report is extremely useful, it focuses primarily on fundamental concepts and unrestrained shrinkage in sealed conditions. The STAR report suggested that more research is needed in several areas. For example, page 139 stated that "...drying shrinkage is not the central focus of the report and this problem needs to be given proper treatment and solved in the future." On page 140, it is stated that more information is needed about "... relation of early age concrete behavior and the durability of mature concrete." Although not explicitly stated in the RILEM report, another area where more research is needed is in the potential for using internal curing to delay or prevent the formation of plastic shrinkage cracks as this has been alluded to by Holm et al. (2003) but not shown. This research can be used to help determine the proportions of LWA used as an internal curing agent needed to reduce shrinkage in sealed and unsealed curing conditions.

This research is not intended to be a repeat of previous work, but rather it is intended to show the influence of internal curing in areas were more research is needed. The desire to move internal curing from the laboratory to the field and the need for more research in these areas is the impetus for this work.

\subsection{Research Objectives}

The main objectives of this project are to provide information on development, manufacture, and performance of self-curing concrete made using local materials. Local materials are characterized to demonstrate which materials show the greatest potential for use in the production of self-curing concrete. High 
performance concrete mixtures are developed with self-curing capabilities using typical local materials. The benefits of self-curing are evaluated using both small and large scale laboratory testing. Finally, technology transfer has been performed to enable these materials to be developed, specified, and implemented in Indiana.

\subsection{Research Approach}

The goal of this project was to provide the Indiana Department of Transportation (INDOT) with information on the development, manufacture, and performance of self-curing concrete made using local materials. Specific tasks considered in this project are outlined as follows:

\subsubsection{Task 1: Literature Review}

The first task of this study was to perform a review of literature pertaining to the development, testing, and use of self-curing concrete. The main objective of this review was to:

- Assemble papers related to the development of self-curing concrete. This included information about previous scientific developments, mixture proportioning procedures, materials that have been used successfully, and applications of self-curing concrete. This study was expedited with information from the RILEM state of the art report on self-curing concrete.

- Assemble a complete listing of test procedures to evaluate self-curing concrete. The procedures were reviewed both based on their ability to determine theoretically fundamental properties as well as the ability to be used for performing practical field tests. 
- Assemble information on the locally available constituent materials and concrete mixture proportions that would be available for the production of self-curing concrete.

\subsubsection{Task 2: Characterization of Local Materials}

Task 2 details important physical properties of the LWA that are needed so that the LWA can be used as an effective internal curing agent. The two main physical properties of the LWA are that the LWA must be able to absorb a large volume of water and be able to desorb (i.e., give back) that water to the concrete as self-desiccation occurs. The testing procedures used to determine these properties of lightweight aggregate; most specifically focusing on absorption procedures is presented. Highlighted in this task are the inadequacies of these testing procedures, the variability which can result, and the influence of these properties on the shrinkage performance. The results of Task 2 are presented in CHAPTER 2 of this report.

\subsubsection{Task 3: Evaluating Self-Curing Concrete Made with Local Materials}

Numerous laboratory methods were utilized to evaluate self curing concrete made with local materials. This task is divided into the following sections which each consist of a separate chapter within this report as indicated below.

- CHAPTER 3 - Mechanisms of Internal Curing: The mechanism which drives internal curing, specifically the creation of vapor filled voids due to self-desiccation is discussed. The process of using LWA as an agent to supply water to fill the voids is described. As such, the IC water can be described in three main ways: (1) the volume of water available for IC, (2) the ability of the water to leave the prewetted lightweight aggregate (SLWA) when needed for IC, and (3) the distribution of the SLWA so that it 
is well-dispersed and its water can readily travel to all of the sections in the paste where it is needed. The difference between the behavior of sealed and unsealed systems is discussed.

- CHAPTER 4 - Monitoring Water Movement From Prewetted Lightweight Aggregate Using X-Ray Absorption: This section examines the timing of the release of water from prewetted lightweight aggregate and the distance that water can travel using $\mathrm{x}$-ray absorption. Experimental details associated with the $x$-ray technique are provided to describe the importance of specimen alignment. The importance of water movement is discussed in terms of mixture proportioning and its influence on shrinkage and stress development.

- CHAPTER 5 - Shrinkage of Cementitious Systems Containing Prewetted Lightweight Aggregate: The shrinkage behavior of IC in mixtures using composite models such as Pickett's equation and Mackenzie's equation is discussed. The results indicate that while the inclusion of prewetted LWA reduces the stiffness of the mortar, the reduction in shrinkage of the paste due to the supply of additional water is more crucial in reducing the overall shrinkage of the mortar. The size of the pores emptied due to selfdesiccation is calculated and used to describe the shrinkage of mortar using Mackenzie's equation.

- CHAPTER 6 - Measuring Volume Change and Cracking Under Sealed and Unsealed Conditions: This section provides measures of unrestrained shrinkage for mortars made with different volume replacements of LWA under sealed and unsealed curing conditions. In addition, this section assesses the cracking potential of mixtures that are restrained from shrinking freely. This section presents data for the mixtures made with different volume replacements of SLWA. The results indicate the inclusion of a sufficient volume of SLWA can significantly 
reduce self-desiccation, reduce autogenous shrinkage, and delay the time to cracking.

- CHAPTER 7 - Plastic Shrinkage Cracking in Mixtures Containing Prewetted Lightweight Aggregate: IC mixtures are measured for its effect on plastic shrinkage properties. Settlement and plastic shrinkage cracking are investigated. The results indicate that when LWA is added to concrete, the formation of plastic shrinkage cracks are reduced. The crack widths of mixtures with LWA were reduced and if sufficient volume of LWA was used, the cracks could be eliminated.

- CHAPTER 8 - Monitoring Water Absorption in Cementitious Systems Made With Prewetted Lightweight Aggregate: This section provides measures of the performance of IC mixtures under different curing conditions in fluid absorption tests. The results indicate that the absorption is reduced with increasing replacement volume of LWA. It is indicated that several possible explanations exists for this. Mixtures with IC show an increased degree of hydration which will reduce the interconnectivity of the pore structure. The dense interfacial transition zone between LWA and cement paste may prohibit water from being absorbed back into LWA.

- CHAPTER 9 - Mechanical Properties of IC Systems Made with Prewetted Lightweight Aggregate: This section provides measures of the compressive strength, split tensile strength, and elastic modulus of IC mixtures under differing replacement volumes of LWA. The results indicate that increasing the replacement volume of LWA decreases the stiffness and strength of mixture.

- CHAPTER 10 - The Effect of Temperature Change on IC Systems Made with Prewetted Lightweight Aggregate: The majority of testing in this report was performed in isothermal conditions. Shrinkage induced by 
temperature change is important because it is additive to the other volume changing mechanisms such as autogenous and drying shrinkage. Thermal shrinkage may cause the overall shrinkage behavior of the concrete to crack when restrained. The sensitivity of thermal cracking in IC mixtures was studied using a newly developed dual restraining ring testing device. Mixtures with IC show a decreased propensity to early age thermal cracking due to the combined effects of reduced residual stress from autogenous shrinkage and lower modulus of elasticity.

- CHAPTER 11 - Evaluating Internally Cured Mixtures With a Temperature Drop Approach: This section presents a temperature reduction technique using the dual ring device presented in CHAPTER 10 to quantify the reserve cracking strength of a fresh mixture over the first of several days after casting. Data is presented that show using lightweight aggregate reduces early age cracking propensity.

- CHAPTER 12 - Freeze Thaw Resistance of Mixtures Containing Prewetted Lightweight Aggregate: This section outlines testing performed to quantify the freeze thaw performance of high performance mixtures containing prewetted lightweight aggregate. Experimental results are presented that show freeze thaw performance of the given low $\mathrm{w} / \mathrm{c}$ mixtures is neither enhanced nor reduced by the addition of lightweight aggregate.

\subsubsection{Tasks 4 and 5: Full Scale Trial Batch and Testing}

The results of the tests performed in Task 3 were analyzed and used for the development of a full scale batch of IC concrete that was used in a newly developed large scale slab cracking device. The details of this testing device are discussed in CHAPTER 13. Results of testing indicate that IC improved the cracking propensity of the concrete mixture. 


\section{CHAPTER 2. CHARACTERIZATION OF LOCAL MATERIALS}

\subsection{Introduction}

Many of the advancements in concrete technology have come from a better understanding of cement chemistry and the introduction of supplementary cementitious materials or chemical admixtures. These materials however, only typically occupy a minor part of the volume of concrete. The majority of the volume of concrete is occupied by aggregate. In conventional concrete mixtures, the aggregate is relatively inert and intended to be stable. New methods are being developed where the aggregate plays an integral role in the development of the paste microstructure. This method, known as internal curing, uses prewetted lightweight aggregate as a shrinkage mitigation strategy to reduce the effects of self-desiccation (RILEM 2007; Henkensiefken et al. 2008b).

The benefits of internal curing as a shrinkage mitigation strategy is well documented in laboratory studies (Jensen et al. 2001a; Zhutovsky et al. 2002; Lura 2003a; Cusson et al. 2006; Lopez et al. 2008; Henkensiefken et al. 2008b; Radlinska et al. 2008b). Several departments of transportations are now considering internal curing (Weiss et al. 2007; Bentz et al. 2008b).

In internal curing (IC), the aggregates are not considered to be inert; rather they play an integral role in hydration. To properly proportion mixtures to obtain the benefits of internal curing, it is essential that the properties of LWA be well understood. The absorption and desorption properties of LWA are two of the 
properties that are important in determining the effectiveness of LWA as an internal curing agent (Kim et al. 2008; Radlinska et al. 2008b). However, the current techniques for determining these properties are not clear and can lead to significant variability (Kleiger et al. 1994). This chapter will discuss different measurement techniques to determine the absorption and pore size distribution of LWA. The implications on the variability of these measurements on the shrinkage performance will be presented. This paper will also present information on selecting LWA which has properties which will be beneficial in internal curing.

\subsection{Techniques for Determining the Absorption of LWA}

Low water-to-cement ratio mixtures tend to self-desiccate. Traditional curing techniques have proven to be ineffective to provide extra water because the low permeability of these mixtures does not allow significant penetration of external water (Sant et al. 2008a). Prewetted inclusions, typically LWA, have been introduced into the mixture to uniformly distribute the water internally. Bentz and coauthors (Bentz et al. 1999b; Bentz et al. 2005) presented an equation to determine the amount of LWA needed to counteract the effects of selfdesiccation.

$M_{L W A}=\frac{C_{f} \times C S \times \alpha_{\max }}{S \times \phi_{L W A}}$

Equation 2-1

where: $\mathrm{M}_{\mathrm{LWA}}\left(\mathrm{kg} / \mathrm{m}^{3}\right.$ or $\left.\mathrm{lbs} / \mathrm{yd}^{3}\right)$ is the mass of LWA (in a dry state) that needs to be prewetted to provide water to fill in the voids created by chemical shrinkage, $\mathrm{C}_{f}\left(\mathrm{~kg} / \mathrm{m}^{3}\right.$ or lbs/yd $\left.{ }^{3}\right)$ is the cement content of the mixture, CS ( $\mathrm{g}$ of water per $\mathrm{g}$ of cement or $\mathrm{lb}$ of water per lb of cement) is the chemical shrinkage of the cement, $\alpha_{\max }$ (unitless) is the expected maximum degree of hydration (0 to 1 ), $S$ (unitless) is the expected degree of saturation of the LWA (0 to 1$)$, and $\Phi_{\mathrm{LWA}}$ ( $\mathrm{kg}$ of water $/ \mathrm{kg}$ of dry LWA or lb/lb) is the absorption capacity of the LWA. One critical 
aspect of this equation is to be able to properly determine the absorption capacity of the LWA. If the absorption is not determined correctly, an incorrect volume of LWA would be added to the mixture which could prove to be too little or too much LWA. The correct absorption must be determined to optimize the mixture. However, determining the absorption of the LWA is difficult using current techniques. The following section will discuss the most common techniques. It should be noted that in these techniques, $24 \mathrm{~h}$ absorption is often taken to be saturated ${ }^{1}$. This however is not entirely accurate since the pore structure of LWA allows the LWA to absorb water for an extended period of time (Holm et al. 2006). Depending on the aggregate, this time could be several weeks or months. The term "prewetted" in this report refers to the $24 \mathrm{~h}$ absorption of the LWA.

Four different aggregates were used in this study. Two of the aggregates were manufactured rotary kiln expanded shale LWA, designated as LWA-K and LWA$\mathrm{H}$. They had an apparent specific gravity of 1.56. The approximate chemical composition of the expanded shale is $57.6 \% \mathrm{SiO}_{2}, 9.6 \% \mathrm{Fe}_{2} \mathrm{O}_{3}, 19.4 \% \mathrm{Al}_{2} \mathrm{O}_{3}$, $3.4 \% \mathrm{CaO}, 2.6 \% \mathrm{MgO}, 0.6 \% \mathrm{SO}_{3}$ and $5.6 \% \mathrm{Na}_{2} \mathrm{O}$ by mass fraction (HPB 2004). The third aggregate was a crushed returned concrete aggregate (CRCA) and it had a specific gravity of 2.04 (Kim et al. 2008). The key difference between these aggregates is how the pore volume is created. During the manufacturing of the LWA the gases expand in the shale which creates an interconnected pore network (ESCSI 1971). Crushed returned concrete aggregate on the other hand has a pore structure similar to that of cement paste

${ }^{1}$ The term saturated is used to describe the degree of saturation in the LWA. It does not necessarily indicate all pores in the LWA are filled with water. 
since paste provides the primary pore-containing component in the crushed paste/aggregate product. The fourth aggregate is a conventional aggregate that is natural river sand with an apparent specific gravity of 2.58. The gradation of the two LWA used can be seen in Figure 2-1.

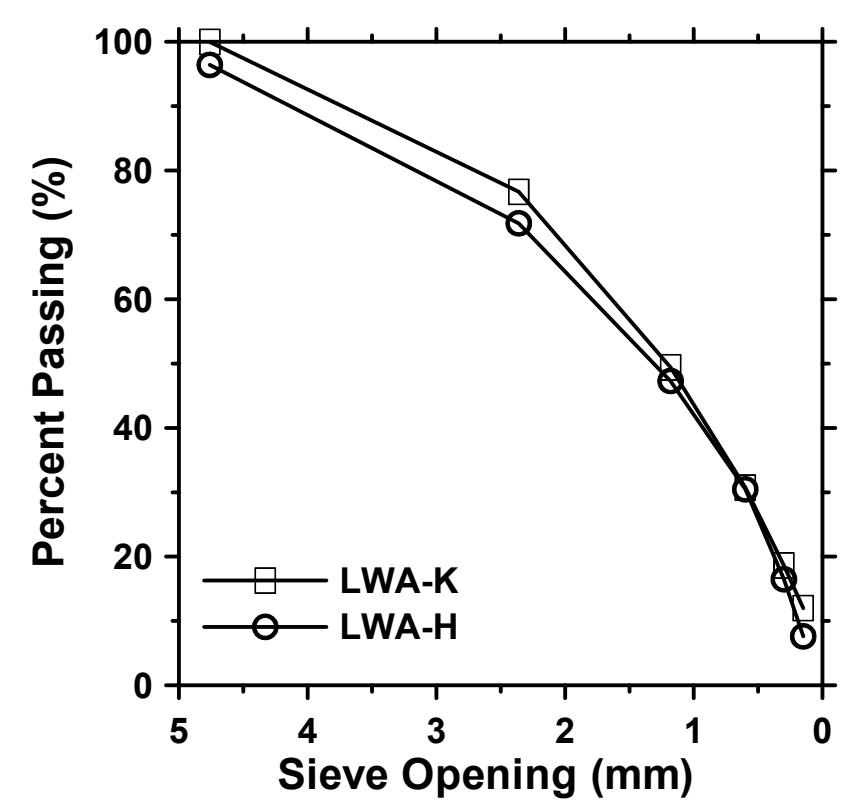

Figure 2-1 - Gradation of LWA-K and LWA-H

\subsubsection{ASTM C128}

One of the most commonly used methods to determine the absorption of aggregate is ASTM C128. It should be noted that this standard is not intended to be used for determining the absorption of LWA as stated in section 1.3 and 5.5 of the standard (2007), however in lieu of a more appropriate method for LWA, it is commonly used. The sample is immersed in water for $24 \mathrm{~h}$ after which time the water is decanted and the surface of the aggregate is dried. The difficulty that arises is determining when there is no more surface moisture on the aggregate. Three different procedures are suggested in the standard to determine this condition; the standard cone test, the provisional cone test and the paper towel test. The cone tests are similar to each other; however the compaction method 
is slightly different. In the standard cone test, aggregate is placed in a cone (like a slump cone for concrete, but smaller) and tamped 25 times. The provisional cone test still involves tamping the aggregate 25 times, however aggregate is added at intermediate times and tamped. Both cone tests rely on the principle of surface tension for determining when the surface moisture disappears. When moisture is still on the surface of the aggregate, surface tension of the water will hold the particles in the form of the cone after the cone is removed. When no moisture is present on the surface, slight slumping of the aggregate will occur. An example of slight slumping is shown in Figure 2-2. The paper towel method involves touching a paper towel to the aggregate and when it appears the paper towels is no longer picking up moisture from the aggregate, a surface dry condition has been reached.

At least two potential issues arise from these cone tests. The first is that LWA is typically manufactured and crushed, resulting in angular aggregate. The angularity of the aggregate could lock the particles together such that when the cone is lifted the shape is retained after surface moisture is gone. The larger issue is that slight slumping is a subjective term and the variation of absorption for person to person could be rather large. The $24 \mathrm{~h}$ absorption for LWA-K using the standard cone test was determined to be $10.5 \%$ with a standard deviation of $0.15 \%$. The vacuum absorption for LWA-K using the standard cone test was determined to be $15.0 \%$ with a standard deviation of $0.15 \%$. A full list of the absorption values for different testing methods can be found in Table 2-1. 


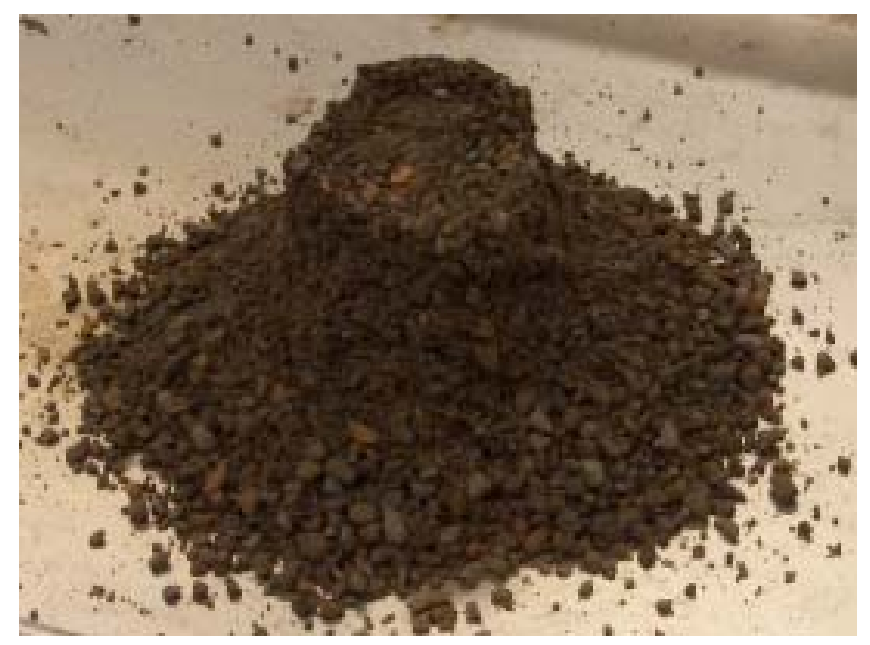

Figure 2-2 - Slight slumping of LWA using the standard cone test in ASTM C128. The slight slumping indicates no surface moisture on the LWA

Table 2-1 - Absorptions of different LWA using different methods

\begin{tabular}{|c|cc|c|c|c|}
\hline \multirow{2}{*}{ Method } & \multicolumn{2}{|c|}{ LWA-K } & LWA-H & CRCA & Sand \\
\cline { 2 - 6 } & $\mathbf{2 4} \mathbf{h}$ & Vacuum & $\mathbf{2 4} \mathbf{~ h}$ & $\mathbf{2 4} \mathbf{h}$ & $\mathbf{2 4 \mathbf { h }}$ \\
\hline $\begin{array}{c}\text { C128 Cone } \\
\text { Method }\end{array}$ & $10.5 \%$ & $15.0 \%$ & $5.8 \%$ & $9.8 \%$ & $1.8 \%$ \\
\hline $\begin{array}{c}\text { C128 Provisional } \\
\text { Cone Method }\end{array}$ & $9.4 \%$ & $14.3 \%$ & $4.6 \%$ & $8.8 \%$ & $1.7 \%$ \\
\hline $\begin{array}{c}\text { C128 Paper } \\
\text { Towel Method }\end{array}$ & $20.8 \%$ & $24.9 \%$ & $15.7 \%$ & $17.5 \%$ & $1.9 \%$ \\
\hline $\begin{array}{c}\text { Absorption Rate } \\
\text { Method }\end{array}$ & $5.0 \%$ & N/A & $1.0 \%$ & $1.1 \%$ & $0.8 \%$ \\
\hline $\begin{array}{c}\text { Cobalt Chloride } \\
\text { Method }\end{array}$ & $11.0 \%$ & - & - & - & - \\
\hline $\begin{array}{c}\text { Pycometer } \\
\text { Method }\end{array}$ & $7.9 \%$ & N/A & $5.7 \%$ & $3.1 \%$ & $1.0 \%$ \\
\hline
\end{tabular}

\subsubsection{Cobalt(II) Chloride Method}

Cobalt(II) chloride $\left(\mathrm{CoCl}_{2}\right)$ can be used to indicate the state of moisture in aggregate (O'Neil et al. 2001). Cobalt(II) chloride changes color in the presence of moisture from blue in the anhydrous (i.e., dry) form to pink when it reacts with 
water. This test was performed with LWA used in conjunction with the ASTM C128 test. Portions of LWA-K were removed and cobalt(II) chloride in powder form was scattered on the surface of the aggregate. An image was captured after $1 \mathrm{~min}$ of placing the cobalt(II) chloride on the surface of the aggregate. After the photo was taken, the cobalt(II) chloride was removed from the surface of the aggregate and the aggregate was placed in the oven to determine the moisture content. When there is a higher moisture content, more water is on the surface of the aggregate and therefore more water can react with the cobalt(II) chloride, resulting in a deeper red color. As the aggregate dries and the moisture content decreases a lower extent of reaction occurs resulting in the cobalt(II) chloride appearing more blue.

The images of cobalt(II) chloride on the surface of the aggregate were analyzed in ImageJ@ image processing software. The original RGB images were split into three separate images according to the red, green and blue color. The area of the cobalt(II) chloride on the surface of the aggregate of the blue image was selected and the average hue of the cobalt(II) chloride was determined. Figure 2-3 shows the average hue of blue obtained from the image analysis as a function of the moisture content in the aggregate. The average hue will increase since unreacted (i.e., dry) cobalt(II) chloride is blue and only the blue image was processed. An image was also captured of cobalt(II) chloride in the unreacted (i.e, dry) state as to determine the upper bound of the hue of blue. The upper bound represents the theoretical maximum hue that could be reached. The aggregate begins to reach this value at low moisture contents. This is because no water is on the surface of the aggregate to react with the cobalt(II) chloride. A lower bound was also determined by combining cobalt(II) chloride with water and letting a complete reaction take place. The towel method and standard cone method are also shown on Figure 2-3. The color change does not appear to begin to stabilize (which would indicate no water is on the surface) until 
approximately $11 \%$. This is close to the $10.5 \%$ indicated by the standard cone method.

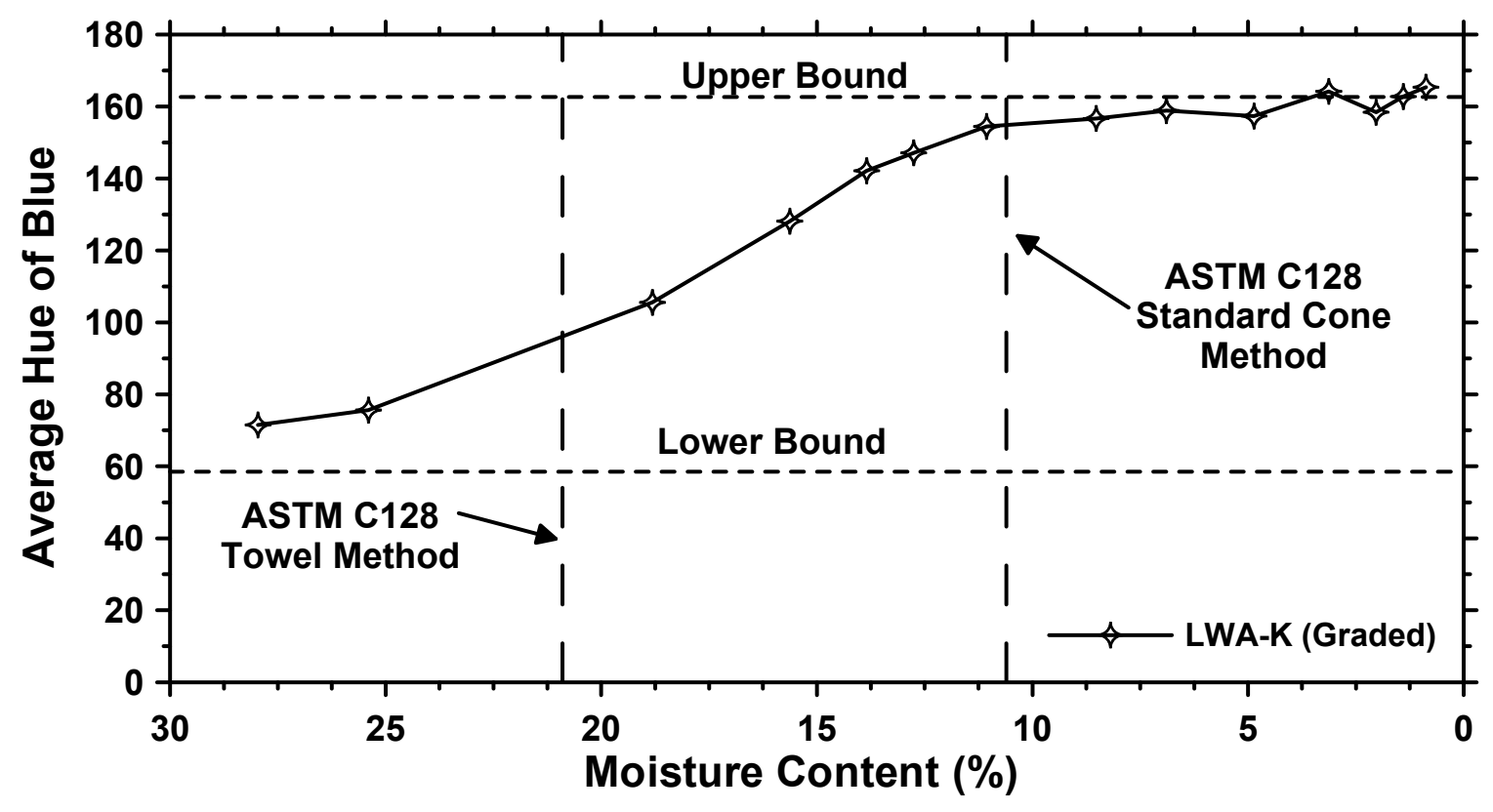

Figure 2-3 - Average hue of blue at different moisture contents for LWA-K using the Cobalt(II) Chloride method

\subsubsection{Absorption Rate Method}

The previous two methods rely on the disappearance of surface moisture for determining the absorption capacity of the LWA. Other methods that exist involve immersing the aggregate in water and monitoring the mass of water absorbed. One method explored was immersing aggregate in a water bath and monitoring the change in mass as water was absorbed. Using this method, the absorption rate of the LWA was determined. The schematic of the setup for this experiment can be seen in Figure 2-4. The LWA was oven-dried and immersed in water maintained at $23.0^{\circ} \mathrm{C} \pm 0.1^{\circ} \mathrm{C}$ and the weight was recorded using a balance with a sensitivity of $0.0001 \mathrm{~g}$ in $1 \mathrm{~min}$ intervals. The absorption for different particle sizes of LWA-K and a graded sample can be found in Figure 2-5(a). The larger the particle size, the higher the absorption, however particle 
sizes smaller than $0.60 \mathrm{~mm}$ appear to have similar absorption rates. The absorption of the graded sample at $24 \mathrm{~h}$ is approximately $5.0 \%$.

Figure 2-5(b) shows the absorption of the graded sample along with the theoretical absorption of a sample if the individual grain size absorptions were combined. The theoretical absorption was calculated by combining the absorption of each of the different grain sizes and the gradation of the LWA. The absorption of the two methods are similar. Figure 2-5(c) shows the absorption of the particles divided by the particle diameter. The absorption rate plotted as a function of the square root of time is shown in Figure 2-5(d). This plot shows a linear relationship between the absorption and the square root of time.

One drawback to this test method is that measurements cannot start immediately when the LWA starts to absorb water. This is because the water needs to be added slowly to the sample so no particles are lost. If the water is added too quickly, the particles will be flushed out of the dish because of the sudden rush of water. This results in the first $15 \mathrm{~s}$ of absorption not being captured. This missing data could explain why this method yields a lower absorption at $24 \mathrm{~h}$ than other methods.

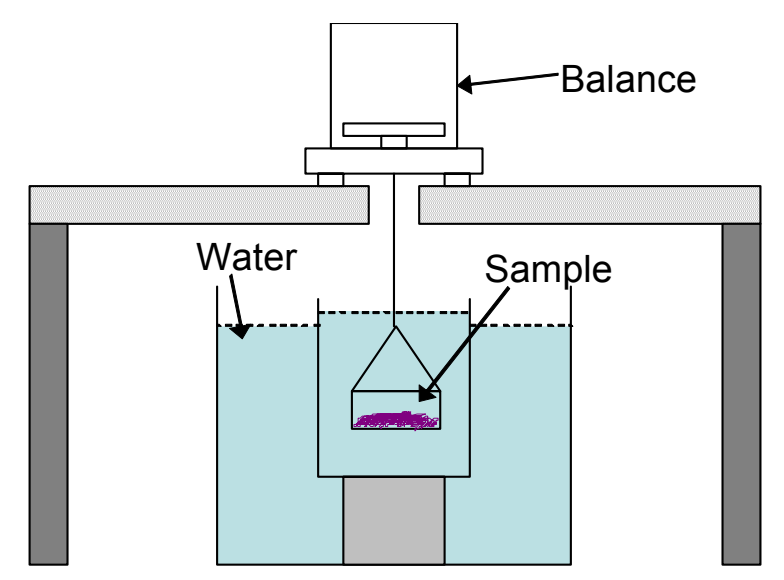

Figure 2-4 - Experimental Setup for the absorption rate method 


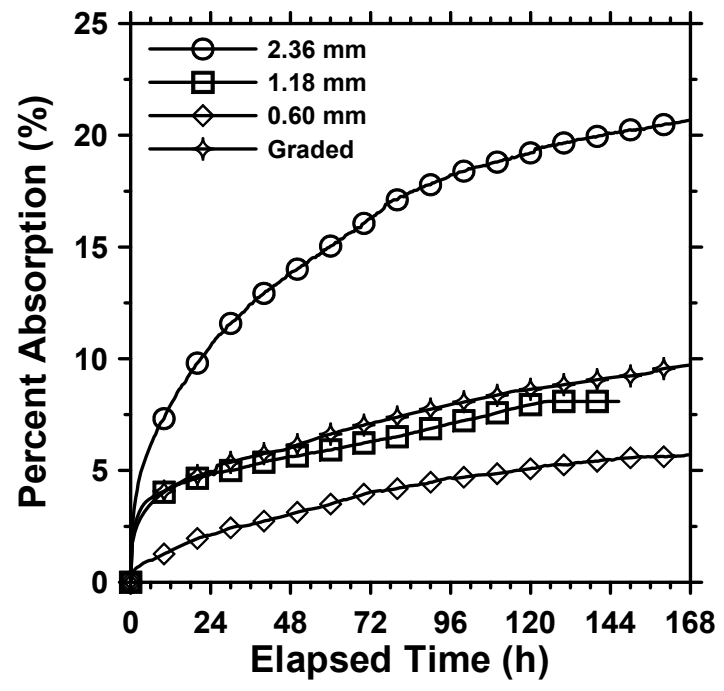

(a)

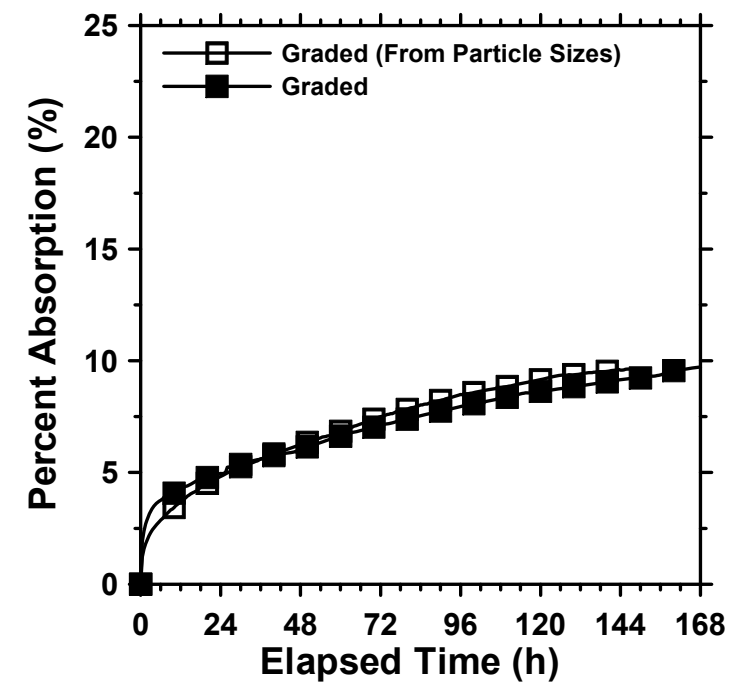

(b)

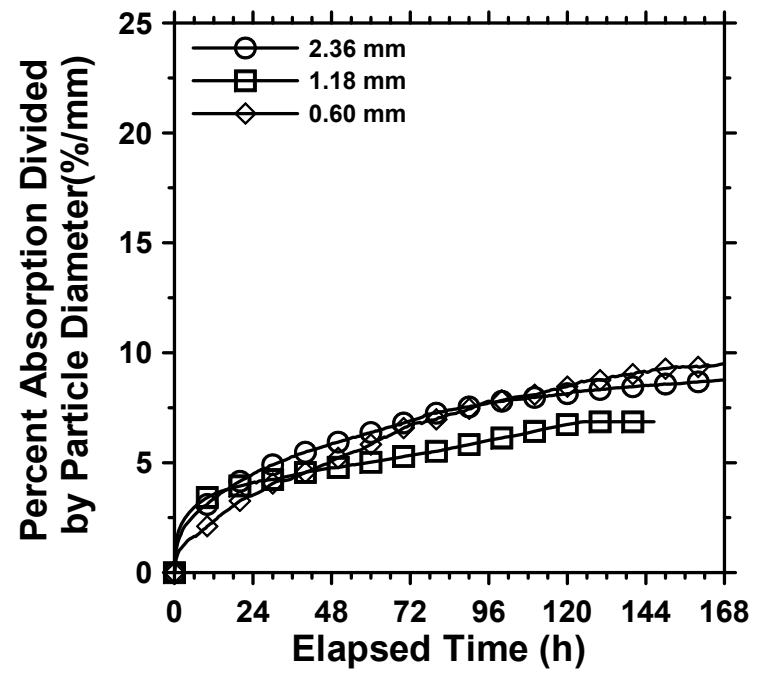

(c)

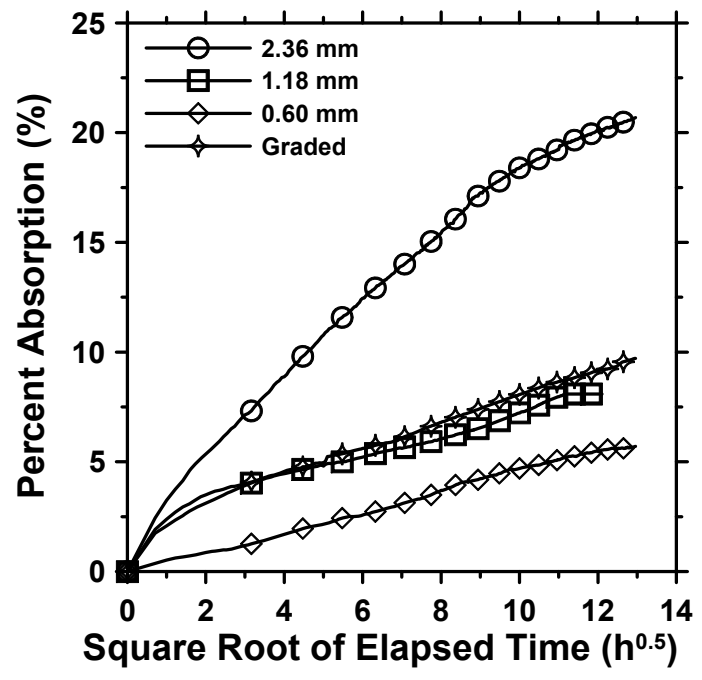

(d)

Figure 2-5 - (a) the absorption of different grain sizes of LWA-K along with a graded sample, (b) the absorption of the graded sample along with a graded sample reconstructed for the individual grain size absorption, (c) absorption divided by the particle diameter as a function of time (d) the absorption of LWA-K as a function of the square root of time 


\subsubsection{Pycnometer Method}

Another method which does not involve drying the aggregate until surface moisture is gone is the pycnometer method. The pycnometer used is shown in Figure 2-6, and had a volume of $50 \mathrm{~mL}$. When the stopper is put in, any water in excess of $50 \mathrm{~mL}$ is forced out through the small capillary opening in the stopper, resulting in a constant volume of $50 \mathrm{~mL}$. A known mass of oven-dried aggregate was placed in the pycnometer to which deionized water was added. The pycnometer was quickly shaken to remove the air bubbles, and the stopper was inserted resulting in a volume in the pycnometer of exactly $50 \mathrm{~mL}$. As the LWA absorbs water, the volume in the pycnometer falls below $50 \mathrm{~mL}$. After $24 \mathrm{~h}$, water was added to the pycnometer to fill in the space of the water absorbed by the LWA. The $24 \mathrm{~h}$ absorption of LWA-K was determined to be $7.9 \%$ with a standard deviation between measurements of $0.15 \%$.

The drawback to this method is that there was approximately $15 \mathrm{~s}$ between the time water was added the time the stopper was inserted, so again the initial absorption was not captured. Black (Black 1986) and Saxer (Saxer 1956) suggested that attempts to measure aggregate absorptions by immersion techniques will not succeed unless it is possible to begin measuring milliseconds after the aggregate comes in contact with water. This could explain the lower absorptions measured by these methods. 


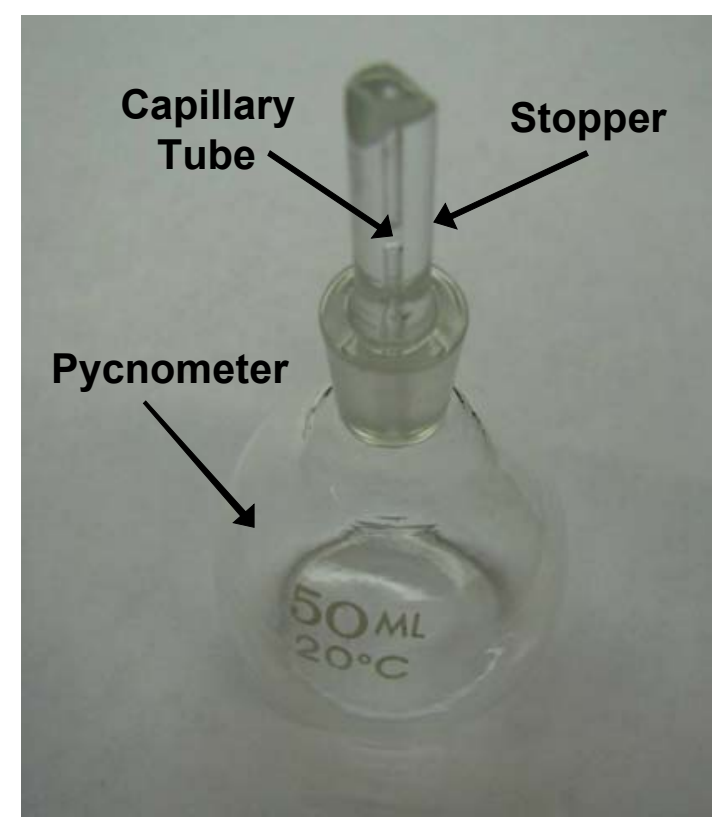

Figure 2-6 - Pycnometer used to determine absorption of LWA

\subsubsection{Comments and Recommendations}

From Table 2-1, it can be seen that the results between these techniques can vary substantially. The immersion techniques yield a lower absorption than the drying techniques. This is because the initial absorption cannot be captured, and these techniques should not be used unless this period can be accurately captured. The drying techniques seem to produce similar results, however it cannot be substantiated that this is the true absorption value. Further efforts are needed to determine the actual absorptions of LWA since they can have an effect on the shrinkage performance. This effect will be discussed in 2.4.

\subsection{Techniques for Determining the Pore Size Distribution}

Not only is it important that the LWA absorb a significant portion of water to be effective, it must be able to release that water to the cement paste. One key feature that is needed for a LWA to be an effective internal curing agent is that it 
must have pores that are larger than the pores of the cement paste. This enables the LWA to release its water at high relative humidities as the cement paste self-desiccates. Two methods are commonly used to determine if a LWA posses these properties. These two methods will be discussed in the following sections.

\subsubsection{Mercury Intrusion Porosimetry}

Mercury intrusion porosimetry (MIP) was used to estimate the pore size distribution of LWA-K and LWA-H. Mercury intrusion porosimetry is based on the principle that a non-wetting liquid will only intrude capillaries under pressure. The relationship between the pressure and capillary diameter is described by the Washburn equation (Washburn 1921) (Equation 2-2).

$P=\frac{-4 \gamma \cos \theta}{d}$

Equation 2-2

where: $\mathrm{P}(\mathrm{Pa})$ is the pressure, $\gamma(\mathrm{N} / \mathrm{m})$ is the surface tension of mercury, $\theta$ (radians) is the contact angle (assumed to be 117 degrees), and $d(\mathrm{~m})$ is the diameter of the pore being intruded with mercury. Using the Washburn equation the pore size distribution was determined for LWA-K and LWA-H. Figure 2-7(a) shows the results of MIP for the LWA along with a $7 \mathrm{~d}$ old w/c 0.30 cement paste. From this it can be seen that the pores of the LWA are larger than the pores of the cement paste. The major advantage of using MIP for determining the pore size distribution is that it is a simple and quick technique.

There are several drawbacks of using mercury intrusion porosimetry for determining the pore size distribution (Diamond 2000). The first is that the Washburn equation assumes cylindrical interconnected pores. As a result, MIP provides a coarse determination of the pore size distribution for LWA. Mercury intrusion porosimetry does not measure the true distribution of pore size 
geometries. If large internal pores are accessible by very narrow openings, MIP misrepresents the size of these pores as having the diameter of the openings, this is known as the ink bottle effect (Diamond 1970; Lura 2003a).

There can also be significant particle-to-particle variation when using MIP to determine the pore size distribution of LWA. Figure 2-7(b) shows the pore size distribution of two single particles of LWA-K. Due to the firing and crushing process during manufacturing, some particles will have lower and more closed porosity (Spaulding 2007). In order to reduce the variability of the measurements, multiple particles are placed in a single penetrometer, as to determine the average of all particles. This method does introduce new variability. As mercury is introduced into the penetrometer, the interparticle spaces could be confused with pores of the LWA.

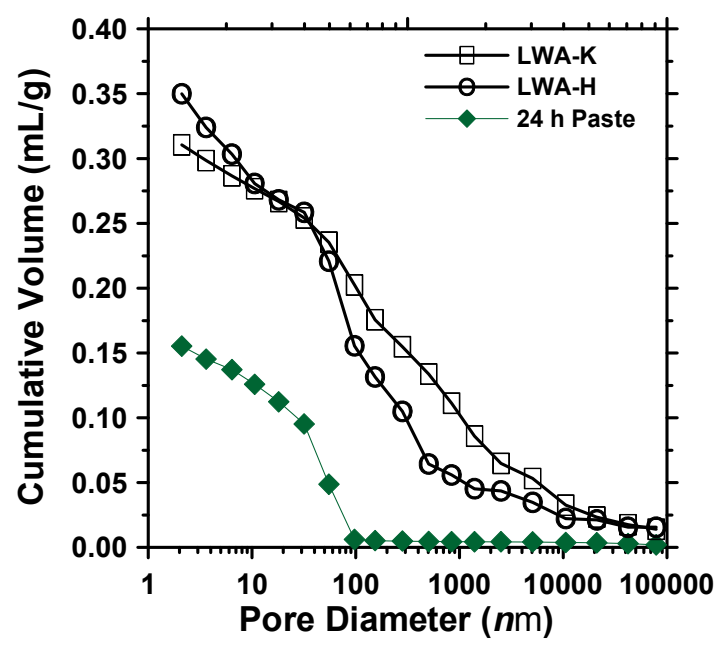

(a)

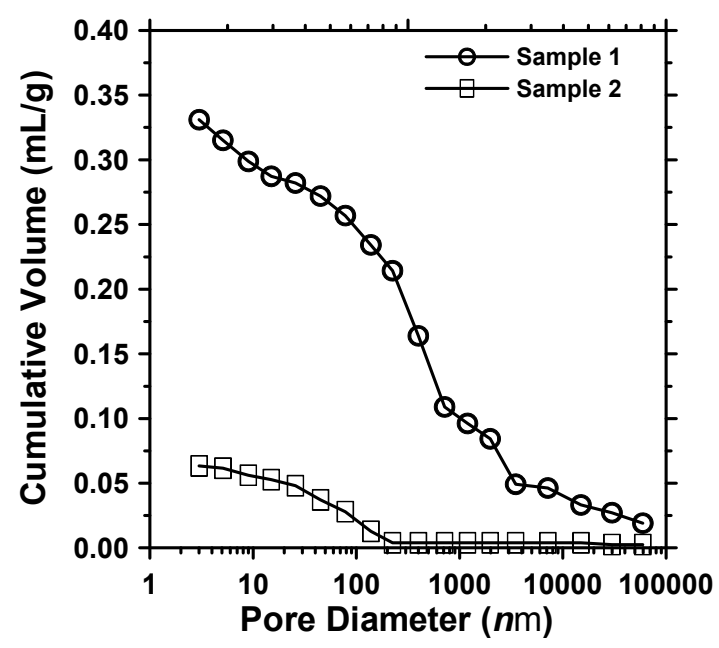

(b)

Figure 2-7 - Pore size distribution from mercury intrusion porosimetry for (a) LWA-K, LWA-H, and a $24 \mathrm{~h}$ old cement paste with a w/c of 0.30 and (b) two different LWA-K samples showing the variation in pores of LWA particles 


\subsubsection{Desorption Isotherm}

Figure 2-8(a) shows the desorption isotherm of LWA-K, LWA-H and CRCA. A majority of water is lost at a high relative humidity ( $\mathrm{RH}>96 \%)$ for the LWA-K and the LWA-H, implying the pores in the LWA are large $(>25 \mathrm{~nm})$ and the water is available to be lost from the aggregate to the cement paste at high relative humidities, which is preferred for internal curing. It is also important to note that the samples release over $96 \%$ of their moisture when a relative of humidity of $92 \%$ is reached, which implies that the water will leave the pores of the LWA if a large enough suction pressure (i.e., a low enough internal relative humidity) exists. Less than half of the water in the CRCA is available at high relative humidities, making it less suitable as an aggregate for internal curing (Kim et al. 2008). The pore size distribution can be back-calculated from the desorption isotherm using the Kelvin-Young-Laplace equation. Figure 2-8(b) shows the pore size distribution of LWA-K and LWA-H from MIP and the desorption isotherm. It should be noted that each pore size distribution is normalized to its own maximum cumulative volume. Figure 2-8(b) shows that MIP and desorption isotherms yield similar pore size distributions for LWA. Additional recent studies have utilized an automated machine called the Q5000 to determine the desorption isotherm (Castro et al. 2010). 


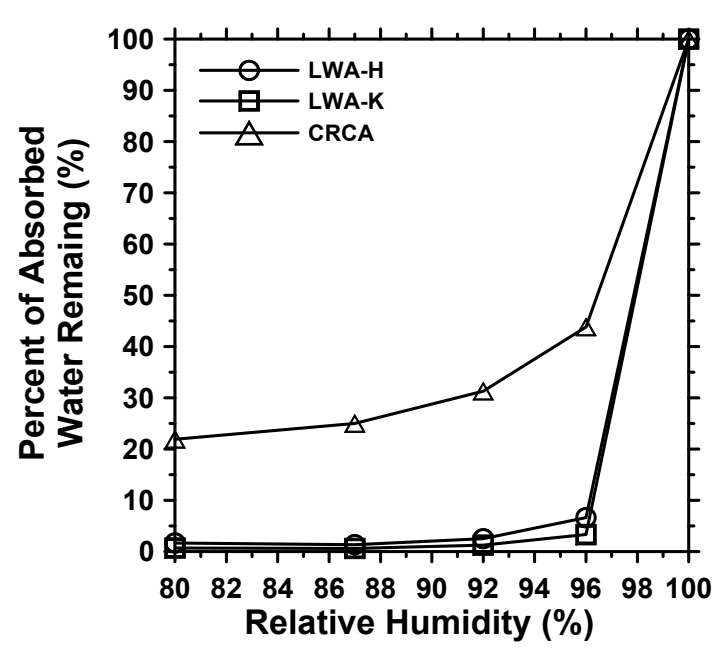

(a)

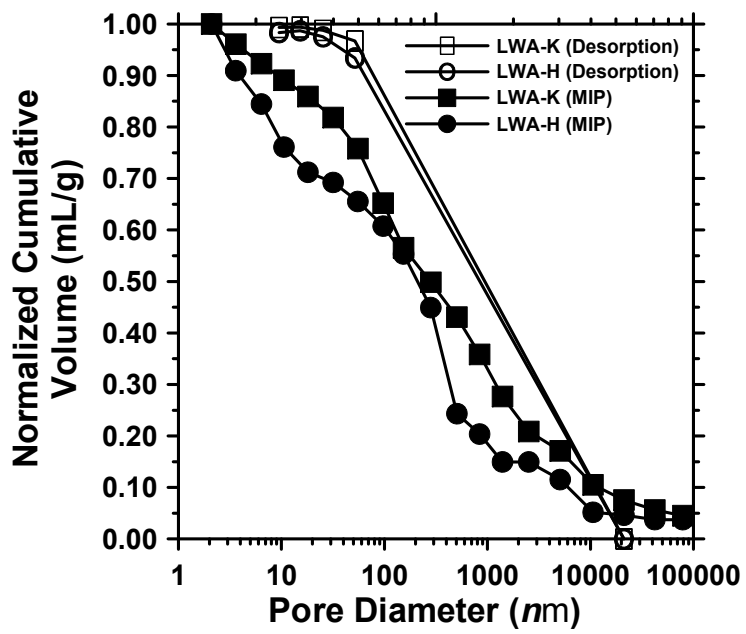

(b)

Figure 2-8 - (a) desorption isotherm of different aggregate, and (b) the pore size distribution of the LWA determined from the desorption isotherm

\subsection{Implications of These Properties on Shrinkage}

\subsubsection{Materials and Testing Procedures}

Four mortar mixtures were made using different aggregate (LWA-K, LWA-H, CRCA and normal weight sand) and the unrestrained shrinkage was measured using the corrugated tube protocol (Sant et al. 2006). The mixture proportions can be found in Table 2-2. The mixtures had a consistent volume of paste and a portion of the normal weight fine aggregate was replaced with LWA. The $25.3 \% \mathrm{k}$ and $25.3 \%$ hixtures had similar replacement volumes of LWA, but because the LWA-H absorbs less water, it provides less additional water for internal curing. The $25.3 \% \mathrm{k}$ and $19.4 \% \mathrm{CRCA}$ mixtures provided a similar volume of water but because of the different absorption and specific gravities of the aggregates, the amount of LWA used is slightly different. From a volumetric perspective, the mixtures were the same. It should be noted that the $25.3 \% \mathrm{k}$ and $19.4 \% \mathrm{CRCA}$ volume replacements correspond to the amount of LWA necessary to eliminate 
self-desiccation from Equation 2-1 (Bentz et al. 1999b; Bentz et al. 2005) using the absorption as determined from ASTM C128. Though there is a similar absorption between the LWA-K and the CRCA, the difference in density requires a different volume replacement between the LWA-K mixture and the CRCA mixture.

Table 2-2 - Mixture proportions for LWA variability study

\begin{tabular}{|c|c|c|c|}
\hline Material & $\mathbf{2 5 . 3 \% k}$ & $\mathbf{2 5 . 3 \% h}$ & $\mathbf{1 9 . 4 \% C R C A}$ \\
\hline Cement $\left(\mathbf{k g} / \mathbf{m}^{\mathbf{3}}\right)$ & 728 & 728 & 728 \\
\hline Water $\left(\mathbf{k g} / \mathbf{m}^{\mathbf{3}}\right)$ & 218 & 218 & 218 \\
\hline Fine Aggregate $\left(\mathbf{k g} / \mathbf{m}^{\mathbf{3}}\right)$ & 808 & 808 & 917 \\
\hline Dry LWA $\left(\mathrm{kg} / \mathbf{m}^{\mathbf{3}}\right)$ & 369 & 369 & 396 \\
\hline $\begin{array}{c}\text { Additional Water Provided } \\
\text { by LWA }\left(\mathbf{k g} / \mathbf{m}^{\mathbf{3}}\right)\end{array}$ & 39 & 22 & 39 \\
\hline
\end{tabular}

\subsubsection{Results and Discussion}

Figure 2-9 shows the unrestrained shrinkage measurements for the first $96 \mathrm{~h}$. The implication of the variability of the absorption can be shown by observing the $25.3 \% \mathrm{k}$ and $25.3 \% \mathrm{~h}$ mixtures. While LWA-K and LWA-H have similar desorption responses, LWA-H consistently absorbs less water than LWA-K using different testing methods. The $25.3 \% \mathrm{k}$ and $25.3 \% \mathrm{~h}$ mixtures show a similar expansion during the first $12 \mathrm{~h}$. However the $25.3 \% \mathrm{k}$ mixture continues to expand while the $25.3 \% \mathrm{~h}$ mixture stabilizes. At this point the LWA-H has likely released a majority of its water, while the LWA-K still retains water in its pores (Henkensiefken et al. 2008d).

The $19.4 \%$ CRCA has a vastly different shrinkage response from the other two mixtures. This mixture has considerable shrinkage strain even though a large 
amount of water is supplied. This is due to the different desorption response of the CRCA. This aggregate releases $55 \%$ of its water at high relative humidities (>92 \%) as opposed to the other LWA that release $96 \%$ of their moisture. Thus it is important not only to select an aggregate that absorbs a large amount of water, but is able to release that water at early ages.

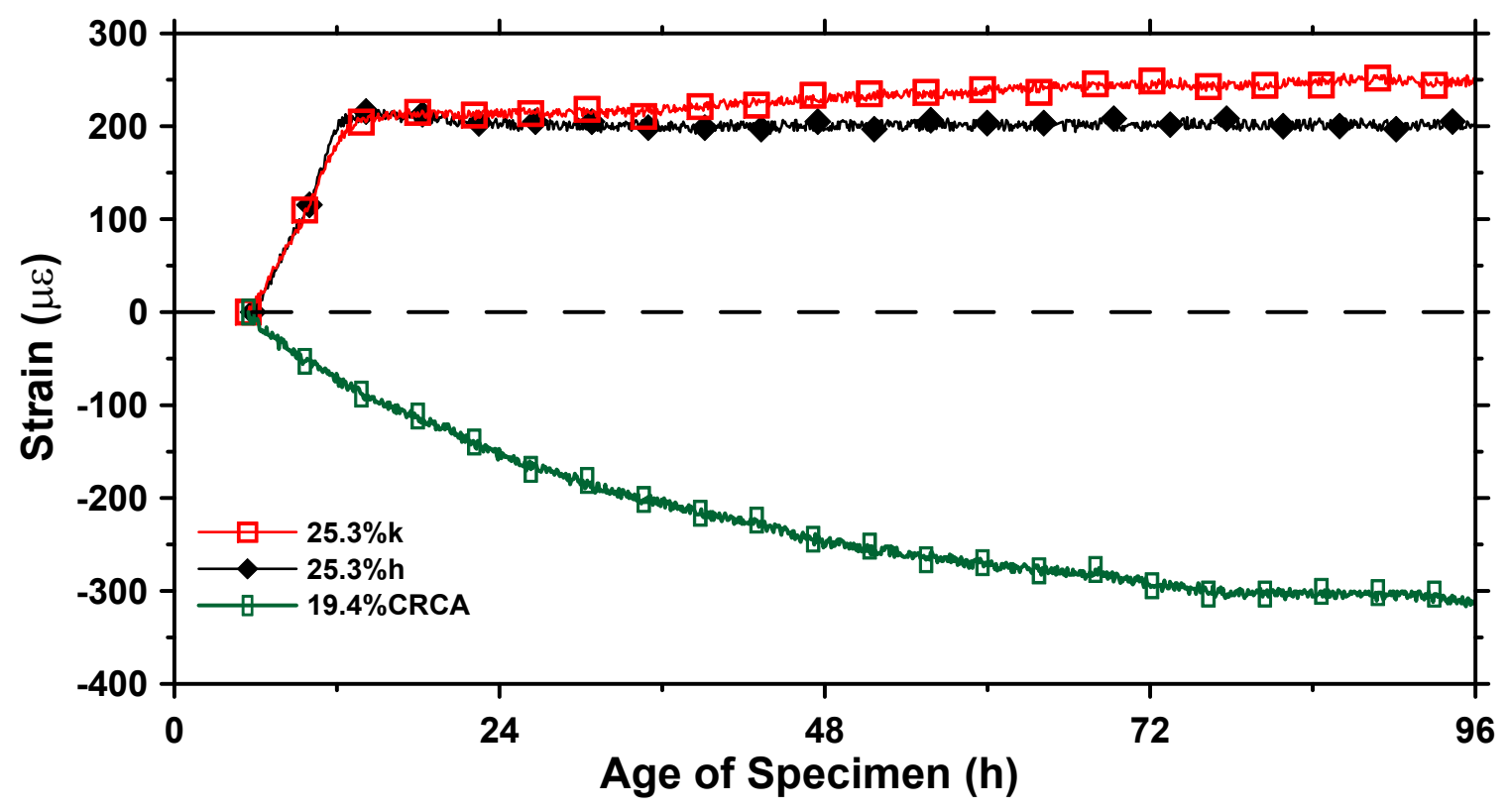

Figure 2-9 - Unrestrained shrinkage measurements for mortar made with different LWA during the first $96 \mathrm{~h}$

\section{5. $\underline{\text { Conclusions }}$}

This chapter has presented different techniques for determining the absorption and pore size distribution of different LWA. The variability of the absorption capacity obtained using different techniques was presented. Different methods were presented, surface drying methods and submersion methods. Because of the difficulty to capture the first seconds of absorption from submersion methods, the submersion methods should not be used. The cobalt(II) chloride method and the standard cone method provide similar results. The standard cone method is 
a simple method and should be used in lieu of a more appropriate method. Two different techniques for measuring the pore size distribution were presented. The importance of the absorption capacity on the shrinkage performance was presented. If the aggregate selected does not have the necessary properties, the shrinkage performance can be adversely affected. Criteria for selecting aggregates that will release water and thus be effective internal curing agents were presented. 


\section{CHAPTER 3. MECHANISMS OF INTERNAL CURING}

Water is released from water-filled prewetted lightweight aggregate (SLWA) when it is used for internal curing. As such, the IC water needs to be described in three main ways: (1) the volume of water available for IC, (2) the ability of the water to leave the SLWA when needed for IC, and (3) the distribution of the SLWA so that it is well-dispersed and its water can readily travel to all of the sections in the paste where it is needed. This section provides a brief overview of each of these topics.

\subsection{Volume of Water Needed for IC}

In order to determine the total volume of water that is needed for IC, two terms must be defined, chemical shrinkage and autogenous shrinkage.

Chemical shrinkage, the primary cause of autogenous shrinkage and selfdesiccation, is a naturally occurring process that has been known for over 100 years (La Chatelier 1900; Powers 1935; L'Hermite 1960; Geiker 1983). Chemical shrinkage describes the total volume reduction of the cement system occurring during hydration of cement (Sant et al. 2006), since the volume of the hydration products is less than the volume of the reactants. It has been reported that this reduction can be as much as $8 \%$ to $10 \%$ by volume in a mature paste (Jensen et al. 2001a; Jensen et al. 2001b). Chemical shrinkage describes the total volume reduction of the system and is typically measured by placing cement paste in water and measuring the volume change of the cement-water system 
(Knudsen et al. 1982). When water is able to permeate the paste, it enables the total volume change to be measured (it is important to note that in low $\mathrm{w} / \mathrm{c}$ systems the depercolation of the paste's capillary porosity may occur and water cannot travel through the paste) (Knudsen et al. 1982). Work by Sant has shown that the depercolation occurs for a $3 \mathrm{~mm}$ cement paste sample with a w/c of 0.30 at approximately $24 \mathrm{~h}$ (Sant 2007). A typical chemical shrinkage result is shown in Figure 3-1 for a paste with w/c of 0.30 by mass fraction (made with the same cement as used in this study). While the buoyancy measurement technique was used to obtain the data in Figure 3-1 (Sant et al. 2006), other procedures could be used such as the ASTM C1608 standard test method for the measurement of chemical shrinkage (2005).

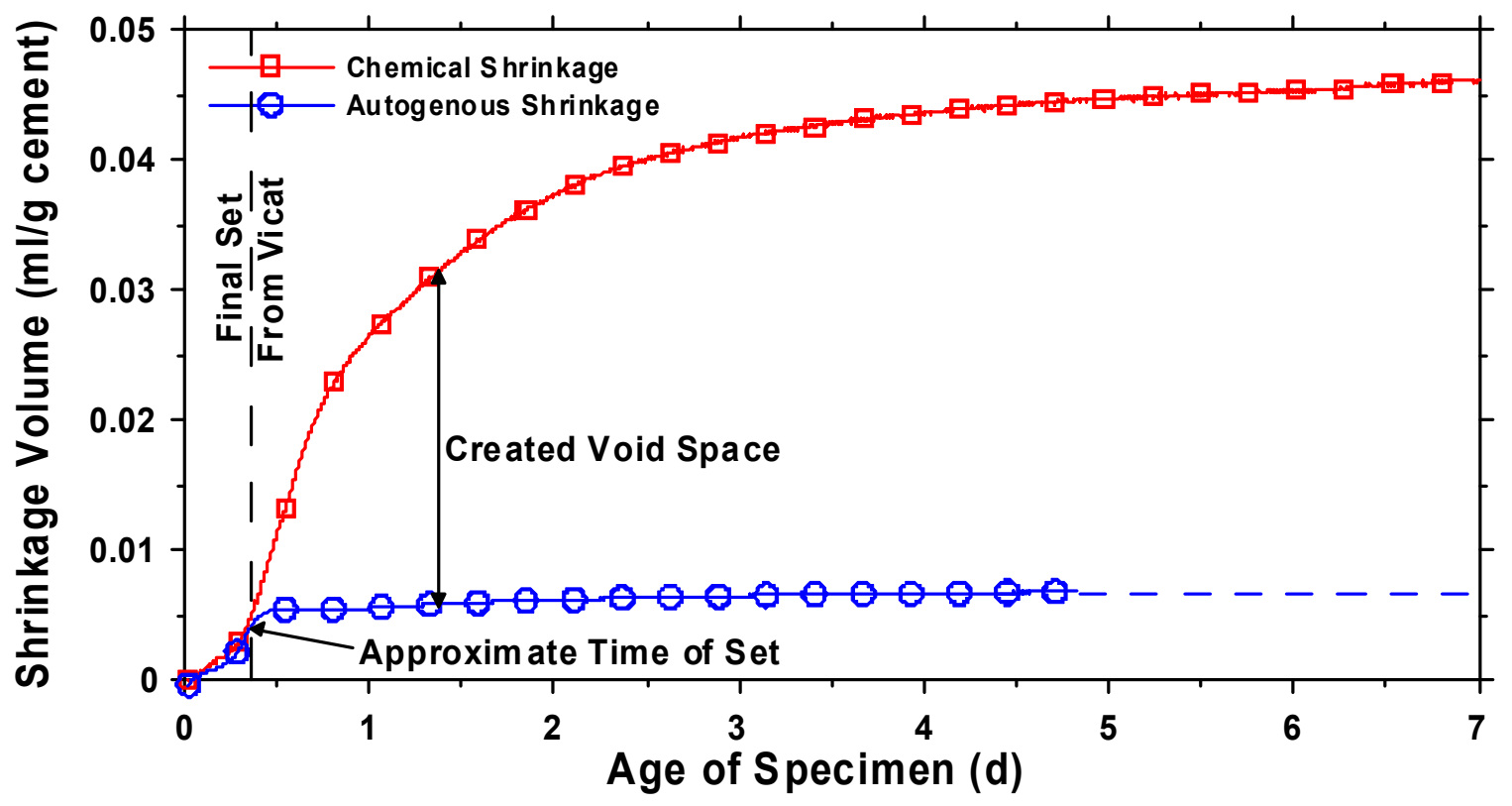

Figure 3-1 - Chemical shrinkage and autogenous shrinkage volumes during hydration of a paste with a w/c of 0.30 (Henkensiefken et al. 2008f)

Autogenous shrinkage can be described as the external volume reduction of a cement paste as it hydrates (Jensen 2005). Before set, the volumes of chemical shrinkage and autogenous shrinkage are the same. This occurs since the 
system is fluid and collapses on itself as it shrinks (Barcelo et al. 2000; Bjøntegaard et al. 2004; Sant et al. 2006). At the time of set, however, the volumes of chemical shrinkage and autogenous shrinkage diverge, as shown in Figure 3-1 (Henkensiefken et al. 2008f). The solidification of the matrix prevents the bulk system from shrinking as chemical shrinkage occurs. This results in an underpressure in the fluid that eventually cavitates vapor-filled space in the pore system (Couch et al. 2006). As hydration proceeds, these voids grow and penetrate smaller and smaller pores. The internal relative humidity can be measured in these concretes to assess the pressure that develops in the fluid.

The concept in using SLWA for IC is that the SLWA can be used as internal reservoirs that can reduce the pressure in the pore fluid by 'replenishing the vapor-filled voids,' effectively eliminating their creation within the hydrating cement paste. While strictly speaking, the volume of water that is needed is only the difference between these curves, accounting for the behavior prior to set will generally be only a minor correction and may be unnecessary for field applications. Therefore, the volume of chemical shrinkage may be approximated as the volume of water that needs to be supplied by the IC agent.

Bentz and Snyder (Bentz et al. 1999b) used this principle to develop a method to estimate the volume of IC water that is needed and the mass of lightweight aggregate (LWA) that is needed to hold the IC water (Bentz et al. 1999b; Bentz et al. 2005).

$$
M_{L W A}=\frac{C_{f} \times C S \times \alpha_{\max }}{S \times \phi_{L W A}}
$$

where: $\mathrm{M}_{\mathrm{LWA}}\left(\mathrm{kg} / \mathrm{m}^{3}\right.$ or $\left.\mathrm{lbs} / \mathrm{yd}^{3}\right)$ is the mass of LWA (in a dry state) that needs to be prewetted to provide water to fill in the voids created by chemical shrinkage, $\mathrm{C}_{f}\left(\mathrm{~kg} / \mathrm{m}^{3}\right.$ or $\left.\mathrm{lbs} / \mathrm{yd}^{3}\right)$ is the cement content of the mixture, CS ( $\mathrm{g}$ of water per $\mathrm{g}$ of 
cement or $\mathrm{lb}$ of water per $\mathrm{lb}$ of cement) is the chemical shrinkage of the cement, $\alpha_{\max }$ (unitless) is the expected maximum degree of hydration (0 to 1 ), $S$ (unitless) is the expected degree of saturation of the LWA ( 0 to 1$)$ and was taken to be 1 in this study when the LWA was soaked for $24 \mathrm{~h}$, and $\Phi_{\text {LWA }}$ (kg of water $/ \mathrm{kg}$ of dry LWA or $\mathrm{lb} / \mathrm{lb}$ ) is the absorption capacity of the LWA (taken here as the $24 \mathrm{~h}$ absorption). An absorption period of $24 \mathrm{~h}$ was chosen to represent "saturated" conditions in this study. Care must be taken when using the term "saturated" when describing LWA. Because of the pore structure of LWA, it is likely that the LWA is not in a truly saturated state. The pores of the LWA could continually absorb water for several weeks or months if left submerged. When the term "saturated" is used here, it is not intended to mean all the pores of the LWA are filled with water, but rather to a specific degree of saturation (Henkensiefken et al. 2008c). Further details can be found in chapter 2 of this thesis. A more correct approach to determining $\Phi_{\mathrm{LWA}}$ would be to use the measured desorption capacity of the LWA from saturation down to $92 \% \mathrm{RH}$ for example, as in an actual concrete, since the LWA is initially "saturated" and undergoes desorption during IC (Bentz et al. 2005).

\subsection{Ability of Water to Leave the LWA}

Water leaves the SLWA due to the suction (underpressure) that develops in the pore fluid within the hydrating cement paste due to its chemical shrinkage and self-desiccation. The consequence of this water movement from SLWA to surrounding paste is frequently quantified in mortar as an increase in internal relative humidity and an increase in the critical pore size that remains prewetted (Lura 2003a; Henkensiefken et al. 2008f). The basic principle for internal curing is that the largest pores will lose water first as the capillary stress that develops will be minimized when pores are emptied in this order. The LWA pores are generally larger than the pores of the surrounding cement paste. The pore size distributions of hydrated cement paste at three different ages and of two different 
LWA were determined using mercury intrusion porosimetry (MIP), the results of which can be seen in Figure 3-2. Figure 3-2 demonstrates that the pores of both the LWAs used in this study are larger than the pores of the cement paste, even at early hydration ages. It should be noted that the maximum standard deviation of the MIP results for the LWA and the cement paste (based on the total absorption) was $0.03 \mathrm{~mL}$ per gram of sample and $0.01 \mathrm{~mL}$ per gram of cement, respectively. Another important feature from the MIP pore size distribution is that the pores of the paste decrease in size as the specimen ages (or as hydration continues). As the pores in the paste decrease in size, the capillary pressure that would develop upon their emptying increases, effectively increasing the driving force that pulls water out of the LWA. This implies that as the specimen ages, the hydrating paste can pull water out of successively smaller pores of the LWA. Figure 3-3 illustrates desorption isotherms of two LWA (LWA-K and LWA$\mathrm{H}$ ). The standard deviation between duplicate samples was $0.3 \%$. A majority of water is lost at a high relative humidity $(\mathrm{RH}>96 \%)$ implying the pores in the LWA are large $(>25 \mathrm{~nm})$ and the water is available to be lost at high relative humidities, which is preferred for internal curing. It is also important to note that the samples release almost all (96\%) of their moisture when a relative of humidity of $92 \%$ is reached, which implies that the water will leave the pores of the LWA if a large enough suction pressure (or a low enough internal relative humidity) exists. It should be noted that this favorable desorption behavior is not characteristic of all LWA (Lura 2003a). 


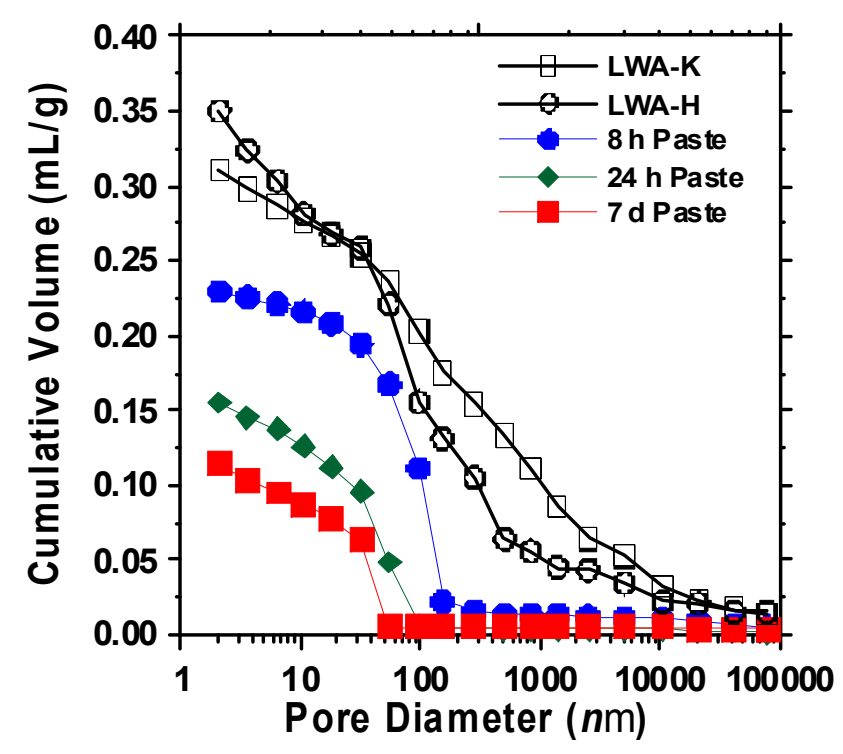

Figure 3-2 - Pore size distribution for cement paste with a $w / c$ of 0.30 at three different ages and for two different LWA measured using mercury intrusion porosimetry

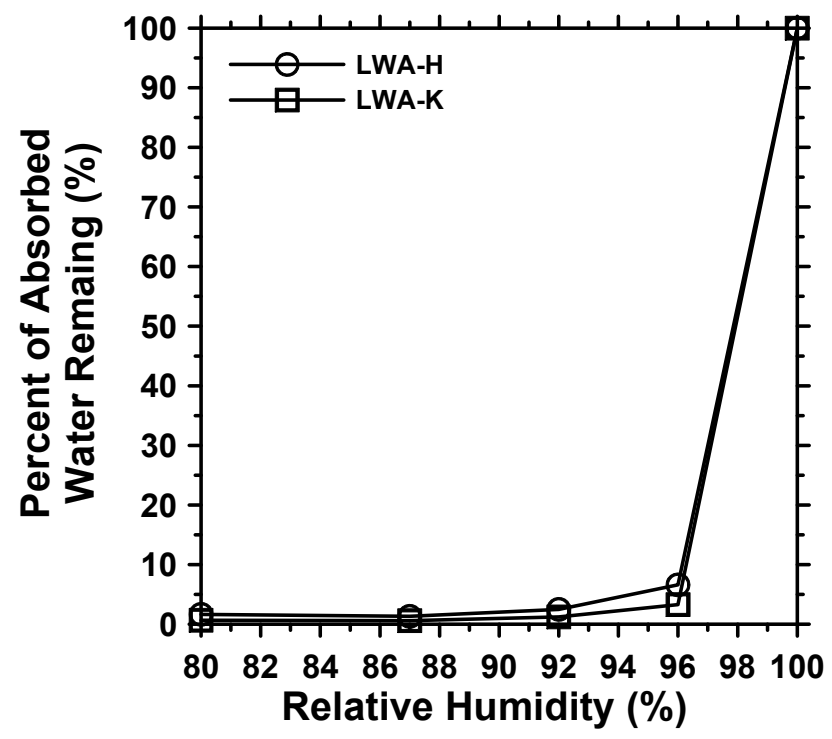

Figure 3-3 - Desorption isotherm of two different LWA

The formation of the liquid-vapor menisci in the paste pores can ultimately lead to shrinkage of the three-dimensional microstructure. These menisci represent a 
suction pressure that developed in the fluid and is higher than the pressure in the vapor. As a result, the radius of curvature of the meniscus (critical Kelvin radius) that develops can be approximated as the size of the largest pore that is still prewetted. The Young-Laplace equation can be used to relate the size of the menisci to the capillary pressure (Equation 3-2):

$\sigma_{\text {cap }}=-\frac{2 \gamma \cos (\theta)}{r}$

Equation 3-2

where: $\sigma_{\text {cap }}(\mathrm{Pa})$ is the capillary pressure, $\gamma(\mathrm{N} / \mathrm{m})$ is the surface tension of pore fluid, $\theta$ (radians) is the liquid-solid contact angle (assumed to be 0 radians), and $r$ $(\mathrm{m})$ is the radius of curvature of the meniscus or the pore size. It can be argued that the smaller meniscus radius in the low $w / c$ mixtures corresponds to a higher capillary pressure, resulting in higher shrinkage. The capillary pressure can also be related to the internal equilibrium relative humidity as represented by Kelvin's equation (Equation 3-3):

$\sigma_{\text {cap }}=\frac{R T \ln (R H)}{V_{m}}$

Equation 3-3

where: $R$ is the universal gas constant $(8.314 \mathrm{~J} / \mathrm{mol} \cdot \mathrm{K}), T(\mathrm{~K})$ is the thermodynamic temperature, $R H(\%)$ is the internal relative humidity, and $V_{m}(\approx$ $18 \times 10^{-6} \mathrm{~m}^{3} / \mathrm{mol}$ ) is the molar volume of pore solution. The capillary pressure that develops can be related to material behavior such as the shrinkage strain, $\varepsilon_{\mathrm{p}}$, using a modified version of Mackenzie's equation which is described in Equation 3-4 (Bentz et al. 1998):

$\varepsilon_{p}=\frac{S}{3} \sigma_{c a p}\left(\frac{1}{K}-\frac{1}{K_{s}}\right)$

Equation 3-4

where: $S$ (unitless) is the degree of saturation of the cement paste, $K(\mathrm{~Pa})$ is the paste's or mortar's bulk modulus, and $K_{s}(\mathrm{~Pa})$ is the modulus of the solid skeleton of the cement paste or mortar. Mackenzie's equation is not the only model that 
can describe the shrinkage of cement paste. Recently another model has been developed to describe the shrinkage of cement paste due to capillary stresses (Vlahinic et al. 2008). Though Equation 3-4 describes the shrinkage of the paste, the shrinkage of concrete can be described using Pickett's Equation (Henkensiefken et al. 2008f). The continued consumption of water due to hydration results in the volume of vapor filled voids progressively increasing and extending into smaller voids, corresponding to smaller radii of curvature. This results in a continuous increase in the capillary pressure, a reduction in the equilibrium relative humidity, and an increase in autogenous shrinkage.

As previously mentioned, the largest pores will empty first so that the LWA acts as a sacrificial water reservoir that can be used to replenish the pores of the paste. This concept is illustrated in Figure 3-4 (Radlinska et al. 2008b; Henkensiefken et al. 2008e). In a sealed system without LWA, pores smaller than a specific size $\left(r_{1}\right)$ will remain saturated. In a LWA system, the emptying pores can be replenished from water in the LWA, provided the water is stored in a larger pore $\left(r_{2}\right)$. It should be noted that this is for conceptual purposes and that the pores of the LWA may eventually empty, depending on the extent of selfdesiccation/hydration. However, in that case, the size of the pores that are still saturated will be larger in the LWA system than in the system without LWA. 

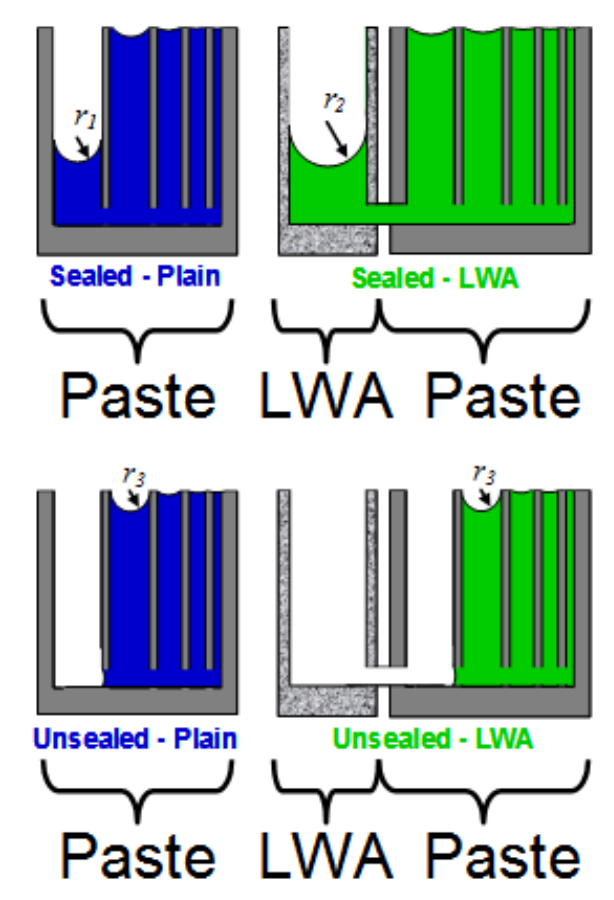

Figure 3-4 - Conceptual illustration of the menisci formation in sealed and unsealed specimens made of plain and SLWA concrete (Henkensiefken et al. 2008e)

\subsection{LWA Spacing}

Knowing how much water is needed for IC (Section 3.1) and knowing how much of that water will likely leave the aggregate (Section 3.2), the last issue that needs to be understood is the distribution of the SLWA throughout the microstructure. Even if a sufficient volume of water is supplied to a system, as determined from Equation 3-1, if the distribution of the water (i.e., SLWA) is insufficient, the system will likely exhibit poor shrinkage performance. This has been shown by comparing the effectiveness of coarse SLWA and fine SLWA when the same volume of water is considered (van Breugel et al. 2000; Zhutovsky et al. 2002). Though the volume of water may be the same, the distribution of the SLWA particles will be much different, resulting in a different volume of protected paste (i.e., the volume fraction of the paste within a given distance from a SLWA particle). The coarse SLWA proved to be less effective 
than the fine SLWA even though they had the same volume of water because of the increased spacing between the aggregate.

The concept of protected paste volume can also be applied when different volumes of fine SLWA are considered. For a constant LWA particle size distribution, when the volume of SLWA is increased, not only is the volume of water increased, but the distribution of water is also improved, which results in a larger fraction of protected paste. The effect of different volume replacements on the protected paste volume was simulated using the hard core/soft shell model developed at the National Institute of Standards and Technology (NIST) (Lu et al. 1992; Maekawa et al. 1999; Bentz et al. 1999a). The results of simulations for the mortars investigated in this study are shown in Figure 3-5. Figure 3-5 describes the volume fraction of cement paste that is within a given distance from the surface of a SLWA particle. It is not intended to show that the paste is protected by internal water stored in the SLWA, since properties like absorption and desorption of the aggregate as well as paste permeability would have to be known to determine whether the water in the SLWA could reach the paste. As the volume replacements are increased, the fraction of paste within a specified proximity of a SLWA also increases. When lower replacements are incorporated in a mixture, there is a rapid increase in the fraction of paste within $1.0 \mathrm{~mm}$ of a SLWA particle. At low replacement volumes, if the water in the SLWA can travel up to $1.0 \mathrm{~mm}$, a majority of the paste will be protected. When higher replacement volumes are used, a larger fraction of paste is within $0.20 \mathrm{~mm}$, which may be important at later ages (RILEM 2007). This is because water cannot travel as far in a mature paste, and the distribution of the SLWA becomes important. This implies that if water is able to travel a large distance, a low replacement volume can protect a majority of the paste (assuming the SLWA can provide enough water). However, if the water cannot travel large distances, a higher replacement volume is needed to protect a majority of the paste. 


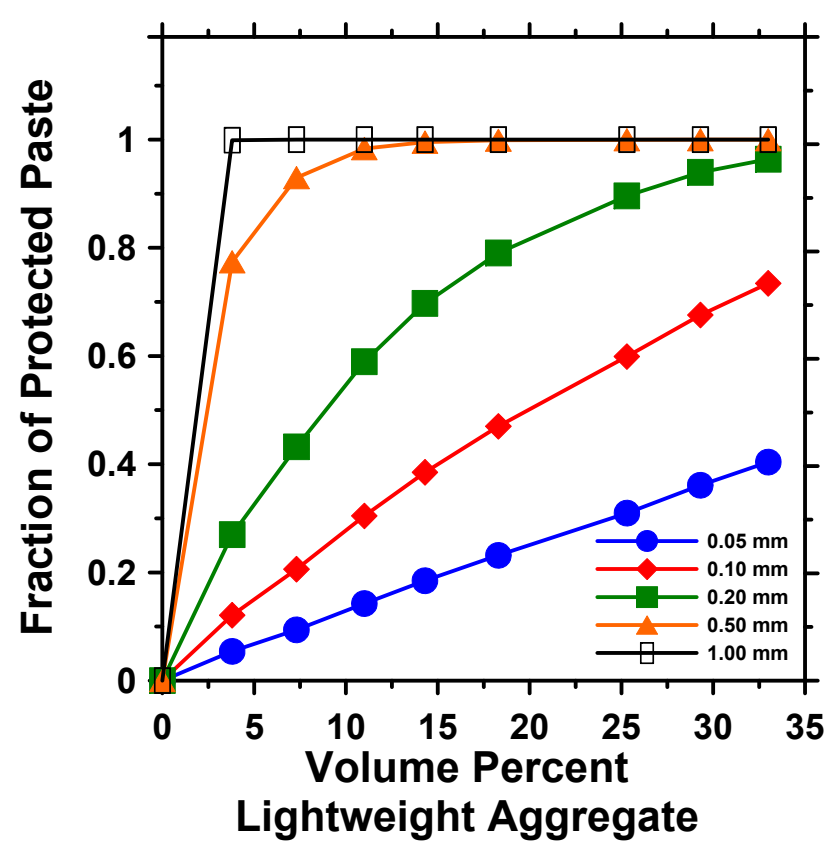

Figure 3-5 - Volume fraction of paste within a specified distance of a SLWA. This plot does not show whether water can reach the paste. Volume and travel distance of water need to be considered to determine this

Figure 3-6 shows the protected paste concept when the same volume replacement of LWA is presented. Figure 3-6(a) indicates the protected paste volume when coarse aggregate is used. Figure $3-6(\mathrm{~b})$ has the same replacement volume of aggregate; however the aggregate replaced is the fine aggregate. A clear distinction can be made in the protected paste volume in these two figures. Because of the better particle distribution, the fine aggregate has more potential to protect the surrounding cement paste than coarse aggregate. Using fine aggregate instead of coarse aggregate could have implications on the strength of the mixture. Replacing the coarse normal weight aggregate with coarse LWA could have detrimental effects on the strength of the concrete. When dealing with higher strength concretes, the aggregate particles will likely be the region of failure, and introducing large weak particles into the system could reduce the strength. By replacing the fine normal weight aggregate 
with fine LWA, the effects if including a weaker aggregate could be reduced since the fine aggregate does not affect the strength of the concrete as much as the coarse aggregate.

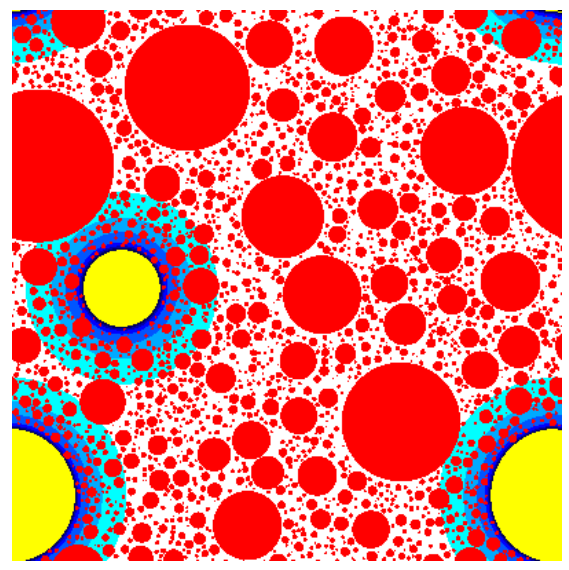

(a)
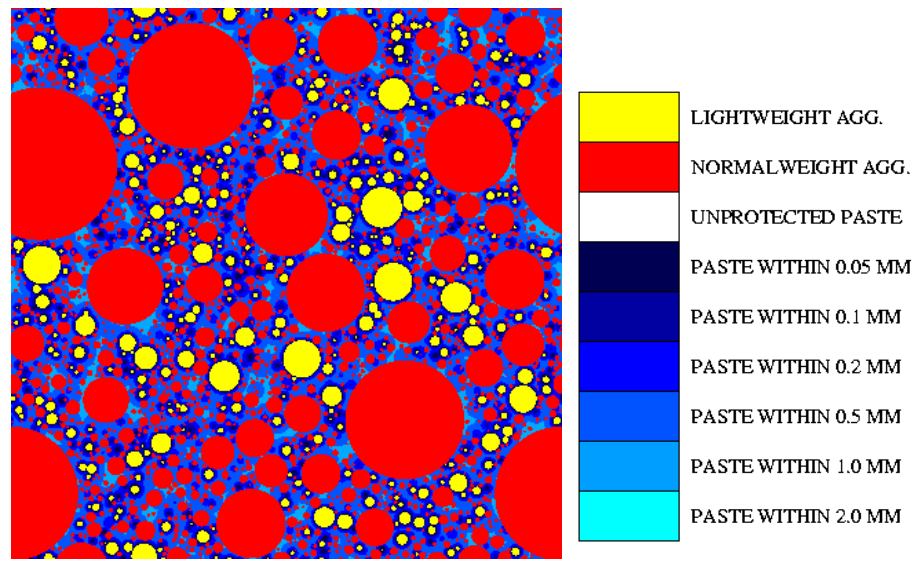

(b)

Figure 3-6 - Illustrations showing the protected paste volume concept of two mixtures with similar LWA replacements volumes, but one replacement is of (a) coarse aggregate, and the other of (b) fine aggregate (Henkensiefken 2008a)

\subsection{The Drying Mechanisms in Sealed and Unsealed Systems}

\subsubsection{Sealed System}

As previously discussed, vapor-filled spaces are created in the paste due to chemical shrinkage, as shown in Figure 3-7(a). The creation of vapor-filled spaces results in the reduction of the internal relative humidity of a sealed sample, called self-desiccation, and could be considered similar to 'internal drying'. In low w/c mixtures, the radius of curvature of these vapor-filled voids would be smaller than in higher $w / c$ mixtures due to the decreased interparticle spacing, resulting in a significant shrinkage-inducing capillary tension $\left(\sigma_{c a p}\right)$ from Equation 3-2. 


\subsubsection{Unsealed System}

For an unsealed specimen that loses moisture externally, liquid-vapor menisci also form (Radlinska et al. 2008b). However, in this case, they form at the surface of the concrete. This is schematically shown in Figure 3-7b and Figure 3-7(c). Figure 3-7(b) shows a hypothetical case in which only external drying occurs and self-desiccation is not included. Figure 3-7(c) shows a more realistic picture of a cement paste that is unsealed at one surface. In this case, while external drying occurs at the exposed surface, self-desiccation develops throughout the interior of the material. This suggests that while a sealed specimen only experiences internal drying (Figure 3-7(a)), an unsealed system would simultaneously undergo both internal and external drying (Figure 3-7(c)).

The formation of a liquid-vapor menisci corresponds with a reduction in the internal relative humidity of cement paste. In an unsealed specimen, as moisture evaporates from the surface, the radius of the menisci continues to decrease. This causes the drying front to penetrate into smaller openings and to gradually grow from the surface towards the interior of the material. The internal relative humidity is proportional to the menisci radius and thus decreases continuously as drying progresses until the internal relative humidity of the cement paste reaches equilibrium with the ambient relative humidity. Since any system will ultimately come to equilibrium with the ambient relative humidity, this will occur at an equal pore radius $\left(r_{3}\right)$, as illustrated in Figure 3-4. In an IC concrete however, the water must leave the LWA first, which could result in a reduction in the rate of shrinkage. 


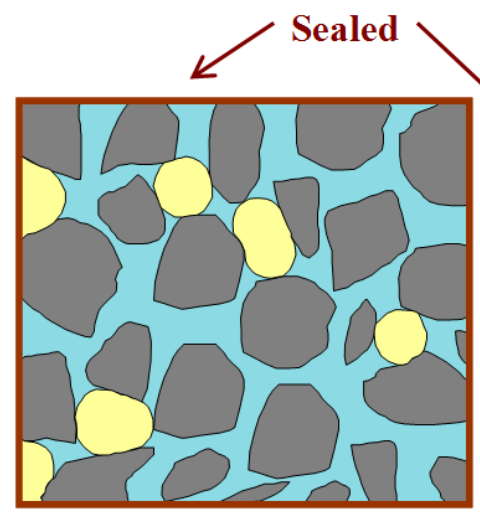

(a) Sealed

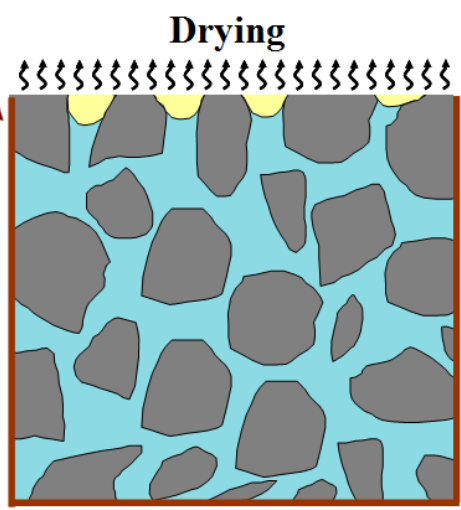

(b) External Drying Only liquid

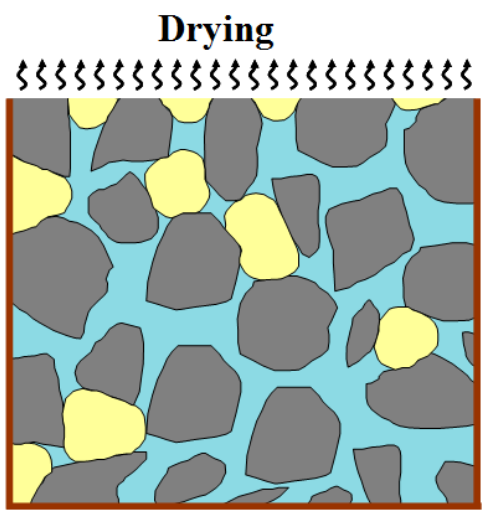

(c) Unsealed solid vapor

Figure 3-7 - Illustration of drying mechanisms in sealed and unsealed systems: (a) Sealed - only internal drying, (b) Only external drying, (c) Unsealed - internal plus external drying (Radlinska et al. 2008b) 


\title{
CHAPTER 4. MONITORING WATER MOVEMENT FROM PREWETTED LIGHTWEIGHT AGGREGATE USING X-RAY ABSORPTION
}

\begin{abstract}
4.1. Introduction
Internal curing (IC), as described in this chapter, uses prewetted (water-filled) lightweight aggregates (SLWA) to supply additional water to the cement paste as it hydrates (Philleo 1991; ACl 2004). Internal curing can be used to produce a dense crack-free microstructure, which is the desire of using a low $w / c$, by supplying additional water to avoid the detrimental effects of autogenous shrinkage (Jensen 2005). Significant research has been performed on the topic of internal curing (Al-Khaiat et al. 1998; Jensen et al. 2001a; Lura et al. 2004; RILEM 2007; Radlinska et al. 2008b; Henkensiefken et al. 2008e). Benefits of internal curing have been shown to include reduced shrinkage of sealed concrete (Lura et al. 2004; Henkensiefken et al. 2008e), increased compressive or flexural strength (especially at later ages) (Al-Khaiat et al. 1998), reduced potential for cracking (Radlinska et al. 2008b; Henkensiefken et al. 2008e) and increased durability (RILEM 2007). Far fewer studies have been conducted to examine other aspects of internal curing, including the timing of water release from the lightweight aggregate and the distance the water travels through the surrounding cement paste.
\end{abstract}

The goal of this study is to build on earlier studies (Bentz et al. 2006a; Lura et al. 2006a; Bentz et al. 2006b) and provide information that can lead to an improved understanding of the timing and distance of water movement from prewetted lightweight aggregates to the paste in concrete mixtures with internal curing. Specifically, the objectives of this chapter are: 
- to use x-ray absorption measurements to trace changes in sample density associated with moisture movement from prewetted lightweight aggregate into the surrounding paste,

- to demonstrate the importance of sample alignment in x-ray absorption measurement and data interpretation,

- to relate moisture transport with the time of paste solidification and chemical shrinkage measurements,

- to provide experimental measures of moisture transport distance from LWA to the surrounding cement paste that can indicate how far water can travel in a hydrating system, and

- to show how particle spacing influences gradation selection, mixture proportioning, and mixture design for IC concrete.

\subsection{Background on Internal Curing}

The volume of cementitious systems typically reduces as the cement reacts with water (hydration). This process is called chemical shrinkage (La Chatelier 1900; L'Hermite 1960; Sant et al. 2006). Before the paste hardens (sets), chemical shrinkage causes the paste to collapse upon itself resulting in an external reduction in volume (autogenous shrinkage) that is equal to the chemical shrinkage. After the paste has set however, the system can no longer collapse upon itself due to the resistance provided by the hardening paste structure. Since hydration still occurs after set, in the absence of an additional supply of "curing" water, the volume reduction due to chemical shrinkage results in the creation of vapor-filled void spaces in the pore structure of the paste (Couch et al. 2006), as shown in Figure 4-1 (Henkensiefken et al. 2008b). The volume of the vapor-filled space increases over time. As the vapor invades smaller and smaller pores, an increased pressure develops in the remaining fluid. This 
pressure can be substantial in low $w / c$ mixtures. Lightweight aggregate can be used to supply water to fill the vapor-filled space during hydration to mitigate the effects of self-desiccation (i.e., reduce the pressure) in low $w / c$ concrete mixtures.

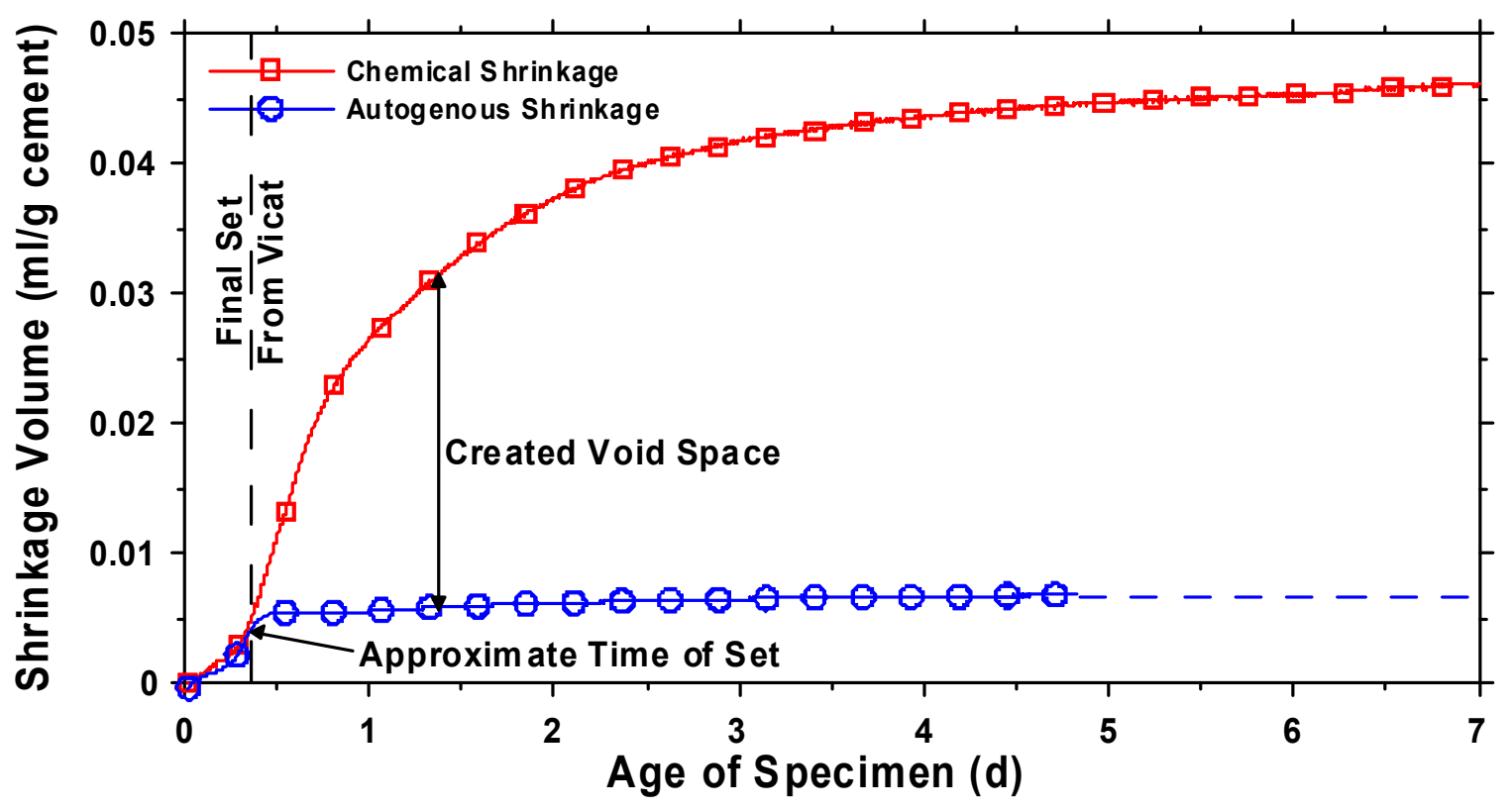

Figure 4-1 - Chemical shrinkage and autogenous shrinkage volumes during hydration of a paste with a $w / c$ of 0.30

The foremost process only focuses on the volume of water needed to fill the voids created by chemical shrinkage. However, as cement paste hydrates, it becomes significantly less permeable and water may only be able to travel distances of a few millimeters. Because of this, the LWA volume fraction and LWA particle size distribution need to be carefully considered during mixture proportioning to ensure that a majority of the paste is within this travel distance of the SLWA (water). For this reason, finer and better dispersed SLWA particles may provide more effective internal curing (van Breugel et al. 2000). 


\subsection{Background on the Detection of Water Movement Using X-ray Absorption}

X-ray absorption can be used to detect a change in density that occurs as water enters or leaves the pores in a material (Lura et al. 2006a). In general, a less dense material absorbs less $\mathrm{x}$-ray radiation so that more radiation is transmitted through the sample. Therefore, as water is removed from the LWA, the density of the composite material would decrease and less x-ray radiation would be absorbed. X-ray absorption will be used in this work to track moisture movement from LWA to the surrounding cement paste.

Equation 4-1 describes the relationship between the incident intensity $x$-ray radiation $\left(\mathrm{I}_{0}\right)$ and the intensity of the radiation that is transmitted (passes) through a sample which contains LWA and cement paste (I $I_{\text {Measured }}$ ) (Sant et al. 2008a; Sant et al. 2008c).

$I_{\text {Measured }}=I_{0} \cdot \exp \left[-\left(\mu_{L W A} \cdot V_{L W A}+\mu_{\text {Paste }} \cdot V_{\text {Paste }}+\mu_{W} \cdot V_{W}+\mu_{V} \cdot V_{V}\right) \cdot t\right] \quad$ Equation 4-1

where: $\mu_{\mathrm{LWA}}$ and $V_{\mathrm{LWA}}$ are the linear attenuation coefficient $\left(\mathrm{cm}^{-1}\right)$ and the volume proportion (fraction) of the LWA, $\mu_{\text {Paste }}$ and $V_{\text {Paste }}$ are the linear attenuation coefficient $\left(\mathrm{cm}^{-1}\right)$ and the volume proportion (fraction) of the cement paste, $\mu_{\mathrm{W}}$ and $V_{W}$ are the linear attenuation coefficient $\left(\mathrm{cm}^{-1}\right)$ and volume proportion (fraction) of water contained in the pores in the LWA, $\mu_{V}$ and $V_{V}$ are the linear attenuation coefficient $\left(\mathrm{cm}^{-1}\right)$ and volume proportion (fraction) of vapor filled pores of the paste or LWA specimen, and $t$ is the thickness of the sample. As water is removed from the lightweight aggregate, the density (and overall $x$-ray attenuation) of the LWA decreases and the transmitted intensity of $x$-ray radiation measured at the camera (detector) increases. Consequently, the level of saturation at any time can be measured and quantified using this technique. 


\subsection{Previous Studies Using X-ray Absorption}

Bentz et al. (Bentz et al. 2006a) used x-ray microtomography to monitor water movement from SLWA particles to the surrounding hydrating cement paste in a w/c of 0.35 mortar containing a mixture of SLWA and normal weight sand. X-ray microtomography enabled water movement from the SLWA particles to be detected within a 3D system. Results indicated that much of the water in the SLWA was drawn out during the first $24 \mathrm{~h}$ of curing. In fact, the water detected as leaving the SLWA was in direct proportion to the measured chemical shrinkage of the hydrating cement paste. However, the distance of water movement into the cement paste could not be determined using this technique, only the rate at which water was being drawn out of the SLWA.

Another recent study (Lura et al. 2006a) addressed the distance water could travel. In this study, a paste ( $w / c$ of 0.30 ) was cast next to a single prismatic LWA particle. The sample was subsequently sealed and water movement was monitored over a $10 \mathrm{~d}$ period. It was observed that a majority of the water emptied from the pores of the LWA during the first few days of hydration and was able to travel at least $4 \mathrm{~mm}$ into the surrounding cement paste. A large 'interface' existed between the SLWA particle and the cement paste, which was proposed to be due to (1) the LWA not being perfectly prismatic, (2) the imperfect alignment between the LWA/paste interface and the x-ray beam, and (3) the resolution of the equipment used (approximately $160 \mu \mathrm{m}$ ).

This investigation builds on the previous studies. This chapter investigates the time at which water begins to leave the LWA and how far that water can travel into the surrounding cement paste. Special techniques will be used to address issues raised by the other studies, which include rotating the sample for proper alignment and employing an x-ray system with a finer resolution. 


\subsection{Improvement to Measuring Techniques}

In this work, the LWA particle to be tested was cut from a larger LWA particle using a small diamond saw. To align the LWA/paste interface with the x-ray beam, the composite sample was placed on a rotating table, as shown in Figure 4-2. The rotation is an important feature of this procedure and is illustrated in Figure 4-3. The sample in Figure 4-3(a) shows a misaligned sample, where the transmitted $x$-rays pass through both paste and LWA. In this case both the attenuation of the paste and LWA must be considered in the composite theory model (Equation 4-1). If the sample is properly oriented, as in Figure 4-3(b), and the interface is perfectly planar, the transmitted $x$-rays pass through either the paste or LWA.

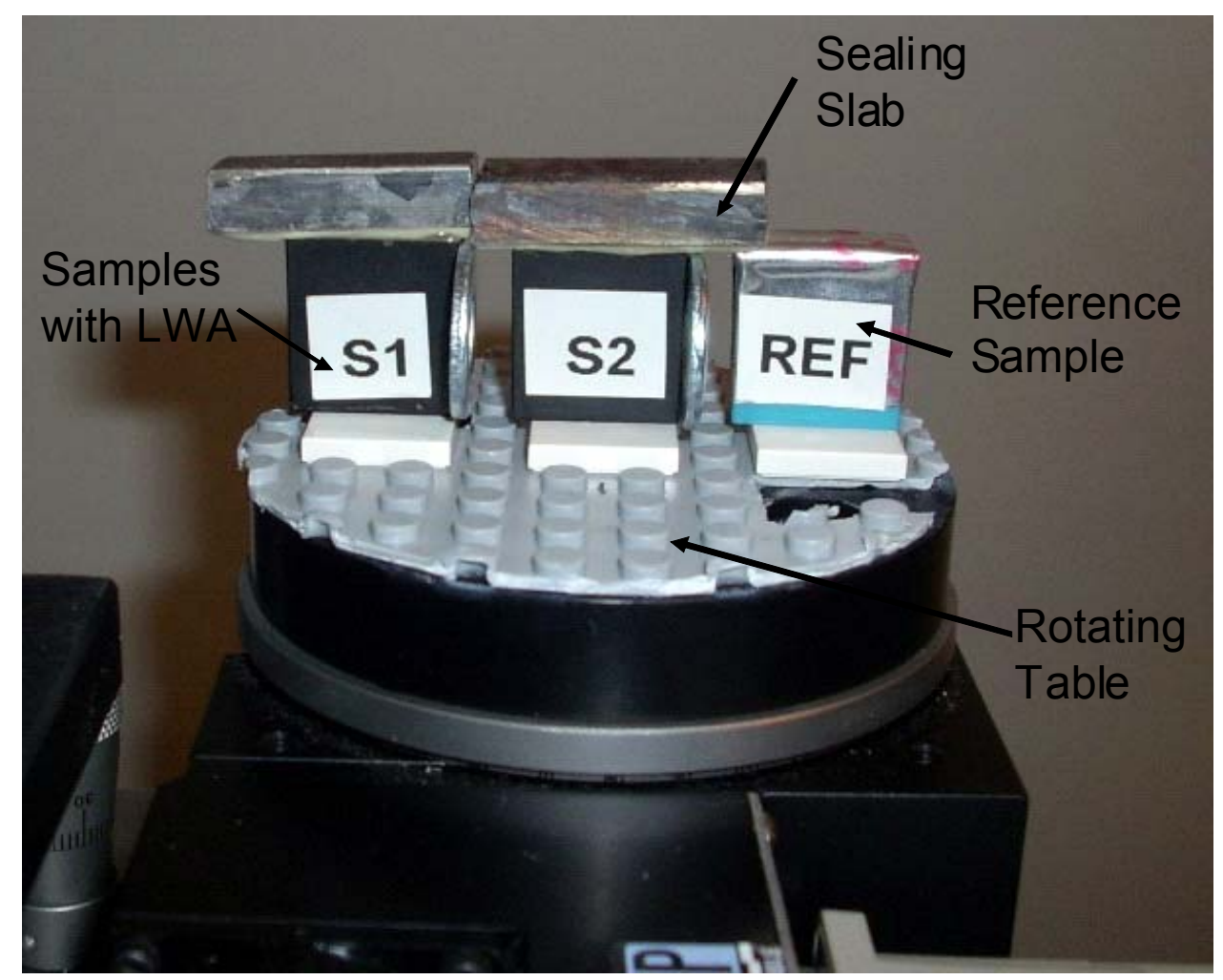

Figure 4-2 - Samples (used for determining travel distance of water) mounted on rotating table inside $x$-ray chamber 


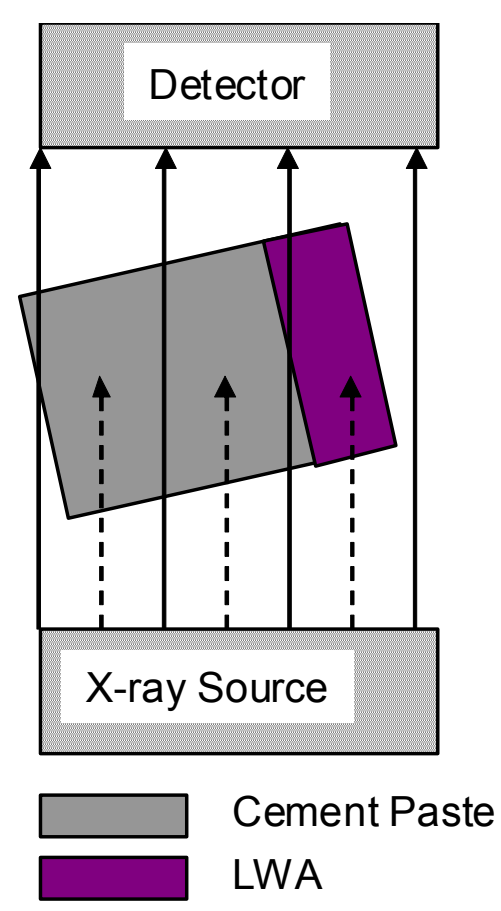

(a)

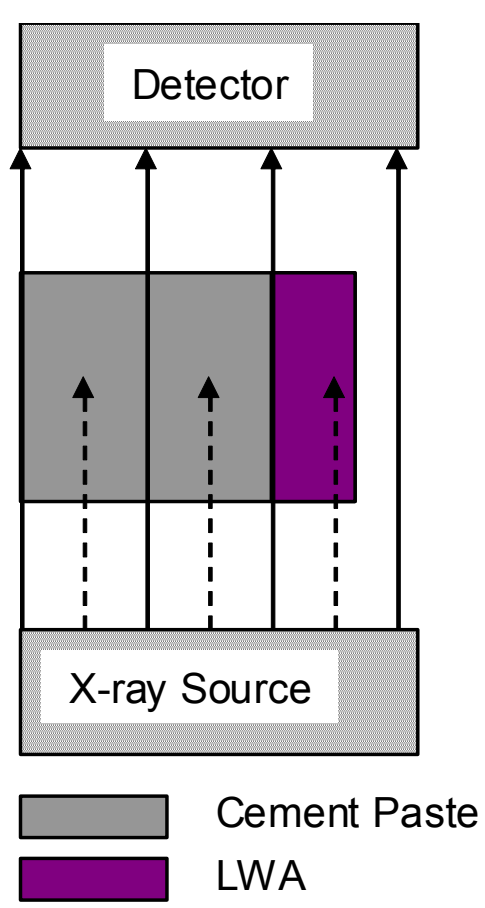

(b)

Figure 4-3 - Schematic (used for detecting travel distance of water) of (a) misaligned sample and (b) a properly oriented sample

The importance of the concept can be seen in Figure 4-4(a) through Figure 4-4(d). If the sample is not properly oriented, a blurry edge exists between the paste and LWA, which has been called the 'interface' in previous studies (Lura et al. 2006a). The effect of this on the x-ray measurements can be seen in Figure 4-5. As the sample is rotated to the correct angle of orientation, the region that reflects beam passing through both the paste and LWA is reduced. When the sample is properly oriented, a sharp edge is noticed where the decrease in the counts occurs. It should be noted that a small interface still exists even when the sample is properly aligned using this technique. An interface of approximately $0.20 \mathrm{~mm}$ exists in this experimental setup, as shown in Figure 4-6, for several reasons. Each pixel in the captured images has a resolution of approximately $0.02 \mathrm{~mm}$. Even if the sample were perfectly aligned, an interface of $0.02 \mathrm{~mm}$ 
would exist because at least one pixel would exhibit both the properties of the paste and the properties of the LWA. Though a rotating table was used to align the samples, the rotation was only uniaxial. Slight misalignment of the sample in the vertical direction and a "jagged edge" could result in a larger interface when all the vertical pixels are averaged together. It should be noted the edge of the LWA tested was also very porous. Paste could penetrate these large pores at the interface resulting in a region of mixed properties. The edge of the LWA may not be straight, resulting in a larger interface.

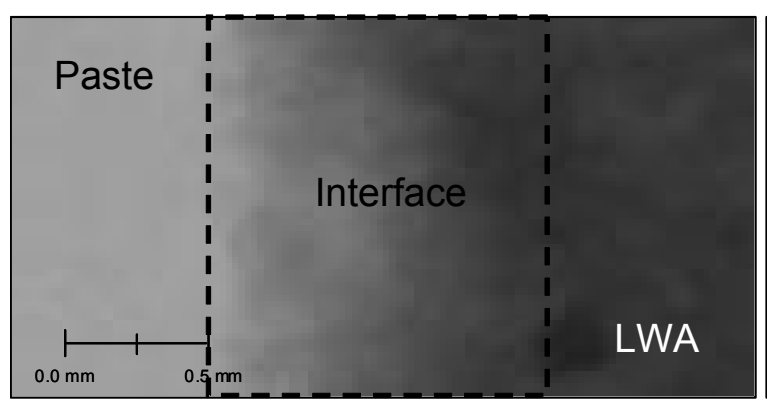

(a)

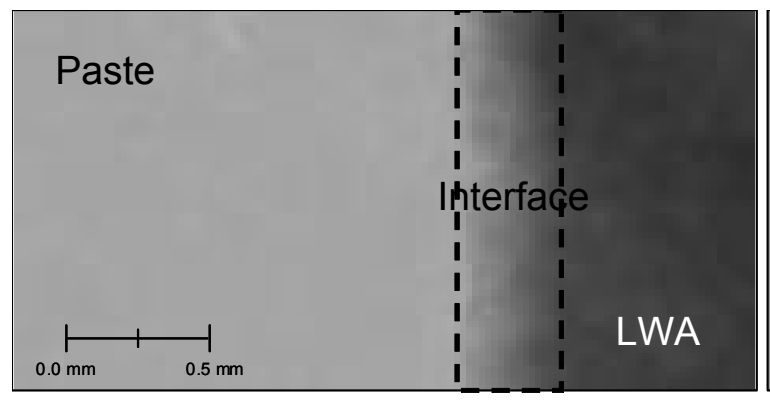

(c)

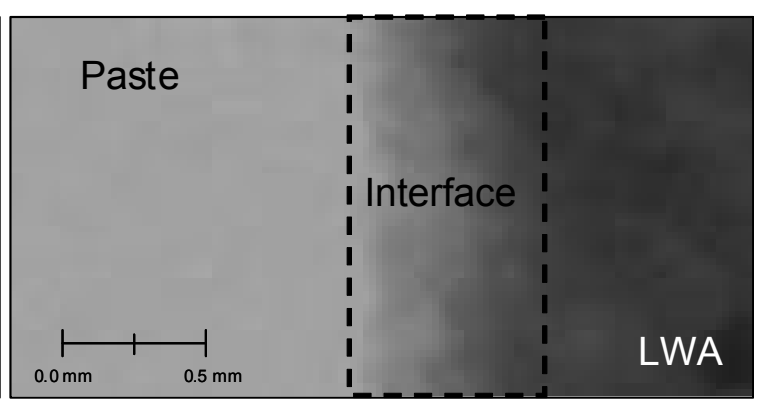

(b)

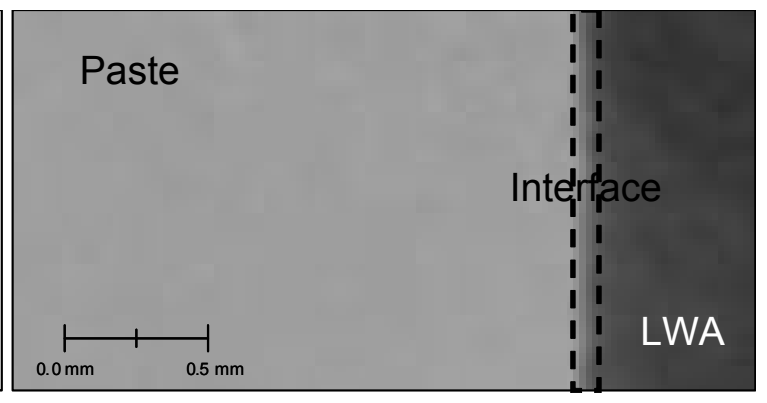

(d)

Figure 4-4 - X-ray image (used for detecting travel distance of water) of samples rotated to orientations of (a) -10.0 degrees, (b) -5.0 degrees, (c) -2.5 degrees and (d) 0.0 degrees 


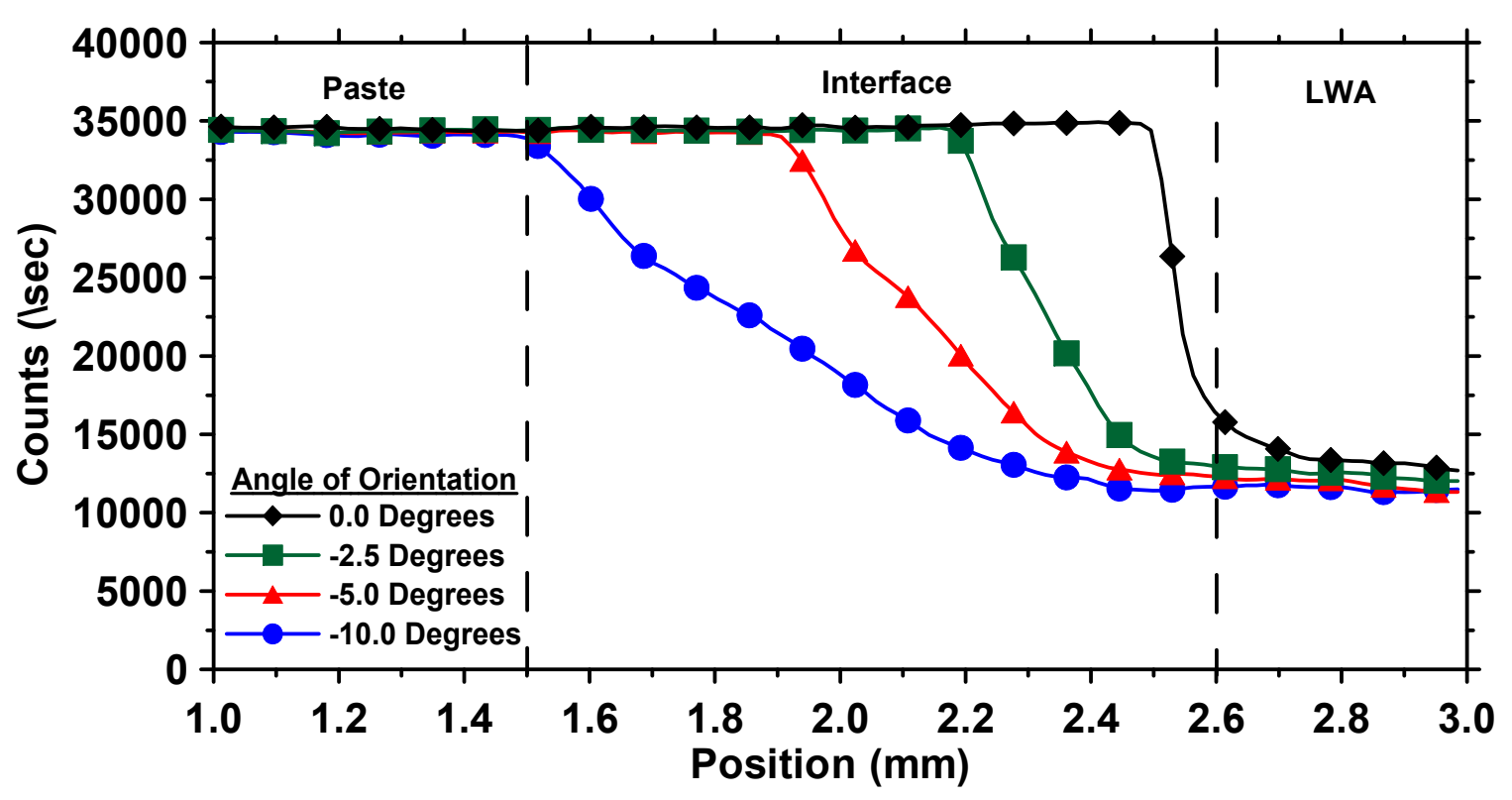

Figure 4-5 - Thickness of the LWA/paste interface as sample is rotated

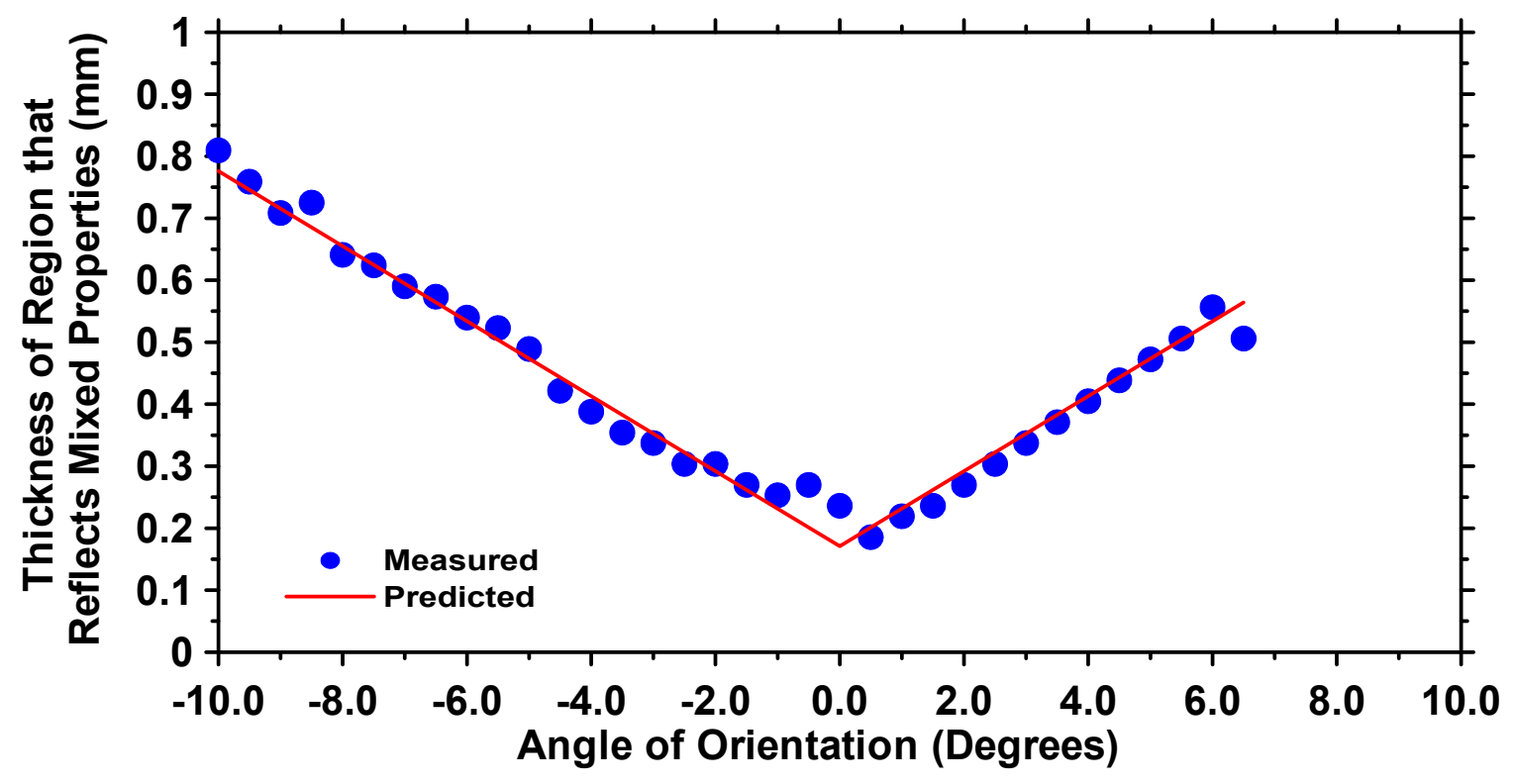

Figure 4-6 - Thickness of LWA/paste interface for different angles of orientation

The effect of rotating the samples from a fundamental perspective can be seen in Figure 4-7(a) that shows a schematic of a sample containing LWA and cement 
paste. Initially, the incident $x$-ray only comes in contact with LWA. If the sample were rotated about point $A$ (while the incident $x$-ray beam stayed fixed), LWA would leave the path of the beam and would be replaced by paste. This region then would exhibit mixed properties. As the sample is rotated, more LWA is replaced by paste, with the most significant effect occurring during the first 0.30 degrees of rotation, as shown in Figure 4-7(b).

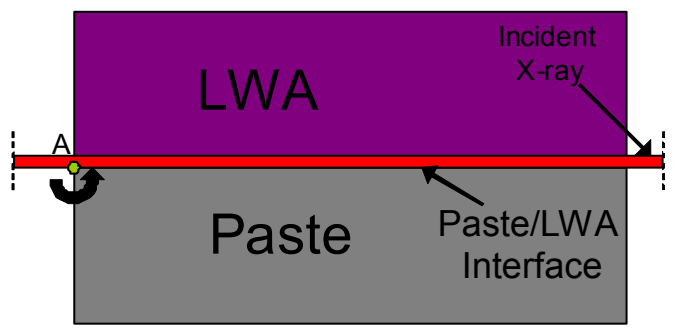

(a)

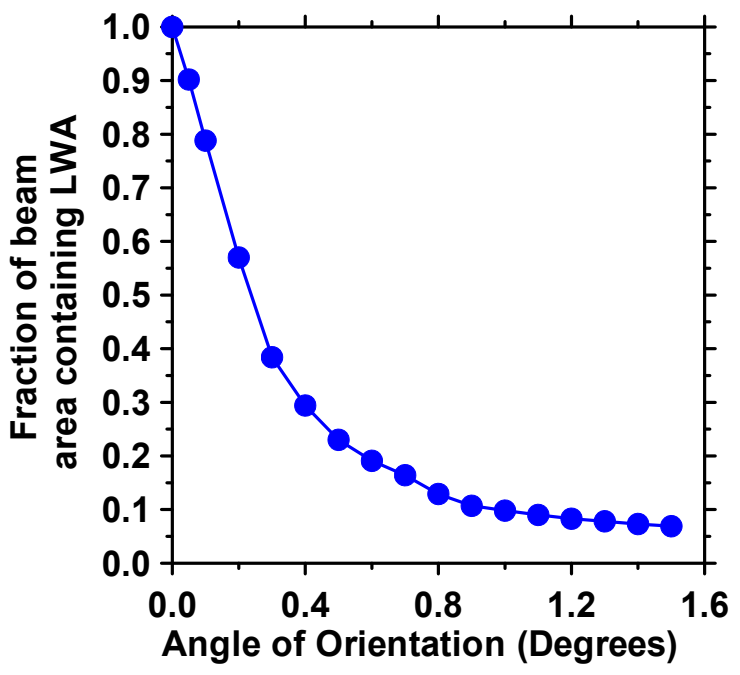

(b)

Figure 4-7 - (a) A schematic illustration of the geometry of the experimental setup (not to scale) and (b) influence of the angle of orientation on the fraction of the $x$-ray beam that contains LWA

In one of the previous studies mentioned (Lura et al. 2006a), a detector was used to monitor the x-ray absorption in the specimen. In the current study, a chargedcouple device (CCD) camera was used. Several differences exist between the detector and the camera, the first being the dimensions of the images captured. The dimensions of the detector are $3.0 \mathrm{~mm} \times 3.0 \mathrm{~mm}$. The dimensions of the camera are $25.0 \mathrm{~mm} \times 25.0 \mathrm{~mm}$. Because of the larger dimensions, the camera is able to capture a larger area of the sample. Often when a larger area is 
considered, resolution is lost. However because of the differences with the way the images are processed, the spatial resolution of the camera is better. This is because the detector averages the photon counts over the $3.0 \mathrm{~mm} \times 3.0 \mathrm{~mm}$ area. The camera however breaks the $25.0 \mathrm{~mm} \times 25.0 \mathrm{~mm}$ area into a grid of $252 \times 256$ pixels. Though the camera captures a $25.0 \mathrm{~mm} \times 25.0 \mathrm{~mm}$ area, this is not necessarily the size of the beam when it interacts with the sample, as seen in Figure 4-8(a). If the sample is placed closer to the x-ray beam source, the size of the beam that interacts with the sample will be smaller, thus resulting in a more precise measurement.

\subsection{Materials and Equipment}

\subsubsection{Materials and Mixture Proportions}

ASTM C150 Type I ordinary portland cement (OPC) was used with a Blaine fineness of $370 \mathrm{~m}^{2} / \mathrm{kg}$ and an estimated Bogue phase composition of $56 \% \mathrm{C}_{3} \mathrm{~S}$, $16 \% \mathrm{C}_{2} \mathrm{~S}, 12 \% \mathrm{C}_{3} \mathrm{~A}, 7 \% \mathrm{C}_{4} \mathrm{AF}$ and a $\mathrm{Na}_{2} \mathrm{O}$ equivalent of $0.68 \%$. A plain paste was prepared with a $w / c$ of 0.30 . A high-range water-reducing admixture (HRWRA) was added at a rate of $0.44 \mathrm{~g}$ per $100 \mathrm{~g}$ of cement. The lightweight aggregate used was a rotary kiln expanded shale with an absorption of $10.5 \%$ per unit dry mass at $24 \mathrm{~h}$ and an apparent specific gravity of 1.56 .

\subsubsection{Description of the X-ray Absorption Equipment}

The equipment used is a GNI x-ray absorption system. The tests were performed in an environmentally controlled (temperature and humidity) chamber (Nielsen 2007). The x-ray intensity that passed through the samples was measured using a charge-coupled device (CCD) (i.e., x-ray camera). The 16-bit CCD camera records the cumulative x-ray intensity at each pixel, for a total surface of 252 
pixels x 256 pixels (Nielsen 2007). The x-ray source and camera can be moved simultaneously using an X-Y positioning table with a precision of $\pm 0.001 \mathrm{~mm}$. The Focus to Detector Distance (FDD) of the equipment can be adjusted from 10 $\mathrm{mm}$ to $500 \mathrm{~mm}$.

\subsection{Experimental Methods}

Two different experimental setups were used in the x-ray measurement system. The first (named the low resolution measurement) consisted of using the x-ray equipment to determine when water movement from the SLWA to the hydrating cement paste occurs under sealed curing conditions. This investigation consisted of placing a SLWA particle in a sample holder with cement paste and fixing the sample holder to a stationary mount. The other investigation (named the high resolution measurement), which has been partially described in Section 4.5, was performed to assess the travel distance of water from the LWA to the surrounding paste.

The key differences between these measurements are that the low resolution measurements were taken with an object to detector distance (ODD) of $100 \mathrm{~mm}$. These measurements can be described as "big picture" measurements. The samples had a fixed position and with the geometry used in this investigation, the single pixel size and spatial resolution was $0.08 \mathrm{~mm}$. These measurements were taken to observe bulk water movement from the LWA to the cement paste. The high resolution measurements can be described as the "close up" measurements. The ODD for these measurements was $400 \mathrm{~mm}$. Because of the close proximity of the sample to the x-ray source, the single pixel size and spatial resolution was $0.02 \mathrm{~mm}$. The samples in this high resolution test were fixed in two directions; however they were rotated to align the interface of the paste and the LWA with the beam. The primary purpose of these measurements 
was to observe the water movement in much finer detail at the interface level. It should be noted that the $x$-ray techniques used in these studies have a massbased moisture content resolution of $0.26 \%$ (g of water/g of sample) (Sant et al. 2008a); this would be consistent with approximately $5 \%$ of the total chemical shrinkage.

\subsubsection{Low Resolution Measurement}

Figure 4-8(b) shows a schematic illustration of the sample holder used in this investigation. The holder was formed from a $5 \mathrm{~mm}$ thick HDPE (high density polyethylene) sheet. The sample holder was $50 \mathrm{~mm} \times 37 \mathrm{~mm}$ with two $12 \mathrm{~mm}$ openings cut out from the center. Two thin clear acetate sheets, approximately $0.5 \mathrm{~mm}$ thick, were attached to the front and back surface of the holder using epoxy to seal the sample holder. A prismatic LWA sample $(2 \mathrm{~mm} \times 5 \mathrm{~mm} \times$ $12 \mathrm{~mm}$ ) cut from a larger specimen was then inserted into a notch in the holder. Before performing the experiments, the LWA was oven-dried at $110^{\circ} \mathrm{C}$ for $24 \mathrm{~h}$ to ensure a uniform moisture condition. The aggregate was then cooled until it reached room temperature and immersed in water for $24 \mathrm{~h}$.

The cement paste was mixed in accordance with the procedure detailed in ASTM C305-06 (2006). To minimize the moisture loss from the LWA, the paste was mixed first and the LWA was removed from the water just prior to placement in the sample holder. The LWA was patted dry using a cotton swab, and then fixed in position in the holder using epoxy. The paste was placed into both openings in the sample holder. One opening contained the test specimen, which included the prewetted aggregate sample and the cement paste, while the other opening contained just the plain cement paste to be used as a reference to account for beam energy variations that may occur during the course of the experiment (Sant et al. 2008c). The design of the sample holder permitted simultaneous testing of 
the test specimen and the reference specimen. After the cement paste was placed, the sample holder was sealed on top with clear tape and the entire sample holder then sealed with aluminum tape to minimize drying. The top surface was sealed with the clear tape first to prevent chemical reactions between the cement paste and the aluminum tape. At this point, the sample holder was placed in the $\mathrm{x}$-ray chamber, which was maintained at $23.0{ }^{\circ} \mathrm{C} \pm$ $0.5^{\circ} \mathrm{C}$. The incident $\mathrm{x}$-rays were produced with a conical spread (Nielsen 2007) using a current of $100 \mu \mathrm{A}$ and a voltage of $50 \mathrm{keV}$.

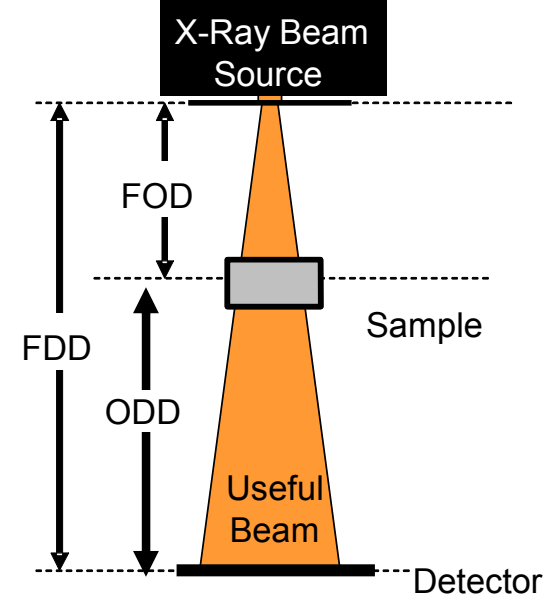

(a)

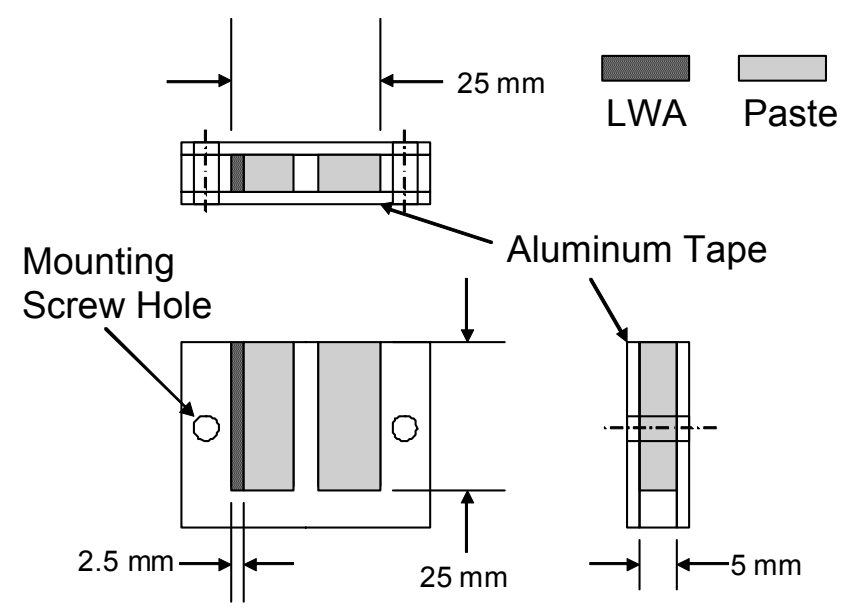

(b)

Figure 4-8 - (a) A schematic illustration of the geometry of the experimental setup (not to scale) and (b) schematic of sample holder with precut LWA and paste (used to detect water movement near the time of set)

\subsubsection{High Resolution Measurement}

The LWA particle, described in Section 4.5, was oven-dried at a temperature of $110^{\circ} \mathrm{C}$ for $24 \mathrm{~h}$. After the aggregate was dried, it was attached inside the sample holder with epoxy glue. Two identical samples with LWA were made along with one reference sample. The absorption of water into the LWA was monitored using x-ray absorption. X-ray measurements began approximately $45 \mathrm{~s}$ after water was added to the empty space in the sample holder. The absorption of 
water into the LWA was monitored for the first $8 \mathrm{~h}$ in $15 \mathrm{~min}$ intervals. The LWA was allowed to absorb water for $24 \mathrm{~h}$ before paste was added. The cement paste was mixed in accordance with the procedure detailed in ASTM C305-06. After the paste was mixed, the water was removed from the sample holder and excess water was removed with a cotton swab. During the process, the sample remained in the $x$-ray chamber so as not to change the position of the sample holder. X-ray measurements began approximately $10 \mathrm{~min}$ after the paste was added. The samples were sealed with putty and a piece of invar. The incident $x-$ rays were produced with a current of $100 \mu \mathrm{A}$ and a voltage of $45 \mathrm{keV}$.

\subsection{Experimental Results}

\subsubsection{Low Resolution Measurement}

While three samples were tested and showed similar trends, Figure 4-9(a) shows a typical result for water movement from the LWA during the first $24 \mathrm{~h}$. The $\mathrm{x}$ axis shows the horizontal position of the specimen, while the y-axis shows the difference in counts (transmitted intensities) normalized to the counts at $3.5 \mathrm{~h}$ (transmitted intensity at $3.5 \mathrm{~h}$ ). The zone between the aggregate and the paste has been labeled as the 'interface' as was done by Lura et al. (Lura et al. 2006a; Henkensiefken et al. 2008g). It is observed Figure 4-9(b) that as the specimen ages, the difference in counts in the LWA becomes more negative, which relates to the removal of water from the prewetted aggregate. Upon further inspection of the information from the first hours when the measurements were taken (i.e., prior to set), the number of counts is observed to remain relatively constant, as seen in Figure 4-9(b). This is expected, as at early ages, the system is saturated (the paste system collapses on itself and does not create vapor-filled space). As a solid skeleton forms in the cement paste, vapor-filled spaces begin to form (cavitate) in the paste system (Couch et al. 2006). This causes a suction 
pressure to develop in the pore fluid which causes water to be drawn out of the aggregate into the paste causing an increase in the measured $x$-ray intensity in the LWA and a decrease in the measured $x$-ray intensity in the paste.

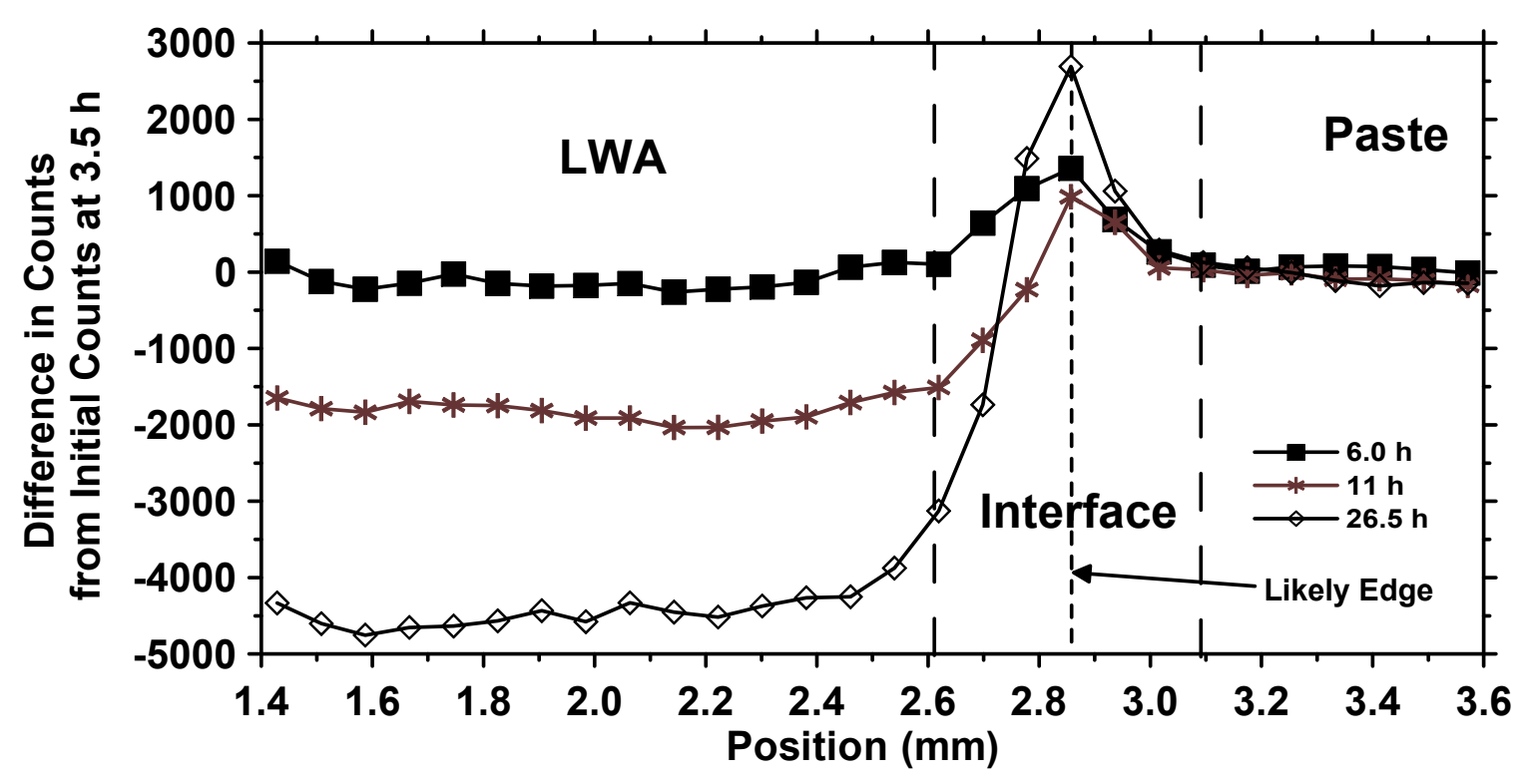

(a)

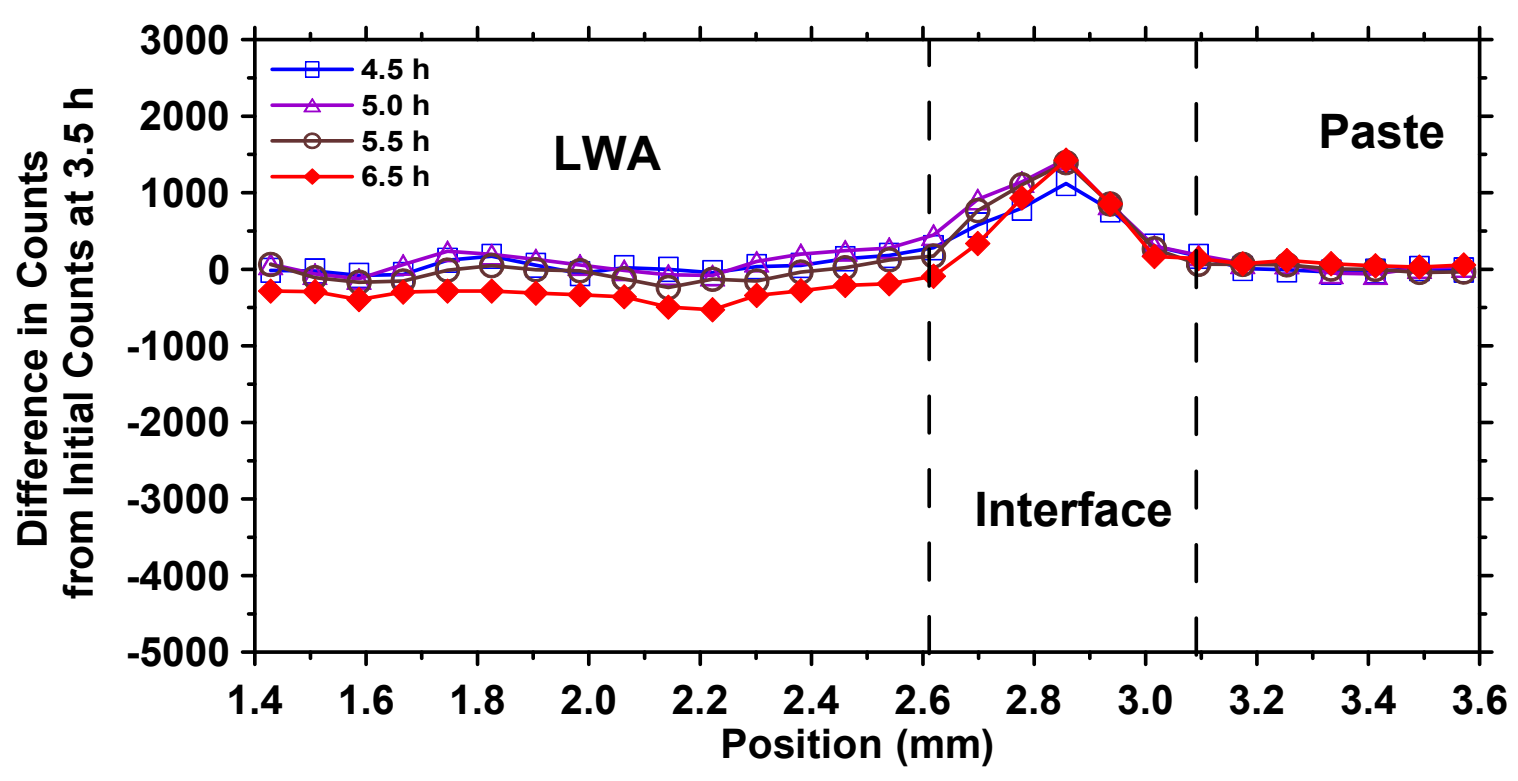

(b) 
Figure 4-9 - (a) X-ray measurements showing water movement from the LWA during the first day, and (b) X-ray measurements during first $6.5 \mathrm{~h}$ for determining when water leaves the LWA

The average of the difference in counts from a position of $2.0 \mathrm{~mm}$ to $2.4 \mathrm{~mm}$ on the specimen is shown as a function of specimen age in Figure 4-10. The measured chemical shrinkage, equivalent to the water demand of the hydrating cement paste, is also shown in Figure 4-10. The counts remain constant until an age of $6 \mathrm{~h}$, indicating that water is not being drawn out of the aggregate until this time. However, after $6 \mathrm{~h}$, the transmitted $\mathrm{x}$-ray intensity is observed to progressively increase in the LWA. This would indicate a loss of water from the pores of the aggregate into the cement paste. The time of first water movement detected by the x-ray measurements $(6 h)$ is similar to the time shown by the initial set as determined with the Vicat needle (6 h) (Henkensiefken et al. 2008g). This is an important observation as it verifies that in a sealed system, a prewetted LWA does not lose significant water until a solid skeleton forms and vapor spaces begin to cavitate, which occurs near the time of set.

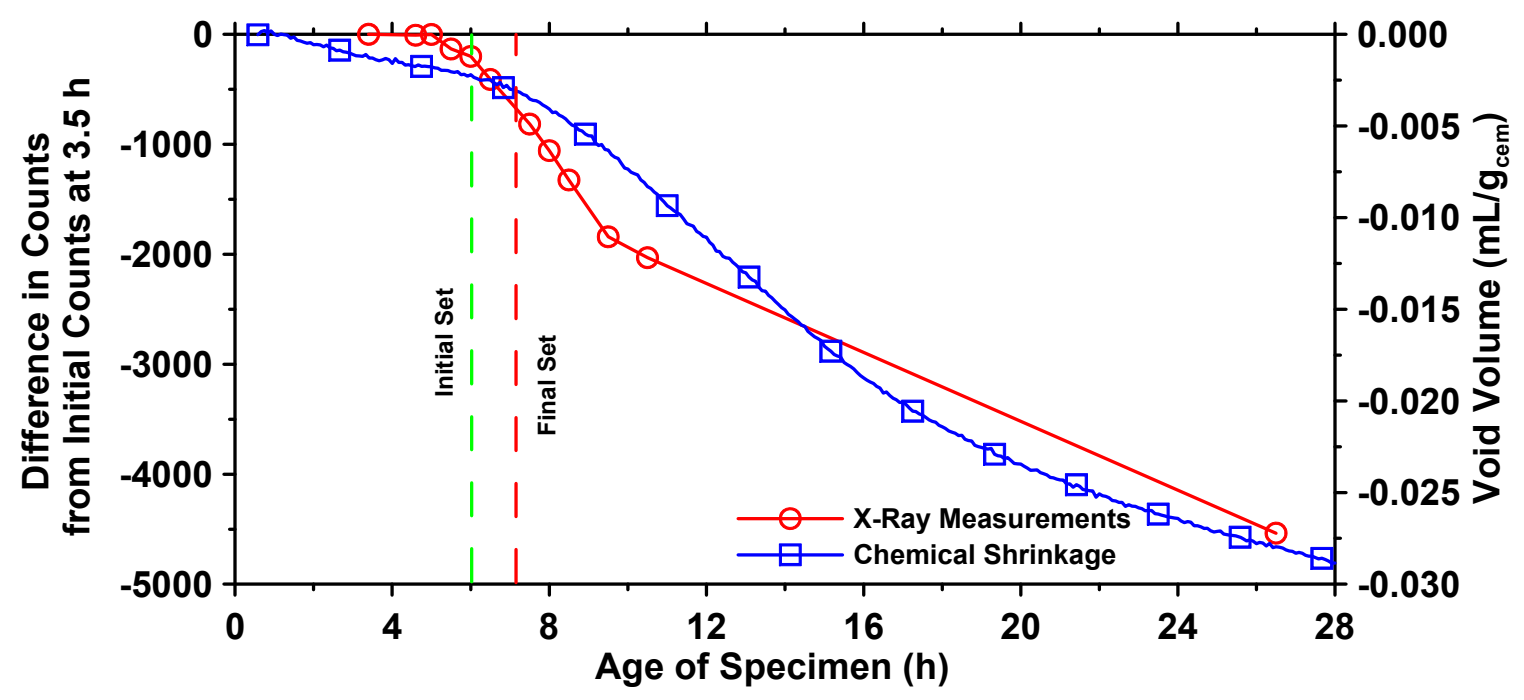

Figure 4-10 - Difference in counts normalized to initial counts averaged from 2.0 $\mathrm{mm}$ to $2.4 \mathrm{~mm}$ in the LWA as a function of age 


\subsubsection{High Resolution Measurement}

Two samples in this investigation were used to detect the distance of water movement, but the results for a typical sample are shown in Figure 4-11. The 'interface' is located at $0.0 \mathrm{~mm}$ and the increasing negative distance is the distance into the hydrating cement paste while a positive distance corresponds to movement into the LWA. Two distinct features can be seen from Figure 4-11. First, as time elapses, the normalized intensity in the paste becomes increasingly positive. This shows that the paste is becoming more dense as water is moving from the LWA to the paste. The second feature that can be seen is a wetting front. It is hard to determine exactly where the wetting front is during the first 9.0 $\mathrm{h}$, as it appears that the high permeability of the fresh cement paste allows fluid movement of more than several $\mathrm{mm}$ at this time. A definite measurement cannot be provided during this time for two reasons. First, it was out of the selected 3 $\mathrm{mm}$ field of view. Second, the changes in intensity are quite low. However, a clearer water front can be seen from $12.0 \mathrm{~h}$ to $75.0 \mathrm{~h}$. The front is shown as a sharp increase in the normalized intensity. This increase indicates the point where the paste goes from being more dense (due to more water) to less dense (due to lack of water). When measurements were taken at $75.0 \mathrm{~h}$, the water was able to move approximately $1.8 \mathrm{~mm}$ into the paste. This distance has implications on the effectiveness of internal curing in systems made with SLWA. 


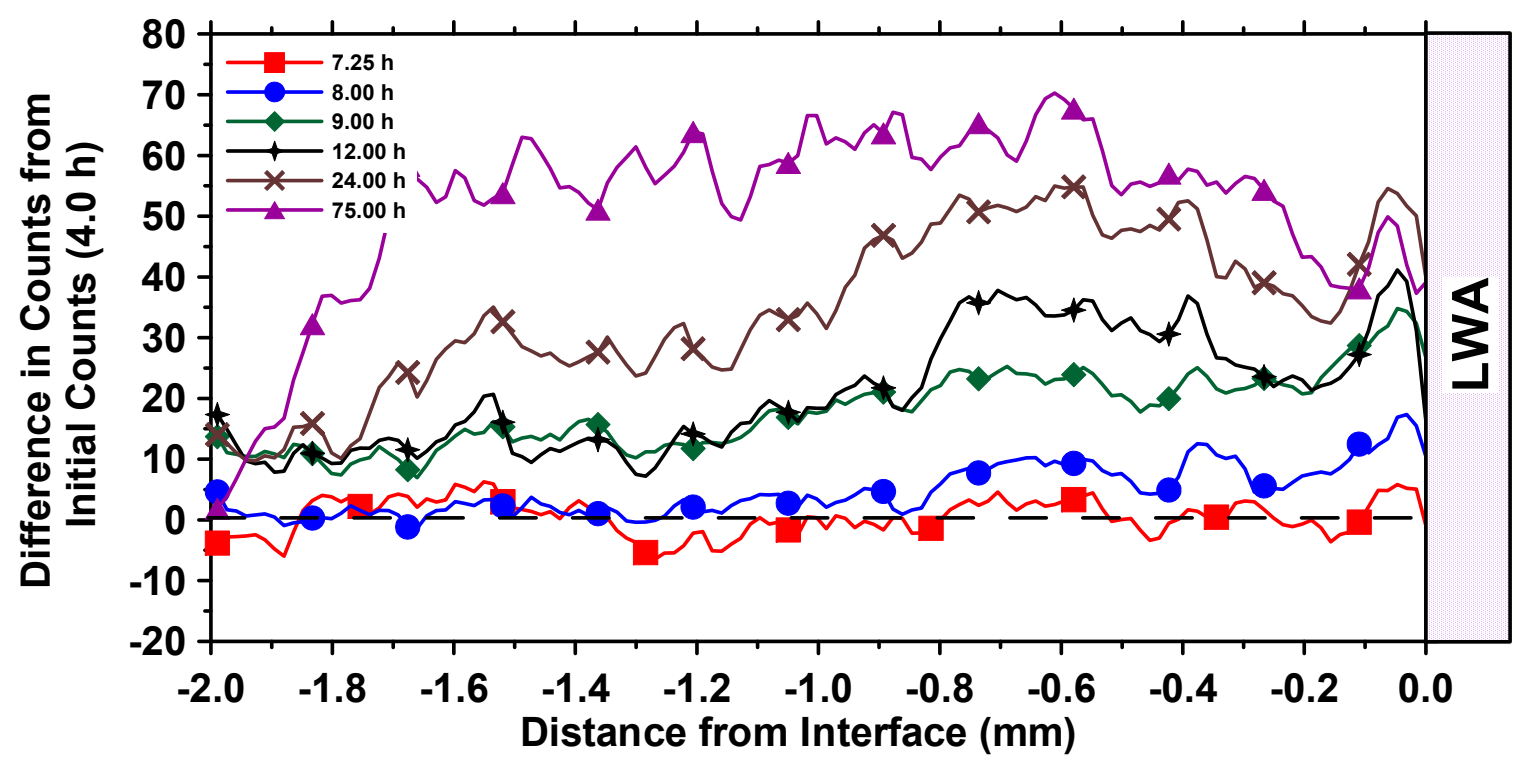

Figure 4-11 - Moisture movement from LWA into the cement paste

The distance water can travel becomes important in selecting the gradation of LWA and the proportions for the mixtures with internal curing. When SLWA is added to concrete, the water that is stored in it must be able to reach the paste which it is meant to protect. If the water stored in the SLWA is unable to penetrate more than $2 \mathrm{~mm}$ into the paste, all the paste in the concrete must be within $2 \mathrm{~mm}$ in order for effective internal curing to take place. The effect of different particle size replacements on the protected paste volume was simulated using the hard core/soft shell model developed at the National Institute of Standards and Technology (NIST) (Lu et al. 1992; Maekawa et al. 1999; Bentz et al. 1999a). Figure 4-12 shows a plot of the volume fraction of cement paste that is within a given distance from the surface of a SLWA particle. For the same volume of LWA, it should be noted that when large particles $(4.76 \mathrm{~mm}$ and 2.36 $\mathrm{mm}$ ) are used, not all of the paste is within $2 \mathrm{~mm}$ of a LWA. As smaller LWA particles $(<2.36 \mathrm{~mm})$ are used, all of the paste is with $2 \mathrm{~mm}$ of a LWA particle. As smaller particles are used, the volume of paste within even smaller distances of a LWA particle surfaces also increases. If a small enough particle is replaced, 
a majority of the paste will be within $0.20 \mathrm{~mm}$, which could be important at later ages (RILEM 2007).

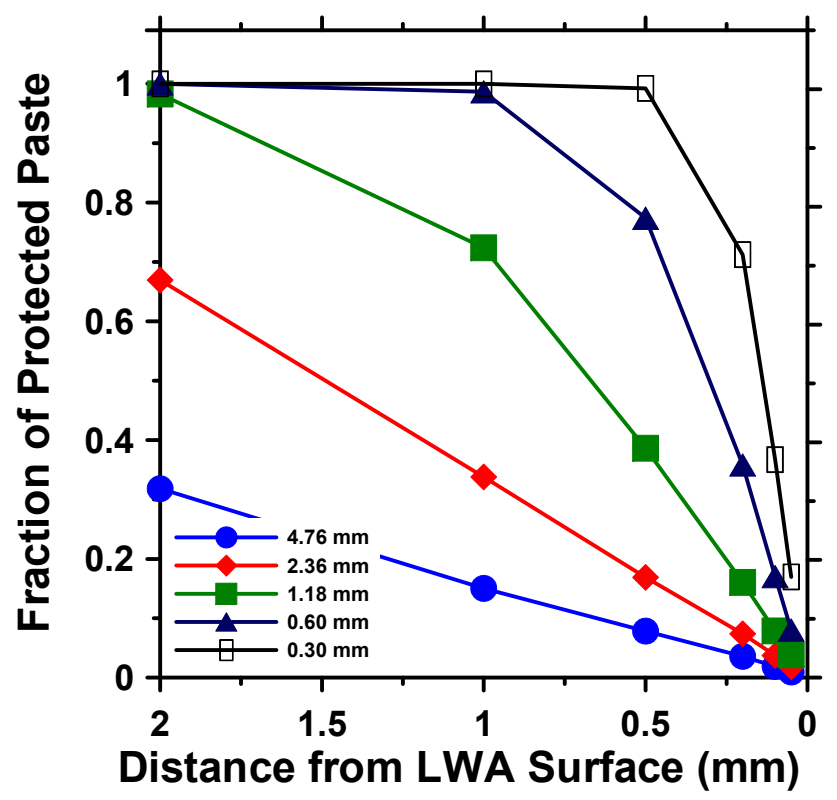

Figure 4-12 - Protected paste volume at different particle replacement sizes

\subsection{Conclusions}

This chapter describes investigations to demonstrate how moisture moves from LWA to the cement paste in an internally cured mixture. The general results of the investigation are:

- the error associated with samples that are not properly oriented was demonstrated using a misaligned interface. These results suggest that sample misalignment would cause incorrect or imprecise determination of travel distance of water,

- $\mathrm{x}$-ray absorption was used to detect that water does not leave the LWA until set has occurred in a sealed system. This is consistent with previous investigations (Bentz et al. 2006a; Henkensiefken et al. 2008g), 
- x-ray absorption was also used to measure the distance that the water moved from the LWA to the surrounding cement paste. In this study, water was determined to move approximately $1.8 \mathrm{~mm}$ into the paste for ages between $24.0 \mathrm{~h}$ and $75.0 \mathrm{~h}$, and

the practical importance of these investigations was also shown by the importance of particle spacing effects. When better particle spacing exists, shrinkage, stress development, and early-age cracking should be reduced. 
CHAPTER 5. DISCUSSION ON THE SHRINKAGE OF THE PASTE IN MORTAR CONTAINING PREWETTED LIGHTWEIGHT AGGREGATE

\subsection{Introduction}

Shrinkage in mortar and concrete is a function of the shrinkage of the paste and the restraint provided by non-shrinking aggregate particles. Attempts have been made to model the shrinkage of concrete using two phase or multi-phase models (Pickett 1956; Hobbs 1969). These models have been used in the past to describe shrinkage in normal weight concrete mixtures (Lura 2003a). In this paper, the autogenous shrinkage of cement paste is calculated based on the autogenous shrinkage of internally cured mortar specimens that contain prewetted LWA using a model developed by Pickett. The overarching goal is to develop tools to describe the shrinkage of the paste when additional water is supplied by the LWA.

\subsection{Constituent Materials}

ASTM C150 Type I ordinary portland cement (OPC) was used, with a Blaine fineness of $370 \mathrm{~m}^{2} / \mathrm{kg}$ and an estimated Bogue phase composition of $56 \% \mathrm{C}_{3} \mathrm{~S}$, $16 \% \mathrm{C}_{2} \mathrm{~S}, 12 \% \mathrm{C}_{3} \mathrm{~A}, 7 \% \mathrm{C}_{4} \mathrm{AF}$ and $\mathrm{Na}_{2} \mathrm{O}$ equivalent of $0.68 \%$.

The sand used was natural river sand with a fineness modulus of 2.71. The sand had a modulus of elasticity of approximately $70 \mathrm{MPa}$ and a Poisson's ratio of 0.25 (Bisschop et al. 2001). Portions of the sand were replaced with manufactured rotary kilned expanded shale with a fineness modulus of 3.10 . 
The $24 \mathrm{~h}$ absorption of the LWA was determined to be $10.5 \%$ according to ASTM C128-07. The LWA was assumed to have a modulus of elasticity of approximately $30 \mathrm{MPa}$ and a Poisson's ratio of 0.22 (Nilsen et al. 1995).

\subsection{Mixture Proportioning}

Four different mixtures were prepared: one plain mortar mixture and three mortar mixtures where a portion of the sand was replaced with prewetted LWA. The mixtures with prewetted LWA replacement were designated as $7.3 \% \mathrm{k}, 14.3 \% \mathrm{k}$ and $25.3 \% \mathrm{k}$, representing the percent of the total volume of the mixture composed of LWA Table 5-1.

Table 5-1 - Mixture proportions for modeling study

\begin{tabular}{|c|c|c|c|c|}
\hline Material & $0.0 \%$ & $7.3 \% \mathrm{k}$ & $14.3 \% k$ & $25.3 \% \mathrm{k}$ \\
\hline Cement $\left(\mathrm{kg} / \mathrm{m}^{3}\right)$ & 728 & 728 & 728 & 728 \\
\hline Water $\left(\mathrm{kg} / \mathrm{m}^{3}\right)$ & 218 & 218 & 218 & 218 \\
\hline Fine Aggregate $\left(\mathrm{kg} / \mathrm{m}^{3}\right)$ & 1418 & 1229 & 1050 & 806 \\
\hline LWA $\left(\mathrm{kg} / \mathrm{m}^{3}\right)$ & 0 & 114 & 223 & 370 \\
\hline HRWRA $\left(g / g_{c e m}\right)$ & 0.44 & 0.44 & 0.50 & 0.66 \\
\hline $\begin{array}{c}\text { Additional Water Provided by } \\
\text { LWA }\left(\mathrm{mL} / \mathrm{g}_{\mathrm{cem}}\right)\end{array}$ & 0 & 0.016 & 0.032 & 0.053 \\
\hline w/c (without additional water) & 0.30 & 0.30 & 0.30 & 0.30 \\
\hline
\end{tabular}

\subsection{Mixing Procedure}

Mixing was performed in accordance with ASTM C192-06. The LWA was oven dried, air cooled, and then submerged in water for $24 \mathrm{~h} \pm 1 \mathrm{~h}$ before mixing. The volume of water used to submerge the LWA included both mixing water and the water the LWA would absorb in $24 \mathrm{~h}$. The mixing water (i.e., the extra water not absorbed in $24 \mathrm{~h}$ ) was then decanted and used in the mixing process. The sand 
was oven-dried and subsequently cooled to room temperature before it was added to the mixture.

\subsection{Experimental Results of Mortar Mixtures}

Autogenous shrinkage measurements for the mortars are shown in Figure 5-1. These results are similar to those obtained by other researchers (RILEM 2007). It can be seen that the volume fraction of LWA contained in the mixture has a significant effect on autogenous shrinkage. The plain mixture begins shrinking from the time of set, however the mixture containing 7.3\%k LWA expands slightly after set before shrinking after approximately $14 \mathrm{~h}$. The $14.3 \% \mathrm{k}$ mixture shows a much more dramatic expansion for the first day before it begins to shrink after 1 d. The $25.3 \% \mathrm{k}$ mixture expands during the first day and continues to expand (but at a lower rate) for the $7 \mathrm{~d}$ it was measured.

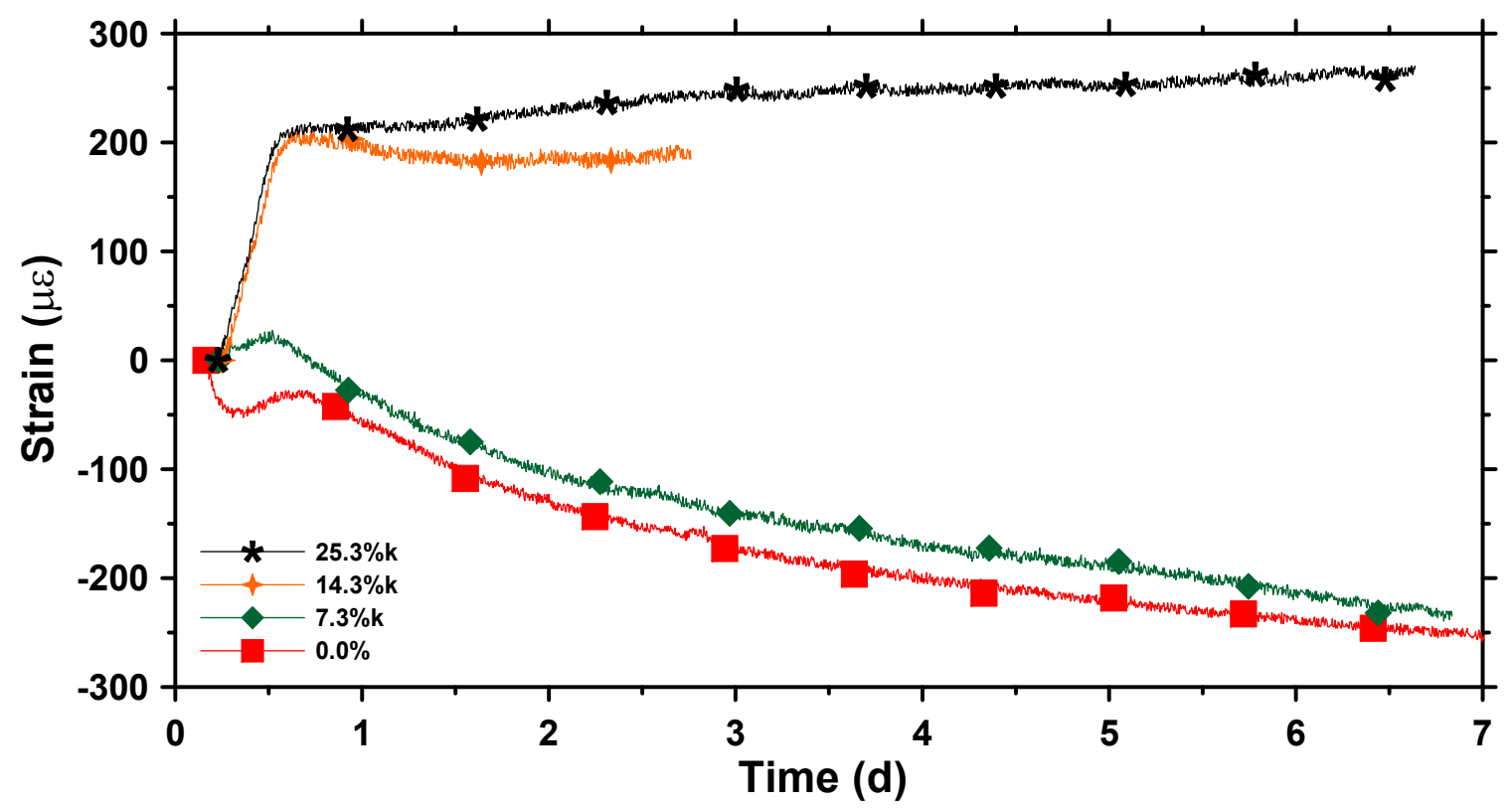

Figure 5-1 - Autogenous shrinkage measurements for mortar specimens

The shrinkage behavior of these mixtures can be explained by considering the water that is provided by the LWA. We can begin by examining the chemical shrinkage of the paste with a $w / c$ of 0.30 as shown in Figure 5-2. As hydration 
occurs, the volume of the specimen is reduced. The chemical shrinkage denotes the entire volume reduction due to the hydration reaction while autogenous shrinkage denotes just the external volume change. The difference between the curves denotes the volume that would be attributed to the cavitation of vapor filled space.

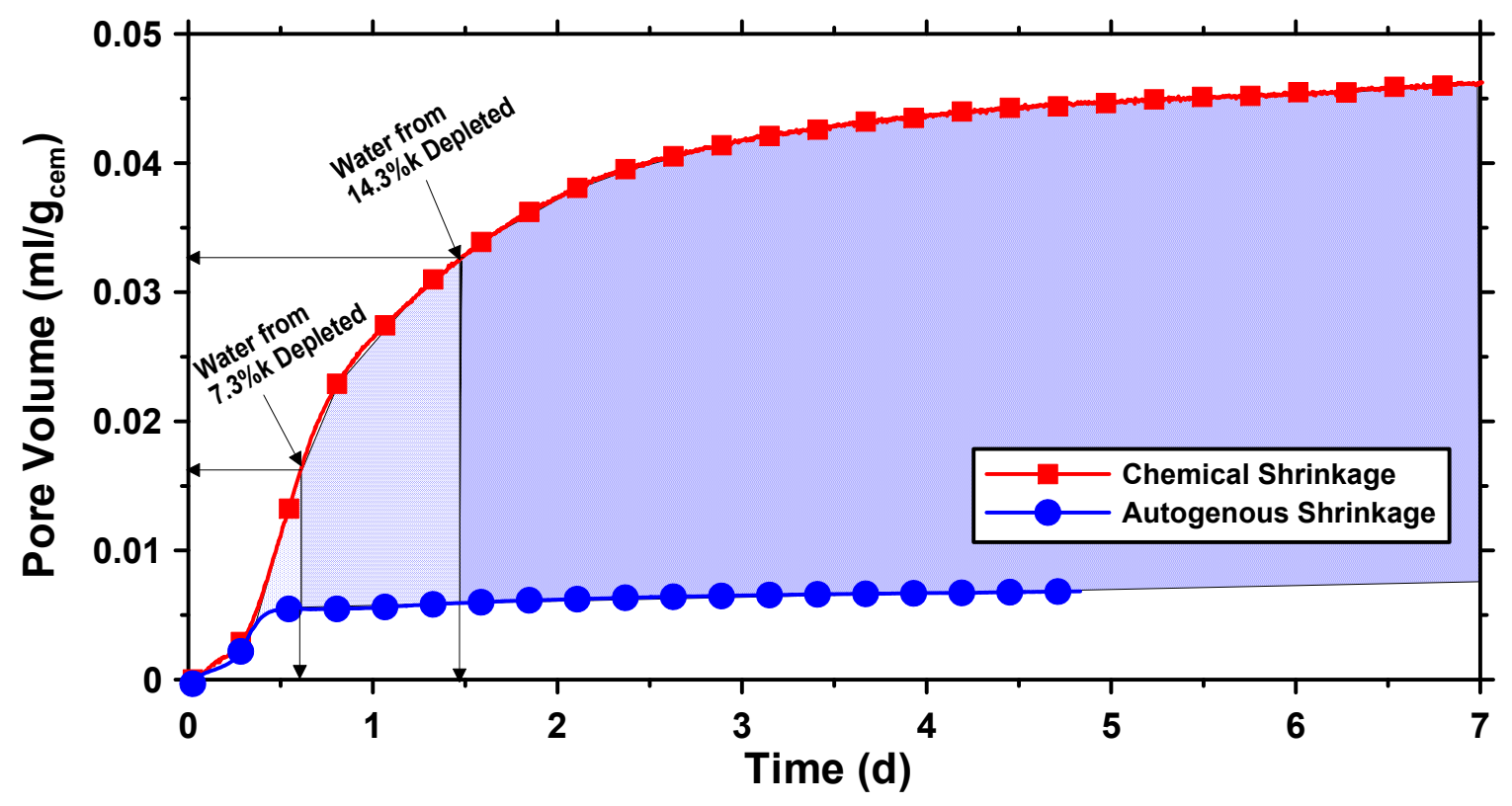

Figure 5-2 - Chemical shrinkage and autogenous shrinkage measurements showing when water depletes from LWA

When prewetted LWA is added to the mixture, the cavitation of vapor filled space will cause a pressure difference resulting in water leaving the LWA and filling the voids created by chemical shrinkage. After the water in the LWA is depleted, shrinkage will occur due to the continued creation of void space. For the $7.3 \% \mathrm{k}$ mixture, water in the LWA is depleted at approximately $14 \mathrm{~h}$, which is when shrinkage begins to occur from Figure 5-1. The 14.3\%k mixture shows that the water will deplete after the first day, which is approximately the time when shrinkage began. From the chemical shrinkage data, it would be expected that the water from the $25.3 \% \mathrm{k}$ mixture does not deplete over the $7 \mathrm{~d}$ time period. 


\subsection{Modeling Concrete Shrinkage from Pickett's Equation}

\subsubsection{General Considerations}

Pickett (Pickett 1956) derived an expression to describe the shrinkage of mortar or concrete as a function of aggregate stiffness and volume as shown in Equation 5-1:

$\varepsilon_{c}=\varepsilon_{p}\left(1-V_{F A}\right)^{n}$

Equation 5-1

where $\varepsilon_{c}(\mathrm{~m} / \mathrm{m})$ is the shrinkage of the concrete/mortar, $\varepsilon_{p}(\mathrm{~m} / \mathrm{m})$ is the shrinkage of the paste, $V_{F A}\left(\mathrm{~m}^{3} / \mathrm{m}^{3}\right)$ is the volume fraction of the aggregate and $n$ is a parameter that describes the aggregate stiffness. The aggregate stiffness term is defined as:

$$
n=\frac{3\left(1-v_{c}\right)}{1+v_{c}+2\left(1-2 v_{a}\right) E_{c} / E_{A}}
$$

where $v_{c}$ and $v_{A}$ are the Poisson's ratio of the concrete/mortar and the aggregate, respectively, and $E_{C}$ and $E_{A}$ are the elastic modulus of the concrete/mortar and the aggregate, respectively. Equation 5-2 applies to concrete consisting of one type of aggregate. To account for the replacement of sand with LWA, Equation 5-1 was modified to the following expression:

$\varepsilon_{c}=\varepsilon_{p}\left(1-V_{F A}\right)^{n_{F A}}\left(1-V_{L W A}\right)^{n_{L W A}}$

where $V_{F A}$ and $V_{L W A}$ are the volume fractions of sand and LWA, respectively. The aggregate stiffness for the fine aggregate and LWA are $n_{\text {FA }}$ and $n_{\text {LWA, }}$ respectively. Several assumptions had to be made in order to use the expression derived from Pickett (Lura 2003a):

- The concrete consists of homogenous materials; paste, fine aggregate and lightweight aggregate.

- The aggregates and paste behave elastically. 
- Microcracking of the paste does not reduce the stiffness.

- The mortar is a two-phase composite consisting of aggregates (normal weight or LWA) and paste with no separate contribution due to interfacial transition zones.

Because shrinkage (or expansion) is a paste property, the resulting shrinkage of the paste could be determined by solving Equation 5-3 for $\varepsilon_{p}$ in terms of the shrinkage of the mortar, the volume fraction of the aggregate and the aggregate stiffness.

$\varepsilon_{p}=\frac{\varepsilon_{c}}{\left(1-V_{F A}\right)^{n_{F A}}\left(1-V_{L W A}\right)^{n_{L W A}}}$

Equation 5-4

\subsubsection{Equivalent Paste Shrinkage}

Figure 5-3 shows the equivalent paste shrinkage which was determined using results from the mortar specimens Equation 5-4. By comparing the experimental and modeling results for the plain mortar and plain paste, it can be noticed that the trend is similar however the experimental value is approximately $80 \mu \varepsilon$ higher at $7 \mathrm{~d}$. This difference may be due to the slight difference in set time. In addition, this difference may be due to the fact that Pickett's model does not account for the interfacial transition zone (Neubauer et al. 1996), changes in bleed water or microcracking (Sant et al. 2008b). The model also shows large expansion of the $14.3 \% \mathrm{k}$ and $25.3 \% \mathrm{k}$ pastes. 


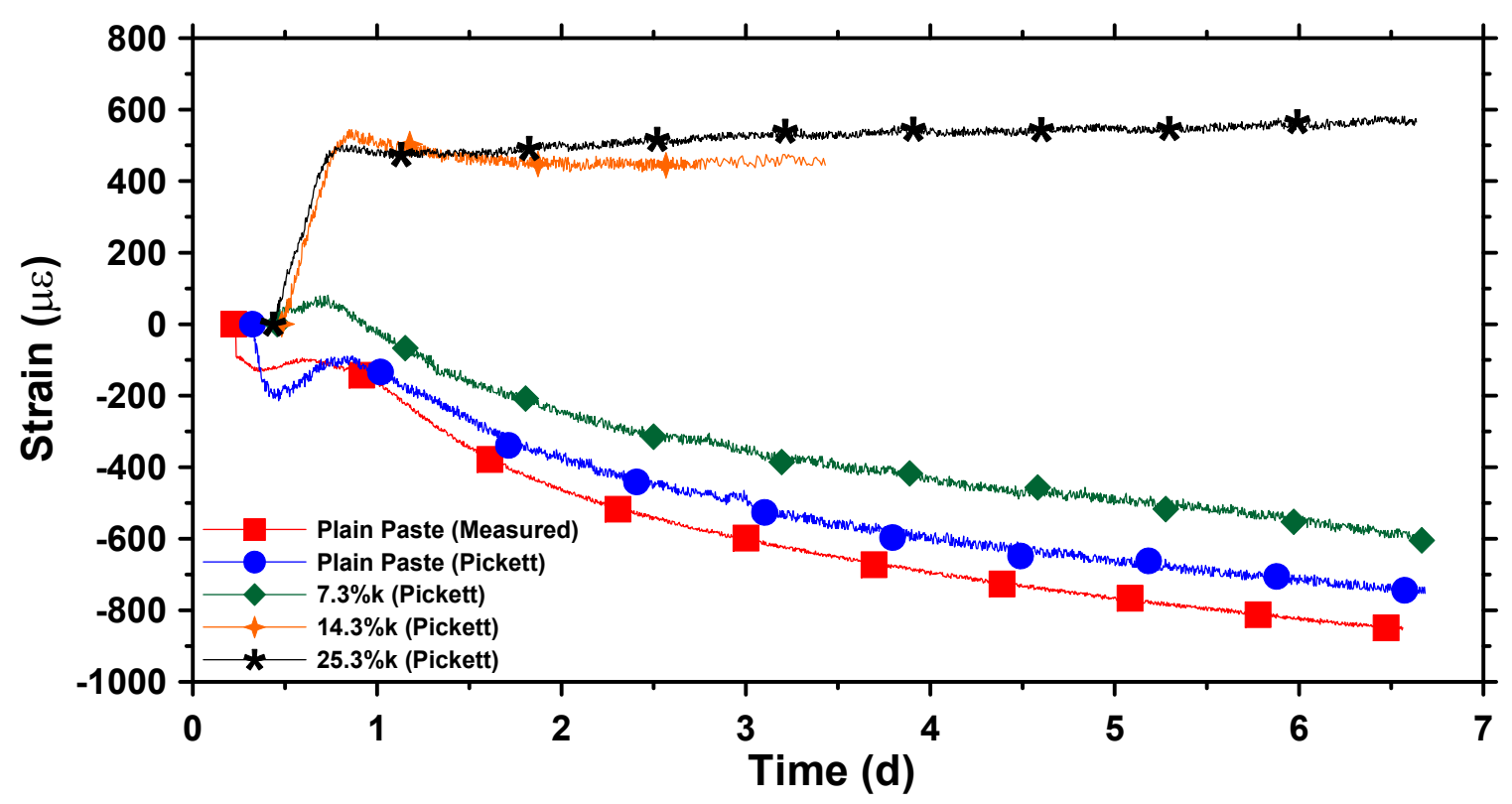

Figure 5-3 - Plain paste measurement and modeling results of plain and LWA pastes using Pickett's Model

\subsection{Describing Shrinkage Using Modified Mackenzie's Equation}

The strain developed in paste can be described using Mackenzie's equation which was modified by Bentz to account for the degree of saturation (Bentz et al. 1998):

$\varepsilon_{p}=\frac{S}{3}\left(\frac{2 \gamma}{r}\right)\left(\frac{1}{K_{p}}-\frac{1}{K_{s}}\right)$

where $S$ (unit less) is the degree of saturation of cement paste, $K_{p}(\mathrm{~Pa})$ is the bulk modulus of the porous body, $K_{s}(\mathrm{~Pa})$ is the bulk modulus of the solid skeleton, $\gamma(\mathrm{N} / \mathrm{m})$ is the surface tension of the pore fluid and $r(\mathrm{~m})$ is the critical Kelvin pore radius. On drawback to this equation is that it is not able to capture expansion that occurs in these mixtures at early ages. 
Figure 5-4 shows the pore size distribution obtained using mercury intrusion porosimetry (MIP) of the plain mortar mixture at $7 \mathrm{~d}$. The pore size of the plain mixture that remains saturated after $7 \mathrm{~d}$ is approximately $7.0 \mathrm{~nm}$ (as determined from internal relative humidity measurements). The volume of water provided by the LWA (Table 5-1) would add water to the system causing the critical (i.e., emptied pore) size to increase to $9.6 \mathrm{~nm}, 11.0 \mathrm{~nm}$ and $19.0 \mathrm{~nm}$ for the mixtures containing $7.3 \% \mathrm{k}, 14.3 \% \mathrm{k}$ and $25.3 \% \mathrm{k}$, respectively. This is shown in Figure 5-5. As expected, the effective pore size depleted due to self-desiccation is larger in a LWA system than in a plain system.

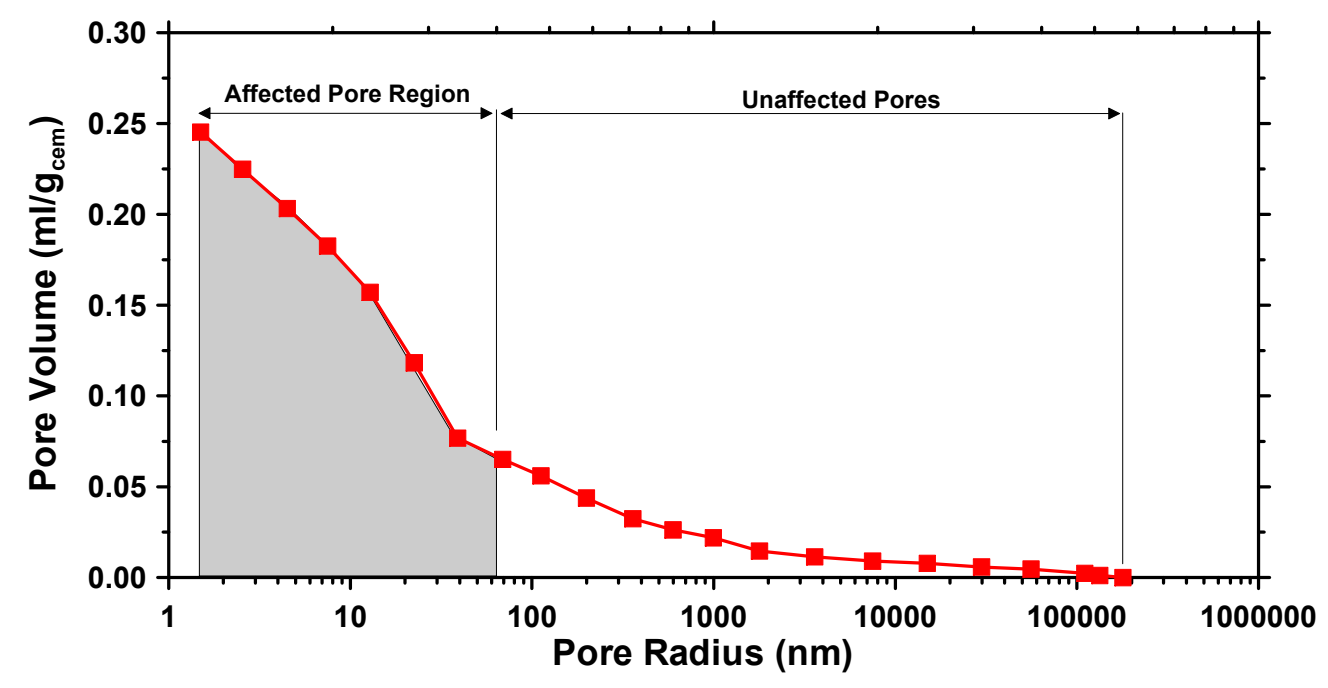

Figure 5-4 - Pore size distribution of plain mortar mixture at $7 d$ 


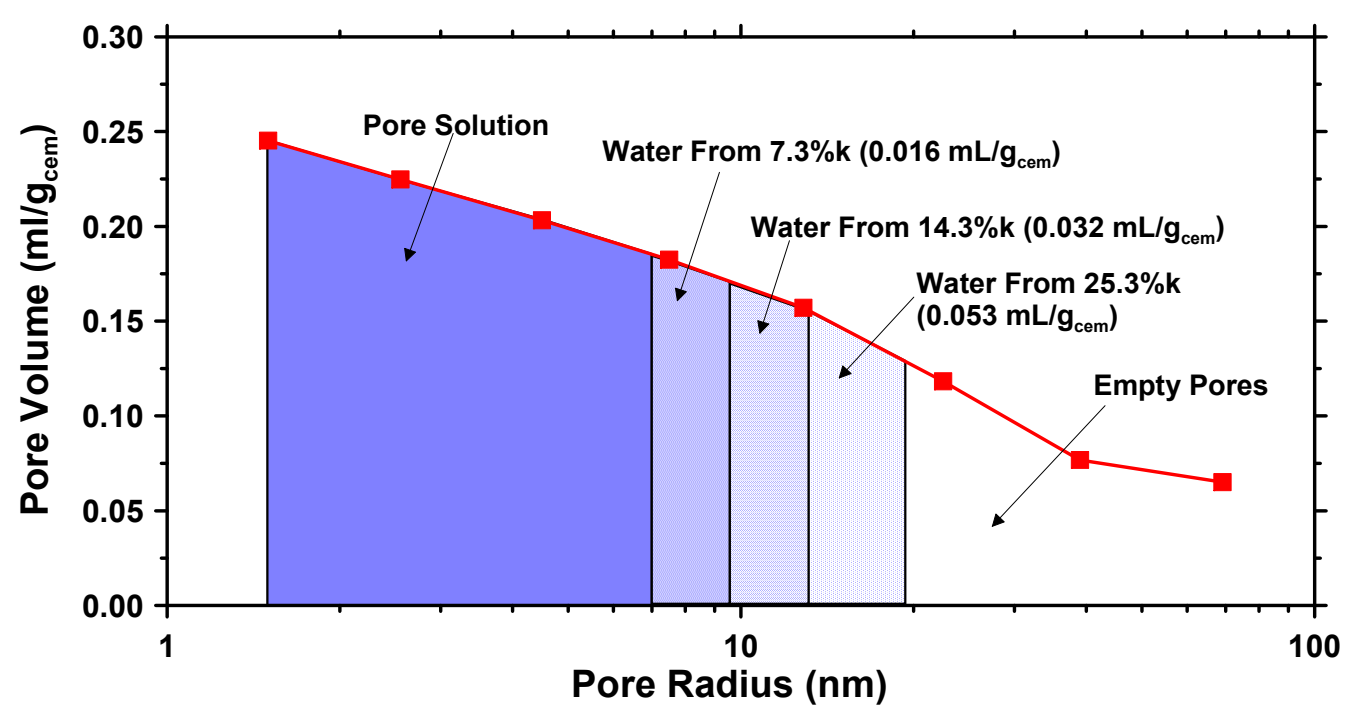

Figure 5-5 - Affected pore size distribution of plain mortar mixture

The critical pore radius (i.e., the depleted pore size) can also be obtained from the internal relative humidity measurements using the Kelvin-Laplace equation (Radlinska et al. 2007):

$r=\left(\frac{2 \gamma}{\ln (R H)}\right)\left(\frac{V_{m}}{R T}\right)$

where $\mathrm{RH}$ (unit less fraction) is the internal relative humidity, $\mathrm{V}_{m}\left(\mathrm{~m}^{3} / \mathrm{mol}\right)$ is the molar volume of the pore solution, $R(\mathrm{~J} / \mathrm{mol} \cdot \mathrm{K})$ is the universal gas constant and $T(\mathrm{~K})$ is the absolute temperature. The measured $\mathrm{RH}$ is a function of the consumption of water due to hydration (Kelvin effect) and the change induced in the activity of the pore solution due to the dissolution of salts (Raoult effect). Raoult's law can then be used to account for the influence of salts in the pore solution to determine the internal $\mathrm{RH}$ purely as a function of water consumption (Kelvin RH), which is slightly higher than the measured RH (Lura 2003a).

The corrected critical pore size determined from the Kelvin-Laplace equation using the Kelvin $\mathrm{RH}$ at $7 \mathrm{~d}$ was $7.0 \mathrm{~nm}, 8.4 \mathrm{~nm}, 9.7 \mathrm{~nm}$ and $16.5 \mathrm{~nm}$ for the $0.0 \%$, 
$7.3 \% \mathrm{k}, 14.3 \% \mathrm{k}$ and $25.3 \% \mathrm{k}$ mixtures, respectively. The experimental results yield a similar albeit slightly smaller pore radius than those obtained from calculations with the MIP data. A comparison between the measurements from the pore size distribution and the $\mathrm{RH}$ measurements (corrected and uncorrected) are detailed in Table 5-2.

Table 5-2 - Kelvin Radius from internal RH and pore size distribution

\begin{tabular}{|l|c|c|c|c|}
\hline Mixture & $\begin{array}{c}\text { From Pore Size } \\
\text { Distribution }\end{array}$ & $\begin{array}{c}\text { From RH } \\
\text { Measurements }\end{array}$ & $\begin{array}{c}\text { From RH Measurements } \\
\text { (corrected) }\end{array}$ & $\begin{array}{c}\text { \% Reduction of } \\
\text { Shrinkage Predicted } \\
\text { using Equation 5-4 }\end{array}$ \\
\hline $0.0 \%$ & 7.0 & 5.3 & 7.0 & $0 \%$ \\
\hline $7.3 \% \mathrm{k}$ & 9.6 & 6.5 & 8.4 & $27 \%$ \\
\hline $14.3 \% \mathrm{k}$ & 11.0 & 7.4 & 9.7 & $36 \%$ \\
\hline $25.3 \% \mathrm{k}$ & 19.0 & 10.8 & 16.5 & $63 \%$ \\
\hline
\end{tabular}

It is evident that if all the other parameters in Equation 5-6 are held constant, while decreasing the radius of the pore size being depleted, the shrinkage increases. Alternatively, if LWA replenishes the pore, the depletion radius will increase resulting in decreased shrinkage. Table 5-2 shows the shrinkage for each the LWA mixtures as a percent reduction of the shrinkage of the plain paste mixture.

\section{8. $\underline{\text { Conclusions }}$}

This chapter discussed the shrinkage of LWA systems. A summary of the observations are:

- Pickett's model can be used to obtain the shrinkage of a paste given the shrinkage of the mortar. 
- The inclusion of a sufficient volume of prewetted LWA can significantly reduce autogenous shrinkage. Though the inclusion of LWA alters the stiffness of the mortars, this effect is less significant than the impact of the release of water from the LWA.

- The inclusion of prewetted LWA will supply water to the paste to increase the effective pore radius that is emptied by self-desiccation, resulting in reduced shrinkage.

- While Mackenzie's modified equation describes the trend in shrinkage, it does not describe the expansion that is observed in the mortars. 


\section{CHAPTER 6. MEASURING VOLUME CHANGE AND CRACKING UNDER SEALED AND DRYING CONDITIONS}

\subsection{Introduction}

High performance concrete (HPC) mixtures have been increasingly promoted for use due to potential long-term benefits in performance (FHWA 2006). HPC mixtures generally have a low water-to-cement ratio $(w / c)$ and may contain finely ground supplementary cementitious materials, (e.g., silica fume). While the fine pore system is beneficial for increased strength (Shah et al. 1994) and reduced fluid transport (El-Dieb et al. 1995; Khan et al. 2002), the finer pore network can lead to increased volumetric changes in sealed systems due to self-desiccation. This volume change is problematic as it can result in cracking if the concrete is restrained from shrinking freely.

Several strategies exist to mitigate autogenous shrinkage such as the use of expansive cements (Saito et al. 1991; ACl 1998), the addition of shrinkage reducing admixtures (Folliard et al. 1997; Bentz et al. 2001b; Weiss et al. 2002; Pease et al. 2005), or the incorporation of water saturated inclusions (Bentz et al. 1999b; Lura 2003a; Lura et al. 2006a; Lura et al. 2007a; Henkensiefken et al. 2008e). The use of prewetted lightweight aggregate (SLWA, known as internal curing), enables water from the SLWA to be supplied to the paste after setting (Henkensiefken et al. 2008g), thereby reducing the effects of self-desiccation. The behavior of SLWA systems is the primary focus of this chapter. 
While several studies have shown the beneficial effects of IC on the shrinkage reduction of high-performance mixtures, few studies have investigated the role of SLWA volume fraction thoroughly (Cusson et al. 2006; Henkensiefken et al. 2008e). While many studies have focused on systems with sealed curing, a recent state-of-the-art report (RILEM 2007) describes how more research is needed to understand the performance when the specimens are exposed to drying. This study provides a substantial data set based on the measured performance of SLWA systems under sealed and drying curing conditions. The performance of SLWA systems is related to fundamental aspects of the pore structure of the paste, the pore structure of the SLWA, and the spacing of the SLWA.

\subsection{Materials}

ASTM C150 Type I ordinary portland cement (OPC) was used, with a Blaine fineness of $370 \mathrm{~m}^{2} / \mathrm{kg}$ and an estimated Bogue composition of $56 \% \mathrm{C}_{3} \mathrm{~S}, 16 \%$ $\mathrm{C}_{2} \mathrm{~S}, 12 \% \mathrm{C}_{3} \mathrm{~A}, 7 \% \mathrm{C}_{4} \mathrm{AF}$ and a $\mathrm{Na}_{2} \mathrm{O}$ equivalent of $0.68 \%$, all by mass fraction.

A high-range water-reducing admixture (HRWRA) was added in varying rates depending on the volume fraction of LWA replacing normal weight sand. For LWA replacement volume fractions up to $7.3 \%$, the HRWRA was added at a dosage of $0.44 \mathrm{~g}$ per $100 \mathrm{~g}$ of cement. For replacement volume fractions of $7.3 \%$ to $18.3 \%$, the HRWRA was added at a dosage of $0.50 \mathrm{~g}$ per $100 \mathrm{~g}$ of cement. For replacement volume fractions larger than $25.3 \%, 0.66 \mathrm{~g}$ per $100 \mathrm{~g}$ of cement was used. Different rates of HRWRA were used to keep similar consistencies of these mixtures.

The normal weight sand used was natural river sand with a fineness modulus of 2.71 and an apparent specific gravity of 2.58. Portions of the normal weight sand 
were replaced with two manufactured rotary kilned expanded shales (LWA-K and LWA-H), with a fineness modulus of 3.10 and an apparent specific gravity of 1.56. The $24 \mathrm{~h}$ absorption of the normal weight sand, LWA-K and LWA-H were determined to be $1.8 \%, 10.5 \%$ and $5.8 \%$ by mass, respectively, according to ASTM C128-07 (2007). The normal weight sand used was natural river sand with a fineness modulus of 2.71 and an apparent specific gravity of 2.58 . Portions of the normal weight sand were replaced with two manufactured rotary kilned expanded shales (LWA-K and LWA-H), with a fineness modulus of 3.10 and an apparent specific gravity of 1.56 . The $24 \mathrm{~h}$ absorption of the normal weight sand, LWA-K and LWA-H were determined to be $1.8 \%, 10.5 \%$ and $5.8 \%$ by mass, respectively, according to ASTM C128-07 (2005). The desorption isotherms for the LWA can be found in Figure 3-3.

\subsection{Mixture Proportioning}

Eleven different mixtures were prepared with an effective $w / c$ of 0.30 : this included a plain mortar mixture designated as $0.0 \%$ and 10 mortar mixtures with varying amounts of sand replaced by prewetted LWA, as shown in Table 6-1. Two types of LWA were used (LWA-K and LWA-H). The LWA-K expanded shale was used for eight of these mixtures which were designated as $3.8 \% \mathrm{k}, 7.3 \% \mathrm{k}$, $11.0 \% \mathrm{k}, 14.3 \% \mathrm{k}, 18.3 \% \mathrm{k}, 25.3 \% \mathrm{k}, 29.3 \% \mathrm{k}$ and $33 \% \mathrm{k}$. The LWA-H expanded shale was used for the remaining two mixtures designated as $25.3 \% \mathrm{~h}$ and $43 \% \mathrm{~h}$. It is important to note that the designations are on a total volume basis. The volume of aggregate (LWA and sand) was maintained constant at $55 \%$ since only the sand was replaced with LWA. The number in the designation represents the total volume of the mixture occupied by the LWA (in percent). The mixture proportions for each mixture are illustrated in Figure 6-1. 
It should be noted that the $25.3 \% \mathrm{k}$ and $43 \%$ h volume replacements correspond to the amount of LWA necessary to eliminate self-desiccation proposed by Bentz et al., (Bentz et al. 1999b; Bentz et al. 2005). The different volumes required for the two aggregates arise from the fact that the LWA-K (10.5\%) can absorb more water in a similar time period as compared to the LWA-H (5.8\%). For this reason, a lower volume of LWA-K was needed to provide the same volume of IC water. This can be noticed by observing the last line in Table 6-1, where the additional water provided for the $25.3 \% \mathrm{k}$ and $43 \% \mathrm{~h}$ mixtures are the same. It is also important to note that one mixture was prepared so that the replacement volume of LWA-K and LWA-H were the same (25.3\%k and 25.3\%h). However LWA-K absorbs more water in $24 \mathrm{~h}(10.5 \%)$ than LWA-H (5.8\%), the volume of IC water for the $25.3 \% \mathrm{k}$ was more than that for the $25.3 \% \mathrm{~h}$. Because of the absorption difference between LWA-K and LWA-H, the volume of IC water for the $25.3 \% \mathrm{~h}$ was similar to the $14.3 \% \mathrm{k}$ mixture, though a smaller volume of aggregate was used.

Table 6-1 - Mixture proportions for volume change and cracking study

\begin{tabular}{|c|c|c|c|c|c|}
\hline Material & $0 \%$ & $3.8 \% \mathrm{k}$ & $7.3 \% \mathrm{k}$ & $11 \% \mathrm{k}$ & $14.3 \% \mathrm{k}^{\mathrm{a}}$ \\
\hline Cement $\left(\mathrm{kg} / \mathrm{m}^{3}\right)$ & 728 & 728 & 728 & 728 & 728 \\
\hline Water $\left(\mathrm{kg} / \mathrm{m}^{3}\right)$ & 218 & 218 & 218 & 218 & 218 \\
\hline Fine Aggregate $\left(\mathrm{kg} / \mathrm{m}^{3}\right)$ & 1418 & 1319 & 1230 & 1135 & 1050 \\
\hline Dry LWA $\left(\mathrm{kg} / \mathrm{m}^{3}\right)$ & 0 & 60 & 114 & 172 & 223 \\
\hline $\begin{array}{c}\text { Additional Water } \\
\text { Provided by LWA }\left(\mathrm{kg} / \mathrm{m}^{3}\right)\end{array}$ & 0 & 6 & 12 & 18 & 23 \\
\hline
\end{tabular}

\begin{tabular}{|c|c|c|c|c|c|c|}
\hline Material & $18.3 \% \mathrm{k}$ & $25.3 \% k^{b}$ & $29.3 \% \mathrm{k}$ & $33 \% k$ & $25.3 \% \mathrm{~h}^{\mathrm{a}}$ & $43 \% h^{b}$ \\
\hline Cement $\left(\mathrm{kg} / \mathrm{m}^{3}\right)$ & 728 & 728 & 728 & 728 & 728 & 728 \\
\hline Water $\left(\mathrm{kg} / \mathrm{m}^{3}\right)$ & 218 & 218 & 218 & 218 & 218 & 218 \\
\hline Fine Aggregate $\left(\mathrm{kg} / \mathrm{m}^{3}\right)$ & 950 & 808 & 667 & 567 & 808 & 310 \\
\hline Dry LWA $\left(\mathrm{kg} / \mathrm{m}^{3}\right)$ & 283 & 369 & 455 & 525 & 369 & 670 \\
\hline $\begin{array}{c}\text { Additional Water } \\
\text { Provided by LWA }\left(\mathrm{kg} / \mathrm{m}^{3}\right)\end{array}$ & 30 & 39 & 48 & 54 & 22 & 39 \\
\hline
\end{tabular}


${ }^{a}$ Denotes an equal volume of water supplied by the $14.3 \% \mathrm{k}$ and $25.3 \% \mathrm{~h}$ mixtures

${ }^{b}$ Denotes an equal volume of water supplied by the $25.3 \% \mathrm{k}$ and $43 \%$ h mixtures

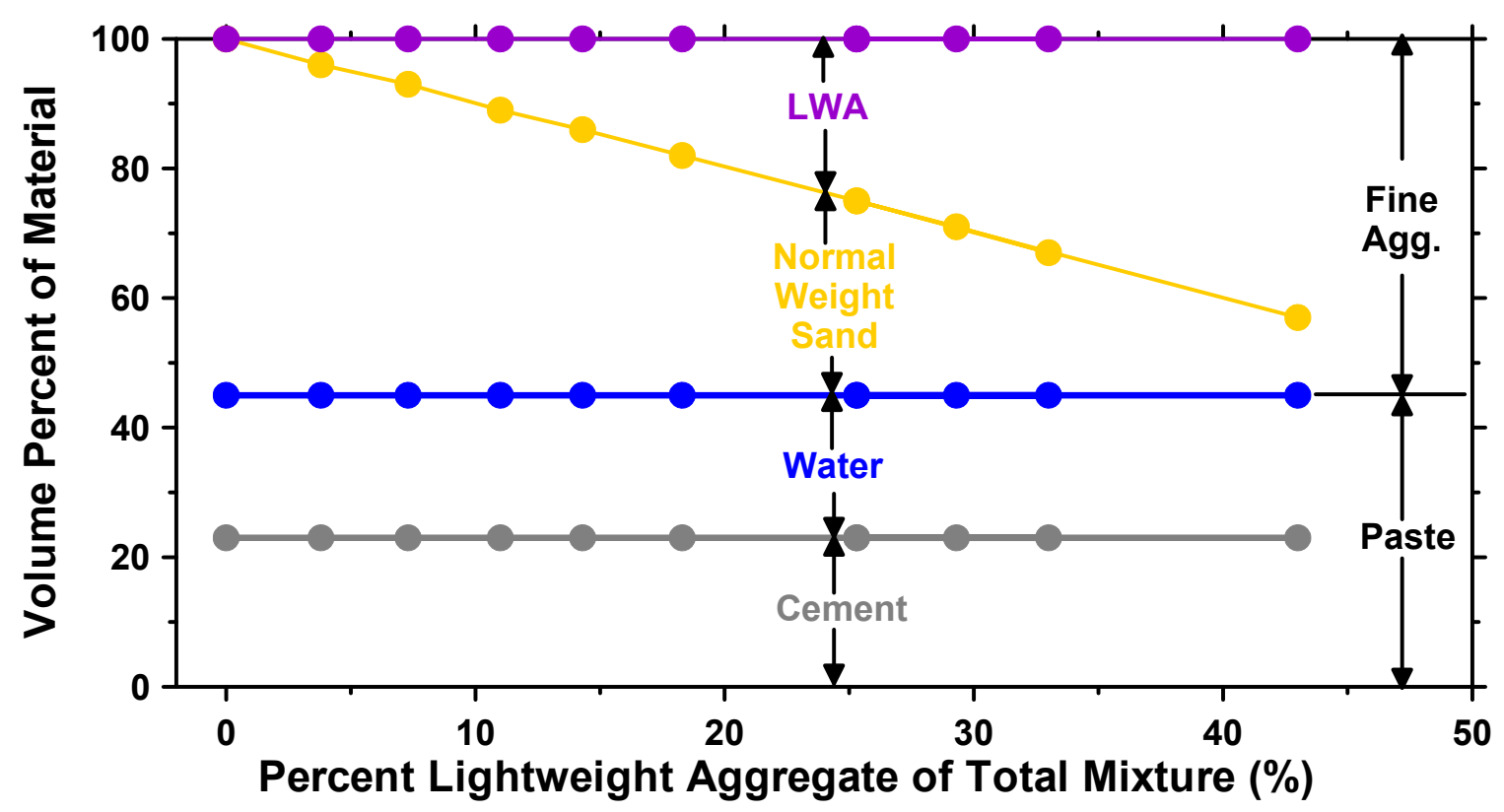

Figure 6-1 - Mixture proportions on a volumetric basis

\subsubsection{Mixing Procedure}

The mixing procedure used was in accordance with ASTM C192-06. The LWA was oven dried, air cooled, and then submerged in water for $24 \mathrm{~h} \pm 1 \mathrm{~h}$ prior to mixing. The volume of water used to submerge the LWA included both mixing water and the water the LWA would absorb in $24 \mathrm{~h}$. The excess water (water not absorbed in $24 \mathrm{~h}$ ) was then decanted and used as the mixing water. The sand was oven dried and cooled for $24 \mathrm{~h}$ before mixing. 


\subsection{Experimental Methods}

\subsubsection{Unrestrained Volumetric Change during the First $24 \mathrm{~h}$}

The autogenous deformation of the mortar specimens during the first $24 \mathrm{~h}$ was measured using the corrugated tube protocol (Jensen et al. 1995; Sant et al. 2006). This procedure consists of placing fresh mortar (approximately $15 \mathrm{~min}$ after water is added to the mixture) in a corrugated polyethylene tube. The length and diameter of the tube was $400 \mathrm{~mm}$ and $30 \mathrm{~mm}$, respectively. The shrinkage strain is measured using two LVDTs. The typical standard deviation after set between two duplicate specimens was $4 \mu \varepsilon$ (microstrain). For each mixture tested, two corresponding corrugated tubes were measured. The specimens were placed in a dilatometer that was maintained at $23^{\circ} \mathrm{C} \pm 1^{\circ} \mathrm{C}$. The transducers were connected to a PC for automated length measurements every $5 \mathrm{~min}$ for the first $7 \mathrm{~d}$.

\subsubsection{Unrestrained Volumetric Change \& Mass Loss after $24 \mathrm{~h}$}

Free shrinkage was measured using $75 \mathrm{~mm} \times 75 \mathrm{~mm} \times 285 \mathrm{~mm}$ prisms according to ASTM C157-06 (2006). Three samples for the sealed condition and three samples for the unsealed condition were prepared. The sealed samples were encased in aluminum tape on all sides. The unsealed samples were sealed on two opposite faces so that they could be directly compared to the restrained shrinkage measurements described in the next section. The length was measured using a comparator beginning $24 \mathrm{~h}$ after casting. The unrestrained volumetric change from the tube protocol for the first $24 \mathrm{~h}$ was added to the prism measurements. The typical standard deviation for unrestrained shrinkage between triplicate specimens was $20 \mu \varepsilon$. The mass loss was recorded for both sealed and unsealed conditions. The mass loss for the sealed samples was measured to ensure a sealed state was maintained during testing. The mass 
loss measurements for the unsealed samples can be used to monitor the extent of drying (water loss) that has occurred. The typical standard deviation for mass loss between triplicate specimens was $0.80 \mathrm{~g}$.

\subsubsection{Measurements of Restrained Shrinkage}

The restrained shrinkage cracking potential was measured using the restrained ring test, in accordance with ASTM C1581-04 (Shah et al. 1998; 2004). Three samples of each mixture were measured. Before casting, molds were conditioned in an environmental chamber at a temperature of $23.0^{\circ} \mathrm{C} \pm 0.1{ }^{\circ} \mathrm{C}$. Each ring was cast in two layers with each layer being vibrated for $15 \mathrm{~s}$. After casting, all samples were kept sealed for $24 \mathrm{~h}$ in an environmental chamber at a constant ambient temperature of $23.0{ }^{\circ} \mathrm{C} \pm 0.1{ }^{\circ} \mathrm{C}$ before they were demolded. After demolding the rings, the sealed ring specimens were sealed with aluminum tape. The unsealed ring specimens were exposed to $50 \% \pm 0.1 \%$ relative humidity at $24 \mathrm{~h}$ and were sealed on the top surface with aluminum tape to limit moisture loss to the outer circumference only (the bottom surface was sealed by the ring's base support). Restrained shrinkage data was measured and collected at $5 \mathrm{~min}$ intervals. The typical standard deviation for restrained shrinkage between triplicate specimens was $4 \mu \varepsilon$ (Radlinska et al. 2008a).

\subsubsection{Internal Relative Humidity of Sealed Specimens}

To measure changes in the internal relative humidity of a sealed sample over time, specimens without normal weight sand were cast in plastic containers which were demolded at an age of $12 \mathrm{~h}$. Approximately $10 \mathrm{~g}$ of the sample was crushed and placed in a glass container with a diameter of $25 \mathrm{~mm}$ and a length of $50 \mathrm{~mm}$ and sealed to prevent loss of moisture. The sealed glass container was kept in an environmental chamber at a constant temperature of $23.0^{\circ} \mathrm{C}$ $\pm 0.1^{\circ} \mathrm{C}$. A temperature and relative humidity meter was used to record relative 
humidity and temperature data inside the sealed container at $2 \mathrm{~h}$ intervals for $7 \mathrm{~d}$. The standard deviation for relative humidity measurements was $1.1 \%$. The sensor was calibrated using saturated salt solutions with known relative humidities in the range of $75 \%$ to $100 \%$ (ASTM E104-02) (2002).

\subsection{Experimental Results and Discussion of Internal Curing Mixtures}

\subsubsection{Different LWA Volume Replacements on Free Shrinkage}

\subsubsection{Sealed Curing Conditions}

The results of free shrinkage tests performed on the plain mixture along with the mixtures containing LWA-K during the first $7 \mathrm{~d}$ and first $28 \mathrm{~d}$ are shown in Figure 6-2 and Figure 6-3, respectively. When a lower replacement volume of LWA is used $(7.3 \% \mathrm{k}, 11.0 \% \mathrm{k})$, the performance is similar to that of a plain mixture (Cusson et al. 2006). When higher volume replacements are used $(14.3 \% \mathrm{k}$, $18.3 \% \mathrm{k}, 25.3 \% \mathrm{k}, 29.3 \% \mathrm{k}, 33.0 \% \mathrm{k}$ ), after the initial expansion, the mixtures remain nearly volumetrically stable. The expansion in these materials during the first hours could be due to osmotic pressure or the formation of ettringite or after, however no experimental data exits to confirm this. Other researchers have seen similar expansions at early ages (Jensen et al. 2001a; Bjøntegaard et al. 2004; Lura et al. 2004). 


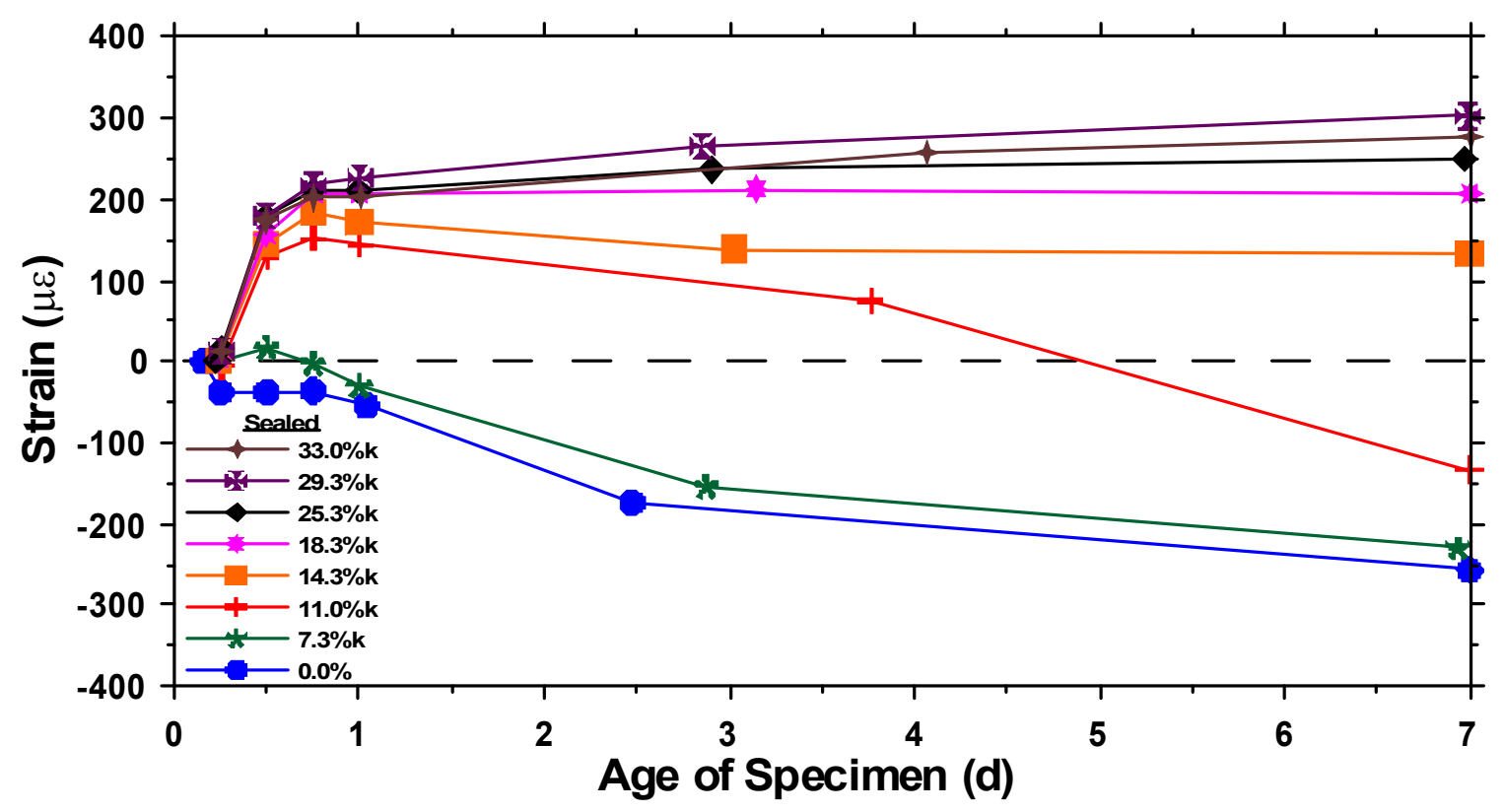

Figure 6-2 - Free shrinkage results of plain and LWA-K mortar mixtures in sealed curing conditions during the first $7 \mathrm{~d}$

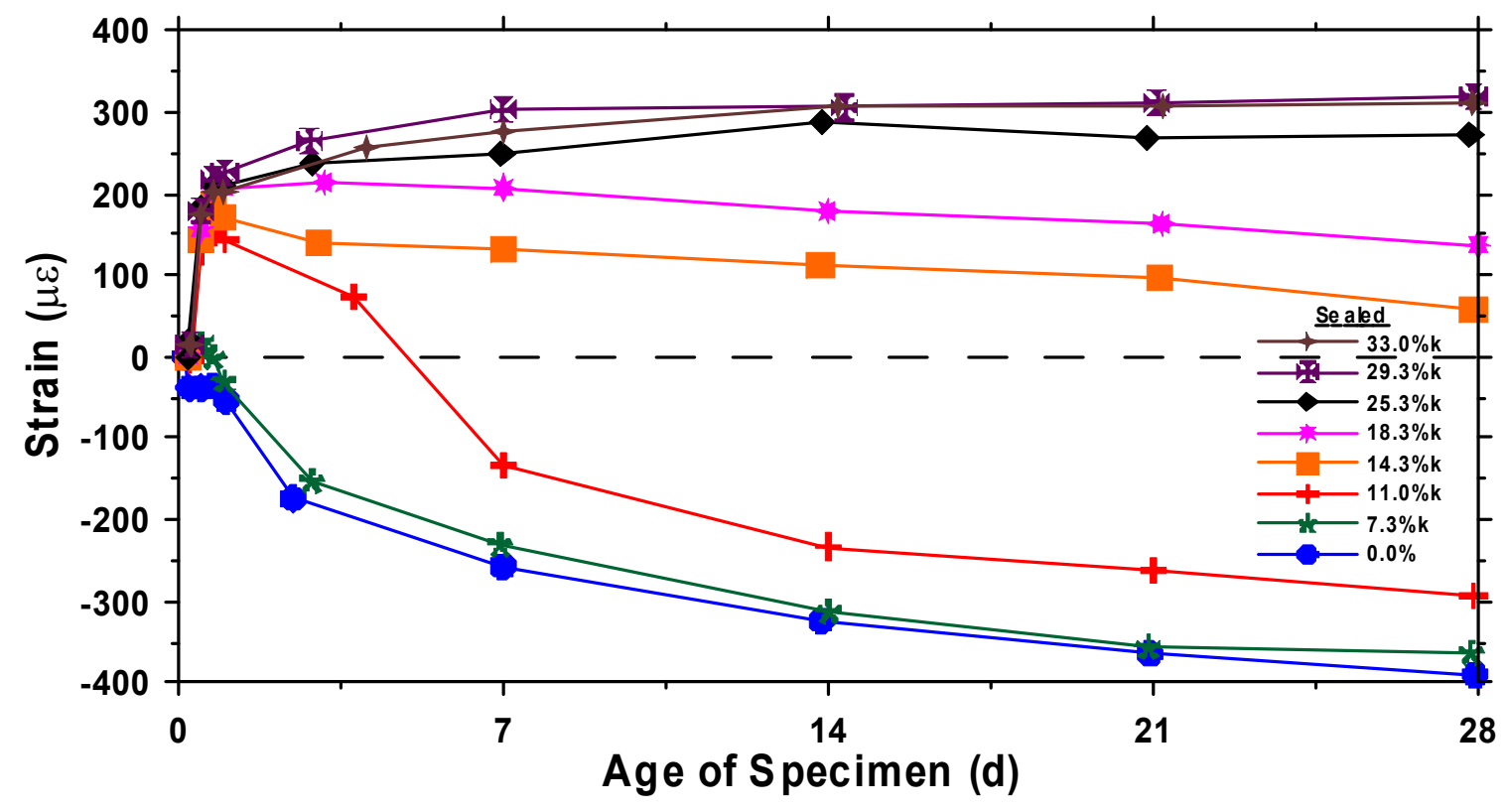

Figure 6-3 - Free shrinkage results of plain and LWA-K mortar mixtures in sealed curing conditions 
The shrinkage can be more thoroughly explained if the volume of void creation is considered as shown in Figure 6-4. The volume of chemical shrinkage minus the volume of autogenous shrinkage is the approximate volume of voids created in the cement paste. The volume of water supplied in the LWA can only fill in voids until all the water in the LWA is depleted. Even if voids are created, the water may not leave the LWA. This could be because the internal relative humidity is not low enough to pull the water from the LWA. When water no longer leaves the LWA, the pores of the paste will begin to empty and significant shrinkage is induced. An explanation for the similar performances of lower replacement volumes could also be explained by their having lower protected paste volume fractions (Figure 3-5). This is not to say that lower replacement volumes of LWA are not beneficial. The $11.0 \% \mathrm{k}$ mixture has a substantially lower rate of shrinkage during the first $7 \mathrm{~d}$. The lower rate of shrinkage could provide enough time for sufficient strength to develop to resist cracking. Indeed, it will be demonstrated later that lower replacement volumes have beneficial effects in restrained shrinkage. 


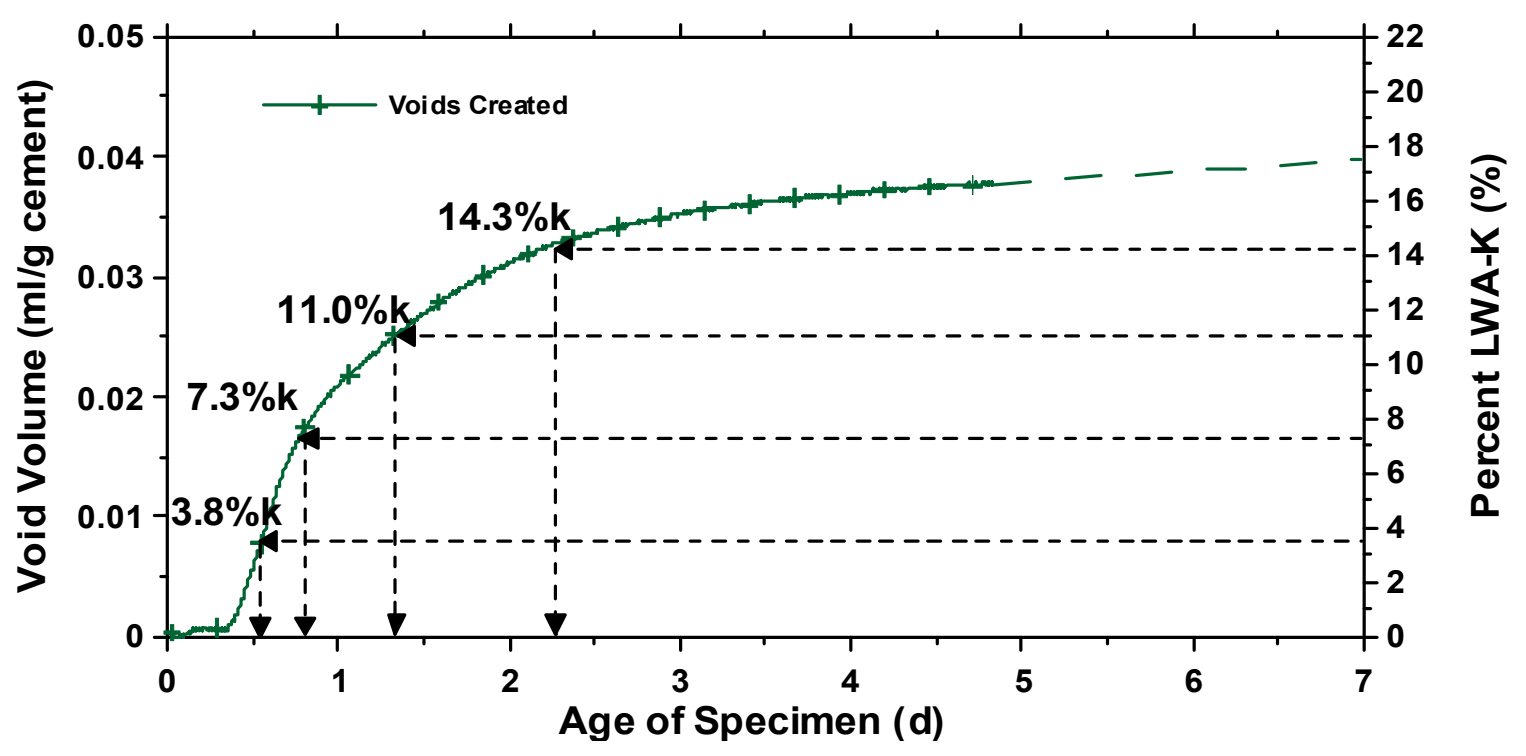

Figure 6-4 - Void creation in cement paste and the time when water depletes from the LWA-K as volume replacements increase. The $18.3 \% \mathrm{k}, 25.3 \% \mathrm{k}$, $29.3 \% \mathrm{k}$ and $33.0 \% \mathrm{k}$ mixtures don't deplete of water before $7 \mathrm{~d}$

Figure 6-5 can be used to provide a better explanation for the shrinkage that occurs in the mixtures with replacement volumes lower than $18.3 \% \mathrm{k}$. This plot shows when the water is expected to deplete from the LWA based on Figure 6-4 along with when the mixtures 'begin to shrink' as measured by the corrugated tubes and sealed prismatic specimens. What is meant by 'begin to shrink' is when the mixture first begins to shrink after its initial expansion, not when the mixture actually shows a negative $(<0)$ value of strain in Figure 6-2 and Figure 6-3. From Figure 6-5, the times at which the water depletes (from Figure 6-4) and when shrinkage occurs in these lower replacement volume mixtures are similar, though not exactly the same. It is expected that the time at which shrinkage would begin would actually occur at a slightly earlier time since all the IC water may not be available in the actual measurements though it is assumed to completely be so in Figure 6-4. The time at which water depletes (from chemical shrinkage) and the time to the beginning of shrinkage is very similar for 
low replacement volumes $(<14.3 \%)$, however, when the volume replacement is larger, the curves diverge.

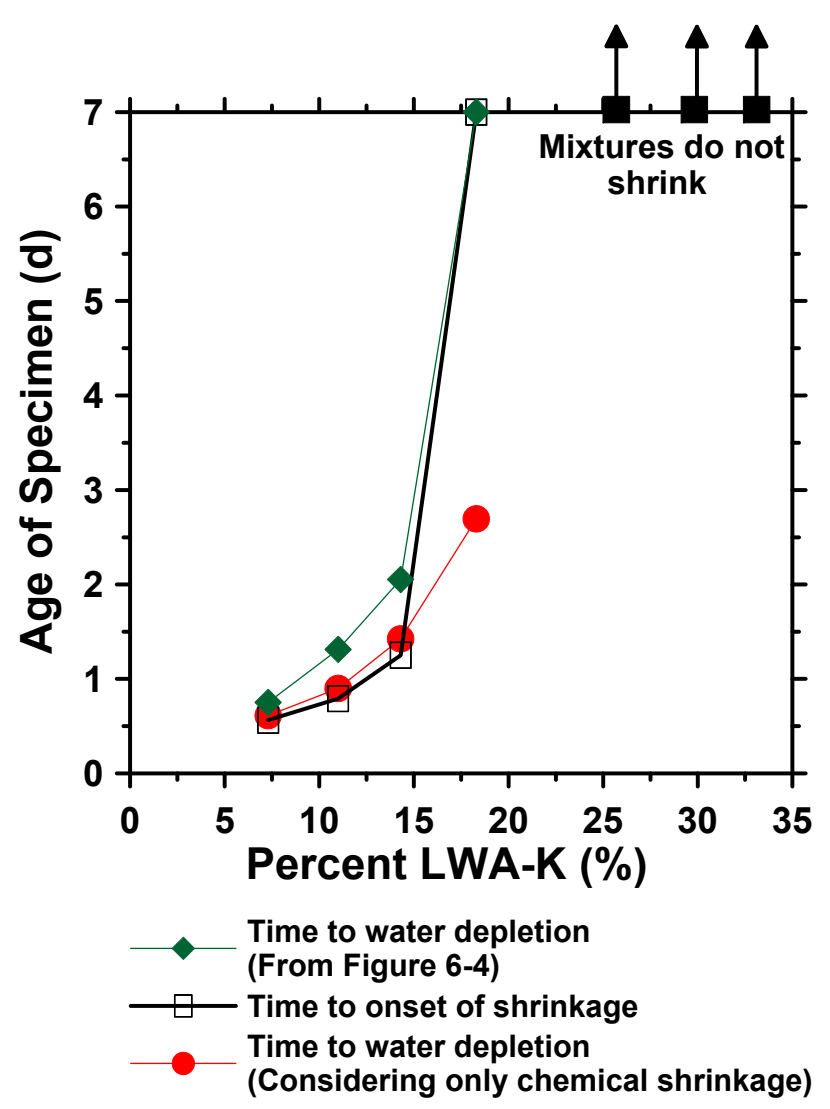

Figure 6-5 - Time of water depletion from the SLWA versus the time when the mixtures begin to shrink as measured by the corrugated tubes and sealed prisms

Another important feature to note is that there is not a significant benefit of using replacement volumes larger than that needed to eliminate self-desiccation (25.3\%k) in the sealed case; however, in the drying case to be presented subsequently, some additional benefits can be observed. The volume and distribution of water in the $25.3 \% \mathrm{k}$ mixture is sufficient to eliminate selfdesiccation. The additional water provided by the $29.3 \% \mathrm{k}$ and $33.0 \% \mathrm{k}$ mixtures would have no voids to fill in the paste and some IC water would simply remain stored in the LWA. 
To better understand the effectiveness of these systems, the $7 \mathrm{~d}$ free shrinkage was plotted against the replacement volume of LWA-K, as shown in Figure 6-6. When a lower replacement volume $(7.3 \% \mathrm{k})$ is used, the shrinkage is very similar to that of the plain mixture. When larger replacement volumes are used, the system becomes more effective in decreasing the shrinkage.

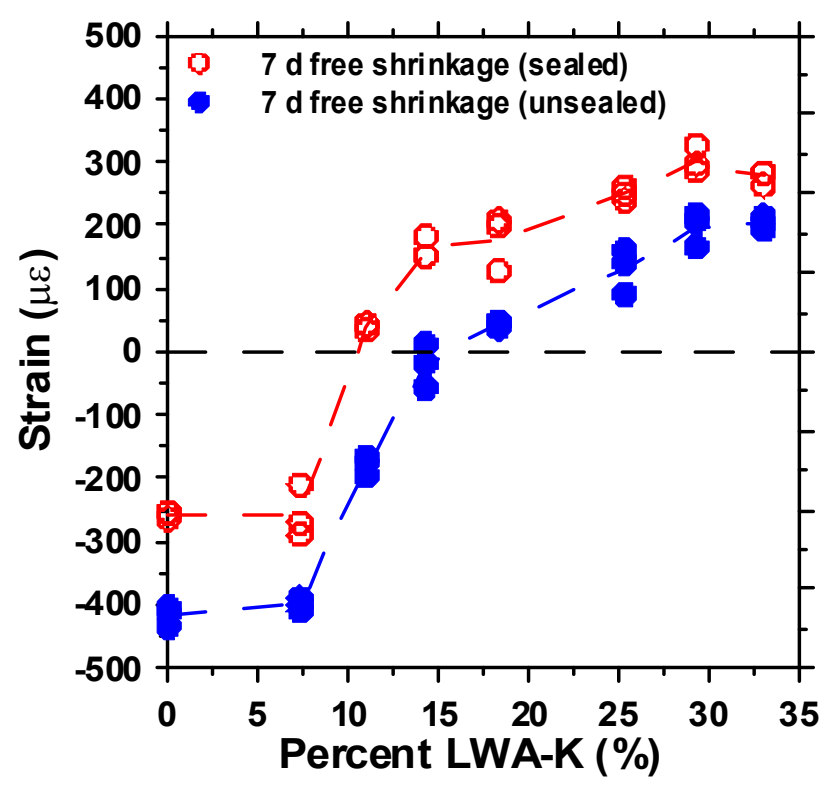

Figure 6-6 - $7 \mathrm{~d}$ free shrinkage measurements plotted as a function of the replacement volume of LWA-K (3 samples are shown for each mixture)

\subsubsection{Unsealed Curing Conditions}

Figure 6-7 shows the average unrestrained length change from three replicate samples of each mixture exposed to drying. The first $24 \mathrm{~h}$ of the unsealed specimens are the same as the sealed specimens (Figure 6-3). After $24 \mathrm{~h}$, the specimens were exposed to drying. As expected, the plain mixture shows the most shrinkage during the first $28 \mathrm{~d}$. The $7.3 \% \mathrm{k}$ mixture performed similar to the plain mixture. The $11.0 \% \mathrm{k}$ mixture has a large expansion during the first day, 
however when the sample was exposed at $24 \mathrm{~h}$, it shrinks rapidly. After $28 \mathrm{~d}$ of testing, the $11.0 \% \mathrm{k}$ mixture has a similar shrinkage performance as the plain mixture. As the LWA replacement volume is increased, the shrinkage performance improves.

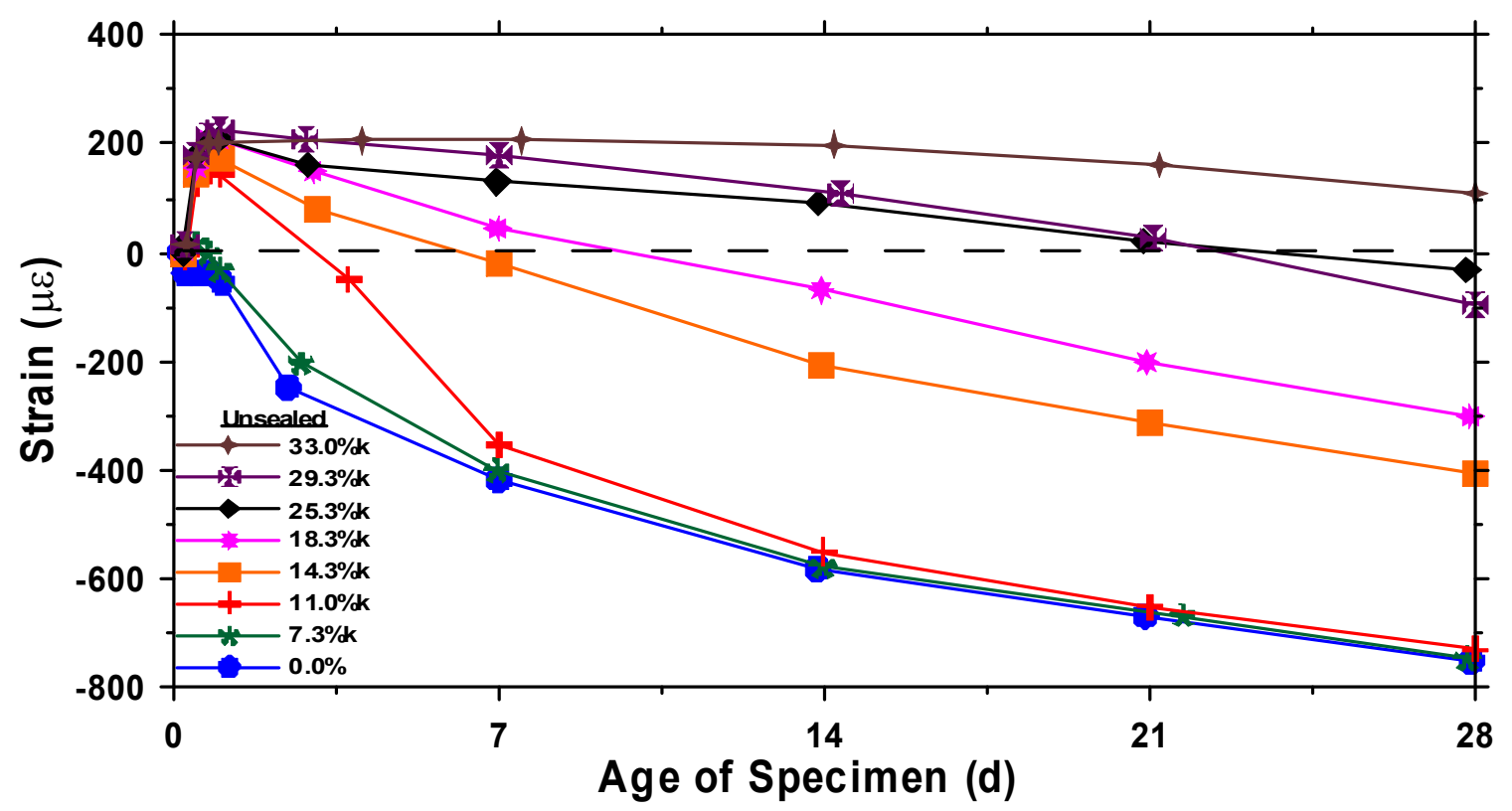

Figure 6-7 - Free shrinkage results of plain and LWA-K mortar mixtures in unsealed curing conditions

Figure 6-6 shows the $7 \mathrm{~d}$ free shrinkage of the unsealed samples. Low replacement volumes proved to be ineffective in mitigating shrinkage caused by the combined effects of external and internal drying. However, when the replacement volume is increased beyond a critical level, the shrinkage is decreased. When high replacement volumes are used, the benefits are similar in Figure 6-6.

The mass loss of the LWA-K samples during testing can be seen in Figure 6-8. The larger the replacement volume the more mass loss occurred. The larger the 
replacement volume the more mass loss occurred. This general trend can be seen in Figure 6-9. This is what would be expected since there is more total (mixture and IC) water which can be lost as drying progresses.

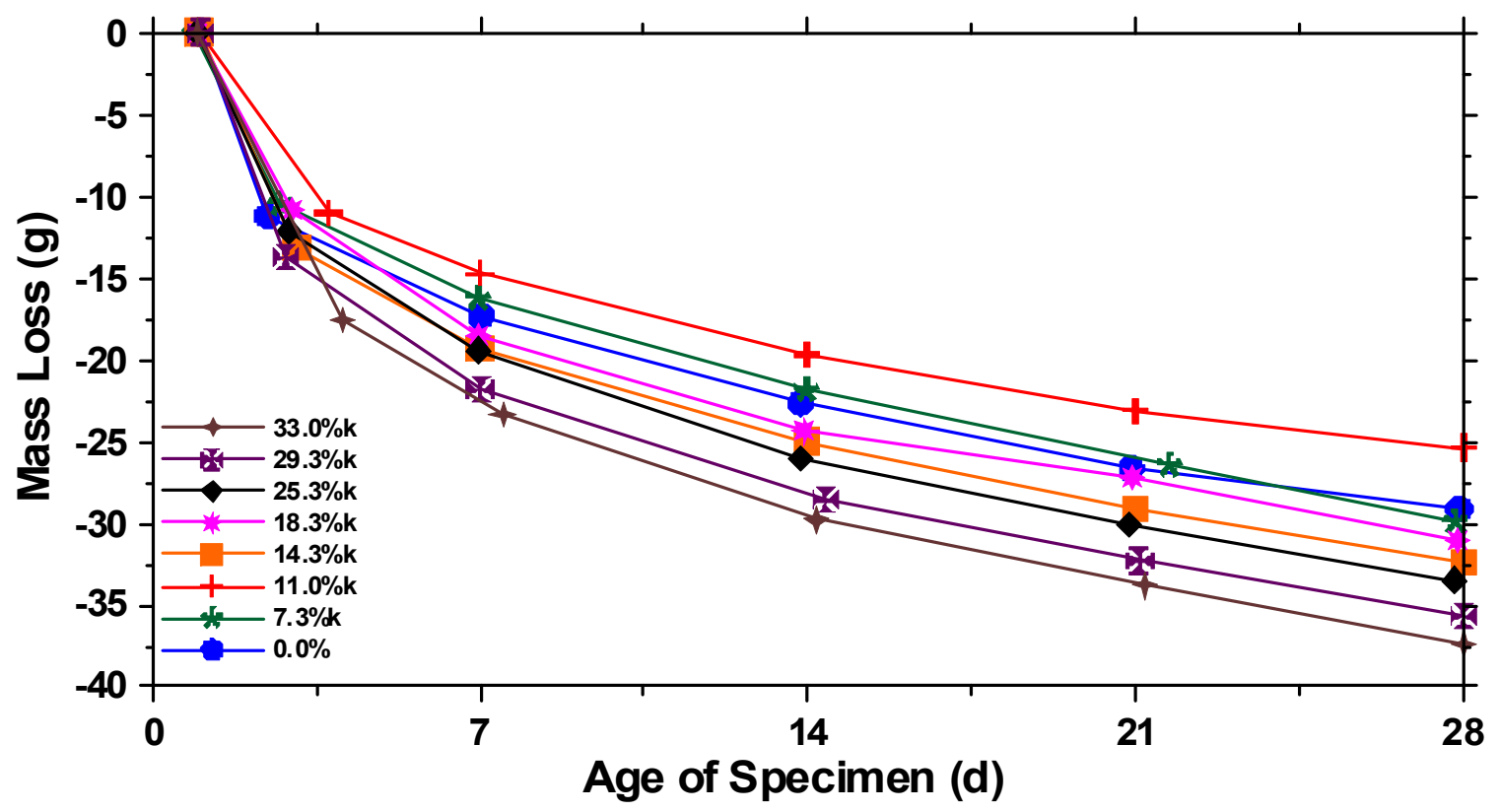

Figure 6-8 - Mass loss of LWA-K free shrinkage samples in unsealed curing conditions 


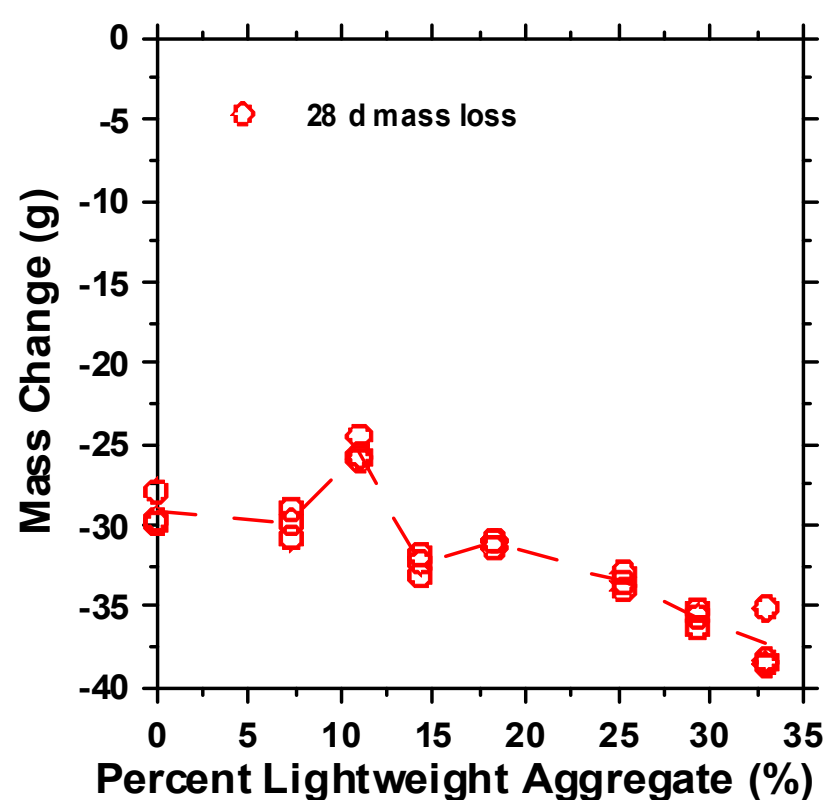

Figure 6-9 - $28 \mathrm{~d}$ mass loss measurements for unsealed free shrinkage measurements plotted as a function of the replacement volume of LWA-K

\subsubsection{Different LWA Volume Replacements on Restrained Shrinkage}

\subsubsection{Sealed Curing Conditions}

The results of the restrained shrinkage tests performed in the plain mixture along with the mixtures containing LWA-K expanded shale are shown in Figure 6-10 for the sealed samples. When small volume replacements of LWA are incorporated, only a small beneficial effect can be observed. For example, the $0.0 \%$ mixture cracked on the sixth day while the mixture containing 3.8\%k LWA cracked at 7.5 $\mathrm{d}$ and the $7.3 \% \mathrm{k}$ mixture cracked at $12 \mathrm{~d}$. The LWA mixtures show a decreasing residual strain at the time of cracking. This may be due to a reduced strength or elastic modulus. When larger replacement volumes are used, it can be seen that cracking can be substantially reduced. Testing of the $14.3 \% \mathrm{k}$ mixture was stopped after $19 \mathrm{~d}$ (with only a residual strain of $-13 \mu \varepsilon$ ). Testing of the $25.3 \% \mathrm{k}$ mixture was stopped after $13 \mathrm{~d}$ and had not developed any residual strain (likely 
due to a slight expansion). It should be noted that the strain measured us the strain in the steel ring, however this can be approximated as the strain in the mortar (Radlinska et al. 2008a).

The performance of the mixtures as a function of SLWA replacement volume fraction is presented in Figure 6-11 for both sealed and unsealed samples. It can be seen that low SLWA volume fractions crack at an early age, however as the replacement volumes are increased to a sufficient percentage, the time to cracking is greatly extended.

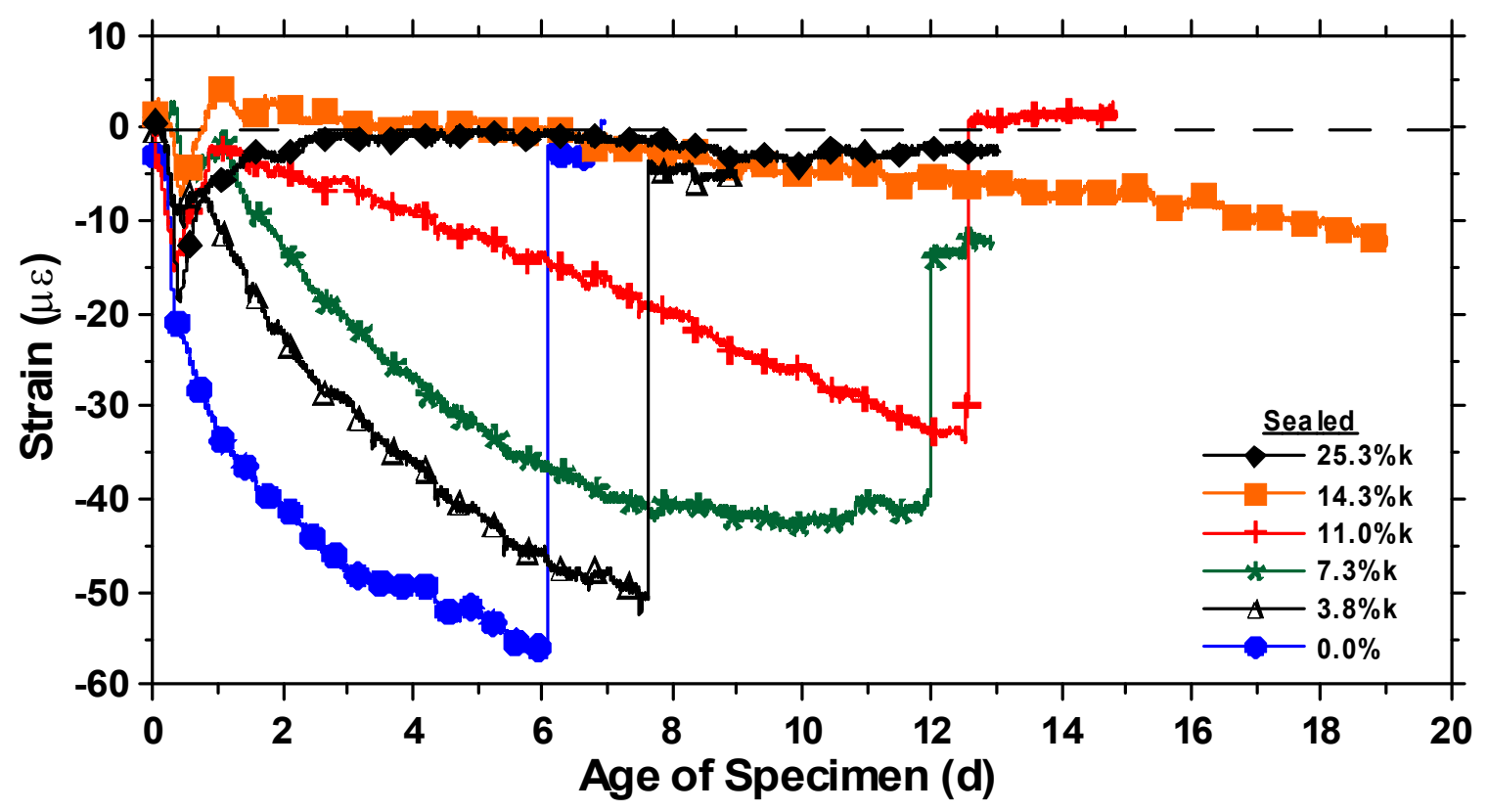

Figure 6-10 - Restrained shrinkage results of plain and LWA-K mortar mixtures in sealed curing conditions (In each case, a typical response is shown from the three samples tested). Sharp vertical rise in strain curve indicates occurrence of cracking in the specimen 


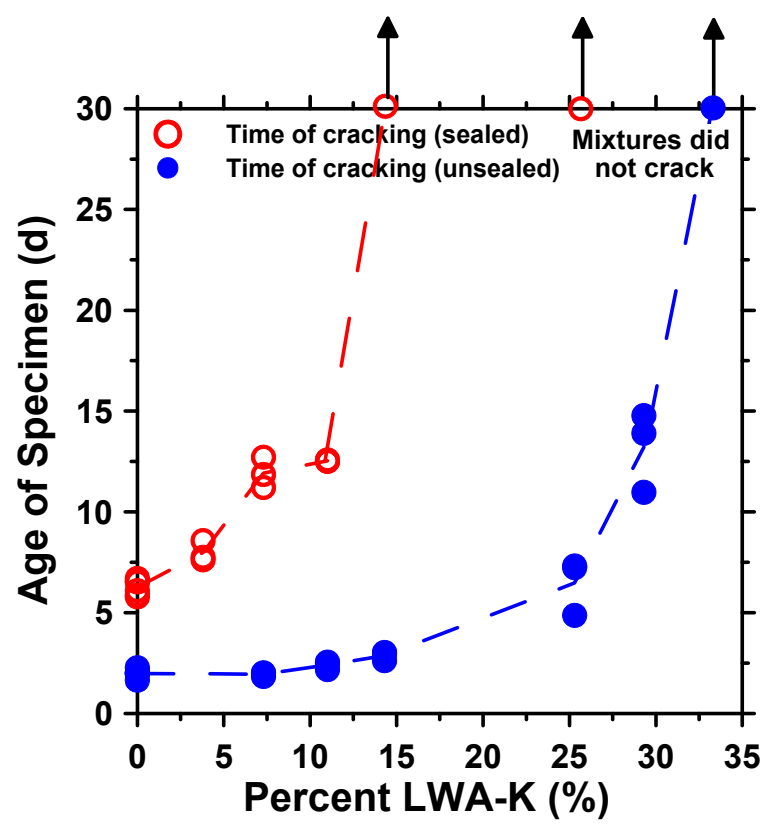

Figure 6-11 - Time of cracking in LWA-K mixtures plotted as a function of replacement volume (3 samples are shown for each mixture)

\subsubsection{Unsealed Curing Conditions}

Figure 6-12 shows the strain measured from the inner surface of the restrained ring. The plain mixture cracks after approximately $2 \mathrm{~d}$. The times to cracking for the mixture with replacement volumes less than $14.3 \%$ were similar to that of the plain mixture, though some cracked at a lower strain. This is likely due to water depleted during the first days due to self-desiccation combined with rapid water loss due to evaporation. When a larger replacement volume was used $(25.3 \% \mathrm{k})$, the time to cracking was prolonged to approximately $8 \mathrm{~d}$. With a further increase in the replacement volume $(29.3 \% \mathrm{k})$, cracking was delayed to $14 \mathrm{~d}$. During the $14 \mathrm{~d}$ of testing, the $33.0 \%$ mixture did not crack and showed little shrinkage strain under these testing conditions. 


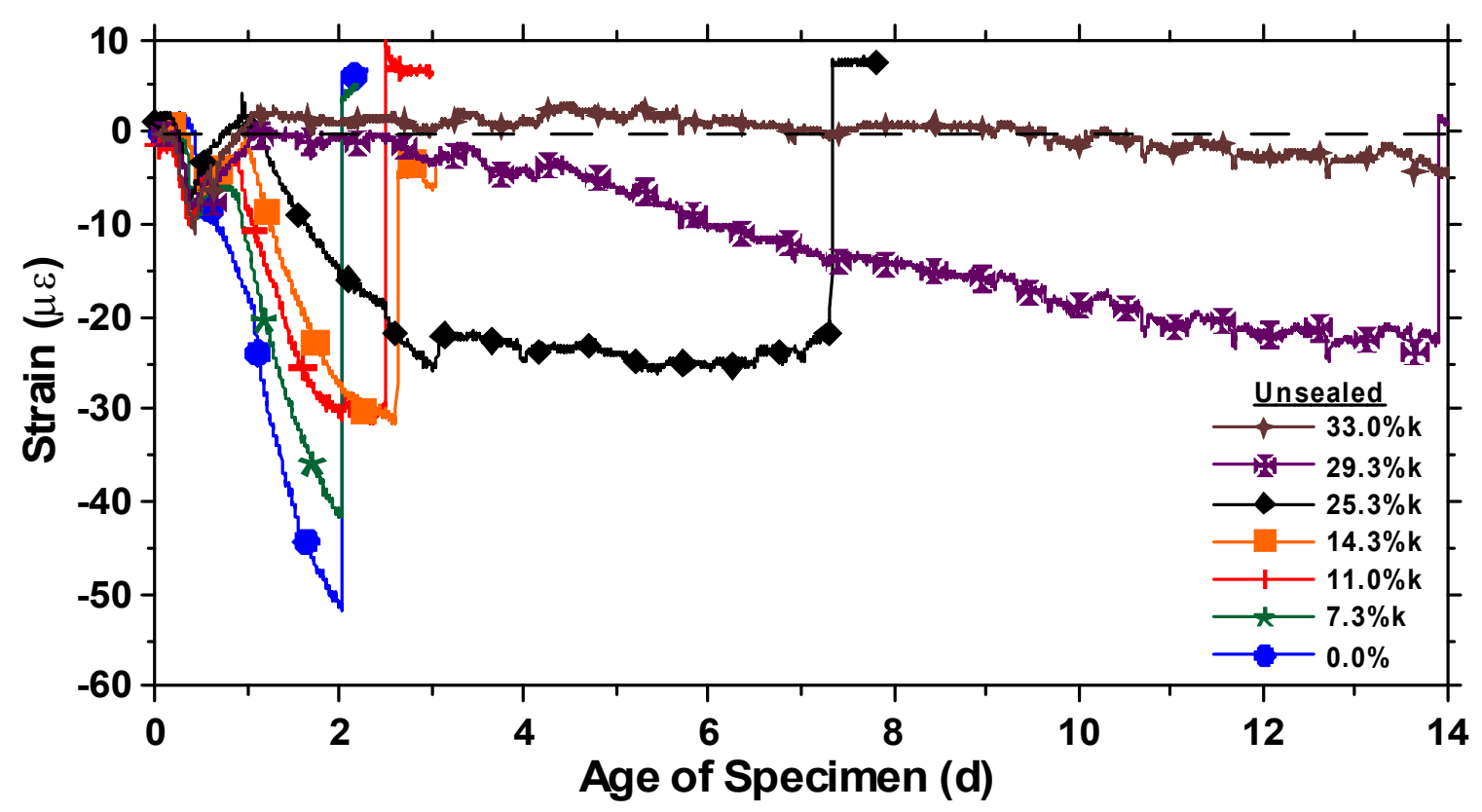

Figure 6-12 - Restrained shrinkage results of plain and LWA-K mortar mixtures in unsealed curing conditions (In each case, a typical response is shown from the three samples tested). Sharp vertical rise in strain curve indicates occurrence of cracking in the specimen

The performance of the mixtures as a function of SLWA volume fraction is presented in Figure 6-11. It can be seen that low SLWA volume fractions crack at an early age; however as the replacement volumes are increased to a sufficient percentage, the time to cracking is greatly extended or cracking is eliminated. Figure 6-13 shows the strain at cracking for both the unsealed and sealed curing conditions. The strain at cracking for each mixture at low replacement volumes is the same regardless of the curing condition (Henkensiefken et al. 2008h) (i.e., strain at cracking for the $11.0 \% \mathrm{k}$ mixture is the same in both sealed and unsealed curing conditions). The mixtures for which the strains do not match are the mixtures that did not crack in sealed curing conditions. It is expected that if the strain for the sealed samples that did not crack $(14.3 \% \mathrm{k}$ and $25.3 \% \mathrm{k})$ were to approach the strain experienced by the 
unsealed samples, they would crack. It can be said that the curing condition does not have an effect on the strain at a cracking event.

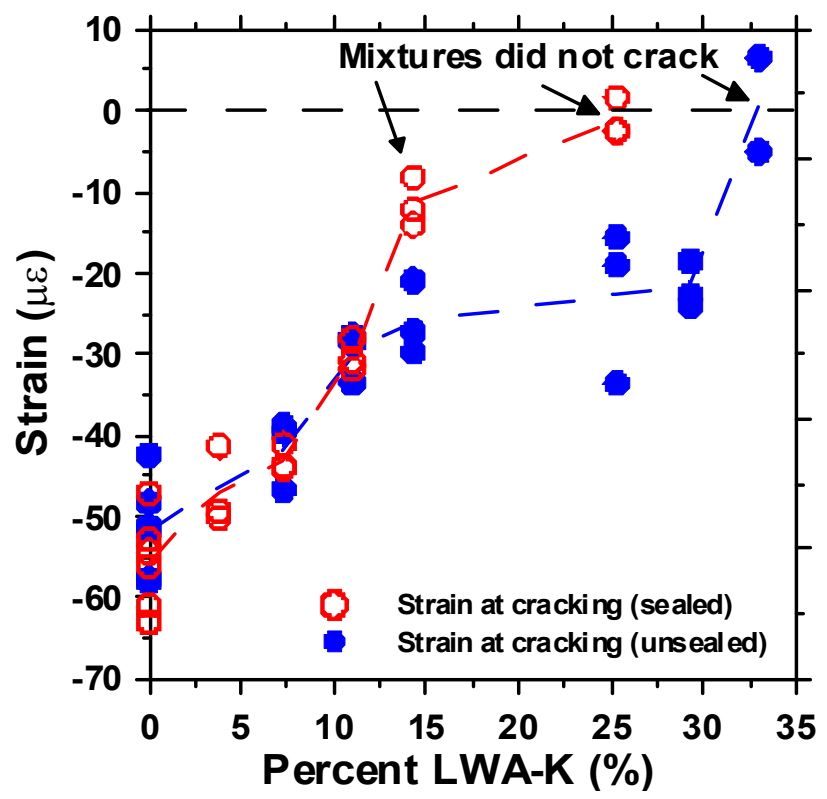

Figure 6-13 - Strain at cracking in LWA-K mixtures plotted as a function of replacement volume (3 samples are shown for each mixture)

\subsubsection{Different Volume Replacements on Internal Relative Humidity}

Figure 6-14 shows the internal relative humidity measurements for the LWA-K mixtures. While the specimens employed for measurement of internal relative humidity contained no normal weight aggregates, the cement paste to SLWA proportion was adjusted to be the same as in the corresponding mortar mixtures. As expected, when higher replacement volumes are used, the internal relative humidity remains higher. Again, this is related to the pore size that remains saturated according to Equation 3-3. Because the internal relative humidity is higher (thus the pore size that is still saturated is larger), it would be expected that the shrinkage for higher replacements volumes in sealed curing conditions would be less. The radius of the meniscus for the pore (approximately the pore 
size still saturated) and the capillary stress are plotted as a function of the replacement volume in Figure 6-15. It can be seen that a critical value exists for the replacement volume of SLWA. When high replacement volumes $(29.3 \% \mathrm{k}$ and $33.0 \% \mathrm{k}$ ) are used the pore size which remains saturated becomes larger. This can be used to explain why these mixtures perform significantly better under restrained shrinkage. The capillary stress is reduced with increasing replacement volume.

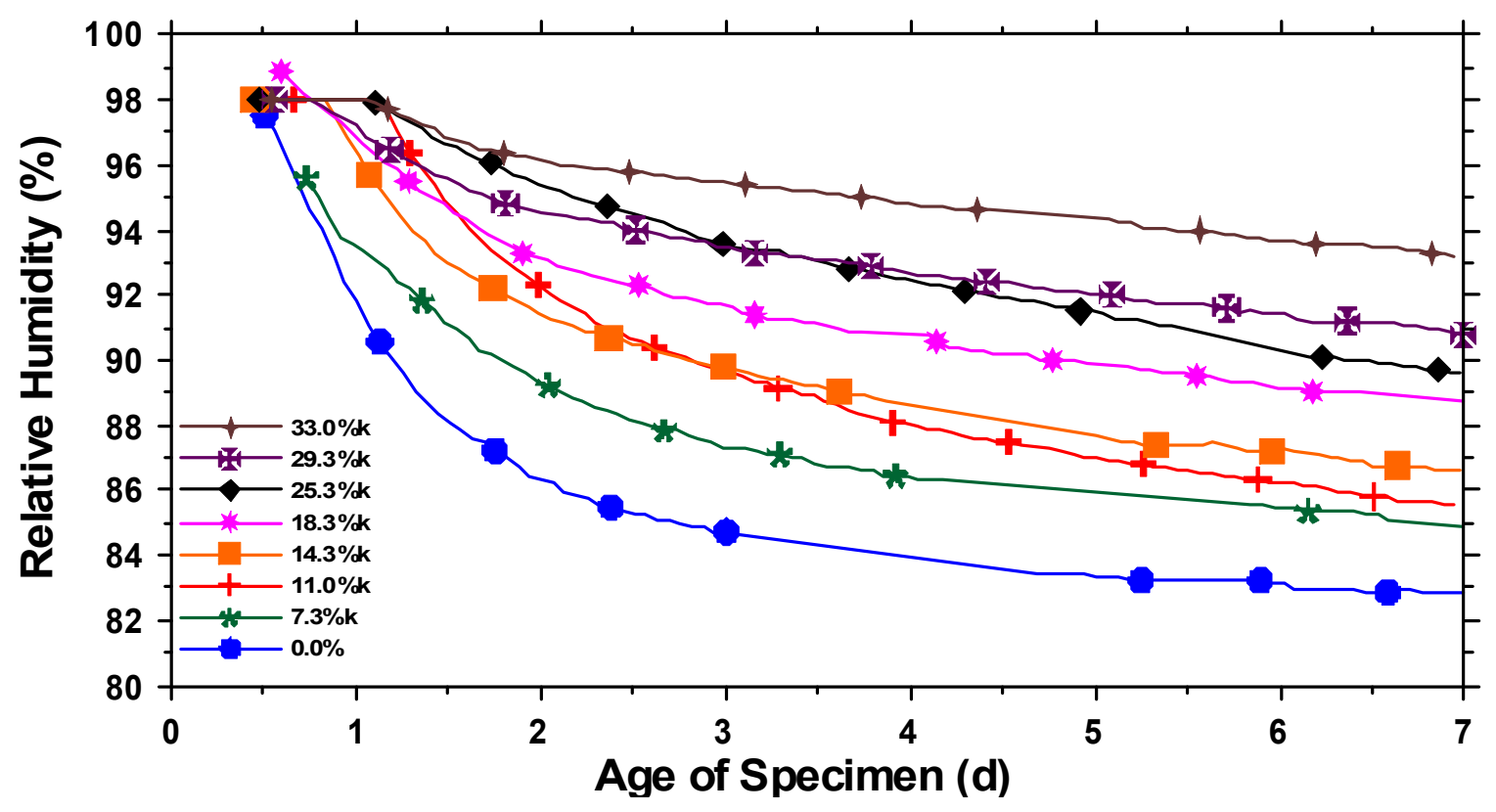

Figure 6-14 - Internal relative humidity measurements for the sealed LWA-K mixtures 


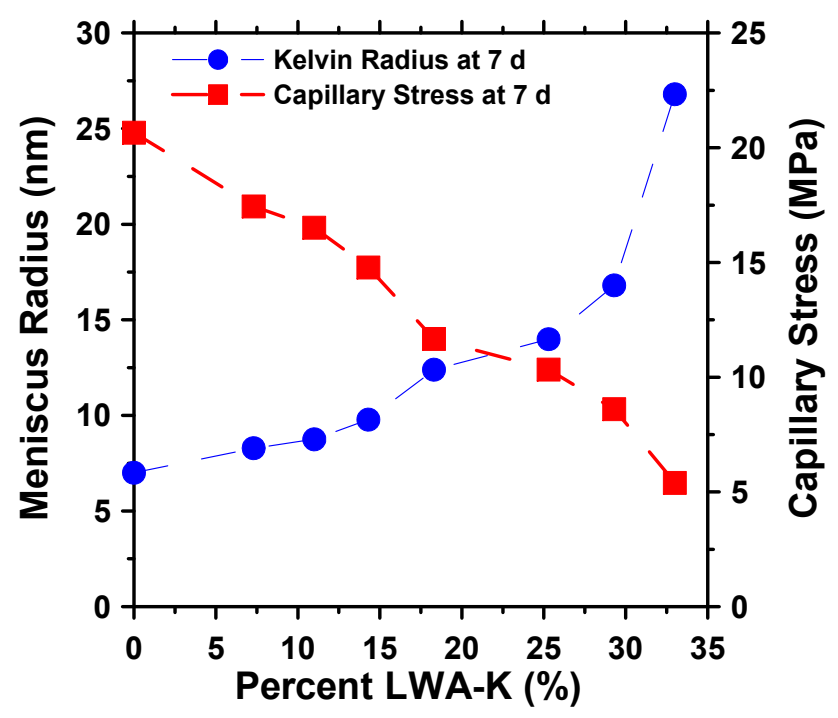

Figure 6-15 - Kelvin Radius and capillary stress as determined from $\mathrm{RH}$ measurements as a function of replacement volume of LWA-K at $7 \mathrm{~d}$

\subsubsection{Effect of Particle Spacing on Internal Curing Effectiveness}

To show the importance of LWA particle size and spacing on the shrinkage performance of internal curing, a second LWA was tested. The aggregates had similar desorption properties, as seen in Figure 3-3, but different absorption volume. This would imply that they would give up water in a similar manner; however the volume of water they could give up would not be the same. The LWA-K had a $24 \mathrm{~h}$ absorption of $10.5 \%$ while the LWA-H was much lower at approximately $5.8 \%$. Two sets of mixtures were selected for this comparison. The first comparison provided enough water to fill in the voids created by chemical shrinkage. The LWA-K mixture was designated as $25.3 \% \mathrm{k}$, and the LWA-H mixture was designated as $43 \% \mathrm{~h}$. Because the LWA-H absorbed less water, more aggregate was needed. The second comparison would have the same replacement volume of LWA. This mixture was designated as $25.3 \% \mathrm{~h}$, to be compared against $25.3 \% \mathrm{k}$. It should be noted that the $25.3 \% \mathrm{~h}$ mixture provided a similar volume of water as the $14.3 \% \mathrm{k}$ mixture. 
Figure 6-16 shows the free shrinkage results for the LWA-K and LWA-H mixtures in sealed curing conditions. It can be seen that when a similar volume of water is supplied (two data sets depicted with diamonds), and when that water is better distributed in the $43 \%$ h mixture, the shrinkage performance is better. Figure 6-17 shows the restrained shrinkage results for the LWA-K and LWA-H mixtures in unsealed curing conditions. When a similar replacement volume is used $(25.3 \% \mathrm{k}$ and $25.3 \% \mathrm{~h}$ ), the strain develops in a similar manner, however the mixture with less water $(25.3 \% \mathrm{~h})$ cracks at an earlier age. This is what would be expected since the mixtures have similar mechanical properties; however the mixture containing LWA-H depletes of water earlier, resulting in earlier cracking. When similar volumes of water $(25.3 \% \mathrm{k}$ and $43 \% \mathrm{~h})$ are used, the mixture with more particles and thus a better particle distribution ( $43 \% \mathrm{~h})$ performs better. The internal relative humidity tests for these mixtures can be found in Figure 6-18. This shows that when similar volumes of water are used, the internal relative humidity is approximately equal, which would imply a similar pore radius that is still saturated.

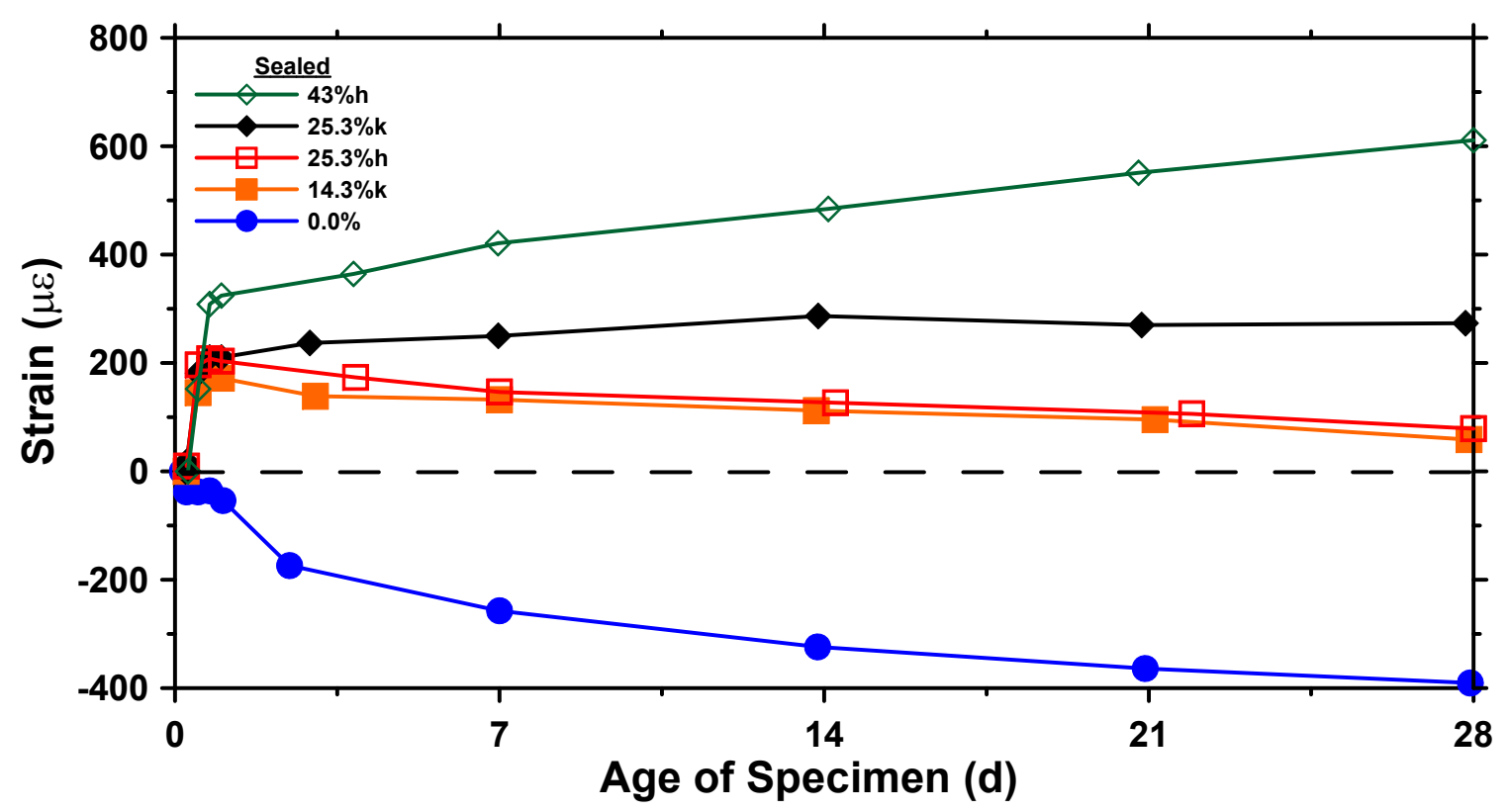

Figure 6-16 - Free shrinkage results of a comparison between LWA-K and LWA$\mathrm{H}$ LWA in sealed curing conditions 


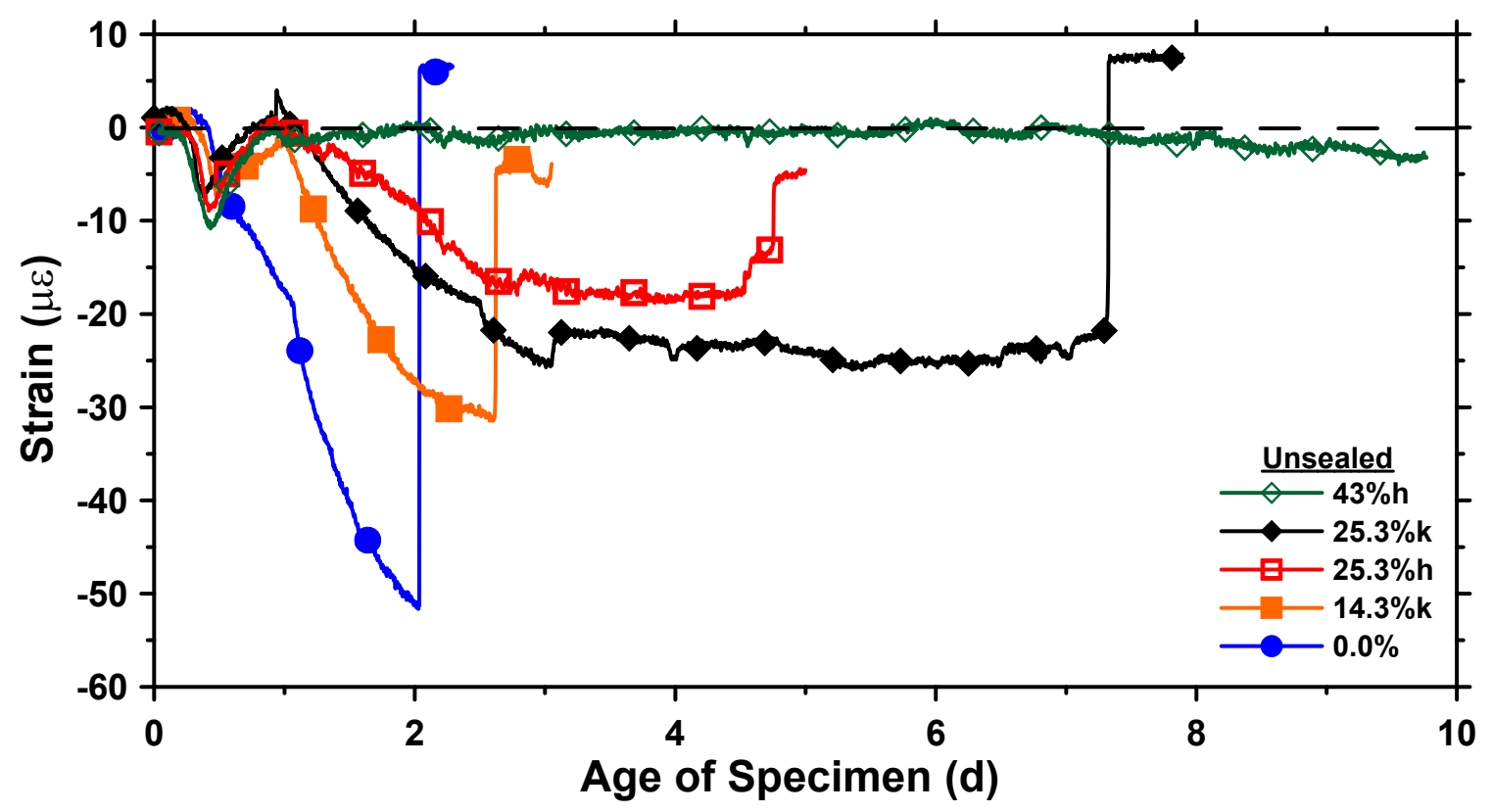

Figure 6-17 - Restrained shrinkage results of a comparison between LWA-K and LWA-H LWA in unsealed curing conditions

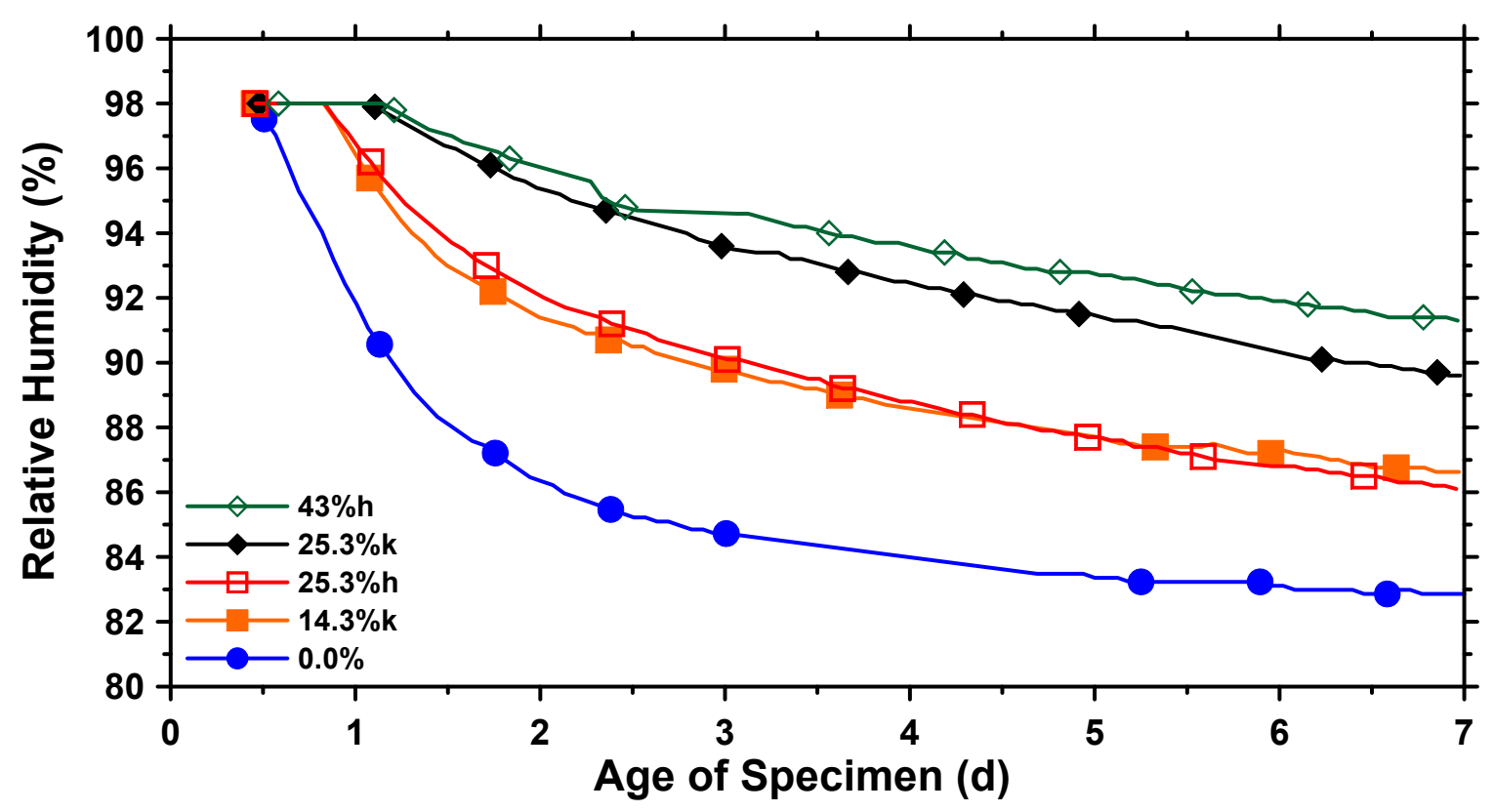

Figure 6-18 - Internal relative humidity results of a comparison between LWA-K and LWA-H SLWA 


\subsection{Conclusions}

This chapter has presented a comprehensive data set that describes the behavior of sealed and unsealed mortars made with SLWA. The chapter provides an understanding of what is occurring in the mixtures with different volume replacements of SLWA. The influence of boundary conditions (sealed or unsealed) on the performance of self-curing concrete is described. The inclusion of a sufficient volume of SLWA can significantly reduce self-desiccation, reduce autogenous shrinkage and delay the time of cracking. A summary of the general conclusions from the data presented in this chapter are:

\section{Sealed Specimens}

- Specimens with larger volume fractions of SLWA showed a greater reduction in both the rate of shrinkage and the autogenous shrinkage. This can be attributed to the ability of the SLWA to supply water to the hydrating paste, which acts to reduce the effects of self-desiccation and maintains the internal relative humidity at a higher level.

- Similar free shrinkage and cracking behavior was observed between a plain mixture and mixtures with low volumes of SLWA replacement. This may be due to the fact that the SLWA particles are spaced too far apart to effectively supply water to all the paste or that the IC water supply is depleted too early in the hydration process. The time to cracking can be prolonged if a sufficiently high replacement volume of SLWA is incorporated.

- Self-desiccation results in a reduction in the internal relative humidity of a low water to cement ratio paste. Specimens with a larger replacement volume of SLWA maintained a higher internal humidity as expected. 
Unsealed Specimens

- The addition of SLWA showed a reduction in the total shrinkage during the first $28 \mathrm{~d}$. This may be due to the initial reduction in selfdesiccation and the additional water that can complete hydration and temporarily replace the water that is lost to the environment.

- A larger mass of water was lost for samples with an increasing volume of SLWA. This is consistent with these mixtures having a larger volume of total (mixture and IC) water.

- The time to cracking is prolonged for mixtures with an increasing volume of SLWA. As illustrated in this study, this is likely due to the reduced shrinkage; in addition, although not specifically examined in this study, reduced elastic modulus, increased relaxation, and increased fracture toughness of the mixtures with SLWA could also contribute to this increase in time to cracking. 


\title{
CHAPTER 7. PLASTIC SHRINKAGE CRACKING IN MIXTURES MADE WITH
} PREWETTED LIGHTWEIGHT AGGREGATE

\begin{abstract}
7.1. Introduction
Concrete can be susceptible to plastic shrinkage cracks if weather conditions between the time of placement to the time of set are too harsh (Powers 1968; Powers et al. 2003a; Powers et al. 2003b). Though these cracks may be unsightly, a larger problem exists as they may lead to the ingress of harmful materials (Qi 2003a). Lightweight aggregate for use in concrete has been increasingly promoted for its benefits of reducing shrinkage cracking caused by self-desiccation. While several studies have shown the beneficial effects of IC on autogenous shrinkage and shrinkage cracking in hardened concrete (Jensen et al. 2001a; Lura 2003a; Henkensiefken et al. 2008b), few, if any, studies have investigated the influence of internal curing on the formation of plastic shrinkage cracking.
\end{abstract}

This chapter provides measurements of plastic shrinkage cracking with different volume replacements of LWA. The results indicate that if a sufficient volume of LWA is added to concrete, plastic shrinkage cracking can be reduced or eliminated.

\subsection{Mechanisms of Plastic Shrinkage}

The formation of plastic shrinkage cracks can be described by the three drying phases which the concrete undergoes (Lura et al. 2006b). During the first drying 
phase the cement and aggregate particles consolidate exuding water to the surface. This water is known as bleed water. This bleed water covers the surface of the concrete and this layer of water is then free to evaporate. The initial evaporation rate from a free surface is from the layer of bleed water that forms and is similar to the evaporation of bulk water (Lura et al. 2006b). This region of evaporation is generally referred to as the first drying region (i.e., phase $(\mathrm{I}))$.

Once the layer of bleed water evaporates, liquid-vapor menisci form at the surface of the concrete (Wittmann 1976). The formation of the liquid-vapor menisci results in capillary pressure. This is commonly referred to as the second drying phase (phase(II)). The Young-Laplace equation can be used to relate the radius of the menisci to the capillary pressure (Equation 7-1):

$\sigma_{\text {cap }}=-\frac{2 \gamma \cos (\theta)}{r}$

Equation 7-1

where: $\sigma_{\text {cap }}(\mathrm{Pa})$ is the capillary pressure, $\gamma(\mathrm{N} / \mathrm{m})$ is the surface tension of pore fluid, $\theta$ (radians) is the liquid-solid contact angle (assumed in this work to be 0 radians), and $r(\mathrm{~m})$ is the radius of curvature of the meniscus. The capillary pressure that develops further compresses the particles more resulting in particle rearrangement and even more pore solution being expelled from the system and being forced to the top surface of the concrete.

With greater capillary pressure, more pore solution (water) will be exuded to the surface and water can be drawn out of the LWA. During this phase, it would be expected that a reduction on the evaporation rate would occur. When the capillary pressure is no longer able to compress the system and the drying front recedes from the surface towards the interior of the concrete, the concrete is susceptible to cracking. The transition that occurs at this point is referred to as 
the critical point (Lura et al. 2006b). The critical point represents the point in time when the concrete is most susceptible to cracking (Cohen et al. 1990).

In the final drying phase, the evaporation and settlement slow down as the drying front penetrates into the interior of the pore system. This final phase of drying is referred to as phase (III).

\subsection{Constituent Materials}

The coarse aggregate used was pea gravel with an apparent specific gravity of 2.58 and a $3 / 8$ inch maximum size aggregate. The normal weight fine aggregate used was natural river sand with a fineness modulus of 2.71 and an apparent specific gravity of 2.58. Portions of the normal weight sand were replaced with a manufactured rotary kilned expanded shale with a fineness modulus of 3.10 and a specific gravity of 1.56 . The $24 \mathrm{~h}$ absorption of the coarse aggregate was determined to be $2.3 \%$ according to ASTM C127-04 (2004). The $24 \mathrm{~h}$ absorption of the normal weight sand and LWA were determined to be $1.8 \%$ and $10.5 \%$, respectively, according to ASTM C128-07 (2007).

For the mortar mixtures, ASTM C150 Type I ordinary portland cement (OPC) was used, with a Blaine fineness of $370 \mathrm{~m}^{2} / \mathrm{kg}$ and an estimated Bogue composition of $56 \% \mathrm{C}_{3} \mathrm{~S}, 16 \% \mathrm{C}_{2} \mathrm{~S}, 12 \% \mathrm{C}_{3} \mathrm{~A}, 7 \% \mathrm{C}_{4} \mathrm{AF}$ and a $\mathrm{Na}_{2} \mathrm{O}$ equivalent of $0.68 \%$. It should be noted that for the concrete mixtures, a different cement was used that also satisfies ASTM C150 (Type I OPC) with a Blaine fineness of $370 \mathrm{~m}^{2} / \mathrm{kg}$ and an estimated Bogue phase composition of $50 \% \mathrm{C}_{3} \mathrm{~S}, 16 \% \mathrm{C}_{2} \mathrm{~S}, 12 \% \mathrm{C}_{3} \mathrm{~A}$, $7 \% \mathrm{C}_{4} \mathrm{AF}$ and $0.68 \%$ of $\mathrm{Na}_{2} \mathrm{O}$. 
A polycarboxylate-based high-range water-reducing admixture (HRWRA) was added to the mortar mixtures in varying rates depending on the volume replacement level of the LWA. For LWA replacement volumes up to $18.3 \%$, the HRWRA was added at a rate of $0.50 \mathrm{~g}$ per $100 \mathrm{~g}$ of cement. For replacement volumes larger than $25.3 \%, 0.66 \mathrm{~g}$ of HRWRA per $100 \mathrm{~g}$ of cement was used. Different rates of HRWRA were used to keep similar consistencies of these mixtures. No HRWRA was used for the concrete mixtures.

\subsection{Mixture Proportioning}

\subsubsection{Mortar Mixture}

Five different mixtures were prepared with a $w / c$ of 0.30 . This included a plain mortar mixture designated as $0.0 \%$ and four mortar mixtures with varying amounts of sand replaced by prewetted LWA, as shown in Table 7-1. The LWA expanded shale was used for the mixtures designated as $11.0 \% \mathrm{k}, 18.3 \% \mathrm{k}$, $25.3 \% \mathrm{k}$, and $33.0 \% \mathrm{k}$. It is important to note that the designations are on a total volume basis. The volume of aggregate (LWA and natural sand) was maintained constant at $55 \%$ since only the sand was replaced with LWA. The number in the designation represents the total volume of the mixture occupied by the LWA (in percent). It should be noted that the $25.3 \% \mathrm{k}$ volume replacement corresponds to the amount of LWA necessary to eliminate self-desiccation (Bentz et al. 1999b; Bentz et al. 2005).

Table 7-1 - Mixture proportions of mortar for settlement study 


\begin{tabular}{|c|c|c|c|c|c|}
\hline Material & $\mathbf{0 \%}$ & $\mathbf{1 1 \% k}$ & $\mathbf{1 8 . 3 \% k}$ & $\mathbf{2 5 . 3 \% k}$ & $\mathbf{3 3 . 0 \% k}$ \\
\hline Cement $\left(\mathbf{k g} / \mathbf{m}^{\mathbf{3}}\right)$ & 728 & 728 & 728 & 728 & 728 \\
\hline Water $\left(\mathbf{k g} / \mathbf{m}^{\mathbf{3}}\right)$ & 218 & 218 & 218 & 218 & 218 \\
\hline Fine Aggregate $\left(\mathbf{k g} / \mathbf{m}^{\mathbf{3}}\right)$ & 1418 & 1135 & 950 & 808 & 567 \\
\hline Dry LWA $\left(\mathbf{k g} / \mathbf{m}^{\mathbf{3}}\right)$ & 0 & 172 & 283 & 369 & 525 \\
\hline $\begin{array}{c}\text { Additional Water } \\
\text { Provided by LWA }\left(\mathbf{k g} / \mathbf{m}^{\mathbf{3}}\right)\end{array}$ & 0 & 18 & 30 & 39 & 54 \\
\hline
\end{tabular}

\subsubsection{Concrete Mixture}

Four different concrete mixtures were prepared with an effective water to cement ratio $(\mathrm{w} / \mathrm{c})$ of 0.55 : this included a plain concrete mixture designated as $0.0 \%$ and three mortar mixtures with varying amounts of sand replaced by prewetted LWA, as shown in Table 7-2. The LWA expanded shale was used for these mixtures designated as $6.0 \% \mathrm{k}, 10.0 \% \mathrm{k}$, and $18.0 \% \mathrm{k}$. It is important to note that the designations are on a total volume basis. The volume of fine aggregate (LWA and sand) was maintained constant at $30 \%$ since only the sand was replaced with LWA. The total aggregate content (coarse aggregate, LWA and sand) for these mixtures was maintained constant at $60 \%$. The number in the designation represents the total volume of the mixture occupied by the LWA (in percent). It should be noted that the $18.0 \% \mathrm{k}$ volume replacement corresponds to the amount of LWA necessary to eliminate self-desiccation (Bentz et al. 1999b; Bentz et al. 2005).

Table 7-2 - Mixture proportions of concrete for plastic shrinkage study 


\begin{tabular}{|c|c|c|c|c|}
\hline Material & $0 \%$ & $6.0 \% \mathrm{k}$ & $10.0 \% k$ & $18.0 \%$ k \\
\hline Cement $\left(\mathrm{kg} / \mathrm{m}^{3}\right)$ & 461 & 461 & 461 & 461 \\
\hline Water $\left(\mathrm{kg} / \mathrm{m}^{3}\right)$ & 254 & 254 & 254 & 254 \\
\hline Fine Aggregate $\left(\mathrm{kg} / \mathrm{m}^{3}\right)$ & 774 & 619 & 518 & 309 \\
\hline Coarse Aggregate $\left(\mathrm{kg} / \mathrm{m}^{3}\right)$ & 825 & 825 & 825 & 825 \\
\hline Dry LWA $\left(\mathrm{kg} / \mathrm{m}^{3}\right)$ & 0 & 94 & 154 & 281 \\
\hline $\begin{array}{c}\text { Additional Water Provided } \\
\text { by LWA }\left(\mathrm{kg} / \mathrm{m}^{3}\right)\end{array}$ & 0 & 9.8 & 16.2 & 29.5 \\
\hline
\end{tabular}

\subsection{Experimental Methods}

\subsubsection{Settlement}

The LWA was oven dried, air cooled, and then submerged in water for $24 \pm 1 \mathrm{~h}$ prior to mixing. The volume of water used to submerge the LWA included both mixing water and the water the LWA would absorb in $24 \mathrm{~h}$ when it is immersed in water. The excess water (water not absorbed in $24 \mathrm{~h}$ ) was then decanted and used as the mixing water. The mixing of the mortar was done in accordance with ASTM C305 (2006), with a modification in the order in which the materials were added. The LWA was added to the pre-wetted bowl, to which the normal weight fine aggregate was added. The aggregate mixture was mixed for $30 \mathrm{~s}$. The cement was added and the mixture was mixed for another $30 \mathrm{~s}$. The water (with HWRWA) was added. The mixture was mixed at slow speed ( 140 rpm) for $30 \mathrm{~s}$ and then on medium speed ( $285 \mathrm{rpm})$ for an additional $30 \mathrm{~s}$. Mixing was stopped for $90 \mathrm{~s}$, after which mixing resumed on medium speed for $60 \mathrm{~s}$. Duplicate samples were cast in $75 \mathrm{~mm} \times 100 \mathrm{~mm}$ (depth $\times$ diameter) cylinders in two layers, each of which was vibrated for $15 \mathrm{~s}$. The samples were placed in an 
environmental chamber at $23.0 \pm 0.1{ }^{\circ} \mathrm{C}$ and a relative humidity of $50 \pm 0.1 \% 10$ $\mathrm{m}$ after addition was water.

Settlement was measured with a non-contact laser (Kayir et al. 2002; Qi et al. 2005), with an accuracy of $0.001 \mathrm{~mm}$, at an interval of $1 \mathrm{~m}$ for the first $6 \mathrm{~h}$. The lasers were situated above the specimens and the laser beam was shot at the surface of the specimen. The shift of laser beam reflected from specimen's surface (while laser projects a laser beam toward the specimen at a small angle) can be used to indicate the consolidation of the sample. This concept can be seen in Figure 7-1. The experimental setup was similar to previous work by Lura et al., (Lura et al. 2006b).
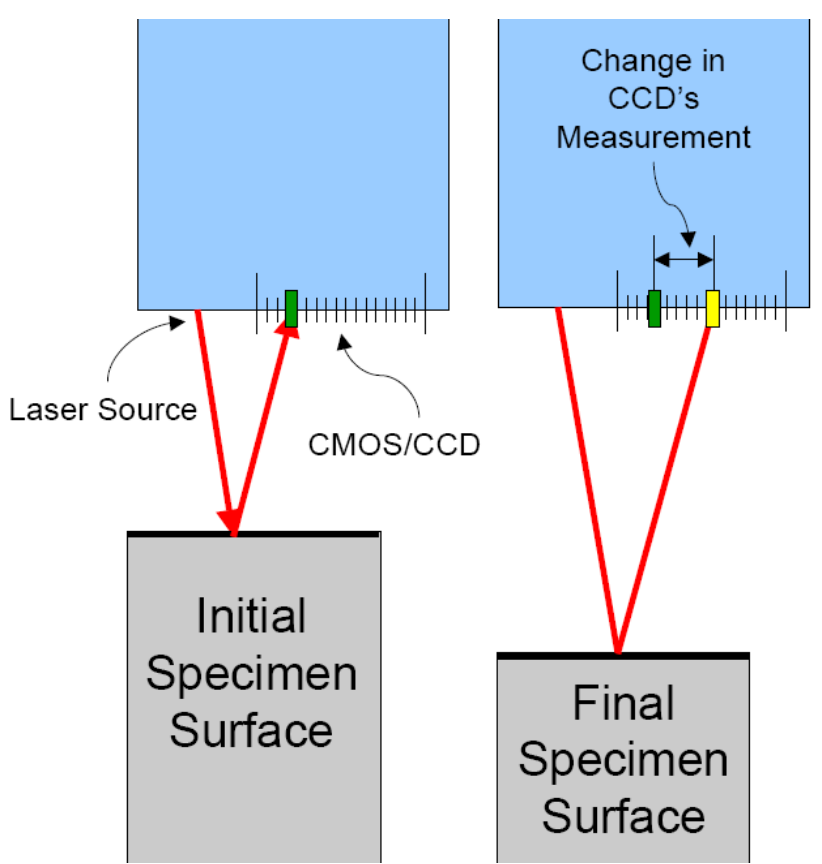

Figure 7-1 - Laser setup for settlement test (Qi et al. 2005) 


\subsubsection{Plastic Shrinkage}

The plastic shrinkage tests were performed in accordance with ASTM C1579-06 (2006). The LWA was prepared exactly like the LWA for the settlement test. The coarse aggregate and normal weight sand were oven dried and cooled for $24 \mathrm{~h}$ before mixing. The coarse aggregate, normal weight sand and lightweight aggregate were placed into the pre-wetted mixing bowl and mixed for $30 \mathrm{~s}$. After this period, cement was added and the decanted water was slowly poured into the dry mixture. The concrete was mixed for $3 \mathrm{~min}$ followed by a 2 min rest and finished by 3 min of mixing.

The concrete was cast into plastic shrinkage molds, shown in Figure 7-2, screeded and finished with a trowel. The samples were put into an environmental chamber 25 min after the addition of water where they were exposed to the following ambient conditions: temperature of $36 \pm 3{ }^{\circ} \mathrm{C}$, relative humidity of $30 \pm 10 \%$ and wind velocity of $24 \pm 2 \mathrm{~km} / \mathrm{h}$. The fans were turned off after $6 \mathrm{~h}$ and for the remaining $18 \mathrm{~h}$ the specimens were exposed to the environment without significant air circulation.

The specimens were taken out of the environmental chamber after $24 \mathrm{~h} \pm 2 \mathrm{~h}$, and pictures were taken of their surfaces. The pictures were always taken from the same height of $184 \mathrm{~mm}$. Subsequently, pictures were analyzed for cracks and crack widths using image software which enables approximately 300 measurements for each specimen, thereby provide statistically valid information about cracks widths and their variability (Qi et al. 2003b). 


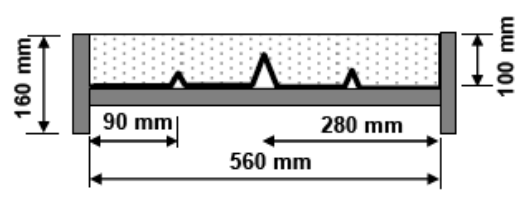

Side View

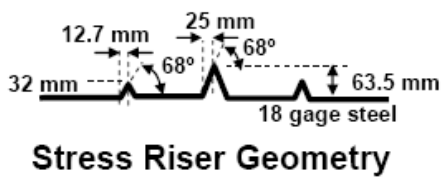

Stress Riser Geometry

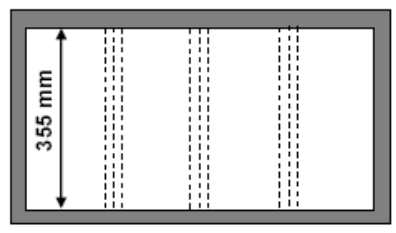

Top View

Figure 7-2 - Geometry of the specimens used for plastic shrinkage investigation (Qi et al. 2003b)

\subsection{Experimental Results and Discussion}

\subsubsection{Settlement}

Figure 7-3 shows the settlement results for the mortar samples over a $6 \mathrm{~h}$ period. The plain mixture shows more consolidation than the mixtures with LWA. It is also interesting to note that as the volume replacement of LWA is increased, the lower the consolidation. This is likely because as capillary stresses increase during phase(II), water is released by the LWA to reduce the capillary stress. The reduction in the capillary stress due to the release of water from the LWA results in less consolidation. 


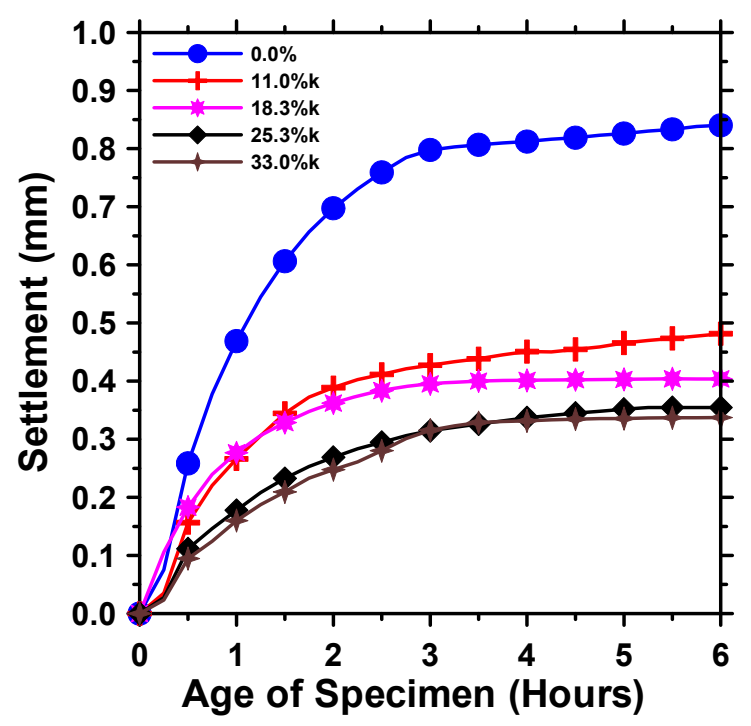

Figure 7-3 - Settlement results for mortar samples

\subsubsection{Plastic Shrinkage}

Figure 7-4 shows the cumulative probability distribution of crack widths occurring in concrete mixtures made with different volume replacements of LWA. The curves shown in Figure 7-4 are the average crack measurement of three panels. The $0.0 \%$ mixture shows the largest crack width. The probability of plastic shrinkage cracks forming decreases as the LWA replacement volume increases. When a sufficient volume of LWA is used $(18.0 \%)$, plastic shrinkage cracking was eliminated. Figure 7-5 shows the probability of cracks widths being smaller than $0.0 \mathrm{~mm}$ and $0.2 \mathrm{~mm}$ as the volume replacement of LWA is increased. By observing the $0.0 \mathrm{~mm}$ curve, it can be seen that cracking can be eliminated if a high enough replacement volume is used. The probability of this critical crack width opening occurring can be significantly reduced or eliminated if a high enough replacement volume of LWA is used. Plastic shrinkage cracking can be reduced or eliminated because the LWA provides additional water that can replace the water that is being evaporated from the surface of the concrete. It should be noted however, that if this water is used to eliminate plastic shrinkage 
cracking, it will not be available to reduce the shrinkage cracking caused by selfdesiccation and drying.

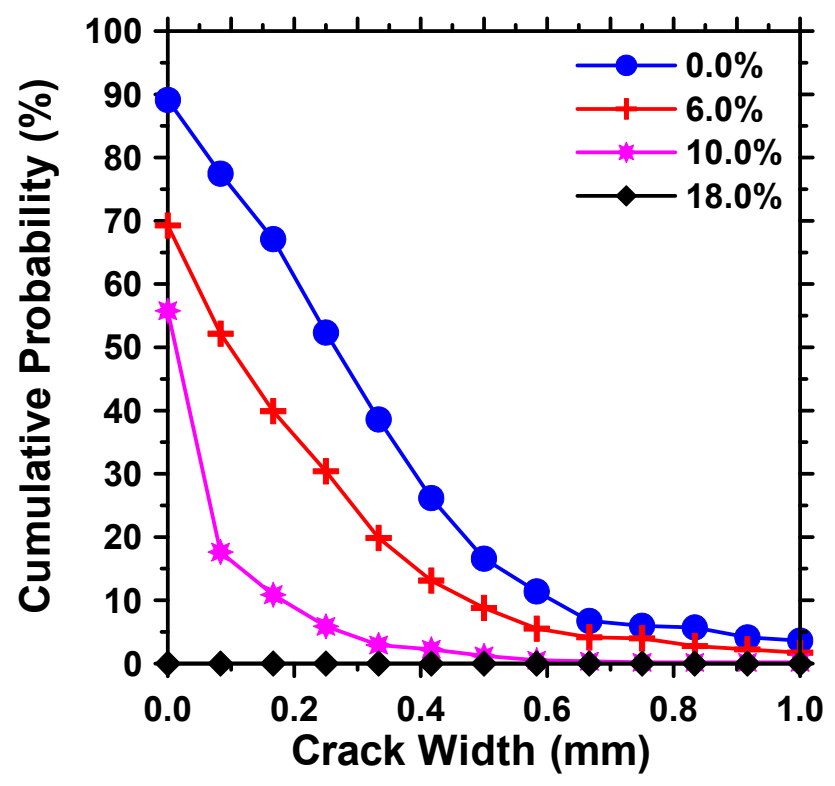

Figure 7-4 - Cumulative distribution of crack width occurrences in concrete with different replacement volumes of LWA

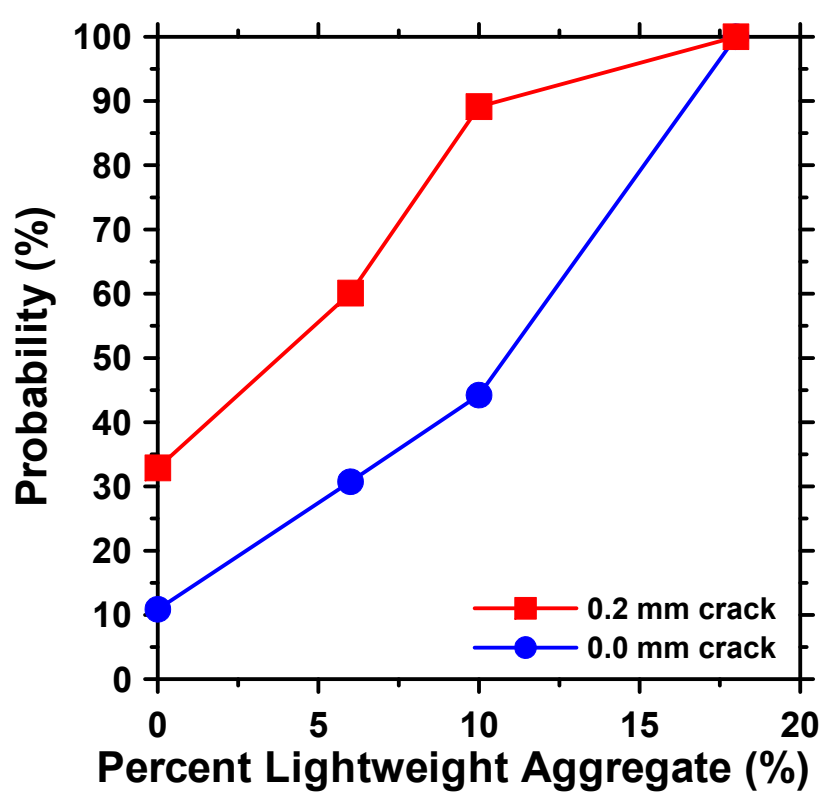


Figure 7-5 - Probability of cracks widths being smaller than $0.0 \mathrm{~mm}$ and $0.2 \mathrm{~mm}$ at different LWA replacement volumes

The crack reducing ratio (CRR) can be calculated according to Equation 7-2.

$C R R=\left[\begin{array}{r}\begin{array}{c}\text { Average Crack Width } \\ \text { of Modified Concrete }\end{array} \\ \begin{array}{c}\text { AverageCrack Width } \\ \text { of Reference Concrete }\end{array}\end{array}\right] \cdot 100 \%$

Equation 7-2

Table 7-3 shows the average crack width and the CCR for the three LWA mixtures tested. The average crack width is reduced as the LWA replacement volume is increased. The plastic shrinkage cracking is reduced as shown by the increase in the crack reducing ratio.

Table 7-3 - Crack reducing ratios for each LWA replacement

\begin{tabular}{|c|c|c|c|}
\hline $\begin{array}{c}\text { Volume of } \\
\text { LWA }\end{array}$ & w/c ratio & $\begin{array}{c}\text { Avg. Cr. W. } \\
\text { (mm) }\end{array}$ & CRR (\%) \\
\hline $\mathbf{0 . 0 \%}$ & 0.55 & 0.487 & 0.00 \\
\hline $\mathbf{6 . 0 \%}$ & 0.55 & 0.199 & 64.77 \\
\hline $\mathbf{1 0 . 0 \%}$ & 0.55 & 0.054 & 88.89 \\
\hline $\mathbf{1 8 . 0 \%}$ & 0.55 & 0 & 100.00 \\
\hline
\end{tabular}

\section{7. $\underline{\text { Conclusions }}$}

This chapter has presented data that describes the behavior of concrete made with prewetted lightweight aggregate. The chapter provides an understanding of what is occurring in these systems during the plastic state and how concrete made with prewetted lightweight aggregate can help eliminate plastic shrinkage cracking. This chapter has shown that if a sufficient volume of prewetted 
lightweight aggregate is provided plastic shrinkage cracking can be reduced or eliminated. It should be noted that the water to reduce plastic shrinkage cracking is unavailable to alleviate the problems associated with self-desiccation and drying. 


\title{
CHAPTER 8. MONITORING WATER ABSORPTION IN CEMENTITIOUS SYSTEMS MADE WITH PREWETTED LIGHTWEIGHT AGGREGATE
}

\begin{abstract}
8.1. Introduction
High performance concrete (HPC) mixtures have been increasingly promoted due to potential long-term benefits in performance (FHWA 2006). HPC mixtures generally have a low water-to-cement ratio $(w / c)$ and may contain finely ground supplementary cementitious materials, (e.g., silica fume). While the fine pore system is beneficial for increased strength (Shah et al. 1994) and reduced fluid transport (El-Dieb et al. 1995; Khan et al. 2002), the finer pore network can lead to increased volumetric changes in sealed systems as a result of self-desiccation (Shah et al. 2000). This volume change (autogenous shrinkage) is problematic as it can result in cracking if the concrete is restrained from shrinking freely.
\end{abstract}

One strategy to mitigate autogenous shrinkage is the incorporation of water filled inclusions (Jensen et al. 2001a; Lura 2003a; Mohr 2005). The use of prewetted lightweight aggregate (SLWA), enables water (from the SLWA) to be supplied to the paste after setting (Henkensiefken et al. 2008g), thereby reducing the effects of self-desiccation. This strategy has proven to be quite effective in reducing autogenous shrinkage and cracking (Henkensiefken et al. 2008b; Henkensiefken et al. 2008e).

The influence of SLWA inclusions on the fluid transport and water absorption properties of concrete is much less understood. This study provides experimental results to quantify the performance of plain and SLWA systems in 
absorption tests and electrical conductivity measurements. The performance of the SLWA systems is related to aspects of the pore structure of the paste, the pore structure of the SLWA, and the pore structure of the interfacial transition zone.

\subsubsection{Background in Sorptivity}

The durability of concrete subjected to aggressive environments depends largely on the penetrability of the pore system (Sabir et al. 1998; 2004). Three mechanisms can be used to describe transport of water in cementitious systems: (1) permeability, (2) diffusion and (3) absorption. Permeability is the measure of the flow of water under a pressure gradient while diffusion is the movement of ions due to a concentration gradient. Absorption can be described as the materials ability to take in water by means of capillary suction. Water absorption is an important factor for quantifying the durability of cementitious systems (Sabir et al. 1998; Yang 2004; Neithalath 2006) and is the primary focus in this study.

The test method that is most commonly used to determine the absorption and rate of absorption (sorptivity) of water in unsaturated hydraulic cement concretes is ASTM C1585 (2004). This test consists of exposing the bottom surface of a sample to water and measuring the increase in mass resulting from absorption. Before the test is conducted, samples are conditioned for 18 days. The samples are placed in a $50{ }^{\circ} \mathrm{C}$ and $80 \% \mathrm{RH}$ environment. After three days of conditioning, the samples are removed from the oven and placed in individually sealed containers where the samples are retained for 15 days to allow internal moisture equilibrium before the test begins. The absorption test involves recording incremental mass change measurements during the first six hours after the sample came in contact with water and taking one measurement ever day for the 
next eight days. The amount of absorbed water is normalized by the crosssection area of the specimen exposed to the fluid using Equation 8-1.

$\mathrm{I}=\frac{\mathrm{m}_{\mathrm{t}}}{(\mathrm{a} \cdot \mathrm{d})}$

Equation 8-1

where: I $\left(\mathrm{mm}^{3} / \mathrm{mm}^{2}\right)$ is the normalized absorbed water, $\mathrm{m}_{\mathrm{t}}(\mathrm{g})$ is the change in specimen mass at time $t ; a\left(\mathrm{~mm}^{2}\right)$ is the exposed area of the specimen (i.e., the size of the bottom face), and $d\left(\mathrm{~g} / \mathrm{mm}^{3}\right)$ is the density of the water (taken to be $0.001 \mathrm{~g} / \mathrm{mm}^{3}$ at $\left.23^{\circ} \mathrm{C}\right)$.

These absorption measurements are then plotted as a function of the square root of time. The initial sorptivity is determined for the slope of the curve during the first six hours, while secondary sorptivity is determined using the slope of the same measurements between one to eight days as outlined in ASTM C1585 (2004).

The initial and secondary sorptivity can be used to evaluate the connectivity of the pore network. Additionally, the secondary sorptivity, combined with exposure conditions, has been used for performing service life predictions (Bentz et al. 2001b).

\subsubsection{Background on the Effects of Internal Curing on Sorptivity}

Low w/c mixtures undergo self-desiccation, even in sealed curing conditions. Self-desiccation occurs as a result of chemical shrinkage. During hydration, the hydration products occupy a volume that is approximately $10 \%$ smaller than the initial reactants (La Chatelier 1900; L'Hermite 1960; Sant et al. 2006). This results in the creation of vapor filled voids inside the concrete (Couch et al. 2006). These vapor-filled voids grow with increased hydration and penetrate 
smaller and smaller pores. Lightweight aggregate can be used as a water reservoir to supply water to replenish the water demanded by the hydrating cement paste thereby reducing the vapor-filled voids and therefore mitigating the effects of self-desiccation in low w/c concrete mixtures. A more thorough description of this process can be found elsewhere (Henkensiefken et al. 2008b; Radlinska et al. 2008b).

The inclusion of SLWA is thought to influence the absorption of water, the explanations can provide expectation for both an increase and/or a decrease in the water absorption. The following section will discuss these explanations.

At first glance, one would think the incorporation of porous inclusions (i.e., LWA) would result in an overall increase in the porosity of the system. The pores in the LWA are large and in the size range of or slightly larger than large capillary pores. As a result, the addition of larger pores into the system could result in higher water absorption.

However, alternate reasons exist to explain why a reduction in the water absorption could also occur. When water leaves the LWA, the paste continues to hydrate resulting in a more dense microstructure in the paste. This can be attributed to additional water being provided to hydrate the paste. This is similar to the supply of extra water from external curing. This concept is shown using Power's Model in Figure 8-1(a) for cement paste with a w/c of 0.30. As the paste hydrates, hydration products are formed. When the water supply is exhausted, hydration ceases. In the case of a paste with a $w / c$ of 0.30 , hydration stops at a degree of hydration of 0.73 for hydration under sealed conditions. 
If the system were not sealed and additional water is supplied, hydration can continue as water can fill in the voids caused by chemical shrinkage. Figure 8-1(b) shows Power's Model for the 11.0\%k mixture, which provides an extra 3.2 $\%$ volume of water (by total volume) to the system. The mixture proportions, which will be covered in a later section can be found in Table 8-1. Note that this water is not included in the total volume used to calculate the porosity (shown as being greater than 1) since it is not part of the paste pore structure at the time of setting when the pore structure has formed. After approximately $32 \%$ hydration, the water in the LWA has been drawn out of the LWA by the underpressure in the fluid to "fill the voids created by chemical shrinkage." During this process, the water from the LWA has resulted in further hydration. The theoretical maximum degree of hydration for the $11.0 \% \mathrm{k}$ mixture would occur when the system runs out of water at approximately 0.77 . The $25.3 \% \mathrm{k}$ mixture provides more water for the cement to hydrate and hydration for the $25.3 \% \mathrm{k}$ mixture is complete at 0.83 . This is shown in Figure 8-1(d). The maximum theoretical degree of hydration increases as the volume replacement increases but comes to a limiting value at $25.3 \% \mathrm{k}$. This is because at that degree of hydration, no pore space remains within the hydrating specimen. It is believed that the water provided does not leave the LWA before set (Henkensiefken et al. 2008g), which explains why the initial porosity would be expected to be the same for the paste in these mixtures. The continued hydration that occurs due to the additional water from the LWA results in a denser microstructure which slows the ingress of water into the system (Bentz et al. 1999b). 
Table 8-1 - Mixture proportions for water absorption study

\begin{tabular}{|c|c|c|c|c|c|}
\hline Material & $55 / 0.25$ & $55 / 0.30$ & $55 / 0.35$ & $11.0 \% \mathrm{k}$ & $25.3 \% k$ \\
\hline Cement $\left(\mathrm{kg} / \mathrm{m}^{3}\right)$ & 793 & 728 & 674 & 728 & 728 \\
\hline Water $\left(\mathrm{kg} / \mathrm{m}^{3}\right)$ & 198 & 218 & 236 & 218 & 218 \\
\hline Fine Aggregate $\left(\mathrm{kg} / \mathrm{m}^{3}\right)$ & 1418 & 1418 & 1418 & 1135 & 808 \\
\hline Dry LWA $\left(\mathrm{kg} / \mathrm{m}^{3}\right)$ & 0 & 0 & 0 & 172 & 369 \\
\hline HRWRA (g/100 g cement) & 0.75 & 0.5 & 0.60 & 0.50 & 0.66 \\
\hline $\begin{array}{c}\text { Additional Water Provided } \\
\text { by LWA }\left(\mathrm{kg} / \mathrm{m}^{3}\right)\end{array}$ & 0 & 0 & 0 & 18 & 39 \\
\hline
\end{tabular}

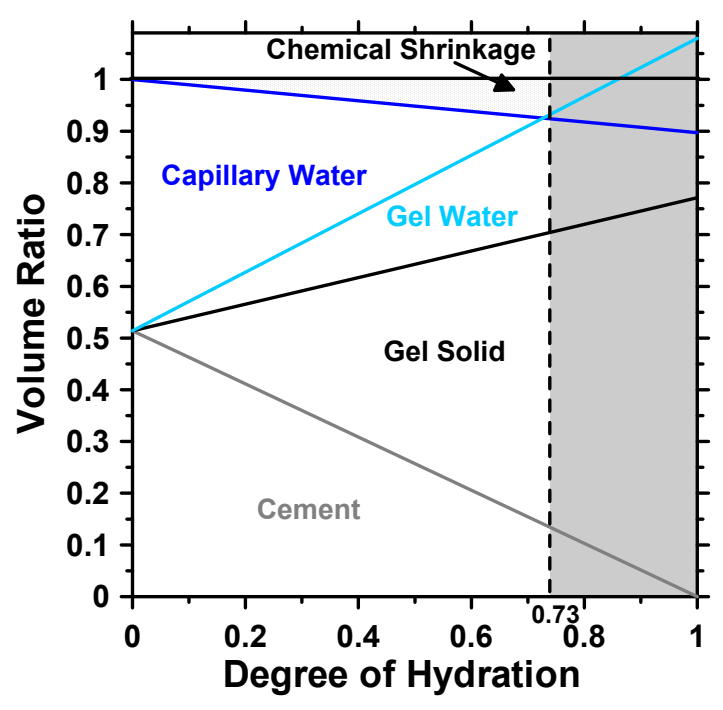

(a)

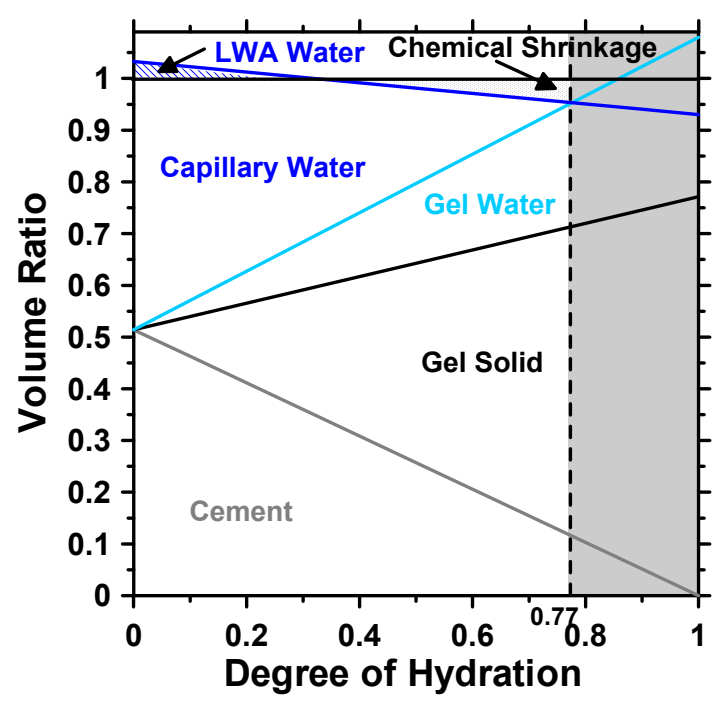

(b) 


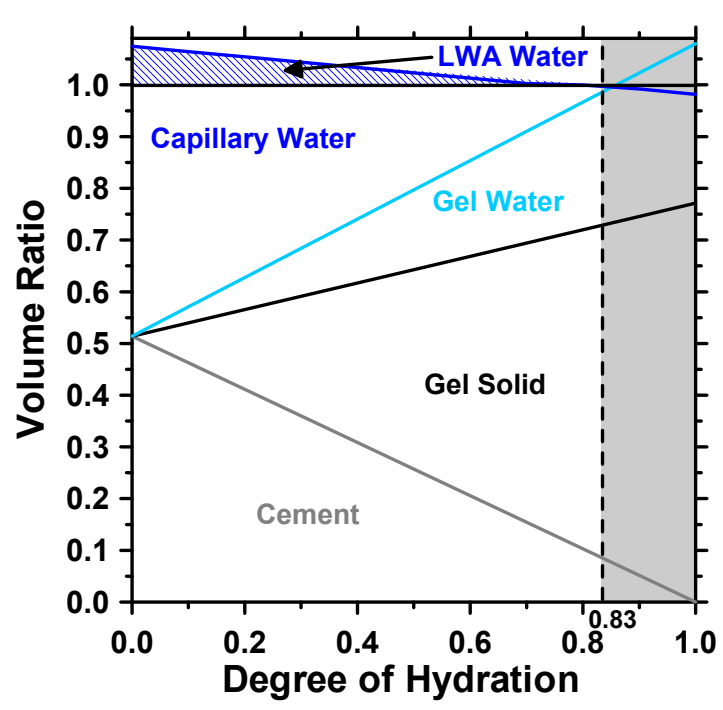

(c)

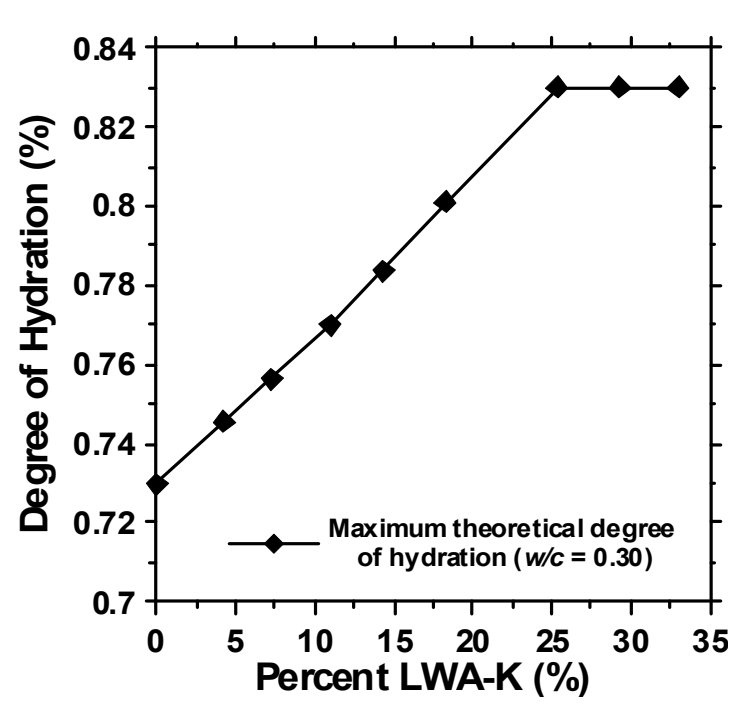

(d)

Figure 8-1 - Powers model showing the phase distribution for (a) 55/0.30, (b) $11.0 \% \mathrm{k}$ and (c) $25.3 \% \mathrm{k}$ mixtures, and (d) the maximum theoretical degree of hydration as a function of LWA volume

The last aspect to consider for a reduction in the water absorption is that the ITZ of the normal weight aggregate (NWA) could be percolated across the 3D microstructure, and the inclusion of the LWA could depercolate the ITZ due to its continued hydration (Winslow et al. 1994; Bentz 2008a). If LWA, with its more dense ITZ (Zhang et al. 1990; Lo et al. 2004), were added to the system, the ITZ may not be percolated as much resulting in a lower absorption. The effect of different volume replacements of LWA on ITZ percolation was simulated using the hard core/soft shell model developed at the National Institute of Standards and Technology (NIST) (Lu et al. 1992; Maekawa et al. 1999; Bentz et al. 1999a). This was similar to the method used by Bentz (Bentz 2008a) to determine the ingress of chloride in mortars. The aggregate particles (both LWA and NWA) are represented as circular 'hard cores' and the ITZ around the normal weight aggregates only is represented as a surrounding ring or 'soft shell'. The results of simulations for the mortars investigated in this study are shown in Figure 8-2. As the replacement volume of LWA increases, the volume 
of ITZ around the NWA decreases (Elsharief et al. 2005). This implies that as more LWA is used, the less porous ITZ around the LWA results in a reduction in absorption. As more LWA is used, the potential for percolation of the NWA ITZ decreases. If enough LWA is used, the ITZ of the NWA would depercolate. For these two reasons, it is expected that the absorption of mortars with LWA could be less.

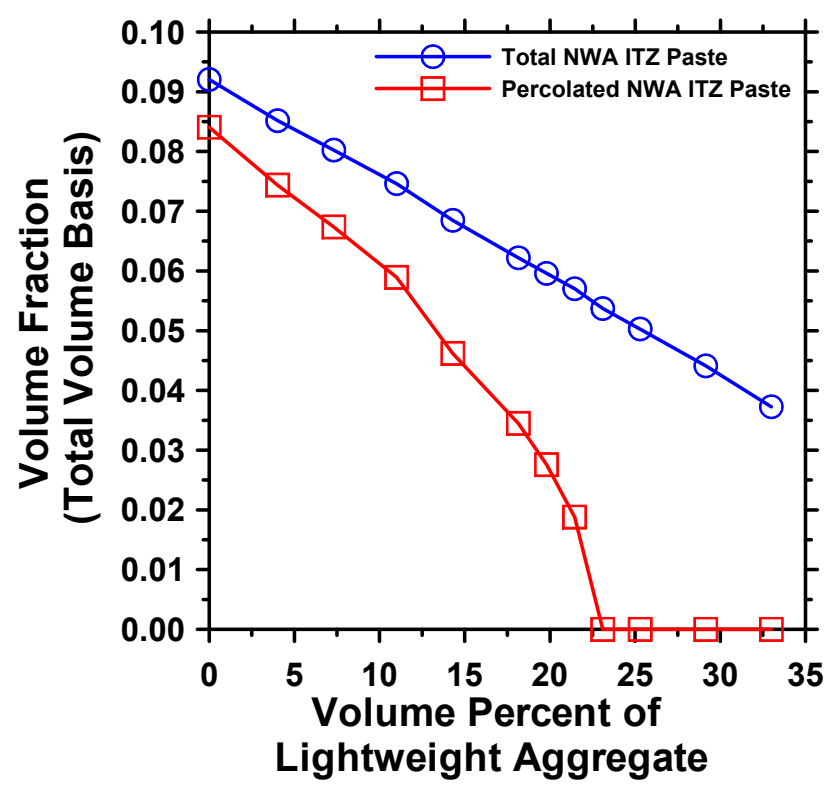

Figure 8-2 - ITZ percolation as a function of replacement volume of LWA

\subsection{Materials}

ASTM C150 Type I ordinary portland cement (OPC) was used in this study, with a Blaine fineness of $370 \mathrm{~m}^{2} / \mathrm{kg}$ and an estimated Bogue composition of $56 \%$ $\mathrm{C}_{3} \mathrm{~S}, 16 \% \mathrm{C}_{2} \mathrm{~S}, 12 \% \mathrm{C}_{3} \mathrm{~A}, 7 \% \mathrm{C}_{4} \mathrm{AF}$ and a $\mathrm{Na}_{2} \mathrm{O}$ equivalent of $0.68 \%$.

A polycarboxylate-based high-range water-reducing admixture (HRWRA) was added in varying rates depending on the mixture. For the mixture with a $w / c$ of 0.25 , the HRWRA was added at a rate of $0.75 \mathrm{~g}$ per $100 \mathrm{~g}$ of cement. For the mixture with a w/c of 0.30 , the HRWRA was added at a rate of $0.70 \mathrm{~g}$ per $100 \mathrm{~g}$ 
of cement. For the mixture with a $w / c$ of 0.35 , the HRWRA was added at a rate of $0.60 \mathrm{~g}$ per $100 \mathrm{~g}$ of cement. For LWA mixtures the HRWRA was added at a rate of $0.50 \mathrm{~g}$ per $100 \mathrm{~g}$ of cement. Different rates of HRWRA were used to keep similar consistencies (i.e., workability) of these mixtures.

The normal weight sand used was natural river sand with a fineness modulus of 2.71 and an apparent specific gravity of 2.58. Portions of the normal weight sand were replaced with rotary kilned expanded shale with a fineness modulus of 3.10 and a specific gravity of 1.56 (LWA). The 24-hour absorption of the LWA was determined to be $10.5 \%$ according to ASTM C128-07 (2007).

\subsubsection{Mixture Proportioning}

Five different mixtures were prepared which consisted of three plain mortar with different $w / c$ and a constant volume fraction of fine aggregate (designated as $55 / 0.25,55 / 0.30$, and 55/0.35) with the number on the left representing the volume fraction of fine aggregate and the number on the right representing $w / c$. Two mortar mixtures were prepared with different volumes of sand replaced by prewetted LWA and a $w / c$ of 0.30 . These mixtures were designated as $11.0 \%$ and $25.3 \%$. It is important to note that though the designations are on a total volume basis, the volume of aggregate (LWA and sand) remained constant at $55 \%$ since only the sand was replaced with LWA. The numbers in the designations for the LWA mixtures represents the total volume of the mixture occupied by the LWA (in percent). It should be noted that the $25.3 \%$ corresponds to the amount of LWA necessary to eliminate self-desiccation. A list of the mixture proportions can be found in Table 8-1. 


\subsubsection{Mixing Procedure}

The mixing procedure used for the mortar was in accordance with ASTM C19206 (2006) while the procedure for the paste was in accordance with ASTM C30506 (2006). The LWA was oven dried, air cooled, and then submerged in water for $24 \pm 1 \mathrm{~h}$ prior to mixing. The volume of water used to submerge the LWA included both mixing water and the water the LWA would absorb in $24 \mathrm{~h}$. The excess water (water not absorbed in $24 \mathrm{~h}$ ) was then decanted and used as the mixing water. The normal weight sand was oven dried and cooled for $24 \mathrm{~h}$ before mixing. For the plain mortar mixtures and the paste, the water and cement was conditioned for $24 \mathrm{~h}$ at room temperature.

\subsection{Experimental Methods}

\subsubsection{Degree of Hydration}

To measure the degree of hydration, fresh cement paste (in the case of LWA mixtures, paste with LWA) was cast that was identical to the mortar without NWA in air-tight plastic vials. At an age of $1 \mathrm{~d}, 7 \mathrm{~d}, 28 \mathrm{~d}$, and $56 \mathrm{~d}$, approximately $10 \mathrm{~g}$ of cement paste was crushed and placed in ceramic crucibles. The samples were placed in an oven at $105^{\circ} \mathrm{C}$ for $24 \mathrm{~h}$. The sample was weighed and then placed in a furnace at $1050^{\circ} \mathrm{C}$ for $3 \mathrm{~h}$. The weights were recorded to monitor the evaporable and non-evaporable water contents. The measurements were corrected to account for loss on ignition of the cement. There is no measured loss-on-ignition for the LWA since it goes through a furnace during manufacturing. 


\subsubsection{Sorptivity}

Five $100 \mathrm{~mm} \times 200 \mathrm{~mm}$ cylinders were cast for each mixture. After one day of curing, the samples were demolded and three $50 \mathrm{~mm} \pm 2 \mathrm{~mm}$ samples were cut from the central portion of each cylinder with a wet saw. The samples were sealed in double plastic bags until they reached ages of $4 \mathrm{~d}, 7 \mathrm{~d}, 28 \mathrm{~d}$, and $90 \mathrm{~d}$. After curing in the plastic bags was complete, samples were conditioned by placing them in a desiccator over a potassium bromide $(\mathrm{KBr})$ salt solution which provided an $80 \%$ relative humidity at $50^{\circ} \mathrm{C}$ (Greenspan 1977). The desiccator was placed in an oven at $50^{\circ} \mathrm{C}$ for three days. The samples were removed then stored in sealed containers for 15 days to allow the moisture to equilibrate within the samples. It should be noted that the $1 \mathrm{~d}$ samples were not stored in plastic bags but were placed directly in the desiccator after cutting. Once the samples were removed from the sealed containers, the side surface was sealed with aluminum tape and the top surface was covered with plastic to avoid evaporation from the sample during testing. After the samples were prepared, testing occurred in accordance with ASTM C1585-04 (2004).

\subsubsection{Electrical Impedance Spectroscopy}

After the sorptivity measurements were completed (120 d), the conductivity of the samples was measured using electrical impedance spectroscopy (EIS). The samples were vacuum saturated using tap water and weighed. After EIS testing, the samples were oven dried at $105^{\circ} \mathrm{C}$ until mass equilibrium was reached (until the difference between two successive $24 \mathrm{~h}$ measurements was less than 0.1 $\%)$. This was done to determine the total volume of water that was in the system after vacuum saturation.

Electrodes were placed on the top and bottom surface of the samples; however contact issues with the samples and electrode existed (Ford et al. 1998; Weiss et 
al. 2008). For this reason, wet sponges were placed between the samples and the electrodes. Two other methods were compared with the wet sponges. The first involved placing conductive silver paint and copper electrodes on the surfaces of the samples before the EIS measurements were taken. The second method involved placing conductive gel (Conductor transmission gel 4238) (Thomas 2008) on the top and bottom surfaces to which the electrodes were attached. The methods were applied on 3 different samples and similar results were achieved. The wet sponge method was selected instead of the conductive gel and conductive silver paint because of its simplicity. After a reliable method was determined all the samples were tested. The impedance measurements were made over a frequency range of $10 \mathrm{MHz}$ to $10 \mathrm{~Hz}$ using a $500 \mathrm{mV} \mathrm{AC}$ signal.

\subsection{Experimental Results and Discussion}

\subsubsection{Effects of Internal Curing on the Degree of Hydration}

Figure 8-3 shows the degree of hydration ( $\mathrm{DOH}$ ) for five of the mixtures tested. As the $w / c$ increases, the $\mathrm{DOH}$ also increases. The $11.0 \% \mathrm{k}$ mixture has slightly higher $\mathrm{DOH}$ than the $55 / 0.30$. This is likely due to the fact that a small volume of additional water in the LWA is added in the $11.0 \% \mathrm{k}$ mixture. The $25.3 \% \mathrm{k}$ mixture has a higher $\mathrm{DOH}$ than the $55 / 0.30$ mixture and it is approaching the $\mathrm{DOH}$ of the $55 / 0.35$ mixture. Again this is due to the extra water provided by the LWA hydrating more cement. 


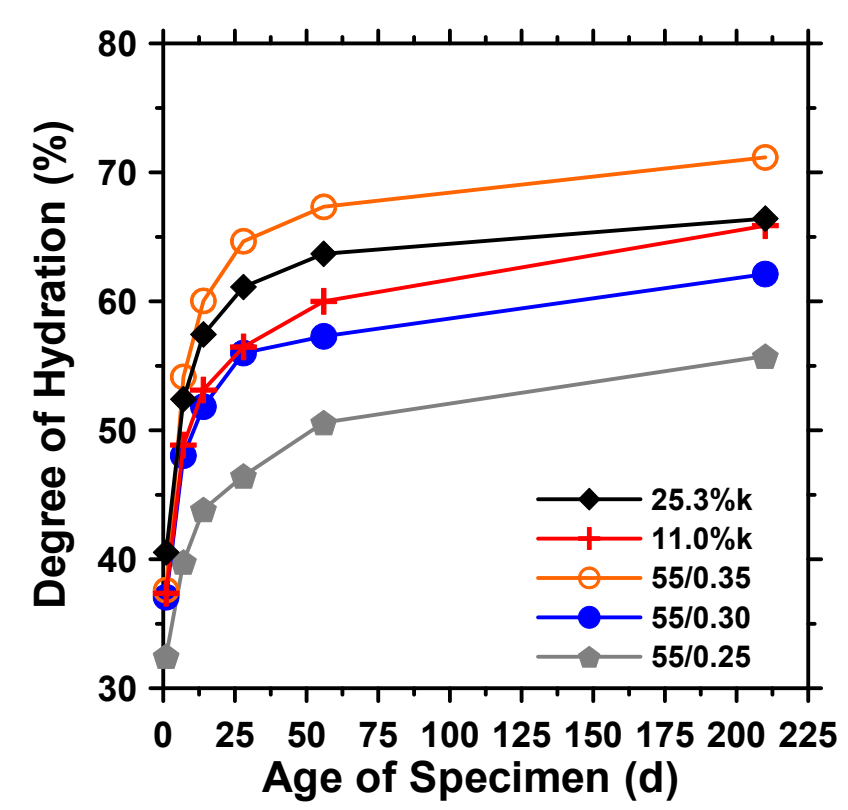

Figure 8-3 - Degree of hydration

\subsubsection{Effects of Internal Curing on Initial and Secondary Sorptivity}

Figure 8-4(a) through Figure 8-4(d) shows the initial and secondary rate of water absorption results for samples cured for 1, 7, 28 and $90 \mathrm{~d}$. The absorbed water is expressed as grams of water per volume of paste. The volume of cement paste in the mortar samples was approximately $45 \%$.

Comparing the mixtures with a similar w/c (55/0.30, $11.0 \% \mathrm{k}, 25.3 \% \mathrm{k})$, it can be seen the absorption for the 55/0.30 was the highest with the absorption decreasing with increasing LWA replacement volume. It can also be seen that the $25.3 \% \mathrm{k}$ mixture has a lower absorption than the 55/0.25 mixture. This behavior was observed on all sorption tests performed on samples cured for different periods. The lower absorptions seen are likely due to the increased hydration, the fact the LWA is not re-saturating due to an improved interfacial transition zone and the depercolation of the interfacial transition zone. 
Figure 8-5(a) shows the initial rate of absorption for samples cured for different periods. The addition of LWA reduces the initial rate of absorption. Comparing the mixtures with a similar $w / c(55 / 0.30,11.0 \% \mathrm{k}, 25.3 \% \mathrm{k})$, the $55 / 0.30$ mixture exhibits the highest initial sorptivity with lower sorptivity being observed with increasing volume replacement of LWA. The $25.3 \% \mathrm{k}$ mixture has an even lower initial sorptivity than the 55/0.25 mixture. Mixture $11.0 \% \mathrm{k}$ has an average reduction of $4 \%$ on initial sorptivity and $1 \%$ on secondary sorptivity. Mixture $25.3 \% \mathrm{k}$ has an average reduction of $46 \%$ on initial sorptivity and $28 \%$ on secondary sorptivity.

Longer curing times reduce the initial sorptivity. However the main portion of the reduction of initial sorptivity in samples containing LWA is reached during the first week of curing. The $11.0 \% \mathrm{k}$ mixtures reach a sorptivity that is similar to the $55 / 0.25$ mixture when samples are cured for $90 \mathrm{~d}$. All the $25.3 \% \mathrm{k}$ mixtures reach lower initial sorptivity values than $55 / 0.25$ mixtures.

When samples without LWA are analyzed a considerable difference is observed when the w/c changes. Mixture with higher w/c (55/0.35) have an important reduction of the rate of absorption when longer curing times are allowed. Mixtures with $w / c 0.3$ show a reduction in the absorption for the first day. The mixture with $w / c 0.25$ does not show a statistically significant difference on sorptivity with the change of curing time. 


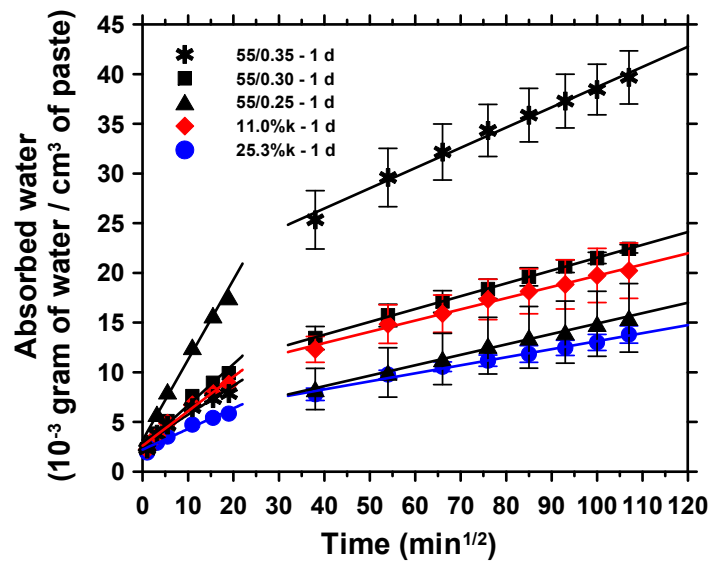

(a)

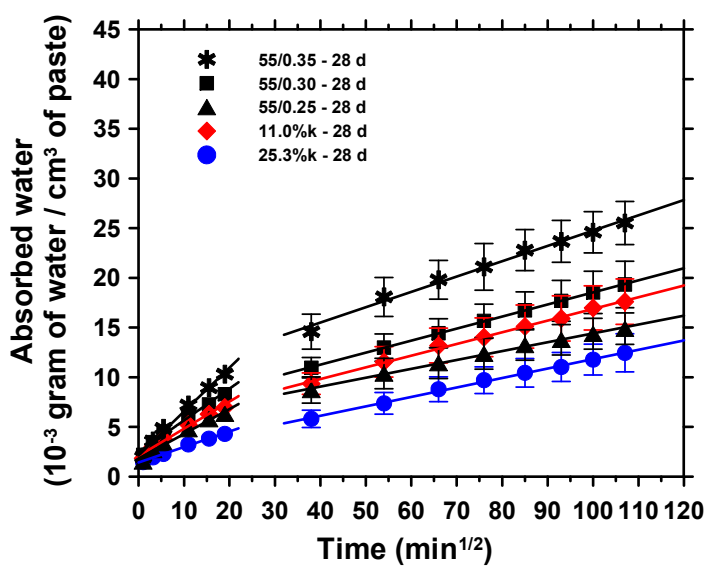

(c)

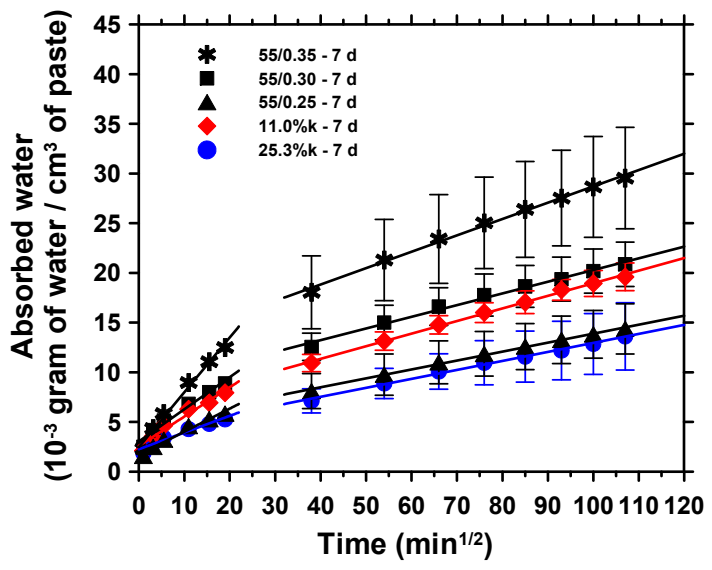

(b)

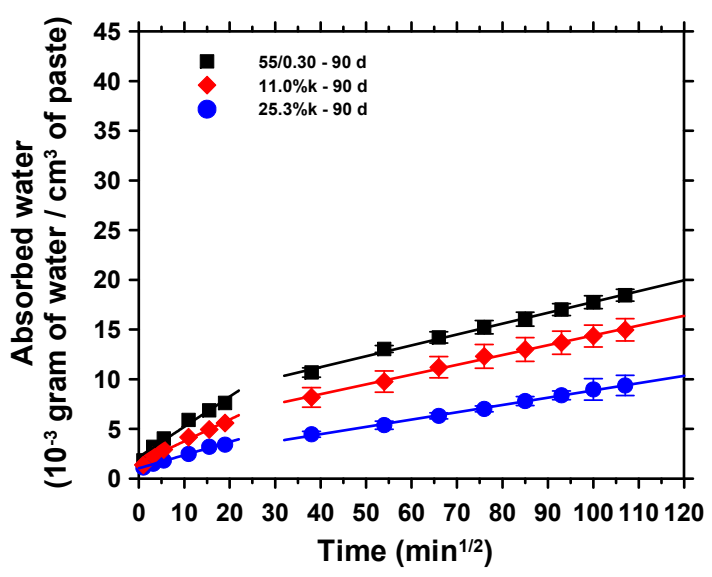

(d)

Figure 8-4 - Sorption test on samples cured for (a) 1d, (b) $7 d$, (c) $28 d$, and (d) $90 \mathrm{~d}$

Figure $8-5(b)$ shows the secondary rate of absorption. Similar to the initial sorptivity, the $25.3 \% \mathrm{k}$ mixture produces a reduction of the secondary rate of absorption. This reduction is comparable with the one obtained using a plain mixture with $w / c$ of 0.25 . Results from $11.0 \% \mathrm{k}$ mixture can not be clearly distinguished from $55 / 0.30$ mixture, likely because of variability of the results. 


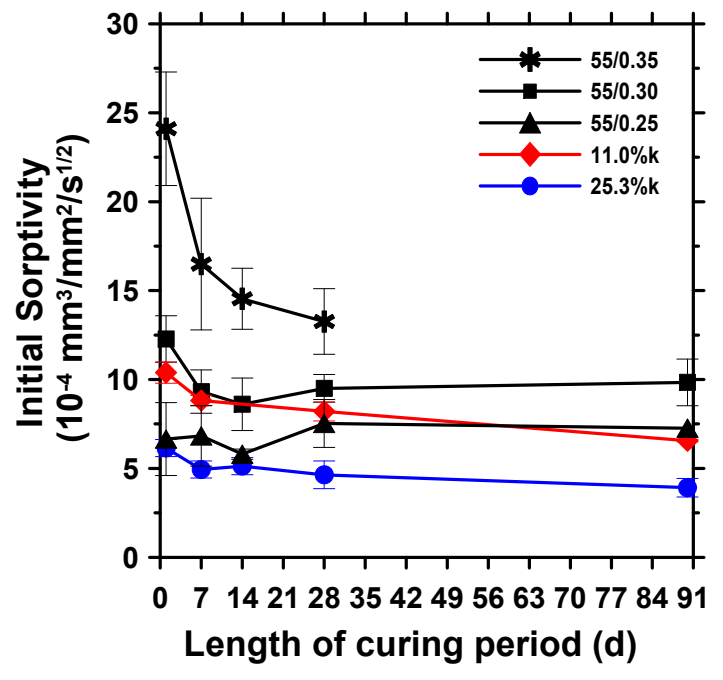

(a)

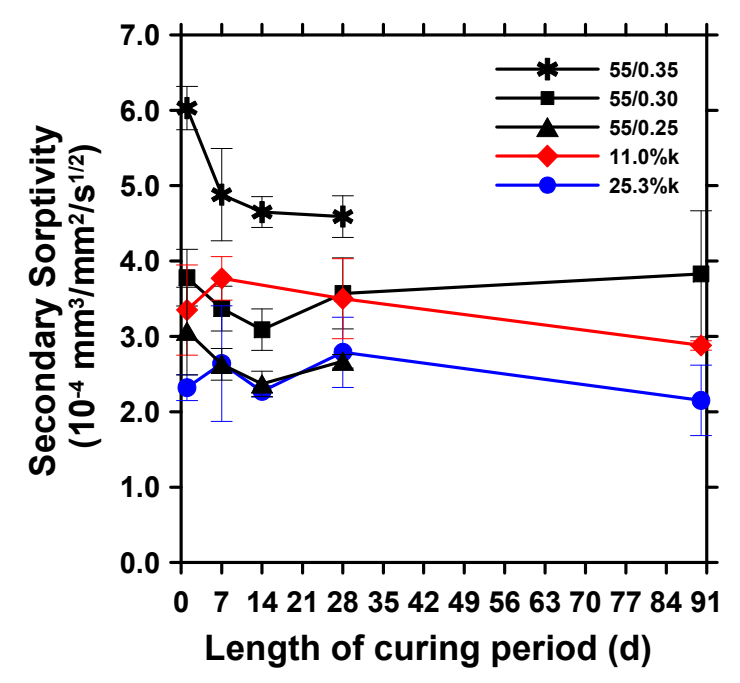

(b)

Figure 8-5 - (a) Initial rate of absorption, and (b) secondary rate of absorption for samples cured for different periods

8.4.3. Effects of Internal Curing on the Amount of Absorbed Water During the Sorption Test

Figure 8-6 shows the cumulative absorbed water after $8 d$ of testing for samples cured for different periods. All the mixtures exhibit a decrease in the total amount of absorbed water when a longer curing period is used. A longer curing period will provide more time to develop a more dense internal structure reducing the size and connectivity of the pore network.

It can be observed that the mixture with a lower replacement volume $(11.0 \% \mathrm{k})$ has a lower absorption than 55/0.30 mixture. The $25.3 \% \mathrm{k}$ mixture has a lower total absorption than the $55 / 0.25$ mixture. Mixture $11.0 \% \mathrm{k}$ has an average reduction of $8 \%$ on absorbed water after $8 \mathrm{~d}$ of test. Mixture $25.3 \% \mathrm{k}$ has an average reduction of $38 \%$ on absorbed water after $8 d$ of test. 


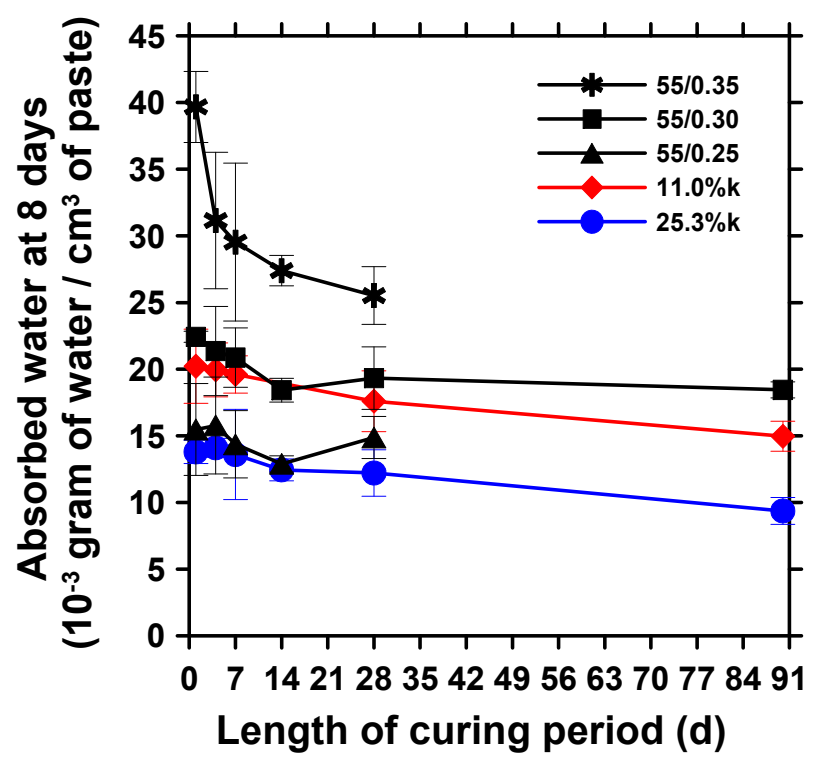

Figure 8-6 - Cumulated absorbed water for samples at $8 \mathrm{~d}$

Figure 8-7(a) shows the relationship between the amount of absorbed water at 8 $\mathrm{d}$ and the $w / c$ for samples cured for $28 \mathrm{~d}$. Because of the continued hydration of the LWA mixtures, they exhibit behavior of a lower w/c mixture. An "equivalent $w / c$ " (i.e., the $w / c$ that would exhibit a similar absorption to the absorption in the LWA mixtures) is shown in Figure $8-7(\mathrm{a})$. The $11.0 \% \mathrm{k}$ has performance similar to that of a $w / c$ of 0.28 , while the $25.3 \% \mathrm{k}$ mixture has a performance equivalent to a $w / c$ of 0.23 . A similar relationship for the conductivity measurements is shown in Figure $8-7(\mathrm{~b})$. The "equivalent $w / c$ " for the $11.0 \% \mathrm{k}$ mixture and $25.3 \% \mathrm{k}$ mixture are 0.29 and 0.23 , which are similar to the results from Figure 8-7(a).

Due to the continued hydration, the porosity of the LWA mixtures will be reduced as compared to the $0.0 \%$ mixture. Figure $8-7$ (c) shows porosity (excluding the gel porosity) of the mixtures as a function of the amount of absorbed water at $8 \mathrm{~d}$ for the samples cured for $28 \mathrm{~d}$. The porosity is also plotted as a function of the conductivity in Figure 8-7(d), and a similar result is seen. Mixture $11.0 \% \mathrm{k}$ and $25.3 \%$ have an average reduction of $13 \%$ and $53 \%$ on conductivity respectively. 


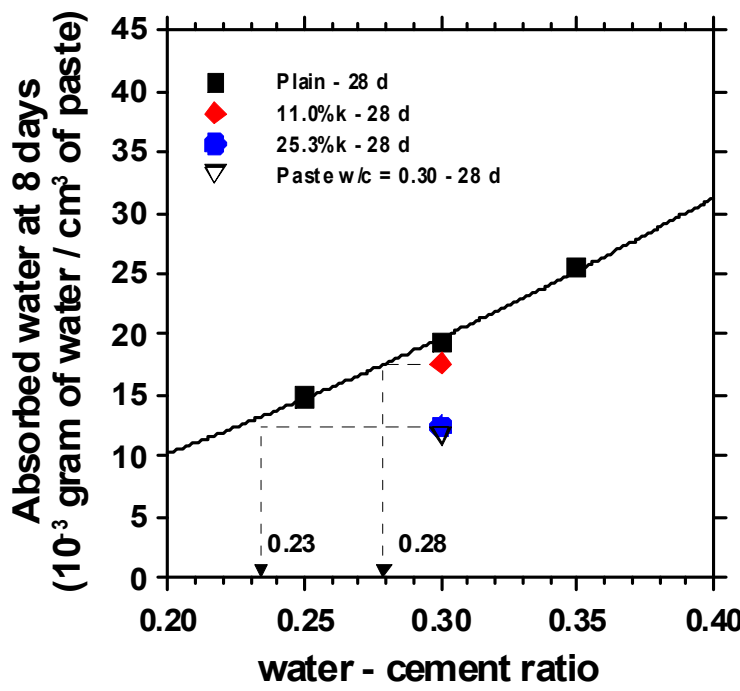

(a)

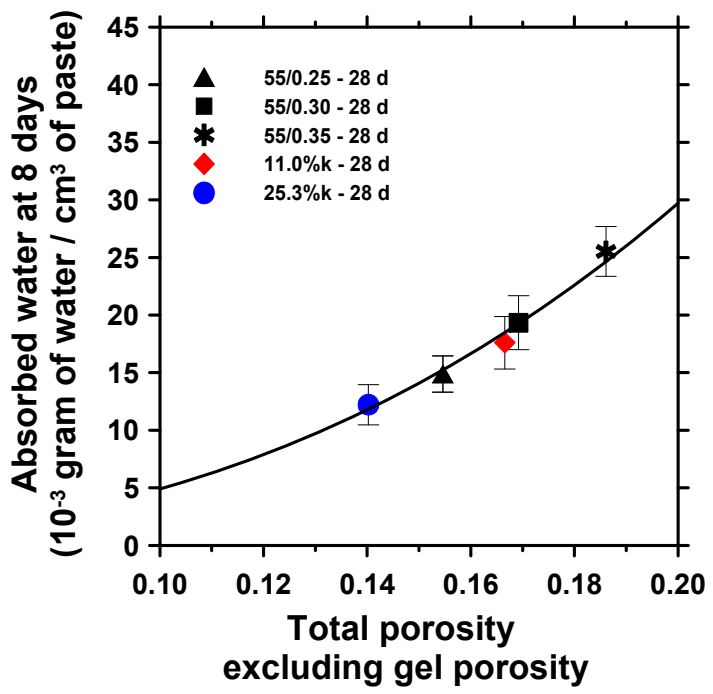

(c)

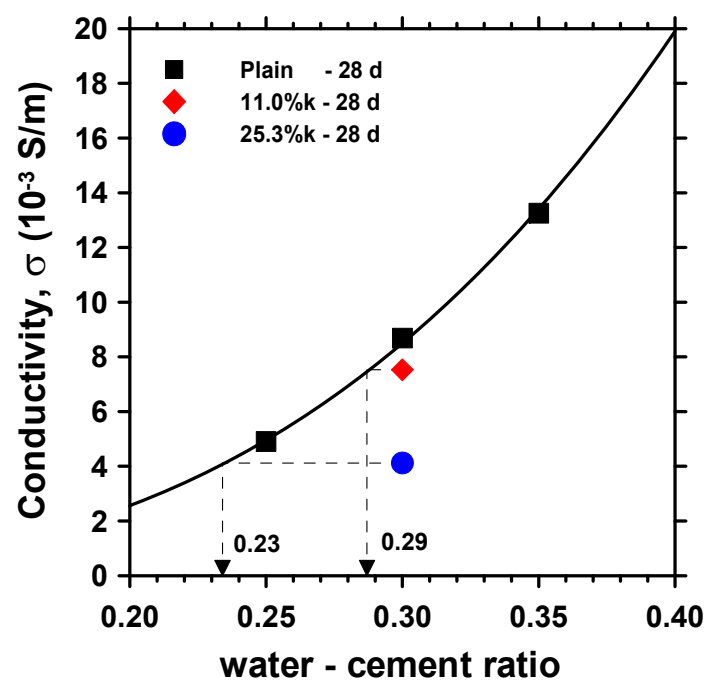

(b)

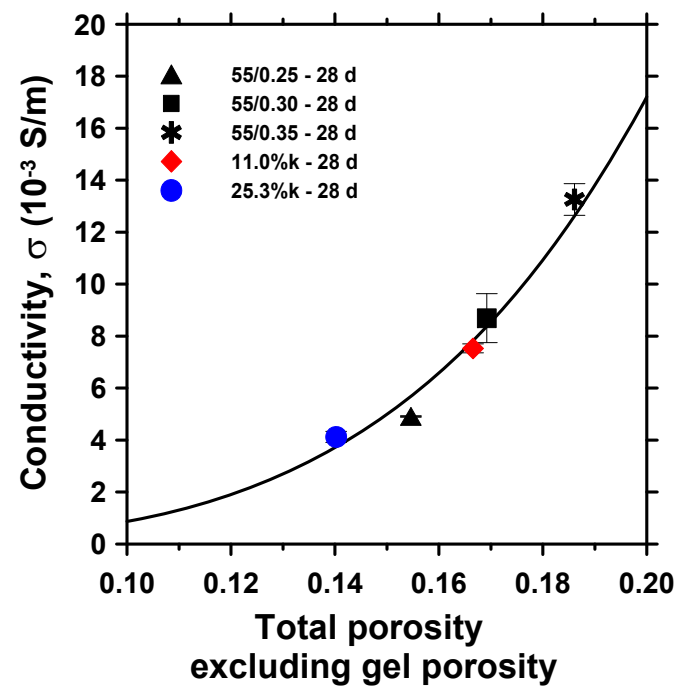

(d)

Figure 8-7 - The equivalent $w / c$ determined from (a) cumulated absorbed water at $8 \mathrm{~d}$ on samples cured for $28 \mathrm{~d}$ and (b) conductivity. Porosity as a function of the (c) cumulated absorbed water at $8 \mathrm{~d}$ on samples cured for $28 \mathrm{~d}$ and (d) conductivity 


\subsubsection{Effects of Available Space on Both Sorptivity and Total Amount of Absorbed Water}

From a fundamental point of view, sorptivity and absorbed water are directly related to the interconnected porosity of the system as this describes the space that is available to be filled with water during the sorption test.

Figure 8-8(a) shows the relationship between the open porosity and the initial sorptivity. The open porosity (i.e., the porosity which can be filled with water during the sorption test) was determined by subtracting the initial water content from the total porosity. The initial water content can be considered the water that is initially contained in the total porosity at the start of the sorption test. The initial water content was determined by subtracting the oven dry weight at the end of the sorption test from the weight at the beginning of the sorption test, resulting in the volume of water initially in the sample. The total porosity was determined by subtracting the oven dry weight after the sorption test from the vacuum saturated weight, resulting in the total volume of water (i.e. the total porosity) in the sample. From Figure 8-8(a), it can be seen that the initial sorptivity is a function of the open porosity of the system. Figure 8-8(b) show the relationship between the open porosity and the total absorbed water after the sorption test. A similar trend can be seen and it can be said that the amount of water absorbed is also a function of the open porosity.

To observe the long term absorption, mixtures cured for $28 \mathrm{~d}$ were monitored past the eight days suggested by ASTM C1585 and were measured for 120 days, which is shown in Figure 8-9(a). This plot indicates that the mixtures will continue to absorb water for an extended period of time. Because the LWA mixtures $(11.0 \% \mathrm{k}$ and $25.3 \% \mathrm{k})$ continually show lower absorption than the plain mixture (55/0.30), it can also be said that during the testing period, it does not appear that the LWA re-saturates. It is shown that it is not until the samples are 
vacuum saturated that the LWA appears to re-saturate. This can be seen in Figure $8-9(b)$ since the LWA mixtures (11.0\%k and $25.3 \% \mathrm{k}$ ) have a higher total vacuum absorption. The LWA likely does not re-saturate during standard testing because of the improved ITZ however it does re-saturate with a large pressure from the vacuum forcing water through the ITZ.

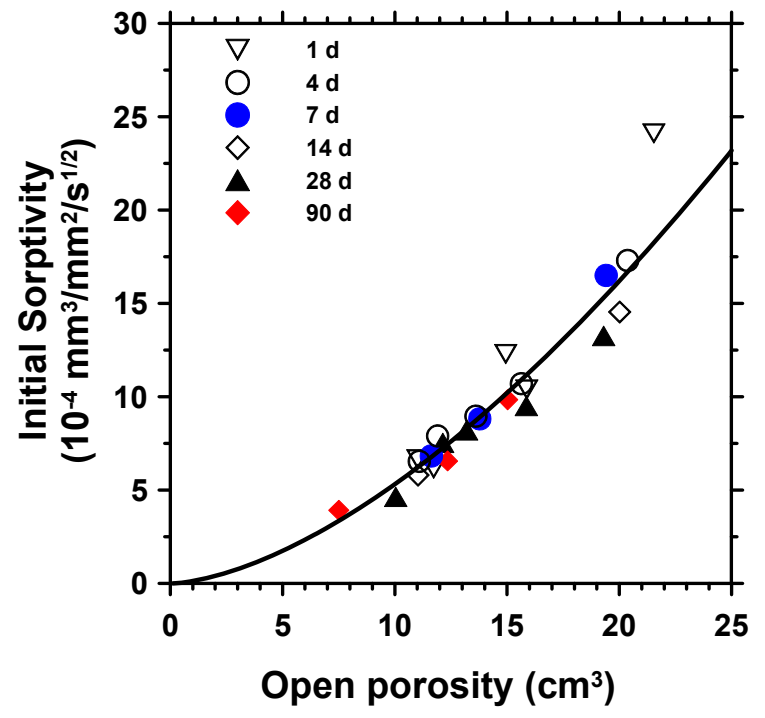

(a)

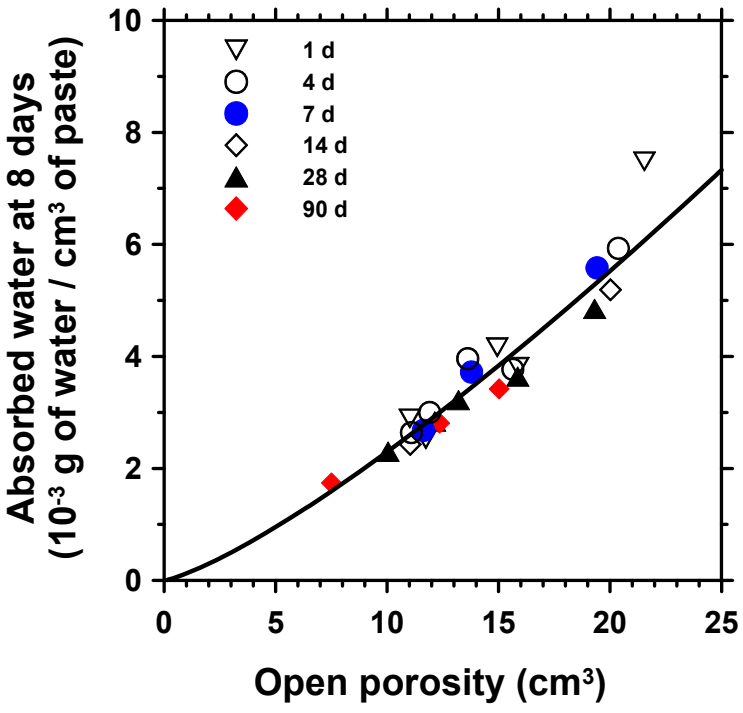

(b)

Figure 8-8 - Relationship between open porosity and (a) initial sorptivity and (b) total absorbed water after 8 days of sorption test 


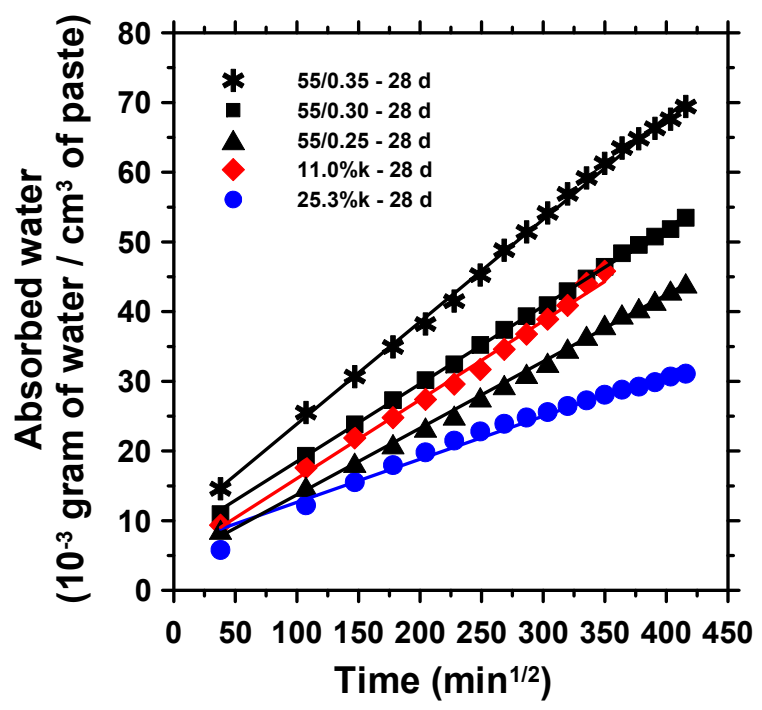

(a)

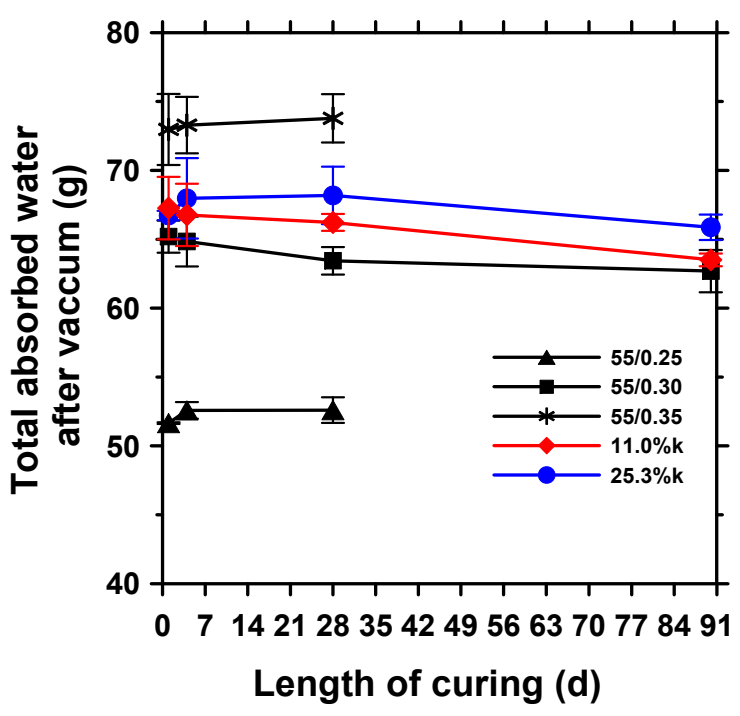

(b)

Figure 8-9 - (a) Long term sorption of samples cured for $28 \mathrm{~d}$ and (b) the total absorption for the samples after vacuum saturation

\subsection{Conclusions}

This chapter has described the absorption behavior of mortars made with SLWA. The inclusion of a sufficient volume of SLWA can significantly reduce water absorption. A summary of the general conclusions from the data presented in this chapter are:

- The addition of LWA as an internal curing agent increases the degree of hydration which results in a more dense microstructure. The more dense microstructure results in less water absorption, less sorptivity and a decrease in the electrical conductivity of the samples, 
- The addition of LWA as an internal curing agent, which has its more dense ITZ due to continued hydration, results in a system with less percolated normal weight aggregate ITZ,

- From a sorptivity and conductivity perspective, the behavior of the mixture with $25.3 \%$ LWA performs better than a mixture with a $w / c=0.25$. The average reduction using this mixture was $46 \%$ on initial sorptivity, $28 \%$ on secondary sorptivity, $38 \%$ on absorbed water after $8 d$ of test; and $53 \%$ on electrical conductivity 


\section{CHAPTER 9. MECHANICAL PROPERTIES OF IC SYSTEMS MADE WITH PREWETTED LIGHTWEIGHT AGGREGATE}

\subsection{Introduction}

The mechanical properties of concrete can play an important role in early age durability performance. Tensile strength can describe how easily a mixture will crack when residual tensile stress develops. Stiffness can describe how quickly a mixture will develop tensile stress if its shrinkage is restrained. Since concrete is a heterogeneous material, mechanical properties are affected by the independent properties of the concrete paste and aggregate. Therefore, substituting lightweight aggregate that is weaker and softer for normal weight aggregate may affect the overall mechanical properties of concrete. In this chapter, the compressive strength, tensile strength, and the modulus of elasticity (E) of internally cured (IC) mixtures using prewetted lightweight aggregate are investigated.

\subsection{Constituent Materials}

Type I ordinary portland cement was used (ASTM C 150-05) with a Blaine fineness of $370 \mathrm{~m}^{2} / \mathrm{kg}$, and an estimated Bogue composition of a $56 \% \mathrm{C}_{3} \mathrm{~S}, 16 \%$ $\mathrm{C}_{2} \mathrm{~S}, 12 \% \mathrm{C}_{3} \mathrm{~A}$ and $7 \% \mathrm{C}_{4} \mathrm{AF}$. Normal weight river sand was used with a fineness modulus of 2.71 and an oven dry specific gravity of 2.58. The lightweight aggregate used was a manufactured rotary kiln expanded shale with a fineness modulus of 4.3 , an oven dry specific gravity of 1.56 , and a 24 hour absorption value of $10.5 \%$ by mass. A high-range water-reducing admixture 
(HRWRA) was added at a rate of $1 \mathrm{~g}$ mass per $100 \mathrm{~g}$ of cement. Mix water consisted of tap water conditioned to $23^{\circ} \mathrm{C} \pm 1^{\circ} \mathrm{C}$.

\subsection{Mixture Proportions}

Three mortar mixtures with varying lightweight aggregate replacement volumes were chosen to study their mechanical properties. They are designated M-0, M11, and M-24 with their proportions provided in Table 9-1. M-0 was a plain mortar while M-11 and M-24 utilized internal curing with pre-wetted fine lightweight aggregate (LWA) at different replacement volumes. All mixtures had a water-to-cement ratio of 0.30 by mass $(w / c=0.30)$ and a paste volume of 45 $\%$. The internally cured mixtures $\mathrm{M}-11$ and $\mathrm{M}-24$ had $11 \%$ and $23.7 \%$ of the total mixture volume comprised of LWA, respectively. It should be noted the $23.7 \%$ replacement value corresponds to the theoretical value of internal curing water that is required to match the chemical shrinkage calculated with Equation 2-1.

Table 9-1 - Mixture proportions for mechanical properties study

\begin{tabular}{|lc|c|c|}
\cline { 2 - 4 } \multicolumn{1}{c|}{} & $\begin{array}{c}\text { M-0 } \\
\text { Plain } \\
\text { Mortar }\end{array}$ & $\begin{array}{c}\text { M-11 } \\
\text { LWA }\end{array}$ & $\begin{array}{c}\text { M-24 } \\
\text { LWA }\end{array}$ \\
\hline \hline Cement (Type I) & 728 & 728 & 728 \\
\hline Water & 218 & 218 & 218 \\
\hline Fine Aggregate & 1444 & 1133 & 821 \\
\hline LWA (SSD) & 0 & 204 & 409 \\
\hline
\end{tabular}




\subsection{Mixing Procedure}

Aggregate for each mixture was batched in the oven dry state. LWA was then presoaked for $24 \mathrm{~h} \pm 0.5 \mathrm{~h}$ in the mix water (including the water for prewetting) while sealed in a container. Mixing was performed in accordance with ASTM C 192-07. First, aggregate was loaded into the slightly dampened mixer. Where applicable, the mixing water was decanted from the LWA. The mixer was started and $50 \%$ of the total water was added. The cement and remaining mix water containing the HRWRA were then added. The mortar was mixed for 3 min, then rested for 3 min while the sides of the mixer were scraped, then mixed for a final 2 min.

\subsection{Mechanical Property Testing}

A set of $100 \mathrm{~mm} \times 200 \mathrm{~mm}$ (4" diameter $\times 6$ " tall) cylinders were cast for each of the three mortar mixtures in order to measure the development of split tensile capacity, compressive strength, and elastic modulus. The cylinders were cast in three lifts, rodded 25 times and vibrated after each lift. The cylinders were then sealed in containers and cured at constant $23^{\circ} \mathrm{C} \pm 1{ }^{\circ} \mathrm{C}$. Testing was performed at ages $1,3,7,28$, and 90 days after mixing. Split tensile capacity was measured in accordance with ASTM C 496/C 496M-04 where three cylinders were loaded perpendicular to their length with a load distribution bar until splitting was observed. Compressive strength was measured in accordance with ASTM C 39. Neoprene end caps were utilized when testing the cylinders.

The elastic modulus was measured in accordance with ASTM C 469-02 where a compressometer was affixed to the test cylinder which was loaded to $40 \%$ of the ultimate load. The compressometer utilized a displacement transducer to measure the change in length of the cylinder due to the applied compression and 
provided continuous measurement during the loading procedure. Two cylinders were tested for elastic modulus for each mixture at each age. No cylinder was tested for elastic modulus at more than one age.

\subsection{Results and Discussion}

\subsubsection{Compressive Strength}

The compressive strength testing results are presented in Figure 9-1. The internally cured mixtures $\mathrm{M}-24$ and $\mathrm{M}-11$ exhibited slightly lower strengths than the plain control mixture M-0 throughout the duration of the testing. Compared to the control M-0 mixture, M-24 showed a $2 \%$ reduction in strength until 28 day and an $8 \%$ reduction in strength at 90 day. Mixture $\mathrm{M}-11$ showed a $6 \%$ reduction in strength until 28 day and a $2 \%$ reduction at 90 day. The reduced strength of the IC mixtures was anticipated, and it can be explained by the replacement of stronger sand with weaker lightweight aggregate. The observed strength of the mixture is a result of the composite behavior of the individual strengths of the paste and aggregate. Although the internal curing water may strengthen the paste by providing additional hydration, the weakening effect by the presence of weaker aggregate dominates the overall behavior. 


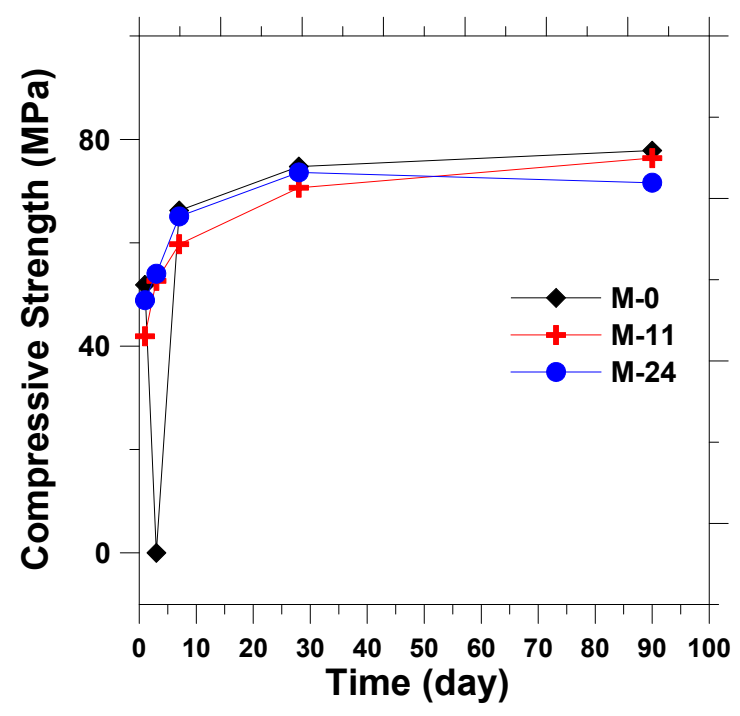

Figure 9-1 - Compressive Strength

\subsubsection{Split Tensile Strength}

Figure 9-2 shows the results of split tensile testing. Generally, the plain mixture was slightly stronger than the IC mixtures, although a trend was difficult to distinguish. The tensile strength of the control $\mathrm{M}-0$ and $\mathrm{M}-11$ mixtures were nearly identical throughout the duration of the test. Mixture M-24 was generally weaker than M-0 and M-11 except at 28 day when it was $10 \%$ stronger. It should be noted that each plotted data point is the average of three split cylinder specimens. Split tensile testing is not precise as the other testing because is highly affected by the ability to visually identify the exact moment splitting occurs. The minimum and maximum splitting capacity for each set of three specimens is indicated by the error bars in the plot. It is likely that trends for split tensile strength are similar to compressive strength where M-24 is the weakest and M-0 is the strongest. 


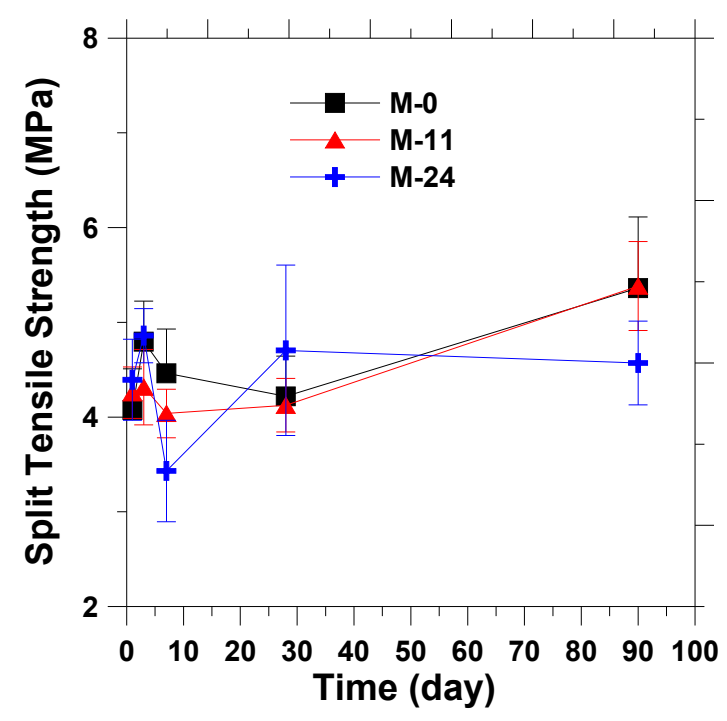

Figure 9-2 - Split Tensile Strength

\subsubsection{Modulus of Elasticity}

Figure 9-3 presents the modulus of elasticity results for the three mortar mixtures $\mathrm{M}-0, \mathrm{M}-11$, and $\mathrm{M}-24$. $\mathrm{M}-0$ was the stiffest mixture and measured $31.0 \mathrm{GPa}$ (4490ksi) at 28 day. Mixture M-11 was about $4 \%$ softer than the control mixture by measuring $29.9 \mathrm{GPa}(4332 \mathrm{ksi})$ at 28 day. Mixture M-24 was the softest mixture and measured $26.9 \mathrm{GPa}(3608 \mathrm{ksi})$ at 28 day which was $13 \%$ less than the control mixture. Similar behavior in all three mixtures occurred at the long term 90 day measurement.

Increasing the lightweight aggregate replacement volume reduces the modulus of elasticity of the mixture at early ages and long term. The observed reduction follows the same trends observed with compressive strength and split tensile strength. This can be explained by the replacement of sand with a relatively softer lightweight material. The effects of modulus reduction on the restrained cracking behavior of the mixture will be discussed later in this report. 


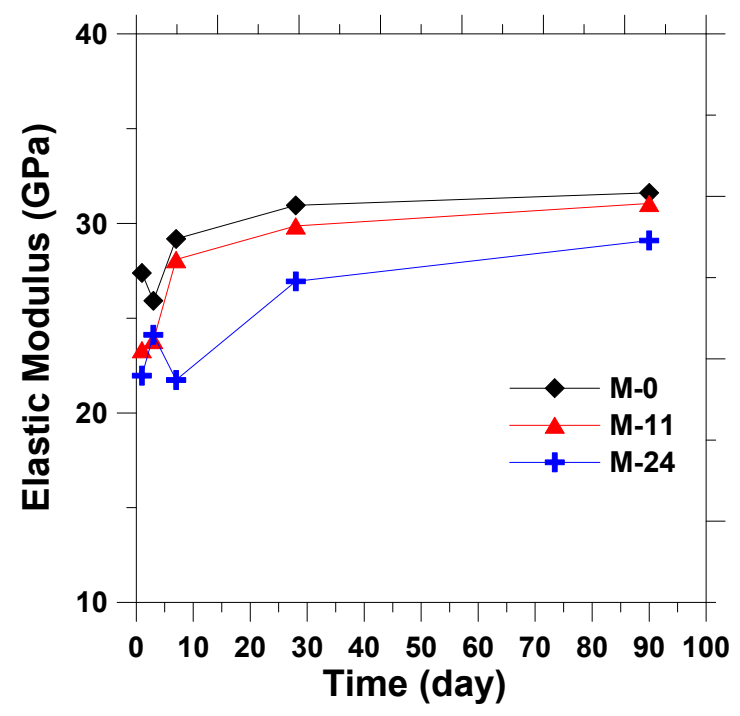

Figure 9-3 - Modulus Of Elasticity

\subsection{Mechanical Properties Conclusions}

This chapter discussed the mechanical properties of mixtures internally cured with prewetted lightweight aggregate. A summary of the observations are:

- The compressive strength of internally cured mixtures with LWA was reduced in the range of $2 \%$ to $8 \%$ as compared to plain mixtures without LWA.

- The split tensile capacity of internally cured mixtures using lightweight aggregate was slightly less than the control mixture but no general trend could be distinguished.

- Internally cured mixtures with LWA were up to $13 \%$ softer than the plain mixture without LWA. 


\section{CHAPTER 10. THE EFFECT OF TEMPERATURE CHANGE ON IC SYSTEMS MADE WITH PREWETTED LIGHTWEIGHT AGGREGATE}

\subsection{Introduction}

Cementitious materials undergo volume change at early ages due to chemical reactions, self desiccation, external drying, and thermal changes (Le Chatelier 1900; Neville et al. 1929; Lynam 1934; Powers 1947; RILEM Commission 42CEA 1981; Wiegrink et al. 1996; Aitcin et al. 1997; Shah et al. 1998; See et al. 2004). Residual stress develops in the cementitious material when this volume change is restrained (Igarashi et al. 2000; Altoubat et al. 2001; See et al. 2004; Radlinska et al. 2008). If the residual tensile stress becomes too large, cracking may occur. This cracking is significant as it inhibits otherwise durable concrete from reaching its potential service life by allowing deleterious materials to enter the cracks, thereby accelerating degradation (Wang et al. 1997; Weiss 1999).

The concerns associated with early-age cracking have spurred the development of many tests to assess a material's cracking potential. Tests based on uniaxial restraint such as cracking frames and temperature-stress testing machines have been implemented by Kovler (Kovler 1994), Springenschmid (Springenschmid et al. 1994), Lange (Altoubat et al. 2001), Marchand (Pigeon et al. 2000), and van Breugel (van Breugel et al. 1998). Another common method that has been used to quantify a mixture's performance is the restrained ring method (Bentz et al. 2008). This approach has been used for nearly a century, and recently the restrained ring test (ASTM C 1581-04) (2004) has been standardized as a procedure to assess the shrinkage cracking potential of cementitious mixtures. This test is performed by casting an annulus of a cementitious mixture (paste, 
mortar, or concrete) around a steel ring. Residual tensile stress is generated in the sample when it attempts to shrink but is restrained by the inner steel ring. A crack results if the residual stress exceeds the developing tensile strength. This is observed to correspond with a sudden drop in the measured strain. Stresses and cracking time in the sample are determined by instrumenting the ring with strain gauges.

The conventional restrained ring test has been widely used to evaluate the restrained shrinkage cracking potential of concrete mixtures but is limited in two significant ways. First, the test can only measure net shrinkage due to its configuration where expanding samples come out of contact with the restraining ring. This limitation becomes significant when studying expansive cements (Sant et al. 2007), shrinkage reducing admixtures (Sant et al. 2006), and internal curing (Henkensiefken et al. 2009) as these technologies may result in early age expansion. The second limitation of the restrained ring test is that it must operate at a constant temperature since the ASTM A53 Grade B steel pipe that is typically used for the restraining rings will expand or contract if the temperature changes. As a result, a temperature change would alter the level of restraint experienced by the cementitious annulus.

This chapter discusses a test method that can evaluate the expansion of internally cured mortars and the effects of varying temperature on restrained cracking propensity. This test, called the dual concentric ring test, uses two concentric restraining rings; one on the outside of the sample and one on the inside of the sample. This enables restraint in both expansion and contraction and measurement of the strain in the restraining elements as samples shrink and expand. The rings are constructed from Invar, a metal with an extremely low coefficient of thermal expansion that allows the temperature to be varied without dramatically altering the degree of restraint. This enables the study of thermally 
induced volume changes in addition to volume change from autogenous shrinkage. A wide variety of temperature profiles can be selected to simulate most field conditions. The ability of the test to induce thermal residual stresses has the potential to quantify the restrained cracking capacity of a mixture at any desired time. This can lead to an approach where a mixture could be tested multiple times in the dual ring and purposely cracked at various ages with temperature reduction to develop a cracking curve.

\subsection{Experimental Equipment}

\subsubsection{Dual Restraining Rings Geometry and Construction}

The restraining rings were fabricated from an Invar Steel alloy instead of conventional ASTM A53 Grade B steel (used for the standard ASTM C 1581-09 single ring test) in order to minimize the effects of temperature variation on the degree of restraint. Invar is a steel nickel alloy with a coefficient of thermal expansion (CTE) at or less than $1.3 \times 10^{-6} \varepsilon /{ }^{\circ} \mathrm{C}$ (Guillaume 1904; Jacobs 1996; Lement et al. 1996), compared to $12.0 \times 10^{-6} \quad \varepsilon /{ }^{\circ} \mathrm{C}$ for conventional steel (Nakamura 1976). For reference, the coefficient of thermal expansion of early age mortar varies (Wittman et al. 1974; Bjontegaard et al. 1998; Sellevold et al. 2006), but is typically within the range of $8 \times 10^{-6} \varepsilon /{ }^{\circ} \mathrm{C}$ to $10 \times 10^{-6} \varepsilon /{ }^{\circ} \mathrm{C}$.

The thermal deformation of the restraining ring directly affects the degree of restraint of the sample during a temperature change. If a material is used for the restraining ring with larger restraining ring CTE values it will produce a greater reduction in the degree of restraint which results in less residual stress development in the sample. This effect is significant because studying the restrained thermal behavior of the sample relies on developing residual stress in the sample during a temperature change. As a result, the more consistent the 
geometry of the restraining element, the easier it becomes to assess the material performance during a temperature change. The lower CTE of an Invar restraining ring stabilizes the degree of restraint during a temperature change and allows residual stress to be induced and measured.

The dual ring geometry is presented in Figure $10-1$ where $R_{O C}$ and $R_{I C}$ are the outer face and inner face radii of the sample respectively, $R_{0 O}$ is the outer face of the outer restraining ring radius, $R_{\|}$is the inner face of the inner restraining ring radius, and $t$ is the thickness of each restraining ring. The dimensions are as follows: $R_{\mathrm{OC}}=203 \mathrm{~mm} \pm 3 \mathrm{~mm}\left(8.0 " \pm 0.12\right.$ "), $\mathrm{R}_{\mathrm{IC}}=165 \mathrm{~mm} \pm 3 \mathrm{~mm}\left(6.5^{\prime \prime} \pm\right.$ $0.12 "), R_{O O}=222 \mathrm{~mm} \pm 3 \mathrm{~mm}\left(8.75^{\prime \prime} \pm 0.12 "\right), R_{\|}=146 \mathrm{~mm} \pm 3 \mathrm{~mm}\left(5.75^{\prime \prime} \pm\right.$ $0.12 "), \mathrm{t}=19 \mathrm{~mm} \pm 3 \mathrm{~mm}\left(0.75^{\prime \prime} \pm 0.12 "\right)$, and $76.2 \mathrm{~mm} \pm 3 \mathrm{~mm}\left(3^{\prime \prime} \pm 0.12 "\right)$ tall. The measured weight of the inner ring is $11.5 \mathrm{~kg}(25.4 \mathrm{lb})$ and that of the outer ring is $16.0 \mathrm{~kg}(35.2 \mathrm{lb})$.

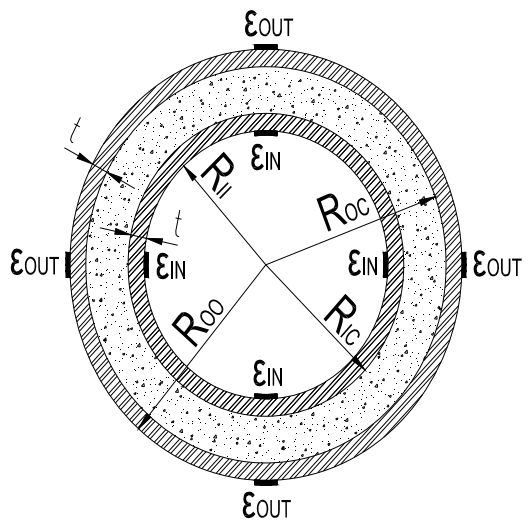

Figure 10-1 - Geometry of the Dual Ring Test

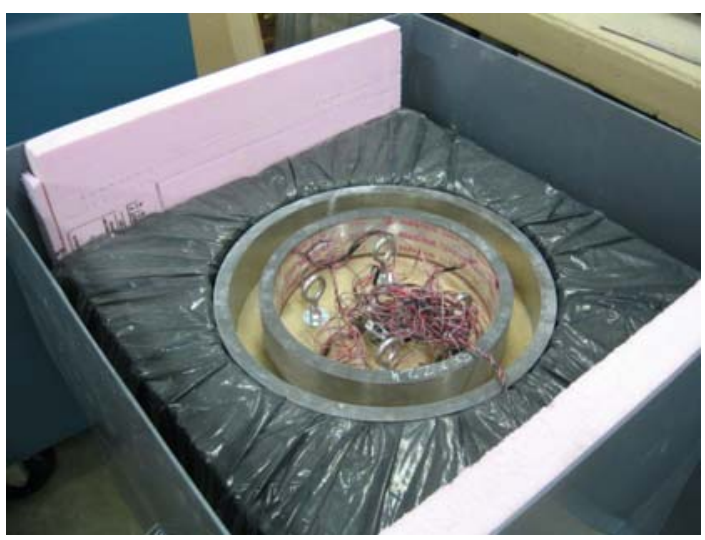

Figure 10-2 - Ring in Insulation Chamber with Top Insulation Removed

It should be noted that the thickness of the restraining ring is slightly different than the current ASTM standard. This difference is intentional since the elastic 
modulus of Invar $^{\odot} \mathrm{E}_{\mathrm{INVAR}}=141 \mathrm{GPa}(20450 \mathrm{ksi})$ (Lement et al. 1996) is less than that of the conventional steel, $E_{\text {STEEL }}=200 \mathrm{GPa}(29000 \mathrm{ksi})$. As a result, an Invar ring of the same size as the standard steel ring would provide less restraint to shrinkage. The goal of the design of the ring for this study was to match the degree of restraint of the dual ring to that of the conventional ASTM C 1581-09 restrained ring test. The degree of restraint of a restraining ring can be calculated by Equation 1 (Moon et al. 2006):

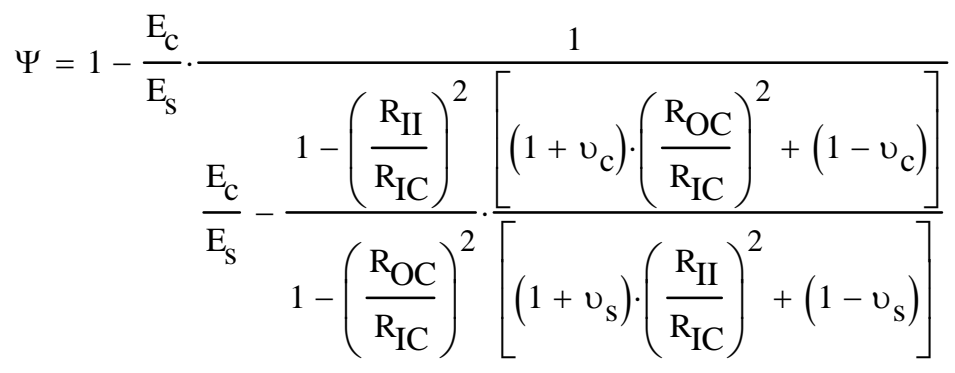

where $E_{c}$ represents the elastic modulus of the cementitious mixture, $E_{s}$ represents the elastic modulus of the ring metal, $v_{c}$ represents Poisson's ratio for concrete, $v_{s}$ represents Poisson's ratio for the ring metal, and $\Psi$ is the degree of restraint. In order to compare ring geometries, the modulus of elasticity and Poisson's ratio for the sample were assumed as constant values of $E_{c}=32 \mathrm{GPa}$ $(4641 \mathrm{ksi})$, and $\mathrm{v}_{\mathrm{c}}=0.18$. Poisson's ratio for both the steel and Invar were selected as $v_{s}=0.3$.

Figure 10-3 shows the relationship between the Invar ring thickness and the degree of restraint calculated with Equation 10-1 and the above mentioned specimen values. The degree of restraint increases as the restraining ring is thickened. The Invar ring thickness that corresponds to a degree of restraint equal to that of the ASTM C 1581-09 (72\%) was found to be $19 \mathrm{~mm}$. 


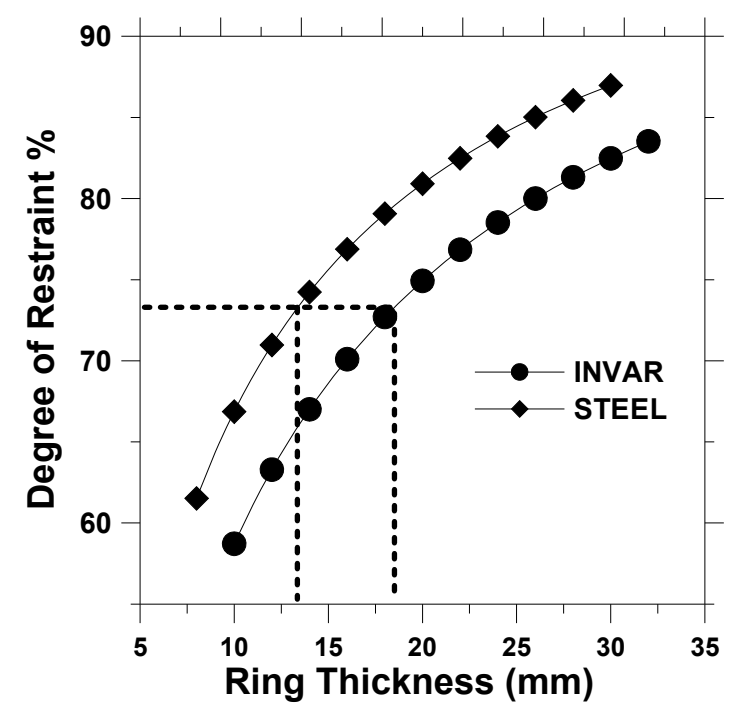

Figure 10-3 - Effect of Invar Ring Thickness on DOR

\subsubsection{Dual Ring Instrumentation and Data Acquisition}

The stress that develops within the cementitious sample is determined by measuring the strain in the Invar rings. Both of the Invar rings were instrumented with four opposing CEA-00 series strain gauges (Vishay Measurements Group 2007) that were mounted at mid height. Figure 10-1 illustrates gauge locations. The inner ring gauges were installed at mid height on the inner face of the inner ring while outer ring gauges were installed at mid height on the outer face of the outer ring. All gauges are operated as shunt calibrated quarter bridge circuits. The gauges have a grid resistance equal to $120 \% \pm 0.3 \% \Omega$, Gage Factor 2.05 $\% \pm 0.5 \%$ at $24{ }^{\circ} \mathrm{C}$, temperature correction of Gage Factor $+1.2 \pm 0.2$, and an operating temperature range of $-75{ }^{\circ} \mathrm{C}$ to $+175{ }^{\circ} \mathrm{C}$ (Vishay Measurements Group 2007).

The gauges were installed with a Vishay ${ }^{\odot}$ installation kit (Vishay Measurements Group 2005; Vishay Measurements Group 2008). Each mounting area was sanded smooth and cleaned with an acidic solution. After neutralizing the area 
treated with acid, the gauges were installed with M-Bond-200 modified alkyl cyanoacrylate adhesive. The gauges were covered with Teflon tape, butyl rubber sealant, neoprene rubber, and sealed with aluminum tape. Strain relief folds were applied to the gauge lead wires.

A Vishay ${ }^{\odot}$ system 5000 data scanner paired with Strain Smart $^{\odot}$ software was used to automatically record strain gauge data at five minute intervals. Data recording initiates immediately after the sample is sealed in the insulation chamber which typically occurs about 30 min after water contacts cement during mixing.

\subsubsection{Strain Gauge Temperature Calibration}

General practice shows that output from bonded strain gauges can change significantly when exposed solely to a temperature change (Hannah et al. 1992; Vishay Measurements Group 2007). This is caused by the concurrent effects of changes in the gauge's grid resistivity and the mismatch in thermal expansion coefficients between the strain gage and substrate (in this case the ring). Therefore, even though the Invar ring dimensions are nominally stable throughout the test's temperature range, the gauges still require thermal correction. It should be noted that the gauges used in this test are specifically designed for use on substrates with low thermal coefficients to minimize error caused by temperature change. Several methods are available to correct thermal output with varying degrees of confidence and accuracy. First hand corrective measurement was chosen to correct the dual rings because it is the most accurate method of thermal correction as it accounts for many gauge specific issues (Hannah et al. 1992; Vishay Measurements Group 2007). This involved generating a correction curve for each gauge by varying the temperature of the rings in the unstressed state and recording the resulting 
output. Figure $3 \mathrm{a}$ and Figure $3 \mathrm{~b}$ illustrate the strain correction curves for gauges on the inner and outer rings, respectively. It should be noted that the curved shape of the plot is expected and is typical (Vishay Measurements Group 2007). Strain Smart $^{\oplus}$ has a function that corrects the strain output based on these curves from temperature readings taken on the ring surface. This is accomplished by taping a type $T$ twisted pair thermocouple to the surface of the ring. Since the thermal uniformity of the rings is high, as discussed later in this paper, it is not necessary to measure temperature at every strain gauge. The correction curves were verified by subjecting the rings to a temperature change without a sample. Figure $3 \mathrm{c}$ illustrates the corrected output, and it can be seen that once the correction is applied the gauge output varied less than $4 \mu \varepsilon$ when subjected to a $45^{\circ} \mathrm{C}$ temperature change.

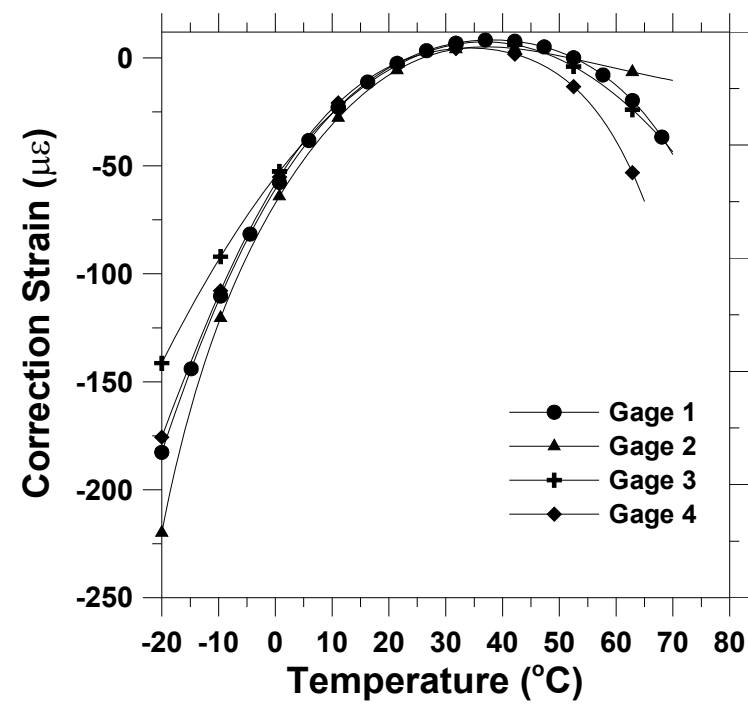

Figure 10-4 - Inner Ring Thermal Correction

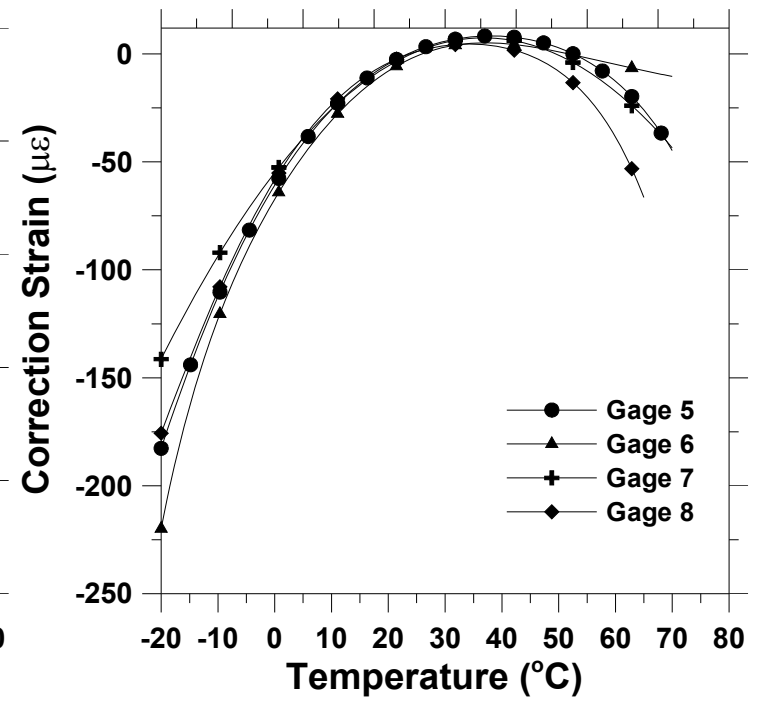

Figure 10-5 - Outer ring Thermal Correction

\subsubsection{Temperature Control System}

Temperature control is provided via a programmable $28 \mathrm{~L}$ water bath system pumping an ethylene glycol water mixture at $24 \mathrm{~L} / \mathrm{min}$ through a looped copper 
coil inside the chamber. The ethylene glycol mixture enables the bath temperature to be lowered below freezing to approximately $-20{ }^{\circ} \mathrm{C}$. Figure $4 \mathrm{~b}$ shows the placement of this coil on top of the restraining rings. A circular $3.2 \mathrm{~mm}$ $(1 / 8$ ) thick sheet of aluminum is placed on top of the rings to provide a rigid platform for the coil. The water bath continuously monitors the temperature delivered to the chamber by using an external probe mounted on the inlet of the coil. This enables the system to automatically compensate for heat loss or gain through the tubing connecting the bath to the chamber. In-line valves are installed on the external tubing to allow the user to turn the coil flow on or off.

The system's heating and cooling capabilities were determined by placing four type $T$ thermocouples on an aged mortar sample at room temperature and varying the bath temperature as quickly as possible. The heating test targeted an end temperature of $50{ }^{\circ} \mathrm{C}$ and it can be seen in Figure $5 a$ that the maximum temperature change the system can generate is about $1.8^{\circ} \mathrm{C}$ per hour at a 20 degree differential between the bath and sample. The cooling test targeted an end temperature of $13^{\circ} \mathrm{C}$ as shown in Figure $5 \mathrm{a}$. It can be seen the temperature change rate decreases as the difference between bath and sample temperature decreases. The maximum thermal differential recorded in the sample during forced heating and cooling between two points spaced vertically or horizontally on the ring was $0.3^{\circ} \mathrm{C}$. This indicates that forced heating and cooling produces uniform temperatures in the sample.

\subsection{Experimental Procedures}

\subsubsection{Mixture Proportions}

Three mortar mixtures with varying shrinkage performance were chosen to study several capabilities of the dual ring test. They are designated $\mathrm{M}-0, \mathrm{M}-11$, and $\mathrm{M}-$ 
24 with their proportions provided in Table 10-1. M-0 was a plain mortar while M-11 and M-24 utilized internal curing with pre-wetted fine lightweight aggregate (LWA). All mixtures had a water-to-cement ratio of 0.30 by mass $(w / c=0.30)$ and a paste volume of $45 \%$. The internally cured mixtures M-11 and M-24 had $11 \%$ and $23.7 \%$ of the total mixture volume comprised of LWA, respectively. It should be noted the $23.7 \%$ replacement value corresponds to the theoretical value of internal curing water that is required to match the chemical shrinkage (Bentz et al. 1999).

\begin{tabular}{|c|c|c|c|}
\hline & $\begin{array}{c}\text { M-0 } \\
\text { Plain } \\
\text { Mortar }\end{array}$ & $\begin{array}{l}\text { M-11 } \\
\text { LWA }\end{array}$ & $\begin{array}{l}\text { M-24 } \\
\text { LWA }\end{array}$ \\
\hline Material & $\left(\mathrm{kg} / \mathrm{m}^{3}\right)$ & $\left(\mathrm{kg} / \mathrm{m}^{3}\right)$ & $\left(\mathrm{kg} / \mathrm{m}^{3}\right)$ \\
\hline Cement (Type I) & 728 & 728 & 728 \\
\hline Water & 218 & 218 & 218 \\
\hline Fine Aggregate & 1444 & 1133 & 821 \\
\hline LWA (SSD) & 0 & 204 & 409 \\
\hline
\end{tabular}

\subsubsection{Materials}

Type I ordinary portland cement was used (ASTM C 150-05) with a Blaine fineness of $370 \mathrm{~m}^{2} / \mathrm{kg}$, and an estimated Bogue composition of a $56 \% \mathrm{C}_{3} \mathrm{~S}, 16 \%$ $\mathrm{C}_{2} \mathrm{~S}, 12 \% \mathrm{C}_{3} \mathrm{~A}$ and $7 \% \mathrm{C}_{4} \mathrm{AF}$. Normal weight river sand was used with a fineness modulus of 2.71 and an oven dry specific gravity of 2.58. The lightweight aggregate used in $\mathrm{M}-11$ and $\mathrm{M}-24$ was a manufactured rotary kiln expanded shale with a fineness modulus of 4.3 , an oven dry specific gravity of 1.56, and a 24 hour absorption value of $10.5 \%$ by mass. A high-range waterreducing admixture (HRWRA) was added at a rate of $1 \mathrm{~g}$ mass per $100 \mathrm{~g}$ of cement. Mix water consisted of tap water conditioned to $23^{\circ} \mathrm{C} \pm 1^{\circ} \mathrm{C}$. 
10.3.3. Dual Ring Specimen Mixing, Casting, and Testing Procedure Aggregate for each mixture was batched in the oven dry state. LWA was presoaked for $24 \mathrm{~h} \pm 0.5 \mathrm{~h}$ in the mix water (including the water for prewetting) while sealed in a container. Mixing was performed in accordance with ASTM C 192-07. First aggregate was loaded into the "buttered" mixer. Where applicable, the mixing water was decanted from the LWA. The mixer was started and $50 \%$ of the total water was added. The cement and remaining mix water containing the HRWRA were then added. The mortar was mixed for $3 \mathrm{~min}$, then rested for 3 min while the sides of the mixer were scraped, then mixed for a final 2 min.

The instrumented rings were prepared by coating the surfaces that would make contact with the sample with form release and a layer of acetate sheet in order to minimize friction on the sample. The fresh mortar was then cast between the two rings in two layers. A handheld vibrator was used to consolidate each layer. The top of the sample was struck level with the top of the ring and then capped with the separator plate and temperature control coil. The top layers of insulation and chamber cap were then installed to effectively seal the sample in the chamber to control its temperature and to prevent drying shrinkage.

The temperature control system was operated at $23{ }^{\circ} \mathrm{C} \pm 0.2{ }^{\circ} \mathrm{C}$ for a minimum of $8 \mathrm{~h}$ prior to casting, to bring the rings and chamber to a consistent temperature. After casting the temperature history follows the plot in Figure 10-6. It operated at $23{ }^{\circ} \mathrm{C} \pm 0.2{ }^{\circ} \mathrm{C}$ until $8 \mathrm{~d}$ when the temperature was reduced at a rate of $1{ }^{\circ} \mathrm{C} / \mathrm{h}$. The temperature reduction attempted to induce a crack in the sample by utilizing thermal shrinkage to produce additional tensile stress. The minimum temperature applied to the sample in this study is $-5^{\circ} \mathrm{C} \pm 0.2{ }^{\circ} \mathrm{C}$. 


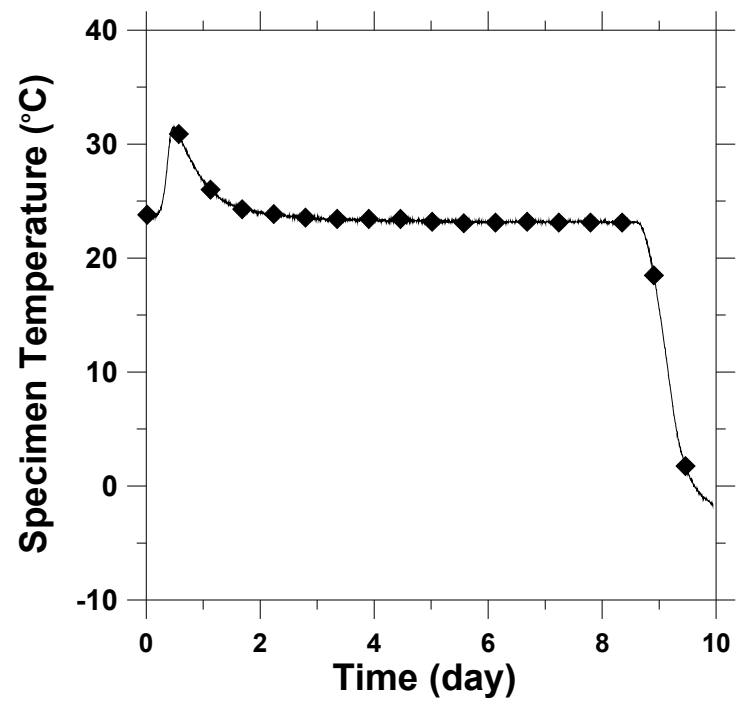

Figure 10-6 - Applied Temperature in Dual Ring Testing

The strain measured on the inner ring $\left(\varepsilon_{\mathrm{IN}}\right)$ and outer ring $\left(\varepsilon_{\mathrm{OUT}}\right)$ is reported as the average output of the four gauges on each ring. The precision of the average strains from subsequent tests of the same mixture deviated less than $6 \mu \varepsilon$. These strains can be used to determine the time of cracking by observing a sudden drop in readings. In addition, they can be used to calculate the residual stress that develops in the sample (Sant et al. 2007; Henkensiefken et al. 2009). First the pressure exerted by the sample on the inner ring $P_{I_{N}}$ can be calculated using Equation 10-2 (Moon et al. 2006):

$$
\mathrm{P}_{\mathrm{IN}}=-\varepsilon_{\mathrm{IN}} \cdot \mathrm{E}_{\mathrm{INVAR}} \cdot\left(\frac{\mathrm{R}_{\mathrm{IC}}{ }^{2}-\mathrm{R}_{\mathrm{II}}{ }^{2}}{2 \cdot \mathrm{R}_{\mathrm{IC}}{ }^{2}}\right)
$$

The pressure exerted by the sample on the outer ring $\mathrm{P}_{\mathrm{OUT}}$ is calculated by Equation 10-3 (Moon et al. 2006):

$$
\mathrm{P}_{\mathrm{OUT}}=\varepsilon_{\mathrm{OUT}} \cdot \mathrm{E}_{\mathrm{INVAR}} \cdot\left(\frac{\mathrm{R}_{\mathrm{OO}}{ }^{2}-\mathrm{R}_{\mathrm{OC}}{ }^{2}}{2 \cdot \mathrm{R}_{\mathrm{OC}}^{2}}\right)
$$


The two pressures obtained from ring strains are then used to calculate the circumferential "residual" stress in the sample at $R_{I C}\left(\sigma_{S}\left(R_{I C}\right)\right)$ by Equation 10-4:

$$
\sigma_{\theta}\left(\mathrm{R}_{\mathrm{IC}}\right)=\mathrm{P}_{\mathrm{IN}}\left[\frac{\mathrm{R}_{\mathrm{OC}}^{2}+\mathrm{R}_{\mathrm{IC}}^{2}}{\left(\mathrm{R}_{\mathrm{OC}}^{2}-\mathrm{R}_{\mathrm{IC}}^{2}\right)}\right]-\mathrm{P}_{\mathrm{OUT}}\left[\frac{2 \cdot \mathrm{R}_{\mathrm{OC}}^{2}}{\left(\mathrm{R}_{\mathrm{OC}}^{2}-\mathrm{R}_{\mathrm{IC}}^{2}\right)}\right]
$$

Equation 10-4

Substituting Equation 10-2 and Equation 10-3 into Equation 10-4 yields Equation $10-5$ for the ring geometry employed in this study. The residual stress $\left(\sigma_{\ominus}\right)$ is output in units of MPa or PSI when units of MPa or PSI are used in the $\mathrm{E}_{\text {INVAR }}$ terms. The values for $\varepsilon_{\text {IN }}$ and $\varepsilon_{O U T}$ are input as actual strain.

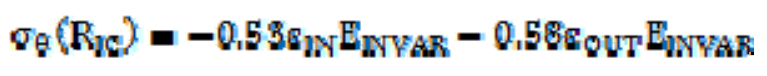

Equation 10-5

\subsection{Results and Discussion}

The inner and outer ring strain history is illustrated in Figure 10-7 and Figure 10-8, respectively. Residual stress development is shown in Figure 10-9. It can be seen the plain mortar $\mathrm{M}-0$ only develops strain in the inner ring, indicating that it only shrinks. This was anticipated as the free shrinkage as discussed previously in this report only exhibit shrinkage. M-0 continues shrinking until it develops a stress of $3.6 \mathrm{MPa}$ (tension) at approximately $4 \mathrm{~d}$, at which time it cracks. 


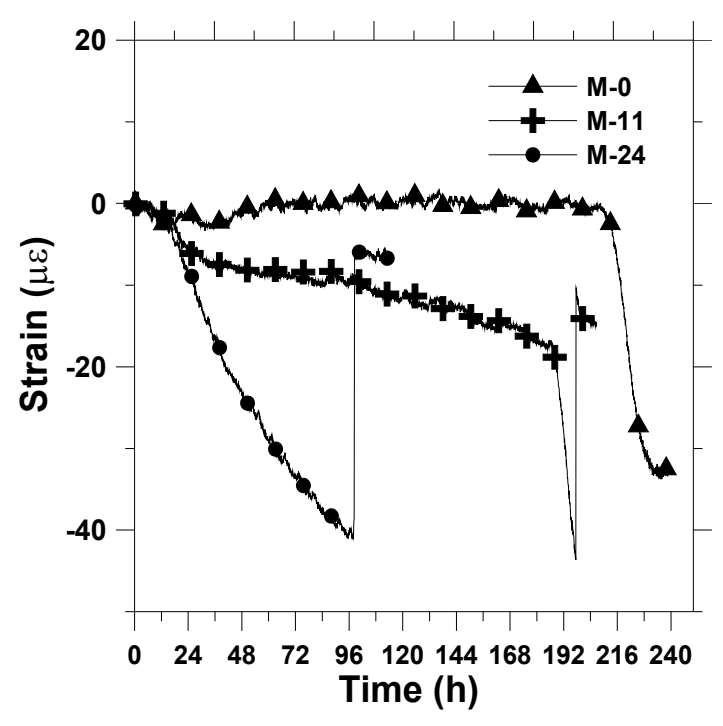

Figure 10-7 - Inner Ring

Strain History

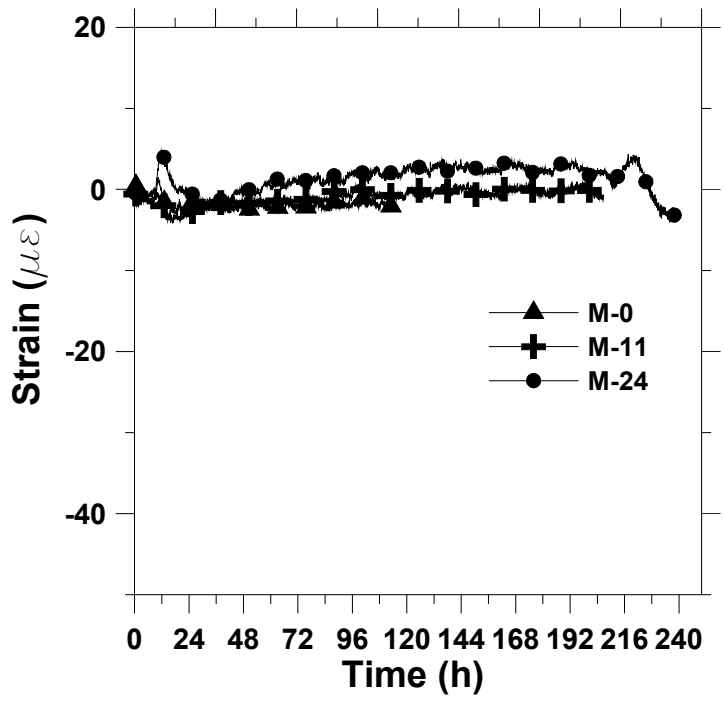

Figure 10-8 - Outer Ring Strain History

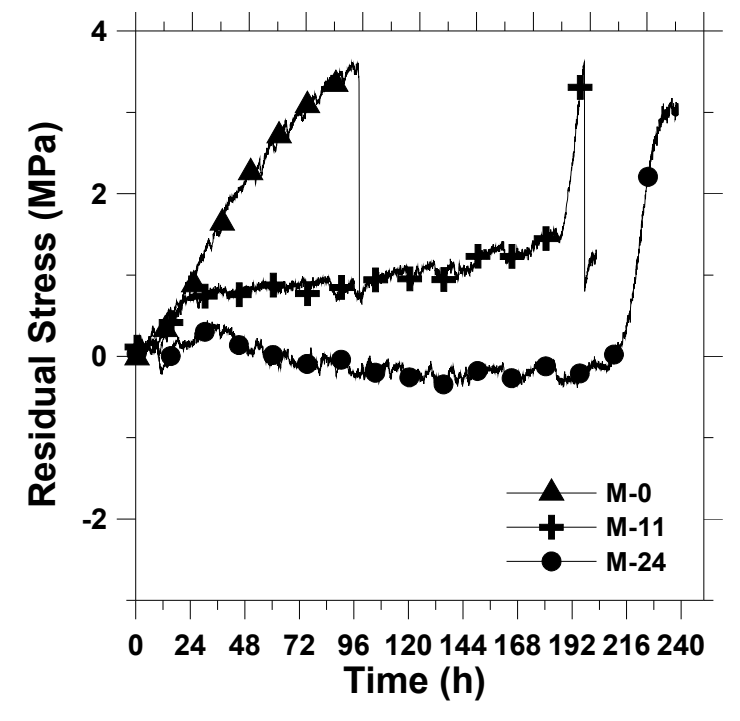

Figure 10-9 - Specimen Stress History

Mixture M-11 develops no expansive stress and develops a residual tensile tress of $1.4 \mathrm{MPa}$ at $8 \mathrm{~d}$ while operating at constant $23^{\circ} \mathrm{C} \pm 0.2^{\circ} \mathrm{C}$. The temperature was then decreased at a rate of $1^{\circ} \mathrm{C} / \mathrm{h}$ and the resulting increase in residual stress can be seen until the sample cracks at $3.6 \mathrm{MPa}$ at $8.2 \mathrm{~d}\left(14.3^{\circ} \mathrm{C}\right)$. This 
indicates that the samples restrained shrinkage only developed $39 \%$ of the stress required to induce cracking. Also, approximately $6 \mu \varepsilon$ of ring strain or 0.2 MPa residual stress in the sample is generated per $1^{\circ} \mathrm{C}$ of temperature change.

It can be seen that mixture M-24 develops a slight expansive strain in the outer ring shortly after the measured time of set of $6 \mathrm{~h}$. The expansion peaks at $4 \mu \varepsilon$ or $0.15 \mathrm{MPa}$ at $11.8 \mathrm{~h}$ and then decreases over a period of $7 \mathrm{~h}$, without producing an opposing reaction on the inner ring. It is likely that this reduction in stress is due to stress relaxation while the modulus of elasticity is low and the creep is high in the sample at early age. Neither shrinkage nor expansion is observed for the remainder of the test while temperature is held at a constant $23{ }^{\circ} \mathrm{C} \pm 0.5{ }^{\circ} \mathrm{C}$. The free shrinkage history during this time shows a subtle expansion which may not be large enough to overcome the rate of creep under restrained conditions. After approximately $8 \mathrm{~d}$, the temperature in the sample is decreased at a rate of $1^{\circ} \mathrm{C} / \mathrm{h}$ and an increase in shrinkage strain and residual tensile stress is observed. It can be seen that the rate of ring strain and residual stress development is the same in both the M-11 and M-24 samples. The M-24 sample does not generate a crack by the time it reaches the minimum temperature limit of $-5{ }^{\circ} \mathrm{C}$.

Relative cracking potentials of $\mathrm{M}-11$ and $\mathrm{M}-24$ can be determined by comparing the amount of additional residual stress required to crack the sample by decreasing temperature. The M-11 sample required an additional 2.2 MPa of tensile stress to induce cracking, while M-24 requires at least 3.0 MPa since it did not crack. Therefore it can be concluded that M-11 would have a greater potential to crack due thermal shrinkage than M-24. This can be attributed to the amount of tensile stress in the sample immediately before the temperature begins to decrease. Since the M-11 sample begins the cooling period with approximately $1.5 \mathrm{MPa}$ of tensile stress more than that of $\mathrm{M}-24$, it is closer to its cracking capacity. While the slight early age expansion is generally more desired 
than shrinkage as seen in the M-24 sample, it can be seen that the volume stability of the sample after $12 \mathrm{~h}$ has a greater contribution to reducing cracking potential.

\subsection{Thermal Study Summary}

This chapter has described the development of a 'Dual Concentric Ring Testing Device' that enables the residual stress that develops in concrete rings to be quantified as concrete expands and contracts due to chemical reaction, hygral shrinkage, or thermal movements. The ring used here is designed to provide a similar degree of restraint when compared with the ASTM C 1581-09 standard test geometry; however conceptually any geometry can be used.

The results of cooling specimens in the dual ring indicate that the internally cured samples are more resistant to thermal cracking than plain mixtures. Larger replacement volumes of prewetted lightweight aggregate provided more resistance. This increased resistance of IC mixtures can be attributed to the reduced residual stress development due to non thermal shrinkage and reduced modulus of elasticity. 


\section{CHAPTER 11. EVALUATING INTERNALLY CURED MIXTURES WITH A TEMPERATURE DROP APPROACH}

\subsection{Introduction}

This chapter will focus on the development of a temperature reduction technique using the dual ring device presented in CHAPTER 10 to quantify the reserve cracking strength of a fresh mixture over the first of several days after casting. This technique is significant because it provides a more complete understanding of the cracking propensity of a mixture as compared to the standardized restrained ring test. This technique allows crack sensitive periods of time to be identified and the effects of sustained stress on cracking strength to be quantified. This chapter will show and discuss the results of the temperature drop technique used on one plain mortar mixture and two internally cured mortar mixtures using prewetted lightweight aggregate.

\subsection{Experimental Approach}

By using the temperature control system to quickly lower the temperature of the device, additional residual stress can be generated to crack the specimen at any time. The temperature drop approach involves casting successive specimens of the same mixture design and forcing cracking to occur at different early ages. A cracking stress curve for the mixture design is then generated by plotting the cracking stress at each age. The difference in the cracking stress curve and the residual stress history of the specimen tested at constant temperature produces a reserve stress capacity curve. 
Figure 11-1 illustrates how temperature reduction of the dual ring specimen may be used to induce cracking. In this example, the temperature control system was operated at $23{ }^{\circ} \mathrm{C}$ until $30.5 \mathrm{hr}$ when the temperature was reduced. It can be noted that the heat of hydration is not completely eliminated. Tensile residual stress in the specimen increases as the temperature decreases. Cracking is observed at $43.9 \mathrm{hr}$ which is indicated by the sudden drop in residual stress. The magnitude of temperature reduction required to induce cracking was $14.6{ }^{\circ} \mathrm{C}$. The reserve stress may be calculated by subtracting the residual stress while operating at constant temperature $\left(\sigma_{S}\right)$ from the cracking stress $\left(\sigma_{C}\right)$. In this example, the reserve stress at $43.9 \mathrm{hr}$ is $3.1 \mathrm{MPa}$.

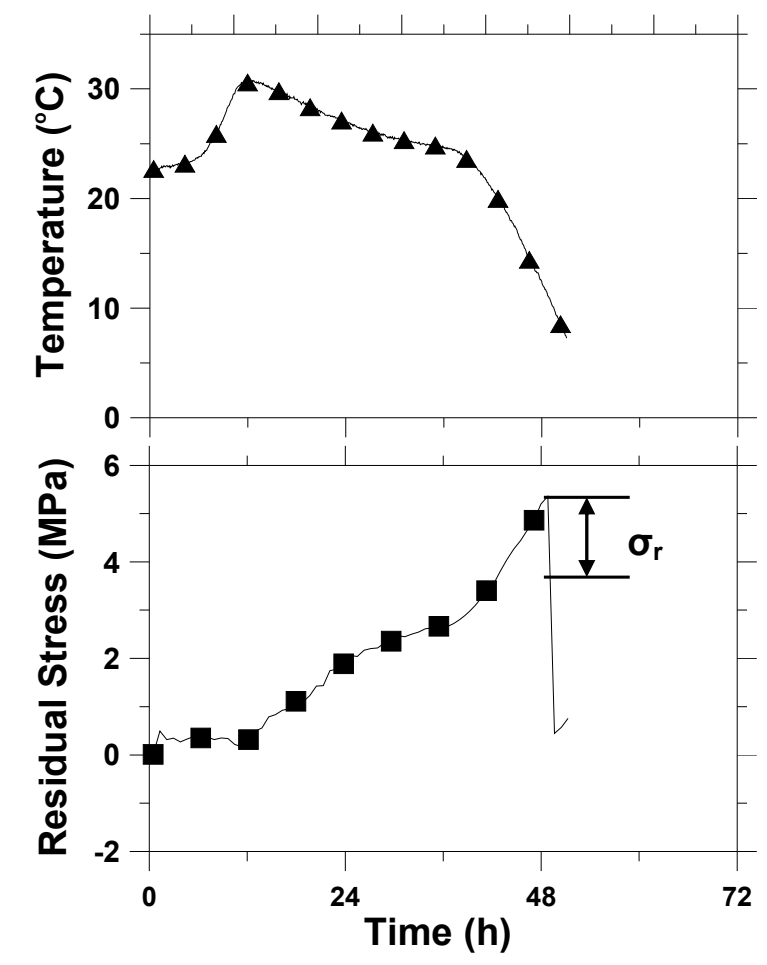

Figure 11-1 - Example Temperature Reduction 


\subsection{Experimental Program}

\subsubsection{Mixture Proportions}

Three low w/c mortar mixtures were used in this study to evaluate internal curing with the temperature drop approach. Mixture proportions are designated as M-0, $\mathrm{M}-11$, and M-24 are presented in Table 11-1.

\begin{tabular}{|l|c|c|c|}
\cline { 2 - 4 } \multicolumn{1}{c|}{} & $\begin{array}{c}\text { M-0 } \\
\text { Plain Mortar }\end{array}$ & $\begin{array}{c}\text { M-11 } \\
\text { SLWA }\end{array}$ & $\begin{array}{c}\text { M-24 } \\
\text { SLWA }\end{array}$ \\
\hline Material & $\begin{array}{c}\text { Amount } \\
\mathbf{k g} / \mathbf{m}^{3}(\mathbf{l b s} / \mathbf{y d} 3)\end{array}$ & $\begin{array}{c}\text { Amount } \\
\mathbf{~ k g} / \mathbf{m}^{3}(\mathbf{l b s} / \mathbf{y d} 3)\end{array}$ & $\begin{array}{c}\text { Amount } \\
\mathbf{~ k g} / \mathbf{m}^{3}(\mathbf{l b s} / \mathbf{y d} 3)\end{array}$ \\
\hline \hline Cement (Type I) & $729(1228)$ & $728(1228)$ & $728(1228)$ \\
\hline Water & $218(368)$ & $218(368)$ & $218(368)$ \\
\hline IC Water & 0 & $19.46(32.82)$ & $38.82(65.49)$ \\
\hline Fine Aggregate & $1444(2435)$ & $1126(1900)$ & $810(1366)$ \\
\hline FLWA & 0 & $189(318)$ & $376(635)$ \\
\hline
\end{tabular}

* Water absorbed in FLWA at set

Table 11-1 -Mixture Proportions for Temperature Drop Approach

Mixture M-0 was a plain mortar while mixtures $M-11$ and $M-24$ utilize internal curing using pre-wetted lightweight sand aggregate (LWA). All mixtures had a water-to-cement ratio of 0.30 by mass $(w / c=0.30)$ and a paste volume of $45 \%$. The internally cured mixtures $\mathrm{M}-11$ and $\mathrm{M}-24$ had $11 \%$ and $23.7 \%$ of the total mixture volume comprised of LWA, respectively. It should be noted the $23.7 \%$ LWA replacement value corresponds to the theoretical value of internal curing water that is required to match the chemical shrinkage (Bentz et al. 1999).

\subsubsection{Materials}

Type I ordinary portland cement was used (ASTM C 150-05) with a Blaine fineness of $370 \mathrm{~m}^{2} / \mathrm{kg}\left(1809 \mathrm{ft}^{2} / \mathrm{lb}\right)$, and an estimated Bogue composition of a 56 
$\% \mathrm{C}_{3} \mathrm{~S}, 16 \% \mathrm{C}_{2} \mathrm{~S}, 12 \% \mathrm{C}_{3} \mathrm{~A}$ and $7 \% \mathrm{C}_{4} \mathrm{AF}$. Normal weight river sand was used with a fineness modulus of 2.71 and an oven dry specific gravity of 2.58. The lightweight aggregate used in $\mathrm{M}-11$ and $\mathrm{M}-24$ was a manufactured rotary kiln expanded shale with a fineness modulus of 4.3 , an oven dry specific gravity of 1.56 , and a 24 hour absorption value of $10.5 \%$ by mass. A high-range waterreducing admixture (HRWRA) was added at a rate of $1 \mathrm{~g}(0.0022 \mathrm{lb})$ mass per $100 \mathrm{~g}(0.22 \mathrm{lb})$ of cement. Mix water consisted of tap water conditioned to $23{ }^{\circ} \mathrm{C}$ $\pm 1^{\circ} \mathrm{C}\left(73.4^{\circ} \mathrm{F} \pm 1.8^{\circ} \mathrm{F}\right)$.

\subsubsection{Dual Ring Testing Protocol}

The restraining rings were prepared by coating the surfaces that would make contact with the sample with form release and a layer of acetate sheet in order to minimize friction on the sample. The fresh mortar was then cast between the two rings in two layers. A handheld vibrator was used to consolidate each layer. The top of the sample was struck level with the top of the rings and then capped with the separator plate and temperature control coil. The top layers of insulation were then installed. Strain gauge data is automatically recorded at five minute intervals beginning immediately after the sample is sealed in the insulation chamber which typically occurs about 30 min after water contacts cement during mixing.

The temperature control system was operated at $23{ }^{\circ} \mathrm{C} \pm 0.2{ }^{\circ} \mathrm{C}$ for a minimum of $8 \mathrm{~h}$ prior to casting to bring the rings and chamber to a consistent temperature. After casting, the system operated at $23{ }^{\circ} \mathrm{C} \pm 0.2{ }^{\circ} \mathrm{C}$ until the test ended due to the sample cracking or when a temperature reduction was desired. The temperature was reduced at a rate of $1^{\circ} \mathrm{C} / \mathrm{h}$ to a minimum temperature of $-5{ }^{\circ} \mathrm{C} \pm$ $0.2^{\circ} \mathrm{C}$. 
11.3.4. Split Tensile, Compressive Strength, and Elastic Modulus Tests A series of $100 \mathrm{~mm} \times 200 \mathrm{~mm}$ cylinders were cast for each of the three mortar mixtures to determine the early age split tensile strength, compressive strength, and elastic modulus. The cylinders were cast according to ASTM C192. Testing was performed at ages $1,3,5$, and 7 days after mixing on three samples at each age. Split tensile capacity was measured in accordance with ASTM C 496/C 496M-04. Compressive strength was measured in accordance with ASTM C 39. The elastic modulus was measured in accordance with ASTM C 469-02.

\subsection{Experimental Results}

The temperature drop approach was applied to the four mixture designs in this study. Eight M-0 specimens were tested and their residual stress histories are presented in Figure 11-2. The temperature was reduced to produce cracking at 33 hr, $38 \mathrm{hr}, 43 \mathrm{hr}, 48 \mathrm{hr}, 72 \mathrm{hr}$, and $79 \mathrm{hr}$. One M-0 specimen was allowed to crack without reducing temperature at $120 \mathrm{hr}$.

Six M-11 specimens were tested and their residual stress histories are presented in Figure 11-3. All M-11 specimens underwent temperature reductions and were forced to crack at $42 \mathrm{hr}, 50 \mathrm{hr}, 72 \mathrm{hr}, 89 \mathrm{hr}, 108 \mathrm{hr}$, and $120 \mathrm{hr}$.

Five M-24 specimens were tested and their residual stress histories are presented in Figure 11-4. All M-24 specimens underwent temperature reductions but cracking was only produced at $127 \mathrm{hr}$ and $197 \mathrm{hr}$. The three earliest aged specimens reached the lower temperature limit of the control system before cracking occurred. 


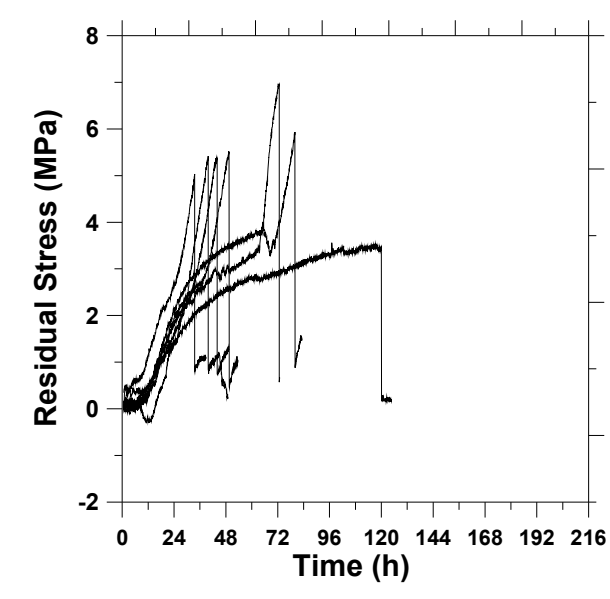

Figure 11-2 - M-0 Residual Stress

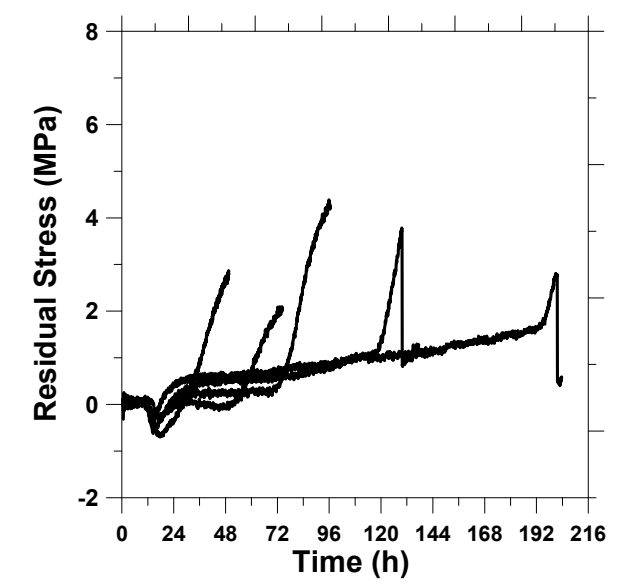

Figure 11-4 - M-24 Residual Stress

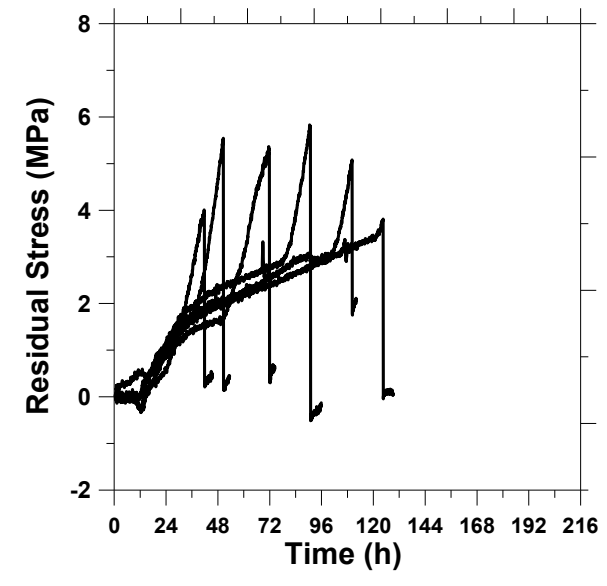

Figure 11-3 - M-11 Residual Stress

The split tensile, modulus of elasticity, and compressive strength results for the four mixtures are presented in Figure 11-5, Figure 11-6 and Figure 11-7. 


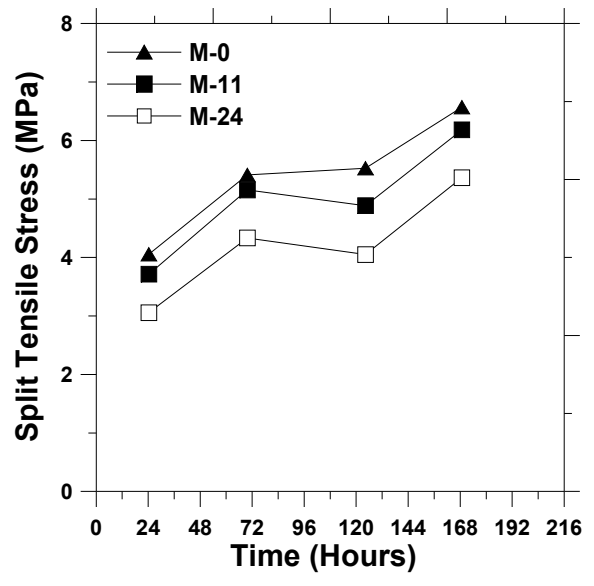

Figure 11-5 - Split Tensile Capacity, $f_{t}$

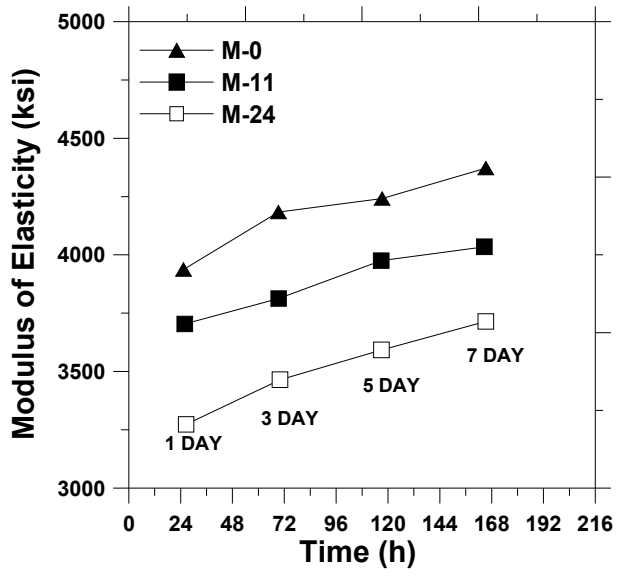

Figure 11-6 - Modulus of Elasticity, E

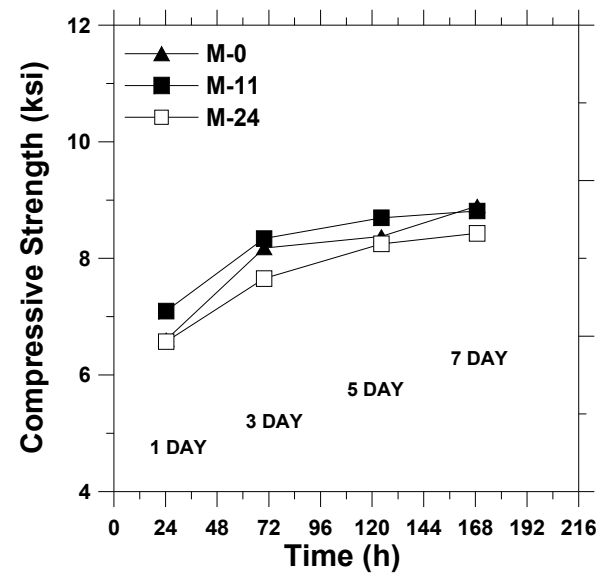

Figure 11-7 - Compressive Strength, $f_{c}$

\subsection{Discussion}

Internal curing reduces or eliminates the amount of shrinkage that results in tensile stress when it is restrained. Previous work has shown that the amount and dispersion of the internal curing water affects its ability to mitigate shrinkage (Jensen et al. 2001; Lura et al. 2007; Henkensiefken et al. 2009). Therefore, mixture M-24 will reduce more shrinkage than the other internally cured mixture $\mathrm{M}-11$. Adding internal curing agents can lower the tensile and compressive 
capacity of the concrete. Figure 11-5 and Figure 11-7 shows the tensile and compressive strength reduction. Although these two properties are reduced, internal curing also reduces modulus of elasticity and autogenous shrinkage which reduces the cracking propensity of the concrete. The following discussion will outline how the temperature drop approach in dual ring can be used to show this potential benefit.

The temperature reduction required to induce cracking can provide an idea of how much thermal change a concrete in the field may be able to sustain. Figure 11-8 summarizes the temperature reduction histories of the four mixture designs from the temperature drop approach. The allowable thermal change is not constant and changes as the mixtures age. Each mixture achieved its largest thermal reduction prior to about $96 \mathrm{hr}$ and then decreased. This indicates that all four mixtures are more susceptible to thermal cracking after $96 \mathrm{hr}$. Mixture M-24 requires the greatest amount of temperature change to induce cracking and is approximately $3 x$ greater than the plain $\mathrm{M}-0$ mixture. This behavior indicates the M-24 mixture design can sustain larger thermal swings than the M-0 mixture. The lower level of internal curing provided by mixture M-11 is most beneficial prior to $96 \mathrm{hr}$. 


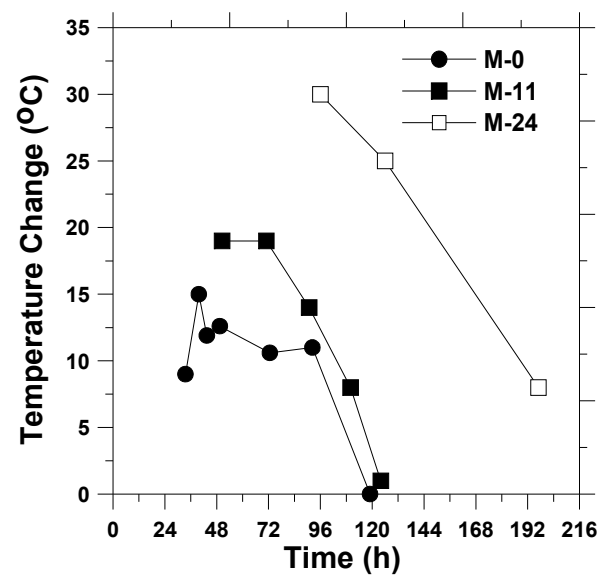

Figure 11-8 - Required Temperature Change to Induce Cracking

The behavior of the cracking stress as a mixture ages in the dual ring can show the effects of sustained residual stress. The cracking stress histories of the four mixture designs by using the temperature drop approach are presented in Figure 11-9. It shows the cracking stress decreased for all mixtures as they aged. All three mixtures produced a similar rate of declining cracking stress. Mixtures M-0 and M-11 produced similar cracking stress profiles where their maximum cracking capacities occurred prior to $96 \mathrm{hr}$ and then began to decline.

Figure 11-5 presents the split tensile capacity histories which indicated an opposite trend of cracking strength development. The split tensile capacity increased as the samples aged instead of decreasing as seen in the dual ring. This difference indicates that microstructural damage (microcracking) occurred in the dual ring samples as result of sustained residual stress. Since split tensile samples are cured in the unstressed state they are not subjected to this damage. This discrepancy indicates that the split tensile cracking stresses may not provide a clear representation of field performance of concrete which is typically in the stressed state. 
The cracking stress histories can also show the effect of internal curing. Mixture M-24 (which had the largest amount of internal curing) cracked at later ages than $\mathrm{M}-0$ and $\mathrm{M}-11$ but it cracked at lower stresses. This reduced stress capacity can be attributed to a combination of the relatively lower split tensile cracking stresses and the longer duration the specimen was stressed. This behavior indicates that the performance of internal curing may be limited to earlier ages if it is not used in a great enough quantity to maintain a low residual stress state.

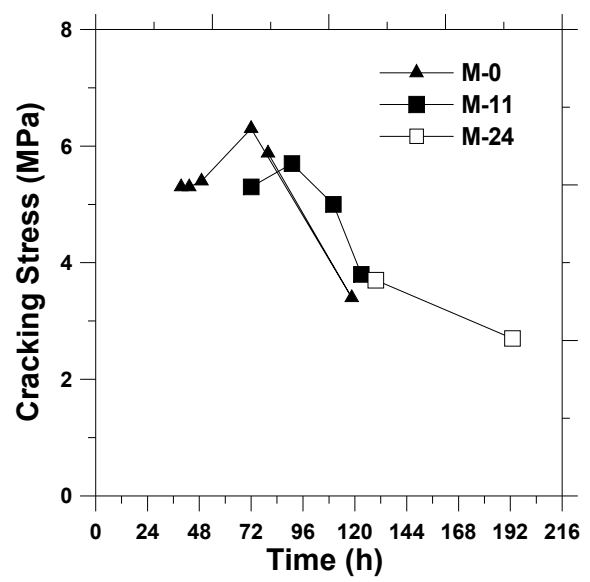

Figure 11-9 - Cracking Stress History

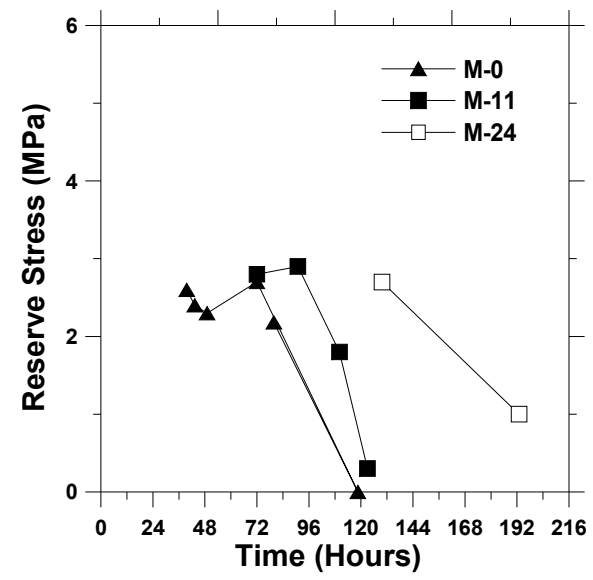

Figure 11-10 - Reserve Stress Capacity

The temperature drop approach can also be used to determine the reserve stress capacity of a mixture. Reserve stress represents the difference between the cracking capacity and the residual stress due to autogenous shrinkage. Therefore it can describe the concurrent effects of a mixture's tensile capacity and autogenous shrinkage. Figure 11-10 presents the reserve stress for the four mixtures. Mixture M-24 produces greater reserve stresses at later ages which indicate a greater ability to prevent cracking. Mixture $\mathrm{M}-11$ show slight improvement in cracking performance than the plain mixture $\mathrm{M}-0$. 
A factor of safety to cracking (FSC) can be calculated by dividing reserve stress capacity by the cracking stress. This can be used to provide a better representation of how close a mixture is to cracking than either the cracking stress or reserve stress separately. The FSC accounts for the reduced cracking capacity that occurs at later ages and due to internal curing.

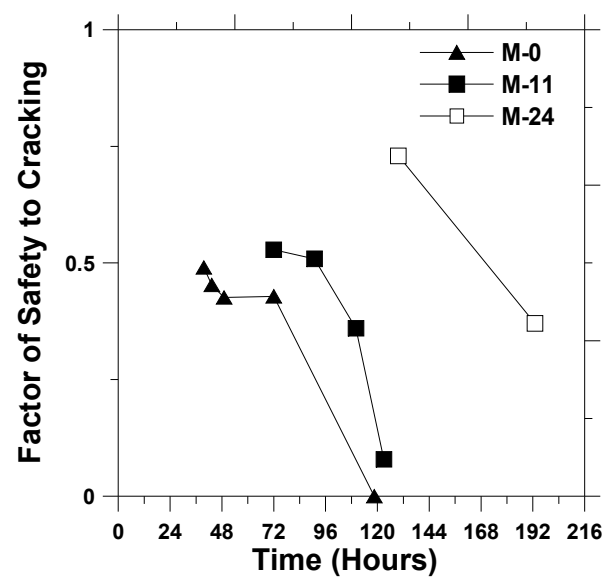

Figure 11-11 - Factor of Safety To Cracking

\subsection{Summary of Temperature Drop Approach}

The temperature drop approach used with the dual ring device provides several new ways to observe the cracking propensity of concretes and how internal curing may improve their performance in the following ways:

- The dual ring can describe the ability of a concrete to sustain temperature swings. The internally cured mixtures sustained up to $3 x$ the amount of temperature swings than plain mixtures any given age.

- The dual ring can show the effect of sustained residual stress on cracking capacity. Mixtures that are loaded in tension longer exhibit reduced tensile strength. 
- It can be used to calculate the reserve stress providing a more clear idea of how restrained specimens perform during early ages. Internally cured mixtures with lightweight aggregate have a larger reserve stress and are less likely to crack. 


\section{CHAPTER 12. FREEZE THAW RESISTANCE OF MIXTURES CONTAINING PREWETTED LIGHTWEIGHT AGGREGATE}

\subsection{Introduction}

Concrete can be susceptible to damage caused by repeated freezing and thawing cycles (Powers 1950; Penttala et al. 2002). The propensity of the mixture to undergo freeze-thaw damage is based on several factors but in general is due to how well the mixture avoids reaching a critical degree of saturation (Fagerlund 1977). Mixtures with low permeability require longer periods of wetting to reach this point. Some concern exists with using lightweight aggregates in freeze-thaw applications because they are porous and can absorb water which may cause greater freezing damage (Roberts 1995). The porosity of the paste plays a large role in the water movement within the sample. Therefore, mixtures with low w/c may sufficiently prevent external water from reaching the lightweight aggregates. This chapter investigates the freeze-thaw resistance of high performance mixtures containing prewetted lightweight aggregate using ASTM C 666 (ASTM 2008).

\subsection{Materials}

ASTM C150 Type I ordinary portland cement (OPC) was used, with a Blaine fineness of $370 \mathrm{~m}^{2} / \mathrm{kg}$ and an estimated Bogue composition of $56 \% \mathrm{C}_{3} \mathrm{~S}, 16 \%$ $\mathrm{C}_{2} \mathrm{~S}, 12 \% \mathrm{C}_{3} \mathrm{~A}, 7 \% \mathrm{C}_{4} \mathrm{AF}$ and a $\mathrm{Na}_{2} \mathrm{O}$ equivalent of $0.68 \%$, all by mass fraction.

A high-range water-reducing admixture (HRWRA) was added in varying rates depending on the volume fraction of LWA replacing normal weight sand in order to maintain similar mixture consistencies. 
The normal weight sand used was natural river sand with a fineness modulus of 2.71 and an apparent specific gravity of 2.58. Portions of the normal weight sand were replaced with manufactured rotary kilned expanded shale (LWA-K), with a fineness modulus of 3.10 and an apparent specific gravity of 1.56 . The $24 \mathrm{~h}$ absorption of the normal weight sand, and LWA-K were determined to be $1.8 \%$ and $10.5 \%$ by mass, respectively, according to ASTM C128-07 (2007). The normal weight sand used was natural river sand with a fineness modulus of 2.71 and an apparent specific gravity of 2.58 .

\subsection{Mixture Proportions}

Three low w/c mortar mixtures were used in this study to evaluate the freeze thaw resistance of mixtures with internal curing. Mixture proportions are designated as $\mathrm{M}-0 \mathrm{k}, \mathrm{M}-11 \mathrm{k}$, and $\mathrm{M}-24 \mathrm{k}$ and are presented in Table 12-1.

Table 12-1 - Mixture Proportions for Freeze Thaw Study

\begin{tabular}{|c|c|c|c|}
\hline & $\begin{array}{c}\text { M-0 } \\
\text { Plain Mortar }\end{array}$ & $\mathrm{M}-11 \mathrm{k}$ & M-24k \\
\hline Material & $\begin{array}{c}\text { Amount } \\
\mathrm{kg} / \mathrm{m}^{3}(\mathrm{lbs} / \mathrm{yd} 3)\end{array}$ & $\begin{array}{c}\text { Amount } \\
\mathrm{kg} / \mathrm{m}^{3} \text { (Ibs/yd3) }\end{array}$ & $\begin{array}{c}\text { Amount } \\
\mathrm{kg} / \mathrm{m}^{3}(\mathrm{lbs} / \mathrm{yd} 3)\end{array}$ \\
\hline Cement (Type I) & $728(1228)$ & $728(1228)$ & $728(1228)$ \\
\hline Water & $218(368)$ & $218(368)$ & $218(368)$ \\
\hline IC Water* & 0 & $19.46(32.82)$ & $38.82(65.49)$ \\
\hline Fine Aggregate & $1444(2435)$ & 1155 (1948) & $818(1381)$ \\
\hline FLWA & 0 & $188(317)$ & 408 (689) \\
\hline
\end{tabular}

${ }^{*}$ Water absorbed in LWA at set

Mixture M-0 was a plain mortar while mixtures $M-11 k$ and $M-24 k$ utilize internal curing using pre-wetted fine lightweight aggregate (LWA). All mixtures had a water-to-cement ratio of 0.30 by mass $(w / c=0.30)$ and a paste volume of $45 \%$. The internally cured mixtures $\mathrm{M}-11 \mathrm{k}$ and $\mathrm{M}-24 \mathrm{k}$ had $11 \%$ and $23.7 \%$ of the total mixture volume comprised of LWA, respectively. It should be noted the $23.7 \%$ 
LWA replacement value corresponds to the theoretical value of internal curing water that is required to match the volume caused by chemical shrinkage.

\subsection{Mixing and Freeze Thaw Testing Procedure}

Aggregate for each mixture was prepared and batched in the oven dry state. The LWA was then presoaked for $24 \mathrm{~h} \pm 0.5 \mathrm{~h}$ in the mix water (including the water for prewetting) while sealed in a container. Mixing was performed in accordance with ASTM C 192-07. First, aggregate was loaded into the slightly dampened mixer. Where applicable, the mixing water was decanted from the LWA. The mixer was started and $50 \%$ of the total water was added. The cement and remaining mix water containing the HRWRA were then added. The mortar was mixed for $3 \mathrm{~min}$, then rested for $3 \mathrm{~min}$ while the sides of the mixer were scraped, then mixed for a final 2 min.

Three $75 \mathrm{~mm} \times 405 \mathrm{~mm} \times 100 \mathrm{~mm}$ (3"x16"x4") prisms were cast for each mixture and were sealed in plastic for 14 days after casting. For one mixture, M-11k, an additional three prisms were cast and wet cured for the first 14 days after casting. After 14 days, all samples were submerged in water and subjected to freezing and thawing cycles at a rate of 2 per day. The temperature cycled from $10^{\circ} \mathrm{C}$ to $18^{\circ} \mathrm{C}$. Periodic measurements of the dynamic modulus and weight were taken while the specimens were in the thawed state.

\subsection{Results and Discussion}

Figure 12-1 presents the average relative dynamic modulus of the three mixtures. The relative dynamic modulus indicates that none of the mixtures underwent freezing and thawing damage. Damage would have produced a 
sudden drop of the dynamic modulus which was not seen in any of the mixtures. It is likely that the low w/c of all three mixtures produced a dense enough paste that it could not reach the critical saturation level required to cause freeze thaw damage. It can be noted that the lower relative dynamic modulus observed for the mixtures with lightweight aggregate is only an indication that they have a lower modulus of elasticity. This was expected and is otherwise discussed in CHAPTER 9.

The amount of weight gain of each mixture is presented in Figure 12-2 indicates how much water the specimens absorbed. IC specimens with prewetted lightweight aggregate absorbed over $2 x$ less water than the plain mixture. This may be an indication that the plain mortar had a lower internal $\mathrm{RH}$ after the 14 day curing period which would allow it to reabsorb more water. Internal relative humidity measurements presented in CHAPTER 6 agree with this reasoning. More water could be absorbed by the drier sample. If the initial weight gain due to the lower $\mathrm{RH}$ is normalized, the longer term weight gain would match $\mathrm{M}-11 \mathrm{k}$ and M-24k. This indicates that the overall porosity of the mixtures may be similar.

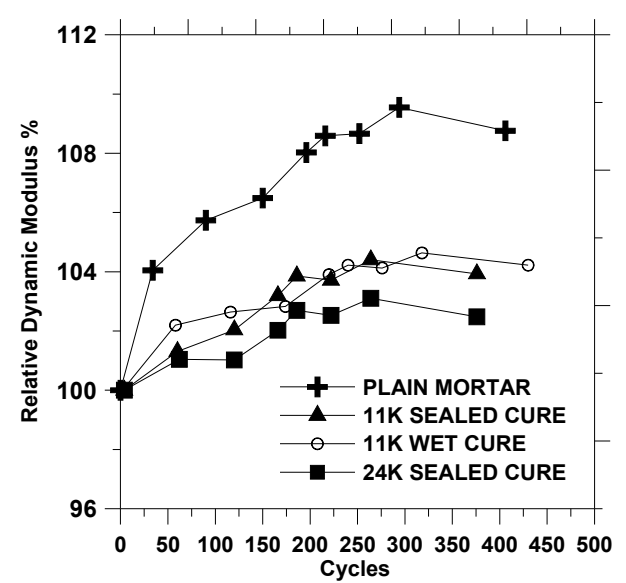

Figure 12-1 - Relative Dynamic Modulus

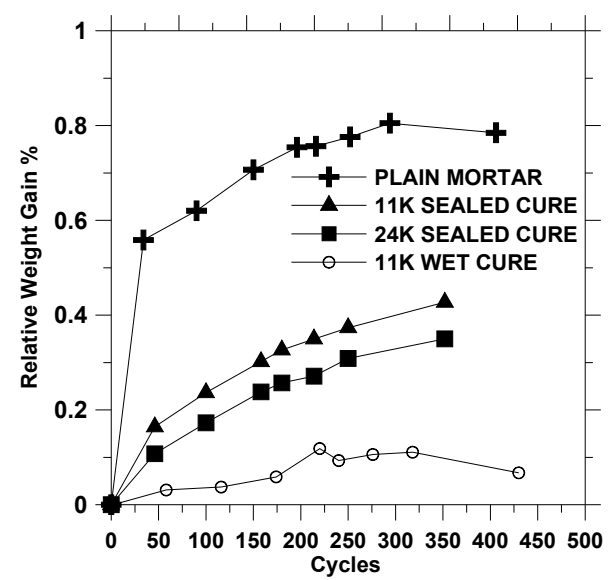

Figure 12-2 - Relative Weight Gain 


\section{6. $\underline{\text { Conclusions }}$}

This chapter has described the freeze thaw behavior of mortars made with prewetted lightweight aggregate. The inclusion of larger volumes of LWA in these particular mixture proportions did not increase or decrease freeze thaw performance. It is likely that the low w/c of the selected mixtures prevented the degree of saturation from reaching a level where freeze thaw damage would occur. 


\section{CHAPTER 13. FULL SCALE BATCHING AND TESTING OF INTERNALLY CURED MIXTURES USING PREWETTED LIGHTWEIGHT AGGREGATE}

\subsection{Introduction}

Shrinkage is an inherent behavior of current portland cement based materials (RILEM Commission 42-CEA 1981). Chemical reactions, self desiccation, external drying, and thermal variations are factors that contribute to the overall shrinkage behavior. The vast majority of concrete elements in the field are at least partially restrained from shrinking. That is, other elements physically prevent part of the shrinkage deformation from occurring. Examples include slabs-on-grade, where the subgrade restrains the concrete shrinkage through friction, concrete bridge decks restrained by the supporting girders and abutments, or elevated composite deck slabs restrained by the composite beams and deck.

Restraint plays a key role in the serviceability of concrete applications. Restrained shrinkage generates residual tensile stress in the shrinking concrete member. Cracking results if the residual internal stress exceeds the concrete's tensile capacity. This cracking is significant as it inhibits otherwise durable concretes from reaching their potential service life by allowing deleterious materials to enter the cracks, thereby accelerating corrosion and degradation (Wang et al. 1997; Hobbs 1999; Weiss 1999) .

Researchers have utilized multiple testing techniques in the last two decades to evaluate in-situ shrinkage cracking performance of concrete mixtures (RILEM 
TC-181 EAS 2002). Most of the tests are performed at a relatively small scale (e.g. ASTM C1581-09). While these techniques provide valuable information on restrained shrinkage cracking potential of concrete mixtures, they lack the ability to simulate issues that only a full scaled specimen can provide, such as the role of reinforcement for obtaining a specific crack width in the field (Raoufi 2010).

This chapter describes the design and implementation of a large scale testing system to evaluate the restrained shrinkage cracking propensity of internally cured concrete mixtures. The test involves casting a concrete sample slab on top of an aged concrete base. The base restrains the concrete sample slab and results in residual stress development to possibly crack the sample.

\subsection{Large Scale Testing Approach}

The large scale restrained slab testing device presented in this paper was operated inside a large $15^{\prime} \times 45^{\prime}$ environmentally controlled chamber and was designed to enable the test conditions (relative humidity and temperature) to be well controlled, specified, and repeated. This large scale test provides a unique opportunity to determine the effectiveness of technologies that mitigate early age shrinkage cracking of concrete mixtures. Information from the large scale tests can be used to calibrate finite element models and the standard restrained ring tests.

The geometry of the specimen and restraining base was determined with a finite element model capable of considering age dependant properties of concrete and non linear fracture mechanics concepts. Effects of various parameters such as length of slab, thickness of base and base rigidity on residual stress development and cracking behavior of concrete slabs are discussed. 
The test was then constructed based on the geometry established with the model. Details of the test construction, mixture selection, and the results of the test are discussed.

\subsection{Design of the Large Scale Restrained Slab Test}

\subsubsection{Modeling Approach}

A commercial finite element package, FEMMASSE MLS 8.5, was used for the design of the large scale slab test. The program is used to investigate the influence of the length and thickness of concrete slab and the rigidity of the restraining base on stress development and cracking behavior of the concrete slab. The software enables age-dependent material properties such as strength, elastic modulus, fracture toughness and autogenous length change to be directly input to study stress development and cracking behavior of concrete at early ages. Non-linear fracture mechanics, strain softening, creep and stress relaxation are considered in the analysis. Various environmental conditions such as temperature, wind speed and relative humidity could also be considered in the analysis. Stress, displacement, temperature rise and cracking were calculated throughout the model.

In this analysis, concrete behaves as a linear elastic material until the tensile strength of concrete is reached. If the tensile strength is reached a crack is formed normal to the direction of the maximum principal stress when the maximum principal stress reaches the strength of materials (Mode I fracture behavior). The cohesive zone model was used to model the cracking of concrete (Hillerborg et al. 1976). Once a crack is formed, as shrinkage increases, the 
width of crack increases and stress gradually decreases to zero following the strain softening relationship. Since stress in the softening relationship of concrete is related to age-dependent strength of concrete, age-dependent fracture toughness of concrete is also considered, thereby enabling the simulation of crack development at early ages. Further details of the modeling approach can be found in the FEMMASSE manual (B.V 2006) and in (Raoufi 2010).

\subsubsection{Model Properties}

The model consists of a concrete sample slab cast on an aged concrete base slab as seen in Figure 13-1. The aged concrete base restrains the shrinkage of the concrete slab. The restraining base slab would be cast several weeks in advance of casting the sample slab and is treated as a mature concrete (with constant material properties) in the model. To minimize the potential for cracking near ends of the slab, each end of the concrete slab flares out and tapers down as it wraps down over the edge of the restraining base slab.

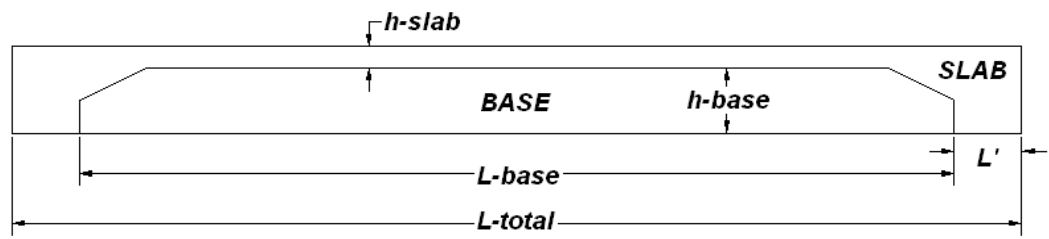

(a)

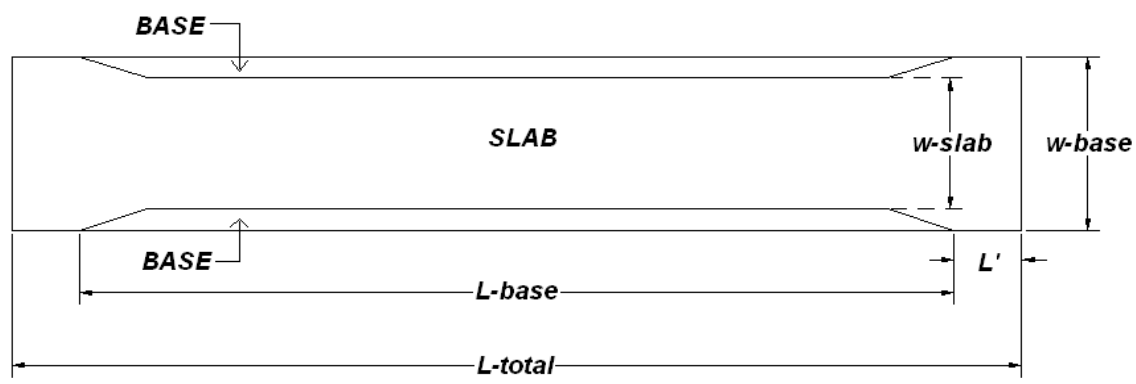

(b) 
Figure 13-1 - Basic Test Geometry (a) Longitudinal Cross Section View

(b) Plan View

The width of the restraining slab (as shown by "w-base" in Figure 13-1) is $0.8 \mathrm{~m}$ and was selected based on logistics related to the environmental chamber. Since the analysis software is two-dimensional based the concrete slab and the base were modeled in a half width along the longitudinal centerline in this analysis. The restraining base slab mesh elements were defined as $0.4 \mathrm{~m}$ deep. The depths of the sample slab mesh elements were varied to simulate changes in cross section width near the ends as shown in Figure 13-1. The analysis was performed at a constant temperature of $23^{\circ} \mathrm{C}$ and the model was completely sealed from the environment (i.e., no temperature and moisture variations were allowed). Although the concrete slab is subjected to uniform shrinkage in this analysis, similar behavior would be expected in the case of drying concrete specimens.

\subsubsection{Modeled Concrete Properties Used to Design Large Scale Test Geometry} Age-dependent tensile strength (Hossain et al. 2004), elastic modulus (Dehrdrai 2008) shown in Figure 13-2, and autogenous shrinkage (Radlinska 2008) shown in Figure 13-3, were used to model a concrete with a water-to-cement ratio of 0.30 and $55 \%$ fine aggregate (with maximum aggregate diameter of $3 \mathrm{~mm}$ ) by volume. A setting time of 5.9 hours were used while simulations started at the time of casting. An age-dependent Maxwell Chain Model was used to model creep and stress relaxation of concrete. A bilinear softening (stress-crack width) relationship was used to model fracture behavior of concrete. The knee point in the bilinear softening relationship was characterized by a stress-to-strength ratio of 0.35 and crack width of $0.01 \mathrm{~mm}$ while a crack width of $0.08 \mathrm{~mm}$ defined the critical crack width beyond which crack surfaces become traction free. A $10 \%$ 
randomly distributed variation in the tensile strength of concrete was considered (Radlinska 2008).

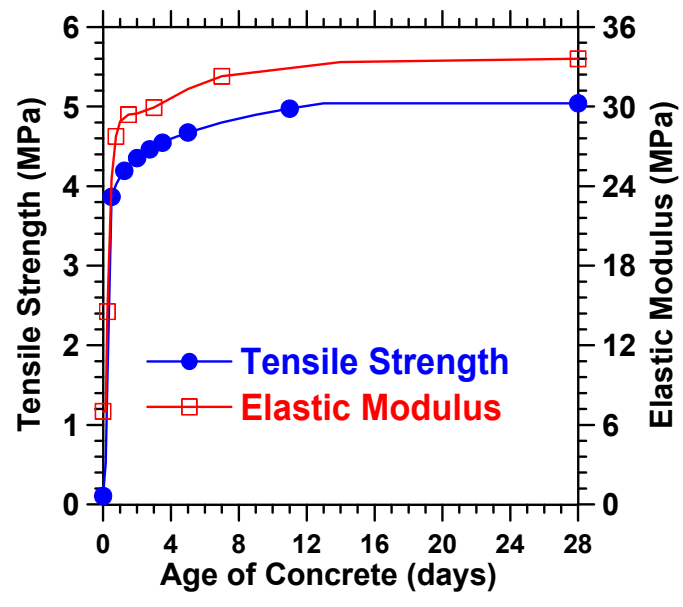

(a)

Figure 13-2 - Elastic Modulus and Tensile Strength Used in Model

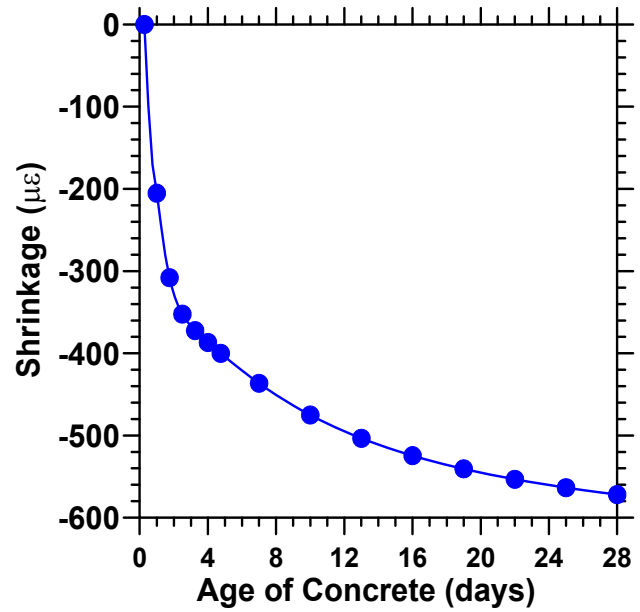

(b)

Figure 13-3 -Autogenous Shrinkage Used in Model

The restraining base slab was modeled as an aged concrete with a constant tensile strength of $6 \mathrm{MPa}$, compressive strength of $80 \mathrm{MPa}$ and density of 2100 $\mathrm{kg} / \mathrm{m}^{3}$. The elastic modulus of the restraining base slab was modeled as a constant $35 \mathrm{GPa}$ except where the study investigated the effects it had on the performance of the test. The middle section of concrete slab (the section with uniform cross section) was connected to the base layer by a series of frictionless and cohesiveless interface elements. The base layer was bonded to the concrete slab at its ends to minimize 'slipping' of the concrete slab. A plain stress condition was assumed in this study. Quadrilateral four-node elements were used to mesh the model while the mesh in concrete slab was refined to enhance numerical accuracy. 


\subsubsection{Results of Modeling and Selected Geometry}

A large number of simulations were performed to gain an understanding of the influence of geometry and stiffness on the behavior of the system while the slab shrank. Two main features were observed in the response. First the slabs curled. This curling occurred since moment is generated at both ends of the slabs from the force couple of the shrinkage force and restraining force. Since the sample slab is cast on top of the restraining slab, the two forces are offset by a depth " $d$ " as seen in Figure 13-4. The term $F_{\text {shrink }}$ represents the shrinkage force and the term $F_{\text {restraint }}$ represents the restraint force from the restraining base slab. The moment bends the sample and restraining base slabs in a concave upward shape as exaggerated in the section view in Figure 13-5. This curvature was quantified by measuring the vertical deflections along the length of the slab model.

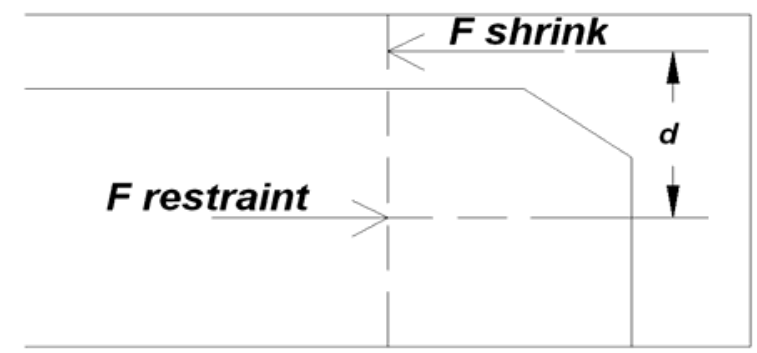

Figure 13-4 - Longitudinal Section at End of Slabs Depicting Shrinkage and Restraining Forces

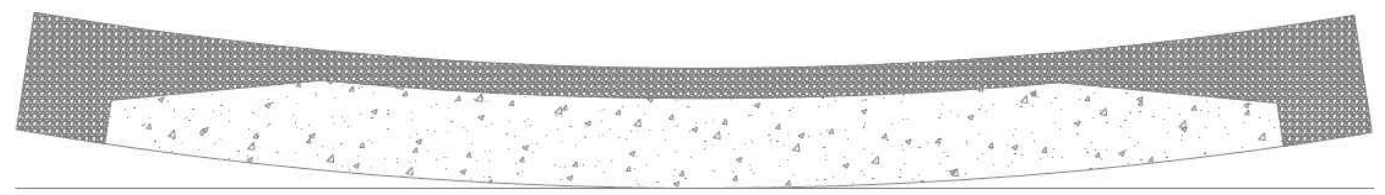

Figure 13-5 - Exaggerated Longitudinal Section View of Slab Curvature During Test Specimen Shrinkage 
The second feature from the test is that the restraining slab is that it undergoes axial shortening. This elastic response occurs as the restraining slab is axially loaded by the shrinking sample slab. The axial shortening of the restraining slabs was quantified by measuring the horizontal displacements of the restraining slab in the model.

The two observed features, slab curling and axial shortening, directly affected the degree of restraint (DOR) of the sample slab because they effectively 'give' in to the sample slab deformation. The DOR is a percentage that quantifies the amount of shrinkage deformation is prevented by the restraining element. A DOR of $0 \%$ would indicate the sample is completely free to deform (unrestrained) while a DOR of $100 \%$ would indicate the sample is not allowed to deform. Increased slab curling and axial shortening work to decrease the DOR which in turn lengthens the time to possible cracking.

Three parameters were identified that influence the behavior of the test. They include the restraining base thickness, sample slab length, and elastic modulus of the restraining slab. FEMMASSE analyzed the influence of each variable and the results will be discussed in the following sections.

\subsubsection{Influence of Restraining Slab Base Thickness}

The thickness of the restraining base slab was varied to study its effect on the test deformation, stress uniformity over the depth of the sample, time until cracking occurs, and the degree of restraint. Figure 13-6 summarizes the results from the simulations. Increasing the restraining base slab thickness decreases both curling and axial shortening. Figure 13-6(a) plots the vertical deflection of the sample slab along its length from a point at mid slab. Thickening the restraining base increases its flexural stiffness and in turn reduces the vertical 
deflections (curvature). It also increases the self weight of the sample/restraining slab assembly which counteracts the curling moment. Reducing the curvature of the two slab assembly improves the stress uniformity of the sample slab as shown in Figure 13-6(c) which depicts the stress variation across the depth of a section at mid slab. It can also be seen that thickening the base slab becomes less effective for thicknesses greater than $305 \mathrm{~mm}$. This occurs because the stiffening effect of a deeper restraining slab is offset by the increased moment couple arm " $\mathrm{d}$ " between the shrinkage and restraint forces. The increased couple moment in turn increases the curling moment the slab assembly must resist.

The time to cracking can be seen in Figure 13-6(b) which details the average stress in the sample slab. The sudden reduction in average stress indicates the sample slab cracks. Thickening the base slab decreases the time until cracking. A $152 \mathrm{~mm}$ thick restraining slab did not crack the sample while a $457 \mathrm{~mm}$ thick restraining slab cracked it at 3 days.

Increasing the base thickness also increases its axial stiffness. As the restraining slab becomes stiffer, the Degree of Restraint (DOR) increases. Figure 13-7 plots the DOR of the test against the thickness of the restraining slab. 


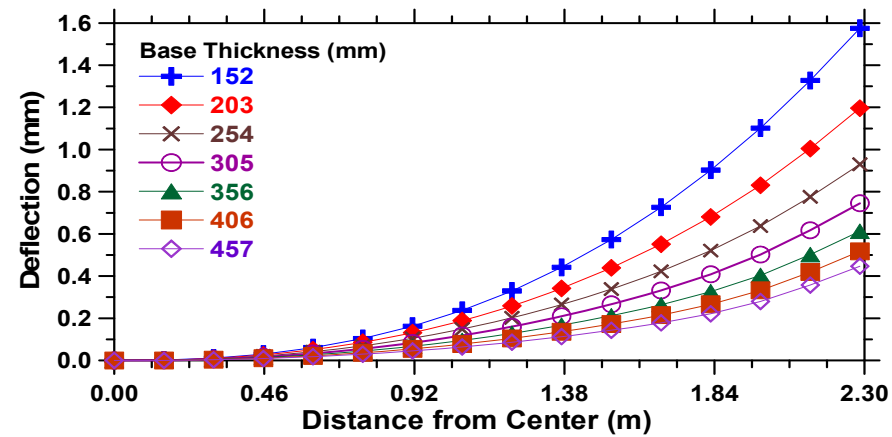

(a)

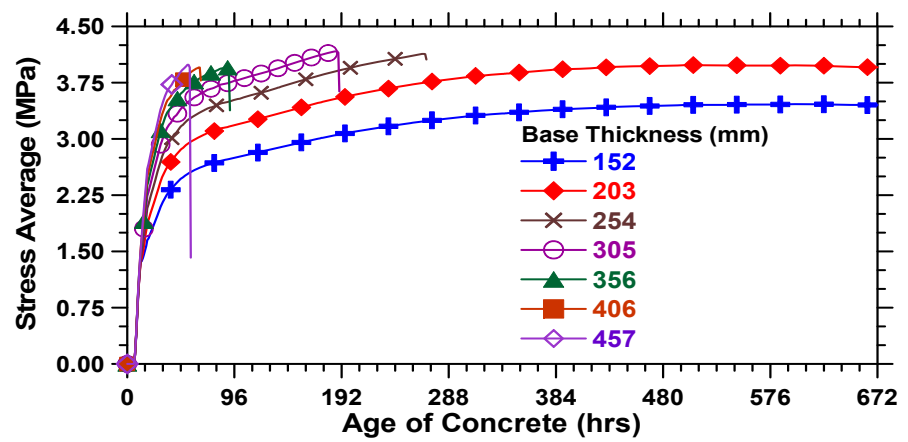

(b)

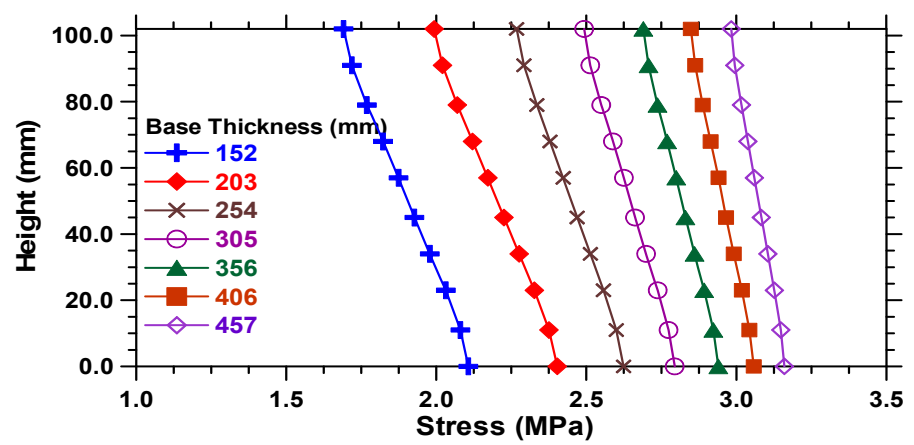

(c)

Figure 13-6 - Restraining Base Thickness Influence on (a) Deflection (b) Average Stress (c) Stress Uniformity 


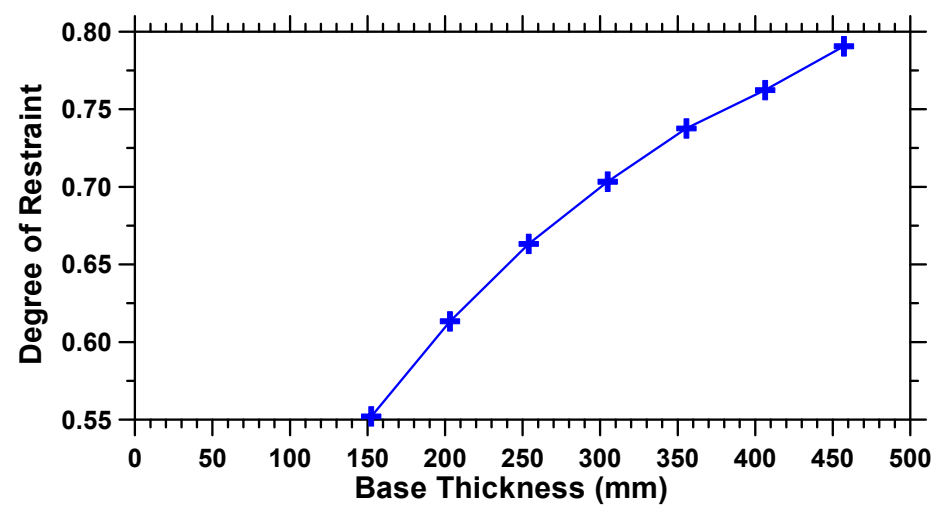

Figure 13-7 - Effect of Restraining Base Thickness on Degree of Restraint

\subsubsection{Influence of Test Slab Length}

The effect of the sample and restraining slab lengths were also analyzed and the results are presented in Figure 13-8 for a restraining slab depth of $305 \mathrm{~mm}$. Longer slabs create a more uniform stress distribution towards the mid length of the sample slab as can be seen in Figure 13-8(a). For example, a slab that is $9.1 \mathrm{~m}$ long has a flat region that is $4.0 \mathrm{~m}$ long whereas a $3.0 \mathrm{~m}$ long slab does not have any flat region. This behavior can be attributed to the action self weight has in counteracting the curling moment. Slab curling lifts the end portions of slabs from contacting the supporting base thereby creating a cantilever. The moment generated by the cantilever opposes the curling moment. The longer slabs were able to develop enough cantilever length to overcome the curling moment. It can be seen in Figure 13-8(a) that $7.6 \mathrm{~m}$ was the minimum length required to develop enough self weight cantilever moment to overcome the effects of curling at the center of the slab. The tensile stress distribution for the longer slabs is more uniform as seen in Figure 13-8(c). Greater stress uniformity occurs because the longer slabs have less curvature. Since less tensile stress is relieved by the effect of curvature the longer slabs crack sooner as depicted in Figure 13-8(b). 
A slab length of $4.6 \mathrm{~m}$ was chosen for the test design geometry because it provides relatively good tensile stress uniformity and fits logistically within the testing facility.

\subsubsection{Influence of Restraining Base Elastic Modulus}

The elastic modulus of the restraining base slab can be manipulated by the type of aggregates used in the concrete mixture. It is common to find the elastic modulus for concrete in the range of $30 \mathrm{GPa}$ to $40 \mathrm{GPa}$. Therefore, its influence on the test behavior was also investigated. Increasing the restraining slab modulus increases the both the flexural and axial restraining stiffness. This is similar to deepening the restraining slab except that curling moment is not increased. Figure 13-9 details the effect elastic modulus of the restraining slab has on the vertical end deflection of the test. The modulus was varied while holding the restraining slab depth constant at $305 \mathrm{~mm}$ and slab length constant at $4.9 \mathrm{~m}$.

Varying the elastic modulus within the range of $30 \mathrm{GPa}$ to $40 \mathrm{GPa}$ had a sizeable effect on the vertical deflection response. Increasing the modulus by $10 \mathrm{GPa}$ decreased the vertical end deflections by $0.06 \mathrm{~mm}$. This has a positive influence by reducing the curvature of the two slab assembly and creating more uniform stress at mid length of the slab. An elastic modulus of 35GPa was selected for the restraining base slab but may be modified once the actual slab tests are performed. This allowance for adjustment may provide a method to change the DOR without modifying the geometry of the sample slab. 


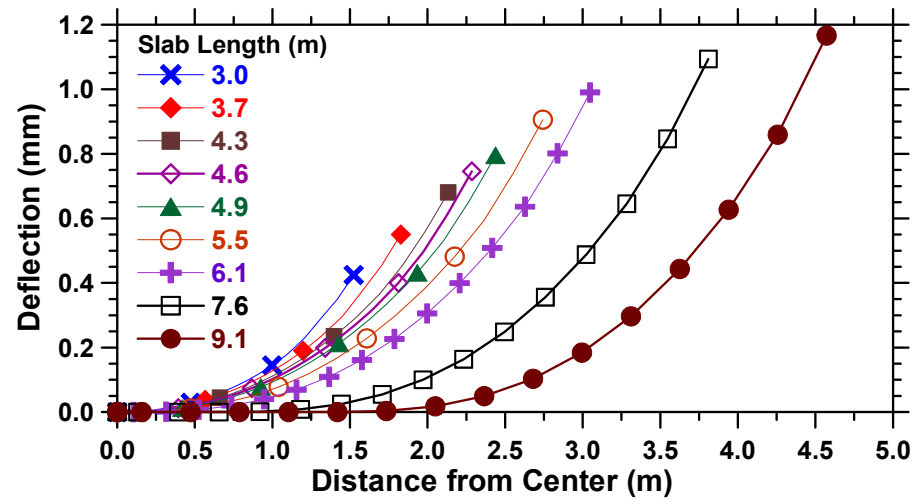

(a)

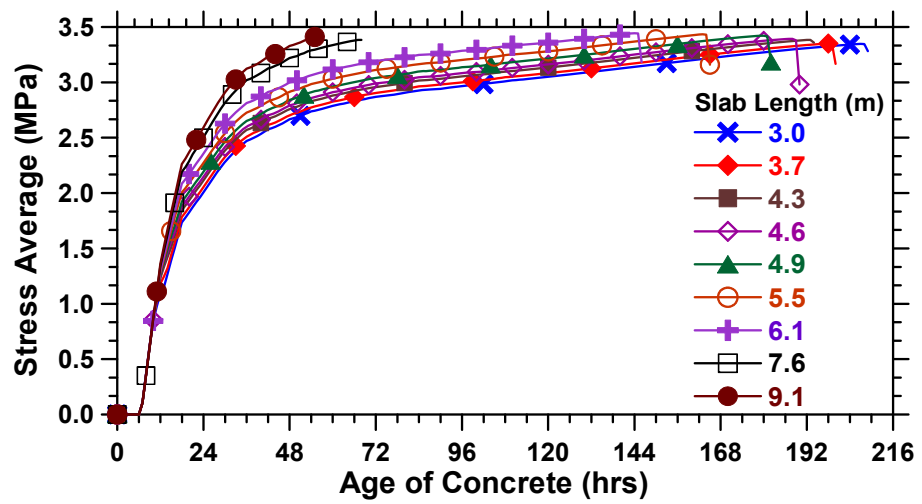

(b)

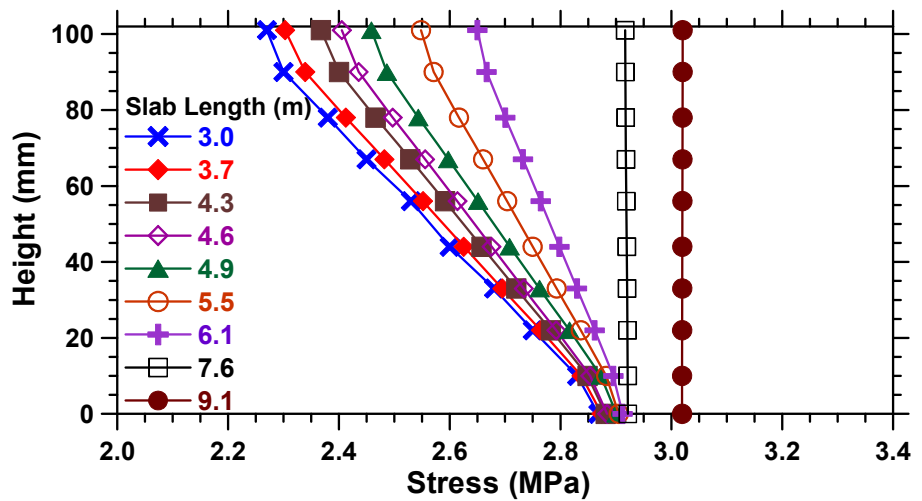

(c)

Figure 13-8 - Test Slab Length influence on (a) Vertical Deflection (b) Average Stress (c) Stress Uniformity 


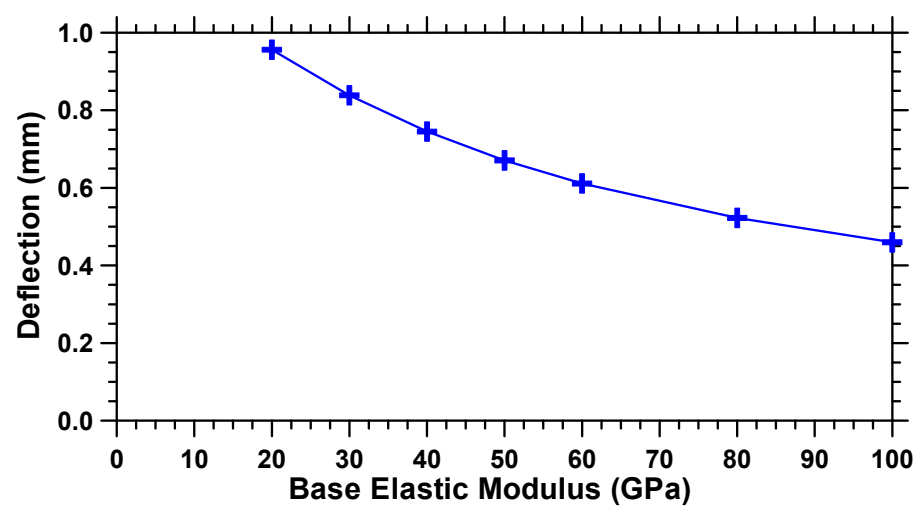

Figure 13-9 - Effect of Restraining Slab Modulus on Vertical Deflection at End of Slab

\subsubsection{Summary of Large Scale Test Design}

The simulations described in this chapter were used to select the final geometry of the testing device that will be used in assessing restrained shrinkage cracking in internally cured mixtures. It was determined that a sample slab that is $4.6 \mathrm{~m}$ long sitting on a 305mm thick base with a $35 \mathrm{GPa}$ base elastic modulus produced an adequate behavior. Thinner and shorter restraining base slabs were found to be susceptible to excessive curling which would lower the degree of restraint and prevent the specimen from cracking.

\subsection{Large Scale Testing of Internally Cured Proportions}

\subsubsection{Restraining Base Slab Construction}

The restraining base slabs were cast in advance of the test specimens. A local ready mix concrete producer supplied plain normalweight concrete proportioned as shown in Table 13-1. Restraining base slabs were cast directly from the ready mix truck, finished, and then covered with visqueen plastic sheeting. They 
were then cured inside the environmental chamber which was maintained at an $\mathrm{RH}$ of $50 \% \pm 2 \%$ and a temperature of $23^{\circ} \mathrm{C} \pm 1{ }^{\circ} \mathrm{C}\left(73.4{ }^{\circ} \mathrm{F} \pm 1.8^{\circ} \mathrm{F}\right)$. A set of 6"x12" cylinders were cast according to ASTM C192 to monitor the elastic modulus and strength development of the base restraining slabs. The elastic modulus was measured in accordance with ASTM C 469-02. Compressive strength was measured in accordance with ASTM C 39. At an age of 14 days, the elastic modulus of the restraining base slabs was measured as $34 \mathrm{GPa}$ (4937ksi). The compressive strength was measured as $30.5 \mathrm{GPa}(4.4 \mathrm{ksi})$. These two values were acceptable and provided relatively good agreement with the material properties used in the model. It should be noted that the restraining base slabs were maintained in sealed conditions (covered in visqueen sheeting) throughout all testing in this report.

Table 13-1 - Mixture Proportions for Restraining Base Slab

\begin{tabular}{|l|c|}
\cline { 2 - 2 } \multicolumn{1}{c|}{} & $\begin{array}{c}\text { Restraining } \\
\text { Base Concrete }\end{array}$ \\
\hline Material & $\begin{array}{c}\text { Amount } \\
\mathbf{k g} / \mathbf{m}^{3} \text { (lbs/yd3) }\end{array}$ \\
\hline \hline Cement (Type I) & $334(564)$ \\
\hline Water & $164(276)$ \\
\hline IC Water & 0 \\
\hline Fine Aggregate & $842(1420)$ \\
\hline FLWA & 0 \\
\hline Coarse Aggregate & $1800(1067)$ \\
\hline
\end{tabular}

\subsubsection{Mixture Proportioning for Test Slabs}

Two large scale test slabs were used to show the effectiveness of internal curing with prewetted lightweight aggregate. The mixture proportions for the test slabs are presented in Table 13-2. The control mixture, designated as mixture C-0, was a plain concrete with water-to-cement ratio of 0.30 by mass $(w / c=0.30)$. Mixture C-0 volumetrically had $40 \%$ paste, $35 \%$ fine aggregate (sand), and $25 \%$ 
coarse aggregate. The internally cured mixture was designated as mixture C-21. This mixture had a water-to-cement ratio of 0.30 by mass $(w / c=0.30)$ with volumetric proportions of $40 \%$ paste, $35 \%$ fine aggregate (sand), and $25 \%$ coarse aggregate. Fine lightweight aggregate (LWA) replaced $61 \%$ of the volume of sand or $21.5 \%$ of the total volume of the mixture. This LWA replacement value reduced the overall batch weight of the mixture by $9.3 \%$. It should be noted that the term "Water" in the mixture proportioning Table 13-2 is adjusted to compensate for absorption values of the normal weight sand and coarse aggregate. This is done so the absorption of the normal weight aggregates do not inadvertently lower the water-to-cement ratio.

The fine lightweight aggregate replacement value was determined with Equation 3-1 which supplies a volume of internal curing water that matches the volume of chemical shrinkage voids that are created after set. The term "IC Water" in the mixture proportioning Table 13-2 represents this volume of water. The chemical shrinkage was assumed to be $0.064 \mathrm{lb}$ of water per $\mathrm{lb}$ of cement. The degree of saturation for the LWA was taken as 1 because the batched lightweight would be soaked for 24 hours and the absorption value was based on a 24 hour soak.

A high-range water-reducing admixture (HRWRA) was added in varying rates to maintain similar consistencies between the two mixtures. For mixture $\mathrm{C}-0$ the HRWRA was added at a dosage of $0.40 \mathrm{~g}$ per $100 \mathrm{~g}$ of cement. For mixture C21 the HRWRA was added at a dosage of $0.70 \mathrm{~g}$ per $100 \mathrm{~g}$ of cement. 
Table 13-2 -Mixture Proportions for Large Scale Testing

\begin{tabular}{|l|c|c|}
\cline { 2 - 3 } \multicolumn{1}{c|}{} & $\begin{array}{c}\text { C-0 } \\
\text { Plain Concrete }\end{array}$ & $\begin{array}{c}\text { C-21 } \\
\text { IC Concrete }\end{array}$ \\
\hline \hline Material & $\begin{array}{c}\text { Amount } \\
\mathbf{k g} / \mathbf{m}^{3} \text { (lbs/yd3) }\end{array}$ & $\begin{array}{c}\text { Amount } \\
\mathbf{k g} / \mathbf{m}^{3} \text { (lbs/yd3) }\end{array}$ \\
\hline Cement (Type I) & $647(1091)$ & $647(1091)$ \\
\hline Water & $194(327)$ & $194(327)$ \\
\hline IC Water & 0 & $38.48(64.90)$ \\
\hline Fine Aggregate & $916(1546)$ & $354(598)$ \\
\hline FLWA & 0 & $334(564)$ \\
\hline Coarse Aggregate & $692(1168)$ & $692(1168)$ \\
\hline
\end{tabular}

* Water absorbed in FLWA at set

Table 13-3 - Coarse Aggregate Gradation for Large Scale Testing (Indiana \#9)

\begin{tabular}{|c|r|r|}
\hline Sieve Size & $\begin{array}{c}\text { Percent } \\
\text { Passing }\end{array}$ & $\begin{array}{c}\text { Percent } \\
\text { Necessary }\end{array}$ \\
\hline 1.00 & - & - \\
\hline 0.75 & $100 \%$ & $28 \%$ \\
\hline 0.50 & $73 \%$ & $28 \%$ \\
\hline 0.375 & $45 \%$ & $38 \%$ \\
\hline$\# 4$ & $8 \%$ & $3 \%$ \\
\hline$\# 8$ & $5 \%$ & $5 \%$ \\
\hline
\end{tabular}

\subsubsection{Materials}

Type I ordinary portland cement was used (ASTM C 150-05) with a Blaine fineness of $370 \mathrm{~m}^{2} / \mathrm{kg}\left(1809 \mathrm{ft}^{2} / \mathrm{lb}\right)$, and an estimated Bogue composition of a 56 $\% \mathrm{C}_{3} \mathrm{~S}, 16 \% \mathrm{C}_{2} \mathrm{~S}, 12 \% \mathrm{C}_{3} \mathrm{~A}$ and $7 \% \mathrm{C}_{4} \mathrm{AF}$. Normal weight river sand was used with a fineness modulus of 2.71 and an oven dry specific gravity of 2.58 , and an absorption of $1.6 \%$ by mass. The fine lightweight aggregate was a manufactured rotary kiln expanded shale with a fineness modulus of 4.3 , an oven dry specific gravity of 1.4 , and a 24 hour absorption value of $11.5 \%$ by mass. Mix water consisted of tap water conditioned to $23^{\circ} \mathrm{C} \pm 1{ }^{\circ} \mathrm{C}\left(73.4{ }^{\circ} \mathrm{F} \pm 1.8^{\circ} \mathrm{F}\right)$. The coarse aggregate was a locally supplied limestone with an oven dry specific gravity of 
2.75 and a measured absorption of $0.8 \%$. The coarse aggregate was graded to an Indiana \#9 gradation (Table 13-3).

\subsubsection{Mixing Procedure}

All mixing and aggregate preparation was performed in the laboratory. Aggregates were weighed in the oven dry state and air cooled prior to mixing. The LWA was oven dried, air cooled, and then submerged in the batched mix water for $24 \mathrm{~h} \pm 1 \mathrm{~h}$ before mixing. The volume of water used to submerge the LWA included both mixing water and the water the LWA would absorb in $24 \mathrm{~h}$ (IC water). The entire volume of water was required in order to fully submerge the LWA. After remaining submerged for $24 \mathrm{~h}$, the remaining unabsorbed water was then decanted and used in the mixing process.

Mixing was performed in a drum mixer with a maximum $6 \mathrm{cu} \mathrm{ft}$ capacity of 9 total cu ft. The sand, limestone, and decanted LWA were first added to the "buttered" mixer. The cement and water were then added. The water-reducing admixture was added along with the last $1 / 3$ of the mix water. Once all the materials were added to the mixer, it was operated for 5 minutes at full speed.

\subsubsection{Large Scale Casting Procedure}

Prior to casting the test slab, a layer of 0.006 " thick visqueen plastic sheeting was placed on the top face of the restraining slab and can be seen in Figure 13-10. This plastic was placed in order to reduce adhesion between the test slab and restraining slab and to facilitate demolding the test slab once the test completed. 


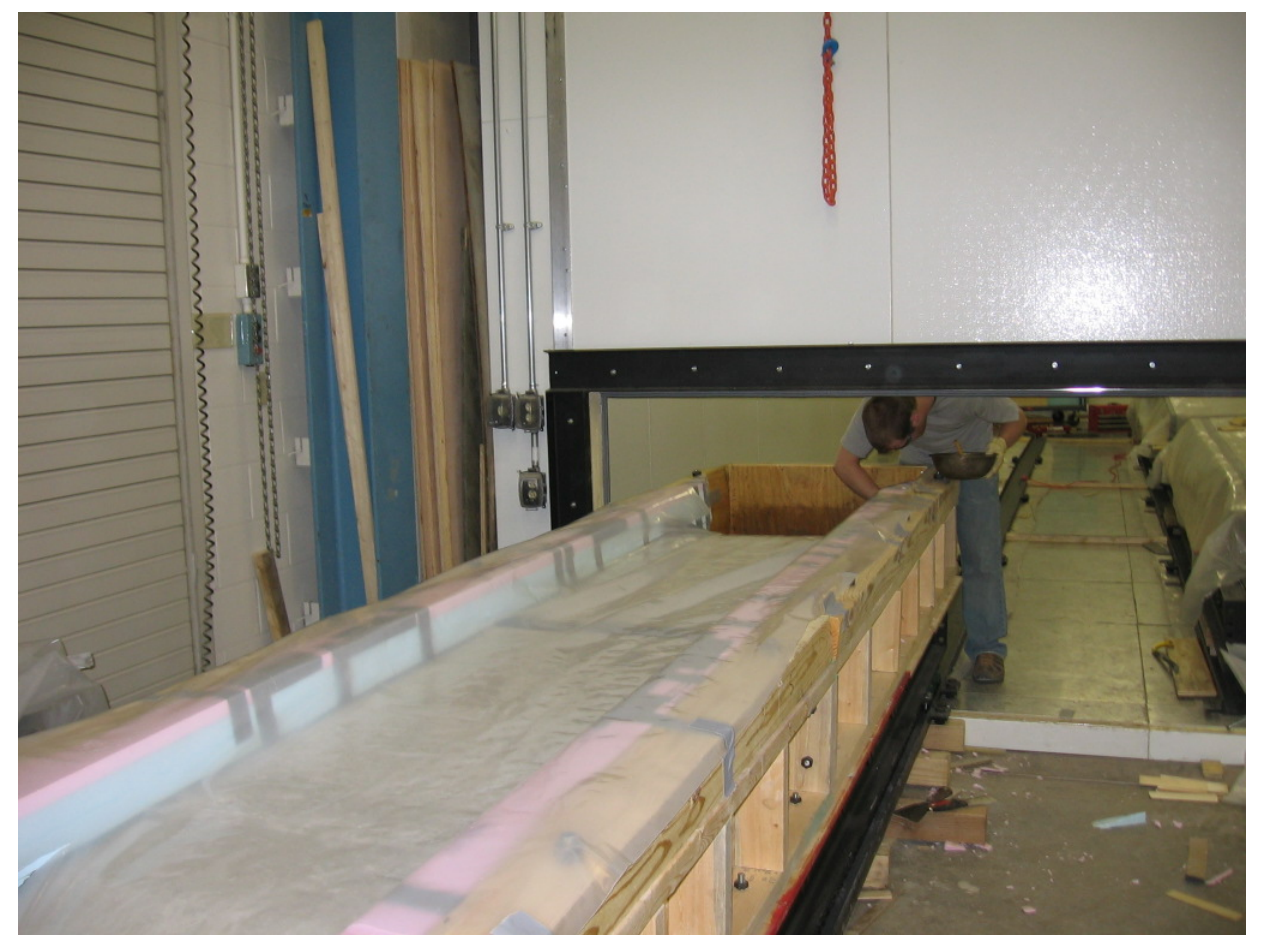

Figure 13-10 - Photo of Formwork Prepared for Large Scale Test Slab

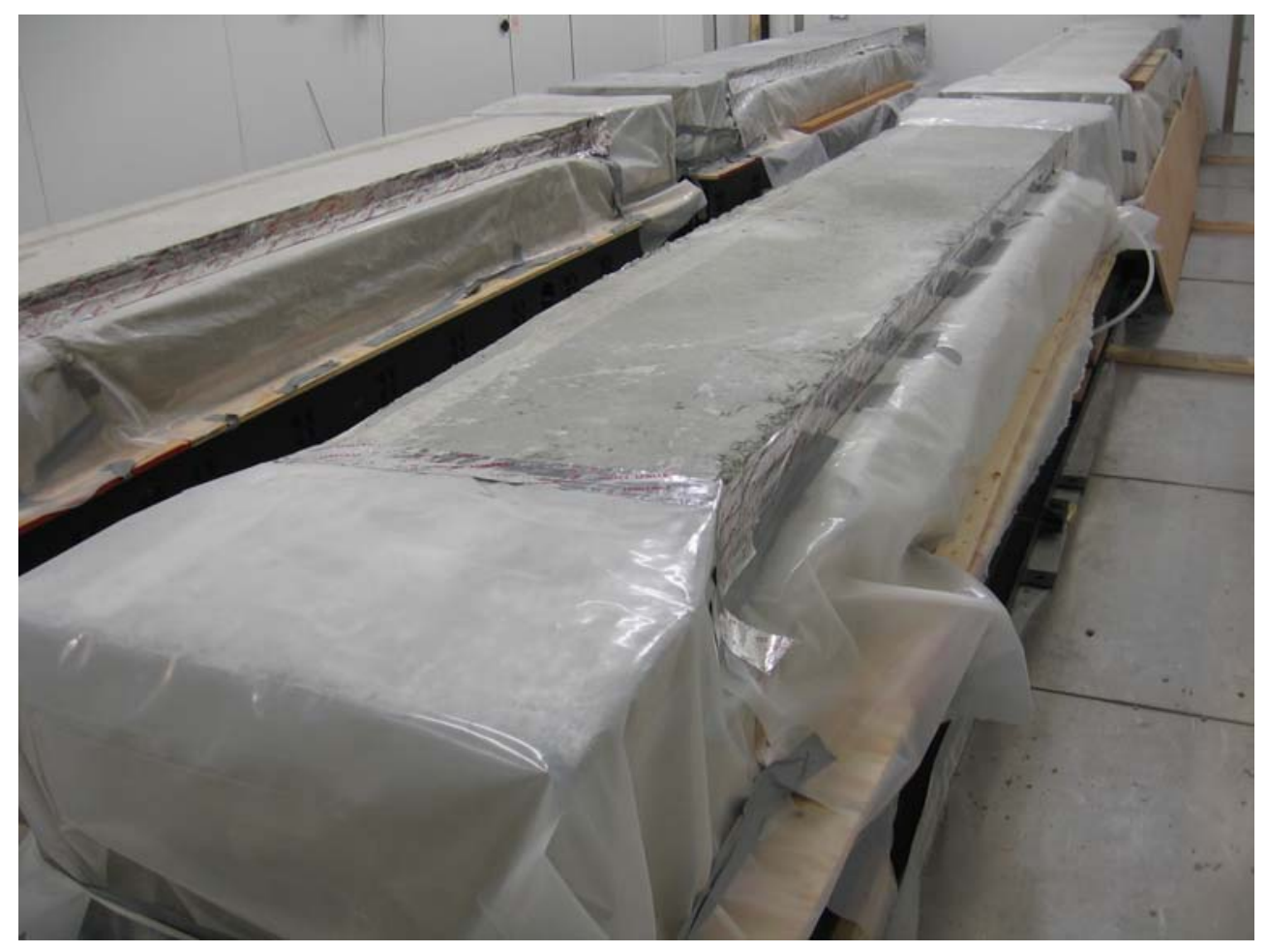

Figure 13-11 - Photo of Finished Test Slab 
The test slabs were consolidated with a commercial hand vibrator. The top face of the slabs were screeded and then finished with hand trowels to produce a smooth surface. The slabs were then placed the environmental chamber which operated at a constant $\mathrm{RH}$ of $50 \% \pm 2 \%$ and temperature of $23{ }^{\circ} \mathrm{C} \pm 1{ }^{\circ} \mathrm{C}\left(73.4^{\circ} \mathrm{F}\right.$ $\pm 1.8^{\circ} \mathrm{F}$ ). Initial curing during the first two days was sealed with visqueen plastic After two days, the formwork was removed and the plastic was removed from the test slab except for at each end. The two sides of the test slabs were sealed with a layer of aluminum tape in order to allow external drying to occur from the top surface. A photo of the finished slab is provided in Figure 13-11.

\subsubsection{Experimental Results and Discussion}

The test slabs were visually monitored to detect cracking. Mixture C-0 produced a crack at 11 days after casting. The crack was full depth and full width across the test slab and occurred at a distance 9'-7" from the end of the test slab. The orientation of the crack was nearly perpendicular to the length of the slab. A photo of the crack is presented in Figure 13-12. The crack width was then monitored for the following 17 days with a crack width microscope. Width measurements were taken at 4 points along the crack and the results are presented in Figure 13-14. The initial average crack width was $0.72 \mathrm{~mm}$ and grew to $1.22 \mathrm{~mm}$ during the monitoring period.

Mixture C-21 produced a crack at 42 days after casting. A photo of the crack is presented in Figure 13-13. The crack was full depth and full width across the test slab and occurred at a distance 11'-3" from the end of the test slab. The orientation of the crack was nearly perpendicular to the length of the slab. The average crack width was measured as $0.34 \mathrm{~mm}$. 
The free shrinkage of the restraining slabs and test slabs was measured with sets of $100 \mathrm{~mm} \times 100 \mathrm{~mm} \times 285 \mathrm{~mm}$ (4"x4"x11 1/4") prisms per ASTM C157. Prisms were subjected to the same environmental conditions as their representative slabs. The restraining slab prisms were cured in sealed conditions and the test slab prisms were allowed to undergo external drying. Figure 13-15 shows the individual free shrinkage results of mixture C-0 and its restraining base slab (Base Slab 1). At the time of cracking, the plain C-0 mixture measured a free shrinkage value of 360 microstrain. The restraining base under mixture C-0 (Base Slab 1) measured a free shrinkage value of 125 microstrain at the time of cracking.

The free shrinkage measurements for the internally cured mixture C-21 and its restraining slab (Base Slab 4) are presented in Figure 13-16. At the time of cracking, mixture C-21 measured a free shrinkage value of 420 microstrain. The restraining base under mixture C-21 (Base Slab 4) measured a free shrinkage value of 80 microstrain at the time of cracking.

The internally cured concrete test slab cracked approximately $4 \mathrm{x}$ later than the plain concrete test slab. This extension in cracking time indicates that internal curing reduced the cracking propensity of the mixture. It is likely that this is due to a combination of better mechanical properties and improved shrinkage behavior. The internally cured mixture measured a larger free shrinkage strain at the time of cracking but measured smaller crack widths on the test slab. This indicates a larger amount of stress relaxation is occurring as compared to the plain mixture. Observations of the mechanical properties of IC mixtures in CHAPTER 9 agree with this reasoning. Free shrinkage measurements of C-21 show it shrinks at a slower rate and shrinks later than the plain mixture $\mathrm{C}-0$. Both of these features improve cracking propensity. Shrinkage at a slower rate will allow relaxation to reduce tensile stress. Shrinkage at a later age allows the 
mixture to develop more tensile strength before being loaded with residual tension.

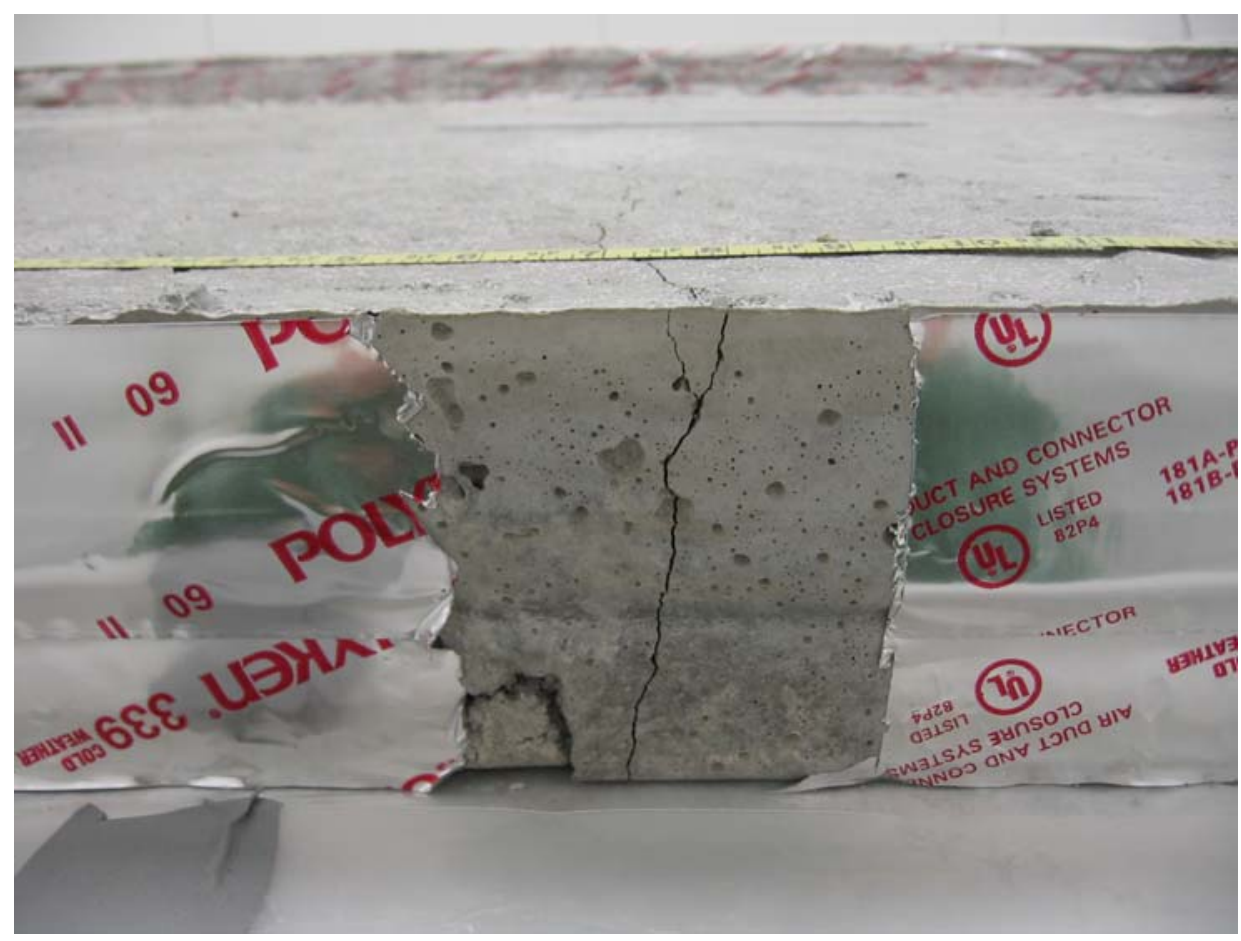

Figure 13-12 - Mixture C-0 Test Slab Crack at 12 Day

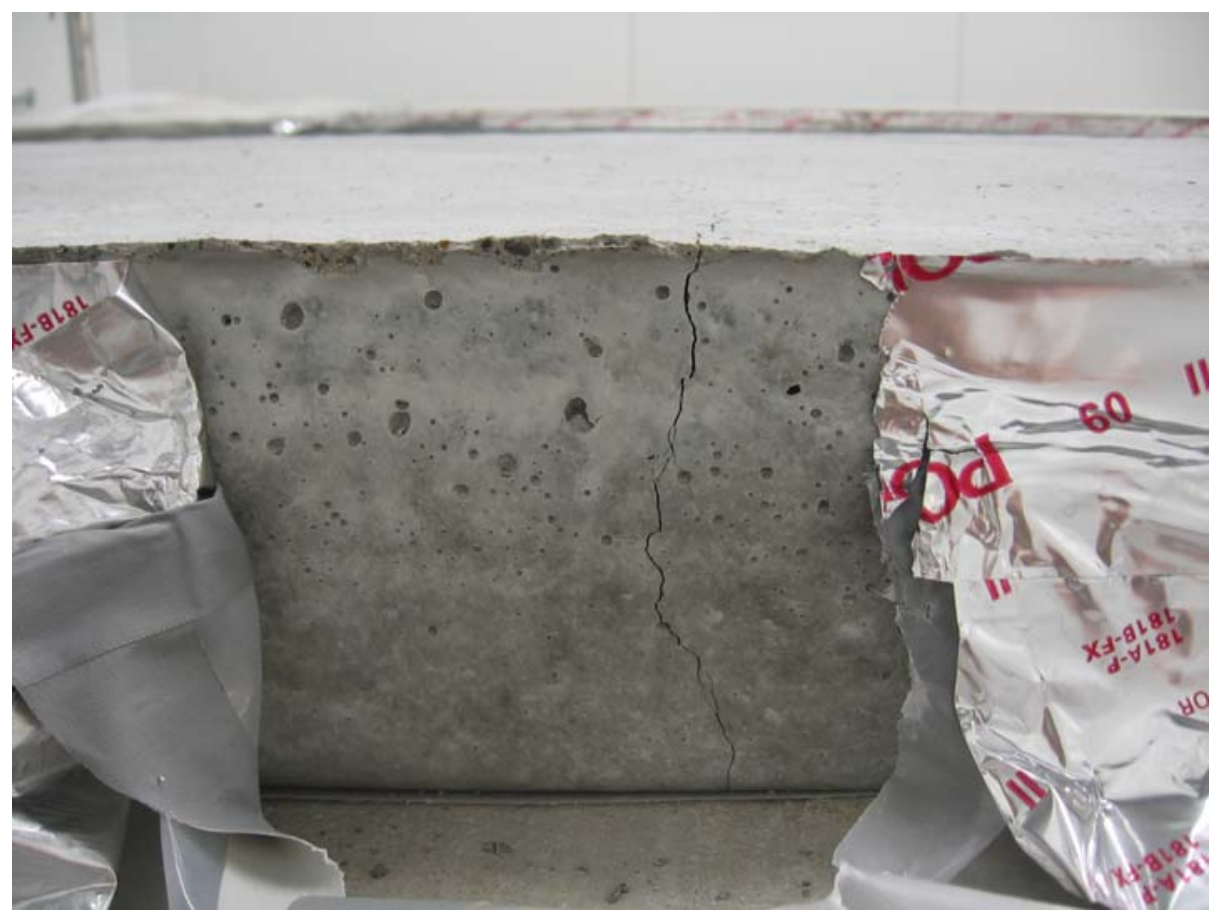


Figure 13-13 - Mixture C-21 Test Slab Crack at 42 day

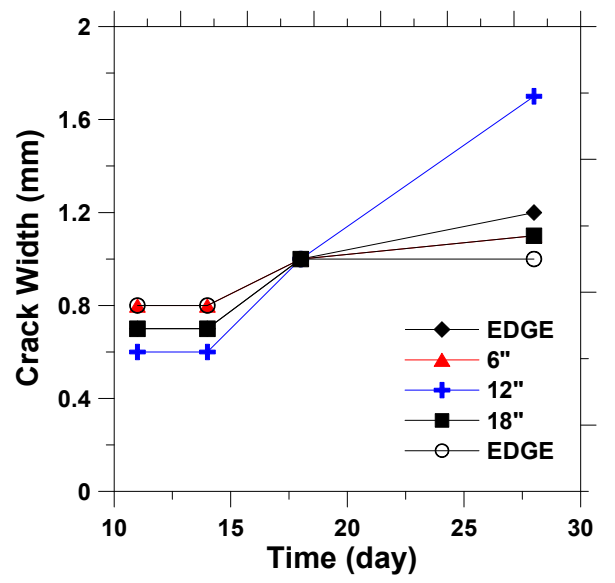

Figure 13-14 - C-0 Crack Width History

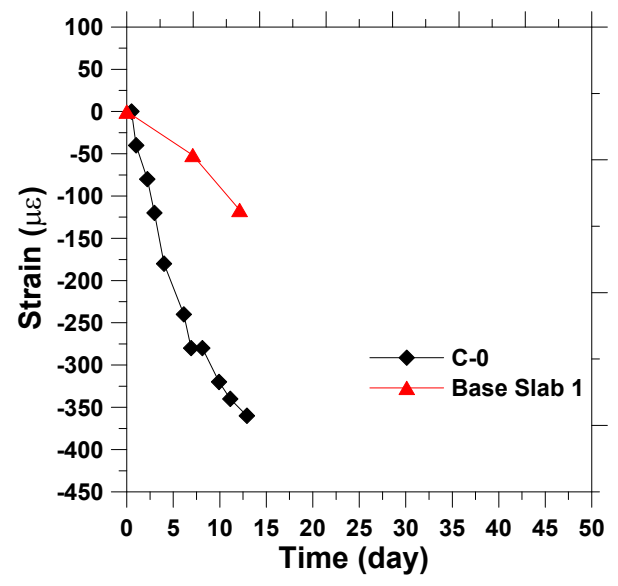

Figure 13-16 - C-21 Free Shrinkage History

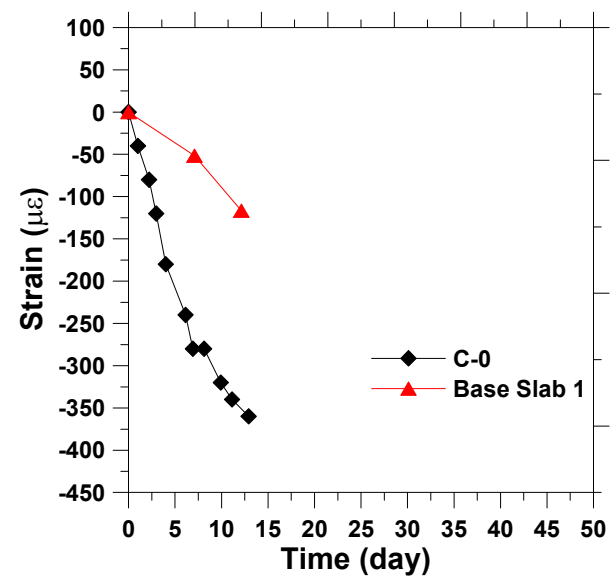

Figure 13-15 - C-0 Free Shrinkage History

\subsection{Large Scale Testing Conclusions}

This chapter has presented the design a large scale slab testing device and how the device was used to investigate the behavior of self curing concrete made with prewetted lightweight aggregate. This chapter outlined the method used to 
proportion a large batch of self curing concrete. Results of the large scale testing show that using a sufficient amount of lightweight aggregate replacement volume will improve the restrained cracking behavior. The self cured mixture cracked at later ages and produced smaller crack widths. 


\section{CHAPTER 14. SUMMARY AND CONCLUSIONS}

\subsection{Summary}

Internally cured concrete (IC) mixtures utilize prewetted lightweight aggregates (LWA) to supply water during hydration thereby enabling a more dense microstructure and a reduced autogenous shrinkage. These internally cured mixtures have demonstrated that they can provide many desired benefits. This report characterizes the local materials (Chapters 2 and 3 ), studies how well they work as internal curing agents (Chapter 4), and then investigates how they influence the durability and long term performance (Chapters $5-13$ ).

Several factors should be considered when selecting a particular LWA in order for internal curing to be effective. Specifically, the following three characteristics of the LWA must be taken into consideration:

- The LWA must be able to absorb an appropriate volume of curing water. This volume is determined based the amount of internal void space created by the hydrating cement. This volume can be approximated as the volume of chemical shrinkage, however strictly speaking; it is the difference in volume between the chemical shrinkage and autogenous shrinkage. In this study, different LWA's were characterized based on their 24 hour absorption values, but conceptually other time durations may be used (Castro et al. 2010).

- The LWA must be able to desorb (i.e, release) a large portion of its absorbed water during the hydration of the cement paste. To 
accomplish this, the pores of the LWA must be larger than the pores of the hydrating cement paste. If the pores in the LWA are not larger than the pores in the paste, the water will remain in the pores of the LWA and cannot be used for internal curing. Determining this characteristic is more difficult than absorption but it is equally, if not more important. Castro has shown in a related study that $92 \%$ of the absorbed water is released by $92 \% \mathrm{RH}$ for common expanded clays, shales, and slates.

- The LWA must be well dispersed throughout the system so that the water can physically travel to all regions of the paste. It was shown that water could travel on the order of $2 \mathrm{~mm}$ after set during the first $75 \mathrm{~h}$ in cement paste with a $w / c$ of 0.30 . Better dispersion is achieved by using finer LWA aggregates and higher LWA replacement volumes. Therefore, fine aggregates typically will perform far better than coarse aggregates. Higher absorption LWA may suffer from a lack of dispersion as these materials require lower replacement volumes for a given amount of chemical shrinkage.

Internal curing with prewetted LWA can improve the shrinkage and cracking performance (i.e., reduce shrinkage and reduce or eliminate cracking) of concrete. When the specimens were in a sealed curing condition, providing enough water to fill in the vapor-filled space cause by chemical shrinkage during the first days of hydration could significantly improve the unrestrained length change (i.e. shrinkage performance) and reduce or eliminate cracking in a restrained specimen. When the specimens were in an unsealed condition, the additional external drying effect required that a larger replacement volume of lightweight aggregate to obtain a similar improvement in performance. This is because specimens experience the combined effect of self-desiccation and external drying. If a sufficient volume of lightweight aggregate is supplied and they are dispersed properly, the shrinkage can be reduced. The incorporation of prewetted lightweight aggregate into a mixture can also help reduce and prevent 
the formation of plastic shrinkage cracks. The following summarizes the observed benefits internal curing had on shrinkage and cracking performance in this study:

- When no LWA was used the unrestrained shrinkage strain at $28 \mathrm{~d}$ in a sealed condition was $-390 \mu \varepsilon$. When $14.3 \%$ of the total mixture volume consists of LWA, the $28 \mathrm{~d}$ strain was an expansion of $59 \mu \varepsilon$.

- When no LWA was used the unrestrained shrinkage strain at $28 \mathrm{~d}$ in an unsealed condition was $-750 \mu \varepsilon$. When $33.0 \%$ of the total mixture volume contained LWA, the $28 \mathrm{~d}$ strain was $110 \mu \varepsilon$.

- When the mixture contained no LWA, cracking occurred at approximately $6 \mathrm{~d}$ in sealed conditions. However when $14.3 \%$ of the total volume of the mixture contained LWA, cracking did not occur during the $20 \mathrm{~d}$ of testing in sealed conditions and virtually no residual stress developed in the system.

- When the mixture contained no LWA, cracking occurred at approximately $2 \mathrm{~d}$ in unsealed conditions. However when $33.0 \%$ of the total mixture volume contained LWA, cracking did not occur during the $14 \mathrm{~d}$ of testing in unsealed conditions.

- When no LWA was in the system, the mixture cracked during the plastic state. When $18.0 \%$ of the total mixture was LWA, plastic shrinkage cracking was eliminated.

- Mixtures that contained prewetted LWA required up to $3 x$ the amount of temperature change (i.e. a plain specimen required a $10^{\circ} \mathrm{C}$ change to crack while the IC specimen required a $30^{\circ} \mathrm{C}$ change) to crack at a given time as compared to plain mixtures without LWA. 
Internal curing with prewetted LWA can also reduce the transport of fluid through (i.e, reduce permeability) concrete mixtures. This is likely due to an increase in the degree of hydration which densifies the bulk paste microstructure and interfacial transition zone between the LWA and the cement paste. The following item summarizes the observed benefits internal curing had on fluid transport:

- When no LWA was in the system, the degree of hydration for a mortar with a w/c of 0.30 was approximately 0.62 at $210 \mathrm{~d}$ of sealed hydration. When $25.3 \%$ of the mixture was LWA, the degree of hydration for a $w / c$ of 0.30 was approximately 0.66 at $210 \mathrm{~d}$ of sealed hydration.

- $\quad$ The water absorbed during eight days of testing for the mixture with no LWA was approximately $21 \mathrm{~g}$ per $\mathrm{cm}^{3}$ of paste when the sample was cured for $28 \mathrm{~d}$ in sealed conditions. Using the same curing and testing conditions when $25.3 \%$ of the mixture was LWA, the volume of absorbed water was reduced to $14 \mathrm{~g}$ per $\mathrm{cm}^{3}$ of paste.

- When no LWA is in the system, approximately $91 \%$ of the ITZ paste is percolated across the sample. When $25.3 \%$ of the total mixture is LWA, the ITZ is depercolated.

- $\quad$ From a sorptivity and conductivity perspective, the behavior of the mixture with $25.3 \%$ LWA ( $w / c$ of 0.30 ) has a performance of a plain mortar mixture with a $w / c$ of 0.23 .

Internal curing with prewetted LWA has effects on the mechanical performance of concrete. The compressive strength for the mixture containing $23.7 \%$ LWA was $2 \%$ to $8 \%$ less than the plain mixture with no LWA. Short term split tensile measurements made during the first 7 days showed a mixture with $23.7 \%$ LWA was $11 \%$ weaker than the plain mixture. This is likely due to the low strength of the LWA. The long term split tensile testing did not show a strong trend that 
mixtures with LWA were weaker than plain mixtures with no LWA. As a result, it is believed that the low strength of the LWA is offset by increased hydration of the cement paste. The modulus of elasticity of the mixture with 23.7 had was up to $13 \%$ less than the plain mixture with no LWA. The freeze thaw performance of the tested $0.30 \mathrm{w} / \mathrm{c}$ mixtures was not increased or reduced by the addition of LWA.

\section{2. $\underline{\text { Conclusions }}$}

The results of this investigation indicate that internally cured concrete has great potential for use in transportation structures. This occurs specifically due to the reduced potential for shrinkage and thermal cracking, the reduced fluid transport, and the increased densification of the matrix. It is recommended that field trials be performed with instrumentation, documentation and monitoring to enable Indiana to quantify the benefits of this material and to enable it to be more commonly used. It is recommended that a performance based specification be developed that would enable these materials to be used and specified more widely in Indiana. It is recommended that a training video be developed that highlights the benefits of this material, describes its use, and discusses important features associated with placement and testing. 


\section{LIST OF REFERENCES}

(1997, 1999, 2002, 2005). Self Dessiccation and Its Importance in Concrete Technology. Proceedings of Inernational Research Seminars.

(2002). Standard practice for maintaining constant relative humidity by means of aqueous solutions. ASTM G109-09a, ASTM International.

(2004). ASTM, Standard C 1581-04. Standard Test Method for Determining Age at Cracking and Induced Tensile Stress Characteristics of Mortar and Concrete under Restrained Shrinkage. ASTM International, West Conshohocken, PA Vol 4(2): 787-792.

(2004). Standard Test Method for Density, Relative Density (Specific Gravity), and Absorption of Coarse Aggregate. ASTM C127-04, ASTM International.

(2004). Standard Test Method for Determining Age at Cracking and Induced Tensile Stress Characteristics of Mortar and Concrete under Restrained Shrinkage. ASTM C1581-04, ASTM International.

(2004). Standard Test Method for Measurement of Rate of Absorption of Water by Hydraulic-Cement Concretes. ASTM C1585-04, ASTM International.

(2005). Standard Test Method for Chemical Shrinkage of Hydraulic Cement Paste. ASTM C1608-05 ASTM International.

(2006). Standard practice for making and curing concrete test specimens in the laboratory. ASTM C192-06, ASTM International.

(2006). Standard Practice for Mechanical Mixing of Hydraulic Cement Pastes and Mortars of Plastic Consistency. ASTM C305-06, ASTM International.

(2006). Standard practice for mechanical mixing of hydraulic cement pastes and mortars of plastic consistency, ASTM International.

(2006). Standard Test Method for Length Change of Hardened Hydraulic-Cement Mortar and Concrete. ASTM C157-06, ASTM International. 
(2007). Standard Test Method for Density, Relative Density (Specific Gravity), and Absorption of Fine Aggregate. ASTM C128-07, ASTM International.

$\mathrm{ACl}$ (1998). Standard practice for the use of shrinkage-compensating concrete. Farmington Hill, Ml, American Concrete Institute: 1-28.

ACI, Ed. (2004). ACI SP-220 Autogenous Deformation of Concrete. Farmington Hill, MI, American Concrete Institute.

Aitcin, P.-C., et al. (1997). "Integrated view of shrinkage deformation." Concrete International 19(9): 35-41.

Al-Khaiat, H. and M. N. Haque (1998). "Effect of initial curing on early strength and physical properties of a lightweight concrete." Cement and Concrete Research 28(6): 859.

Altoubat, S. A. and D. A. Lange (2001). "Creep, shrinkage, and cracking of restrained concrete at early age." ACI Materials Journal 98(Compendex): 323-331.

Altoubat, S. A. and D. A. Lange (2001). "Creep, shrinkage, and cracking of restrained concrete at early age." $\mathrm{ACI}$ Materials Journal 98(4): 323-331.

ASTM (2008). ASTM, Standard C 666. Standard Test Method for Resistance of Concrete to Rapid Freezing and Thawing. ASTM International, West Conshohocken, PA Vol 4(2).

B.V, F. (2006). FEMMASSE MLS 8.5 User Manual, "Finite Element Modules for Material Science and Structural Engineering". Netherland.

Barcelo, L., et al. (2000). Early age shrinkage of concrete: back to physical mechanisms. Materials aspects of concrete repair and rehabilitation, Mont-Tremblant, RILEM.

Bentz, D. P. (2008a). "Influence of internal curing using lightweight aggregates on interfacial transition zone percolation and chloride ingress in mortars." Cement and Concrete Composites: Submitted.

Bentz, D. P., et al. (1998). "Modelling drying shrinkage in reconstructed porous materials: application to porous Vycor glass." Modelling Simul. Mater. Sci. Eng. 6: 211-236.

Bentz, D. P., et al. (1999a). "A hard core/soft shell microstructural model for studying percolation and transport in three-dimensional composite media." 
Bentz, D. P., et al. (2001b). "Shrinkage-reducing admixtures and early-age desiccation in cement pastes and mortars." Cement and Concrete Research 31(7): 1075.

Bentz, D. P., et al. (2006a). Four-dimensional x-ray microtomography study of water movement during internal curing. Proceedings of the International RILEM Conference - Volume Changes of Hardening Concrete: Testing and Mitigation.

Bentz, D. P., et al. (2006b). Water movement during internal curing: Direct observation using x-ray microtomography. Concrete International. 28: 3945.

Bentz, D. P. and O. M. Jensen (2004). "Mitigation strategies for autogenous shrinkage cracking." Cement and Concrete Composites 26(6): 677-685.

Bentz, D. P., et al. (2005). Mixture proportioning for internal curing. Concrete International. 27: 35-40.

Bentz, D. P., et al. (2008b). Reducing Early-Age Cracking in Concrete Today. West Lafayette, IN, Purdue University.

Bentz, D. P., et al. (2008). "Reducing Early-Age Cracking in Concrete Today." West Lafayette, IN, Purdue University.

Bentz, D. P. and K. A. Snyder (1999). "Protected paste volume in concrete: Extension to internal curing using saturated lightweight fine aggregate." Cement and Concrete Research 29(11): 1863-1867.

Bentz, D. P. and K. A. Snyder (1999b). "Protected paste volume in concrete: Extension to internal curing using saturated lightweight fine aggregate." Cement and Concrete Research 29(11): 1863.

Bisschop, J., et al. (2001). "Shrinkage microcracking in cement-based materials with low water-cement ratio." Concrete Science and Engineering 3(11): 151-156.

Bjontegaard and E. J. Sellevold (1998). Thermal dilation-autogenous shrinkage: How to separate. Autogenous Shrinkage of Concrete: Proceedings of the Int. Workshop organized by the $\mathrm{JCl}$, Hiroshima.

Bjøntegaard, Ø., et al. (2004). "On the measurement of free deformation of early age cement paste and concrete." Cement and Concrete Composites 26(5): 427.

Black, R. W. (1986). "The Determination of Specific Gravity Using the SiphonCan Method." Cement, Concrete, and Aggregates: 46. 
Castro, J., et al. (2010). "Drying and Wetting of Concrete in the Presence of Deicing Solutions (In Production)."

Cohen, M. D., et al. (1990). "Mechanism of plastic shrinkage cracking in portland cement and portland cement-silica fume paste and mortar." Cement and Concrete Research 20(1): 103.

Couch, J., et al. (2006). Use of acoustic emission to detect cavitation and solidification (time zero) in cement pastes. International RILEM Conference on Volume Changes of Hardening Concrete: Testing and Mitigation.

Cusson, D. and T. Hoogeveen (2006). Preventing autogenous shrinkage of highperformance concrete structures by internal curing. Measuring, Monitoring and Modeling Concrete Properties: 83.

Dehrdrai, M. (2008). Health Monitoring of Civil Engineering Materials. West Lafayette, Purdue University. MS.

Delatte, N., et al. (2007). Evaluation of High Absorptive Materials to Improve Internal Curing of Low Permeability Concrete. State Job Number: 134227, for the Ohio Department of Transportation: 1-142.

Diamond, S. (1970). "Pore Size Distribution in Clays." Clay and Clay Minerals 18: 7-23.

Diamond, S. (2000). "Mercury porosimetry: An inappropriate method for the measurement of pore size distributions in cement-based materials." Cement and Concrete Research 30(10): 1517.

El-Dieb, A. S. and R. D. Hooton (1995). "Water-permeability measurement of high performance concrete using a high-pressure triaxial cell." $\underline{\text { Cement }}$ and Concrete Research 25(6): 1199.

Elsharief, A., et al. (2005). "Influence of lightweight aggregate on the microstructure and durability of mortar." Cement and Concrete Research 35(7): 1368.

ESCSI (1971). Lightweight Concrete, History, Applications, Economics, Expanded Shale, Clay and Slate Institute: 1-43.

Fagerlund, G. (1977). "The Critical Degree of Saturation Method of Assessing Freeze/Thaw Resistance of Concrete." Materiaux et constructions 58.

FHWA (2006). Compilation and evaluation of results from high-performance concrete bridge projects, volume 1: Final Report. McLean, VA, U.S. Department of Transportation: 180. 
Folliard, K. J. and N. S. Berke (1997). "Properties of high-performance concrete containing shrinkage-reducing admixture." Cement and Concrete Research 27(9): 1357.

Ford, S. J., et al. (1998). "Assignment of features in impedance spectra of the cement-paste/steel system." Cement and Concrete Research 28(12): 1737.

Geiker, M. (1983). Studies of Portland cement hydration by measurements of chemical shrinkage and a systematic evaluation of hydration curves by means of the dispersion model, Technical University of Denmark. Ph. D.

Greenspan, L. (1977). "Humidity fixed points of binary saturated aqueous solutions." Journal of Research of the National Bureau of Standards Physics and Chemistry 81A(1).

Guillaume, C. E. (1904). "Invar and its applications." Nature.

Hannah, R. L. and S. E. Reed (1992). Strain gage users' handbook.

Henkensiefken, R. (2008a). Volume Change and Cracking in Internally Cured Mixtures Made with Saturated Lightweight Aggregate Under Sealed and Drying Conditions, Presented at ACl Fall Convention, St. Louis, MO.

Henkensiefken, R., et al. (2009). "Volume change and cracking in internally cured mixtures made with saturated lightweight aggregate under sealed and unsealed conditions." Cement and Concrete Composites 31(7): 427-437.

Henkensiefken, R., et al. (2008b). "Volume change and cracking in internally cured mixtures made with saturated lightweight aggregate under sealed and drying conditions." Cement and Concrete Composites: Submitted.

Henkensiefken, R., et al. (2008c). "The Absorption Properties of Lightweight Aggregate: A Discussion on Critical Aspects of Moisture of Absorption for use in Proportioning Internal Curing Concrete." Journal of ASTM International.

Henkensiefken, R., et al. (2008d). "Saturated lightweight aggregate for internal curing in low w/c mixtures: Monitoring water movement using x-ray absorption." Strain: Submitted.

Henkensiefken, R., et al. (2008e). Reducing Restrained Shrinkage Cracking in Concrete: Examining the behavior of self-curing concrete made using different volumes of saturated lightweight aggregate. National Concrete Bridge Conference, St. Louis, MO. 
Henkensiefken, R., et al. (2008f). Comments on the shrinkage of paste in mortar containing saturated lightweight aggregate. CONMOD. Delft, The Netherlands.

Henkensiefken, R., et al. (2008g). Detecting solidification using moisture transport from saturated lightweight aggregate. Technical session on "Transition from Fluid to Solid Re-examining the Behavior of Concrete at Early Ages" at the ACI Spring 2009 Convention. San Antonio, TX.

Henkensiefken, R. and J. Weiss (2008h). Effects of Cure Time on Internal Curing, Purdue University.

Hillerborg, A., et al. (1976). "Analysis of crack formation and crack growth in concrete by means of fracture mechanics and finite elements." Cement and Concrete Research 6(6): 163-168.

Hobbs, D. W. (1969). "Bulk Modulus and Thermal Expansion of a Two Phase Material." Nature 222: 849-851.

Hobbs, D. W. (1999). "Aggregate influence on chloride ion diffusion into concrete." Cement and Concrete Research 29(12): 1995-1998.

Holm, T. A. and T. W. Bremner (August 2000). State-of-the-art-report on highstrength, high-durability structural low-density concrete for applications in severe marine environments: 116

Holm, T. A. and J. P. Ries (2006). Lightweight concrete and aggregates. Significance of Tests and Properties of Concrete and Concrete-Making Materials, West Conshohocken, United States, American Society for Testing and Materials.

Hossain, A. B. and J. Weiss (2004). "Assessing residual stress development and stress relaxation in restrained concrete ring specimens." Cement and Concrete Composites 26(5): 531-540.

HPB (2004). General Haydite Properties - Chemical Analysis, Hydraulic Press Brick Company.

Igarashi, S.-I., et al. (2000). "Autogenous shrinkage and induced restraining stresses in high-strength concretes." Cement and Concrete Research 30(11): 1701-1707.

Jacobs, S. F. (1996). Measuring Invar's dimensional stability. Invar Effect. A Centennial Symposium. Proceedings of an International Symposium on the Invar Effect held on the occasion of the 100th Anniversary of its Discovery, 7-8 Oct. 1996, Warrendale, PA, USA, TMS. 
Jensen, O. M. (2005). Autogenous phenomena in cement-based materials. Department of Building Technology and Structural Engineering, Aalborg University.

Jensen, O. M. and P. F. Hansen (1995). "A dilatometer for measuring autogenous deformation in hardening portland cement paste." Materials and Structures 28(7): 406.

Jensen, O. M. and P. F. Hansen (2001). "Water-entrained cement-based materials: I. Principles and theoretical background." Cement and Concrete Research 31(4): 647-654.

Jensen, O. M. and P. F. Hansen (2001a). "Autogenous deformation and RHchange in perspective." Cement and Concrete Research 31(12): 1859.

Jensen, O. M. and P. F. Hansen (2001b). "Water-entrained cement-based materials: I. Principles and theoretical background." Cement and Concrete Research 31(4): 647.

Kayir, H. and J. Weiss (2002). A fundamental look at settlement in fresh systems: role of mixing time and high range water reducers. First North American Conference on Self-Consolidating Concrete. Chicago, IL.

Khan, M. I. and C. J. Lynsdale (2002). "Strength, permeability, and carbonation of high-performance concrete." Cement and Concrete Research 32(1): 123.

Kim, H. and D. Bentz (2008). Internal curing with crushed returned concrete aggregate for high performance concrete. NRMCA Concrete Technology Forum: Focus on Sustainable Development.

Kleiger, P. and J. F. Lamond (1994). Significance of Tests and Properties of Concrete and Concrete-Making Materials. Fredericksburg, VA.

Knudsen, T. and M. Geiker (1982). Chemical shrinkage as an indicator of the stage of hardening. International RILEM Conference on Concrete of Early Ages, Paris.

Kovler, K. (1994). "Testing system for determining the mechanical behaviour of early age concrete under restrained and free uniaxial shrinkage." Materials and Structures 27(6): 324-330.

L'Hermite, R. G. (1960). Volume changes of concrete. 4th International Symposium on the Chemistry of Cement, Washington D.C. 
La Chatelier, H. (1900). Sur les changements de volume qui accompagent le durcissement des ciments. Bulletin Societe de l'encouragemnent pour l'industrie nationale, Paris.

Le Chatelier, H. (1900). "Sur les changements de volume qui accompagnent le durcissement des ciments." Bulletin de la Societe d'Encouragement pour l'Industrie Nationale: $54-57$.

Lement, B. S., et al. (1996). The dimensional behavior of Invar. Invar Effect. A Centennial Symposium. Proceedings of an International Symposium on the Invar Effect held on the occasion of the 100th Anniversary of its Discovery, 7-8 Oct. 1996, Warrendale, PA, USA, TMS.

Lo, T. Y. and H. Z. Cui (2004). "Effect of porous lightweight aggregate on strength of concrete." Materials Letters 58(6): 916.

Lopez, M., et al. (2008). The influence of internal curing on basic creep and autogenous shrinkage. Internal Curing of High-Performance Concretes: Laboratory and Field Experiences - ACI Fall Convention 2007, October 14, 2007 - October 18, 2007, Fajardo, Puerto rico, American Concrete Institute.

Lu, B. and S. Torquato (1992). "Nearest-surface distribution functions for polydispersed particle systems." Physical Review A 45(8): 5530.

Lura, P. (2003). Autogenous deformation and internal curing of concrete. Delft University of Technology. Delft PhD.

Lura, P. (2003a). Autogenous deformation and internal curing of concrete, Technical University of Delft. PhD Dissertation.

Lura, P., et al. (2006a). "Measurement of water transport from saturated pumice aggregates to hardening cement paste." Materials and Structures 39(9): 861.

Lura, P. and J. Bisschop (2004). "On the origin of eigenstresses in lightweight aggregate concrete." Cement and Concrete Composites 26(5): 445.

Lura, P., et al. (2007a). "Experimental observation of internal water curing of concrete." Materials and Structures 40(2): 211.

Lura, P., et al. (2007). "Experimental observation of internal water curing of concrete." Materials and Structures/Materiaux et Constructions 40(Compendex): 211-220.

Lura, P., et al. (2006b). Evaporation, settlement, temperature evolution, and development of plastic shrinkage cracks in mortars with shrinkage- 
reducing admixtures. ConcreteLife'06 - International RILEM-JCI Seminar on Concrete Durability and Service Life Planning: Curing, Crack Control, Performance in Harsh Environments.

Lynam, C. G. (1934). Growth and movement in Portland cement concrete. London, England, Oxford University Press.

Maekawa, K., et al. (1999). Modelling of concrete performance: Hydration, microstructure and mass transport. London, Taylor and Francis.

Mohr, B. J. (2005). Durability of pulp fiber-cement composites, Georgia Institute of Technology. Ph. D.

Mohr, B. J., et al. (June 2005). Examination of wood-derived powders and fibers for internal curing of cement-based materials. 4rd International research seminar on self-desiccation and its importance in concrete technology, Lund University, Lund, Sweden.

Moon, J.-H., et al. (2006). "Quantifying the influence of specimen geometry on the results of the restrained ring test." Journal of ASTM International 3(8).

Nakamura, Y. (1976). "The Invar problem." IEEE Transactions on Magnetics MAG-12(Copyright 1976, IEE): 278-291.

Neithalath, N. (2006). "Analysis of moisture transport in mortars and concrete using sorption-diffusion approach." ACI Materials Journal 103(3): 209-218.

Neubauer, C. M., et al. (1996). "A three-phase model of the elastic and shrinkage properties of mortar." Advanced Cement Based Materials 4(1): 6.

Neville, H. A. and H. C. Jones (1929). "The study of hydration changes by a volume-change method." Lehigh University -- Publication 3(1): 309-318.

Nielsen, G. (2007). X-Ray CT System - Users Manual' GNI/XRAS/UM.003.

Nilsen, A. U., et al. (1995). "Estimation of the elastic moduli of lightweight aggregate." Cement and Concrete Research 25(2): 276.

O'Neil, M. J., et al. (2001). The Merck Index. Rahway, New Jersey, John Wiley \& Sons.

Pease, B., et al. (2005). "Shrinkage behavior and residual stress development in mortar containing shrinkage reducing admixtures (SRAs)." SP-227 227.

Penttala, V. and F. Al-Neshawy (2002). "Stress and strain state of concrete during freezing and thawing cycles." Cement and Concrete Research 32(Compendex): 1407-1420. 
Philleo, R. (1991). Concrete science and reality. Materials Science of Concrete II, Westerville, $\mathrm{OH}$, American Ceramic Society.

Pickett, G. (1956). "Effect of Aggregate on Shrinkage of Concrete and Hypothesis Concerning Shrinkage." Journal of ACI 52: 581-590.

Pigeon, M., et al. (2000). "Equipment for the analysis of the behaviour of concrete under restrained shrinkage at early ages." Magazine of Concrete Research 52(Compendex): 297-302.

Powers, T. C. (1935). "Absorption of Water by Portland Cement Paste during the Hardening Process." Ind. Eng. Chem. 27(7): 790-794.

Powers, T. C. (1947). "A discussion of cement hydration in relation to the curing of concrete." Proc. Highw. Res. Board 27 178-188.

Powers, T. C. (1950). Air requirement of frost-resistant concrete: 1-19.

Powers, T. C. (1968). The Properties of Fresh Concrete. New York, John Wiley and Sons.

Powers, T. C. and T. L. Brownyard (2003a). Landmark Series: Studies of the Physical Properties of Hardened Portland Cement Paste. Concrete International. 25.

Powers, T. C. and T. L. Brownyard (2003b). Landmark Series: Studies of the Physical Properties of Hardened Portland Cement Paste, Part 2. Concrete International. 25.

Qi, C. (2003a). Quantitative assessment of plastic shrinkage cracking and its impact on the corrosion of steel reinforcement. School of Civil Engineering. West Lafayette, Purdue University. Ph. D.

Qi, C., et al. (2003b). "Characterization of plastic shrinkage cracking in fiber reinforced concrete using image analysis and a modified Weibull function." Materials and Structures 36(6): 386.

Qi, C., et al. (2005). Assessing the settlement of fresh concrete using a noncontact laser profiling approach. International Conference on Construction Materials: ConMat'05. Vancouver, BC, Canada.

Radlinska, A. (2008). Reliability-Based Analysis Of Early-Age Cracking In Concrete. West Lafayette, Purdue University. PhD.

Radlinska, A., et al. (2008). "Comments on the interpretation of results from the restrained ring test." Journal of ASTM International 5(Copyright 2009, The Institution of Engineering and Technology): JAl101944 (101912 pp.). 
Radlinska, A., et al. (2008a). "Comments on the Interpretation of Results From the Restrained Ring Test." Journal of ASTM International.

Radlinska, A., et al. (2007). "Shrinkage Mitigation Strategies in Cementitious Systems: a Closer Look at Differences in Sealed and Unsealed Behavior." Accepted by Transportation Research Board.

Radlinska, A., et al. (2008b). Shrinkage mitigation strategies in cementitious systems: A closer look at sealed and unsealed material behavior. Accepted for publication in the Transportation Research Record.

Raoufi, K. (2010). Shrinkage Cracking of Concrete Systems: The Influence of Damage Localization On Crack Widths. West Lafayette, Purdue University. PhD.

RILEM (2007). Internal curing of concrete, Editor: Kovler, K. and O.M. Jensen, RILEM, ICC 196.

RILEM Commission 42-CEA (1981). "Properties of set concrete at early ages (state of the art report)." Mate'r. Const 84 399-460.

RILEM Report 41 (2007). Internal Curing of Concrete. K. Kovler and O. M. Jensen.

RILEM TC-181 EAS (2002). RILEM Report 25: Early age shrinkage induced stresses and cracking in cementitous systems. RILEM Technical Committee 181-EAS. A. Bentur.

Roberts, J. (1995). Freeze-thaw durability of concretes using lightweight aggregate of differing absorption characteristics. First Int. Conf. on Concrete under Severe Conditions-Environment and Loading. Sapporo, Japan: 139-146.

Sabir, B., et al. (1998). "A water sorptivity test for mortar and concrete." Materials and Structures 31(8): 568.

Saito, M., et al. (1991). "Role of aggregate in the shrinkage of ordinary portland and expansive cement concrete." Cement and Concrete Composites 13(2): 115.

Sant, G. (2007). Examining Volume Changes, Stress Development and Cracking in Cement Based Systems. West Lafayette, Purdue University. MSCE.

Sant, G., et al. (2008a). "The influence of shrinkage reducing-admixtures (SRAs) on moisture absorption in cementitious materials at early-ages." submitted to the ASCE Journal of Materials in Civil Engineering (2008). 
Sant, G., et al. (2007). "Examining residual stress development in cementitious materials experiencing an early-age expansion." Proc., 9th CANMET-ACI Int. Conf.-T.C. Holland Symp: 21-29.

Sant, G., et al. (2006). "Measurement of volume change in cementitious materials at early ages: Review of testing protocols and interpretation of results." Transportation Research Record 1979.

Sant, G., et al. (2006). "Examining Early Age Expansion In Pastes Containing Shrinkage Reducing Admixtures (SRA's)." Advances in Concrete through Science and Engineering, RILEM Quebec: abstract p. 353 (electronic proceedings pp. 311).

Sant, G. and J. Weiss (2008b). "Unpublished Results."

Sant, G. and W. Weiss (2008c). "The use of x-ray absorption for assessing moisture movement in cementitious materials." submitted to the Journal of ASTM International (2008).

Saxer, E. L. (1956). A Direct Method for Determining the Absorption and Specific Gravity of Aggregates. Rock Products: 78.

See, H. T., et al. (2004). "Potential for restrained shrinkage cracking of concrete and mortar." Cement, Concrete and Aggregates 26(2): 123-130.

Sellevold, E. J. and Bjontegaard (2006). Coefficient of thermal expansion of cement paste and concrete: Mechanisms of moisture interaction, Van Godewijckstraat 30, Dordrecht, 3311, Netherlands, Springer Science and Business Media Netherlands.

Shah, S. and S. H. Ahmad (1994). High performance concretes and applications, Butterworth-Heinemann.

Shah, S. P. and J. Weiss (2000). High Strength Concrete: Strength, Permeability, and Cracking. Proceedings of the PCI/FHWA International Symposium on HPC, Orlando, Florida.

Shah, S. P., et al. (1998). Shrinkage Cracking -- Can It Be Prevented? Concrete International. 20: 51-55.

Shah, S. P., et al. (1998). "Shrinkage cracking - can it be prevented?" Concrete International 20(4): 51-55.

Spaulding, J. (2007). Manufacturing of Lightweight Aggregate. R. Henkensiefken. Brooklyn, IN. 
Springenschmid, R., et al. (1994). Development of the cracking frame and the temperature-stress testing machine. In: Proceedings of the International RILEM Symposium: Thermal Cracking in Concrete at Early Ages.

Thomas, M. (2008). Use of Conductive Gel. J. Weiss.

van Breugel, K. and H. de Vries (1998). Mixture Optimization of Low Water/Cement Ratio, High-Strength Concretes in View of Reduction of Autogenous Shrinkage. Proceedings International Symposium on HighPerformance and Reactive Powder Concretes, University of Sherbrooke, Quebec, Canada.

van Breugel, K. and P. Lura (2000). Effect of Initial Moisture Content and Particle Size Distribution of Lightweight Aggregates on Autogenous Deformation. Proceedings of the 2nd International Symposium on Structural Lightweight Aggregate Concrete, Kristiansand, Norway.

Vishay Measurements Group (2005). "Application Note VMM-19 'Surface Preparation'."

Vishay Measurements Group (2007). "Tech Note TN-504-1 'Strain Gage Thermal Output and Gage Factor Variation With Temperature'."

Vishay Measurements Group (2007). "Tech Note TN-505-4 'Strain Gage Selection Criteria Procedures Recommendations'."

Vishay Measurements Group (2008). "Instruction Bulletin B-127-14 'M-Bond 200 Installation Instructions'."

Vlahinic, I., et al. (2008). "A constitutive model for drying shrinkage of a partially saturated porous material." Journal of the Mechanics and Physics of Solids.

Wang, K. and S. P. Shah (1997). "Permeability study of cracked concrete." Cement and Concrete Research 27(3): 381-393.

Washburn, E. W. (1921). "The Dynamics of Capillary Flow." Physical Review 17(3): 273.

Weber, S. and H. W. Reinhardt (1995). A blend of Aggregates to Support Curing of Concrete. Proceedings on Structural Lightweight Aggregate Concrete International Symposium, Sandefjord, Norway.

Weiss, J. (1999). Prediction of Early-Age Shrinkage Cracking in Concrete. Evanston, IL, Northwestern University. 
Weiss, J. and T. Nantung (2007). Proposal for a Research Study on the Development of Self-Curing Concrete for Increased Service Life. SPR3211, Joint Transportation Research Program: 1-13.

Weiss, W. and S. Shah (2002). "Restrained shrinkage cracking: the role of shrinkage reducing admixtures and specimen geometry." Materials and Structures 35(2): 85.

Weiss, W. J., et al. (2008). Aspects of monitoring moisture changes using electrical impedance spectroscopy. 2nd Symposium on Self-Desiccation and Its Importance in Concrete Technology, Lund, Sweden.

Wiegrink, K., et al. (1996). "Shrinkage cracking of high-strength concrete." $\underline{\mathrm{ACl}}$ Materials Journal 93(5): 409-415.

Winslow, D. N., et al. (1994). "Percolation and pore structure in mortars and concrete." Cement and Concrete Research 24(1): 25.

Wittman, F. and J. Lukas (1974). "Experimental Study of Thermal Expansion of Hardened Cement Paste." 7(Compendex): 247-252.

Wittmann, F. H. (1976). "On the action of capillary pressure in fresh concrete." Cement and Concrete Research 6(1): 49.

Yang, Z. (2004). Assessing cumulative damage in concrete and quantifying its influence on life cycle performance modeling. West Lafayette, Purdue University. Ph. D.

Zhang, M.-H. and O. E. Gjørv (1990). "Microstructure of the interfacial zone between lightweight aggregate and cement paste." Cement and Concrete Research 20(4): 610 .

Zhutovsky, S., K. Kovler, A. Bentur, (2002). "Efficiency of lightweight aggregates for internal curing of high strength concrete to eliminate autogenous shrinkage." Materials and Structures 35(2): 97-101.

Zhutovsky, S., et al. (2002). "Efficiency of lightweight aggregates for internal curing of high strength concrete to eliminate autogenous shrinkage." Materials and Structures 35(2): 97. 


\section{Appendix A. Calculation of LWA needed from Equation 2-1}

1. Start with the initial mixture proportions:

\begin{tabular}{|c|c|c|c|c|c|}
\hline & & & & & \\
\hline & & Batch Size $\left(\mathrm{ft}^{3}\right)$ & 27.000 & & \\
\hline & & w/c ratio & 0.3 & & \\
\hline & & $\%$ FA & $55 \%$ & & \\
\hline & & $\% \mathrm{CA}$ & $0 \%$ & & \\
\hline & & Total Agg & $55 \%$ & & \\
\hline Materials & $\begin{array}{l}\text { Specific } \\
\text { Gravity }\end{array}$ & $\begin{array}{l}\text { Amount } \\
\left(\mathrm{lbs} / \mathbf{y d}^{3}\right)\end{array}$ & $\begin{array}{l}\text { Volume } \\
\left(\mathrm{ft}^{3} / \mathbf{y d}^{3}\right)\end{array}$ & $\begin{array}{c}\text { Batch Volume } \\
\left(\mathrm{ft}^{3}\right) \\
\end{array}$ & $\begin{array}{c}\text { Batch Weight } \\
\text { (lbs) }\end{array}$ \\
\hline Cement (Type I) & 3.15 & 1227.87 & 6.25 & 6.25 & 1227.87 \\
\hline Water & 1 & 368.36 & $\overline{5.90}$ & 5.90 & 368.36 \\
\hline Fine Aggregate & 2.58 & 2390.73 & 14.85 & 14.85 & 2390.73 \\
\hline Course Aggregate & 2.75 & 0.00 & 0.00 & 0.00 & 0.00 \\
\hline
\end{tabular}

2. Determine the amount of dry LWA to add:

$$
M_{L W A}=\frac{C_{f} \times C S \times \alpha_{\max }}{S \times \phi_{L W A}} \Rightarrow \frac{1228 \mathrm{lbs} / y d^{3} \times 0.064 \mathrm{lbs} / \mathrm{lbs} \times 0.83}{1 \times 0.105 \mathrm{lbs} / \mathrm{lbs}}=623.68 \mathrm{lbs} / \mathrm{yd}^{3}
$$

3. Replace equal volume of sand by using the ratio of specific gravities

$$
M_{F A}=\frac{S G_{F A}}{S G_{L W A}} \times M_{L W A}=\frac{2.58}{1.56}=1031.47 \mathrm{lbs} / y d^{3}
$$

4. Replace that volume of fine aggregate with LWA yielding final mixture proportions 


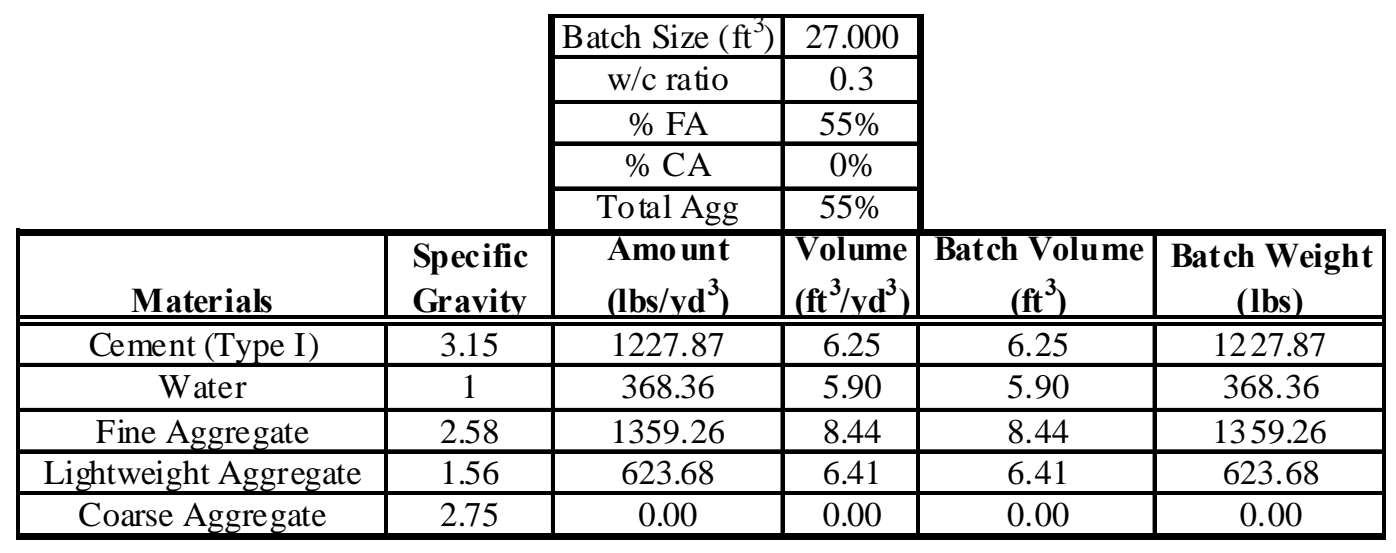




\section{Appendix B. Unrestrained Shrinkage Measurements}

Table B-1 - All unrestrained shrinkage measurements in sealed conditions

\begin{tabular}{|c|c|c|c|c|c|c|c|c|c|c|c|}
\hline \multicolumn{4}{|c|}{$0.0 \%$} & \multicolumn{4}{|c|}{$7.3 \% \mathrm{k}$} & \multicolumn{4}{|c|}{$11.0 \% \mathrm{k}$} \\
\hline Time (d) & Strain $(\mu \varepsilon)$ & Strain $(\mu \varepsilon)$ & Strain $(\mu \varepsilon)$ & Time (d) & Strain $(\mu \varepsilon)$ & Strain $(\mu \varepsilon)$ & Strain $(\mu \varepsilon)$ & Time (d) & Strain $(\mu \varepsilon)$ & Strain $(\mu \varepsilon)$ & Strain $(\mu \varepsilon)$ \\
\hline 0.16 & 0.00 & 0 & 0 & 0.25 & 0.00 & 0.00 & 0.00 & 0.25 & -4.64 & -4.64 & -4.64 \\
\hline 0.25 & -38.00 & -38 & -38 & 0.50 & 17.00 & 17.00 & 17.00 & 0.50 & 129.86 & 129.86 & 129.86 \\
\hline 0.50 & -38.00 & -38 & -38 & 0.75 & -2.00 & -2.00 & -2.00 & 0.75 & 151.26 & 151.26 & 151.26 \\
\hline 0.75 & -36.00 & -36 & -36 & 1.00 & -30.00 & -30.00 & -30.00 & 1.00 & 143.56 & 143.56 & 143.56 \\
\hline 1.04 & -54.00 & -54 & -54 & 2.88 & -210.00 & -120.00 & -130.00 & 3.77 & 63.56 & 83.56 & 73.56 \\
\hline 2.47 & -164.00 & -174.00 & -184.00 & 6.94 & -290.00 & -190.00 & -210.00 & 7.00 & 43.56 & 43.56 & 33.56 \\
\hline 7.00 & -254.00 & -264.00 & -254.00 & 14.02 & -380.00 & -270.00 & -290.00 & 14.00 & -66.44 & -36.44 & -76.44 \\
\hline 13.86 & -334.00 & -304.00 & -334.00 & 20.90 & -430.00 & -310.00 & -330.00 & 21.00 & -96.44 & -76.44 & -96.44 \\
\hline 20.92 & -354.00 & -354.00 & -384.00 & 27.84 & -430.00 & -320.00 & -340.00 & 27.90 & -146.44 & -96.44 & -116.44 \\
\hline 27.93 & -374.00 & -384.00 & -414.00 & & & & & & & & \\
\hline
\end{tabular}

\begin{tabular}{|c|c|c|c|c|c|c|c|c|c|c|c|}
\hline \multicolumn{4}{|c|}{$14.3 \% \mathrm{k}$} & \multicolumn{4}{|c|}{$18.3 \% k$} & \multicolumn{4}{|c|}{$25.3 \% k$} \\
\hline Time (d) & Strain $(\mu \varepsilon)$ & Strain $(\mu \varepsilon)$ & Strain $(\mu \varepsilon)$ & Time (d) & Strain $(\mu \varepsilon)$ & Strain $(\mu \varepsilon)$ & Strain $(\mu \varepsilon)$ & Time (d) & Strain $(\mu \varepsilon)$ & Strain $(\mu \varepsilon)$ & Strain $(\mu \varepsilon)$ \\
\hline 0.24 & 0.00 & 0.00 & 0.00 & 0.24 & 3.02 & 3.02 & 3.02 & 0.23 & 0.00 & 0.00 & 0.00 \\
\hline 0.50 & 145.00 & 145.00 & 145.00 & 0.50 & 157.38 & 157.38 & 157.38 & 0.25 & 17.00 & 17.00 & 17.00 \\
\hline 0.75 & 185.00 & 185.00 & 185.00 & 0.75 & 207.81 & 207.81 & 207.81 & 0.50 & 181.00 & 181.00 & 181.00 \\
\hline 1.00 & 172.00 & 172.00 & 172.00 & 1.00 & 207.81 & 207.81 & 207.81 & 0.75 & 210.00 & 210.00 & 210.00 \\
\hline 3.03 & 122.00 & 152.00 & 142.00 & 3.14 & 217.81 & 127.81 & 207.81 & 1.00 & 210.00 & 210.00 & 210.00 \\
\hline 7.00 & 112.00 & 152.00 & 132.00 & 7.00 & 197.81 & 97.81 & 217.81 & 2.91 & 240.00 & 210.00 & 260.00 \\
\hline 13.83 & 92.00 & 122.00 & 122.00 & 13.98 & 167.81 & 57.81 & 187.81 & 6.97 & 260.00 & 240.00 & 250.00 \\
\hline 21.13 & 82.00 & 102.00 & 102.00 & 21.18 & 147.81 & 37.81 & 177.81 & 13.87 & 300.00 & 270.00 & 290.00 \\
\hline 27.90 & 32.00 & 82.00 & 62.00 & 28.00 & 127.81 & 7.81 & 147.81 & 20.85 & 290.00 & 240.00 & 280.00 \\
\hline & & & & & & & & 27.83 & 280.00 & 260.00 & 280.00 \\
\hline & & & & $\%$ k & & & & $\%$ \%k & & & \\
\hline & & Time (d) & Strain $(\mu \varepsilon)$ & Strain $(\mu \varepsilon)$ & Strain $(\mu \varepsilon)$ & Time (d) & Strain $(\mu \varepsilon)$ & Strain $(\mu \varepsilon)$ & Strain $(\mu \varepsilon)$ & & \\
\hline & & 0.25 & 13.81 & 13.81 & 13.81 & 0.25 & 13.71 & 13.71 & 13.71 & & \\
\hline & & 0.50 & 180.15 & 180.15 & 180.15 & 0.50 & 174.04 & 174.04 & 174.04 & & \\
\hline & & 0.75 & 216.77 & 216.77 & 216.77 & 0.75 & 201.41 & 201.41 & 201.41 & & \\
\hline & & 1.00 & 225.91 & 225.91 & 225.91 & 1.00 & 203.00 & 203.00 & 203.00 & & \\
\hline & & 2.85 & 255.91 & 285.91 & 255.91 & 4.06 & 243.00 & 273.00 & 253.00 & & \\
\hline & & 6.99 & 285.91 & 325.91 & 295.91 & 7.00 & 263.00 & 283.00 & 283.00 & & \\
\hline & & 14.37 & 285.91 & 325.91 & 305.91 & 14.20 & 303.00 & 303.00 & 313.00 & & \\
\hline & & 21.09 & 295.91 & 315.91 & 325.91 & 21.22 & 303.00 & 303.00 & 313.00 & & \\
\hline & & 27.90 & 305.91 & 335.91 & 315.91 & 27.90 & 313.00 & 303.00 & 323.00 & & \\
\hline
\end{tabular}




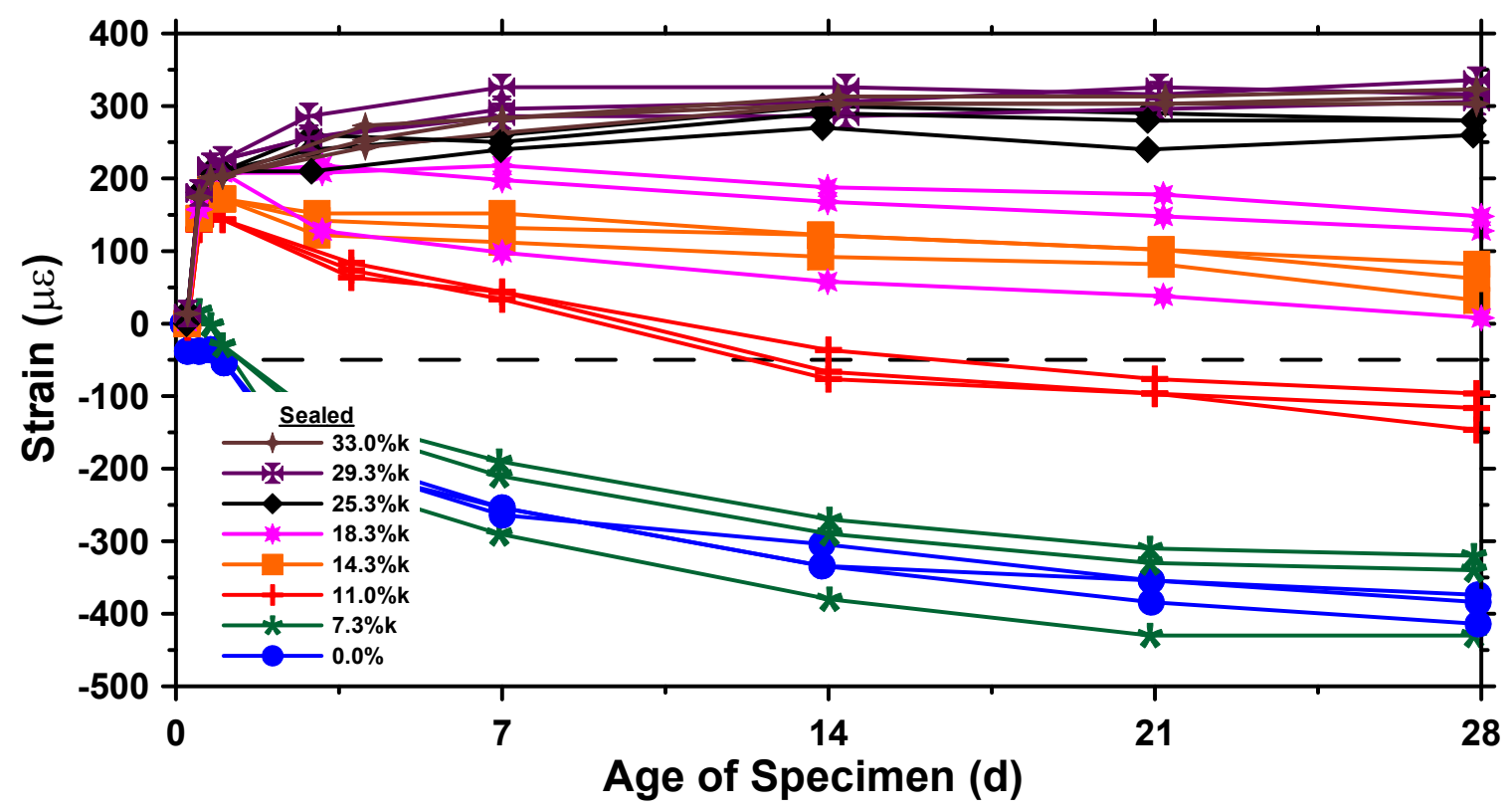

Figure B-1 - All unrestrained, sealed shrinkage measurements

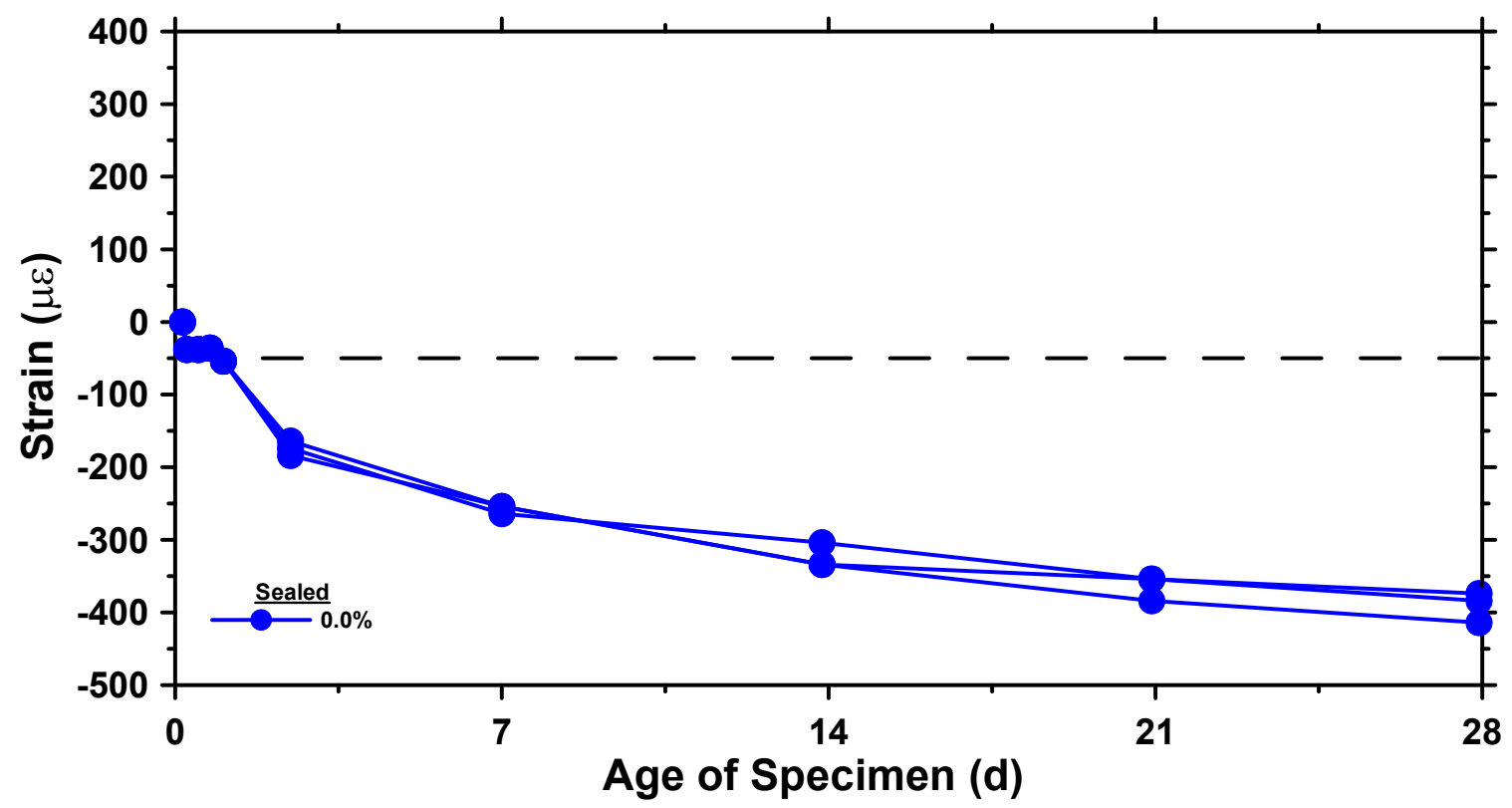

Figure B-2 - Unrestrained, sealed shrinkage for $0.0 \%$ mixture 


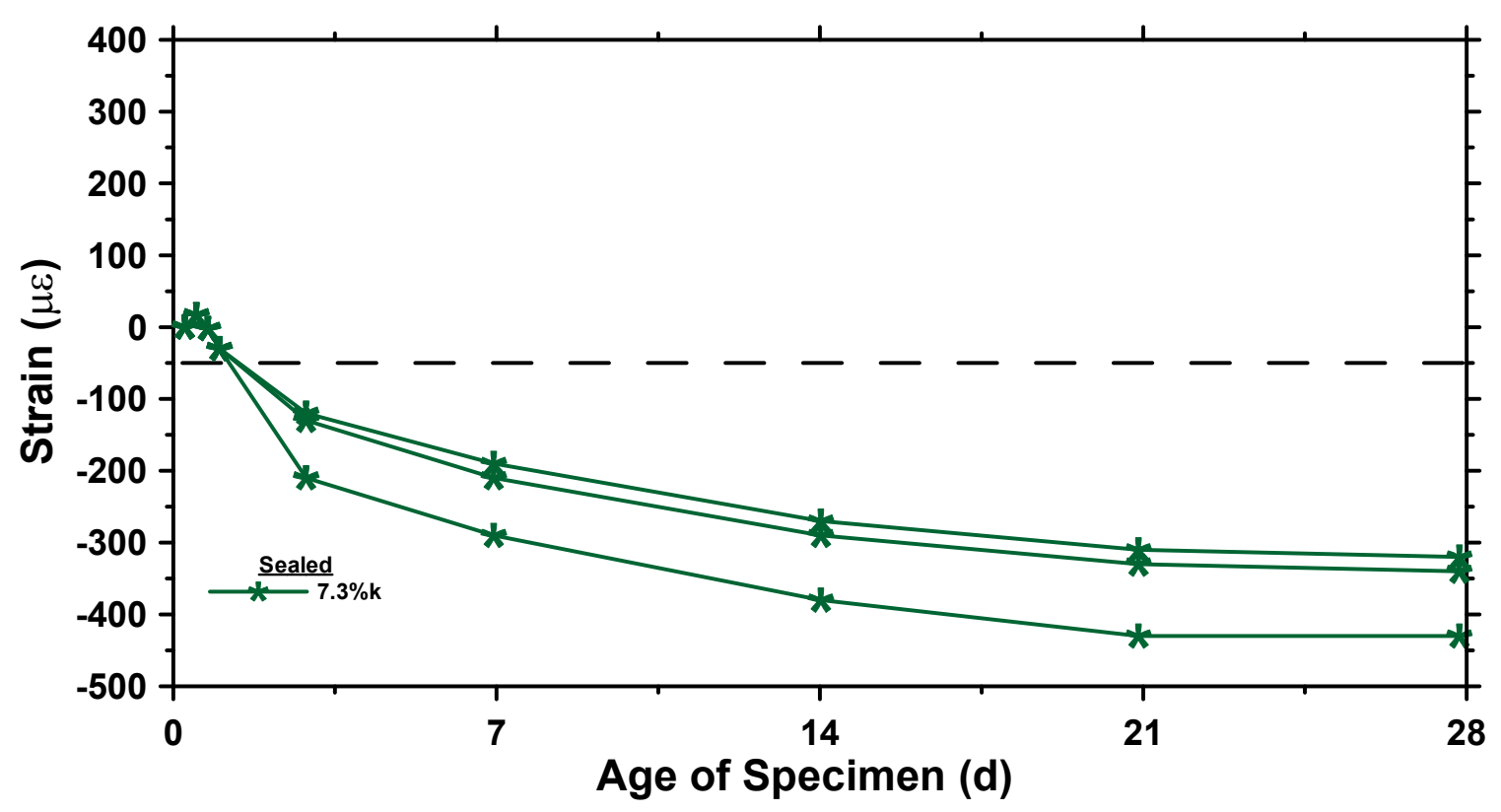

Figure B-3 - Unrestrained, sealed shrinkage for $7.3 \%$ mixture

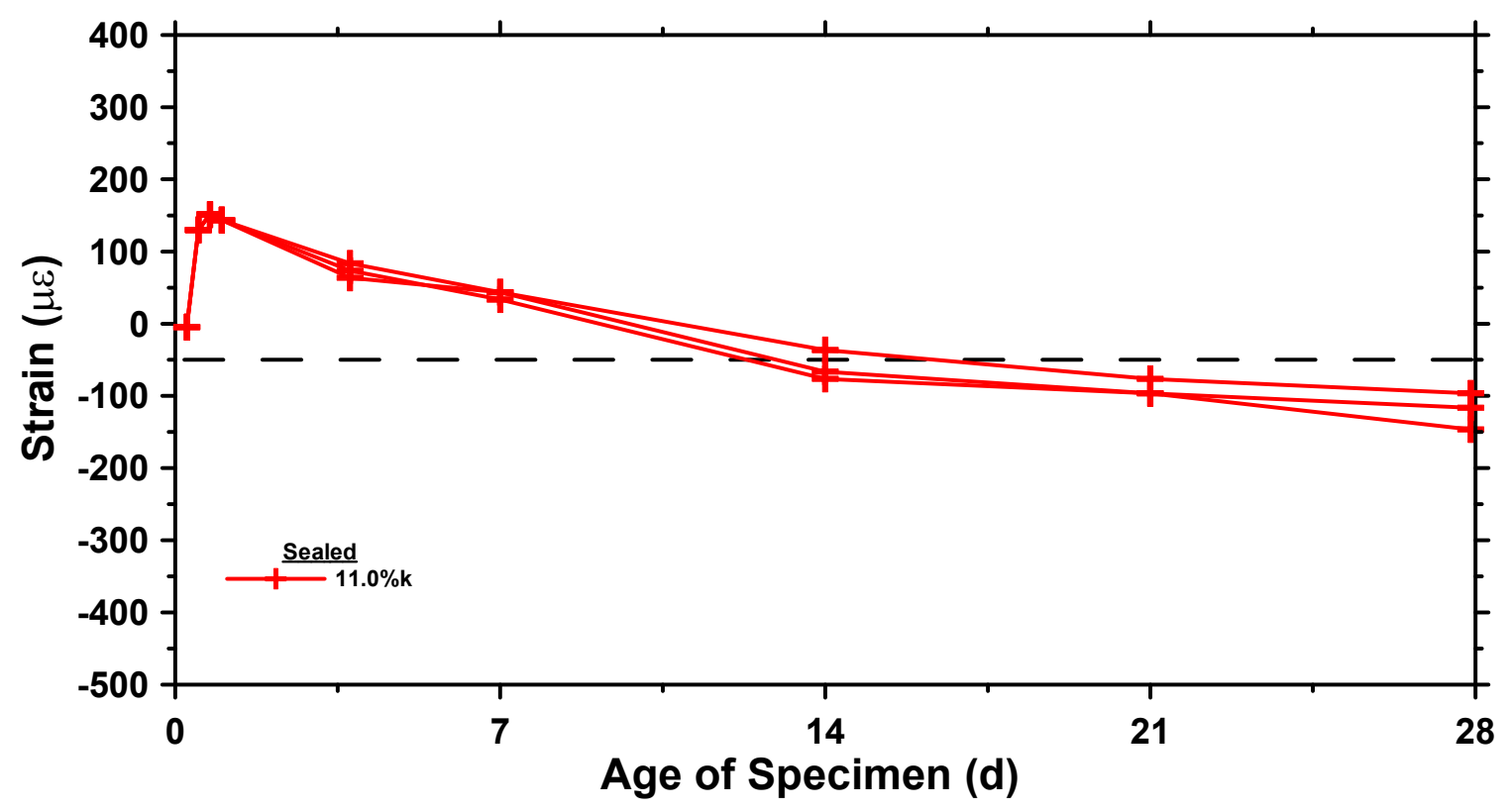

Figure B-4 - Unrestrained, sealed shrinkage for $11.0 \% \mathrm{k}$ mixture 


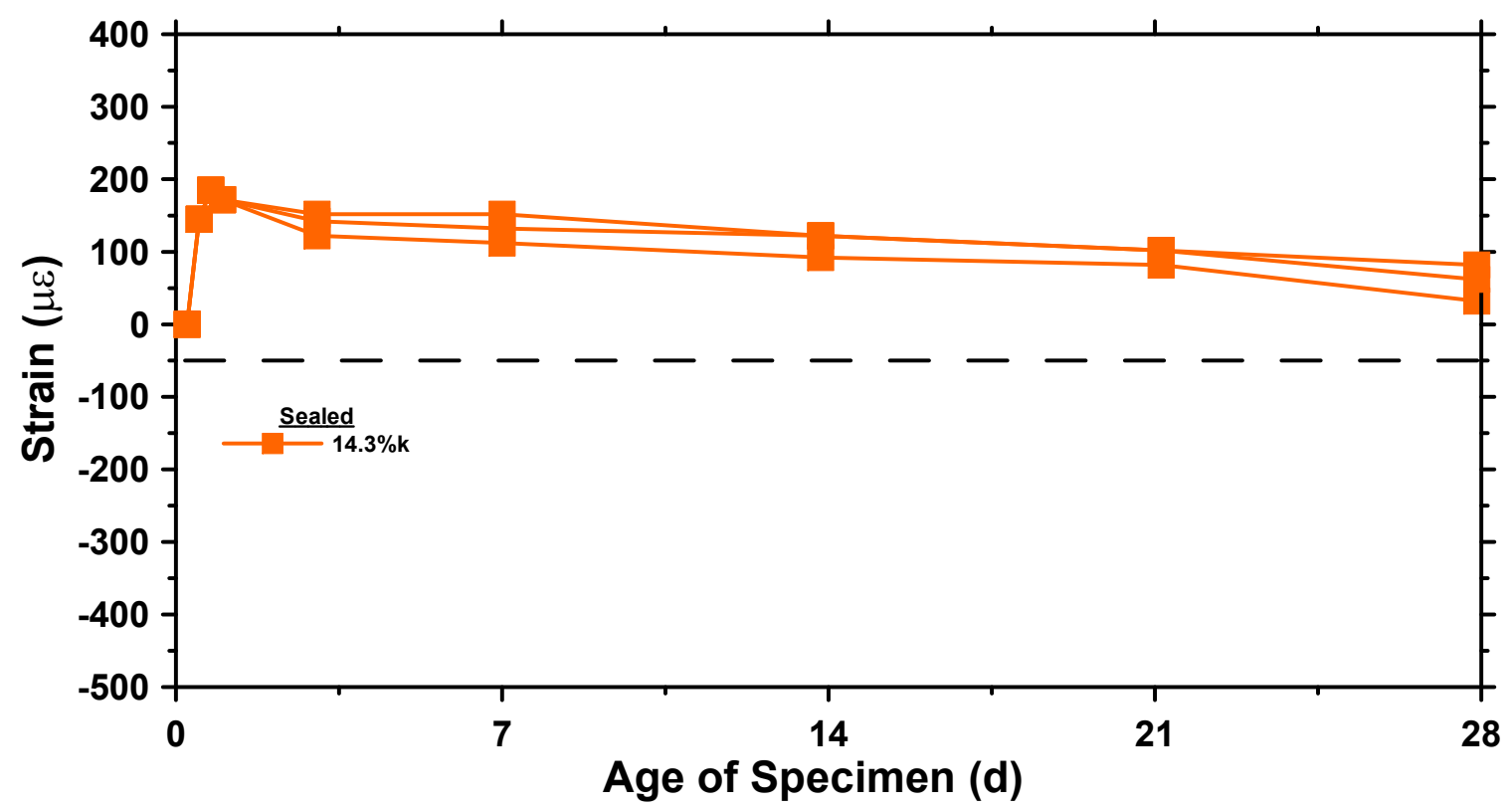

Figure B-5 - Unrestrained, sealed shrinkage 14.3\%k mixture

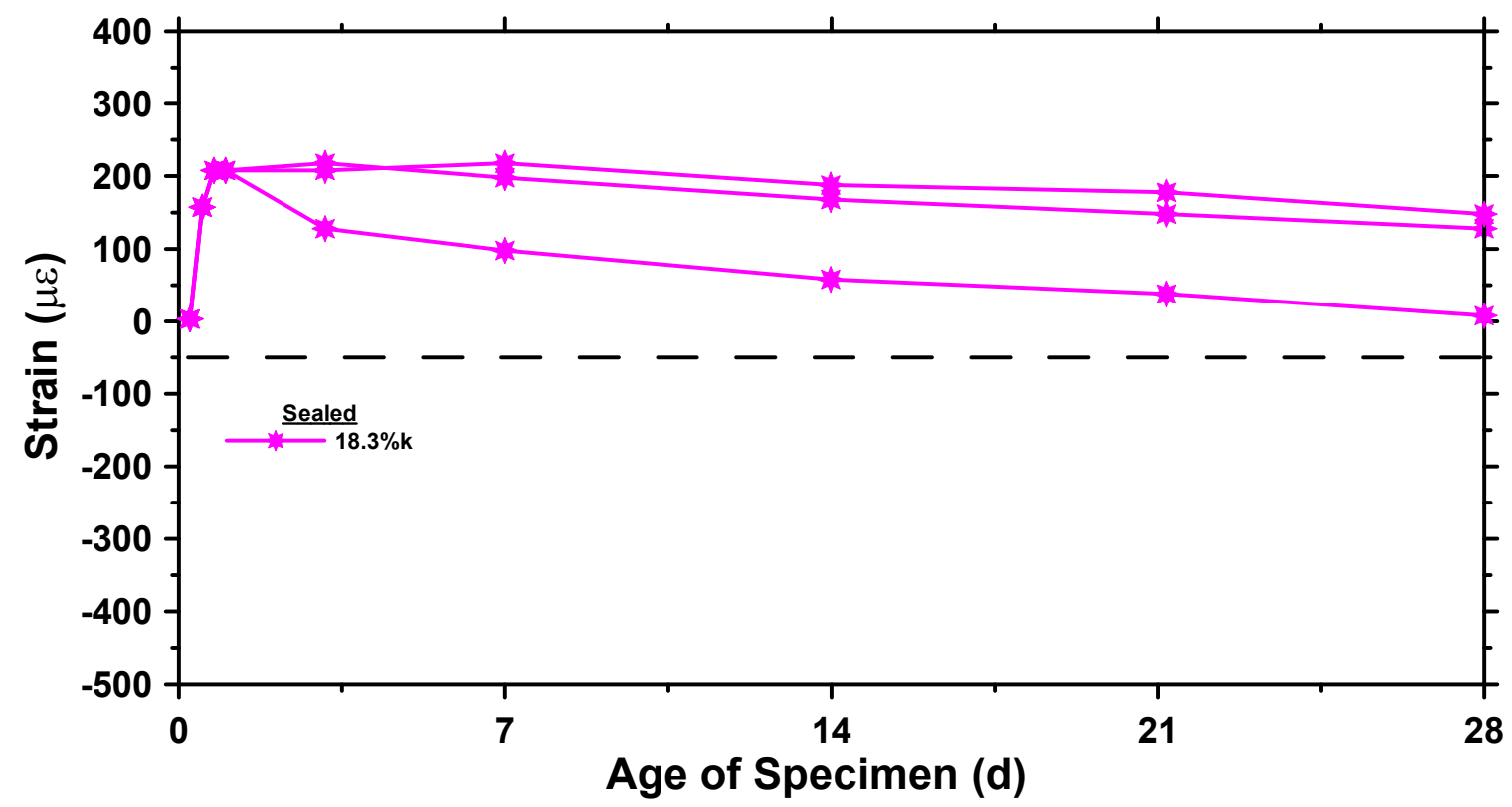

Figure B-6 - Unrestrained, sealed shrinkage for 18.3\%k mixture 


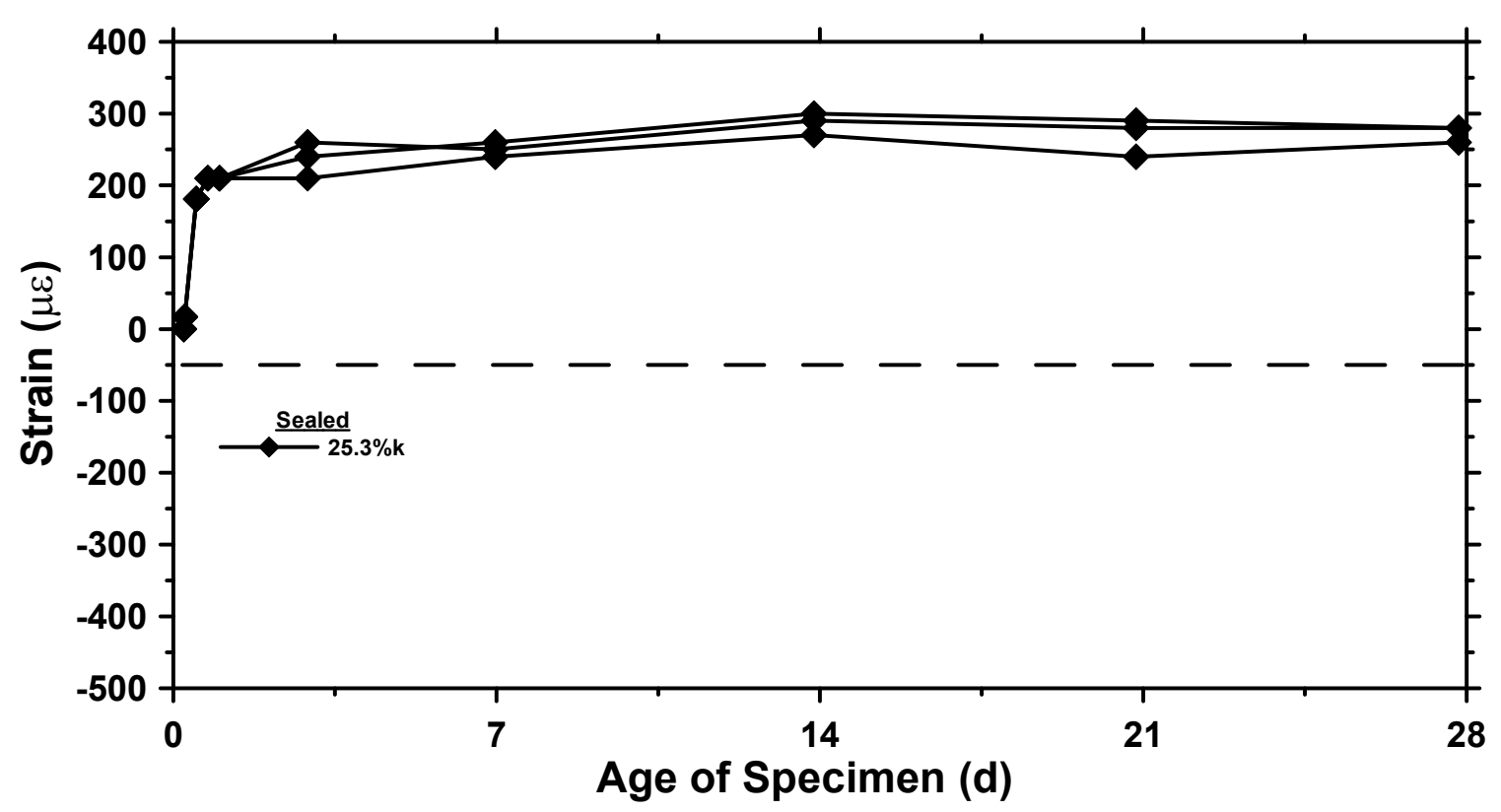

Figure B-7 - Unrestrained, sealed shrinkage 25.3\%k mixture

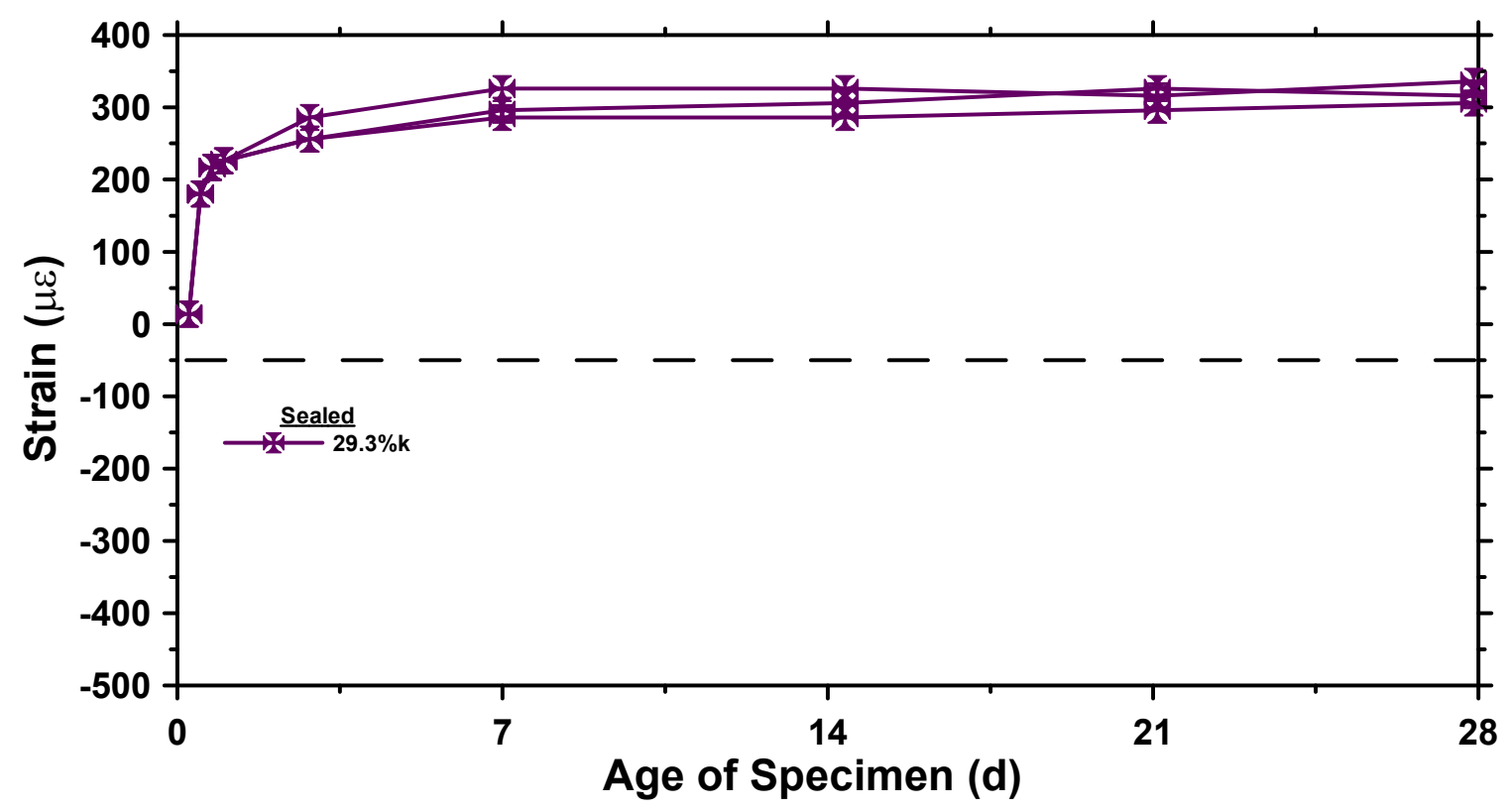

Figure B-8 - Unrestrained, sealed shrinkage for 29.3\%k mixture 


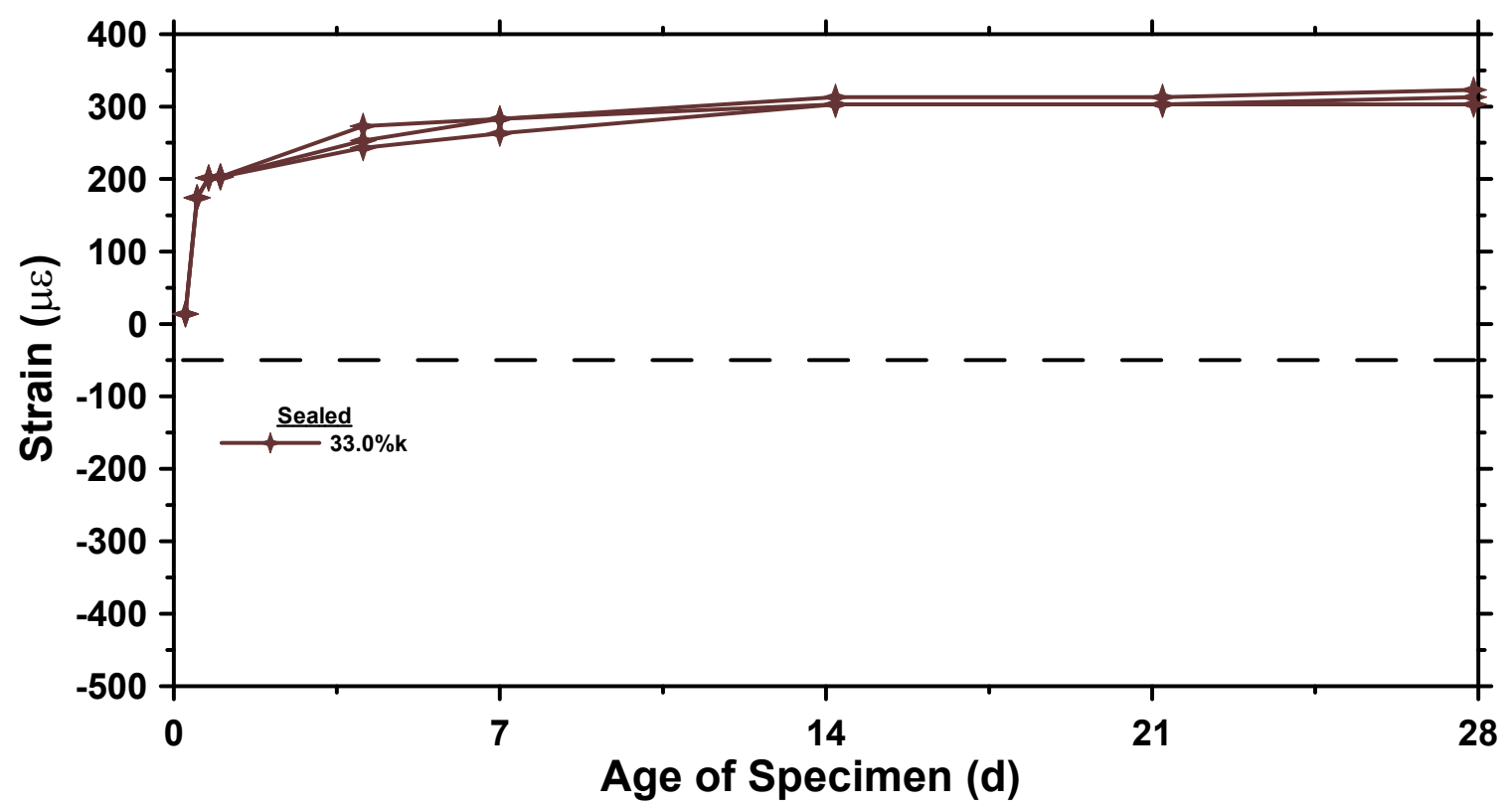

Figure B-9 - Unrestrained, sealed shrinkage for 33.0\%k mixture 
Table B-2 - All unrestrained shrinkage measurements in unsealed conditions

\begin{tabular}{|c|c|c|c|c|c|c|c|c|c|c|c|}
\hline \multicolumn{4}{|c|}{$0.0 \%$} & \multicolumn{4}{|c|}{$7.3 \% \mathrm{k}$} & \multicolumn{4}{|c|}{$11.0 \% \mathrm{k}$} \\
\hline Time (d) & Strain $(\mu \varepsilon)$ & Strain $(\mu \varepsilon)$ & Strain $(\mu \varepsilon)$ & Time (d) & Strain $(\mu \varepsilon)$ & Strain $(\mu \varepsilon)$ & Strain $(\mu \varepsilon)$ & Time (d) & Strain $(\mu \varepsilon)$ & Strain $(\mu \varepsilon)$ & Strain $(\mu \varepsilon)$ \\
\hline 0.16 & 0.00 & 0.00 & 0.00 & 0.25 & 0.00 & 0.00 & 0.00 & 0.25 & -4.64 & -4.64 & -4.64 \\
\hline 0.25 & -38.00 & -38.00 & -38.00 & 0.50 & 17.00 & 17.00 & 17.00 & 0.50 & 129.86 & 129.86 & 129.86 \\
\hline 0.50 & -38.00 & -38.00 & -38.00 & 0.75 & -2.00 & -2.00 & -2.00 & 0.75 & 151.26 & 151.26 & 151.26 \\
\hline 0.75 & -36.00 & -36.00 & -36.00 & 1.00 & -30.00 & -30.00 & -30.00 & 1.00 & 143.56 & 143.56 & 143.56 \\
\hline 1.04 & -54.00 & -54.00 & -54.00 & 2.74 & -210.00 & -180.00 & -220.00 & 3.75 & -220.00 & -240.00 & -210.00 \\
\hline 2.46 & -274.00 & -234.00 & -234.00 & 6.96 & -410.00 & -390.00 & -400.00 & 6.99 & -370.00 & -350.00 & -340.00 \\
\hline 7.00 & -434.00 & -404.00 & -414.00 & 13.97 & -600.00 & -570.00 & -560.00 & 13.98 & -560.00 & -560.00 & -530.00 \\
\hline 13.84 & -624.00 & -564.00 & -554.00 & 21.72 & -690.00 & -650.00 & -660.00 & 20.98 & -670.00 & -660.00 & -620.00 \\
\hline 20.91 & -704.00 & -654.00 & -644.00 & 27.83 & -760.00 & -740.00 & -740.00 & 28.00 & -750.00 & -720.00 & -720.00 \\
\hline 27.92 & -794.00 & -744.00 & -714.00 & & & & & & & & \\
\hline
\end{tabular}

\begin{tabular}{|c|c|c|c|c|c|c|c|c|c|c|c|}
\hline \multicolumn{4}{|c|}{$14.3 \% \mathrm{k}$} & \multicolumn{4}{|c|}{$18.3 \% \mathrm{k}$} & \multicolumn{4}{|c|}{$25.3 \% \mathrm{k}$} \\
\hline Time (d) & Strain $(\mu \varepsilon)$ & Strain $(\mu \varepsilon)$ & Strain $(\mu \varepsilon)$ & Time (d) & Strain $(\mu \varepsilon)$ & Strain $(\mu \varepsilon)$ & Strain $(\mu \varepsilon)$ & Time (d) & Strain $(\mu \varepsilon)$ & Strain $(\mu \varepsilon)$ & Strain $(\mu \varepsilon)$ \\
\hline 0.24 & 0.00 & 0.00 & 0.00 & 0.24 & 3.02 & 3.02 & 3.02 & 0.23 & 0.00 & 0.00 & 0.00 \\
\hline 0.50 & 145.00 & 145.00 & 145.00 & 0.50 & 157.38 & 157.38 & 157.38 & 0.25 & 17.00 & 17.00 & 17.00 \\
\hline 0.75 & 185.00 & 185.00 & 185.00 & 0.75 & 207.81 & 207.81 & 207.81 & 0.50 & 181.00 & 181.00 & 181.00 \\
\hline 1.00 & 172.00 & 172.00 & 172.00 & 1.00 & 207.81 & 207.81 & 207.81 & 0.75 & 210.00 & 210.00 & 210.00 \\
\hline 3.09 & -18.00 & -38.00 & -8.00 & 3.00 & 127.81 & 127.81 & 122.81 & 1.00 & 210.00 & 210.00 & 210.00 \\
\hline 6.99 & -168.00 & -148.00 & -138.00 & 6.97 & 47.81 & 47.81 & 37.81 & 2.91 & 170.00 & 130.00 & 180.00 \\
\hline 13.96 & -358.00 & -358.00 & -328.00 & 13.94 & -72.19 & -62.19 & -72.19 & 6.97 & 140.00 & 90.00 & 160.00 \\
\hline 20.98 & -468.00 & -458.00 & -418.00 & 20.93 & -202.19 & -192.19 & -212.19 & 13.87 & 110.00 & 50.00 & 120.00 \\
\hline 28.00 & -548.00 & -518.00 & -518.00 & 27.90 & -302.19 & -272.19 & -322.19 & 20.85 & 20.00 & 0.00 & 50.00 \\
\hline & & & & & & & & 27.83 & -50.00 & -50.00 & 10.00 \\
\hline
\end{tabular}

\begin{tabular}{|c|c|c|c|c|c|c|c|}
\hline \multicolumn{9}{|c|}{$29.3 \% \mathrm{k}$} & \multicolumn{4}{c|}{$33.0 \% \mathrm{k}$} \\
\hline Time (d) & Strain $(\boldsymbol{\mu} \varepsilon)$ & Strain $(\boldsymbol{\mu \varepsilon})$ & Strain $(\boldsymbol{\mu} \varepsilon)$ & Time $(\mathbf{d})$ & Strain $(\boldsymbol{\mu \varepsilon})$ & Strain $(\boldsymbol{\mu \varepsilon})$ & Strain $(\boldsymbol{\mu \varepsilon})$ \\
\hline 0.25 & 13.81 & 13.81 & 13.81 & 0.25 & 13.71 & 13.71 & 13.71 \\
\hline 0.50 & 180.15 & 180.15 & 180.15 & 0.50 & 174.04 & 174.04 & 174.04 \\
\hline 0.75 & 216.77 & 216.77 & 216.77 & 0.75 & 201.41 & 201.41 & 201.41 \\
\hline 1.00 & 225.91 & 225.91 & 225.91 & 1.00 & 203.00 & 203.00 & 203.00 \\
\hline 2.85 & 245.91 & 165.91 & 215.91 & 4.06 & 213.00 & 203.00 & 203.00 \\
\hline 7.01 & 215.91 & 165.91 & 205.91 & 7.48 & 203.00 & 213.00 & 193.00 \\
\hline 14.37 & 155.91 & 115.91 & 195.91 & 14.20 & 193.00 & 193.00 & 203.00 \\
\hline 21.09 & 145.91 & 105.91 & 175.91 & 21.22 & 173.00 & 163.00 & 153.00 \\
\hline 28.00 & 105.91 & 65.91 & 145.91 & 28.00 & 173.00 & 143.00 & 143.00 \\
\hline
\end{tabular}




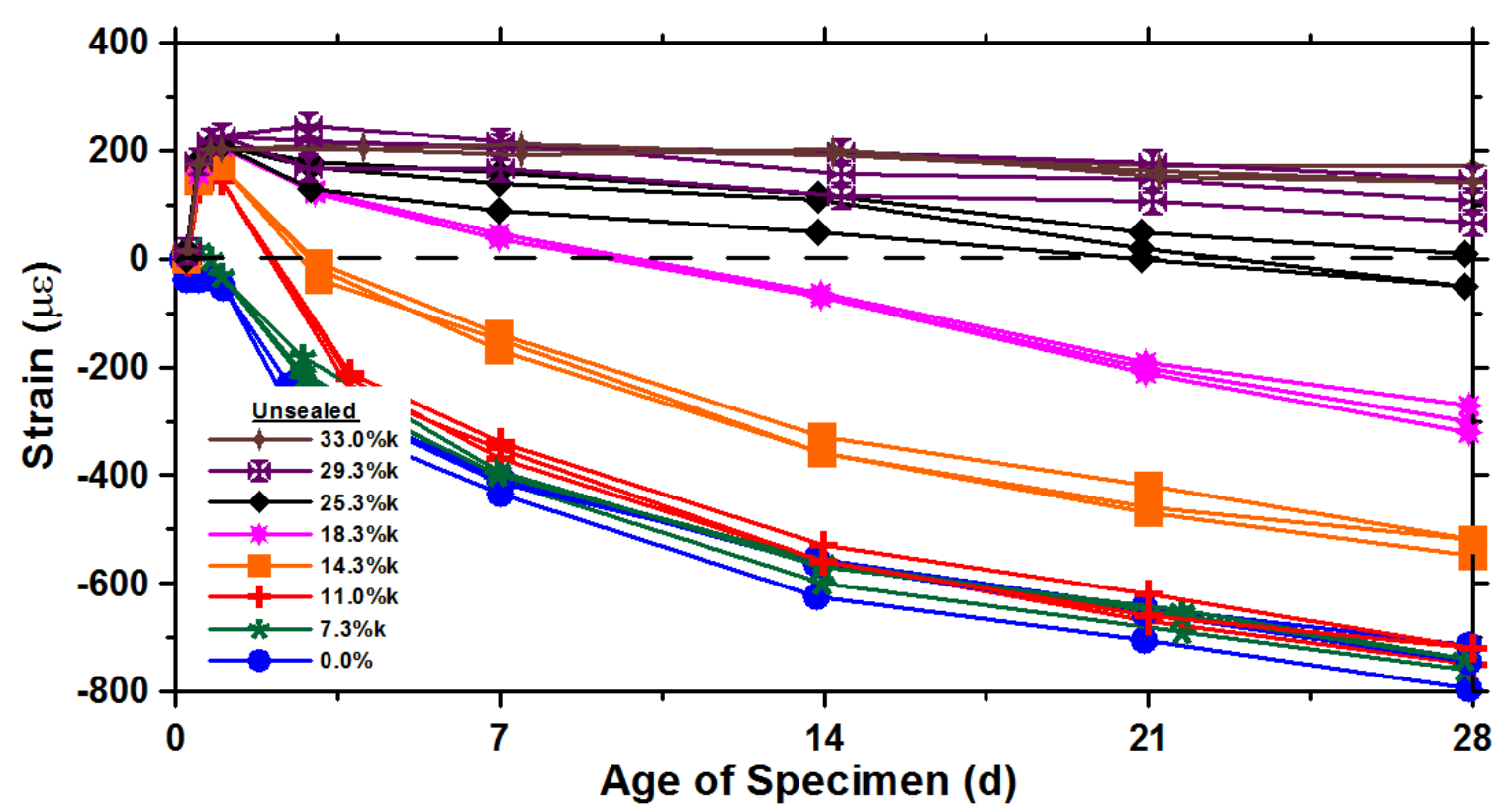

Figure B-10 - All unrestrained, unsealed shrinkage measurements

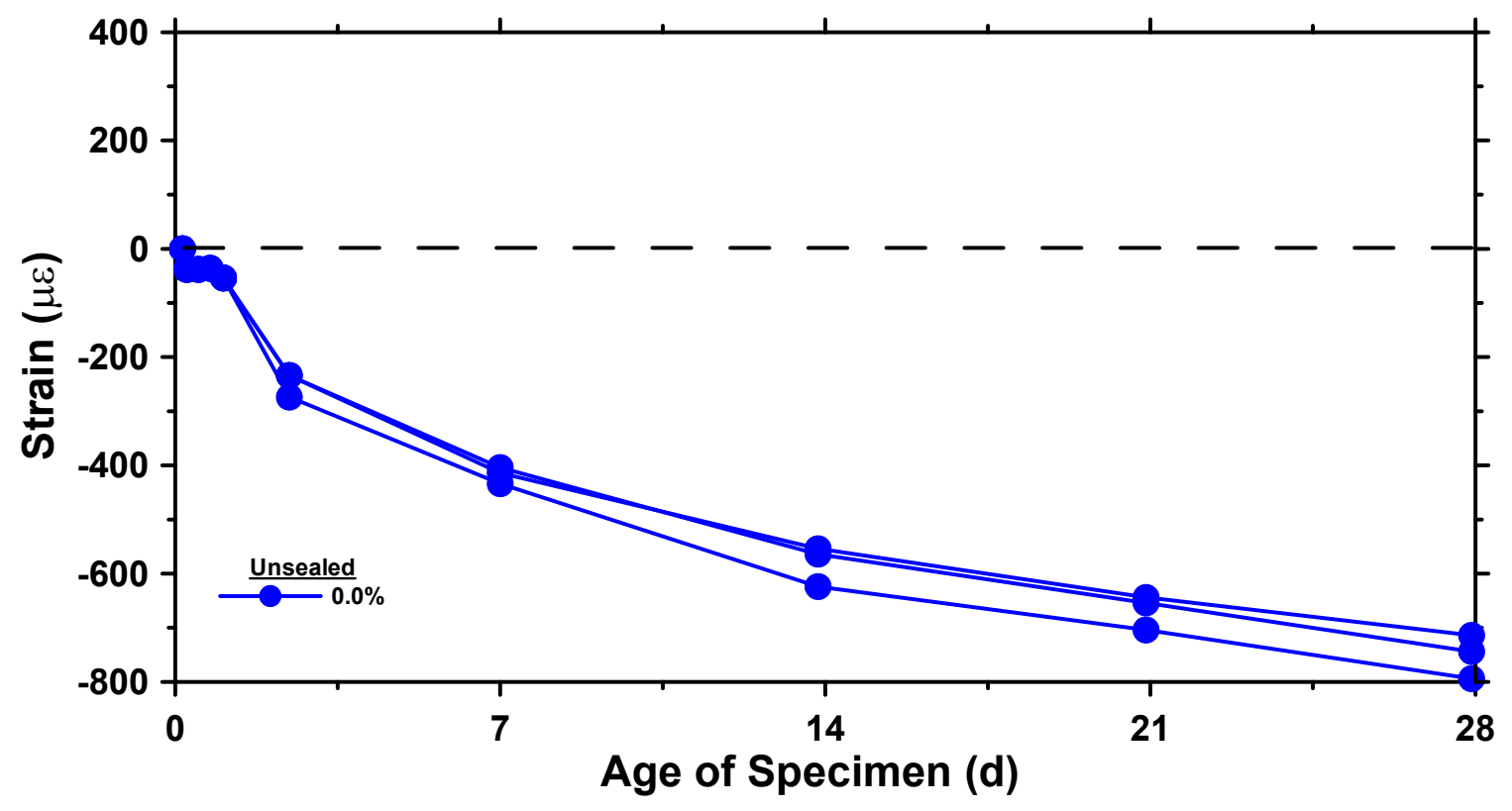

Figure B-11 - Unrestrained, unsealed shrinkage for $0.0 \%$ mixture 


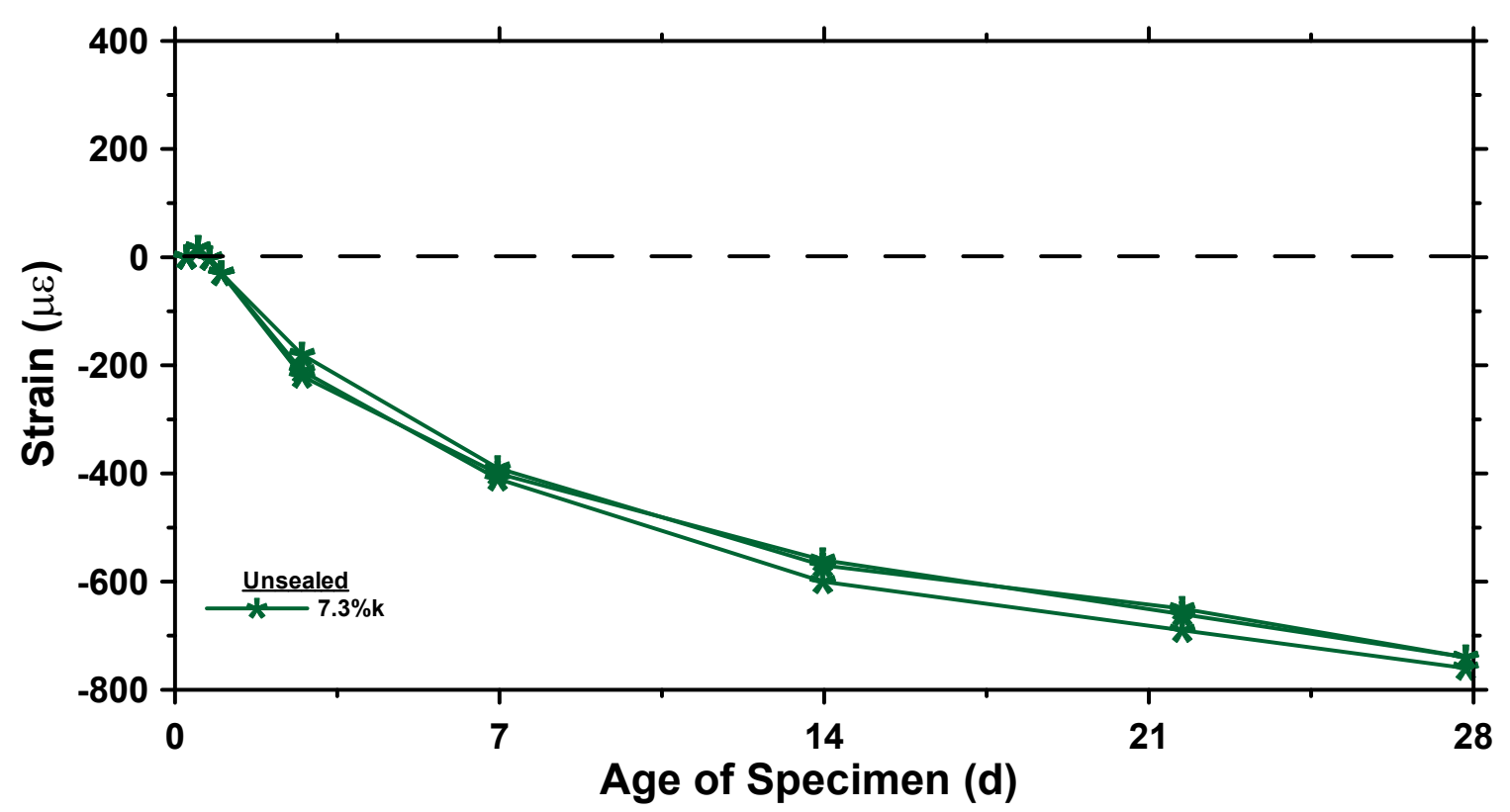

Figure B-12 - Unrestrained, unsealed shrinkage for 7.3\%k mixture

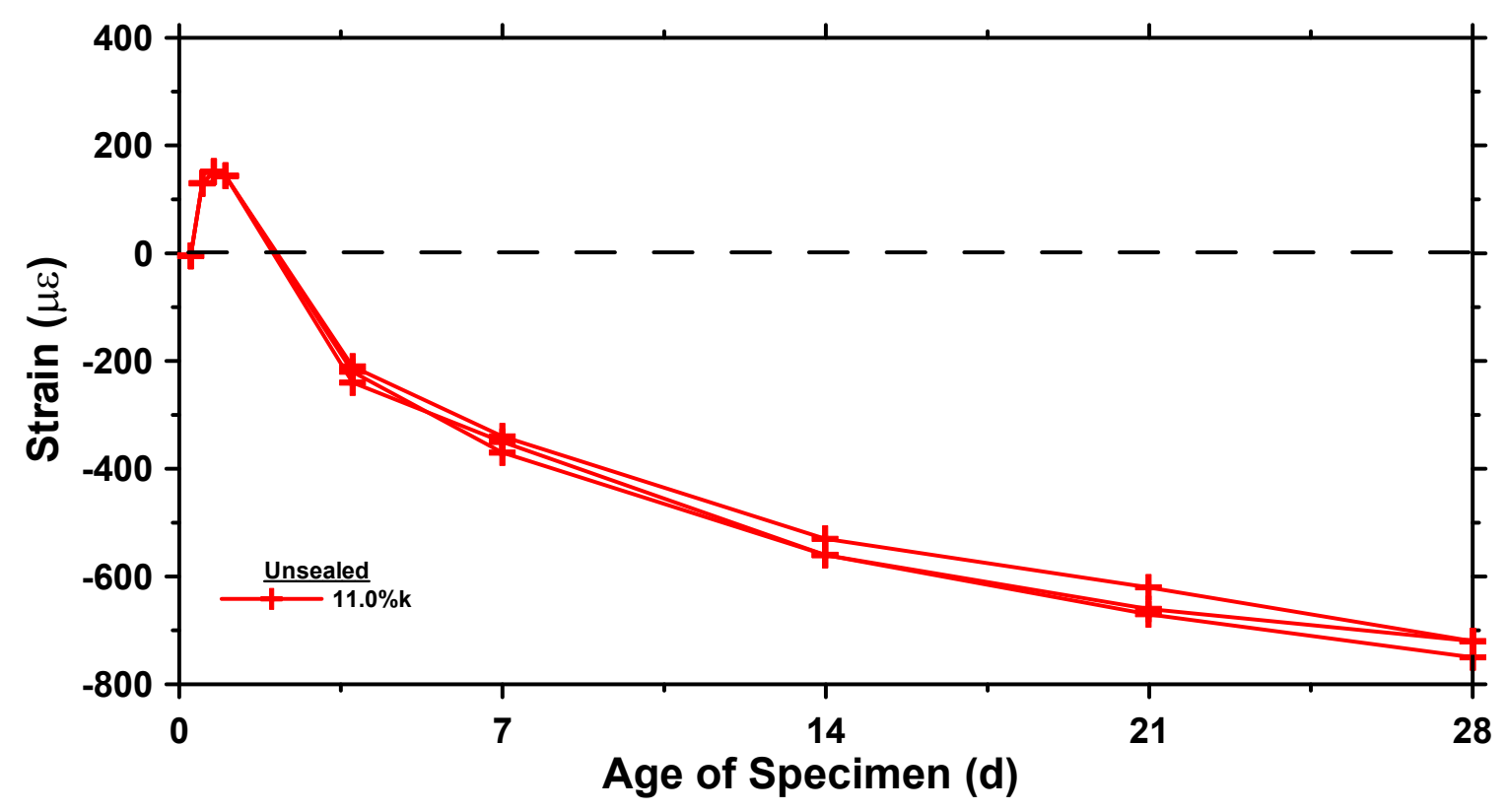

Figure B-13 - Unrestrained, unsealed shrinkage for $11.0 \% \mathrm{k}$ mixture 


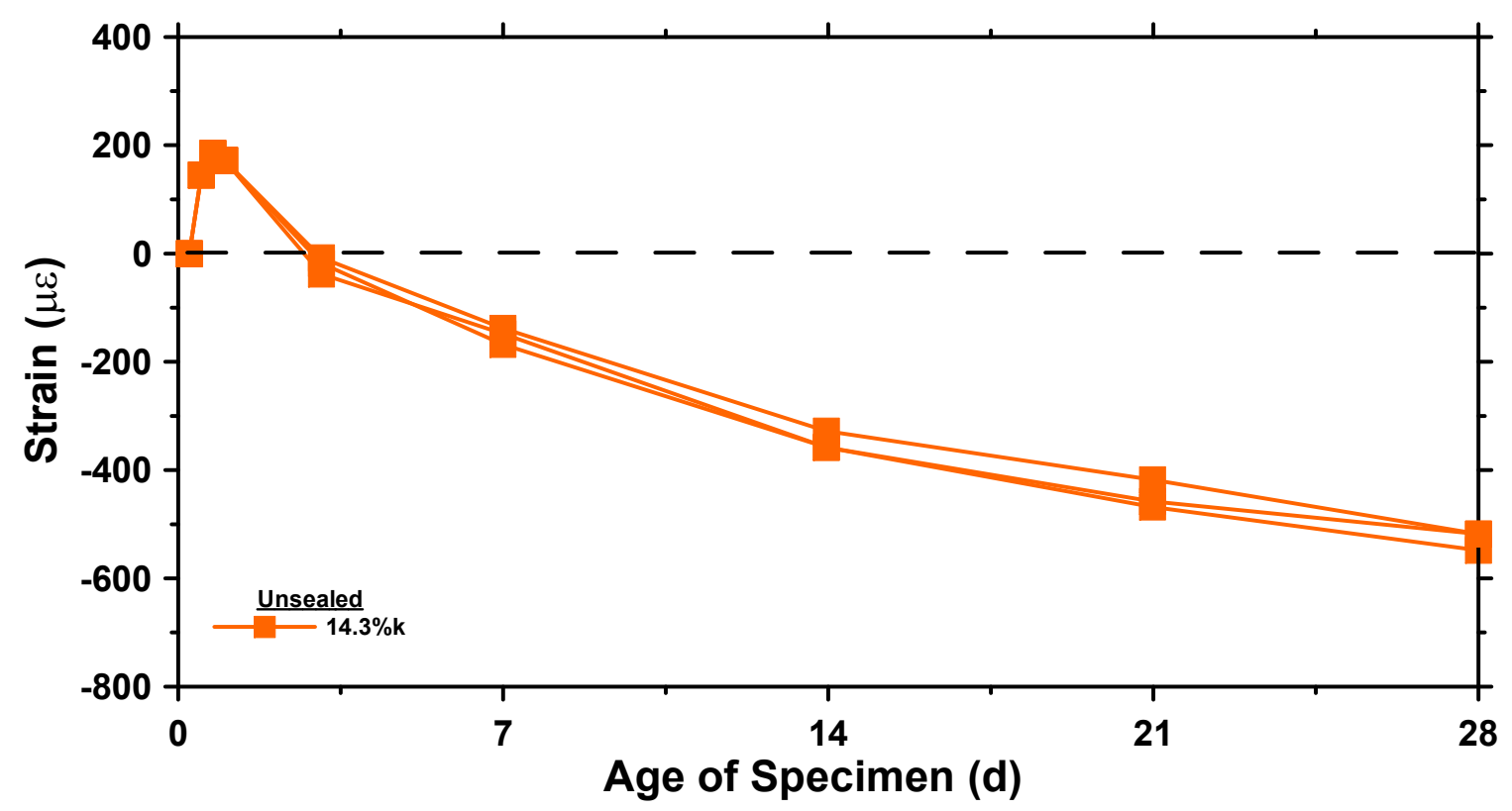

Figure B-14 - Unrestrained, unsealed shrinkage for $14.3 \% \mathrm{k}$ mixture

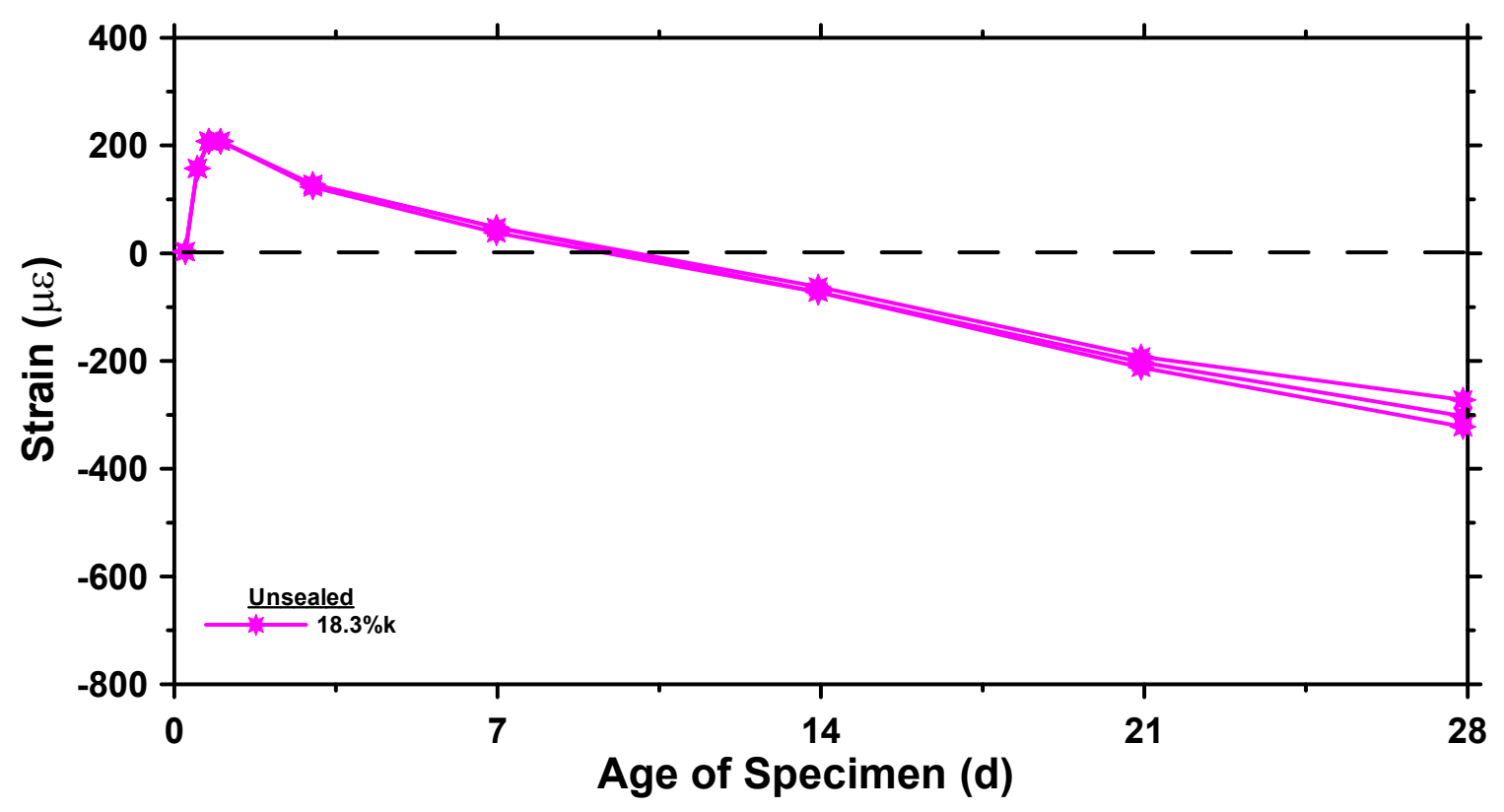

Figure B-15 - Unrestrained, unsealed shrinkage for $18.3 \% \mathrm{k}$ mixture 


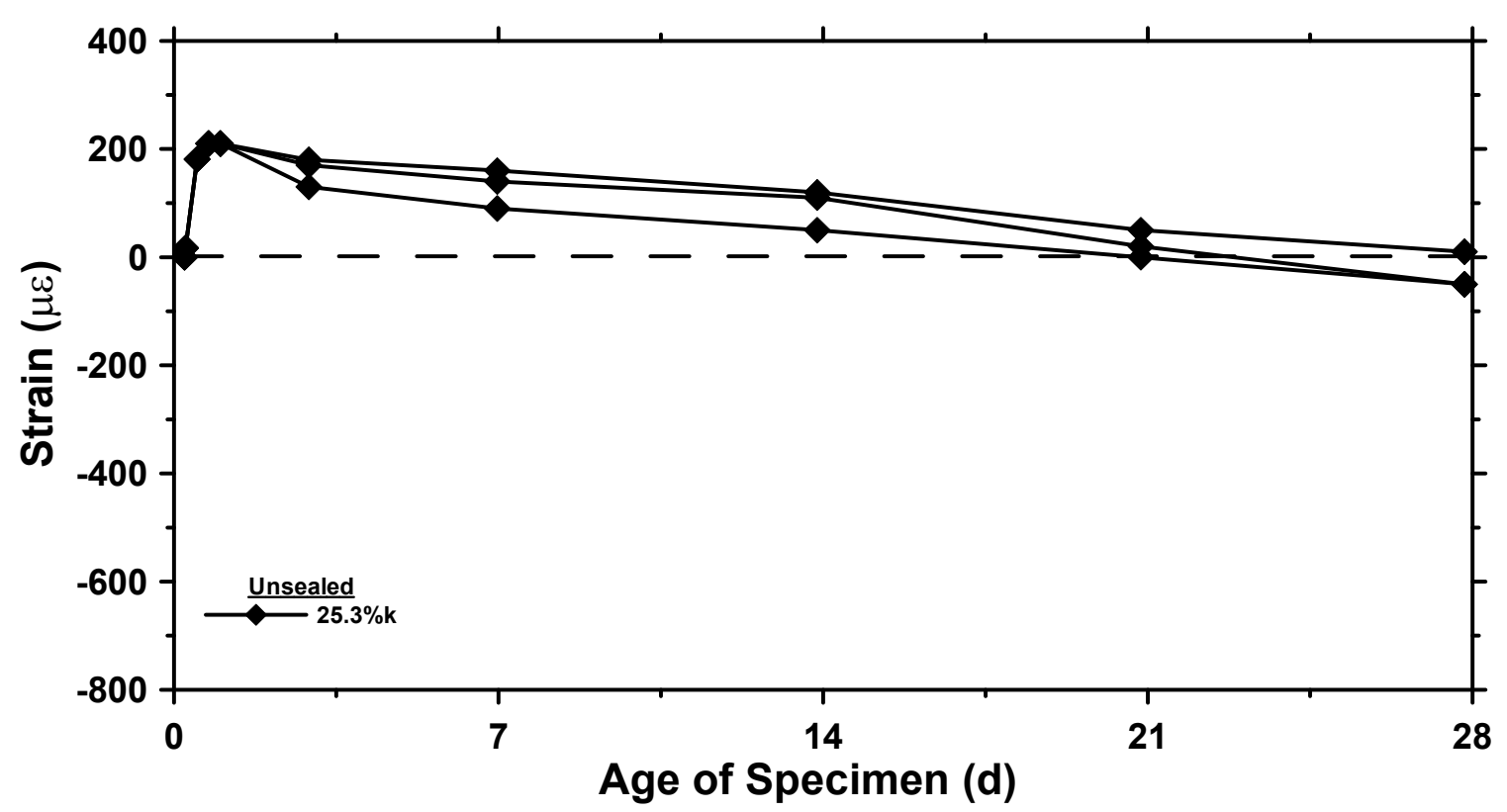

Figure B-16 - Unrestrained, unsealed shrinkage for $25.3 \%$ k mixture

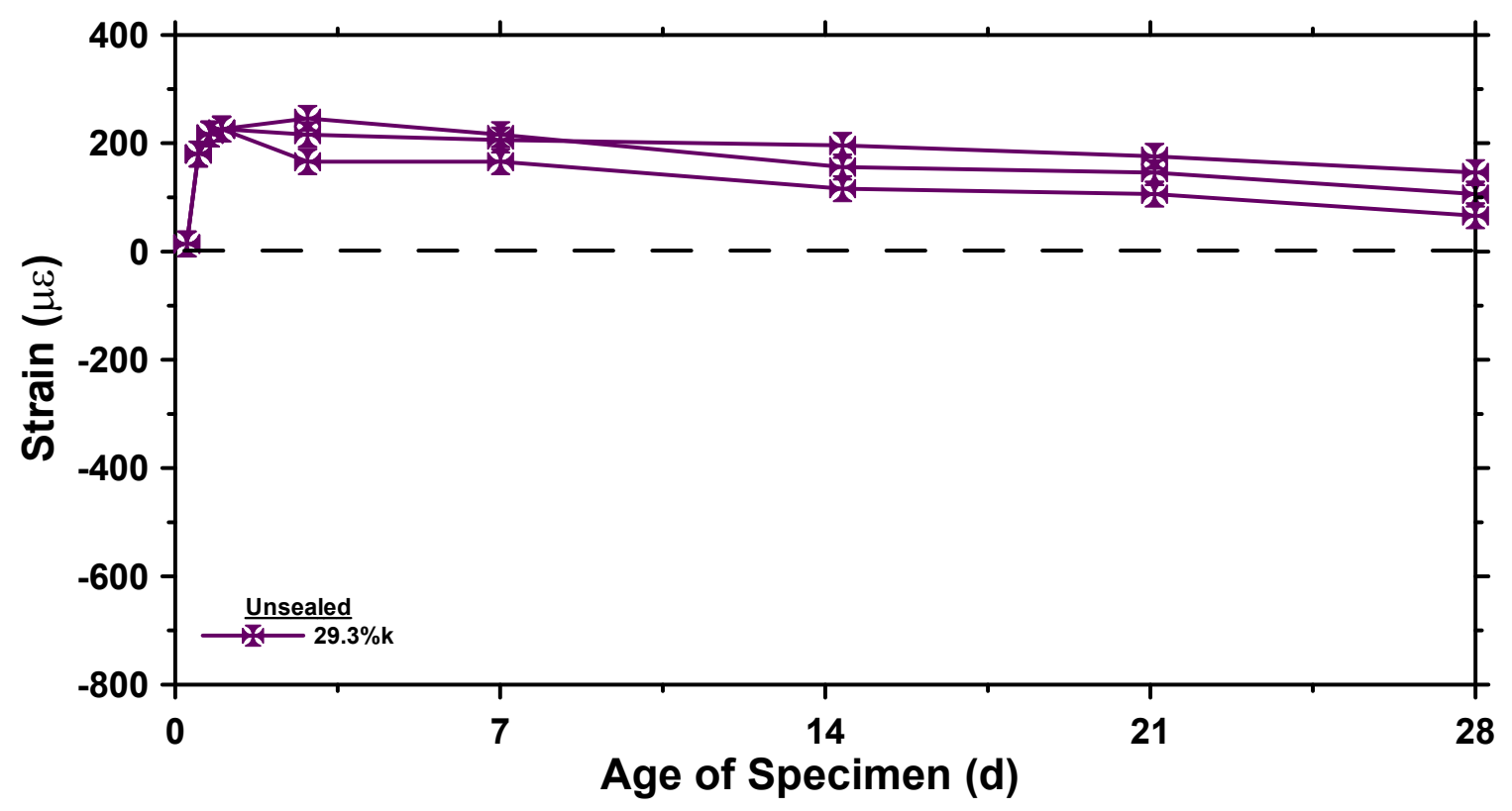

Figure B-17 - Unrestrained, unsealed shrinkage for 29.3\%k mixture 


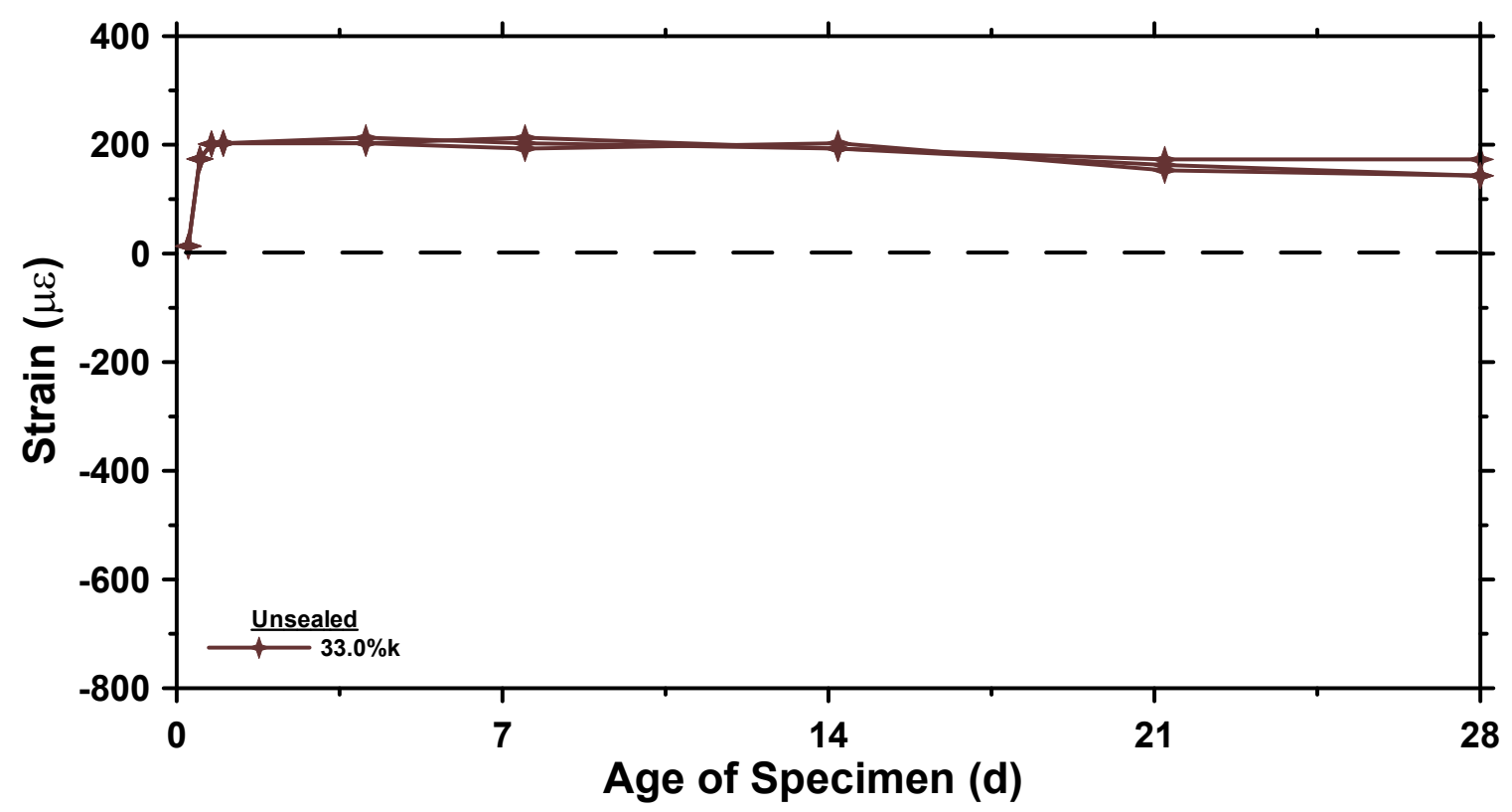

Figure B-18 - Unrestrained, unsealed shrinkage for 33.0\%k mixture 
Appendix C. Restrained Shrinkage Measurements

Table C-1 - Restrained, sealed measurements for $0.0 \%$ mixture

\begin{tabular}{|c|c|c|c|c|c|c|}
\hline \multicolumn{7}{|c|}{$\mathbf{0 . 0 \%}$} \\
\hline Days & {$[\mu \varepsilon]$} & {$[\mu \varepsilon]$} & {$[\mu \varepsilon]$} & {$[\mu \varepsilon]$} & {$[\mu \varepsilon]$} & {$[\mu \varepsilon]$} \\
\hline 0.00 & 0.00 & 0.00 & 0.00 & 0.00 & 0.00 & 0.00 \\
\hline 0.25 & -7.84 & -7.58 & -9.15 & -6.26 & -8.79 & -8.79 \\
\hline 0.50 & -24.36 & -21.52 & -18.00 & -20.42 & -23.55 & -19.72 \\
\hline 0.75 & -29.61 & -26.22 & -21.60 & -24.87 & -28.60 & -24.19 \\
\hline 1.00 & -34.96 & -30.27 & -25.52 & -28.19 & -32.64 & -29.22 \\
\hline 1.25 & -38.47 & -32.83 & -27.85 & -30.64 & -35.08 & -33.49 \\
\hline 1.50 & -41.23 & -34.76 & -30.86 & -32.56 & -37.36 & -36.63 \\
\hline 1.75 & -43.75 & -37.53 & -33.51 & -34.61 & -39.42 & -39.42 \\
\hline 2.00 & -45.19 & -38.98 & -34.96 & -35.34 & -41.11 & -41.72 \\
\hline 2.25 & -47.60 & -40.43 & -36.77 & -37.15 & -42.55 & -44.02 \\
\hline 2.50 & -49.53 & -42.60 & -39.30 & -39.08 & -44.73 & -46.68 \\
\hline 2.75 & -51.09 & -43.56 & -40.99 & -40.89 & -45.93 & -48.25 \\
\hline 3.00 & -52.41 & -45.25 & -43.16 & -42.94 & -47.26 & -50.19 \\
\hline 3.25 & -54.10 & -46.57 & -45.10 & -43.91 & -48.10 & -52.37 \\
\hline 3.50 & -55.42 & -47.30 & -45.46 & -44.39 & -48.95 & -52.97 \\
\hline 3.75 & -55.90 & -48.02 & -46.54 & -45.36 & -49.67 & -53.94 \\
\hline 4.00 & -56.26 & -47.66 & -45.22 & -44.39 & -48.95 & -53.09 \\
\hline 4.25 & -57.22 & -48.87 & -46.30 & -44.75 & -49.67 & -54.06 \\
\hline 4.50 & -58.91 & -50.67 & -48.71 & -47.53 & -51.72 & -56.85 \\
\hline 4.75 & -59.87 & -51.39 & -48.95 & -47.89 & -52.33 & -56.85 \\
\hline 5.00 & -58.91 & -51.15 & -48.11 & -46.93 & -52.09 & -56.48 \\
\hline 5.25 & -59.99 & -52.48 & -49.56 & -47.05 & -53.05 & -57.57 \\
\hline 5.50 & -61.67 & -53.56 & -51.49 & -48.74 & -54.62 & -59.51 \\
\hline 5.75 & -61.67 & -54.52 & -51.49 & -49.10 & -55.58 & -59.75 \\
\hline 6.00 & -60.75 & -0.88 & -51.41 & -2.30 & -56.35 & -60.64 \\
\hline 6.25 & -61.11 & -0.76 & -51.65 & -2.30 & -2.53 & -60.52 \\
\hline 6.50 & -60.63 & -1.37 & -53.22 & -3.38 & -3.38 & -62.58 \\
\hline 6.75 & -22.51 & -0.88 & -0.12 & -3.02 & -3.02 & -59.79 \\
\hline 7.00 & 0.72 & 3.51 & 0.84 & 0.72 & 0.59 & 2.54 \\
\hline & & & & & & \\
\hline
\end{tabular}


Table C-2 - Restrained, sealed measurements for 3.8\%k mixture

\begin{tabular}{|c|c|c|c|}
\hline \multicolumn{4}{|c|}{$3.8 \%$} \\
\hline Days & {$[\mu \varepsilon]$} & {$[\mu \varepsilon]$} & {$[\mu \varepsilon]$} \\
\hline 0.00 & 0.00 & 0.00 & 0.00 \\
\hline 0.25 & -2.07 & -2.73 & -4.51 \\
\hline 0.50 & -10.55 & -8.82 & -8.66 \\
\hline 0.75 & -15.69 & -13.93 & -6.84 \\
\hline 1.00 & -21.47 & -20.22 & -10.54 \\
\hline 1.25 & -23.19 & -24.41 & -13.91 \\
\hline 1.50 & -26.36 & -28.43 & -17.13 \\
\hline 1.75 & -28.37 & -31.00 & -19.87 \\
\hline 2.00 & -30.30 & -33.10 & -21.80 \\
\hline 2.25 & -32.23 & -35.84 & -25.51 \\
\hline 2.50 & -34.28 & -38.58 & -27.61 \\
\hline 2.75 & -35.49 & -39.88 & -28.09 \\
\hline 3.00 & -35.61 & -40.84 & -29.22 \\
\hline 3.25 & -38.38 & -43.58 & -32.12 \\
\hline 3.50 & -40.44 & -45.03 & -33.25 \\
\hline 3.75 & -41.40 & -46.00 & -35.19 \\
\hline 4.00 & -42.37 & -47.29 & -35.99 \\
\hline 4.25 & -44.30 & -47.77 & -37.28 \\
\hline 4.50 & -46.23 & -49.71 & -39.70 \\
\hline 4.75 & -43.21 & -50.35 & -40.35 \\
\hline 5.00 & -43.45 & -50.19 & -41.15 \\
\hline 5.25 & -44.54 & -52.45 & -42.76 \\
\hline 5.50 & -43.94 & -53.42 & -44.54 \\
\hline 5.75 & -44.91 & -52.94 & -45.51 \\
\hline 6.00 & -43.02 & -52.90 & -45.95 \\
\hline 6.25 & -43.98 & -53.05 & -47.40 \\
\hline 6.50 & -44.10 & -53.22 & -48.37 \\
\hline 6.75 & -43.86 & -54.66 & -48.69 \\
\hline 7.00 & -41.81 & -53.54 & -47.40 \\
\hline 7.25 & -41.81 & -54.02 & -49.50 \\
\hline 7.50 & -44.22 & -56.60 & -51.92 \\
\hline 7.75 & -41.33 & -54.18 & -4.19 \\
\hline 8.00 & -3.31 & -49.67 & -4.83 \\
\hline 8.25 & -2.22 & -49.03 & -4.35 \\
\hline 8.50 & -3.31 & -49.67 & -5.32 \\
\hline 8.75 & -3.55 & -5.35 & -5.16 \\
\hline 9.00 & -3.43 & -4.86 & -4.99 \\
\hline & & & \\
\hline
\end{tabular}


Table C-3 - Restrained, sealed measurements for $7.3 \% \mathrm{k}$ mixture

\begin{tabular}{|c|c|c|c|}
\hline \multicolumn{4}{|c|}{$7.3 \%$} \\
\hline Days & {$[\mu \varepsilon]$} & {$[\mu \varepsilon]$} & {$[\mu \varepsilon]$} \\
\hline 0.00 & 0.00 & 0.00 & 0.00 \\
\hline 0.25 & 1.28 & 1.57 & -0.77 \\
\hline 0.50 & -9.94 & -5.87 & -11.85 \\
\hline 0.75 & -4.52 & -3.21 & -6.80 \\
\hline 1.00 & -2.13 & -3.10 & -2.85 \\
\hline 1.25 & -4.29 & -4.23 & -6.83 \\
\hline 1.50 & -6.81 & -8.57 & -11.30 \\
\hline 1.75 & -7.30 & -9.86 & -13.35 \\
\hline 2.00 & -10.66 & -12.92 & -15.88 \\
\hline 2.25 & -12.59 & -15.01 & -18.78 \\
\hline 2.50 & -14.27 & -17.75 & -22.16 \\
\hline 2.75 & -15.11 & -18.40 & -23.36 \\
\hline 3.00 & -15.71 & -20.97 & -25.53 \\
\hline 3.25 & -17.52 & -22.42 & -27.71 \\
\hline 3.50 & -19.32 & -24.19 & -30.24 \\
\hline 3.75 & -19.80 & -25.48 & -32.17 \\
\hline 4.00 & -20.77 & -27.41 & -32.77 \\
\hline 4.25 & -22.21 & -28.22 & -34.70 \\
\hline 4.50 & -23.65 & -29.99 & -37.72 \\
\hline 4.75 & -24.37 & -31.44 & -38.81 \\
\hline 5.00 & -25.22 & -31.93 & -37.72 \\
\hline 5.25 & -26.42 & -33.54 & -39.77 \\
\hline 5.50 & -27.98 & -34.99 & -42.06 \\
\hline 5.75 & -28.58 & -35.63 & -42.91 \\
\hline 6.00 & -29.11 & -37.04 & -43.07 \\
\hline 6.25 & -30.19 & -37.20 & -44.76 \\
\hline 6.50 & -31.03 & -37.53 & -45.73 \\
\hline 6.75 & -32.35 & -38.65 & -46.69 \\
\hline 7.00 & -33.68 & -40.26 & -47.53 \\
\hline 7.25 & -33.44 & -40.10 & -47.17 \\
\hline 7.50 & -34.04 & -40.27 & -47.20 \\
\hline 7.75 & -34.16 & -41.24 & -47.32 \\
\hline 8.00 & -35.00 & -40.92 & -48.04 \\
\hline 8.25 & -35.60 & -40.59 & -46.59 \\
\hline 8.50 & -36.20 & -40.60 & -46.35 \\
\hline 8.75 & -36.80 & -41.08 & -46.95 \\
\hline 9.00 & -37.76 & -42.20 & -46.47 \\
\hline & & & \\
\hline
\end{tabular}

\begin{tabular}{|c|c|c|c|}
\hline \multicolumn{4}{|c|}{$\mathbf{7 . 3 \% k}$} \\
\hline Days & {$[\mu \varepsilon]$} & {$[\mu \varepsilon]$} & {$[\mu \varepsilon]$} \\
\hline 9.25 & -37.04 & -41.88 & -45.63 \\
\hline 9.50 & -37.76 & -41.56 & -45.99 \\
\hline 9.75 & -37.64 & -42.53 & -46.35 \\
\hline 10.00 & -38.12 & -42.53 & -45.99 \\
\hline 10.25 & -40.05 & -42.21 & -44.66 \\
\hline 10.50 & -40.05 & -42.05 & -44.54 \\
\hline 10.75 & -40.77 & -41.90 & -44.90 \\
\hline 11.00 & -43.53 & -39.80 & -43.69 \\
\hline 11.25 & -20.09 & -40.12 & -42.25 \\
\hline 11.50 & -21.29 & -41.08 & -44.06 \\
\hline 11.75 & -20.33 & -40.60 & -43.58 \\
\hline 12.00 & -19.85 & -13.50 & -43.22 \\
\hline 12.25 & -18.40 & -13.49 & -43.58 \\
\hline 12.50 & -18.52 & -13.82 & -43.82 \\
\hline 12.75 & -18.28 & -11.88 & -35.01 \\
\hline 13.00 & -18.88 & -12.21 & -35.49 \\
\hline
\end{tabular}


Table C-4 - Restrained, sealed measurements for $11.0 \% \mathrm{k}$ mixture

\begin{tabular}{|c|c|c|c|}
\hline \multicolumn{4}{|c|}{$\mathbf{1 1 . 0 \% k}$} \\
\hline Days & {$[\mu \varepsilon]$} & {$[\mu \varepsilon]$} & {$[\mu \varepsilon]$} \\
\hline 0.00 & 0.00 & 0.00 & 0.00 \\
\hline 0.25 & -10.50 & -13.35 & -7.85 \\
\hline 0.50 & -10.63 & -13.85 & -9.55 \\
\hline 0.75 & -6.54 & -12.53 & -12.83 \\
\hline 1.00 & -2.36 & -10.53 & -9.02 \\
\hline 1.25 & -2.72 & -10.78 & -13.36 \\
\hline 1.50 & -3.92 & -12.70 & -15.65 \\
\hline 1.75 & -4.41 & -12.70 & -17.22 \\
\hline 2.00 & -5.13 & -13.18 & -17.10 \\
\hline 2.25 & -5.61 & -14.01 & -18.67 \\
\hline 2.50 & -6.09 & -14.13 & -19.51 \\
\hline 2.75 & -5.85 & -14.01 & -19.39 \\
\hline 3.00 & -5.73 & -13.77 & -18.42 \\
\hline 3.25 & -6.94 & -14.97 & -20.11 \\
\hline 3.50 & -8.14 & -15.93 & -21.32 \\
\hline 3.75 & -8.50 & -15.09 & -22.17 \\
\hline 4.00 & -8.98 & -15.33 & -22.40 \\
\hline 4.25 & -9.70 & -16.17 & -23.13 \\
\hline 4.50 & -10.78 & -17.01 & -24.46 \\
\hline 4.75 & -11.03 & -17.25 & -24.58 \\
\hline 5.00 & -11.39 & -17.37 & -24.46 \\
\hline 5.25 & -12.35 & -17.97 & -25.78 \\
\hline 5.50 & -13.31 & -19.17 & -27.47 \\
\hline 5.75 & -14.15 & -19.89 & -28.44 \\
\hline 6.00 & -13.31 & -18.81 & -26.99 \\
\hline 6.25 & -14.88 & -20.25 & -28.20 \\
\hline 6.50 & -15.96 & -21.21 & -28.80 \\
\hline 6.75 & -16.08 & -20.98 & -29.65 \\
\hline 7.00 & -16.44 & -20.86 & -28.20 \\
\hline 7.25 & -17.89 & -21.70 & -30.01 \\
\hline 7.50 & -20.17 & -23.98 & -33.15 \\
\hline 7.75 & -19.57 & -23.26 & -31.46 \\
\hline 8.00 & -19.81 & -23.49 & -32.06 \\
\hline 8.25 & -20.41 & -23.98 & -32.18 \\
\hline 8.50 & -21.85 & -25.18 & -34.35 \\
\hline 8.75 & -23.18 & -25.78 & -35.20 \\
\hline 9.00 & -24.02 & -26.49 & -35.20 \\
\hline & & & \\
\hline
\end{tabular}

\begin{tabular}{|c|c|c|c|}
\hline \multicolumn{4}{|c|}{$\mathbf{1 1 . 0 \% k}$} \\
\hline Days & {$[\boldsymbol{\mu \varepsilon}]$} & {$[\boldsymbol{\mu \varepsilon ]}$} & {$[\boldsymbol{\mu \varepsilon}]$} \\
\hline 9.25 & -24.50 & -26.74 & -35.68 \\
\hline 9.50 & -25.47 & -27.10 & -36.41 \\
\hline 9.75 & -25.83 & -27.10 & -35.92 \\
\hline 10.00 & -25.59 & -27.10 & -35.68 \\
\hline 10.25 & -27.27 & -28.65 & -39.90 \\
\hline 10.50 & -28.35 & -28.17 & -42.67 \\
\hline 10.75 & -28.83 & -29.14 & -45.20 \\
\hline 11.00 & -29.32 & -29.02 & -45.20 \\
\hline 11.25 & -30.28 & -29.86 & -46.04 \\
\hline 11.50 & -31.61 & -30.46 & -46.16 \\
\hline 11.75 & -31.60 & -30.33 & -46.28 \\
\hline 12.00 & -32.57 & -31.54 & -47.13 \\
\hline 12.25 & -32.81 & -31.17 & -45.56 \\
\hline 12.50 & -33.17 & -31.78 & -46.64 \\
\hline 12.75 & 0.97 & -20.76 & -31.24 \\
\hline 13.00 & 0.73 & -17.50 & -30.92 \\
\hline 13.25 & 1.45 & -13.02 & -30.44 \\
\hline 13.50 & 1.45 & -7.31 & -30.60 \\
\hline 13.75 & 1.45 & -6.68 & -29.63 \\
\hline 14.00 & 1.21 & -6.80 & -29.95 \\
\hline 14.25 & 1.69 & -6.43 & -28.66 \\
\hline 14.50 & 1.69 & -5.83 & -28.18 \\
\hline 14.75 & 1.45 & 0.91 & -28.34 \\
\hline 15.00 & 1.93 & 0.54 & -28.82 \\
\hline
\end{tabular}


Table C-5 - Restrained, sealed measurements for $14.3 \% \mathrm{k}$ mixture

\begin{tabular}{|c|c|c|c|}
\hline \multicolumn{4}{|c|}{$\mathbf{1 4 . 3 \% k}$} \\
\hline Days & {$[\mu \varepsilon]$} & {$[\mu \varepsilon]$} & {$[\mu \varepsilon]$} \\
\hline 0.00 & 0.00 & 0.00 & 0.00 \\
\hline 0.25 & 0.38 & -0.66 & -2.03 \\
\hline 0.50 & -5.73 & -8.14 & -9.81 \\
\hline 0.75 & -0.17 & -1.62 & -4.51 \\
\hline 1.00 & 4.25 & 4.37 & 0.52 \\
\hline 1.25 & 2.45 & 2.85 & -1.17 \\
\hline 1.50 & 1.01 & 2.53 & -1.90 \\
\hline 1.75 & 2.33 & 3.01 & -0.68 \\
\hline 2.00 & 2.09 & 3.01 & -1.65 \\
\hline 2.25 & 1.49 & 2.21 & -2.62 \\
\hline 2.50 & 0.65 & 1.24 & -3.35 \\
\hline 2.75 & 1.61 & 1.89 & -2.62 \\
\hline 3.00 & 0.88 & 1.56 & -2.63 \\
\hline 3.25 & 1.01 & 1.73 & -3.35 \\
\hline 3.50 & -0.20 & 0.60 & -4.32 \\
\hline 3.75 & 0.16 & 1.09 & -4.56 \\
\hline 4.00 & 0.64 & 1.57 & -3.59 \\
\hline 4.25 & 0.53 & 1.57 & -3.83 \\
\hline 4.50 & -0.32 & 0.92 & -4.80 \\
\hline 4.75 & 0.88 & 1.57 & -3.59 \\
\hline 5.00 & 0.53 & 1.73 & -4.56 \\
\hline 5.25 & 0.40 & 1.73 & -4.32 \\
\hline 5.50 & -0.92 & 0.76 & -4.80 \\
\hline 5.75 & 0.04 & 1.09 & -4.56 \\
\hline 6.00 & -0.25 & 1.44 & -4.60 \\
\hline 6.25 & -0.61 & 0.97 & -5.09 \\
\hline 6.50 & -1.45 & 0.48 & -5.09 \\
\hline 6.75 & -2.18 & -0.32 & -5.57 \\
\hline 7.00 & -2.42 & -0.48 & -6.30 \\
\hline 7.25 & -1.69 & 0.16 & -5.57 \\
\hline 7.50 & -2.18 & -0.32 & -5.57 \\
\hline 7.75 & -2.78 & -0.16 & -6.06 \\
\hline 8.00 & -3.02 & -0.32 & -6.30 \\
\hline 8.25 & -2.42 & -0.32 & -6.06 \\
\hline 8.50 & -2.90 & -0.64 & -5.57 \\
\hline 8.75 & -3.38 & -0.64 & -6.30 \\
\hline 9.00 & -4.35 & -1.44 & -6.54 \\
\hline 9.25 & -4.11 & -0.80 & -6.30 \\
\hline 9.50 & -4.23 & -1.12 & -6.54 \\
\hline 9.75 & -4.47 & -1.28 & -7.27 \\
\hline 10.00 & -4.59 & -1.12 & -6.78 \\
\hline & & & \\
\hline
\end{tabular}

\begin{tabular}{|c|c|c|c|}
\hline \multicolumn{4}{|c|}{$\mathbf{1 4 . 3 \%}$} \\
\hline Days & {$[\boldsymbol{\mu}]$} & {$[\boldsymbol{\mu \varepsilon}]$} & {$[\boldsymbol{\mu \varepsilon}]$} \\
\hline 10.25 & -4.83 & -1.44 & -7.02 \\
\hline 10.50 & -4.23 & -1.12 & -7.27 \\
\hline 10.75 & -4.71 & -0.80 & -7.27 \\
\hline 11.00 & -4.35 & -0.15 & -6.78 \\
\hline 11.25 & -4.95 & -1.12 & -7.02 \\
\hline 11.50 & -6.52 & -1.92 & -7.75 \\
\hline 11.75 & -5.43 & -1.60 & -7.27 \\
\hline 12.00 & -5.07 & -1.44 & -7.03 \\
\hline 12.25 & -5.67 & -1.60 & -7.51 \\
\hline 12.50 & -6.03 & -1.60 & -8.24 \\
\hline 12.75 & -5.31 & -1.44 & -8.00 \\
\hline 13.00 & -6.27 & -1.76 & -9.93 \\
\hline 13.25 & -5.91 & -1.76 & -9.45 \\
\hline 13.50 & -6.27 & -1.60 & -9.20 \\
\hline 13.75 & -7.00 & -2.73 & -10.42 \\
\hline 14.00 & -7.00 & -2.24 & -10.17 \\
\hline 14.25 & -6.76 & -2.09 & -9.93 \\
\hline 14.50 & -6.52 & -2.25 & -10.42 \\
\hline 14.75 & -7.36 & -3.05 & -11.38 \\
\hline 15.00 & -5.92 & -3.05 & -10.42 \\
\hline 15.25 & -6.28 & -2.73 & -9.93 \\
\hline 15.50 & -8.69 & -4.82 & -12.11 \\
\hline 15.75 & -8.81 & -4.50 & -11.87 \\
\hline 16.00 & -7.73 & -3.53 & -11.14 \\
\hline 16.25 & -7.60 & -4.50 & -11.14 \\
\hline 16.50 & -8.69 & -4.34 & -11.63 \\
\hline 16.75 & -9.90 & -5.94 & -12.60 \\
\hline 17.00 & -9.42 & -6.26 & -12.35 \\
\hline 17.25 & -9.90 & -5.95 & -13.08 \\
\hline 17.50 & -9.66 & -5.63 & -12.60 \\
\hline 17.75 & -10.86 & -7.39 & -14.29 \\
\hline 18.00 & -10.02 & -6.10 & -12.84 \\
\hline 18.25 & -10.86 & -6.91 & -13.32 \\
\hline 18.50 & -11.47 & -7.23 & -13.08 \\
\hline 18.75 & -11.59 & -8.19 & -14.05 \\
\hline 19.00 & -12.08 & -8.52 & -14.30 \\
\hline & & & \\
\hline
\end{tabular}


Table C-6 - Restrained, sealed measurements for $25.3 \% \mathrm{k}$ mixture

\begin{tabular}{|c|c|c|c|}
\hline \multicolumn{4}{|c|}{$\mathbf{2 5 . 3 \% \mathbf { k }}$} \\
\hline Days & {$[\boldsymbol{\mu} \varepsilon]$} & {$[\boldsymbol{\mu \varepsilon}]$} & {$[\boldsymbol{[ \mu \varepsilon ]}$} \\
\hline 0.00 & 0.00 & 0.00 & 0.00 \\
\hline 0.25 & -3.89 & -4.74 & -3.08 \\
\hline 0.50 & -15.30 & -15.19 & -13.32 \\
\hline 0.75 & -7.54 & -7.42 & -5.76 \\
\hline 1.00 & -6.10 & -5.50 & -3.72 \\
\hline 1.25 & -4.92 & -3.97 & -2.91 \\
\hline 1.50 & -2.90 & -2.31 & -0.97 \\
\hline 1.75 & -2.62 & -2.39 & -1.00 \\
\hline 2.00 & -3.46 & -2.39 & -1.49 \\
\hline 2.25 & -1.65 & -1.90 & -0.36 \\
\hline 2.50 & -0.80 & -1.18 & 0.61 \\
\hline 2.75 & -0.44 & -0.94 & 1.09 \\
\hline 3.00 & -0.80 & -0.70 & 0.61 \\
\hline 3.25 & -0.93 & -0.94 & 1.25 \\
\hline 3.50 & -1.04 & -0.82 & 0.93 \\
\hline 3.75 & -0.68 & -1.42 & 0.61 \\
\hline 4.00 & -1.05 & -0.82 & 0.61 \\
\hline 4.25 & -0.92 & -1.30 & 1.26 \\
\hline 4.50 & -0.92 & -1.30 & 1.26 \\
\hline 4.75 & -0.44 & -1.18 & 1.42 \\
\hline 5.00 & -0.20 & -0.46 & 2.06 \\
\hline 5.25 & -0.68 & -0.94 & 1.41 \\
\hline 5.50 & -1.16 & -1.06 & 1.42 \\
\hline 5.75 & -1.29 & -1.54 & 0.93 \\
\hline 6.00 & -0.61 & -0.86 & 1.94 \\
\hline 6.25 & -0.72 & -0.62 & 1.78 \\
\hline 6.50 & -1.09 & -1.22 & 1.46 \\
\hline 6.75 & -1.09 & -1.22 & 1.13 \\
\hline 7.00 & -1.09 & -0.74 & 1.61 \\
\hline 7.25 & -1.33 & -1.35 & 1.45 \\
\hline 7.50 & -1.09 & -1.10 & 1.62 \\
\hline 7.75 & -1.21 & -0.98 & 1.62 \\
\hline 8.00 & -2.05 & -1.82 & 0.81 \\
\hline 8.25 & -1.81 & -2.07 & 1.29 \\
\hline 8.50 & -2.54 & -2.67 & 0.49 \\
\hline 8.75 & -2.42 & -2.67 & 0.97 \\
\hline 9.00 & -2.90 & -3.15 & 0.16 \\
\hline & & & \\
\hline
\end{tabular}

\begin{tabular}{|c|c|c|c|}
\hline \multicolumn{4}{|c|}{$\mathbf{2 5 . 3 \% \mathbf { k }}$} \\
\hline Days & {$[\boldsymbol{\mu \varepsilon}]$} & {$[\boldsymbol{\mu \varepsilon}]$} & {$[\boldsymbol{[ \mu \varepsilon ]}$} \\
\hline 9.25 & -2.78 & -2.91 & 0.81 \\
\hline 9.50 & -3.38 & -2.79 & 0.00 \\
\hline 9.75 & -3.02 & -3.39 & -0.65 \\
\hline 10.00 & -3.63 & -3.27 & -0.16 \\
\hline 10.25 & -3.02 & -2.91 & 0.49 \\
\hline 10.50 & -2.54 & -2.43 & 0.97 \\
\hline 10.75 & -2.42 & -2.43 & 0.65 \\
\hline 11.00 & -3.02 & -2.79 & 0.65 \\
\hline 11.25 & -2.90 & -2.79 & 0.81 \\
\hline 11.50 & -2.54 & -2.55 & 0.81 \\
\hline 11.75 & -2.66 & -2.07 & 1.45 \\
\hline 12.00 & -2.30 & -2.19 & 1.45 \\
\hline 12.25 & -2.29 & -2.67 & 1.29 \\
\hline 12.50 & -2.17 & -1.82 & 1.13 \\
\hline 12.75 & -1.93 & -2.19 & 1.29 \\
\hline 13.00 & -2.66 & -2.43 & 1.29 \\
\hline
\end{tabular}




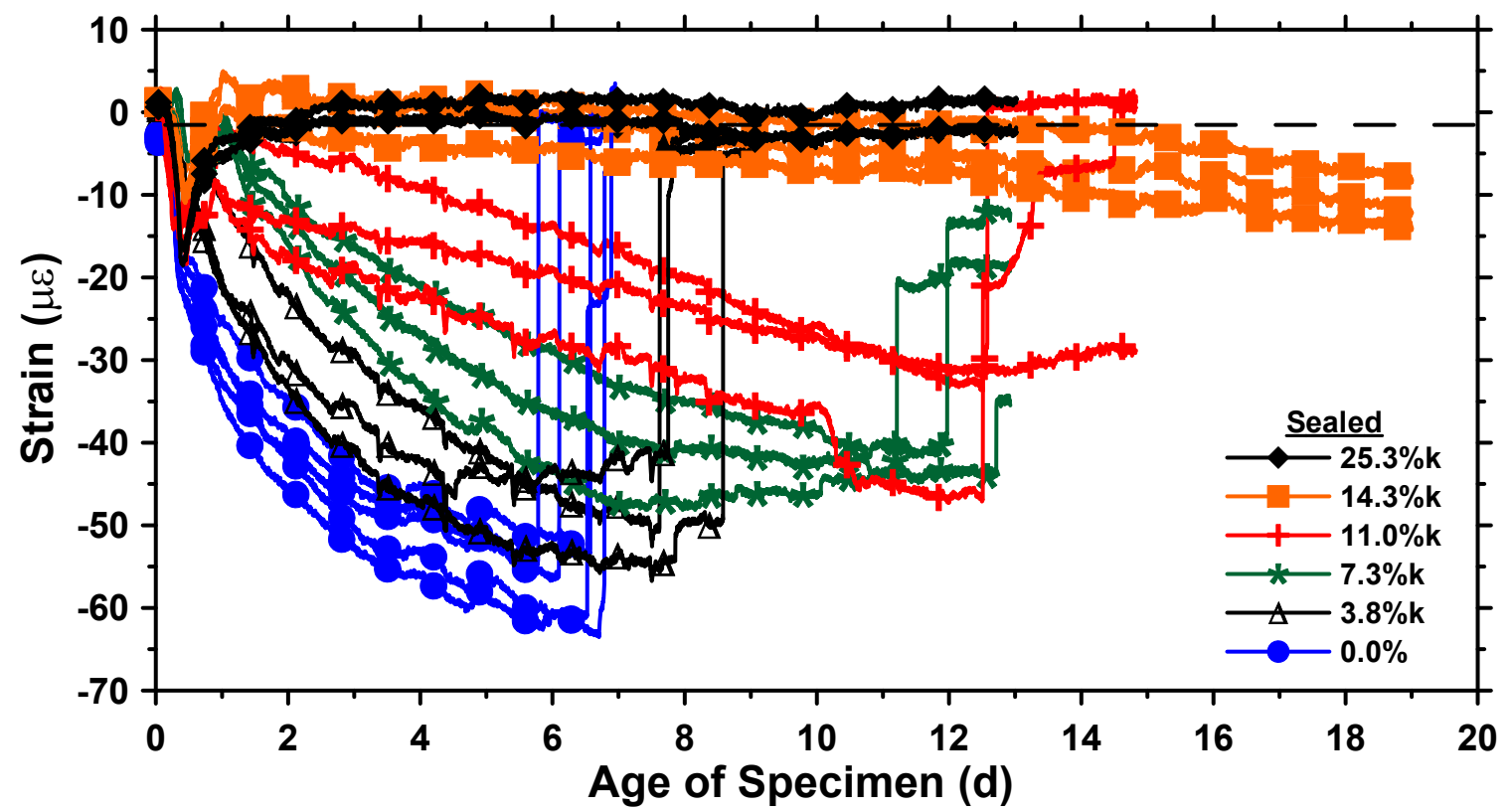

Figure C-1 - All restrained, sealed shrinkage measurements

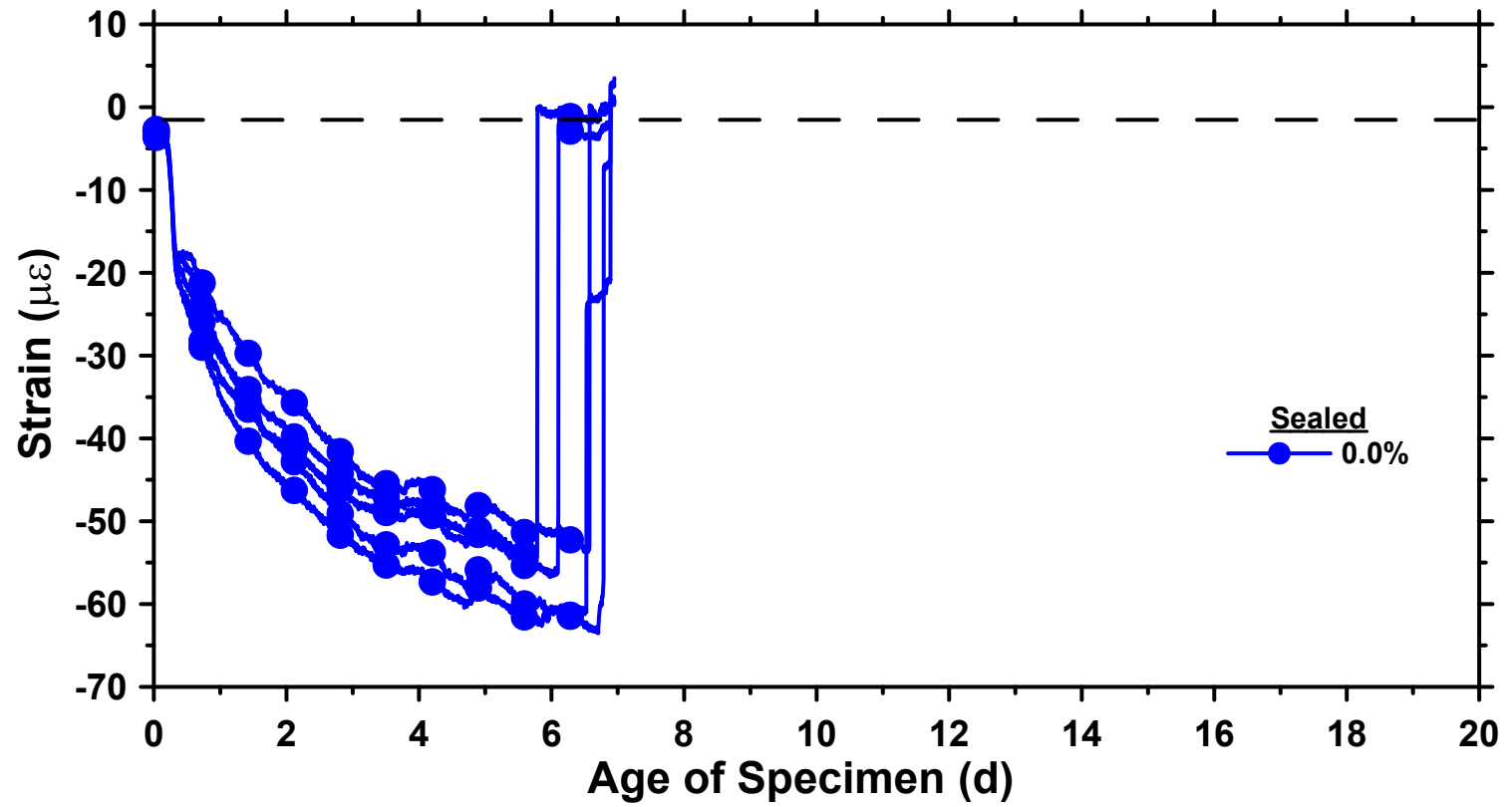

Figure C-2 - Restrained, sealed shrinkage for $0.0 \%$ mixture 


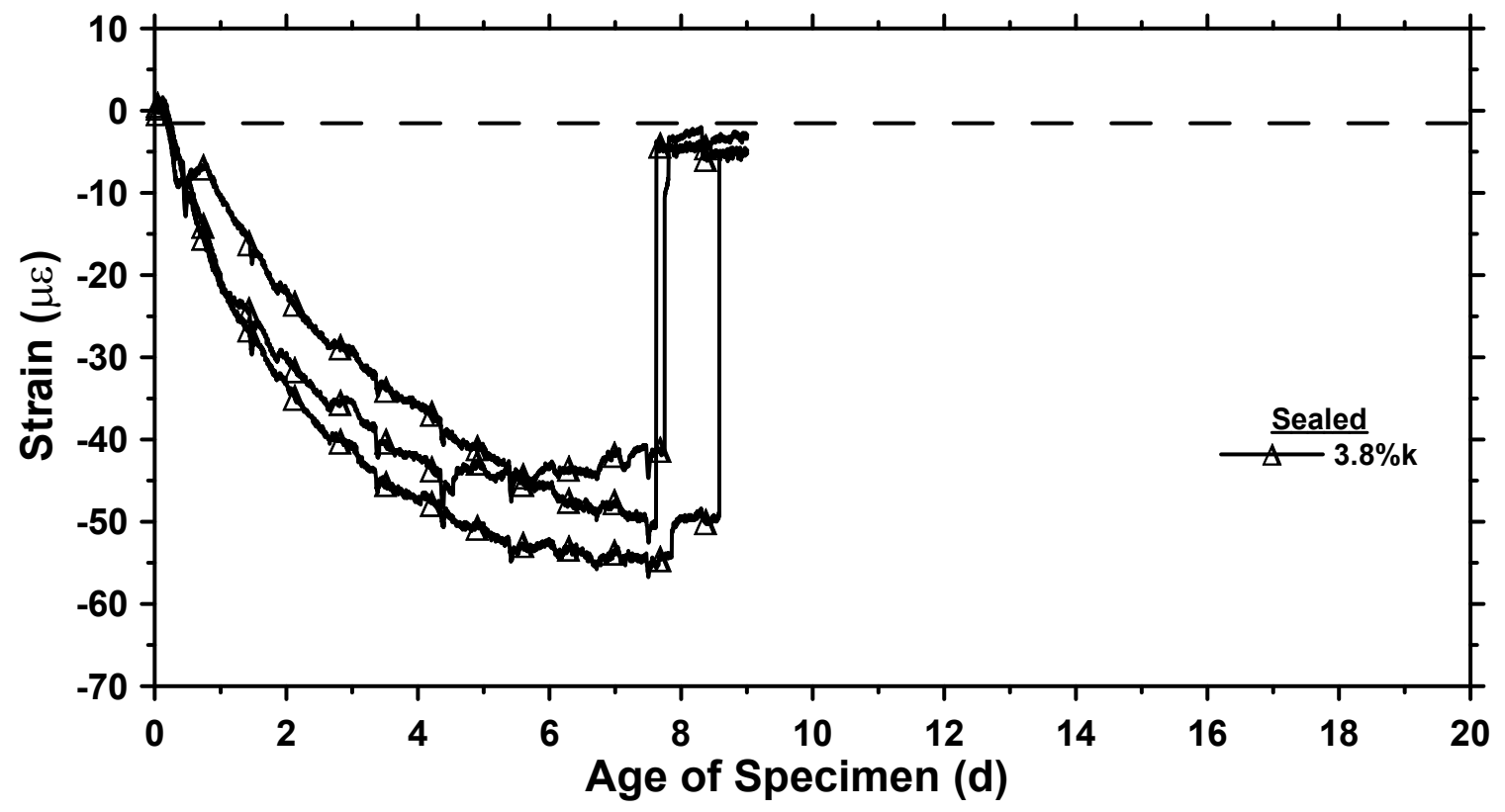

Figure C-3 - Restrained, sealed shrinkage for 3.8\%k mixture

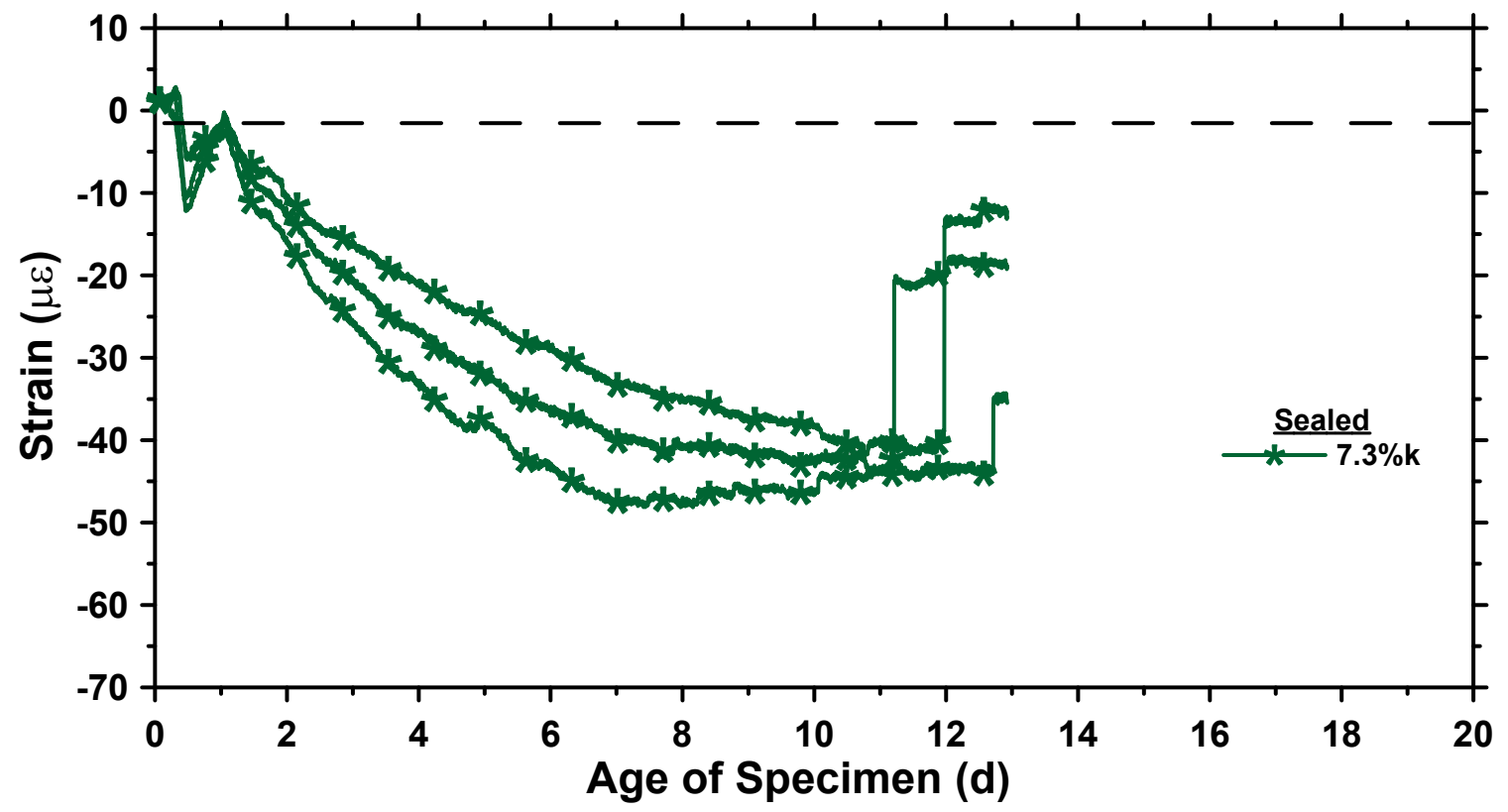

Figure C-4 - Restrained, sealed shrinkage for $7.3 \% \mathrm{k}$ mixture 


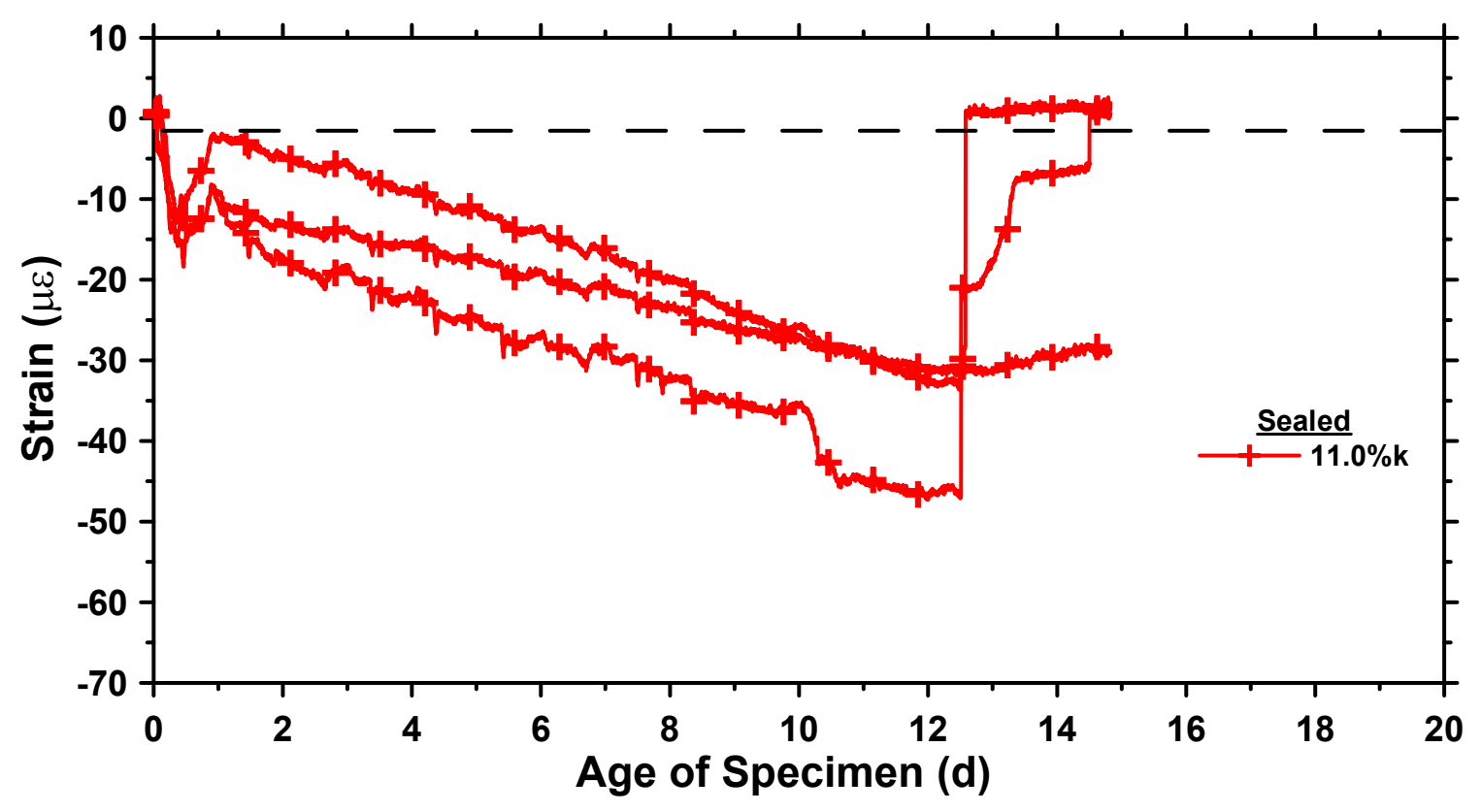

Figure C-5 - Restrained, sealed shrinkage for $11.0 \% \mathrm{k}$ mixture

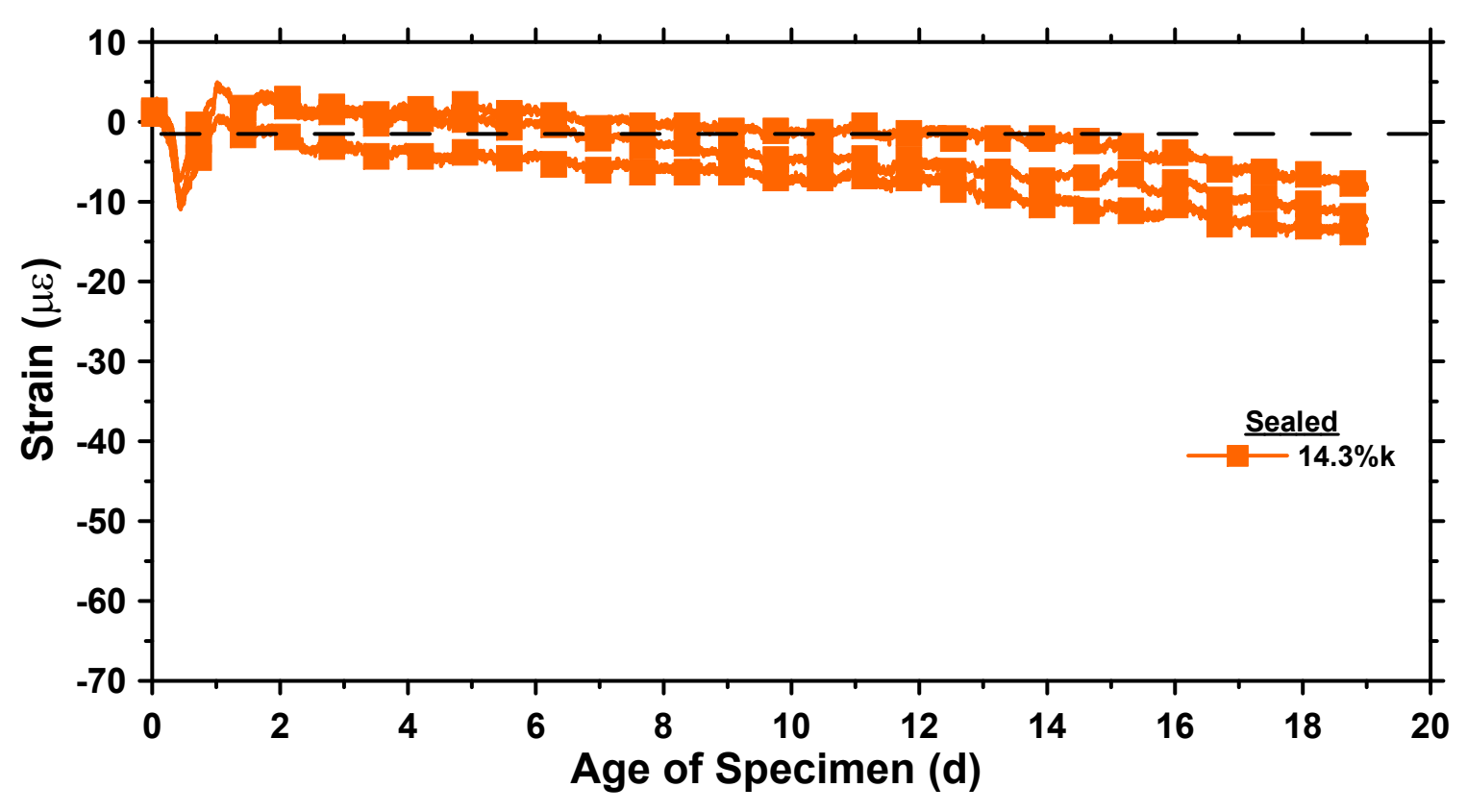

Figure C-6 - Restrained, sealed shrinkage for $14.3 \% \mathrm{k}$ mixture 


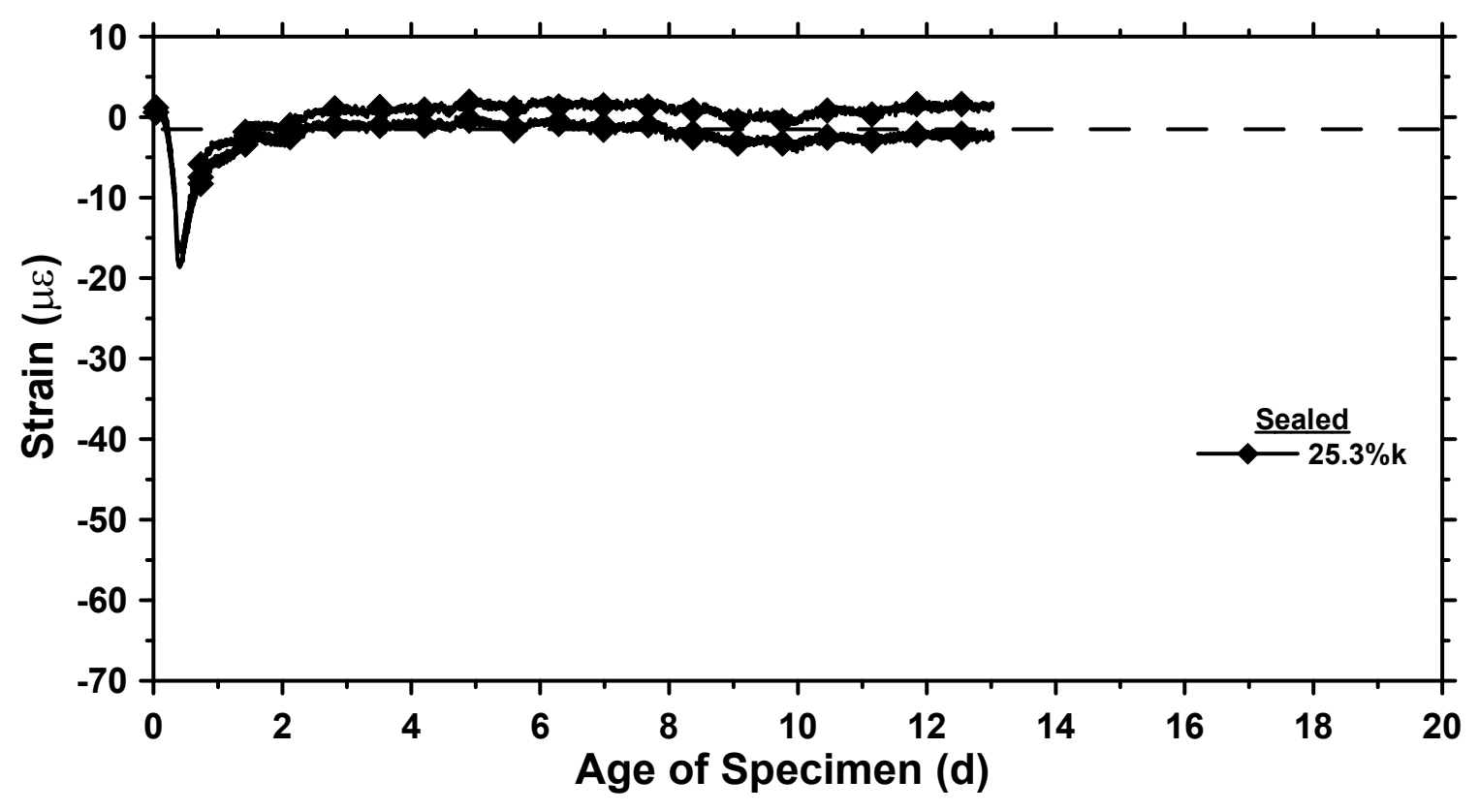

Figure C-7 - Restrained, sealed shrinkage for $25.3 \%$ k mixture 
Table C-7 - Restrained, unsealed measurements for $0.0 \%$ mixture

\begin{tabular}{|c|c|c|c|c|c|c|}
\hline \multicolumn{7}{|c|}{$\mathbf{0 . 0 \%}$} \\
\hline Days & {$[\boldsymbol{\mu \varepsilon}]$} & {$[\boldsymbol{\mu \varepsilon}]$} & {$[\boldsymbol{\mu \varepsilon}]$} & {$[\boldsymbol{\mu \varepsilon}]$} & {$[\boldsymbol{\mu \varepsilon}]$} & {$[\boldsymbol{\mu \varepsilon}]$} \\
\hline 0.00 & 0.00 & 0.00 & 0.00 & 0.00 & 0.00 & 0.00 \\
\hline 0.25 & 1.23 & 2.46 & 0.64 & 2.93 & 1.00 & 2.94 \\
\hline 0.50 & -7.33 & -4.06 & -4.55 & -5.76 & -4.55 & 0.17 \\
\hline 0.75 & -12.54 & -9.79 & -9.43 & -17.04 & -11.12 & -6.54 \\
\hline 1.00 & -18.74 & -16.26 & -15.77 & -25.32 & -17.34 & -13.63 \\
\hline 1.25 & -31.26 & -28.59 & -28.57 & -37.76 & -30.87 & -27.68 \\
\hline 1.50 & -40.03 & -37.53 & -37.14 & -46.93 & -40.29 & -37.48 \\
\hline 1.75 & -46.90 & -44.29 & 0.40 & -54.66 & -47.29 & -45.35 \\
\hline 2.00 & -51.95 & -50.80 & 3.42 & 5.24 & -50.91 & 8.52 \\
\hline 2.25 & -56.88 & 6.09 & 5.96 & 5.48 & 6.57 & 8.76 \\
\hline 2.50 & 5.94 & 6.33 & 6.20 & 5.48 & 6.57 & 9.12 \\
\hline 2.75 & 5.94 & 6.33 & 6.20 & 5.48 & 6.57 & 9.12 \\
\hline 3.00 & 5.94 & 6.33 & 6.20 & 5.48 & 6.57 & 9.12 \\
\hline
\end{tabular}

Table C-8 - Restrained, unsealed measurements for $7.3 \% \mathrm{k}$ mixture

\begin{tabular}{|c|c|c|c|}
\hline \multicolumn{4}{|c|}{$\mathbf{7 . 3 \% k}$} \\
\hline Days & {$[\boldsymbol{\mu \varepsilon}]$} & {$[\boldsymbol{\mu} \varepsilon]$} & {$[\boldsymbol{\mu \varepsilon}]$} \\
\hline 0.00 & 0.00 & 0.00 & 0.00 \\
\hline 0.25 & 1.06 & 0.64 & 0.94 \\
\hline 0.50 & -8.36 & -7.42 & -6.65 \\
\hline 0.75 & -5.45 & -6.25 & -4.13 \\
\hline 1.00 & -11.90 & -14.05 & -10.12 \\
\hline 1.25 & -22.69 & -24.55 & -23.23 \\
\hline 1.50 & -30.74 & -32.05 & -33.12 \\
\hline 1.75 & -36.51 & -37.82 & -41.31 \\
\hline 2.00 & -41.68 & 3.64 & 4.75 \\
\hline 2.25 & 5.10 & 3.33 & 5.84 \\
\hline 2.50 & 5.10 & 3.33 & 5.84 \\
\hline 2.75 & 5.10 & 3.33 & 5.84 \\
\hline 3.00 & 5.10 & 3.33 & 5.84 \\
\hline
\end{tabular}


Table C-9 - Restrained, unsealed measurements for $11.0 \% \mathrm{k}$ mixture

\begin{tabular}{|c|c|c|c|}
\hline \multicolumn{4}{|c|}{$\mathbf{1 1 . 0 \% k}$} \\
\hline Days & {$[\boldsymbol{\mu \varepsilon}]$} & {$[\boldsymbol{\mu \varepsilon}]$} & {$[\boldsymbol{\mu \varepsilon}]$} \\
\hline 0.00 & 0.00 & 0.00 & 0.00 \\
\hline 0.25 & -0.85 & -4.09 & -5.14 \\
\hline 0.50 & -2.14 & -6.46 & -7.75 \\
\hline 0.75 & 0.11 & -4.21 & -4.86 \\
\hline 1.00 & -4.13 & -8.95 & -6.88 \\
\hline 1.25 & -13.79 & -15.36 & -17.02 \\
\hline 1.50 & -21.38 & -23.56 & -26.45 \\
\hline 1.75 & -26.67 & -28.14 & -32.09 \\
\hline 2.00 & -31.01 & -30.07 & -36.60 \\
\hline 2.25 & 4.98 & -30.79 & -37.73 \\
\hline 2.50 & 5.22 & -28.63 & -33.22 \\
\hline 2.75 & 4.98 & 6.84 & 2.38 \\
\hline 3.00 & 5.58 & 6.11 & 2.38 \\
\hline
\end{tabular}

Table C-10 - Restrained, unsealed measurements for $14.3 \% \mathrm{k}$ mixture

\begin{tabular}{|c|c|c|c|}
\hline \multicolumn{4}{|c|}{$\mathbf{1 4 . 3 \% k}$} \\
\hline Days & {$[\boldsymbol{\mu \varepsilon}]$} & {$[\boldsymbol{\mu} \varepsilon]$} & {$[\boldsymbol{\mu} \varepsilon]$} \\
\hline 0.00 & 0.00 & 0.00 & 0.00 \\
\hline 0.25 & 1.99 & 1.87 & 1.31 \\
\hline 0.50 & -5.52 & -6.84 & -6.51 \\
\hline 0.75 & 0.05 & -0.31 & -3.24 \\
\hline 1.00 & 0.82 & 2.40 & -1.15 \\
\hline 1.25 & -6.84 & -4.77 & -9.54 \\
\hline 1.50 & -12.51 & -11.16 & -17.12 \\
\hline 1.75 & -17.34 & -16.23 & -22.91 \\
\hline 2.00 & -20.59 & -21.66 & -27.27 \\
\hline 2.25 & -23.49 & -26.00 & -29.52 \\
\hline 2.50 & -24.57 & -28.78 & -30.81 \\
\hline 2.75 & -26.38 & -29.27 & -3.73 \\
\hline 3.00 & 7.65 & -0.21 & -5.83 \\
\hline
\end{tabular}


Table C-11 - Restrained, unsealed measurements for $25.3 \% \mathrm{k}$ mixture

\begin{tabular}{|c|c|c|c|}
\hline \multicolumn{4}{|c|}{$\mathbf{2 5 . 3 \% k}$} \\
\hline Days & {$[\boldsymbol{\mu \varepsilon}]$} & {$[\boldsymbol{\mu \varepsilon}]$} & {$[\boldsymbol{\mu \varepsilon}]$} \\
\hline 0.00 & 0.00 & 0.00 & 0.00 \\
\hline 0.25 & -0.29 & -0.02 & -1.72 \\
\hline 0.50 & -3.65 & -4.21 & -7.13 \\
\hline 0.75 & 0.57 & -0.01 & -2.68 \\
\hline 1.00 & 2.65 & 1.82 & 1.09 \\
\hline 1.25 & -1.20 & -3.14 & -0.97 \\
\hline 1.50 & -5.41 & -7.85 & -6.52 \\
\hline 1.75 & -9.02 & -11.60 & -10.26 \\
\hline 2.00 & -11.67 & -15.22 & -13.76 \\
\hline 2.25 & -14.67 & -17.15 & -16.29 \\
\hline 2.50 & -16.72 & -19.09 & -17.98 \\
\hline 2.75 & -21.17 & -23.31 & -26.07 \\
\hline 3.00 & -23.82 & -25.24 & -29.45 \\
\hline 3.25 & -21.29 & -22.24 & -23.17 \\
\hline 3.50 & -22.25 & -21.87 & -22.09 \\
\hline 3.75 & -24.06 & -22.72 & -22.93 \\
\hline 4.00 & -26.10 & -24.53 & -26.19 \\
\hline 4.25 & -26.94 & -23.21 & -23.06 \\
\hline 4.50 & -27.79 & -23.33 & -21.62 \\
\hline 4.75 & -27.91 & -22.85 & -19.32 \\
\hline 5.00 & -28.75 & -23.94 & 5.67 \\
\hline 5.25 & -29.95 & -25.39 & 5.19 \\
\hline 5.50 & -31.27 & -25.16 & 5.55 \\
\hline 5.75 & -32.96 & -25.28 & 5.19 \\
\hline 6.00 & -34.76 & -25.52 & 4.83 \\
\hline 6.25 & -34.52 & -25.28 & 4.59 \\
\hline 6.50 & -37.05 & -25.04 & 4.71 \\
\hline 6.75 & -35.37 & -23.47 & 4.59 \\
\hline 7.00 & -37.30 & -24.44 & 1.93 \\
\hline 7.25 & 4.33 & -22.39 & 4.59 \\
\hline 7.50 & 4.57 & 7.73 & 5.19 \\
\hline & & & \\
\hline & & \\
\hline
\end{tabular}


Table C-12 - Restrained, unsealed measurements for $29.3 \% \mathrm{k}$ mixture

\begin{tabular}{|l|l|l|l|}
\hline \multicolumn{4}{|c|}{$\mathbf{2 9 . 3 \% k}$} \\
\hline Days & {$[\boldsymbol{\mu \varepsilon}]$} & {$[\boldsymbol{\mu \varepsilon}]$} & {$[\boldsymbol{\mu \varepsilon}]$} \\
\hline 0.00 & 0.00 & 0.00 & 0.00 \\
\hline 0.25 & -2.24 & -1.15 & -1.87 \\
\hline 0.50 & -5.26 & -7.41 & -6.44 \\
\hline 0.75 & -2.04 & -3.60 & -4.57 \\
\hline 1.00 & -0.23 & -1.30 & -1.55 \\
\hline 1.25 & -0.20 & -0.55 & 0.29 \\
\hline 1.50 & -0.20 & -0.43 & -0.32 \\
\hline 1.75 & -1.53 & -1.53 & -1.53 \\
\hline 2.00 & -3.46 & -2.37 & -0.68 \\
\hline 2.25 & -4.67 & -3.21 & -1.41 \\
\hline 2.50 & -4.55 & -3.34 & -0.32 \\
\hline 2.75 & -6.96 & -4.79 & -2.02 \\
\hline 3.00 & -9.02 & -5.75 & -3.59 \\
\hline 3.25 & -7.81 & -5.15 & -2.02 \\
\hline 3.50 & -8.90 & -5.51 & -2.02 \\
\hline 3.75 & -12.04 & -7.43 & -4.68 \\
\hline 4.00 & -11.68 & -7.19 & -4.31 \\
\hline 4.25 & -10.83 & -6.47 & -3.47 \\
\hline 4.50 & -11.67 & -7.32 & -4.07 \\
\hline 4.75 & -13.24 & -8.04 & -5.52 \\
\hline 5.00 & -15.06 & -9.13 & -6.37 \\
\hline 5.25 & -14.09 & -8.89 & -5.76 \\
\hline 5.50 & -16.26 & -9.85 & -7.82 \\
\hline 5.75 & -17.71 & -11.30 & -8.91 \\
\hline 6.00 & -18.80 & -11.90 & -10.12 \\
\hline 6.25 & -18.56 & -11.90 & -10.60 \\
\hline 6.50 & -20.00 & -12.63 & -11.33 \\
\hline 6.75 & -19.40 & -13.23 & -11.57 \\
\hline 7.00 & -20.97 & -14.43 & -12.30 \\
\hline 7.25 & -22.05 & -14.80 & -13.99 \\
\hline 7.50 & -20.85 & -14.19 & -13.50 \\
\hline 7.75 & -20.72 & -14.19 & -13.99 \\
\hline 8.00 & -21.33 & -14.32 & -14.23 \\
\hline 8.25 & -21.32 & -14.56 & -14.95 \\
\hline 8.50 & -22.17 & -14.56 & -15.07 \\
\hline 8.75 & -21.69 & -14.43 & -15.56 \\
\hline 9.00 & -22.17 & -15.16 & -15.92 \\
\hline
\end{tabular}

\begin{tabular}{|c|c|c|c|}
\hline \multicolumn{4}{|c|}{$\mathbf{2 9 . 3 \% k}$} \\
\hline Days & {$[\boldsymbol{\mu \varepsilon}]$} & {$[\boldsymbol{\mu \varepsilon}]$} & {$[\boldsymbol{\mu \varepsilon}]$} \\
\hline 9.25 & -21.93 & -14.92 & -15.92 \\
\hline 9.50 & -22.89 & -16.73 & -17.37 \\
\hline 9.75 & -23.25 & -17.70 & -18.10 \\
\hline 10.00 & -23.61 & -18.06 & -18.94 \\
\hline 10.25 & -22.16 & -16.85 & -17.85 \\
\hline 10.50 & -22.41 & -17.94 & -19.06 \\
\hline 10.75 & -24.09 & -19.14 & -20.64 \\
\hline 11.00 & 1.50 & -19.14 & -21.36 \\
\hline 11.25 & -0.92 & -18.18 & -19.91 \\
\hline 11.50 & 1.14 & -18.30 & -20.03 \\
\hline 11.75 & 0.89 & -18.90 & -21.48 \\
\hline 12.00 & -0.07 & -19.02 & -21.48 \\
\hline 12.25 & 1.62 & -18.66 & -21.60 \\
\hline 12.50 & 2.35 & -18.54 & -21.72 \\
\hline 12.75 & 1.50 & -19.27 & -22.69 \\
\hline 13.00 & 0.53 & -19.15 & -22.32 \\
\hline 13.25 & 1.86 & -17.47 & -22.08 \\
\hline 13.50 & 2.47 & -17.35 & -21.96 \\
\hline 13.75 & 1.98 & -18.20 & -21.60 \\
\hline 14.00 & 0.77 & -19.40 & 0.77 \\
\hline 14.25 & 2.59 & -18.08 & 1.14 \\
\hline 14.50 & 3.19 & -18.44 & 0.66 \\
\hline 14.75 & 2.23 & -18.44 & 1.26 \\
\hline 15.00 & 2.83 & -18.56 & 1.14 \\
\hline
\end{tabular}


Table C-13 - Restrained, unsealed measurements for 33.0\%k mixture

\begin{tabular}{|c|c|c|c|}
\hline \multicolumn{4}{|c|}{$\mathbf{3 3 . 0 \% k}$} \\
\hline Days & {$[\boldsymbol{\mu} \boldsymbol{]}]$} & {$[\boldsymbol{\mu \varepsilon}]$} & {$[\boldsymbol{\mu \varepsilon}]$} \\
\hline 0.00 & 0.00 & 0.00 & 0.00 \\
\hline 0.25 & -7.18 & -2.61 & -2.76 \\
\hline 0.50 & -14.91 & -9.12 & -6.55 \\
\hline 0.75 & -9.63 & -6.50 & -2.89 \\
\hline 1.00 & -6.97 & -2.40 & 0.73 \\
\hline 1.25 & -8.13 & -2.13 & 2.05 \\
\hline 1.50 & -8.86 & -0.92 & 1.73 \\
\hline 1.75 & -10.31 & -0.92 & 1.57 \\
\hline 2.00 & -10.55 & -0.92 & 1.25 \\
\hline 2.25 & -10.55 & -0.68 & 0.92 \\
\hline 2.50 & -9.58 & -0.20 & 1.57 \\
\hline 2.75 & -11.99 & -0.44 & 0.44 \\
\hline 3.00 & -12.71 & -1.17 & 0.28 \\
\hline 3.25 & -10.55 & 0.52 & 2.05 \\
\hline 3.50 & -10.07 & 0.52 & 1.89 \\
\hline 3.75 & -12.23 & -0.20 & 0.60 \\
\hline 4.00 & -11.75 & 0.52 & 1.09 \\
\hline 4.25 & -11.27 & 1.24 & 2.37 \\
\hline 4.50 & -12.23 & 1.00 & 2.21 \\
\hline 4.75 & -13.43 & 0.28 & 2.21 \\
\hline 5.00 & -14.63 & -0.20 & 1.08 \\
\hline 5.25 & -12.95 & 1.24 & 2.85 \\
\hline 5.50 & -14.63 & 0.28 & 1.89 \\
\hline 5.75 & -15.60 & -0.20 & 1.25 \\
\hline 6.00 & -16.08 & -0.20 & 0.76 \\
\hline 6.25 & -16.32 & 1.00 & 1.40 \\
\hline 6.50 & -17.52 & 0.76 & 0.44 \\
\hline 6.75 & -18.48 & 1.24 & 0.76 \\
\hline 7.00 & -19.44 & 1.00 & 0.28 \\
\hline 7.25 & -19.44 & 0.04 & -0.37 \\
\hline 7.50 & -19.44 & 1.24 & 0.28 \\
\hline 7.75 & -19.20 & 1.73 & 0.60 \\
\hline 8.00 & -19.44 & 1.48 & 0.60 \\
\hline 8.25 & -19.92 & 1.97 & 0.76 \\
\hline 8.50 & -20.40 & 1.97 & 0.60 \\
\hline 8.75 & -20.40 & 2.21 & 0.76 \\
\hline 9.00 & -21.12 & 2.21 & 0.43 \\
\hline
\end{tabular}

\begin{tabular}{|c|c|c|c|}
\hline \multicolumn{4}{|c|}{$\mathbf{3 3 . 0 \% k}$} \\
\hline Days & {$[\boldsymbol{\mu \varepsilon}]$} & {$[\boldsymbol{\mu \varepsilon}]$} & {$[\boldsymbol{\mu \varepsilon}]$} \\
\hline 9.25 & -19.44 & 2.20 & 0.75 \\
\hline 9.50 & -20.88 & 2.20 & -0.21 \\
\hline 9.75 & -21.36 & 1.24 & -0.70 \\
\hline 10.00 & -21.84 & 1.24 & -1.02 \\
\hline 10.25 & -20.64 & 2.44 & -0.21 \\
\hline 10.50 & -21.84 & 1.24 & -1.02 \\
\hline 10.75 & -23.29 & 1.72 & -2.14 \\
\hline 11.00 & -23.53 & 1.24 & -2.31 \\
\hline 11.25 & -22.32 & 3.17 & -1.34 \\
\hline 11.50 & -23.53 & 2.69 & -1.82 \\
\hline 11.75 & -23.05 & 2.69 & -2.14 \\
\hline 12.00 & -24.01 & 2.20 & -3.11 \\
\hline 12.25 & -23.05 & 2.93 & -1.82 \\
\hline 12.50 & -24.01 & 2.20 & -2.95 \\
\hline 12.75 & -24.25 & 2.45 & -3.11 \\
\hline 13.00 & -24.25 & 2.44 & -2.95 \\
\hline 13.25 & -23.29 & 2.68 & -2.15 \\
\hline 13.50 & -24.49 & 3.17 & -2.31 \\
\hline 13.75 & -25.21 & 2.45 & -2.79 \\
\hline 14.00 & -26.41 & 2.69 & -4.56 \\
\hline 14.25 & -24.97 & 3.41 & -2.95 \\
\hline 14.50 & -25.93 & 3.65 & -3.27 \\
\hline 14.75 & -26.41 & 3.17 & -3.11 \\
\hline 15.00 & -28.10 & 2.69 & -4.56 \\
\hline 15.25 & -25.45 & 2.69 & -4.40 \\
\hline 15.50 & -26.65 & 3.65 & -4.40 \\
\hline 15.75 & -26.17 & 3.17 & -4.89 \\
\hline 16.00 & -25.93 & 2.93 & -5.05 \\
\hline 16.25 & -24.97 & 5.10 & -4.24 \\
\hline 16.50 & -24.73 & 5.82 & -3.92 \\
\hline 16.75 & -25.21 & 4.86 & -4.08 \\
\hline 17.00 & -25.45 & 4.86 & -4.56 \\
\hline 17.25 & -24.49 & 5.82 & -3.92 \\
\hline 17.50 & -24.73 & 5.10 & -4.88 \\
\hline 17.75 & -25.69 & 5.10 & -5.69 \\
\hline 18.00 & -25.45 & 5.34 & -5.21 \\
\hline & & & \\
\hline
\end{tabular}




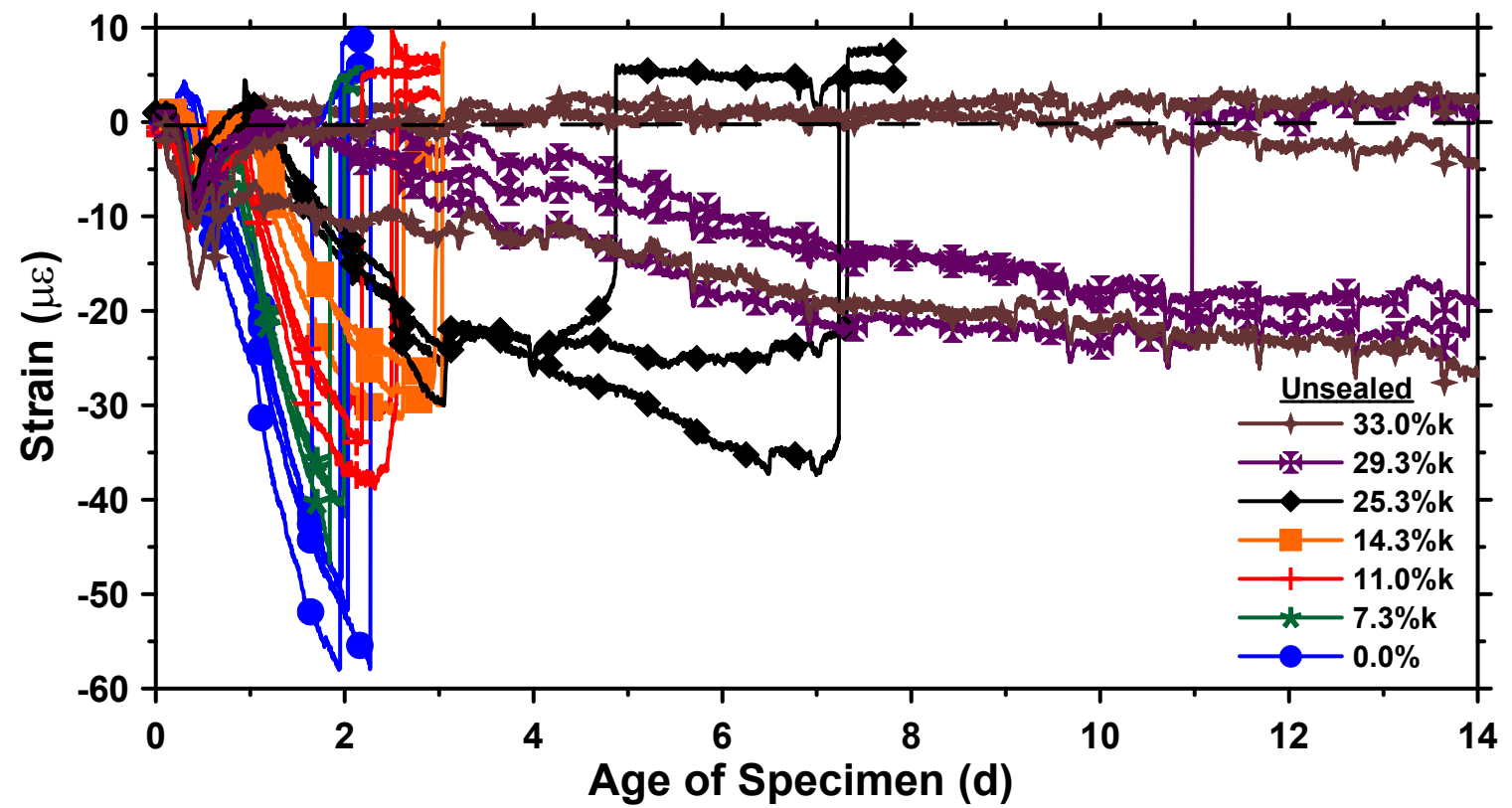

Figure C-8 - All restrained, unsealed shrinkage measurements

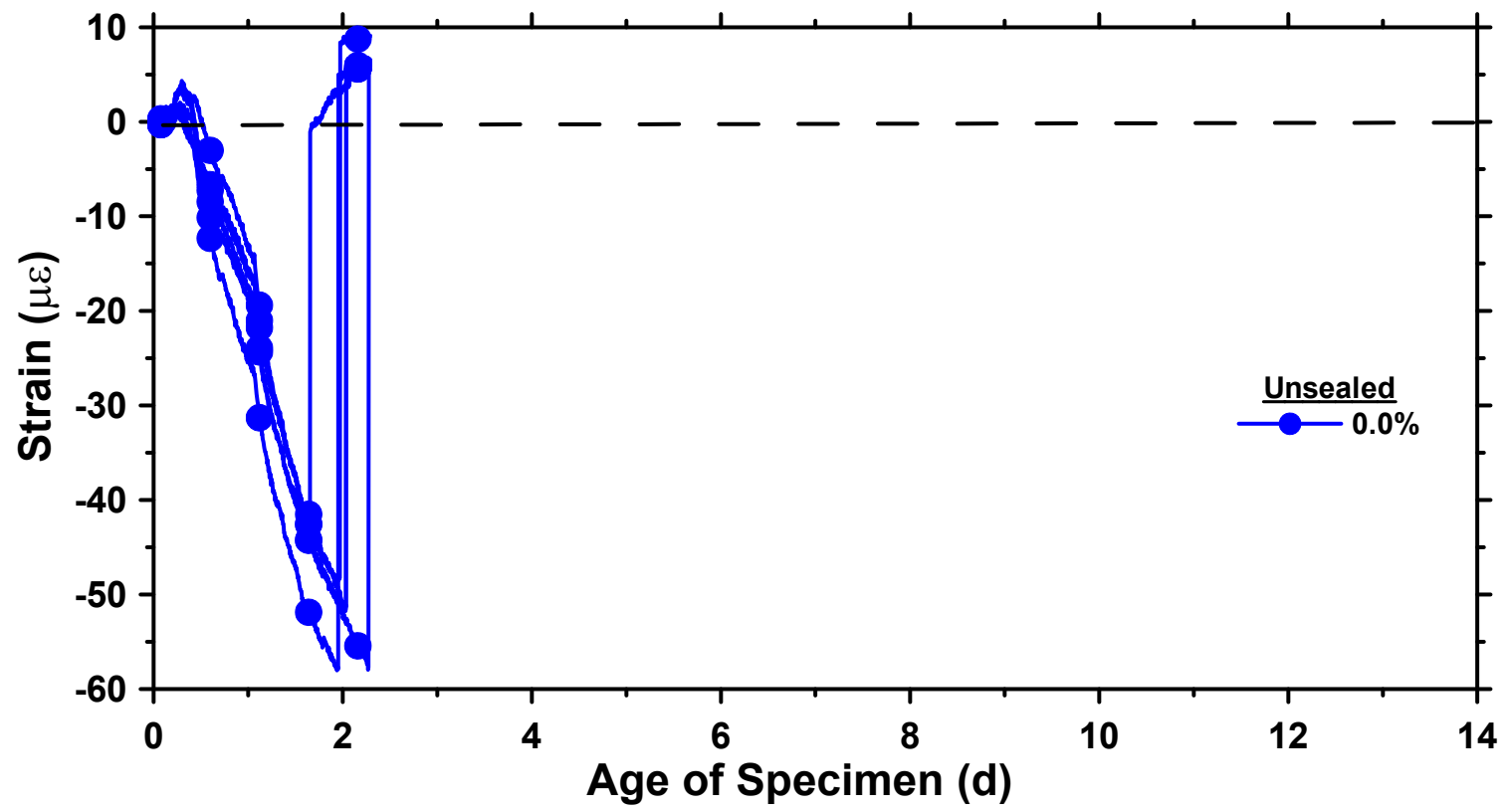

Figure C- 9 - Restrained, unsealed shrinkage for $0.0 \%$ mixture 


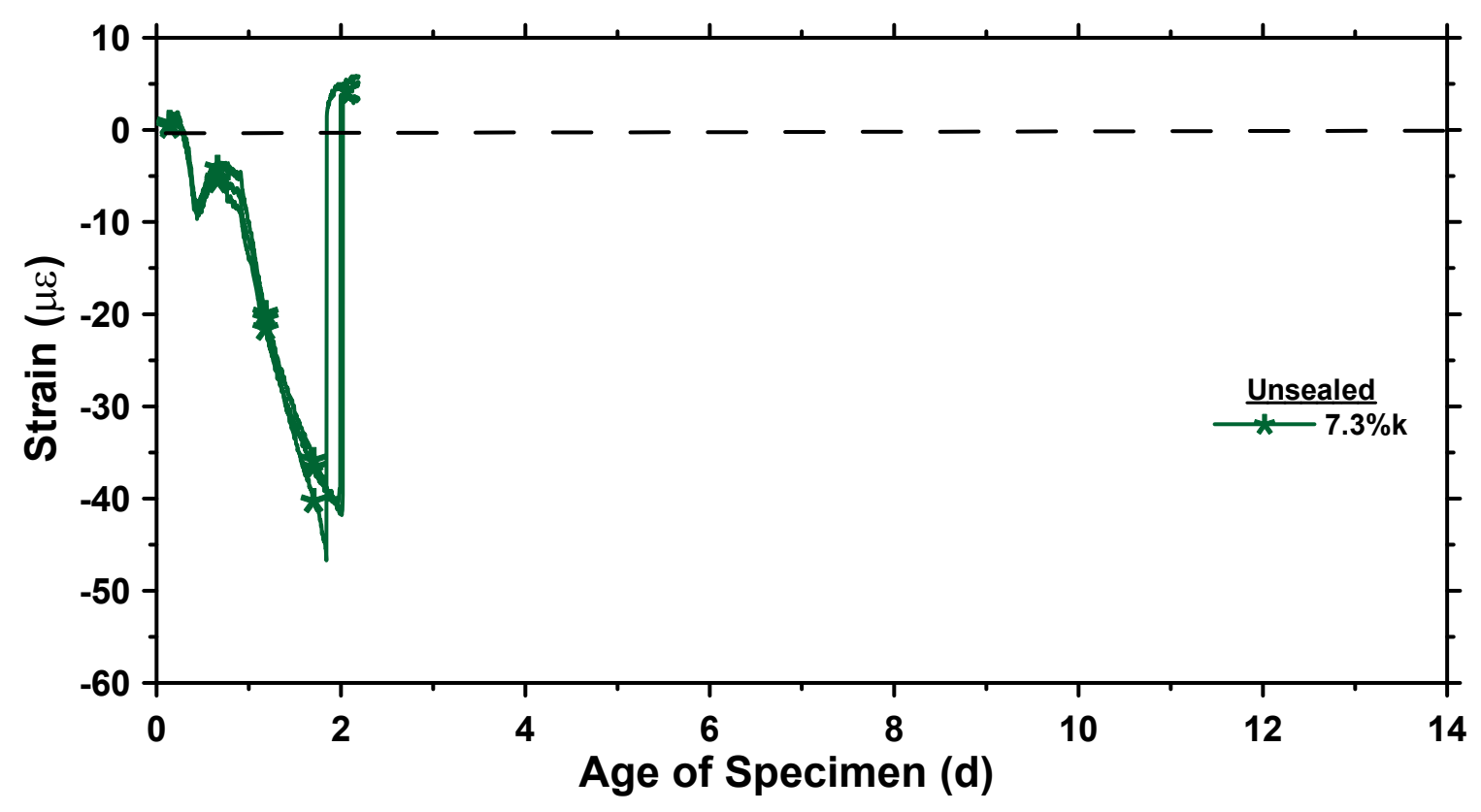

Figure C-10 - Restrained, unsealed shrinkage for $7.3 \%$ k mixture

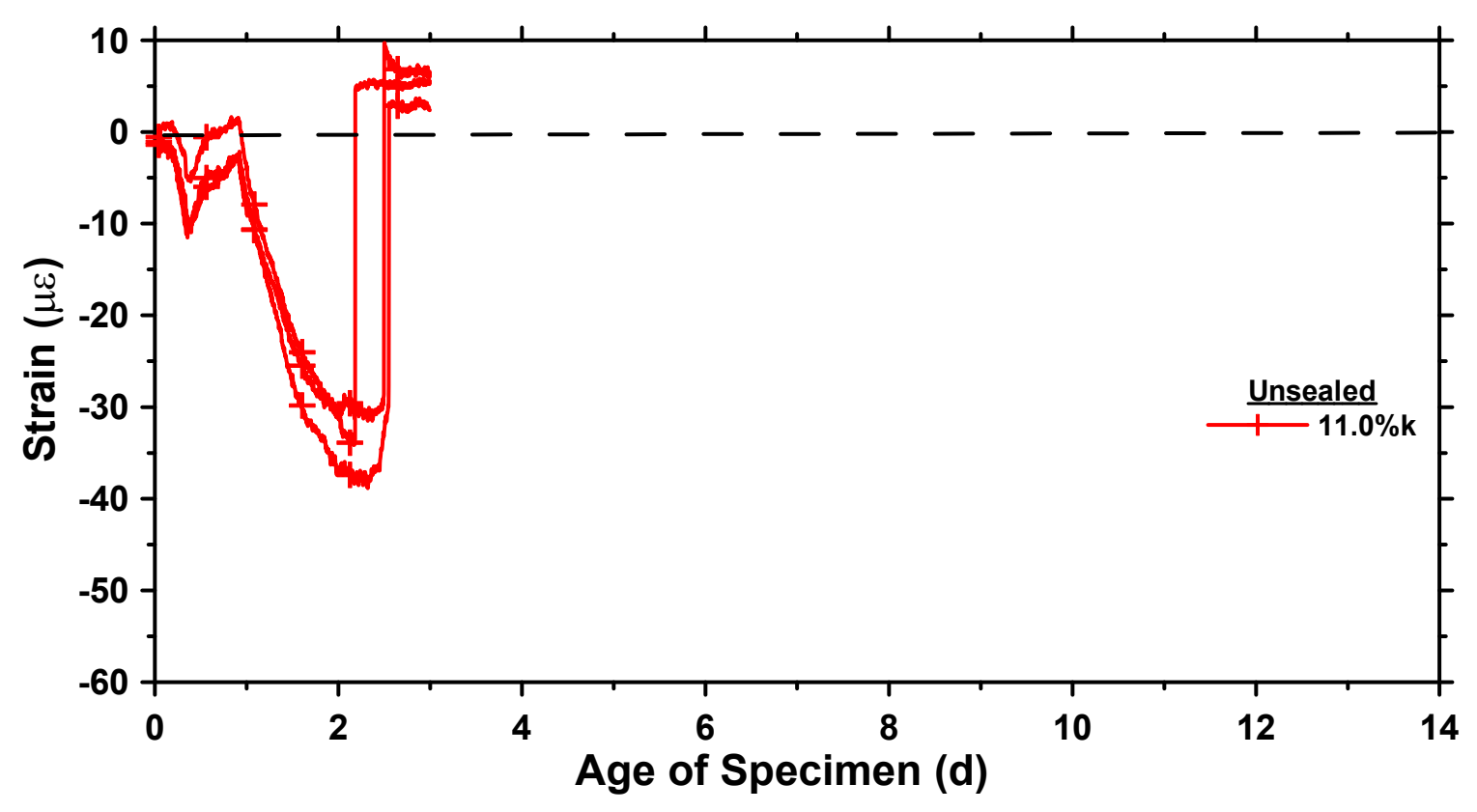

Figure C-11 - Restrained, unsealed shrinkage for $11.0 \% \mathrm{k}$ mixture 


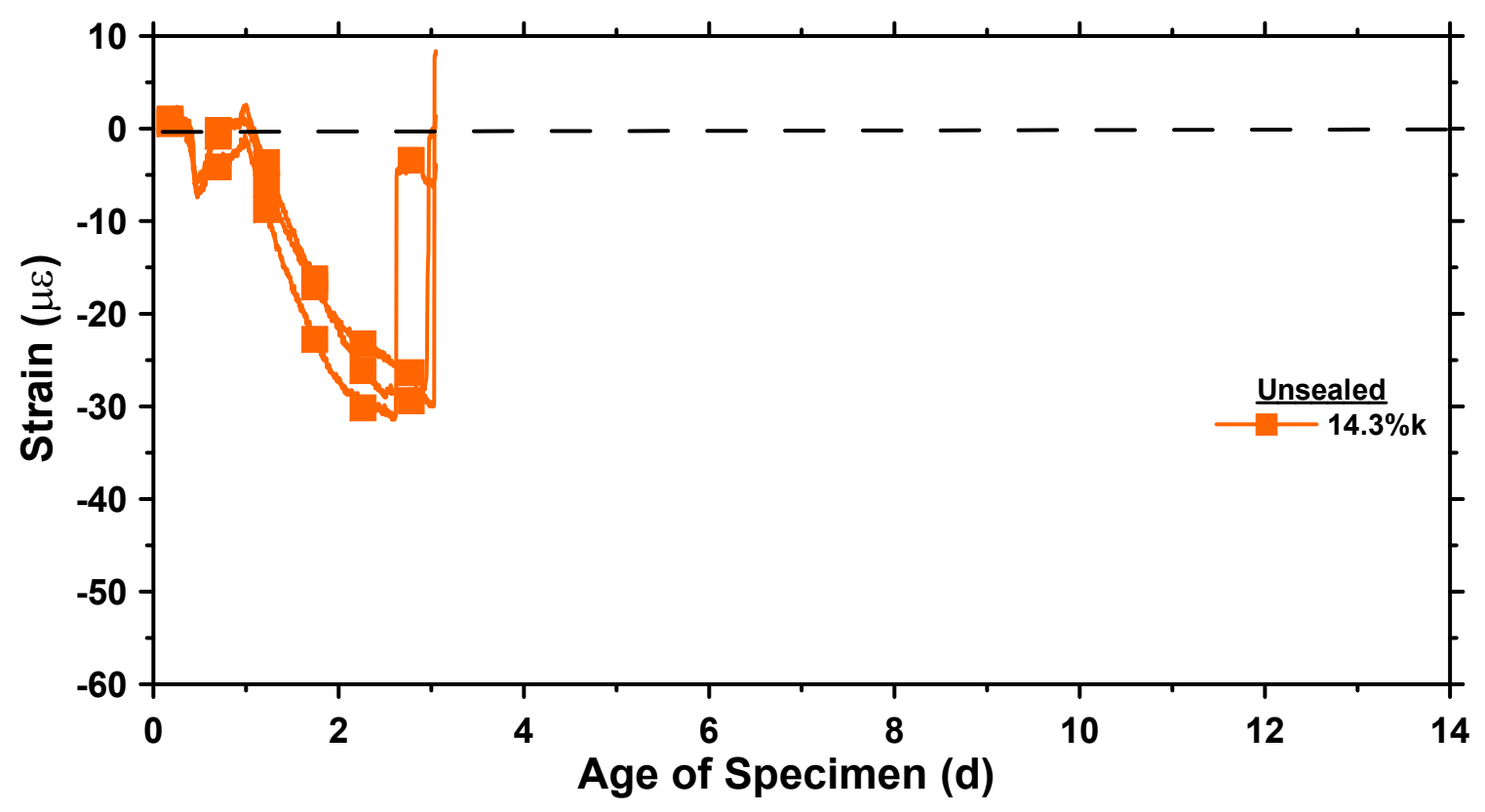

Figure C-12 - Restrained, unsealed shrinkage for $14.3 \% \mathrm{k}$ mixture

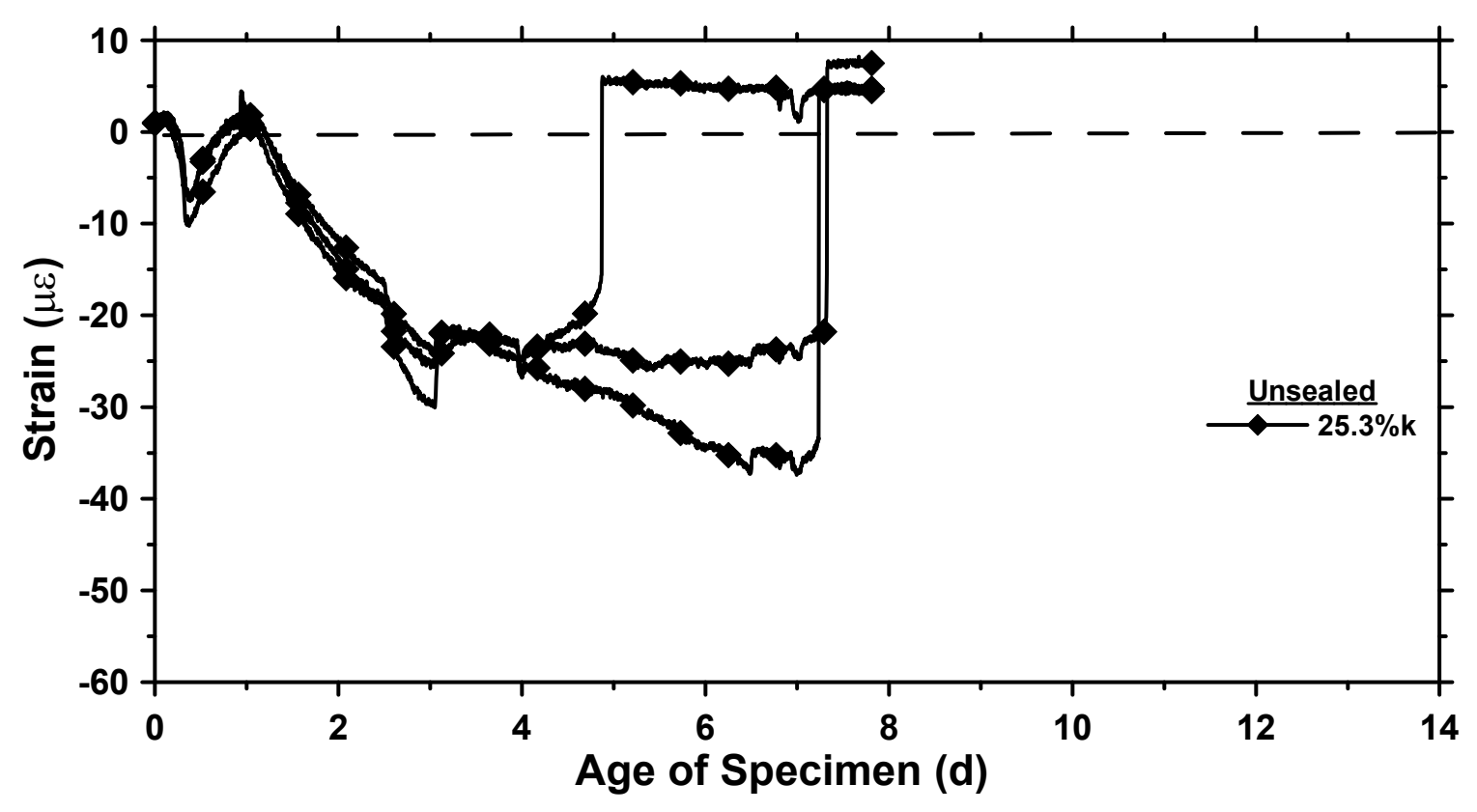

Figure C-13 - Restrained, unsealed shrinkage for $25.3 \% \mathrm{k}$ mixture 


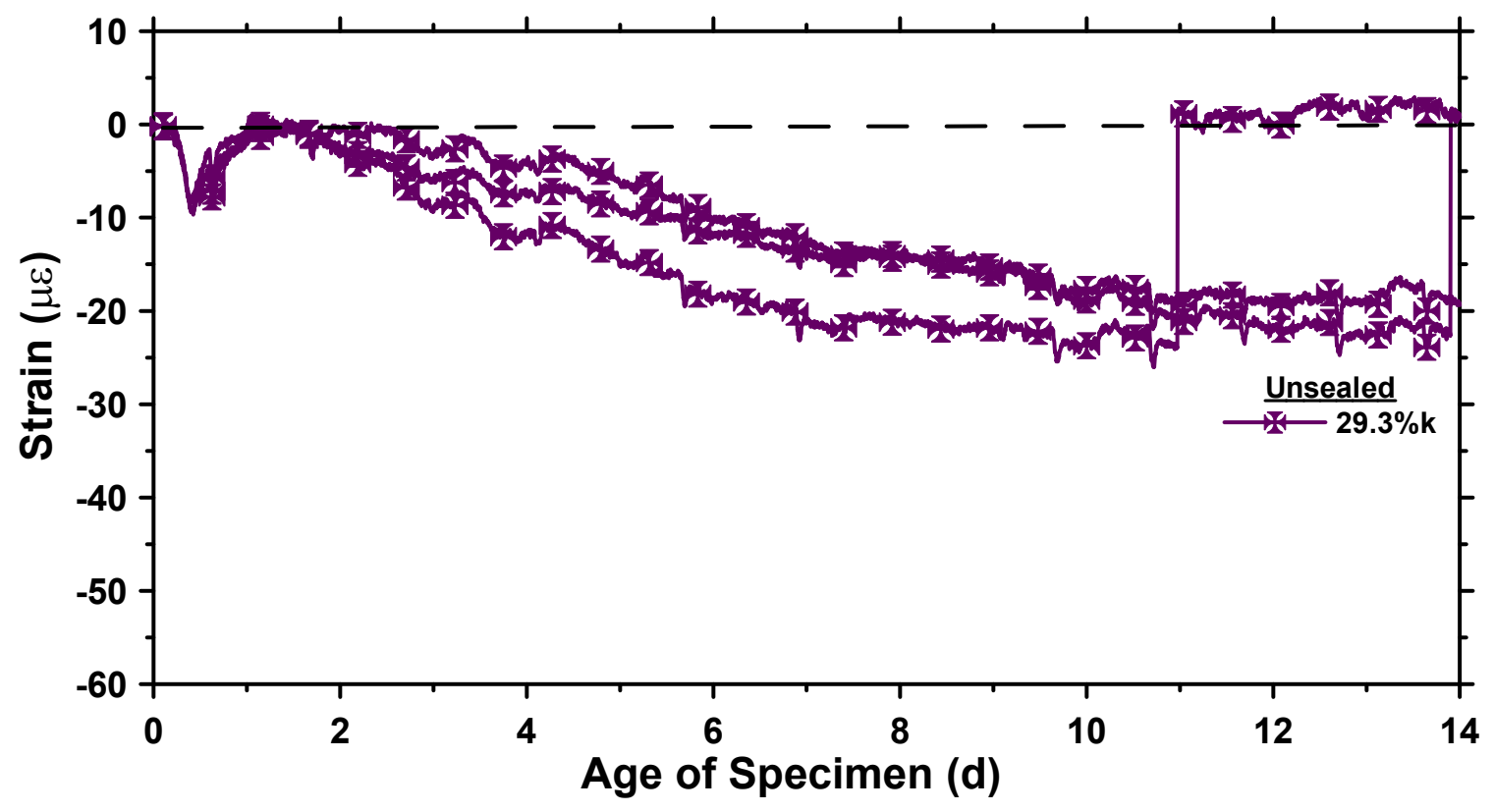

Figure C-14 - Restrained, unsealed shrinkage for $29.3 \%$ k mixture

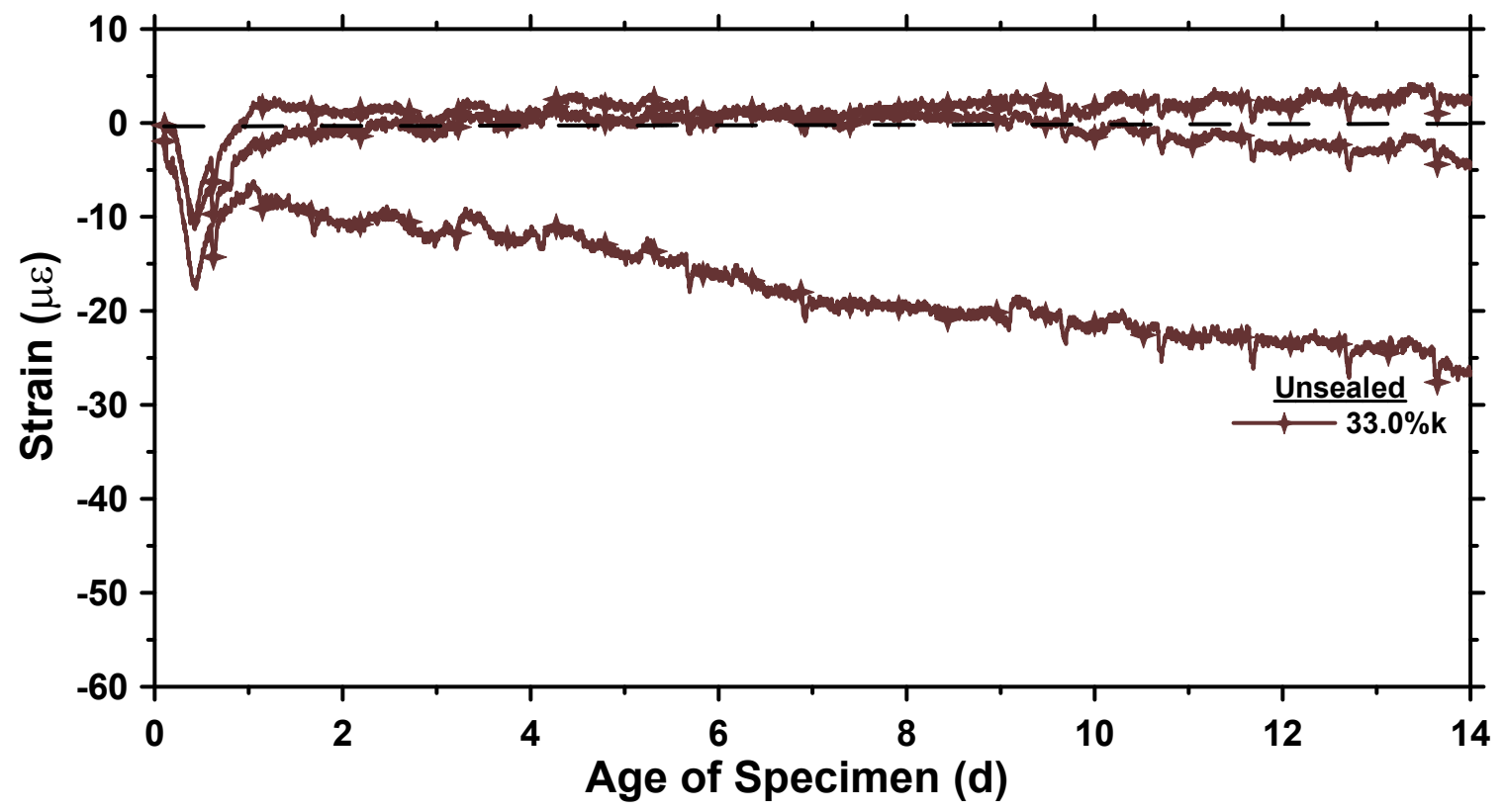

Figure C-15 - Restrained, unsealed shrinkage for 33.0\%k mixture 
Appendix D. MECHANICAL PROPERTIES DATA

Table D-1 - Mixture M-0 Mechanical Properties

\begin{tabular}{|c|c|c|c|c|c|c|}
\hline \multicolumn{8}{|c|}{ M-0 } \\
\hline & Compressive Strength & \multicolumn{2}{c|}{ Tensile Strength } & \multicolumn{2}{c|}{ Modulus } \\
\cline { 2 - 7 } Age (d) & Mpa & psi & Mpa & psi & GPa & ksi \\
\hline \hline 1 & 51.9 & 7522 & 4.08 & 591 & 27 & 3972 \\
\hline 3 & N/A & N/A & 4.80 & 696 & 26 & 3760 \\
\hline 7 & 66.3 & 9611 & 4.46 & 647 & 29 & 4233 \\
\hline 28 & 74.8 & 10844 & 4.22 & 612 & 31 & 4490 \\
\hline 90 & 77.8 & 11290 & 5.36 & 778 & 32 & 4586 \\
\hline
\end{tabular}

Table D-2 - Mixture M-11 Mechanical Properties

\begin{tabular}{|c|c|c|c|c|c|c|}
\hline \multicolumn{7}{|c|}{ M- 11} \\
\hline & \multicolumn{2}{|c|}{ Compressive Strength } & \multicolumn{2}{c|}{ Tensile Strength } & \multicolumn{2}{c|}{ Modulus } \\
\cline { 2 - 7 } Age (d) & Mpa & psi & Mpa & psi & GPa & ksi \\
\hline \hline 1 & 41.9 & 6079 & 4.26 & 617 & 23 & 3385 \\
\hline 3 & 52.6 & 7632 & 4.32 & 626 & 24 & 3455 \\
\hline 7 & 59.7 & 8662 & 4.04 & 586 & 28 & 4075 \\
\hline 28 & 70.6 & 10247 & 4.13 & 598 & 30 & 4332 \\
\hline 90 & 76.4 & 11081 & 5.38 & 781 & 31 & 4506 \\
\hline
\end{tabular}

Table D-3 - Mixture M-24 Mechanical Properties

\begin{tabular}{|c|c|c|c|c|c|c|}
\hline \multicolumn{7}{|c|}{ M-24 } \\
\hline & Compressive Strength & \multicolumn{2}{c|}{ Tensile Strength } & \multicolumn{2}{c|}{ Modulus } \\
\cline { 2 - 7 } Age (d) & Mpa & psi & Mpa & psi & GPa & ksi \\
\hline \hline 1 & 48.9 & 7091 & 4.39 & 637 & 22 & 3187 \\
\hline 3 & 54.0 & 7834 & 4.86 & 705 & 24 & 3499 \\
\hline 7 & 65.1 & 9442 & 3.43 & 498 & 22 & 3153 \\
\hline 28 & 73.6 & 10678 & 4.70 & 682 & 27 & 3908 \\
\hline 90 & 71.6 & 10389 & 4.57 & 663 & 29 & 4221 \\
\hline
\end{tabular}


Appendix E. DUAL RING DATA

Table E-1 - Mixture M-0 7-Day Mechanical Properties

\begin{tabular}{|c|c|c|c|c|c|c|}
\hline \multicolumn{9}{|c|}{ M-0 } \\
\hline \multirow{2}{*}{ Age (d) } & \multicolumn{2}{|c|}{ Compressive } & \multicolumn{2}{c|}{ Tensile } & \multicolumn{2}{c|}{ Modulus } \\
\hline \hline 1.01 & Mpa & psi & Mpa & psi & GPa & ksi \\
\hline 2.51 & 56.37 & 6603 & 4.05 & 588 & 27.14 & 3937 \\
\hline 5.18 & 57.75 & 8377 & 5.41 & 785 & 28.84 & 4183 \\
\hline 7.03 & 61.35 & 8899 & 6.57 & 801 & 29.24 & 4241 \\
\hline
\end{tabular}

Table E-2 - Mixture M-11 7-Day Mechanical Properties

\begin{tabular}{|c|c|c|c|c|c|c|}
\hline \multicolumn{9}{|c|}{ M- 11} \\
\hline \multirow{2}{*}{ Age (d) } & \multicolumn{2}{|c|}{ Compressive } & \multicolumn{2}{c|}{ Tensile } & \multicolumn{2}{c|}{ Modulus } \\
\hline \hline 1.01 & Mpa & psi & Mpa & psi & GPa & ksi \\
\hline 2.91 & 57.50 & 7096 & 3.71 & 539 & 25.53 & 3704 \\
\hline 5.18 & 59.95 & 8696 & 5.15 & 748 & 26.29 & 3813 \\
\hline 7.03 & 60.76 & 8813 & 6.18 & 709 & 27.41 & 3975 \\
\hline
\end{tabular}

Table E-3 - Mixture M-24 7-Day Mechanical Properties

\begin{tabular}{|c|c|c|c|c|c|c|}
\hline \multicolumn{9}{|c|}{ M-24 } \\
\hline \multirow{2}{*}{ Age (d) } & \multicolumn{2}{|c|}{ Compressive } & \multicolumn{2}{c|}{ Tensile } & \multicolumn{2}{c|}{ Modulus } \\
\cline { 2 - 7 } & Mpa & psi & Mpa & psi & GPa & ksi \\
\hline 1.01 & 45.31 & 6572 & 3.06 & 443 & 22.56 & 3273 \\
\hline 2.91 & 52.76 & 7654 & 4.33 & 629 & 23.89 & 3465 \\
\hline 5.18 & 56.88 & 8250 & 4.05 & 587 & 24.77 & 3592 \\
\hline 7.03 & 58.11 & 8429 & 5.36 & 778 & 25.61 & 3714 \\
\hline
\end{tabular}


Table E-4 - Mixture M-0 Dual Ring Data

\begin{tabular}{|c|c|c|c|c|c|}
\hline \multicolumn{7}{|c|}{ M-0 } \\
\hline $\begin{array}{c}\text { Age } \\
(\mathbf{h r})\end{array}$ & $\begin{array}{c}\mathbf{\Delta T} \\
\left({ }^{\circ} \mathbf{C}\right)\end{array}$ & $\begin{array}{c}\text { Cracking } \\
\text { Stress } \\
(\mathbf{M p a})\end{array}$ & $\begin{array}{c}\text { Isothermal } \\
\text { Stress } \\
\text { (Mpa) }\end{array}$ & $\begin{array}{c}\text { Reserve } \\
\text { Stress } \\
\text { (Mpa) }\end{array}$ & $\begin{array}{c}\text { Factor } \\
\text { Of } \\
\text { Safety }\end{array}$ \\
\hline \hline 33.5 & 20.1 & 4.6 & 2.5 & 2.1 & 0.46 \\
\hline 39.7 & 15.0 & 5.3 & 2.7 & 2.6 & 0.49 \\
\hline 43.4 & 11.9 & 5.3 & 2.9 & 2.4 & 0.45 \\
\hline 49.4 & 12.6 & 5.4 & 3.1 & 2.3 & 0.43 \\
\hline 72.6 & 10.6 & 6.3 & 3.6 & 2.7 & 0.43 \\
\hline 119.0 & 0.0 & 0.0 & 0.0 & 0.0 & 0.00 \\
\hline
\end{tabular}

Table E-5 - Mixture M-11 Dual Ring Data

\begin{tabular}{|c|c|c|c|c|c|}
\hline \multicolumn{7}{|c|}{ M-11 } \\
\hline $\begin{array}{c}\text { Age } \\
(\mathbf{h r})\end{array}$ & $\begin{array}{c}\mathbf{\Delta T} \\
\left({ }^{\circ} \mathbf{C}\right)\end{array}$ & $\begin{array}{c}\text { Cracking } \\
\text { Stress } \\
\text { (Mpa) }\end{array}$ & $\begin{array}{c}\text { Isothermal } \\
\text { Stress } \\
\text { (Mpa) }\end{array}$ & $\begin{array}{c}\text { Reserve } \\
\text { Stress } \\
\text { (Mpa) }\end{array}$ & $\begin{array}{c}\text { Factor } \\
\text { Of } \\
\text { Safety }\end{array}$ \\
\hline \hline 40.2 & 20.1 & 4.0 & 1.9 & 2.1 & 0.53 \\
\hline 50.5 & 19.0 & 5.5 & 2.1 & 3.4 & 0.62 \\
\hline 71.0 & 19.0 & 5.3 & 2.5 & 2.8 & 0.53 \\
\hline 90.8 & 14.0 & 5.7 & 2.8 & 2.9 & 0.51 \\
\hline 110.0 & 8.0 & 5.0 & 3.2 & 1.8 & 0.36 \\
\hline 124.0 & 1.0 & 3.8 & 3.5 & 0.3 & 0.08 \\
\hline
\end{tabular}

Table E-6 - Mixture M-24 Dual Ring Data

\begin{tabular}{|c|c|c|c|c|c|}
\hline \multicolumn{7}{|c|}{ M-24 } \\
\hline $\begin{array}{c}\text { Age } \\
(\mathbf{h r})\end{array}$ & $\begin{array}{c}\boldsymbol{\Delta} \mathbf{T} \\
\left({ }^{\circ} \mathbf{C}\right)\end{array}$ & $\begin{array}{c}\text { Cracking } \\
\text { Stress } \\
(\mathbf{M p a})\end{array}$ & $\begin{array}{c}\text { Isothermal } \\
\text { Stress } \\
\text { (Mpa) }\end{array}$ & $\begin{array}{c}\text { Reserve } \\
\text { Stress } \\
\text { (Mpa) }\end{array}$ & $\begin{array}{c}\text { Factor } \\
\text { Of } \\
\text { Safety }\end{array}$ \\
\hline \hline 96.0 & 30.0 & 0.0 & 0.0 & 0.0 & 0.00 \\
\hline 126.0 & 25.0 & 3.7 & 1.0 & 2.7 & 0.73 \\
\hline 197.0 & 8.0 & 2.7 & 1.7 & 1.0 & 0.37 \\
\hline
\end{tabular}


Appendix F. FREEZE THAW DATA

Table F-1 - Mixture M-0 Freeze Thaw Data

\begin{tabular}{|c|c|c|c|}
\hline \multicolumn{5}{|c|}{ Age } & & $\begin{array}{c}\text { \% } \\
\text { Mass } \\
\text { (d) }\end{array}$ & Cyles & Pc & Change \\
\hline \hline 34 & 0 & 100.00 & 0.00 \\
\hline 37 & 6 & 101.13 & 0.36 \\
\hline 45 & 22 & 102.59 & 0.43 \\
\hline 51 & 34 & 104.05 & 0.56 \\
\hline 62 & 56 & 104.77 & 0.60 \\
\hline 72 & 76 & 105.32 & 0.59 \\
\hline 79 & 90 & 105.74 & 0.62 \\
\hline 86 & 104 & 106.39 & 0.65 \\
\hline 99 & 130 & 106.17 & 0.68 \\
\hline 109 & 150 & 106.49 & 0.71 \\
\hline 125 & 182 & 107.36 & 0.74 \\
\hline 128 & 188 & 107.32 & 0.74 \\
\hline 132 & 196 & 108.03 & 0.75 \\
\hline 135 & 202 & 107.51 & 0.73 \\
\hline 139 & 210 & 107.76 & 0.75 \\
\hline 142 & 216 & 108.59 & 0.76 \\
\hline 149 & 230 & 108.34 & 0.78 \\
\hline 156 & 244 & 108.05 & 0.77 \\
\hline 160 & 252 & 108.66 & 0.78 \\
\hline 167 & 266 & 108.69 & 0.79 \\
\hline 174 & 280 & 108.69 & 0.81 \\
\hline 181 & 294 & 109.55 & 0.81 \\
\hline 208 & 348 & 109.25 & 0.79 \\
\hline 225 & 382 & 110.27 & 0.85 \\
\hline 237 & 406 & 108.76 & 0.78 \\
\hline 274 & 480 & 111.46 & 0.83 \\
\hline & & & \\
\hline \hline 25 & \\
\hline
\end{tabular}


Table F-2 - Mixture M-11 Sealed Cure Freeze Thaw Data

\begin{tabular}{|c|c|c|c|}
\hline \multicolumn{4}{|c|}{ M-11 Sealed Cure } \\
\hline $\begin{array}{c}\text { Age } \\
\text { (d) }\end{array}$ & Cycles & Pc & $\begin{array}{c}\text { \%o } \\
\text { Mass } \\
\text { Change }\end{array}$ \\
\hline 31 & 0 & 0.00 & 0.00 \\
\hline 33 & 4 & 100.00 & 0.10 \\
\hline 44 & 26 & 100.82 & 0.15 \\
\hline 54 & 46 & 101.19 & 0.16 \\
\hline 61 & 60 & 101.31 & 0.18 \\
\hline 68 & 74 & 101.85 & 0.19 \\
\hline 81 & 100 & 101.78 & 0.24 \\
\hline 91 & 120 & 102.04 & 0.26 \\
\hline 107 & 152 & 102.67 & 0.28 \\
\hline 110 & 158 & 102.77 & 0.30 \\
\hline 114 & 166 & 103.19 & 0.32 \\
\hline 117 & 172 & 102.84 & 0.30 \\
\hline 121 & 180 & 103.22 & 0.33 \\
\hline 124 & 186 & 103.86 & 0.31 \\
\hline 131 & 200 & 103.29 & 0.33 \\
\hline 138 & 214 & 103.41 & 0.35 \\
\hline 142 & 222 & 103.72 & 0.35 \\
\hline 149 & 236 & 103.76 & 0.37 \\
\hline 156 & 250 & 103.95 & 0.37 \\
\hline 163 & 264 & 104.41 & 0.39 \\
\hline 190 & 318 & 104.14 & 0.40 \\
\hline 207 & 352 & 104.95 & 0.43 \\
\hline 219 & 376 & 103.93 & 0.40 \\
\hline 256 & 450 & 105.86 & 0.46 \\
\hline
\end{tabular}


Table F-3 - Mixture M-24 Sealed Cure Freeze Thaw Data

\begin{tabular}{|c|c|c|c|}
\hline \multicolumn{4}{|c|}{ M-24 Sealed Cure } \\
\hline $\begin{array}{c}\text { Age } \\
\text { (d) }\end{array}$ & Cycles & Pc & $\begin{array}{c}\text { \% } \\
\text { Mass } \\
\text { Change }\end{array}$ \\
\hline 31 & 0 & 0.00 & 0.00 \\
\hline 33 & 4 & 100.00 & 0.08 \\
\hline 44 & 26 & 100.71 & 0.12 \\
\hline 54 & 46 & 101.05 & 0.11 \\
\hline 62 & 62 & 101.05 & 0.13 \\
\hline 68 & 74 & 101.43 & 0.15 \\
\hline 81 & 100 & 101.02 & 0.17 \\
\hline 91 & 120 & 101.02 & 0.19 \\
\hline 107 & 152 & 101.64 & 0.23 \\
\hline 110 & 158 & 101.76 & 0.24 \\
\hline 114 & 166 & 102.03 & 0.25 \\
\hline 117 & 172 & 101.64 & 0.24 \\
\hline 121 & 180 & 102.12 & 0.26 \\
\hline 124 & 186 & 102.70 & 0.25 \\
\hline 131 & 200 & 102.41 & 0.28 \\
\hline 138 & 214 & 102.38 & 0.27 \\
\hline 142 & 222 & 102.53 & 0.28 \\
\hline 149 & 236 & 102.48 & 0.29 \\
\hline 156 & 250 & 102.89 & 0.31 \\
\hline 163 & 264 & 103.11 & 0.31 \\
\hline 190 & 318 & 103.06 & 0.33 \\
\hline 207 & 352 & 103.59 & 0.35 \\
\hline 219 & 376 & 102.48 & 0.31 \\
\hline 256 & 450 & 104.58 & 0.39 \\
\hline
\end{tabular}


Table G-1 - Large Scale Restraining Slab 1 Unrestrained Shrinkage

\begin{tabular}{|c|c|}
\hline \multicolumn{2}{|c|}{ Restraining Slab 1 } \\
\hline Time $(\mathbf{d})$ & Strain $(\boldsymbol{\mu} \boldsymbol{\varepsilon})$ \\
\hline 0.00 & 0.00 \\
\hline 7.08 & -51.58 \\
\hline 12.13 & -116.58 \\
\hline 22.19 & -171.58 \\
\hline 29.97 & -161.58 \\
\hline 40.92 & -196.58 \\
\hline
\end{tabular}

Table G-2 - Large Scale Restraining Slab 4 Unrestrained Shrinkage

\begin{tabular}{|c|c|}
\hline \multicolumn{2}{|c|}{ Restraining Slab 4 } \\
\hline Time (d) & Strain $(\boldsymbol{\mu} \boldsymbol{\varepsilon})$ \\
\hline 0.00 & 0.00 \\
\hline 5.08 & -10.67 \\
\hline 10.13 & -37.34 \\
\hline 20.19 & -54.01 \\
\hline 27.97 & -54.01 \\
\hline 38.92 & -84.01 \\
\hline
\end{tabular}

Table G-3 - Large Scale Mixture C-0 Unrestrained Shrinkage

\begin{tabular}{|c|c|}
\hline \multicolumn{2}{|c|}{ C- 0} \\
\hline Time (d) & Strain $(\boldsymbol{\mu} \boldsymbol{\varepsilon})$ \\
\hline 0.51 & 0.00 \\
\hline 1.01 & -40.00 \\
\hline 2.20 & -80.00 \\
\hline 2.98 & -120.00 \\
\hline 4.00 & -180.00 \\
\hline 6.13 & -240.00 \\
\hline 6.92 & -280.00 \\
\hline 8.13 & -280.00 \\
\hline 9.92 & -320.00 \\
\hline 11.14 & -340.00 \\
\hline 12.91 & -360.00 \\
\hline
\end{tabular}


Table G-4 - Large Scale Mixture C-21 Unrestrained Shrinkage

\begin{tabular}{|c|c|}
\hline \multicolumn{2}{|c|}{ C- 21} \\
\hline Time (d) & Strain $(\boldsymbol{\mu} \boldsymbol{\varepsilon})$ \\
\hline 1.01 & 0.00 \\
\hline 2.20 & 53.33 \\
\hline 2.94 & 70.00 \\
\hline 5.06 & 16.67 \\
\hline 5.85 & -50.00 \\
\hline 7.06 & -83.33 \\
\hline 8.85 & -146.67 \\
\hline 10.07 & -153.33 \\
\hline 12.00 & -173.33 \\
\hline 12.86 & -203.33 \\
\hline 23.79 & -330.00 \\
\hline
\end{tabular}

